\title{
98. Deutscher Röntgenkongress
}

8. gemeinsamer Röntgenkongress der DRG und ÖRG

Datum/Ort:

24.-27. Mai 2017, Leipzig
Kongresspräsidenten:

Prof. Dr. Ernst J. Rummeny, München

o. Univ.-Prof. Dr. Werner Jaschke, Innsbruck
Vortrag (Wissenschaft)

S2 Bildverarbeitung/IT/Software/Gerätetechnik/

S9 Experimentelle Radiologie

S15 Gastro- und Abdominaldiagnostik

S22 Herzdiagnostik/Gefäßdiagnostik

S35 Interventionelle Radiologie

S49 Kinderradiologie

S53 Kontrastmittel

S55 Kopf/Hals-Diagnostik

S58 Mammadiagnostik

S61 Molekulare Bildgebung

S65 Muskuloskelettale Radiologie

S72 Neuroradiologie

S83 Notfalldiagnostik/Intensivmedizin

S86 Onkologische Bildgebung/Onkologie

S92 Strahlenschutz

S95 Thoraxradiologie

S101 Uroradiologie/Urogenitaldiagnostik

Poster (Wissenschaft)

S107 Bildverarbeitung/IT/Software/Gerätetechnik/ Qualitätsmanagement

S108 Gastro- und Abdominaldiagnostik

S109 Herzdiagnostik/Gefäßdiagnostik
S109 Interventionelle Radiologie

S111 Kinderradiologie

S111 Kopf/Hals-Diagnostik

S111 Molekulare Bildgebung

S112 Muskuloskelettale Radiologie

S112 Neuroradiologie

S113 Thoraxradiologie

S113 Ultraschall

Poster (Fortbildung)

S115 Ganzkörperdiagnostik/Kohortenstudien

S116 Interventionelle Radiologie

S116 Kopf/Hals-Diagnostik

S116 Muskuloskelettale Radiologie

S117 Notfalldiagnostik/Intensivmedizin

S117 Uroradiologie/Urogenitaldiagnostik

Nachtrag Poster-Ausstellung (Wissenschaft)

S118 Bildverarbeitung/IT/Software/Gerätetechnik/ Qualitätsmanagement

S119 Experimentelle Radiologie

S121 Ganzkörperdiagnostik/Kohortenstudien

S121 Gastro- und Abdominaldiagnostik

S121 Herzdiagnostik/Gefäßdiagnostik

S123 Interventionelle Radiologie 


\section{S124 Mammadiagnostik}

S124 Molekulare Bildgebung

\section{S125 Muskuloskelettale Radiologie}

S125 Neuroradiologie

S126 Onkologische Bildgebung/Onkologie

S127 Ultraschall

S127 Uroradiologie/Urogenitaldiagnostik

S128 Ausbildung und Beruf

Nachtrag Poster-Ausstellung (Fortbildung)

S129 Gastro- und Abdominaldiagnostik

S130 Herzdiagnostik/Gefäßdiagnostik

S130 Interventionelle Radiologie

S131 Kinderradiologie

S131 Muskuloskelettale Radiologie

S132 Neuroradiologie

S133 Thoraxradiologie

S133 Notfalldiagnostik/Intensivmedizin

\section{S134 Namenverzeichnis}

\section{Vortrag (Wissenschaft)}

Bildverarbeitung/IT/Software/Gerätetechnik/

Qualitätsmanagement

WISS 207.2 Echtzeit-Monitoring von Wartezeit, Terminplan und Befundfreigabezeit mithilfe eines RISbasierten Dashboards

Autoren Bassing $T^{1}$, Krämer $P^{2}$, Nagel $A^{3}$, Cavallaro $A^{3}$

Institut 1 Universitätsklinikum Erlangen, Radiologisches Institut, Erlangen;

2 Siemens Healthcare $\mathrm{GmbH}$, Transformation and Advisory, Erlangen;

3 Universitätsklinikum Erlangen, Radiologisches Institut

DOI 10.1055/s-0037-1600165

Zielsetzung Für die Überwachung der Patientenwartezeit und des Terminplans wurde eine Softwareanwendung zur täglichen Nutzung in einer radiologischen Einrichtung erstellt. Ziel ist die Schaffung von Transparenz in radiologischen Arbeitsabläufen. Die Offenlegung und anschließende gezielte Beseitigung eventueller Problemfelder wird so ermöglicht. Relevante Informationen müssen dazu quasi in Echtzeit verfügbar und über mobile Endgeräte abrufbar sein.

Material und Methodik Ein Business Intelligence (BI) Dashboard zu interaktiven Datenanalyse wurde in QlikSense (QlikTech, USA) erstellt. Es erlaubt auf einfache Weise die Auswahl relevanter Dimensionen in den dargestellten Informationen sowie die Anzeige gewünschter Kennzahlen. Eine Analyse auf mobilen Endgeräten wird durch eine automatische Skalierung auf die jeweilige Anzeigeauflösung ermöglicht. Als Datenquelle wurde das RIS genutzt, da es alle Daten für eine umfassende Auswertung enthält.

Ergebnisse In Kooperation mit dem radiologischen Institut wurde als Proof of Concept ein individuell angepasstes Dashboard erstellt. Es stellt Informationen zum Erfüllungsgrad des Terminplans, den Patientenwartezeiten und den offenen Befunden dar. Die verantwortlichen Radiologen sind so in der Lage, auftretende Verzögerungen auszumachen. Die Möglichkeit lange Befundfreigabezeiten nach verschiedenen Dimensionen zu filtern, hilft deren Ursache offenzulegen. Ergänzt werden diese echtzeitnahen Ansichten durch weitere, prozessorientierte Darstellungen für die Modalitäten CT und MR u.a. mit Terminvergabe-, Warte-, Untersuchungs- und Befundfreigabezeit. Durch die Gesamtheit der dargestellten aktuellen Informationen besteht jederzeit die Möglichkeit, alle Arbeitsabläufe transparent darzustellen und zu quantifizieren. Schlussfolgerungen Mit überschaubarem Aufwand lässt sich eine Bl-Anwendung für tägliche Analysen implementieren. Grenzen setzen vor allem die Leistungsfähigkeit des IT Systems hinsichtlich Aktualität und die schwankende Qualität der RIS Daten hinsichtlich der Exaktheit bestimmter Metriken.

WISS 203.3 Dual-Layer Spectral Computed Tomografy: Reduction of metallic artefacts from posterior spinal fusions using virtual monoenergetic imaging

Authors Dangelmaier $]^{1}$, Schwaiger $B^{2}$, Renz $M^{2}$, Sauter $A^{2}$, Riederer $I^{2}$, Münzel $D^{2}$, Braren $R^{3}$, Fingerle $A^{2}$, Rummeny $E^{2}$, Noël $P^{3}$ Institute 1 Klinikum rechts der Isar der Technischen Universität München, Institut für diagnostische und interventionelle Radiologie, München;

2 Klinikum Rechts der Isar der TU München, Diagnostische und Interventionelle Radiologie, München; 3 Klinikum Rechts der Isar der TU München, Diagnostische und Interventionelle Radiologie DOI 10.1055/s-0037-1600166

Zielsetzung To evaluate the clinical potential of virtual monoenergetic images (monoE) generated with a dual-layer spectral Computed Tomografy (CT) system and determine the optimal settings for reduction of metal artifacts from posterior spinal fusions of diverse extent and different spine levels. 
Material und Methodik Twenty patients with posterior spinal fusion who underwent a spectral CT scan (IQon Spectral CT, Philips Healthcare, USA) for various clinical indications were included into this study. Two independent readers evaluated axial $0.9 \mathrm{~mm}$ slides with a window center of $50 \mathrm{HU}$ and width of $380 \mathrm{HU}$. Image quality of the conventional scan was compared with monoE images at 40,60, 80, 100, 120, 140, 160, 180 and $200 \mathrm{keV}$. A four point Likert-scale was used to document subjective impression of diagnostic image quality and the Hounsfield unit of the area with the most pronounced streak artefact was documented for every keV-setting. Additionally, the optimal monoE (OPTkeV) setting for all patients was determined.

Ergebnisse Quantitative and qualitative analysis showed statistically significant artefact reduction for higher monoE levels and especially for the OPTkeV compared to conventional images. Optimal keV-settings ranged from 120 to $140 \mathrm{keV}$. Our results reveal high inter-reader agreement for quantitative and qualitative evaluations.

Schlussfolgerungen Virtual monoenergetic images of higher energy levels $(120-140 \mathrm{keV})$ provide significant reduction of metallic artefacts from posterior spinal fusions compared to conventional scans. Compared to previous results, with dual-layer spectral CT our findings can be applied to the whole field-of-view and to all patients without any compromises.

\section{WISS 203.5 Systematische Evaluation virtuell monoenergetischer Bilder aus Spectral Detector CT Daten in der Bildgebung des Abdomens in venöser Kontrastmittelphase: erste in-vivo Ergebnisse}

Autoren Dörner $\rfloor^{1}$, Byrtus $\rfloor^{1}$, Hauger $\mathrm{M}^{1}$, Große Hokamp $\mathrm{N}^{1}$, Hubois $\mathrm{C}^{1}$, Hickethier $T^{1}$, Wybranski $C^{1}$, Maintz $D^{1}$, Haneder $S^{1}$

Institut 1 Uniklinik Köln, Institut für Diagnostische und Interventionelle Radiologie, Köln

DOI 10.1055/s-0037-1600167

Zielsetzung Dual Energy CT ermöglicht mittels niedrigenergetischer virtuell monoenergetischer (vMEl) Bildgebung die Kontrastanhebung lod-haltiger Strukturen. Dies ist insbesondere für die Beurteilung arterieller Strukturen in venöser Kontrastmittelphase (KM) interessant. Bisherige Dual Energy-Techniken beruhen auf Modulationen an der Röntgenröhre (z.B. Dual Source oder KV-Switching). Seit kurzem ist eine Detektor-basierte Lösung (Spectral Detector $\mathrm{CT}$; SDCT) verfügbar. In abdominellen SDCT-Datensätzen in venöser KMPhase sollen deshalb arterielle Gefäße im Hinblick auf Bildqualitätskriterien evaluiert werden.

Material und Methodik Die SDCT-Datensätze (IQon, Philips; 120KV) von 55 Patienten mit einer venösen KM-Phase des Abdomens wurden mittels dedizierter Software (ISP Spectral, Philips) in folgenden virtuellen keV-Stufen rekonstruiert: 40 bis 120 (10er Schritte), 160 und 200. In jeder Rekonstruktion wurden mittels etablierter Bildqualitätskriterien (SNR und CNR) definierte Gefäßprovinzen (Aorta supra- und infrarenal, A. mesenterica sup. (AMS), Truncus coeliacus, A. hepatica com., A. lienalis) untersucht und intra-individuell mit den konventionellen, polychromatischen Standard-Bildern verglichen.

Ergebnisse Im Vergleich zur konventionellen Bildgebung zeigte sich in allen untersuchten Gefäßprovinzen ein signifikanter Anstieg des SNR für 40 und 50 $\mathrm{KeV}(\mathrm{p}<0.02)$, in der Aorta und der A. lienalis auch für $60 \mathrm{keV}(\mathrm{p}<0.002)$. Signifikant niedrigere SNR zeigten sich für keV ab 120 (bis auf Truncus coelicus und AMS). Ein signifikant höheres CNR zeigte sich in alle Gefäßprovinzen für 40, 50 und $60 \mathrm{keV}(p<0.001)$ mit signifikant niedrigeren Werten ab $110 \mathrm{keV}$ $(\mathrm{p}<0.04)$ bis auf $\mathrm{A}$. lienalis (ab $120 \mathrm{keV})$.

Schlussfolgerungen vMEI bei niedriger keV Stufe aus SDCT Daten in venöser KM-Phase des Abdomens erreichen signifikant höhere Bildqualitätskriterien als die konventionelle Bildgebung und machen niedrige keV Stufen nun diagnostisch nutzbar.

\section{WISS 203.4 Thorako-abdominelles Staging mit einem neuen Dual-Layer CT: Intra-individueller Vergleich der Strahlendosis und der Bildqualität mit 64- und 128-Zeilen Single-Energy CTs}

Autoren Haneder $S^{1}$, Heneweer $C^{1}$, Siedek $F^{1}$ Große Hokamp $N^{1}$, Maintz $D^{1}$, Wybranski $\mathrm{C}^{1}$

Institut 1 Uniklinik Köln, Institut für Diagnostische und Interventionelle Radiologie, Köln

DOI 10.1055/s-0037-1600168

Zielsetzung Seit wenigen Monaten ist ein neuer Dual-Layer Scanner (Spektral Detektor CTs [SDCT]) mit einer Detektor-basierten Dual-Energy Technik klinisch verfügbar. Ziel dieser Studie ist der intra-individuelle Vergleich der Strahlendosis, der objektiven und subjektiven Bildqualität von konventionellen, polychromatischen Bildern dreier CTs desselben Herstellers (68-Zeilen SDCT, 64Zeilen [CT64] und 128-Zeilen [CT128] CT) unter Verwendung von filteredback-projection (FBP) und zweier verschiedener iterativen Rekonstruktionsalgorithmen (IR1, IR2).

Material und Methodik 73 Patienten (32w,41 m) mit einer portal-venösen, klinischen, thorako-abdominellen Staging-SDCT-Untersuchung und einer Voruntersuchung an einem CT64 oder CT128 wurden eingeschlossen. Vier Gruppen mit jeweils 25 Patienten (CT128 IR1, CT128 IR2, CT128 FBP, CT64 FBP) wurden von zwei Radiologen intra-individuell mit dem SDCT in Hinblick auf objektive (Kontrast-zu-Rausch-Verhältnis [CNR] in Leber, Pankreas, Nieren und Aorta) und subjektive (Bildqualität auf einer 5-Punkte-Skala) Bildqualitäts-Parameter, sowie CT dose index (CTDIvol), Dosislängenprodukt und Effektive Dosis normiert auf $68 \mathrm{~cm}$ (DLP68 and ED 68) miteinander verglichen.

Ergebnisse Das SDCT erzielte in allen anatomischen Lokalisationen gegenüber den anderen CTs, unabhängig vom Rekonstruktionsalgorithmus, im konventionellen, polychromatischen Datensatz signifikant höhere CNR Werte $(25,1 \%$ $36,7 \% ; p<0,0001)$ und eine überlegene, hervorragende, subjektive Bildqualiät $(p<0,0001)$ mit einem exzellenten Inter-Reader-Agreement $(\kappa \geq 0,801)$. Zusätzlich war für das SDCT das CTDIvol, DLP68 und ED68 signifikant geringer gegenüber dem CT128, tendenziell jedoch nach Bonferroni Korrektur nicht mehr statistisch signifikant gegenüber dem CT64.

Schlussfolgerungen Das SDCT erzielte eine niedrigere Strahlendosis und eine bessere Bildqualität im konventionellen, polychromatischen Datensatz als ein CT64 und ein CT128 mit verschiedenen Rekonstruktionsalgorithmen und ermöglicht die Anwendung der Dual-Energy Technik in allen CT Scans.

\section{WISS 207.5 Präoperative Simulation von operativen Implantationen mittels Open-Source 3D-Rendering und CAD-Software}

Autoren Haubold $\mathrm{J}^{1}$, Nensa $\mathrm{F}^{1}$, Forsting $\mathrm{M}^{1}$, Ringelstein $\mathrm{A}^{1}$

Institut 1 Uniklinik Essen, Institut für Diagnostische und Interventionelle Radiologie und Neuroradiologie, Essen

DOI 10.1055/s-0037-1600169

Zielsetzung Mit den routinemäßig erfassten 3D- und 4D-Datensätzen (zeitliche Auflösung) in der Computertomografie sind wir in der Lage hochaufgelöste 3D-Modelle der Patientenanatomie zu erstellen. Heute werden diese 3D-Modelle hauptsächlich verwendet, um eine Pathologie zu visualisieren. Die präoperative Planung selbst basiert meist auf Messungen in 2D-Datensätzen, in welcher die Winkel und die daraus resultierende Verzerrung der gemessenen Abstände meist ignoriert werden. Wir haben eine Open-SourceMethode entwickelt, um diese 3D-Datensätze für eine virtuelle Simulation der Implantationen zu verwenden, um die Genauigkeit der präoperativen Planung zu verbessern.

Material und Methodik Wir haben 3D-Modelle von Osteosynthese-Materialien mittels Dual-Source-Computertomografie in einem Somatom Definition Force (Siemens) CT-Scanner mit einer Raumauflösung von 0,125 mm akquiriert. 3D-Modelle der Patientenanatomie wurden mit präoperativen CT-Scans erstellt. Die 3D-Modelle der Patientenanatomie und der Osteosynthese Mate- 
rialien wurden mit 3D-Slicer (www.slicer.org) gerendert und als Stereolithografie-Dateien exportiert. Um eine Implantation zu simulieren, importierten wir diese 3D-Modelle in Open-Source Computer-Aided Design-Software (FreeCAD).

Ergebnisse Durch die Zusammenführung der 3D-Modelle der verschiedenen Implantate mit der Patientenanatomie konnten wir das "ideale" Implantat für jedes Individuum evaluieren. Darüber hinaus konnten wir mögliche Hindernisse eines chirurgischen Eingriffs beurteilen und untersuchen, in welchem Winkel die Implantation erfolgen sollte.

Schlussfolgerungen Die virtuelle Simulation von operativen Verfahren mittels Open-Source-CAD-Software bietet eine kostengünstige Möglichkeit die Genauigkeit der präoperativen Planung zu verbessern. Ob diese Methode die perioperative Morbidität und Mortalität senken kann, wird aktuell in laufenden Studien untersucht.

\section{WISS 207.8 Odontologische Personenidentifizierung mittels merkmalsbasierter Bildanalyse von OPG- Aufnahmen}

Autoren Heinrich $A^{1}$, Wendt $S^{1}$, Teichgräber $U^{1}$, Güttler $F^{1}$

Institut 1 Universitätsklinikum Jena, Institut für Diagnostische und

Interventionelle Radiologie, Jena

DOI 10.1055/s-0037-1600170

Zielsetzung Der Fingerabdruck ist ein solides Mittel um Personen eindeutig zu identifizieren, jedoch sind nicht immer Vergleichswerte vorhanden. Dagegen wird die Orthopantomografie (OPG) routinemäßig beim Zahnarzt durchgeführt. Das Ziel war die Implementierung und Evaluation einer merkmalsbasierten Bildanalyse zum Vergleich von OPG-Aufnahmen zur odontologischen Personenidentifizierung.

Material und Methodik Die Studie umfasste 45.000 OPG-Aufnahmen von 24.500 Patienten in einem zeitlichen Rahmen von ca. 10 Jahren. Die OPGAufnahmen wurden mit Matlab R2014b, sowie den Toolboxen image processing und computer vision system verarbeitet. Um Zahnmerkmale, wie Füllungen und Implantate, zu extrahieren und die Übereinstimmung zu analysieren wurde der Speeded Up Robust Features Algorithmus angewendet. Die Anzahl der Verknüpfungen zwischen zwei verschiedenen OPG-Aufnahmen stellt dabei ein Maß für die Übereinstimmung dar. Mittels dieser Verknüpfungen erfolgte anhand der neusten OPG-Aufnahme eines Patienten $(n=40)$ eine Identifizierung durch ein Abgleich mit dem Datenpool.

Ergebnisse Die eindeutige Identifizierung gelang bei 33 von 40 Patienten (83\%). Für verschiedene Aufnahmen desselben Patienten waren, im Vergleich zu den anderen Patienten, die Anzahl der Verknüpfungen signifikant höher (Mittelwert 40,39, Median 21,25). Die Zeit zwischen den Aufnahmen und das verwendete Aufnahmegerät waren für das Resultat unerheblich, so gelang eine eindeutige Identifizierung auch bei einer Differenz zwischen Such- und Datenbank-Bild von über 8 Jahren. Die Anzahl der Verknüpfungen zwischen OPG-Aufnahmen verschiedener Patienten war durchgehend niedrig (Mittelwert und Median 4, Maximum 12).

Schlussfolgerungen Bei Personen mit Zahnersatz ist eine odontologische Personenidentifizierung durch eine merkmalsbasierte Bildanalyse von OPG-Aufnahmen möglich. Die Zuverlässigkeit dieses Verfahrens ist auch bei großen Datenmengen gegeben.

\section{WISS 210.3 Implementierung sowie erste Ergebnisse einer automatischen größenkorrigierten Dosiserfassung bei CT-Untersuchungen mittels SSDE in der klinischen Routine eines Krankenhauses der Maximalversorgung}

Autoren Heinzler $N^{1}$, Kröpil $P^{1}$, Thomas $C^{1}$, Aissa $J^{1}$, Bethge $O^{1}$, Elisabeth $A^{1}$, Antoch $\mathrm{G}^{1}$, Boos $\mathrm{J}^{1}$

Institut 1 Universitätsklinikum Düsseldorf, Institut für diagnostische und

interventionelle Radiologie, Düsseldorf

DOI 10.1055/s-0037-1600171

Zielsetzung Implementierung einer automatischen Erfassung von SSDE mittels des wasseräquivalenten Durchmessers (Dw) aus allen Schichten des CTScanvolumens zur Dosiserfassung bei CT-Scans in der klinischen Routine und Vergleich der initialen Ergebnisse mit den durch die CT-Scanner ermittelten Dosiswerten (CTDIvol).

Material und Methodik 1476 konsekutive CT-Scans wurden analysiert (905 abdominelle/ 517 thorakale, med. Patientenalter $62 \pm 15$ Jahre, $850 \mathrm{~m} / 626 \mathrm{w}$, BMI 25,5 $\pm 5 \mathrm{~kg} / \mathrm{m} 2$ ). Zur Analyse wurden die Daten vom CT auf einen Server übertragen und mittels eines selbstentwickelten Matlab-Algorithmus (The Mathworks, Natick, MA) eine automatisierte Segmentation sowie Berechnung des Dw aus jeder Schicht des Scanvolumens durchgeführt. Aus den gemittelten Dw-Werten wurde anschließend die SSDE berechnet. Die Effektivität der Segmentierung wurde durch 2 unabhängige Radiologen in repräsentativen Schichten aus 100 CT-Scans überprüft und mittels einer 5-Punkt-Skala bewertet ( 1 = Fehlsegmentation, 5 = perfekte Segmentation). Die SSDE-Werte wurden mit den unkorrigierten CTDIvol-Werten verglichen.

Ergebnisse Die automatische Ermittlung der SSDE war in allen 1476 CT-Scans erfolgreich. Der Segmentationsalgorithmus zeigte eine exzellente Genauigkeit, Fehlsegmentationen traten nicht auf (mediane Bewertung 5, IQR 4-5). Im Vergleich der SSDE mit den durch die CT-Scanner bereitgestellten CTDIvolWerten zeigten sich signifikante Unterschiede der Dosiswerte: Die SSDE waren sowohl bei thorakalen $(5,8 \pm 3,6 \mathrm{mGy}$ vs. 5,4 $\pm 3,2 \mathrm{mGy})$ als auch bei abdominellen Untersuchungen (10,7 $\pm 6,1 \mathrm{mGy}$ vs. $8,8 \pm 5,6 \mathrm{mGy})$ höher als der unkorrigierte CTDIvol ( $p<0,001)$.

Schlussfolgerungen Die automatisierte Berechnung von größenkorrigierten Dosisdaten (SSDE) aus gemittelten Dw-Werten des gesamten CT-Scanvolumens ist in der klinischen Routine technisch umsetzbar und erlaubt eine automatisierte Erfassung von größenkorrigierten Dosisdaten. Die initialen Ergebnisse zeigen signifikant höhere SSDE in abdominellen und thorakalen CTUntersuchungen im Vergleich zu den unkorrigierten CTDIvol-Werten.

\section{SP 105.6 Einfluss von Faltungskern und Schwellenwert auf die CT-basierte automatisierte Quantifizierung des Lungenemphysems}

Autoren Jacob $F^{1}$, Drömann $D^{2}$, Erhardt $J^{3}$, Vogt $F^{4}$, Goltz $J^{4}$, Oechtering $T^{4}$, Anton $\mathrm{S}^{4}$, Barkhausen $\mathrm{J}^{4}$, Frydrychowicz $\mathrm{A}^{4}$

Institut 1 UKSH, Radiologie und Nuklearmedizin, Lübeck; 2 UKSH Campus

Lübeck, Pulmonologie, Lübeck; 3 Universität zu Lübeck, Institut für

Medizinische Informatik, Lübeck; 4 UKSH Campus Lübeck, Radiologie und Nuklearmedizin, Lübeck

DOI 10.1055/s-0037-1600172

Zielsetzung Unterschiedliche Bildrekonstruktionsalgorithmen schränken die retrospektive, quantitative Auswertung von CT-Datensätzen ein. Diese Arbeit analysiert die Abhängigkeit des Emphysemindex (EI) vom Faltungskern (FK) und Emphysemschwellenwert (LAV) bei Patienten mit Lungenemphysem.

Material und Methodik 50 CT-Datensätze (128-Zeilen MSCT, Siemens, 1 mm Schichtdicke) von Patienten mit bekanntem Lungenemphysem wurden mit den Referenzeinstellungen (FK B20) einer kommerziellen Software (Pulmo3D, SyngoVia, Siemens) analysiert. Emphysem wurde basierend auf der Standardabweichung gesunder Patienten als El > 0,64\% (LAV -950 HU) bzW. El >0,21\% 
(LAV -970 HU) definiert. Die Ergebnisse wurden mit Auswertungen basierend auf CT Datensätzen mit mittelweichem (B30), mittlerem (B50) und hartem Faltungskern (B80) verglichen. Zur statistischen Analyse wurden t-Test und Bland-Altman Analyse durchgeführt.

Ergebnisse Unter Verwendung der Referenzwerte wurden 41/50 (EI=10,4 $\pm 9,9 \%$ bei B20/-950) beziehungsweise 30/50 Patienten ( $E \mathrm{l}=5,1 \pm 6,3 \%$ bei B20/-970) korrekt erkannt. Mit der Wahl härterer Faltungskerne stieg der Emphysemindex und damit die Zahl der korrekt erkannten Patienten sowohl bei LAV $-950 \mathrm{HU}$ (B30: $\mathrm{El}=11,1 \pm 10,0 \%, \mathrm{n}=43$; $\mathrm{B} 50$ : $\mathrm{El}=18,1 \pm 9,1 \%, \mathrm{n}=50$; B80: $\mathrm{El}=29,2 \pm 6,2 \%, \mathrm{n}=50$ ) als auch bei LAV $-970 \mathrm{HU}$ (B30: $\mathrm{El}=5,6 \pm 6,4 \%$, $n=31 ; B 50: E l=10,9 \pm 6,7 \%, n=49$ und $B 80: E l=22,1 \pm 5,3 \%, n=50$ ), alle $p<0,05$ gegenüber der Referenzmethode. Die Bland-Altman Analyse bestätigte die höheren Werte für harte Faltungskerne.

Schlussfolgerungen Die Faltungskerne B20 und B30 sind im genutzten Setting für die automatisierte Emphysemanalyse mit beiden Schwellwerten austauschbar. Härtere Faltungskerne führen zu höheren Emphysemindices und möglicherweise auch falsch positiven Befunden. Daher sind eine Korrelation mit der Klinik und der Bodyplethysmografie sowie die Auswertung lungengesunder Patienten notwendig, um diese Diskrepanz zu entschlüsseln.

\section{WISS 203.2 Vergleich von verschiedenen Metallartefaktalgorithmen in Single- und Dual-Source CT Scannern in einem Wirbelkörpermodell}

\author{
Autoren Jagoda $\mathrm{P}^{1}$, Schmitz $\mathrm{D}^{1}$, Bücker $\mathrm{A}^{1}$, Minko $\mathrm{P}^{1}$ \\ Institut 1 Universitätsklinik des Saarlandes, Klinik für Diagnostische und \\ Interventionelle Radiologie, Homburg \\ DOI 10.1055/s-0037-1600173
}

Zielsetzung Vergleich der Algorithmen OMAR und IMAR (Single-Source) sowie der monoenergetischen Nachverarbeitung (MN bei Dual Source) bezüglich ihres Potenzial zur Metallartefaktreduktion und detailgetreuen Darstellung des Gewebes.

Material und Methodik Ein Wirbelkörper vom Rind wurde mit 16 artifiziellen Osteolysen und zwei Osteosyntheseschrauben von $20 \times 4,5 \mathrm{~mm}$ präpariert. Anschließend wurde der Wirbelkörper an zwei Single-Source Scannern (Brilliance 64, Phillips und Somatom AS64, Siemens) bei 80, 100, 120 und $140 \mathrm{kV}$ mit jeweils $150 \mathrm{mAs}$ untersucht. Die Akquisition des Wirbelkörperphantoms am Dual-Source CT (Force, Siemens) erfolgte bei einer Röhrenspannung von 100/150 kV, wobei der Röhrenstrom mit 31, 64, 110 und 168 mAs jeweils so angepasst wurde, dass die Dosis mit der der Single-Source Untersuchungen übereinstimmte. Die Bilddatensätze wurden mit OMAR, IMAR sowie bei dem Dual-Source CT mit einer monoenergetischen Nachverarbeitung rekonstruiert. Drei Radiologen werteten die Datensätze verblindet mit einer 4-Punkte-Skala bezüglich des Ausmaßes der Metallartefakte, der Darstellung von Knochenstruktur, Weichteilgewebe und der Osteosyntheseschrauben aus. Der Schraubendiameter wurde für jede Rekonstruktion ermittelt. Der Kendall Konkordanzkoeffizient (w) und der Intraklassenkorrelationskoeffizient (ICC) wurden zur statistischen Evaluation verwendet.

Ergebnisse MN zeigte mit 1,0 \pm 0 (mean \pm SD) die beste Metallartefaktreduktion, 3,1 $\pm 0,7$ iMAR, 3,7 $\pm 0,5$ O-MAR. MN rekonstruierte die Knochenstruktur in unmittelbarer Nähe zu den Osteosyntheseschrauben am realistischsten $(1,4$ $\pm 0,5 \mathrm{MN}, 3,7 \pm 0,5$ iMAR, $4,0 \pm 0$ OMAR; $w=0,43$ ) und bot die beste Darstellung des Weichteilgewebes $(1,3 \pm 0,5 \mathrm{MN}, 3,2 \pm 0,6$ iMAR, 2,9 $\pm 0,5$ OMAR; $w=0,59)$. MN stellte den Diameter der Osteosyntheseschrauben am realistischsten dar 4,9 $\pm 0,2$, OMAR $7,9 \pm 2,0 \mathrm{~mm}$, iMAR $8,3 \pm 1,9 \mathrm{~mm}$; ICC 0,98 ; $\mathrm{p}<0,0001$.

Schlussfolgerungen MN ist den Algorithmen OMAR und IMAR bezüglich der Metallertefaktreduktion sowie der Darstellung des angrenzenden Weichteilgewebes und des Osteosynthesematerials überlegen.
WISS 210.2 Untersuchung zur aktuellen Situation der Aufklärung am Beispiel der Computertomografie. Wie steht es um Qualität, Aufwand, Ärzte- und Patientenzufriedenheit? Welche Verbesserungsmöglichkeiten gibt es?

Autoren Kildal $D^{1}$, Blasenbrey $\mathrm{T}^{2}$, Schmidt $\mathrm{S}^{1}$, Beer $\mathrm{M}^{1}$

Institut 1 Universitätsklinikum Ulm, Klinik für Diagnostische und

Interventionelle Radiologie, Ulm; 2 NSN Ulm, Ulm

DOI 10.1055/s-0037-1600174

Zielsetzung Die Zahl der in Deutschland durchgeführten CTs und MRTs pro Jahr ist in den letzten Jahren stetig angestiegen. Damit verbunden nehmen die Aufklärungen einen zunehmenden Zeitbedarf im Alltag eines Arztes ein. Dazu kommt, dass in 14\% der Schadensersatzprozesse die Aufklärung beanstandet wird, somit kommt der Korrektheit und Dokumentation der Aufklärung eine hohe Bedeutung zu. Wir untersuchten Qualität, Patientenzufriedenheit, Aufwand und Ärztezufriedenheit und leiteten daraus potentielle Verbesserungsmöglichkeiten ab.

Material und Methodik Es wurden 1438 Aufklärungsbögen von 4 Kliniken verschiedener Versorgungsstufen überprüft und deutschlandweit 512 Patienten und 106 Ärzte zu Ergebnisqualität, Zufriedenheit, Aufwand und Verbesserungsmöglichkeiten bei der Aufklärung für CT-Untersuchungen sowie zur Akzeptanz neuer Medien befragt.

Ergebnisse Bis zu $40 \%$ der Patienten gaben an, nicht von ärztlichem Personal aufgeklärt worden zu sein. Zudem sind Patienten wie Ärzte mit der aktuellen Situation nur mäßig zufrieden, z.B. wünschen sich 22\% der Patienten eine längere Aufklärung. Gleichzeitig gaben 85,3\% der Ärzte an, dass Aufklärungen sie bereits jetzt schon stark im Arbeitsablauf beeinträchtigen. Die Ärzte konzentrieren sich bei der Aufklärung vor allem auf die Kontrastmittelnebenwirkungen. Demgegenüber erinnern sich bis $62 \%$ der Patienten nicht an wichtige Kontrastmittelnebenwirkungen. Es zeigte sich weiterhin, dass die Dokumentation der Aufklärungen durch radiologisch tätige Ärzte weniger Mängel aufwies als die der nicht-radiologisch tätigen Ärzte und dass die Qualität der Aufklärungen deutlich vom Personalschlüssel abhängt.

Schlussfolgerungen Folglich sind Aufklärungen für radiologische Untersuchungen aufwendig und aktuell nur eingeschränkt erfolgreich. Dagegen sind neue Medien wie Videoaufklärungen und PC-gestützte Anamnesesysteme vielversprechend, neue Qualitätsstandards zu setzen und den Aufklärungsaufwand für Ärzte reduzieren; diese haben gleichzeitig eine hohe Akzeptanz bei Patienten und Ärzten.

\section{WISS 210.1 Verbesserung der Ergebnisqualität und Patientenzufriedenheit bei radiologischen Aufklärungen durch den Einsatz von Aufklärungsvideos}

Autoren Kildal $D^{1}$, Blasenbrey $T^{2}$, Schmidt $S^{1}$, Beer $M^{1}$

Institut 1 Universitätsklinikum Ulm, Klinik für Diagnostische und

Interventionelle Radiologie, Ulm; 2 NSN Ulm, Ulm DOI 10.1055/s-0037-1600175

Zielsetzung Der korrekt dokumentierten Aufklärung kommt bei radiologischen Untersuchungen eine hohe Bedeutung zu, sie sind jedoch teuer und zeitaufwendig; zudem wird sie in $14 \%$ der Schadensersatzprozesse beanstandet. Die gesetzlichen Vorgaben und Wünsche der Patientenvertreter sind kaum mehr einzuhalten. Gleichzeitig steigt die Zahl der in Deutschland durchgeführten CTs pro Jahr stark an. Bereits jetzt werden hierzulande pro Jahr ca. 17,7 „Arztarbeitsjahre“ für CT-Aufklärungen aufgewendet. Dennoch sind Patienten und Ärzte mit der Situation nur mäßig zufrieden. Die Studie untersuchte die Akzeptanz von Patienten und Ärzten hinsichtlich der Einführung eines Informationsvideos bei der CT-Aufklärung.

Material und Methodik Es wurden deutschlandweit 512 Patienten und 106 Ärzte zur aktuellen Situation und Zufriedenheit sowie zur Akzeptanz techni- 
scher Neuerungen befragt. Zur Validierung wurden die Teilnehmer zu einer weiteren Umfrage mit einem Informationsvideo eingeladen. Wir analysierten, an welche Risiken und Nebenwirkungen die Patienten sich nach Aufklärungen noch erinnern.

Ergebnisse Es zeigte sich eine hohe Akzeptanz für technische Neuerungen bei Patienten und Ärzten. Bei den 62 Teilnehmern der Videostudie zeigte sich ein signifikanter Anstieg in allen abgefragten Bereichen. So erinnerten sich befragte Patienten nach der konventionellen Aufklärung zwischen 27\% und 71\%, nach der Videoaufklärung dagegen nur zwischen $13 \%$ und $27 \%$ nicht mehr an Strahlenrisiken, Kontrastmittelnebenwirkungen oder allgemeine Risiken der Untersuchung. Die Zufriedenheit mit der Aufklärung in der Videostudie war in allen abgefragten Teilbereichen höher als nach konventioneller Aufklärung. Schlussfolgerungen Die Videoinformation ist sicher zukunftsweisend. Patienten können mit gleichbleibend hoher Qualität besser und zufriedenstellender über die Untersuchung informiert werden. Es ist zu erwarten, dass ärztliche Aufklärungsgespräche besser vorbereitet, fokussierter auf den Fall und dadurch kürzer werden, was zusätzlich zu Kosteneinsparungen führt.

\section{WISS 207.3 IHE-MRRT konforme strukturierte Befundung von konventionellen Röntgen-Thorax- Aufnahmen}

Autoren Kornetzky $N^{1}$, Güttler $F^{1}$, Heinrich $A^{1}$, Rottenbach $C^{1}$, Gabmeyer $P^{1}$, Malouhi $A^{1}$, Bruhn $\mathrm{H}^{1}$, Schleicher $\mathrm{C}^{1}$, Teichgräber $\mathrm{U}^{1}$

Institut 1 Institut für Diagnostische und Interventionelle Radiologie

Uniklinikum Jena, Jena

DOI 10.1055/s-0037-1600176

Zielsetzung Einführung und Evaluation der strukturierten Befundung von konventionellen Röntgen-Thorax-Aufnahmen mittels IHE-MRRT-konformen Templates.

Material und Methodik Mithilfe des Online-Editors T-Rex der RSNA wurde ein MRRT-konformes Template erstellt. Damit erfolgte die strukturierte Befundung durch zwei Assistenzärzte. Die Befunde wurden durch Oberärzte anhand der Kriterien Vollständigkeit und Qualität (mittels Likert-Skala) evaluiert. Zudem erfolgte eine automatisierte Zeitmessung für die strukturierte Befunderstellung im Vergleich zur herkömmlichen Freitexterfassung. Als Vergleich dienten die Freitextbefunde derselben Testärzte.

Ergebnisse Insgesamt wurden 44 strukturierte Befunde mithilfe des Templates erstellt und mit 300 herkömmlichen Freitextbefunden mit Spracherkennung verglichen. Bei allen strukturierten Befunden wurde eine Aussage zu Fremdmaterialien, Pneumothorax, Trachea und Herz getroffen. Zudem wurde bei allen p.a.-Aufnahmen der HTQ bestimmt. Eine Aussage zu pneumonischen Infiltraten fehlte bei zwei, zu Mediastinum, kardiopulmonaler Stauung und Pleuraerguss bei jeweils einem Befund. Auf der Likert-Skala von 1 (trifft nicht zu) bis 5 (trifft zu) lagen der Median und der Modus für alle Qualitätskriterien bei 5. Diese waren: Umfang ausreichend zur Beschreibung der Pathologien, Pathologien detailliert beschrieben, zutreffende/mögliche Differentialdiagnosen gefunden, alle wichtigen Pathologien erfasst und Übersichtlichkeit des Befundes. Bei 10 Befunden (22,7\%) war eine Korrektur nötig. Der durchschnittliche Zeitrahmen für die Befunderstellung lag bei 4 min $21 \mathrm{~s}$. Für herkömmliche Freitextbefunde liegt der Mittelwert bei 3 min $15 \mathrm{~s}$.

Schlussfolgerungen Mithilfe des MRRT-konformen Templates wurden qualitativ hochwertige Befunde zum Röntgen Thorax erstellt. Die Übersichtlichkeit und die Praktikabilität müssen hingegen noch verbessert werden. Darüber hinaus lag der durchschnittliche Zeitrahmen über dem der herkömmlichen Befunderstellung.

\section{WISS 207.6 Evaluation eines CAD-Tools und des Einflusses der Spectral Detector CT bei der automatischen Detektion von pulmonalarteriellen Thromben}

\author{
Autoren Kröger $J^{1}$, Hickethier $T^{1}$, Pahn $G^{2}$, Maintz $D^{1}$, Bunck $A^{1}$
}

Institut 1 Uniklinik Köln, Institut für Diagnostische und interventionelle

Radiologie, Köln; 2 Philips GmbH Market DACH

DOI 10.1055/s-0037-1600177

Zielsetzung Evaluation eines "Computer assisted detection“ (CAD)-Tools zur automatischen Detektion von Thromben in der pulmonalarteriellen Strombahn in CT-Angiografien bei Patienten mit klinischem Verdacht auf eine akute Lungenarterienembolie und Untersuchung des Einflusses unterschiedlicher monoenergetischer Rekonstruktionen auf die Ergebnisse der CAD-Analyse.

Material und Methodik Retrospektiv wurden für 15 Patienten mit nachgewiesener LAE, die mittels eines Spectral Detector CTs (IQon Spectral CT, Philips) untersucht wurden, zusätzliche axiale monoenergetische Bildrekonstruktionen mit $40 \mathrm{keV}$ und $70 \mathrm{keV}$ angefertigt. Eine weitere, individuell für jeden Patienten angepasste keV Stufe wurde auf einen Dichtewert von knapp > $600 \mathrm{HU}$ im Truncus pulmonalis geeicht. Für jeden Datensatz wurde erfasst, wie viele Thromben auf Segment- und Subsegmentebene pro Lungenlappen in der CAD-Analyse detektiert wurden. Die Grundwahrheit bildete eine Auswertung durch einen erfahrenen Radiologen.

Ergebnisse Von 86 Thromben detektierte das CAD-Tool im konventionellen Datensatz 59 (68,6\%), im 70 keV MonoE 57 (66,3\%), im 40 keV MonoE 21 $(24,4 \%)$ und im angepassten MonoE 49 (57\%) Thromben. Während der Unterschied in der patientenbasierten Analyse zwischen dem konventionellen Datensatz und dem $70 \mathrm{keV}$ MonoE nicht signifikant war, wurden im $40 \mathrm{keV}$ MonoE und im angepassten MonoE signifikant weniger Thromben detektiert $(p<0,005)$. Im konventionellen Datensatz wurden mit 43 signifikant mehr falsch positive Befunde im Vergleich zu den monoenergetischen Rekonstruktionen detektiert (70 keV: 26, $\mathrm{p}<0,05 ; 40 \mathrm{keV}: 8, \mathrm{p}<0,005$; angepasster MonoE: 20, $p<0,05)$.

Schlussfolgerungen Das untersuchte CAD-Tool ist mit einer Detektionrate von bis zu $68,6 \%$ für segmentale und subsegmentale Thromben in der pulmonalarteriellen Strombahn eine geeignete Hilfe bei der Befundung. Der Einsatz monoenergetischer Rekonstruktionen führt zu einer reduzierten Detektionsrate senkt allerdings auch die Rate an falsch positiven Befunden signifikant.

\section{WISS 210.4 Image matters - wie sehen Medizinstudenten die Radiologie?}

Autoren Lorch $\mathrm{H}^{1}$

Institut 1 Universitätsklinikum Schleswig-Holstein, Institut für Radiologie,

Aschaffenburg

DOI 10.1055/s-0037-1600178

Zielsetzung Wie bewerten Medizinstudenten Attraktivität, Wichtigkeit und Image der Radiologie? Welche Kompetenzen sind nach Einschätzung von Studenten für Radiologen wichtig, welche eher unwichtig? Was gefällt Studenten an der Radiologie, was nicht? Die Studie beantwortet diese Fragen und untersucht, ob und wie sich sich die Einschätzungen im Laufe des Radiologiekurses im ersten klinischen Studienjahr verändern.

Material und Methodik Schriftliche Befragung von Medizinstudenten vor und nach dem Kursus für Radiologie $(n=91 / 121)$. Die Auswertung erfolgte deskriptiv-statistisch und mit dem t-Test für unverbundene Stichproben $(\alpha<0,05)$.

Ergebnisse Das Fach Radiologie wird vor und nach dem Kurs für sehr wichtig gehalten, seine Attraktivität als mittelmäßig eingeschätzt. Die vor dem Kurs ebenfalls mittelmäßige Einschätzung des Images der Radiologie verbesserte sich während des Kurses signifikant ( $p=0,048$ ). 65,3\% der Studenten gaben an, dass sich ihr Bild von der Radiologie durch den Kurs positiv verändert habe. Medizinstudenten kritisieren in erster Linie den geringen Patientenkontakt in 
der Radiologie, sie schätzen v.a. die Vielseitigkeit des Fachs. Für Radiologen sind gute anatomische und physikalisch-technische Kenntnisse und ein breites Fachwissen wichtig, sozial-kommunikative Kompetenzen sind eher unwichtig, so glauben die Studenten.

Schlussfolgerungen Der direkte Kontakt von Medizinstudenten mit der Radiologie und Radiologen beeinflusst deren Einstellung zur Radiologie positiv, was die Bedeutung eines eigenständigen Radiologiekurses unterstreicht. Radiologie wird nach wie vor als patientenfernes Fach wahrgenommen, für das sozial-kommunikative Kompetenzen unwichtig sind. Das hat Bedeutung für die strategische Positionierung des Fachs und kann - je nach gewählter Zielsetzung - Auswirkungen auf die Auswahl geeigneter Bewerber haben.

\section{WISS 207.7 CAD-basierte Kinetikanalyse beim multiparametrischen Prostata-MRT: prädiktiver Wert zur Malignitätsdiagnostik von Ktrans}

Autoren Malich $A^{1}$, Kovacevic $D^{1}$, Teichgräber $U^{2}$, Papageorgiou I

Institut 1 Südharzklinikum Nordhausen, Radiologie, Nordhausen; 2 Friedrich Schiller Universität, Institut für diagnostische und interventionelle Radiologie, Jena

DOI 10.1055/s-0037-1600179

Zielsetzung In der aktuellen Fasung der PIRADS-Codierung ist die Permeabilitätsanalyse nicht quantitativ verankert, die Dynamikanalyse durch die T2w bzw. DWI/ADC weitgehend in der Bedeutung zurückgesetzt. Mittels CAD ist nicht nur eine Farbcodierung des KM-Uptakes möglich, sondern auch die Bestimmung verschiedener dynamischer Parameter, basierend auf der Tumorneoangiogenese bzw. der Permeabilität. Die vorliegende Studie analysierte zahlreiche quantitative CAD-basiert ermittelte Dynamikparameter an MRbioptisch gesicherten Läsionen, um deren diagnostisches Potential zu analysieren.

Material und Methodik Unter CAD-Anwendung (Aegis Prostate ${ }^{\mathrm{TM}}$ 1.0.0; Sentinelle, Hologic) erfolgte die quantitative Analyse von 81 MR-gestützt gesicherten Läsionen (3T Philips Ingenia). Ktrans, kep, Ve, wash-in; wash-out Zeiten sowie TTP wurden automatisch ermittelt und zur Histologie verglichen. Ergebnisse Ktrans max, Ve max und Wash-in zeit waren significant höher bei PCA ( $p<0.05$, Mann Whitney U-test). kep war insignificantly $(p>0.05)$ und TTP min war kürzer bei PCA $(p<0.05)$. Das prädiktive Potential der ROCanalyse zeigte für Ktrans max den höchsten Wert $(p<0.001)$; AUC-Kurve $1.00 \pm 0.00$ und $95 \%$ iges Konfidenzintervall 1.00-1.00. Unter Verwendung des Cut-off wertes $0.39 \mathrm{~L} / \mathrm{min}$ resultiert eine Sens von $100 \%(\mathrm{Cl} 85.18$ $100 \%$ ); Spec von $98.28 \%$ (Cl 90.76-99.96\%). Ve max/wash-in max zeigten eine AUC von $0.69 \pm 0.06 / 0.65 \pm 0.06$ mit korrespondierender Sens./Spec. von $82.61 \% / 51.02 \%$ bzw. 69.57\%/53.45\%. TTP min war insignificant PCa $(p=0.052)$.

Schlussfolgerungen Ktrans max scheint ein wertvoller leicht automatisch generierbarer Parameter zu sein in der Prädiktion eines PCA und sollte in einer Dynamikanalyse mit einem Cut off von $0.39 \mathrm{~L} / \mathrm{min}$ genutzt werden.

\section{WISS 203.1 Evaluation der Strahlenexposition einer Ganzkörper-Skelett-CT Untersuchung unter der Verwendung eines $100 \mathrm{kV}$ Zinn-Filters im Vergleich zu einem Standardprotokoll}

Autoren Meyer $\mathrm{M}^{1}$, Bardehle $\mathrm{F}^{1}$, Vogler $\mathrm{N}^{1}$, Haubenreisser $\mathrm{H}^{1}$, Mey Lien $\mathrm{M}^{1}$, Schoenberg $S^{1}$, Henzler $\mathrm{T}^{1}$

Institut 1 Universitätsmedizin Mannheim, Medizinische Fakultät Mannheim, Universität Heidelberg, Institut für Klinische Radiologie und Nuklearmedizin, Mannheim

DOI 10.1055/s-0037-1600180

Zielsetzung Evaluation der Strahlenexposition sowie der diagnostischen Genauigkeit einer Ganzkörper-Skelett-CT Untersuchung unter der Verwendung eines $100 \mathrm{kV}$ Zinn-Filters.
Material und Methodik Insgesamt wurden 25 Patienten mit histologisch gesichertem Multiplen Myelom und vorheriger Ganzkörper-Skelett-CT Untersuchung (innerhalb eines Monats) mittels eines Ganzkörper-Skelett-CT Protokolls (120 kV an einem dritte Generations dual-sorce CT) unter der Verwendung eines $100 \mathrm{kV}$ Zinn-Filters untersucht. Alle Untersuchungen wurden an einem dual-source CT der dritten Generation unter der Verwendung der folgenden Parameter untersucht: ref. mAs 280, $192 \mathrm{~mm} \times 0.6 \mathrm{~mm}$ Detektorkollimation, Tischvorschub 1.2. Die subjektive und objektive Bildqualität wurde in verschiedenen anatomischen Regionen untersucht und zum Standard-CT-Protokoll verglichen. Ebenfalls wurde die Strahlenexposition sowie die diagnostische Genauigkeit bezüglich der Detektion von osteolytischen Knochenläsionen zwischen den beiden Protokollen verglichen.

Ergebnisse Alle Untersuchungen wurden als diagnostisch gewertet. Es konnte kein Unterschied in der subjektiven Bildqualität zwischen beiden Protokollen festgestellt werden $(p>0,05)$. Im Vergleich zum Standard-CT-Protokoll resultierten signifikant niedrigere Dichtewerte bei dem 100 kV Zinn-Filter Protokoll für Knochen-, Lungen- und Lebergewebe $(p<0,05)$. Die Strahlendosis für das 100 kV Zinn-Filter Protokoll im Vergleich zum Standard-CT-Protokoll war signifikant niedriger (Mittelwert des Dosislängenprodukt $161 \pm 30 \mathrm{mGy}^{*} \mathrm{~cm}$ gegen $1233 \pm 211$ mGy*cm; p<0,0001). Mit dem 100 kV Zinn-Filter Protokoll konnten alle osteolytischen Knochenläsionen $>5$ mm detektiert werden, ohne dass sich falsch positive Läsionen ergaben.

Schlussfolgerungen Unter der Verwendung eines $100 \mathrm{kV}$ Zinn-Filter Protokolls lässt sich die Strahlenexposition im Vergleich zu einem Standard-CTProtokoll um $88 \%$ reduzieren ohne dabei an diagnostischer Genauigkeit zu verlieren.

\section{SP 105.4 Künstliches neuronales Netz zur automatischen Markierung von Lungenflügeln in CT-Datensätzen}

Autoren Mohammad $\mathrm{Q}^{1}$, Limburg $\mathrm{R}^{1}$, Krombach $\mathrm{G}^{1}$, Obert $\mathrm{M}^{1}$ Institut 1 Universitätsklinikum Gießen, Klinik für Diagnostische und Interventionelle Radiologie, Gießen

DOI 10.1055/s-0037-1600181

Zielsetzung In bisherigen Computer aided diagnosis Projekten der Lunge werden regelbasierte Algorithmen zur Segmentierung eingesetzt, wobei die Segmentierung Voraussetzung zur Bestimmung quantitativer Bildmarker ist. In diesem Projekt wird dagegen ein Lungenmarkierungsansatz evaluiert, der Methoden der „künstlichen Intelligenz“ verwendet.

Material und Methodik Mit der Software OpenCV wurde durch „überwachtes Lernen“ ein künstliches neuronales Netz (KNN) zur automatischen Markierung von Lungenflügeln trainiert. Zum „Anlernen“ wurden hierzu aus 50 Datensätzen Bilder von isolierten Lungenflügeln als Positivgruppe erzeugt und Bilder von CT-Schnittbildebenen, die keinerlei Lungenstruktur enthielten, als Negativgruppe selektiert. Nach abgeschlossener „Lernphase“ wurden Test-Bild-Daten von 13 Personen (7 Normal, 3 Fibrose, 3 Emphysem) mit insgesamt 4378 CT-Schnittbildern mit dem KNN ausgewertet. Testdaten überschnitten sich nicht mit Anlerndaten. Die Anzahl richtig bzw. falsch markierter Lungenflügel wurde bestimmt.

Ergebnisse Die KNN-Methodik konnte erfolgreich eingesetzt werden: 4322 von 4378 untersuchten CT-Datensätze konnten erfolgreich markiert werden. Bei 56 Schnittbildern gelang dies nicht. Falsch positiv wurden 15 und falsch negativ 41 Bilder klassifiziert. Spezifität und Sensitivität sind >99,99\%.

Schlussfolgerungen Das KNN ermöglicht die Erkennung und Markierung von Lungenflügeln, die nicht im Training inbegriffen waren. Damit ist ein Anfangsschritt in der Lungendiagnostik mit künstlicher Intelligenz erfolgreich absolviert. Dies ist wichtig, da die KNN-Methode prinzipiell auch direkt zur automatischen Krankheitserkennung angelernt werden kann, wenn man Bildausschnitte mit Pathologien zum Training einsetzt. Weiterhin ist die automatische Lungenflügelmarkierung unter Minimierung der Schnittstelle zwischen 
Mensch und Maschine für viele Schritte der Weiterverarbeitung einschließlich Radiomics bedeutsam.

\section{WISS 207.4 Strukturierte Befundung und Richtlinie zur Organtransplantation gemäß §16 TPG - erste Erfahrungen}

Autoren Pinto dos Santos $D^{1}$, Mildenberger $P^{2}$, Kloeckner $R^{2}$

Institut 1 Uniklinik Köln, Institut für Diagnostische und Interventionelle Radiologie, Köln; 2 Universitätsmedizin Mainz, Klinik und Poliklinik für Diagnostische und Interventionelle Radiologie, Mainz DOI 10.1055/s-0037-1600182

Zielsetzung Mit Inkrafttreten der novellierten Richtlinie zur Organtransplantation gemäß § 16 TPG wird erstmalig die Erhebung eines strukturierten Befundbogens durch den Radiologen gesetzlich vorgeschrieben. Hierbei müssen bei Patienten mit HCC, welche für eine Lebertransplantation geplant sind, genaue Messungen der Tumoren erhoben und dokumentiert werden. Unbeabsichtigt aber passend hierzu hat der Vorstand der Deutschen Röntgengesellschaft auf dem RöKo 2016 Strukturierte Befundung als ein zentrales Thema für das Jahr 2016 definiert. Es erschien daher naheliegend, den in der Richtlinie veröffentlichten Bogen in ein, entsprechend der Empfehlung der DRG formatiertes, digitales Format zu übertragen.

Material und Methodik Die elektronische Befundvorlage wurde unter Verwendung eines freien HTML-Editors erstellt und anschließend in eine im eigenen Haus entwickelte Befundungsplattform importiert. Nach kurzer Schulung wurden die zuständigen Radiologen angewiesen, die geforderten strukturierten Befundberichte nur unter Verwendung des Templates zu erstellen. Während der 70-tägigen Evaluationsperiode wurde kontinuierliche Feedback gesammelt.

Ergebnisse Insgesamt wurden 44 Anforderungen gestellt, einen strukturierten Befund gemäß der Richtliniennovellierung zu erheben. Sowohl Benutzer als auch Zuweiser berichteten über eine große Zufriedenheit mit der elektronischen Umsetzung des Formulars. Es wurden dabei allerdings mehrere inhaltliche Unklarheiten in der Richtlinie offenbar, die abteilungsübergreifend bearbeitet wurden. Darüber hinaus ließen sich aus den gespeicherten Befunden weitergehende Auswertungen anfertigen, so war die Mehrzahl der HCC-Herde rechtshepatisch lokalisiert und die Herde im Mittel mit $22 \mathrm{~mm}$ in arterieller Phase ausgemessen worden. Etwa die Hälfte aller Patienten hatte zum Zeitpunkt der Befunderhebung keine vitalen HCC-Herde mehr.

Schlussfolgerungen Strukturierte Befundung, wie von der DRG vorgeschlagen, eignet sich zur Umsetzung gesetzlicher Vorgaben. Hersteller sollten entsprechende Produkte anbieten.

\section{WISS 207.1 Erste Erfahrungen mit einer digitalen Patientenaufklärung im klinischen Alltag}

Autoren Raatschen $\mathrm{H}^{1}$, Alikhani $\mathrm{B}^{1}$, Grosser $\mathrm{A}^{1}$, Hensen $\mathrm{B}^{1}$, Cartes $\mathrm{M}^{2}$, von Bestenbostel $\mathrm{M}^{2}$, Wacker $\mathrm{F}^{1}$

Institut 1 Medizinische Hochschule Hannover, Institut für Diagnostische und Interventionelle Radiologie, Hannover; 2 Medizinische Hochschule Hannover, Stabstelle Strategisches Risikomanagement und Patientensicherheit, Hannover DOI 10.1055/s-0037-1600183

Zielsetzung Evaluation des zeitlichen Aufwands einer Tablet-basierten digitalen Patientenaufklärung in der Computertomografie unter Berücksichtigung des Patientenalters.

Material und Methodik Wir berichten über die ersten 301 Aufklärungsgespräche, die in unserem Institut vor diagnostischen CT-Untersuchungen digital mit Unterstützung einer Tablet-basierten Software durchgeführt wurden. Diese Software enthält einen erläuternden Teil, der über die Untersuchung und die damit verbundenen Risiken informiert, und einen Anamnesebogen. Es erfolgte die Einteilung in zwei Altersgrupen: $<60$ Jahre $(n=145)$ und $>60$ Jahre $(n=156)$. Erfasst wurden die Gesamtzeit des Aufklärungsprozesses von der Zuweisung des Formulars bis zur digitalen Unterschrift (Gesamtzeit), der zeitliche Aufwand des aufklärenden Arztes (Arztzeit) sowie die Anzahl der offenen Fragen vor dem Gespräch mit dem Radiologen.

Ergebnisse Das mittlere Alter der Patienten betrug 58,8 $\pm 16,6$ Jahre. Die Gesamtzeit betrug 18,0 $\pm 12,5$ min pro Patient, die Arztzeit 1,5 $\pm 1,9$ min. Beim Vergleich der beiden Patientengruppen ergaben sich weder für die Gesamtzeit (19,0 $\pm 13,6$ vs. $17,2 \pm 11,3 \mathrm{~min})$ noch die Arztzeit ( $2,1 \pm 1,9$ vs. 2,1 $\pm 2,0 \mathrm{~min}$ ) signifikante Unterschiede ( $p=0,22$ und 0,99$)$. $133 / 301(44,2 \%)$ der Patienten benötigten Hilfe beim Ausfüllen des Fragebogens. Diese 133 Patienten hatten insgesamt 241 Fragen, wobei besonders die Fragen zu vorherigen CT-Untersuchungen (24,9\%), Medikamenten (15,4\%) und bekannten gutartigen oder bösartigen Erkrankungen (8,7\%) Schwierigkeiten bereiteten. Die Anzahl der offenen Fragen war in beiden Gruppen ähnlich (1,4 $\pm 1,8$ gegenüber $1,5 \pm 2,0)$.

Schlussfolgerungen Die eingesetzte Software erlaubt eine zeiteffiziente Aufklärung von Patienten vor diagnostischen CT-Untersuchungen und kann in allen Altersgruppen verwendet werden.

\section{SP 105.3 Semi-automatische Software zur zeiteffizienten CT-basierten Quantifizierung des abdominellen Fettgewebes}

Autoren Schaudinn $A^{1}$, Linder $N^{1}$, Hudak $A^{1}$, Stange $R^{1}$, Garnov $N^{1}$, Stocker $\mathrm{C}^{2}$, Hacker $\mathrm{U}^{2}$, Kahn $\mathrm{T}^{1}$, Busse $\mathrm{H}^{1}$

Institut 1 Universitätsklinikum Leipzig, Klinik für Diagnostische und Interventionelle Radiologie, Leipzig; 2 Universitätsklinikum Leipzig,

Universitäts Krebszentrum Leipzig, Leipzig

DOI 10.1055/s-0037-1600184

Zielsetzung Die CT-basierte Quantifizierung des viszeralen und subkutanen Fettgewebes (VAT, SAT) findet klinisch zunehmend Anwendung bei der Risikobeurteilung von Patienten mit metabolischem Syndrom, in der Onkologie oder vor einem abdominellen Eingriff. Ziel dieser Studie war die Validierung einer hauseigenen Software (ATQ, adipose tissue quantification) für die semiautomatische Fettquantifizierung.

Material und Methodik Die CT-Datensätze (Somatom Sensation 64, Siemens, Schichtdicke $5 \mathrm{~mm}$ ) von 20 normal- bis übergewichtigen Patienten (14 männlich, Ø BMl: $29,4 \mathrm{~kg} / \mathrm{m}^{2}$ ) wurden in die retrospektive Analyse eingeschlossen. Für alle Patienten lag ein positives Ethikvotum vor. Das gesamte VAT-Volumen (V-VAT) sowie das abdominelle SAT-Volumen (V-SAT, Zwerchfell bis Beckenboden) wurden mittels semiautomatischer Segmentierung und histogrammgestützter Auswertung bestimmt (ATQ, unter MatLab, MathWorks). Voxel mit einer Dichte zwischen -190 und $30 \mathrm{HU}$ wurden als Fettgewebe quantifiziert. Die Analyse wurde mit einer kommerziellen Segmentierungs-Software wiederholt (SliceOmatic, TomoVision). Zur Bewertung der Übereinstimmung dienten das Bestimmtheitsmaß $R^{2}$ sowie die Standardabweichungen der mittleren Volumendifferenzen (Bland-Altman).

Ergebnisse Die mittleren Volumina betrugen 5,6 L für V-VAT und 8,6 L für V-SAT. Die Bearbeitung eines Datensatzes umfasste im Mittel 83 Schichten und dauerte $15 \mathrm{~min}$ (ATQ) bzw. $41 \mathrm{~min}$ (SliceOmatic) pro Patient. Für beide Fettkompartimente fand sich eine Übereinstimmung von $R^{2}=0,99$. Die Mittelwerte (bias) und Standardabweichungen der jeweiligen Differenzen lagen bei $+0,11 \mathrm{~L}$ und $0,07 \mathrm{~L}(1,2 \%)$ für $\mathrm{V}$-VAT bzw. $-0,22 \mathrm{~L}$ und $0,17 \mathrm{~L}(1,9 \%)$ für $\mathrm{V}$-SAT. Schlussfolgerungen Die vorgestellte Software erlaubt eine einfache und verlässliche CT-Quantifizierung diverser Fettvolumina. Der mittlere Zeitaufwand von 15 min pro Patient ist erheblich kürzer als mit kommerziellen Werkzeugen und wäre auch absolut betrachtet für eine klinische Routineanwendung vertretbar. 


\section{SP 105.5 Vollautomatische, lappenbasierte Segmentierung von MR-Pefusionsmessungen in COPD Patienten mit Methoden des maschinellen Lernens}

\author{
Autoren Winther $\mathrm{H}^{1}$, Hundt $\mathrm{C}^{2}$, Czerner $\mathrm{C}^{1}$, Kaireit $\mathrm{T}^{1}$, Wacker $\mathrm{F}^{1}$, Shin $\mathrm{H}^{1}$, \\ Vogel-Claussen ${ }^{1}$ \\ Institut 1 Institut für Diagnostische und Interventionelle Radiologie, \\ Hannover; 2 Institut für Informatik der Johannes Gutenberg-Universität, \\ Parallel and Distributed Architectures Group, Mainz \\ DOI 10.1055/s-0037-1600185
}

Zielsetzung Die bei COPD auftretenden parenchymalen Lungenperfusionsveränderungen können mit einer dynamischen 4 dimensionalen kontrastverstärkten (DCE) MRT-Untersuchung zuverlässig quantifiziert warden. Die Auswertung der Perfusionsdaten ist complex und erfordert menschliche Interaktion. Dies bindet wertvolle Ressourcen und es besteht das Risiko einer hohen Variabilität zwischen Beobachtern. Eine vollautomatische Segmentierung könnte die Beurteilung erleichtern und die Auswertung verbessern. Ziel dieser Studie war es, zu evaluieren, ob maschinelles Lernen für eine lappenbasierte Segmentierung der Lunge für 4D MR-Perfusionsmessungen verwendet werden kann.

Material und Methodik CT-Datensätze von 11 Studienteilnehmern mit COPD wurden maunell auf Lappenebene segmentiert und als Ground Truth akzeptiert. Diese wurden auf einen korrespondierenden 4D DCE-MRI Datensatz des gleichen Patienten registriert. Die resultierende Segmentierung wurde als MRT Ground Truth angenommen (Atlasbildung). Eine Multi-Atlas-Bildregistrierung der Atlas-MRTs auf die Ziel-MRT wurde durchführt mit anschließendem Majority Label Voting als Label-Fusions-Algorithmus. Hierbei wird aus einem Pool von Vorhersagen für jeden Voxel die Klasse mit den meisten Stimmen akzeptiert.

Ergebnisse Es wurde eine Überlappung der Segmentierung von 92\% für Lungenflügel sowie von $85 \%$ für einzelne Lappen mit der Ground Truth erreicht.

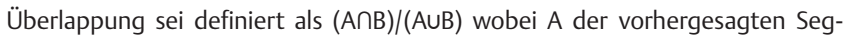
mentierung und B der Ground Truth entspricht (Jaccard index).

Schlussfolgerungen Maschinelles Lernen wurde erfolgreich für die vollautomatische lappenbasierte Segmentierung der Lunge verwendet. Die vorgestellte Methode illustriert die erste praktische Anwendung einer vollautomatischen, lappenbasierten Lungensegmentierung für 4D MR-Perfusionsmessungen.

\section{Experimentelle Radiologie}

\section{WISS 311.3 Frequenzselektive, nichtlineare Fensterung zur Steigerung der Erkennbarkeit von Lungenarterienembolien und venösen Thrombosen in der Computertomografie}

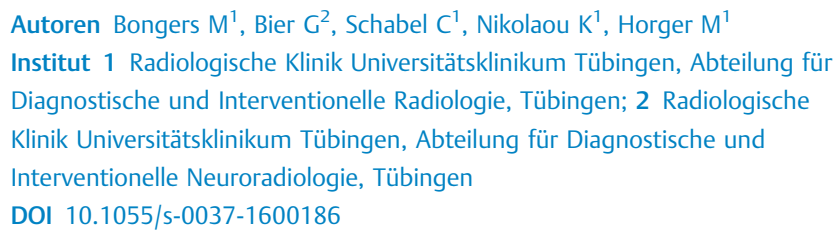

Autoren Bongers $\mathrm{M}^{1}$, Bier $\mathrm{C}^{2}$, Schabel $\mathrm{C}^{1}$, Nikolaou $\mathrm{K}^{1}$, Horger $\mathrm{M}^{1}$ Institut 1 Radiologische Klinik Universitätsklinikum Tübingen, Abteilung für Diagnostische und Interventionelle Radiologie, Tübingen; 2 Radiologische Klinik Universitätsklinikum Tübingen, Abteilung für Diagnostische und Interventionelle Neuroradiologie, Tübingen

DOI 10.1055/s-0037-1600186

Zielsetzung Ziel war es herauszufinden, ob es möglich ist mittels eines neuartigen frequenzselektiven Algorithmus der nichtlinearen Fensterung (NLF) die Erkennbarkeit von Lungenarterienembolien und venösen Thrombosen in portal-venösen Ganzkörper-Staging-CTs zu steigern.

Material und Methodik 67 Patienten mit nebenbefundlichen Lungenarterienembolien und/oder venösen Thrombosen wurden retrospektiv eingeschlossen. Die CTs wurden 65-90 Sekunden nach Kontrastmittelapplikation akquiriert. Ein neuartiger frequenzselektiver Algorithmus der NLF wurde zur Rekonstruktion verwendet. Zwei unabhängige Radiologen ermittelten die am besten geeigneten NLF-Einstellungen (Center, Delta, Slope). Mittels ROI-Messungen wurden der Kontrast, das Bildrauschen und das Kontrast-zu-Rauschen zwischen Thrombus und Gefäß ermittelt. Eine subjektive Bildanalyse wurde bezüglich des Bildrauschens, der Bildqualität und Abgrenzbarkeit der Thromben durchgeführt. Die Parameter wurden mittels Wilcoxon-Vorzeichen-Rang-Test zwischen Standard- und NLF-Bildern verglichen.

Ergebnisse Durch frequenzselektive NLF mit einem Center von 100 HU, Delta von $40 \mathrm{HU}$ und Slope von 5 lässt sich das CNR von Lungenarterienembolien (StandardCNR10[6,16]; NLFCNR22[15,30];p < 0.001) und venösen Thrombosen (StandardCNR8[5,15]; NLFCNR12[7,19]; $\mathrm{p}=0.0007$ ) signifikant steigern. Die mittlere Schwächung im Gefäß war in NLF-Bildern sowohl in Pulmonalarterien (Standard138[118,191]HU;NLF,269[176,329] HU; $\mathrm{p}<0.0001$ ) als auch in Venen (Standard120[103,162]HU;NLF169[132,217]HUp<0.0001) signifikant höher. Das Bildrauschen war nicht signifikant unterschiedlich $(p=0.64-0.88)$. Die Interrater-Reliabilität zeigte eine beachtliche bis fast vollkommene Übereinstimmung. Die Bildqualität $(p<0.004)$ und subjektive Abgrenzbarkeit der Thromben $(p<0.0001)$ konnte durch NLF in beiden Subgruppen signifikant gesteigert werden.

Schlussfolgerungen Mittels frequenzselektiver NLF lässt sich die Bildqualität und Erkennbarkeit von Lungenarterienembolien und venösen Thrombosen in Standard-Ganzkörper-Staging-CTs signifikant steigern.

\section{WISS 304.3 Magnetic Particle Imaging von Lebertumoren im Kleintiermodell}

Autoren Dieckhoff $]^{1}$, Kaul $\mathrm{M}^{1}$, Mummert $\mathrm{T}^{1}$, Jung $\mathrm{C}^{1}$, Salamon $]^{1}$, Adam $\mathrm{G}^{1}$, Knopp $\mathrm{T}^{2}$, Ittrich $\mathrm{H}^{1}$

Institut 1 Universitätsklinikum Hamburg-Eppendorf, Klinik und Poliklinik für Diagnostische und Interventionelle Radiologie und Nuklearmedizin, Hamburg; 2 Universitätsklinikum Hamburg-Eppendorf und Technische Universität Hamburg, Abteilung für experimentelle biomedizinische Bildgebung, Hamburg DOI 10.1055/s-0037-1600187

Zielsetzung Evaluation des in vivo Magnetic Particle Imaging (MPI) zur Abbildung von murinen Lebertumoren durch kombinierte MPI-MRT Messungen. Material und Methodik Gentechnisch veränderte Mäuse (Mdr2 Knockout, $\mathrm{n}=10$ ) wurden ab einem Alter von einem Jahr mittels Kleintier-MRT (ClinScan, Bruker BioSpin $\mathrm{GmbH}$ ) in regelmäßigen Abständen hinsichtlich der Ausbildung von inflammationsassoziierten hepatozellulären Karzinomen (HCC) untersucht. Bei Entwicklung von HCC mit Durchmessern > 5 mm wurden 4 Stunden nach intravenöser Injektion von Ferucarbotran (Resovist ${ }^{\circledR}$, Bayer Schering Pharma AG; 0.5 mmol Eisen pro kg KG) sequentiell MRT- und MPI-Messungen (Bruker BioSpin GmbH/Philips AG; Drive Field: $14 \mathrm{mT}$; Gradient: $1.5 \mathrm{~T} / \mathrm{m}$; FoV: $37.4 \times 37.4 \times 18.7 \mathrm{~mm} 3$ ) vorgenommen. Die gewonnenen MPI-Messdaten wurden mithilfe eines selbstentwickelten Softwareframeworks rekonstruiert und mit den MRT-Daten fusioniert, so dass ein direkter Vergleich der beiden Bildgebungsmodalitäten hinsichtlich der Lebermorphologie ermöglicht wurde. Ergebnisse Es konnte eine gute Korrelation zwischen den MPI- und MRTMessdaten der Leber erreicht werden. Im direkten Vergleich der beiden Modalitäten zeigte sich eine im Vergleich zur Hochfeld-MRT stark reduzierte räumliche Auflösung in den MPI-Daten (verwendete Konfiguration: $-5 \mathrm{~mm}$ ). HCC - charakterisiert durch eine fehlende Ferucarbotran-Akkumulation - verursachten je nach Position und Größe eine Verringerung oder Aussparung des MPI-Messsignals.

Schlussfolgerungen Die ersten Leber-MPI-Messungen an einem Lebertumortragenden Mausmodel demonstrieren die prinzipielle Machbarkeit einer Leberund Lebertumorvisualisierung mittels MPI. 


\section{WISS 311.5 Neuronale Netze zur multiparametrischen Diagnostik experimenteller Maus-Colitiden}

Autoren Ellmann $\mathrm{S}^{1}$, Langer $\mathrm{V}^{2}$, Britzen-Laurent $\mathrm{N}^{2}$, Huber $\mathrm{C}^{3}$, Hildner $\mathrm{K}^{3}$, Stürzl $M^{2}$, Uder $M^{1}$, Bäuerle $T^{1}$

Institut 1 Universitätsklinikum Erlangen, Radiologisches Institut, Erlangen; 2 Universitätsklinikum Erlangen, Molekulare und Experimentelle Chirurgie, Erlangen; 3 Universitätsklinikum Erlangen, Medizinische Klinik 1, Erlangen DOI 10.1055/s-0037-1600188

Zielsetzung MRT ermöglicht bei chronisch-entzündlichen Darmerkrankungen die nichtinvasive Erfassung pathologischer Veränderungen der betroffenen Darmabschnitte. Neben morphologischen Veränderungen können im MRT auch Relaxationszeiten und Diffusionskapazität der Darmwand zur Colitisdiagnostik verwendet werden. Ziel der Studie war die multiparametrische Bildgebung experimenteller Colitiden im Mausmodell unter Zuhilfenahme Neuronaler Netze (NN), um Sensitivitäten und Spezifitäten in der Colitisdiagnostik zu optimieren.

Material und Methodik In 31 Mäusen mit DSS-induzierter Colitis ( $n=13$ in vivo, $n=18$ ex vivo) und 18 Kontrolltieren ( $n=6$ in vivo, $n=12$ ex vivo) wurden mittels MRT Wanddicke, T1-, T2-, T2*-Relaxationszeiten und Diffusionskapazität in der Darmwand bestimmt. ROC-Kurven wurden zur Berechnung von Sensitivität und Spezifität dieser Einzelwerte verwendet. Ein NN wurde mittels Kreuzvalidierung auf die Kombinationen obiger Messwerte trainiert und dessen prädiktive Kapazität mittels McNemar Tests mit denen der einzelnen Parameter verglichen.

Ergebnisse In der in vivo Untergruppe wies kein Einzelparameter kombinierte Sensitivitäten und Spezifitäten $>90 \%$ auf, NN waren mit 91,3\% Sensitivität und $92,3 \%$ Spezifität allen Einzelparametern signifikant überlegen ( $p \leq 0,002)$. In der ex vivo Untergruppe zeigte sich die Wanddicke als bester singulärer Prädiktor (Sensitivität 77,6\%; Spezifität 79,5\%), NN wiesen hier eine Sensitivität von $87,4 \%$ und Spezifität von $82,9 \%$ auf und waren bezüglich Sensitivität signifikant überlegen $(p \leq 0,01)$. In keinem der Vergleiche waren NN Einzelparametern signifikant unterlegen.

Schlussfolgerungen In experimentellen Maus-Colitiden steigert die Kombination multiparametrischer MRT-Messwerte zu einem NN signifikant die diagnostische Genauigkeit im Vergleich zu den Einzelparametern. Diese PilotStudie liefert die Grundlage zur Entwicklung eines MRT-basierten Colitis Scores, der zunächst im Mausmodell, später auch bei klinischen Fragestellungen quantifizierbare Diagnostik und Verlaufsbeurteilungen ermöglichen soll.

\section{WISS 312.3 Erste Ergebnisse der Dunkelfeld- Radiografie des menschlichen Thorax}

Autoren Fingerle $\mathrm{A}^{1}$, Willer $\mathrm{K}^{2}$, Gromann $\mathrm{L}^{2}$, de Marco $\mathrm{F}^{2}$, Herzen $\mathrm{J}^{2}$, Achterhold $K^{2}$, Gleich $B^{2}$, Münzel $D^{1}$, Scherer $K^{2}$, Renz $M^{3}$, Renger $B^{3}$, Kopp $\mathrm{F}^{3}$, Kriner $\mathrm{F}^{4}$, Fischer $\mathrm{F}^{4}$, Braun $\mathrm{C}^{4}$, Auweter $\mathrm{S}^{5}$, Hellbach $\mathrm{K}^{5}$, Reiser $\mathrm{M}^{5}$, Schröter $\mathrm{T}^{6}$, Mohr $\mathrm{J}^{6}$, Yaroshenko $\mathrm{A}^{7}$, Maack $\mathrm{H}^{7}$, Pralow $\mathrm{T}^{7}$, van der Heijden $\mathrm{H}^{7}$, Proksa $\mathrm{R}^{8}$, Koehler $\mathrm{T}^{9}$, Wieberneit $\mathrm{N}^{7}$, Rindt $\mathrm{K}^{7}$, Rummeny $\mathrm{E}^{3}$, Pfeiffer $\mathrm{F}^{10}$, Noël $\mathrm{P}^{11}$

Institut 1 Institut für diagnostische und interventionelle Radiologie, Lehrstuhl für Biomedizinische Physik und Institut für Medizintechnik, Technische Universität München, München; 2 Lehrstuhl für Biomedizinische Physik und Institut für Medizintechnik, Technische Universität München, Garching; 3 Institut für diagnostische und interventionelle Radiologie, Technische Universität München, München; 4 Institut für Rechtsmedizin, LudwigMaximilians-Universität München, München; 5 Institut für klinische Radiologie, Ludwig-Maximilians-Universität München, München; 6 Karlsruher Institut für Technologie, Karlsruhe; 7 Philips Medical Systems DMC GmbH, Hamburg; 8 Philips GmbH Innovative Technologies, Research Laboratories, Hamburg; 9 Philips GmbH Innovative Technologies, Research Laboratories; Institute for Advanced Study, Technische Universität München, Hamburg; 10 Lehrstuhl für Biomedizinische Physik und Institut für Medizintechnik,
Institut für diagnostische und interventionelle Radiologie, Institute for Advanced Study, Technische Universität München, Garching; 11 Institut für diagnostische und interventionelle Radiologie, Lehrstuhl für Biomedizinische Physik und Institut für Medizintechnik, München

DOI 10.1055/s-0037-1600189

Zielsetzung Die Performance eines experimentellen Dunkelfeld-Radiografie Systems für die Thoraxbildgebung im Menschen zu evaluieren und mit konventionellem Röntgen zu vergleichen.

Material und Methodik Die Studie wurde von der Ethikkommission der Technischen Universität München genehmigt. Ein einzelner menschlicher Leichnam (52 Jahre, weiblich, Größe: $173 \mathrm{~cm}$, Gewicht: 84 kg, Brustumfang: $97 \mathrm{~cm}$ ) wurde innerhalb von $24 \mathrm{~h}$ post mortem mit einem experimentellen Dunkelfeld-Radiografie-System untersucht. Als Referenz erfolgte zusätzlich eine Untersuchung an einem klinischen CT. Der gitterbasierte Dunkelfeld-Radiografie Aufbau war mit einem Satz von drei Gittern ausgerüstet, um Dunkelfeld-Röntgenbildgebung zu ermöglichen. Der Prototyp verwendet eine Beschleunigungsspannung von $70 \mathrm{kVp}$ und ein für den klinischen Einsatz geeignetes Bildfeld $(>35 \times 35 \mathrm{~cm} 2)$.

Ergebnisse Ein Dunkelfeld-Röntgenbild des gesamten menschlichen Thorax konnte gemessen werden. Dies zeigt die Übertragbarkeit von Ergebnissen vorangehender tierexperimenteller Studien zur Dunkelfeldbildgebung. Lungengewebe erzeugt eine starke Streuung, die zu einem hohen Dunkelfeld Röntgensignal führt. Rippen und Wirbelsäule erzeugen dagegen ein deutlich geringeres Signal, sind jedoch abgrenzbar. Weichgewebe kommt im Dunkelfeldbild nicht zur Darstellung. Lungenregionen mit ödematösen Flüssigkeitseinlagerungen, nachgewiesen im CT, zeigten ein vermindertes Dunkelfeldsignal im Vergleich zu normalem Lungengewebe.

Schlussfolgerungen Unsere Ergebnisse zeigen den aktuellen Stand der translationalen Dunkelfeld-Röntgenbildgebung von präklinischer (Kleintier)forschung hin zu klinischer Bildgebung (am Patienten). Das experimentelle Dunkelfeld-Radiografie-System ermöglicht zum ersten Mal Multikontraströntgenaufnahmen (Absorption und Dunkelfeld) des menschlichen Thorax.

\section{WISS 312.5 Funktionelle MRT zur Quantifizierung des pharmakotherapeutischen Effektes eines ACE- Hemmers auf die Nierenperfusion und die T1-Relaxationszeit}

Autoren Getzin $\mathrm{T}^{1}$, May $\mathrm{M}^{2}$, Schmidbauer $\mathrm{M}^{3}$, Gutberlet $\mathrm{M}^{3}$, Wacker $\mathrm{F}^{3}$, Schindler $\mathrm{C}^{2}$, Hueper $\mathrm{K}^{3}$

Institut 1 Medizinische Hochschule Hannover, Radiologie, Hannover;

2 Medizinische Hochschule Hannover, CRC Core Facility, Hannover;

3 Medizinische Hochschule Hannover, Diagnostische und Interventionelle

Radiologie, Hannover

DOI 10.1055/s-0037-1600190

Zielsetzung Evaluation der Anwendbarkeit von arterial spin labeling (ASL) und T1mapping zur Quantifizierung des Effektes einer pharmakologischen Intervention mit dem ACE-Hemmer Captopril auf die Nierenperfusion und die T1Zeit des Nierenparenchyms.

Material und Methodik 15 gesunde Probanden ( 22 - 50 Jahre alt) wurden mit einem 1,5T MRT (Siemens Avanto) zweimal nüchtern und 60 Minuten nach oraler Gabe von $50 \mathrm{mg}$ Captopril untersucht. Mittels flow-sensitive alternating inversion-recovery (FAIR) true-FISP ASL und modifizierter look-locker inversion recovery (MOLLI) Sequenz wurden die renale Perfusion und T1-Relaxationszeiten im Nierenkortex bestimmt. Ausgewertet wurden die Reproduzierbarkeit der Daten zwischen zwei Untersuchungen und zwei Untersuchern sowie der pharmakologische Effekt von Captopril auf die MRT-Parameter.

Ergebnisse Die Reproduzierbarkeit der MRT-Parameter zwischen zwei Untersuchungen (Intra-Klassen-Korrelationskoeffizienten ICC $=0,77$, Variationskoeffizient $C V=6,7 \%$ ) und zwei Lesern (ICC =0,97, CV =2,9\%) war exzellent. Die Gabe von Captopril führte bei Probanden zu einem Abfall des Blutdrucks von $120 / 78$ auf $107 / 69 \mathrm{~mm} \mathrm{Hg}(p<0,001)$. Mittlere Captoprilspiegel lagen bei 
$711 \mathrm{ng} / \mathrm{ml}$. Mittels MRT war nach Captopril-Gabe eine signifikante Steigerung der kortikalen Nierenperfusion um $22 \%$ ( $369 \pm 48$ vs. $452 \pm 15 \mathrm{ml} / \mathrm{min} / 100 \mathrm{~g}$, $\mathrm{p}<0,001)$ nachweisbar, während die kortikalen T1-Zeiten stabil blieben (1126 \pm 20 vs. $1161 \pm 18 \mathrm{~ms}$ ).

Schlussfolgerungen Die Messung der renalen Perfusion und T1-Zeit mittels ASL und T1 mapping ist gut reproduzierbar und pharmakotherapeutische Effekte auf die Niere können mit geringer Probandenzahl erfasst werden. Dies ist für den Einsatz der MRT-Techniken zur Beurteilung von Wirkmechanismen und Therapieeffekten entscheidend. In dieser Studie konnte ein hochsignifikanter Perfusionsanstieg durch die orale Gabe von Captopril nachgewiesen werden, der am ehesten durch eine Vasodilatation und Senkung des peripheren renalen Perfusionswiderstandes zu erklären ist.

\section{WISS 304.5 Intra-individuelle quantitative und qualitative Bolus-Analyse von Dreiphasen-3D- und 4D-MRA mit Gadobutrol und Gadoterate Meglumine bei 1,5T und 3Tesla bei Minipigs}

Autoren Hadizadeh $\mathrm{D}^{1}$, Jost $\mathrm{C}^{2}$, Keil $\mathrm{V}^{1}$, Marx $\mathrm{C}^{1}$, Rauch $\mathrm{M}^{1}$, Schmeel $\mathrm{F}^{1}$, Pietsch $\mathrm{H}^{2}$, Schild $\mathrm{H}^{1}$, Willinek $\mathrm{W}^{3}$

Institut 1 Universität Bonn, Radiologie, Bonn; 2 Bayer Pharma AG, MR und CT Kontrastmittel-Forschung, Berlin; 3 Krankenhaus der barmherzigen Brüder

Trier, Radiologie, Neuroradiologie, Sonografie und Nuklearmedizin, Trier DOI 10.1055/s-0037-1600191

Zielsetzung Für MR Angiografie werden räumlich hochaufgelöste Mehrphasen-3D-(MP3D) und zunehmend auch zeitlich hochaufgelöste 4D-Techniken eingesetzt. Bisher existieren kaum systematische Analysen zur Optimierung von Kontrastmittel-Applikationen (KM). Ziel dieser Studie war, quantitative und qualitative Daten zur Applikation von Gadobutrol und Gadoterate Meglumine bei 1,5 und 3T zu erheben.

Material und Methodik 8 Göttinger Minipigs erhielten MP3D- und 4D-MRAUntersuchungen an Ganzkörper-1,5T (Siemens Avanto) und 3T (Philips Intera) MRTs. Zur Anwendung kamen für die MP3D-MRA bei 1,5T 4 Phasen 3D FLASH-(64 Schichten, Voxel $\left.=(1,2 \mathrm{~mm})^{3}\right)$ und bei 3T 3D FFE-Sequenzen (Vo$\left.x e l=(1,2 \times 1,25 \times 1,5) \mathrm{mm}^{3}\right)$. Die 4D-MRA erfolgte bei 1.5T mit TWIST (Bildwiederholrate $(B W R)=1,48 \mathrm{~s}$, Voxel $\left.=(1,7 \mathrm{~mm})^{3}\right)$ und bei 3T mittels 4D-TRAK $\left(B W R=1,5 \mathrm{~s}\right.$, Voxel $\left.=(1,6 \mathrm{~mm})^{3}\right) . \mathrm{KM}: 1 \mathrm{ml} / \mathrm{s}$ Standarddosis Gadobutrol $(0,1$ $\mathrm{mmol} / \mathrm{kg}, \mathrm{sGB})$ und halbe Dosis Gadobutrol (0,05 mmol $/ \mathrm{kg}, \mathrm{hGB}), 2 \mathrm{ml} / \mathrm{s}$ Standarddosis Gadoterate Meglumine (0,1 mmol/kg, sGM); MP3D-MRA: nur sGB und sGM. Quantitative Analyse der MP3D- und 4D-MRA anhand Baselinekorrigierter Signalintensitäten (Aorta, Vena cava inferior, Vena portae). Qualitative Analyse: 3 Radiologen, Sichtbarkeit von Arterien und Venen.

Ergebnisse Mit sGB zeigten sich in allen Untersuchungen die höchsten Signalmaxima. 1,5T 4D-MRA: in allen Gefäßen signifikant höher, 3T 4D-MRA: in Venen signifikant höher. MP3D-MRA: Signifikant höhere Signale mit SGB gegenüber sGM in der venösen Phase bei 1,5T und 3T. In der 4D-MRA bei 3.0T beobachteten wir gekappte Maxima der arteriellen Boli bei $7 / 8$ Tieren mit sGB und SGM. Mit hGB und bei 1,5T zeigten sich hingegen keine gekappten Maxima. Die Bildqualität der MP3D-MRA von Venen wurde bei beiden Feldstärken mit sGB signifikant besser gewertet als mit sGM.

Schlussfolgerungen Mit sGB sind die Maxima in allen Phasen und Gefäßen am höchsten in der MP3D- und 4D-MRA bei 1,5 und 3T. Signalmaxima und Bildqualität von Venen profitieren besonders von SGB. In Arterien zeigen sich in der 4D-MRA mit sGB und sGM gekappte Maxima.

\section{WISS 312.2 Magnetic Particle Imaging: Dynamische Darstellung einer Ballondilatation im Gefäßmodell in Echtzeit}

Autoren Herz $S^{1}$, Vogel $P^{1}$, Philipp $D^{1}, \operatorname{Kampf} \mathrm{T}^{2}$, Kunz $\mathrm{J}^{1}$, Rückert $\mathrm{M}^{2}$, Behr $\mathrm{V}^{2}$, Bley $\mathrm{T}^{1}$

Institut 1 Universitätsklinikum Würzburg, Institut für Diagnostische und

Interventionelle Radiologie, Würzburg; 2 Universität Würzburg,

Experimentelle Physik V, Würzburg

DOI 10.1055/s-0037-1600192

Zielsetzung Ziel dieser Studie war die dynamische Visualisierung der Ballondilatation einer Stenose im Gefäßmodell in Echtzeit mittels Magnetic Particle Imaging (MPI).

Material und Methodik Ein Gefäßmodell aus Polyvinylchlorid mit einem maximalen Innendurchmesser von $8 \mathrm{~mm}$ enthielt eine $50 \%$ ige Stenose. Als Referenzmethode dieser Versuchsanordnung diente die konventionelle Angioplastie (PTA). In das Gefäßlumen wurden zunächst SPIOs (superparamagnetische Eisenoxid Nanopartikel, Ferucarbotran) als Kontrastmittel injiziert und dynamisch im MPI Scanner (FOV $65 \times 25 \times 25$ mm³ $^{3}$ ) dargestellt. Die Bildrekonstruktion in Echtzeit erfolgte mit einem eigens programmierten Auswertungsalgorithmus. In einem zweiten Schritt wurde die Stenose mittels eines PTA-Ballons aufgedehnt. Zur Visualisierung des Führungsdrahts und des Ballon-Katheters wurden diese jeweils mit einem paramagnetischen Lack markiert. Die Stenose wurde MPI-gesteuert mit dem Führungsdraht sondiert und der Ballonkatheter über diesen in der Stenose platziert. Durch das Inflatieren des Ballonkatheters mit Ferucarbotran wurde die Stenose dilatiert.

Ergebnisse MPI ermöglicht die dynamische Visualisierung einer Ballondilatation in Echtzeit. Die Positionierung von Führungsdraht und Ballonkatheter gelingt präzise mittels Markierung mit einem paramagnetischen Lack. Analog zur konventionellen Angioplastie zeigte die MPI-Abschlusskontrolle der untersuchten Versuchsanordnung keine nennenswerte Reststenose.

Schlussfolgerungen Durch die technisch erfolgreich visualisierte Ballondilatation mittels MPI, empfiehlt sich diese neue, strahlenfreie Bildgebungsmethode als vielversprechender Ansatz für die endovaskuläre Intervention.

\section{WISS 304.4 Detektion metaboler Subgruppen des hepatozellulären Karzinoms (HCC) mittels Magnetresonanzspektroskopie (MRSI) mit hyperpolarisiertem 13C-Pyruvat}

Autoren Kaissis $G^{1}$, Bliemsrieder $E^{1}$, Keim $D^{1}$, Gebrekidan $L^{1}$, Topping $G^{2}$, Hundshammer $C^{2}$, Düwel $S^{2}$, Schilling $F^{2}$, Steiger $K^{3}$, Weichert $W^{3}$, Schwaiger $\mathrm{M}^{2}$, Rummeny $\mathrm{E}^{1}$, Braren $\mathrm{R}^{1}$

Institut 1 Klinikum rechts der Isar, Institut für diagnostische und interventionelle Radiologie, München; 2 Klinikum rechts der Isar, Klinik für Nuklearmedizin, München; 3 Klinikum rechts der Isar, Institut für Pathologie, München

DOI 10.1055/s-0037-1600193

Zielsetzung Die Energiegewinnung durch aerobe Glykolyse ("Warburg-Effekt") zeichnet verschiedene Tumorarten aus. Die MRSI mit hyperpolarisierten Substanzen (z. B. C-13-Pyruvat) erlaubt die nicht-invasive Evaluation des Tumormetabolismus. Ziel dieser Arbeit war die Evaluation der 13C-Pyruvat MRSI als Methode zur metabolen Subgruppendetektion im HCC.

Material und Methodik HCCs wurden in Wistar-Ratten mit oralem Diethylnitrosamin (8 Wochen) induziert. 20 HCC tragende und 6 gesunde Kontrolltiere wurden mittels hochaufgelöster T2-gewichteter Bildgebung und Free-Induction-Decay-Chemical Shift Imaging (FID-CSI) untersucht. 5 HCC tragende Tiere wurden zusätzlich mittels 18 F-FDG-PET untersucht. Tumor- und gesundes Lebergewebe wurden histopathologisch untersucht und mit der in-vivo Bildgebung korreliert.

Ergebnisse Der Label-Exchange-Quotient zwischen 13-C-Pyruvat/Laktat und 13-C-Pyruvat/Alanin lag in HCCs signifikant höher als in gesundem Lebergewebe (HCC: $1.69 \pm 0.44$; Leber: $1.05 \pm 0.12, \mathrm{p}=0.002$ ). Es zeigte sich eine 
deutliche intertumorale Heterogenität des Lac/Ala-Quotienten $(\sigma 2=0.19)$ im Vergleich zur gesunden Leber $(\sigma 2=0.016)$. Niedriggradige und hochgradige Tumore zeigten signifikant unterschiedliche Lac/Ala Quotienten. Tumore mit ähnlichem SUV in der PET wiesen deutlich differente Lac/Ala-Quotienten auf. Schlussfolgerungen C-13-Pyruvat-MRSI ermöglicht die Tumordetektion im HCC-Rattenmodell. Ein höheres histopathologisches Tumorgrading korreliert mit höheren Lac/Ala-Quotienten. Die geringe Korrelation der 13C-Pyruvat MRSI mit der 18F-FDG PET Bildgebung ist ein möglicher Indikator für den additiven Wert dieser neuen Bildgebungsmethode.

\section{WISS 211.11 Detektion metaboler Subgruppen des hepatozellulären Karzinoms (HCC) mittels Magnetresonanzspektroskopie (MRSI) mit hyperpolarisiertem 13C-Pyruvat Bewerber für den Young Investigator Award}

Autoren Kaissis $\mathrm{G}^{1}$

Institut 1 Klinikum rechts der Isar, Institut für diagnostische und interventionelle Radiologie, München

DOI 10.1055/s-0037-1600194

Zielsetzung Die Energiegewinnung durch aerobe Glykolyse ("Warburg-Effekt") zeichnet verschiedene Tumorarten aus. Die MRSI mit hyperpolarisierten Substanzen (z. B. C-13-Pyruvat) erlaubt die nicht-invasive Evaluation des Tumormetabolismus. Ziel dieser Arbeit war die Evaluation der 13C-Pyruvat MRSI als Methode zur metabolen Subgruppendetektion im HCC.

Material und Methodik HCCs wurden in Wistar-Ratten mit oralem Diethylnitrosamin (8 Wochen) induziert. $20 \mathrm{HCC}$ tragende und 6 gesunde Kontrolltiere wurden mittels hochaufgelöster T2-gewichteter Bildgebung und Free-Induction-Decay-Chemical Shift Imaging (FID-CSI) untersucht. 5 HCC tragende Tiere wurden zusätzlich mittels 18F-FDG-PET untersucht. Tumor- und gesundes Lebergewebe wurden histopathologisch untersucht und mit der in-vivo Bildgebung korreliert.

Ergebnisse Der Label-Exchange-Quotient zwischen 13-C-Pyruvat/Laktat und 13-C-Pyruvat/Alanin lag in HCCs signifikant höher als in gesundem Lebergewebe (HCC: $1.69 \pm 0.44$; Leber: $1.05 \pm 0.12, p=0.002$ ). Es zeigte sich eine deutliche intertumorale Heterogenität des Lac/Ala-Quotienten $(\sigma 2=0.19) \mathrm{im}$ Vergleich zur gesunden Leber $(\sigma 2=0.016)$. Niedriggradige und hochgradige Tumore zeigten signifikant unterschiedliche Lac/Ala Quotienten. Tumore mit ähnlichem SUV in der PET wiesen deutlich differente Lac/Ala-Quotienten auf. Schlussfolgerungen C-13-Pyruvat-MRSI ermöglicht die Tumordetektion im HCC-Rattenmodell. Ein höheres histopathologisches Tumorgrading korreliert mit höheren Lac/Ala-Quotienten. Die geringe Korrelation der 13C-Pyruvat MRSI mit der 18F-FDG PET Bildgebung ist ein möglicher Indikator für den additiven Wert dieser neuen Bildgebungsmethode.

\section{WISS 311.6 Single-Shot-Diffusionsgewichtete MRT mittels paralleler Bildgebung und 2D-RF-Anregung für die Intravoxel Incoherent Motion (IVIM) Analyse bei 7T im Xenograft-Tumormodell der Maus}

Autoren Kaul $\mathrm{M}^{1}$, Salamon $\mathrm{J}^{1}$, Frenzel $\mathrm{T}^{2}$, Finsterbusch $\mathrm{J}^{3}$, Adam $\mathrm{G}^{1}$, Peldschus $\mathrm{K}^{1}$

Institut 1 Universitätsklinikum Hamburg-Eppendorf, Klinik und Poliklinik für Diagnostische und Interventionelle Radiologie, Hamburg;

2 Universitätsklinikum Hamburg-Eppendorf, Klinik für Strahlentherapie und Radioonkologie, Hamburg; 3 Universitätsklinikum Hamburg-Eppendorf, Institut für Systemische Neurowissenschaften, Hamburg DOI 10.1055/s-0037-1600195

Zielsetzung Im Rahmen der diffusionsgewichten Bildgebung wurden in einem präklinischen Versuch zwei Sequenztechniken, die ohne Multishot-Technik eine Verkürzung der Echozeit erzielen, um verzerrungsfreie Bilder zu gewährleisten, auf ihre Reproduzierbarkeit untersucht.
Material und Methodik Zur Tumorindizierung wurden $10^{6} \mathrm{OH} 1$-Zellen (humane, SCLC-Zelllinie) subkutan in sieben SCID-Mäusen injiziert. MRT Messungen wurden am 15. und 16. Tag an einem präklinischen MRT (7T Clinscan) wiederholend mit einer 8-Kanal Oberflächenspule (Rapid) durchgeführt. Einer transversalen T2-gewichten Sequenz folgten in gleicher Schichtführung zwei DWI Sequenzen nach. Zur Verkürzung des EPI-Auslesezuges wurde bei der ersten Sequenz (1D-RF) ein SENSE-Faktor von 2 gewählt. Bei der anderen Sequenz (2D-RF) wurde ein 2D-RF-Anregungspuls verwendet. Für eine IVIM Analyse wurden b-Werte von 0, 10, 20, 40, 60, 80, 100, 300, 600, 900, $1200 \mathrm{~s} / \mathrm{mm}^{2}$ gewählt. TE betrug bei für die 1D-RF $26 \mathrm{~ms}$, für die 2D-RF 25 ms. Die Matrix betrug $128 \times 128$ respektive $128 \times 48$ Pixel bei einem FOV von $26 \mathrm{~mm}$. Die Bildanalyse erfolgte mittels einer Bilddatenverarbeitung (Imagej) und der Softwareerweiterung (qMapIT). Abschließend wurde eine deskriptive statistische Analyse (Mittelwert \pm Standardabweichung) sowie t-Tests durchgeführt.

Ergebnisse Das mittlere Tumorvolumen betrug $21.4 \pm 2.5 \mathrm{~mm}^{3}$. Die 1DRF-Sequenz zeigte vereinzelt Ghostig-Artefakte, welche bei der 2D-RF-Sequenz nicht auftraten. Im direkten, paarweisen Vergleich zeigte der t-Test signifikante Unterschiede: Diffusion $1317 \pm 118 \mu \mathrm{m}^{2} / \mathrm{s}$ vs. $971 \pm 123 \mu \mathrm{m}^{2} / \mathrm{s} \quad\left(p<1.3^{*}\right.$ $\left.10^{-6}\right)$ und Perfusionsfraktion $20.4 \pm 2.3 \%$ vs. $25.6 \pm 2.8 \%\left(p<1.7^{*} 10^{-7}\right)$. Der mittlere relative Messfehler betrug für die Diffusion $8 \%$ vs. $24 \%$ (infolge eines Ausreißer) und für die Perfusionsfraktion von $8 \%$ vs. $6 \%$.

Schlussfolgerungen Beide DWI Techniken ermöglichen eine verzerrungsarme Bildgebung bei hoher Feldstärke trotz Single-Shot-Akquisition. Trotz der Tendenz zu Anfälligkeit durch Ghosting-Artefakten scheint die parallele Bildgebung die robustere Technik zu sein.

\section{WISS 312.4 Experimentelle ex vivo Flussstudien an Schweinenieren durch eine Multimodale Angiografie unter Verwendung von DSA, MRA und Magnetic Particle Imaging}

Autoren Kaul $\mathrm{M}^{1}$, Molwitz $\mathrm{I}^{1}$, Jung $\mathrm{C}^{1}$, Salamon J $\mathrm{J}^{1}$, Knopp $\mathrm{T}^{2}$, Adam $\mathrm{G}^{1}$, Ittrich $\mathrm{H}^{1}$

Institut 1 Universitätsklinikum Hamburg-Eppendorf, Klinik und Poliklinik für Diagnostische und Interventionelle Radiologie, Hamburg;

2 Universitätsklinikum Hamburg-Eppendorf, Abteilung für Biomedizinische Bildgebung, Hamburg

DOI 10.1055/s-0037-1600196

Zielsetzung Zielsetzung war die multimodale Beurteilung von Fluss und Perfusion in ex-vivo Schweinenieren, insbesondere mittels der neuartigen Bildgebungstechnik Magnetic Particle Imaging (MPI). MPI erfasst mit einer hohen zeitlichen Auflösung die dreidimensionale Verteilung von magnetischen Partikeln im Körper/Organ. Um MPI für die Gefäßdarstellung und für Perfusionsstudien zu testen und mit anderen Modalitäten vergleichen zu können, wurde ein ex vivo Flussphantom etabliert.

Material und Methodik Nieren von Schweinen wurden direkt nach der Schlachtung mit Kochsalz und Heparin perfundiert. Die arteriellen und venösen Hauptgefäße sowie der Ureter wurden mit Schläuchen verbunden. Die Flüsse wurden mit einer Perfusionspumpe generiert. Um eine Verschiebung der Nieren während des Transportes zwischen den Modalitäten zu minimieren, wurden diese auf eine Liege fixiert, welche kompatibel zu den eingesetzten Bildgebungstechniken war. Zum Einsatz kamen eine klinische digitale Subtraktionsangiografie Einheit (Allura, Philips), ein präklinisches Hochfeld Magnet-Resonanz-Tomografie System (7T ClinScan, Bruker) und ein präklinisches MPI System (Bruker/Philips). Mit jeder Modalität wurde eine dynamische Messsequenz durchgeführt. Hierbei wurde ein jeweilig dezidiertes Kontrastmittel (Imeron 300 und Ominscan) bzw. MPI Tracer (Resovist) standarisiert appliziert.

Ergebnisse Es wurden mit allen drei Systemen erfolgreich Angiogramme erstellt. Die Gewebsperfusion ließ sich detektieren. Da der Volumenstrom durch die Pumpe gesteuert wurde, ließ sich bei der MR-Bildgebung zeitliche Auflö- 
sung gegen räumliche Auflösung opfern und eine Submillimeterauflösung erzielen. Auch sind Perfusionsexperimente für die Untersuchung der diffusionsgewichteten Bildgebung zur Intravoxel Incoherent Motion Analyse möglich.

Schlussfolgerungen Das ex vivo Nierenflussphantom wurde erfolgreich etabliert und kann für eine bildgestützte Evaluierung von Bildgebung und Bildanalysetechniken eingesetzt werden. Das MPI bietet das Potential einer direkten Bewertung der Gewebeperfusion.

\section{WISS 311.7 Identifizierung von Mtss1 als neues Kandidaten-Gen für die Entwicklung des kongenitalen kommunizierenden Hydrozephalus - Eine Maus-MRT Analyse}

Autoren Keil $V^{1}$, Wieners $B^{2}$, Vasyukov $M^{2}$, Keil $V^{1}$, Gieseke $J^{1}$, Baader $S^{2}$, Hattingen $E^{1}$, Schild $H^{1}$, Eiberger $B^{2}$

Institut 1 UK Bonn, Radiologie, Bonn; 2 RFWU Bonn, Anatomisches Institut, Anatomie und Zellbiologie, Bonn

DOI 10.1055/s-0037-1600197

Zielsetzung Das multifunktionelle Protein Mtss1, das bisher vor allem im Hinblick auf seine Regulation in verschiedenen Tumoren untersucht wurde, stellt nicht nur eine Verbindung zwischen dem Aktinzytoskelett und der Zellmembran her, sondern wurde auch als Interaktionspartner von Basalkörpern primärer Zilien nachgewiesen. Da Mtss1 im Ependym exprimiert wird, vermuten wir einen Effekt auf die Funktionalität ependymaler Zilien und damit einen Einfluss auf die Ausprägung einer Ventrikulomegalie. Ziel dieser kontrollierten prospektiven Maus-MRT-Studie ist die quantitative und qualitative Untersuchung der Arbeitshypothese.

Material und Methodik Je 12 Mtss1-defiziente und Wildtyp-Kontrollmäuse wurden anästhesiert und bei 3T mittels Mauskopfspule untersucht (sagittale T2 TSE, 27 Schichten, Schichtdicke 0,5 mm, TE $97 \mathrm{~ms}$, TR $3 \mathrm{~s}, 124 \times 117 \mathrm{~mm}$ Matrix, $0.2 \times 0.2 \times 0.5 \mathrm{~mm}$ Voxel, Scandauer 8 Min.; Philips Ingenia MRT, Rapid Biomedical Spule). Jeder Ventrikel wurde manuell individuell volumetriert (Philips Intellispace 5.0) und das Totalvolumen sowie die Einzelvolumina der inneren Liquorräume bestimmt. Die Ausprägung einer Aquäduktstenose oder einer Liquordiapedese wurden ebenfalls untersucht. Es erfolgten zusätzlich funktionelle und histologische Analysen.

Ergebnisse Mtss1-defiziente Mäuse wiesen signifikant vergrößerte Seitenventrikel ohne Vorliegen einer Aquäduktstenose auf (Mittelwert: $21.2 \mathrm{~mm}^{3}$ vs. Wildtyp $\left.6.6 \mathrm{~mm}^{3} ; \mathrm{p}<0.0001\right)$. Eine Liquordiapedese konnte nur in zwei Tieren mit stark ausgeprägtem Hydrozephalus festgestellt werden. Das Ventrikelvolumen korrelierte nicht mit dem Tieralter $(p=0.06)$. Die histologischen und funktionellen Analysen zeigten, dass bei Mtss1-defizienten Tieren Veränderungen der ependymalen Zilien vorlagen.

Schlussfolgerungen Die MRT-basierte Mäusehirnanalyse zeigt, dass eine Mtss1-Defizienz einen Hydrocephalus internus der Seitenventrikel verursacht. Mtss1 kann folglich als neues Kandidatengen für die Entwicklung eines kongenitalen, kommunizierenden Hydrozephalus angesehen werden.

\section{WISS 311.2 Evaluation von DNA- Doppelstrangbrüchen humaner Blutlymphozyten nach zerebralen MRT-Untersuchungen}

\author{
Autoren Lanzman $R^{1}$, Dickmann $C^{1}$, Ljimani $A^{1}$, Aissa J ${ }^{1}$, Schroeder $T^{2}$, \\ Cadeddu $R^{2}$, Kröpil $P^{1}$, Antoch $G^{1}$, Heusch $P^{1}$ \\ Institut 1 Universitätsklinikum Düsseldorf, Institut für Diagnostische und \\ Interventionelle Radiologie, Düsseldorf; 2 Universitätsklinikum Düsseldorf, \\ Klinik für Hämatologie, Düsseldorf \\ DOI 10.1055/s-0037-1600198
}

Zielsetzung Im Rahmen dieser Studie soll untersucht werden, ob nach klinisch indizierten MRT-Untersuchungen des Schädels gehäuft potenziell kanzerogene DNA-Doppelstrangbrüche in humanen Blutlymphozyten auftreten.
Material und Methodik 50 Patienten (24 Männer, 26 Frauen, Alter: 56,7 $\pm 15,3$ Jahre) wurden prospektiv in diese Studie eingeschlossen. Vor und nach einer zerebralen MRT-Untersuchungen bei 1.5T $(n=39)$ oder 3T $(n=11)$ wurde bei allen Patienten eine periphere Blutentnahme durchgeführt. Die Blutproben wurden mithilfe des Antikörpers $\mathrm{\gamma H} 2 \mathrm{AX}$ auf Doppelstrangbrüche untersucht. Dabei wurde eine automatisierte Zählung der Doppelstrangbrüche mittels Durchflusszytometrie (FACSCalibur, BD Bioscience, Heidelberg, Deutschland) und eine manuelle Zählung der Doppelstrangbrüche mittels Fluoreszenzmikroskopie durchgeführt.

Ergebnisse In der Durchflusszytometrie zeigte sich bezüglich der Doppelstrangbrüche kein signifikanter Unterschied vor und nach der MRT-Untersuchung. So betrug der Anteil pH2A.X-positiver Lymphozyten an allen Lymphozyten vor MRT $0,15 \pm 0,20 \%$ und nach MRT auf $0,14 \pm 0,19 \%(p=0,39)$. Mithilfe der Fluoreszenzmikroskopie wurden im Durchschnitt $47 \pm 29$ Lymphozyten ausgezählt. Hier zeigte sich ein geringer, nicht signifikanter Anstieg der Doppelstrangbrüche von $0,049 \pm 0,022$ pro Zelle vor MRT auf $0,051 \pm 0,027$ pro Zelle nach MRT $(p=0,21)$.

Schlussfolgerungen Zerebrale MRT- Untersuchungen führen nicht zu vermehrten DNA-Doppelstrangbrüchen in humanen Blutlymphozyten. Es ergibt sich somit kein Anhalt für eine potentiell karzinogene Wirkung.

\section{WISS 311.4 Verbesserung der Bildqualität zur Detektion intrakranieller Blutungen und hypodenser Defektareale durch monoenergetische Rekonstruktionen der Spektraldetektor- Computertomografie}

Autoren Lennartz $\mathrm{S}^{1}$, Laukamp $\mathrm{K}^{1}$, Neuhaus $\mathrm{V}^{1}$, Große Hokamp $\mathrm{N}^{1}$, Abdullayev $\mathrm{N}^{1}$, Maintz $\mathrm{D}^{1}$, Mpotsaris $\mathrm{A}^{1}$, Borggrefe $\mathrm{J}^{1}$

Institut 1 Universitätsklinikum Köln, Institut für Diagnostische und Interventionelle Radiologie, Köln

DOI 10.1055/s-0037-1600199

Zielsetzung Vergleich der Abgrenzbarkeit von intrakraniellen Blutungs- und Defektarealen in monoenergetischen Rekonstruktionen (MER) und konventionellen gefilterten Rückprojektionen (FBP) der Spektraldetektor-CT (SDCT). Material und Methodik SDCT (IQon ${ }^{\circledR}$, Philips, Amsterdam, NL) von jeweils 29 Patienten mit intrakraniellen Blutungen und hypodensen Defektarealen $(\mathrm{n}=58)$. Protokoll: Natives Schädel-CT mit Schichtdicke $1 \mathrm{~mm}$, pitch 1 , 320 mAs, 120 kVp. Bildrekonstruktion mit FBP und MER (40 - 120 keV; Intervall 10keV). ROI-gestützte Bestimmung der Dichte (HU), des Rauschens (SD der jeweiligen ROI) sowie des Kontrast-zu-Rausch-Verhältnisses (CNR) in folgenden Arealen: 1) Blutung bzw. Defektareal 2) Marklager 3) Rinde 4) Liquor. Ergebnisse Gegenüber $40 \mathrm{keV}$ und FBP zeigte sich bei $120 \mathrm{keV}$ ein signifikant besseres CNR hypodenser Läsionen zum Marklager (MER 120keV: 4,9 2,5, MER 40keV: 1,4 $\pm 2,9(p<0,0001)$, FBP: $3,2 \pm 1,8(p<0,01))$ und hyperdenser Läsionen zur grauen Substanz (MER 120keV: $8,7 \pm 4.27$, MER 40keV: 0,43 $\pm 6,4$ $(p<0,0001)$, FBP: $6,0 \pm 3,1(p<0,01))$. Invers hierzu zeigte sich gegenüber 120 $\mathrm{keV}$ und FBP bei $40 \mathrm{keV}$ ein signifikant besseres CNR hyperdenser Läsionen zum Marklager (MER 40keV: 13,0 $\pm 6,8$, MER 120keV: 9,1 $\pm 3,7$ ( $p<0,05)$, FBP: $9,0 \pm 3,0(p<0,01))$ und hypodenser Läsionen zur grauen Substanz (MER 40keV: 11,2 $\pm 3,7$, MER 120keV: 4,9 $\pm 2,4$ ( $p<0,0001)$, FBP: $5,7 \pm 2,2$ $(p<0,0001))$. Das CNR zwischen grauer und weißer Substanz zeigte einen kontinuierlichen Anstieg mit absteigender keV (MER 40keV: 10,2 $\pm 3,1$, MER 120keV: $0,11 \pm 0,99(\mathrm{p}<0,0001))$.

Schlussfolgerungen MER der SDCT ermöglichen gegenüber FBP eine deutliche CNR-Steigerung. Rekonstruktionen mit niedrigen wie auch hohen keV sind in Abhängigkeit der Läsion und Lokalisation ggf. sinnvolle Ergänzungen zur FBP. MER 120keV zeigen einen hohen Kontrast zur sensitiveren Detektion von hyperdensen und hypodensen Läsionen, insbesondere für hypodense Marklagerläsionen. MER 40keV zeigen eine gute Kontrastierung der grauen Substanz und erlauben hierdurch die präzisere Lokalisation von Läsionen innerhalb der Stammganglien. 
WISS 312.1 Elektrische Impedanz Tomografie (EIT) überwacht valide und robust globale und regionale Ventilation in physiologischen und pathologischen Beatmungszuständen im Schweinemodell

Autoren Reinartz $\mathrm{S}^{1}$, Imhoff $\mathrm{M}^{2}$, Tolba $\mathrm{R}^{3}$, Fischer $\mathrm{F}^{2}$, Fischer $\mathrm{E}^{4}$, Teschner $\mathrm{E}^{2}$, Koch $\mathrm{S}^{3}$, Gärber $\mathrm{I}^{2}$, Isfort $\mathrm{P}^{1}$, Gremse $\mathrm{F}^{5}$

Institut 1 Universitätsklinikum Aachen, Klinik für Diagnostische und Interventionelle Radiologie, Aachen; 2 Fa. Dräger, Lübeck; 3 ILAS, Aachen; 4 Fa. Aix Scientifics (CRO), Aachen; 5 ExMI-Experimental Molecular Imaging, Aachen

DOI 10.1055/s-0037-1600200

Zielsetzung Präzises Beatmungsmonitoring und die frühzeitige, sichere Diagnose pathologischer Beatmungszustände sind entscheident zur Vermeidung eines ARDS in kritisch kranken Patienten. Da EIT diese Information bettseitig kontinuierlich prinzipiell zur Verfügung stellt, wurde EIT systematisch im Tierversuch in physiologischen und pathologischen Beatmungszuständen im Vergleich mit Dual-Energy-CT und Respiratordaten hinsichtlich globaler und regionaler Ventilation validiert.

Material und Methodik Für alle 13 Schweine (dt. Landrasse, ca. 70 kg) wurden insgesamt 18 Teilversuche, zunächst mit abfallendem Tidalvolumen (12, 10 und $8 \mathrm{ml} / \mathrm{kg}$ Körpergewicht) unter verschiedenen PEEP-Niveaus (15, 10, und $5 \mathrm{mmH} 2 \mathrm{O}$ ), unter ein und zwei Lungenventilation sowie nach Lungenlavage mit langsamem Recruitment Maneuver mithilfe eines inkrementellen PEEP von 0 bis $25 \mathrm{mmH} 2 \mathrm{O}$ in $5 \mathrm{mmH} 20$ Schritten durchgeführt. In jeder Beatmungskondition wurden synchrom EIT Signalintensitäten, Respiratorparameter als auch jeweils ein DualEnergy CT des Thorax zur Artefaktreduktion des EIT Gürtels jeweils in endexspiratorischer und endinspiratorischer Atemlage erfasst. DECT: Kollimation $128 \times 0.6 \mathrm{~mm}, 100 / 140 \mathrm{kV}, 380 \mathrm{~mm} 2$ FOV. Virtuell monochromatische Datensätze mit minimalem Artefakt wurden erzeugt und ausgewertet. Das Qualtiätsmanagement wurde durch eine CRO überwacht.

Ergebnisse Die Korrelation zwischen EIT Signal und Respiratormessungen ergibt eine Pearson Koeffizienten von $\mathrm{R}=0,983$ (95\%Cl: $0.979-0.987$, Endpunkt 1). Der Endpunkt 2 zeigt eine signifikante Änderung des EIT Signals bei Ein-Lungenventilation sowie eine Korrelation der Differenzen der endexpiratorischen Lungenvolumina (Delta-EELV) aus der DECT mit den korrespondierenden Differenzen der endexspiratorischer Lungenimpedanz (Delta-EELI) des EIT im Bezug auf 4 Lungenquadranten mit $R=0,94$.

Schlussfolgerungen Im Vergleich mit dem jeweiligen Goldstandard wurde EIT anhand typischer Beatmungssituationen systematisch validiert und eigenet sich somit als robustes Werkzeug zur Überwachung der Beatmung in der Intensivmedizin.

\section{WISS 304.2 Visualisierung des Temperaturverlaufes in einem Lebertumor-Ablationsmodell mittels Magnetic Particle Imaging}

Autoren Salamon $\mathrm{J}^{1}$, Dieckhoff $\mathrm{J}^{1}$, Jung $\mathrm{C}^{1}$, Kaul M${ }^{1}$, Adam $\mathrm{G}^{1}$, Knopp $\mathrm{T}^{2}$, Ittrich $\mathrm{H}^{1}$

Institut 1 Universitätsklinikum Hamburg-Eppendorf, Klinik und Poliklinik für Diagnostische und Interventionelle Radiologie und Nuklearmedizin, Hamburg; 2 Universitätsklinikum Hamburg-Eppendorf und Technische Universität Hamburg, Abteilung für experimentelle biomedizinische Bildgebung, Hamburg

DOI 10.1055/s-0037-1600201

Zielsetzung Evaluation der Machbarkeit der Visualisierung des Temperaturverlaufes während einer Thermoablation in einem in vitro Lebermodell mittels MPI unter Verwendung verschiedener Eisenoxidtracer.

Material und Methodik In vitro Pseudo-Lebergewebs-Phantome mit verschiedenen Eisenoxidtracern (L93, Bayer-Schering; LS008, Load Spin Labs; MM4, TOPASS GmbH, Konzentrationen von $0.1-0.5 \mathrm{mg} / \mathrm{ml}$ ) in einer Proteinmatrix (Chicken White Protein, Sigma Aldrich) wurden durch 1:1 Volumen-Mischung mit Wasser über $15 \mathrm{~min}$. bei $75^{\circ} \mathrm{C}$ in Eppendorf-Tubes generiert. Die Phantome wurden mittels eines eingebrachten Kupferdrahtes ( $1 \mathrm{~mm}$ Durchmesser) durch MPI-induzierte Wirbelströme erhitzt und resultierende Signalveränderungen simultan bildgebend mittels MPI erfasst. Als in vitro Lebertumor-Ablationsmodell wurde Tracer-freies Protein (Pseudotumoren) in mit L93 (c(Fe) $=0.356 \mathrm{mg} / \mathrm{ml}$ ) vermengtem Protein (Pseudo-Lebergewebe) eingebettet. Die Pseudotumoren wurde mittels eines eingebrachten Kupferdrahtes unter simultaner Erfassung des MPI-Signals des umgebenden Pseudo-Lebergewebes erwärmt. Alle Experimente erfolgten an einem kommerziellen MPI System (Philips/Bruker) unter Verwendung eines FoV von $37.3 \times 37.3 \times 18.6 \mathrm{~mm} 3$ und einer Bildfrequenz von 46 Bildern/s.

Ergebnisse Bei allen verwendeten Tracern ließen sich im zeitlichen Verlauf unter Erwärmung MPI-Signalsteigerungen detektieren. LS008 zeigte die höchste Signaländerung. Im Lebertumor-Ablationsmodell war die Erwärmung des Pseudo-Lebergewebes im zeitlichen Verlauf Signaländerungen zu visualisieren.

Schlussfolgerungen MPI eignet sich zur Visualisierung der Temperaturverteilung in einer mit Eisenoxidtracern angereicherten Protein-Wasser-Matrix als Pseudo-Lebergewebe, die entscheidend von eingesetzten Tracer abhängt. Eine Temperaturüberwachung gesunden Gewebes zur optimierten MPI-gestützten Tumorablation in Echtzeit ist denkbar.

\section{WISS 312.6 Einfluss des freien Hämoglobins auf die Manifestation des akuten Nierenversagens im Mausmodell - nicht-invasive Untersuchung mittels ASL und T2-Mapping}

Autoren Schmidbauer $\mathrm{M}^{1}$, Chen $\mathrm{R}^{2}$, Wang $\mathrm{L}^{2}$, Gutberlet $\mathrm{M}^{1}$, Bräsen $\mathrm{J}^{3}$, Meier $\mathrm{M}^{4}$, Madyaningrana $\mathrm{K}^{5}$, Vijith $\mathrm{V}^{5}$, Wacker $\mathrm{F}^{1}$, Immenschuh $\mathrm{S}^{5}$, Gueler $\mathrm{F}^{2}$, Hueper $\mathrm{K}^{1}$

Institut 1 Medizinische Hochschule Hannover, Institut für Diagnostische und Interventionelle Radiologie, Hannover; 2 Medizinische Hochschule Hannover, Institut für Nephrologie, Hannover; 3 Medizinische Hochschule Hannover, Institut für Pathologie, Hannover; 4 Medizinische Hochschule Hannover, Zentrum für Kleintierbildgebung, Hannover; 5 Medizinische Hochschule Hannover, Institut für Transfusionsmedizin, Hannover DOI 10.1055/s-0037-1600202

Zielsetzung Freies Hämoglobin kann als endogenes Nephrotoxin in pathologisch erhöhten Konzentrationen durch seine tubulo-toxische und vasokonstringierende Wirkung zum akuten Nierenversagen (ANV) führen. Ziel dieser Studie war es, den Einfluss freien Häms auf die Entstehung des ANV nach kurzer Ischämie mittels nicht-invasivem Arterial Spin Labeling (ASL) und T2Mapping zu evaluieren.

Material und Methodik Ein subklinischer Ischämie-Reperfusionsschaden (IRSchaden) wurde bei C57BL/6-Mäusen durch bilaterales temporäres Abklemmen der Nierengefäße über 15 Minuten induziert. Bei $n=11$ Tieren erfolgte eine einmalige Applikation von Häm (20 mg/kg i.v., IR-Häm), 10 Tiere erhielten Vehikel (reine Pufferlösung, IR-Vehikel). Als Vergleichsgruppe dienten Mäuse mit Sham Operation ( $n=6$ Sham-Vehikel, $n=5$ Sham-Häm). FAIR ASLund Multi-Echo Spin-Echo-Sequenzen wurden am 7T-MRT einen Tag nach Operation akquiriert und die renale Perfusion und T2-Relaxationszeiten erhoben. Serumkreatininspiegel wurden präoperativ und an Tag 1 postoperativ bestimmt.

Ergebnisse Nach subklinischem IR-Schaden war die Nierenperfusion Häm-behandelter Tiere mit $300 \pm 17 \mathrm{ml} / \mathrm{min} / 100 \mathrm{~g}$ postoperativ signifikant gegenüber allen übrigen Versuchsgruppen reduziert (IR-Vehikel $395 \pm 31$, Sham-Häm 434 \pm 23 , Sham-Vehikel $391 \pm 35 \mathrm{ml} / \mathrm{min} / 100 \mathrm{~g}, \mathrm{p}<0,05)$. Ebenso waren die T2Zeiten in der IR-Häm Gruppe mit $48 \pm 3$ ms erhöht (IR-Vehikel $42 \pm 1$, ShamHäm $40 \pm 1$, Sham-Vehikel $36 \pm 1 \mathrm{~ms}$ ). Korrespondierend zu den MR-Parametern zeigten nur Häm-IR-Tiere einen zum Ausgangswert signifikanten Kreatininanstieg ( $26 \pm 1$ vs. $81 \pm 22 \mu \mathrm{mol} / \mathrm{L}, \mathrm{p}<0,05)$. MRT-Parameter und Kreatininspiegel korrelierten dabei signifikant (Perfusion: $r=-0,48, T 2: r=0,58$ ). 
Schlussfolgerungen Freies Häm führt zur Aggravation eines subklinischen IRSchadens, was anhand einer signifikanten Perfusionseinschränkung und Erhöhung der T2-Relaxationszeit messbar ist. Die Ergebnisse der funktionellen MRT korrespondieren mit laborchemischen Messungen der Nierenfunktion und erlauben zusätzlich eine multiparametrische Analyse und regionale Zuordnung der Schädigung.

\section{WISS 311.1 Diffusionsbildgebung mittels RESOLVE- DWI bei $3 T$ kann zwischen differenzierten und undifferenzierten Schilddrüsenkarzinomen unterscheiden}

Autoren Schob $\mathrm{S}^{1}$, Voigt $\mathrm{P}^{2}$, Höhn $\mathrm{A}^{3}$, Meyer $\mathrm{H}^{4}$, Wickenhauser $\mathrm{C}^{5}$, Behrmann $\mathrm{C}^{4}$, Kachel $\mathrm{P}^{6}$, Dralle $\mathrm{H}^{7}$, Hoffmann $\mathrm{K}^{2}$, Surov $\mathrm{A}^{8}$ Institut 1 Uniklinik Leipzig, Klinik und Poliklinik für diagnostische und interventionelle Radiologie, Abt. für Neuroradiologie, Leipzig; 2 Uniklinik Leipzig, Abteilung für Neuroradiologie, Leipzig; 3 Uniklinik Leipzig, Pathologie, Leipzig; 4 Uniklinik Halle, Radiologie, Halle/Saale; 5 Uniklinik Halle, Pathologie, Halle/Saale; 6 Hämatologie und Onkologie, Universitätsspital Zürich, Zürich; 7 Uniklinik Essen, Endokrine Chirurgie, Essen; 8 Uniklinik Leipzig, Diagnostische und interventionelle Radiologie, Leipzig DOI 10.1055/s-0037-1600203

Zielsetzung Schilddrüsenkarzinome stellen die häufigsten malignen Tumore des endokrinen Systems dar. In jüngeren Studien wurde gezeigt, dass die MRDiffusionsbildgebung (DWI) zwischen malignen und benignen Noduli differenzieren kann. Obgleich diese Unterscheidung zweifellos hilfreich ist, wäre eine weitere Abgrenzbarkeit von undifferenzierten und differenzierten Tumoren wichtig, um den optimalen Behandlungsalgorithmus zu identifizieren. Ziel der Studie war es daher zu untersuchen, ob DWI mittels RESOLVE Sequenz differenzierte von undifferenzierten Schilddrüsenkarzinomen unterscheiden kann.

Material und Methodik 14 Patienten mit unterschiedlichen Schilddrüsenkarzinomen, welche mit präopertiver MRT untersucht wurden, konnten in unsere Studie eingeschlossen werden. In jedem Tumor wurden ADCmin, ADCmean, ADCmax and D nach Koregistrierung auf T1-gewichtete Bilder nach KM-Gabe ermittelt. Alle Tumoren wurden reseziert und histologisch untersucht. Ki-67 Index, p53 Status und Zellularität (Zellzahl, Kernfläche) wurden quantifziert. Ergebnisse ANOVA ergab statistisch signifikante Unterschiede bzgl. der ADCmean Werte von differenzierten vs. undifferenzierten Schilddrüsenkarzinomen $(p=0.022)$. Die Spearman-Rho Berechnung identifizierte signifikante Korrelationen zwischen $A D C$ max und Zellularität $(r=0.541, p=0.046)$ sowie zwischen ADCmean und Kernfläche $(r=0.605, p=0.022)$.

Schlussfolgerungen Die Diffusionsbildgebung mittels RESOLVE-Sequenz bei 3T kann zwischen differenzierten und undifferenzierten Schilddrüsenkarzinomen unterscheiden.

\section{WISS 304.1 Entwicklung und Evaluation eines temporären Embolisationsmaterials zur arteriellen Transkatheter-Applikation im Kaninchenmodell}

\author{
Autoren Stechele $\mathrm{M}^{1}$, Wittgenstein $\mathrm{H}^{1}$, Breinl $\mathrm{J}^{2}$, Stolzenburg $\mathrm{N}^{2}$, \\ Schnapauff $D^{1}$, Rudolph $B^{3}$, Schmidt $C^{4}$, Schnorr $J^{2}$, Hamm $B^{1}$, Günther $R^{1}$, \\ Streitparth $\mathrm{F}^{1}$ \\ Institut 1 Charité Universitätsmedizin Berlin, Klinik für Radiologie, Berlin; \\ 2 Charité Universitätsmedizin Berlin, Institut für Radiologie, Berlin; 3 Charité \\ Universitätsmedizin Berlin, Institut für Pathologie, Berlin; 4 microParticles \\ $\mathrm{GmbH}$, Berlin \\ DOI 10.1055/s-0037-1600204
}

Zielsetzung Evaluation der Embolisationsfähigkeit, Gewebeverträglichkeit und Resorbierbarkeit eines neuen temporären Embolisationsmaterials zur transarteriellen Katheterembolisation im Kaninchenmodell

Material und Methodik Angiografische superselektive Embolisation einer Nierensegmentarterie des Unterpols mit Polydioxanon-Mikrosphären (50-
$500 \mu \mathrm{m}$ ) an 24 Kaninchen (New Zealand White Rabbit; w; 2,5-3,5 kg). Es wurden $\mathrm{n}=15$ blande $\& \mathrm{n}=9$ SPIO-beladene Mikrosphären verwendet. Evaluation des Embolisationseffekts mittels DSA \& MRT (T1-TSE prä/post-KM, T2TSE, T2*-GRE). 4 Tiere wurden direkt nach der Embolisation euthanasiert (Akutgruppe), 20 Tiere wurden nach einem Zeitraum von $1(n=3), 4(n=3)$, $8(n=3), 12(n=8) \& 16$ Wochen $(n=3)$ einem Kontrollangiogramm zur Beurteilung von Embolisationserfolg \& arterieller Reperfusion sowie erneuter MRT unterzogen. Abschließend histopathologische Untersuchung auf Embolisationseffekt, Gewebeverträglichkeit \& Resorbierbarkeit der Mikrosphären.

Ergebnisse In DSA \& MRT zeigte sich eine erfolgreiche superselektive Embolisation bei 18/20 Tieren, bei 2/20 Tieren eine Embolisation der gesamten Niere. Die T2*-GRE erlaubte die Deteketion und Verteilung der SPIO-beladenen Mikrosphären. Bei Vergleich der durchgeführten DSA direkt postinterventionem und zum Zeitpunkt der Euthanasie zeigte sich eine partielle Reperfusion der Gefäße. Die makro- und mikroskopische Auswertung zeigte Infarkt-/ Nekrosezonen in den embolisierten Nierenbereichen sowie leichte akute Entzündungsreaktion in allen Tieren. Fremdkörperreaktionen waren nur bei fortgeschrittenen Beobachtungszeiträumen erkennbar. Mit zunehmenden Beobachtungszeiträumen waren die Mikrosphären mikroskopisch zunehmend abgebaut, zum Zeitpunkt nach 16 Wochen mit einem Degradations-score von $2,9 / 3$.

Schlussfolgerungen Die neuen bioresorbierbaren Polymer-Mikrosphären erzielten einen erfolgreichen Gefäßverschluss mit guter Biokompatibilität. Das Embolisationsmaterial war nach 16 Wochen deutlich resorbiert und bietet somit die Möglichkeit zur Re-Angiografie, z. B. bei der TACE.

\section{Gastro- und Abdominaldiagnostik}

\section{WISS 206.5 Abdominale Computertomografie (CT) zur Detektion von Body-Packing: Wie weit kann die Strahlendosis reduziert werden?}

Autoren Aissa $\rfloor^{1}$, Sawicki $L^{1}$, Heusch $\mathrm{P}^{1}$, Appel $E^{1}$, Kröpil $\mathrm{P}^{1}$, Thomas $\mathrm{C}^{1}$, Antoch $\mathrm{G}^{1}$, Boos ] $^{1}$

Institut 1 Universitätsklinikum Düsseldorf, Institut für Diagnostische und Interventionelle Radiologie, Düsseldorf

DOI 10.1055/s-0037-1600205

Zielsetzung In dieser Studie sollte anhand von Abdomen-CT-Untersuchungen mit simuliert unterschiedlichen Strahlendosisexpositionen ein sinnvolles Ausmaß der Dosisreduktion im Hinblick auf die Detektierbarkeit von ingestierten Drogencontainern und Inzidentalbefunden ermittelt werden.

Material und Methodik Siebenundzwanzig Individuen (20 männlich, 7 weichlich, 37,8 $\pm 11,1$ Jahre) die zwischen 11/2014 und 02/2016 eine AbdominenCT zur Detektion von ingestierten Drogencontainern erhielten $(80 \mathrm{kV}$, automated tube current modulation, eff. dose $2.1 \pm 1.3 \mathrm{mSv}$ ) wurden retrospektiv eingeschlossen. Mithilfe eines validierten Nachverarbeitungsprogramms zur Erhöhung des Bildrauschens (ReconCT 13.8.2.0, Siemens Healthcare GmbH, Erlangen, Deutschland) wurden die CT-Bilder mit simulierten Strahlendosen von $50 \%, 10 \%, 5 \%$ and $1 \%$ der initialen Dosis rekonstruiert. Die originären Rekonstruktionen (100\%) dienten als Referenzstandard. Drei unabhängige und geblindete Radiologen beurteilten alle Rekonstruktionen in zufälliger Reihenfolge im Hinblick auf 1. Die Anwesenheit und Zusammensetzung von Drogencontainern; 2. Die Bildqualität anhand einer 5-Punkt-Skala und 3. Die Anwesenheit von Inzidentalbefunden.

Ergebnisse Zwischen Rekonstruktionen mit 100\% und 50\% Dosis war die Bildqualität vergleichbar $(p>0,05)$. Eine Dosisreduktion von mehr als $50 \%$ führte zu signifikant schlechterer Bildqualität $(p<0,01)$. Der Grenzwert für die korrekte Identifikation von sämtlichen Inzidentalbefunden lag bei $10 \%$ der Ausgangsdosis (eff. Dosis von $0,21 \pm 0,13 \mathrm{mSv}$ ). Der Grenzwert für die korrekte Identifikation aller 8 Individuen mit ingestierten Drogencontainern durch alle drei Radiologen lag bei $5 \%$ der Ausgangsdosis (eff. Dosis $0,1 \mathrm{mSv}$ ); eine Dosisreduktion auf $1 \%$ führte hingegen zu drei falsch-positiven Fällen. 
Schlussfolgerungen Eine effektive Dosis von 0,1 mSv scheint ausreichend zur Detektion von ingestierten Drogencontainern in der Abdomen-CT. Zur sicheren Detektion von Inzidentalbefunden ist jedoch eine höhere Dosis notwendig.

\section{WISS 302.6 Natives Fast-MRI mit DWI zur radiologischen Evaluation einer Appendicitis}

Autoren Goessmann $\mathrm{H}^{1}$, Dendl $\mathrm{L}^{1}$, Pregler $\mathrm{B}^{1}$, Wiggermann $\mathrm{P}^{1}$, Scherer $\mathrm{M}^{2}$, Opitz $S^{2}$, Niessen $C^{1}$, Fellner $C^{1}$, Stroszczynski $C^{1}$, Schreyer $A^{1}$

Institut 1 Universitaetsklinikum Regensburg, Institut fuer

Roentgendiagnostik, Regensburg; 2 Universitaetsklinikum Regensburg, Klinik und Polyklinik für Chirugie, Regensburg

DOI 10.1055/s-0037-1600206

Zielsetzung Evaluation einer schnellen, nativen und sequenzreduzierten MRT („Fast-MRI“) ohne orale, rektale oder i.V. Kontrastierung bezüglich der diagnostische Sicherheit bei klinischem V.a. akute Appendizitis.

Material und Methodik 47 konsekutive Patienten mit klinischem Verdacht auf akute Appendizitis wurden prospektiv in einem Haus der Maximalversorgung in die Studie eingeschlossen. Zusätzlich zum klinischen Standardvorgehen (Labor und klinische Untersuchung) wurde ein „Fast-MRI“ mit 12 Minuten Untersuchungsdauer mit einer orientierenden T2 Haste axial und DWI (b00; 600; 1000) durchgeführt. Eine Diffusionsrestriktion der Appendix wurde dabei als entzündungsverdächtig gewertet und das Ergebnis der MRT gegen den klinischen Verlauf oder den intraoperativen Befund verglichen.

Ergebnisse Von den 47 Patienten wurden 25 operativ versorgt. Bei den operierten Patienten wurden 16 Diffusionsrestriktion und 7 unauffällige DWI Befunde erhoben, wobei 2 Untersuchungen inkonklusiv waren. Die 16 diffusionsgestörten Appendices zeigten operativ in 14 Fällen eine akute Appendizitis und in 2 Fällen einen malignen Befund (1 Appendix-CA, 1 Coecum-CA). In den 7 nicht diffusionsgestörten Patienten wurden in 5 Fällen auch intraoperativ keine akute Appendizitis nachgewiesen. Die 22 konservativ versorgten Patienten wiesen allesamt keine Diffusionsstörung der Appendix auf. Die Sensitivität betrug somit $87,5 \%$ bei einer Spezifität von $93,1 \%$, einem PPV von $87,5 \%$ und einem NPV von $93.1 \%$.

Schlussfolgerungen Die Fast-MRI mit DWI erwies sich in der durchgeführten prospektiven Studie als eine schnelle und strahlungsfreie Methode, um mit hoher Sicherheit eine akute Appendicitis auszuschließen bzw. nachweisen zu können.

\section{WISS 206.2 Auswertung bildmorphologischer Kriterien bei alveolärer Echinokokkose der Leber anhand der EMUC-CT Klassifikation}

Autoren Gräter $\mathrm{T}^{1}$, Kratzer $\mathrm{W}^{2}$, Seufferlein $\mathrm{T}^{2}$, Schmiedberger $\mathrm{J}^{2}$, Leitner $\mathrm{E}^{2}$, Grüner $B^{3}$

Institut 1 Universitätsklinikum Ulm, Klinik für Diagnostische und

Interventionelle Radiologie, Ulm; 2 Universitätsklinikum Ulm, Klinik für Innere Medizin I, Ulm; 3 Universitätsklinikum Ulm, Klinik für Innere Medizin III, Ulm DOI 10.1055/s-0037-1600207

Zielsetzung Die Computertomografie stellt bei Verdacht auf alveoläre Echinokkokkose (AE) eines der wichtigsten diagnostischen Instrumente dar. Die unlängst etablierte "Echinococcus multilocularis Ulm classification for computed tomografy" (EMUC-CT Klassifikation) stellt die morphologisch sehr unterschiedlichen Erscheinungsbilder dieser Parasitose in der Leber heraus. Die EMUC-CT Klassifikation unterscheidet 5 Primärmorphologien, teils mit Subkriterien, sowie 6 Kalzifikationsmuster. Ziel der Arbeit war es mehrere bildmorphologische Kriterien von Leberläsionen bei AE anhand der EMUC-CT Klassifikation zu untersuchen und im Rahmen einer Verlaufsbeurteilung zu vergleichen.

Material und Methodik Retrospektiv wurden von 72 Patienten unter medikamentöser Therapie bei hepatischer AE die nach EMUC klassifizierten CT-Daten- sätze ausgewertet. Die bildmorphologischen Läsionskriterien wurden statistisch ausgewertet und hinsichtlich ihrer Dynamik im Verlauf beurteilt.

Ergebnisse Die Primärmorphologie Typen präsentieren sich im Verlauf sehr konstant, wohingegen die Subkriterien unterschiedliches Verhalten aufweisen. Zwischen den Kalzifikationsmustern sind nur geringe Veränderlichkeiten zu verzeichnen. Der Grad der Kalzifikation nimmt aber deutlich zu. Es kommt unter Therapie zu einer geringen Größenregredienz der Läsionen. Die Größen und die Anzahl von Läsionen der unterschiedlichen Typen unterscheiden sich signifikant. Es bestehen deutliche Unterschiede zwischen den Typen hinsichtlich extrahepatischer sowie vaskulärer und biliärer Beteiligung.

Schlussfolgerungen Die Studie konnte klare Zusammenhänge zwischen unterschiedlichen bildmorphologischen Kriterien der EMUC-CT Klassifikation absolut und in Hinblick auf Veränderlichkeiten im Verlauf darstellen. Klinisch relevant erscheinen insbesondere die Beobachtungen zu Läsionsgrößen und deren Anzahl, sowie zum Vorliegen extrahepatischer und vaskulärer/biliärer Beteiligung. Größere Studien müssen zeigen ob daraus künftig Folgerungen hinsichtlich Prognose und Therapieplanung abgeleitet werden können.

\section{RK 102.3 GD-EOB-DTPA-gestützte MRT zur Beurteilung der Leberfunktion: Vergleich zwischen Signalintensitätsindices und T1 Relaxometrie}

Autoren Haimerl $\mathrm{M}^{1}$, Verloh $\mathrm{N}^{1}$, Fellner $\mathrm{C}^{1}$, Nickel $\mathrm{D}^{2}$, Stroszczynski $\mathrm{C}^{1}$, Wiggermann $\mathrm{P}^{1}$ Institut 1 Universitätsklinikum Regensburg, Institut für Röntgendiagnostik, Regensburg; 2 Siemens AG, Healthcare GmbH, MR Applications Predevelopment, Erlangen DOI 10.1055/s-0037-1600208

Zielsetzung Ziel ist es in der Gd-EOB-DTPA-gestützten MRT Signalintensitäts (SI)-Messungen mit der T1 Relaxometrie hinsichtlich der quantitativen Abschätzung der Leberfunktion zu vergleichen.

Material und Methodik 117 Patienten unterzogen sich sowohl einer Gd-EOBDTPA-gestützten MRT einschließlich MR-Relaxometrie als auch einem ICGLeberfunktionstest. Es wurden sowohl SI-basierte Leberfunktionsindices, einschließlich dem „hepatocellular uptake index“ (HUI) sowie durch Muskulatur und Milz korrigierte SI-indices als auch die Reduktionsrate der T1 Relaxationszeit (rrT1) berechnet. Um T1 maps zu generieren wurde eine 3D VIBESequenz (TR $5.79 \mathrm{~ms}$, TE $2.46 \mathrm{~ms}, \alpha 1^{\circ}, 7^{\circ}, 14^{\circ}$ ) vor und 20 Minuten nach GdEOB-DTPA-Gabe akquiriert und die rrT1 zwischen nativen und Gd-EOB-DTPAgestützten Sequenzen berechnet. Die ICG Plasmaverschwinderate (ICG-PDR) wurde mit den MRT-basierten Leberfunktionsparametern korreliert. Zudem wurde in einer multivariaten Regression der Einfluss des entsprechenden Lebervolumen (LV) auf die MRT-basierten Leberindices evaluiert.

Ergebnisse In einer einfachen linearen Regression zeigte sich ein signifikanter Zusammenhang zwischen ICG-PDR und allen MRT-basierten Leberfunktionsindices $(r=0.624-0.829$; $p<0.001)$, wobei rrT1 $(r=0.829)$, verglichen mit SIbasierten Indices, signifikant besser mit ICG-PDR korrelierte $(p \leq 0.013)$. Unter den SI-basierten Indices korrelierte HUI am besten mit ICG-PDR ( $r=0.735)$ und es zeigte sich zwischen den SI-basierten Indices kein signifikanter Unterschied $(r=0.675-0.735)$. Unter Miteinbeziehung des LV konnte in einem multiplen linearen Regressionsmodell eine jeweils signifikant stärkere Korrelation zwischen ICG-PDR und SI-basierten Indices $(r=0.723-0.767)$ wie auch rrT1 $(r=0.9)$ gezeigt werden $(p<0.001)$.

Schlussfolgerungen Die Leberfunktion kann quantitativ durch Gd-EOB-DTPAgestützte MRT-basierte Leberfunktionsindices abgeschätzt werden. Diesbezüglich ist die T1 Relaxometrie den SI-Messungen überlegen und alle Indices zeigen unter Miteinbeziehung des LV einen diagnostischen Zugewinn. 
WISS 103.5 Perfusionsanalyse des Rektums mittels zeitaufgelöster GRASP bei freier Atmung im Vergleich zur konventionellen TWIST-Angiografie

\author{
Autoren Hausmann $D^{1}$, Liu J $J^{2}$, Riffel $P^{1}$, Budjan J ${ }^{1}$, Schönberg $S^{1}$, \\ Attenberger $U^{1}$ \\ Institut 1 Universitätsmedizin Mannheim, Institut für Klinische Radiologie \\ und Nuklearmedizin, Mannheim; 2 Peking Universtiy First Hospital, \\ Department of Radiology, Beijing, China \\ DOI 10.1055/s-0037-1600209
}

Zielsetzung Qualitativer und quantitativer Vergleich einer prototypischen zeitaufgelösten radialen golden-angle stack-of-stars T1-gewichteten 3D Gradientenecho-Sequenz (GRASP) und einer zeitaufgelösten Angiografie mit stochastischen Trajektorien (TWIST) für die Perfusionsbeurteilung des Rektums.

Material und Methodik 20 Rektumkarzinompatienten wurden nach parenteraler Kontrastmittelgabe untersucht (10 mittels GRASP (zeitliche Auflösung $3,45 \mathrm{~s} ; 10$ mittels TWIST). Eine Kohorte von 20 Patienten (10 GRASP; 10 TWIST) ohne bekannte Rektumpathologie diente als gesunde Kontrollgruppe. Die Rekonstruktionen beider Sequenzen wurden zunächst mittels voxel-basierter Dekonvulotion analysiert, um den rektalen Plasmafluss (rPF) (ml/100 ml/ min) zu beurteilen. Der über ROls in den generierten Perfusionskarten gemessene rPF wurde danach im Tumor sowie im gesunden Rektum (mittleres, oberes, unteres Drittel) verglichen. Zudem erfolgte eine subjektive Beurteilung der Qualität der Perfusionskarten, der arterial input functions (AIFs) und der morphologischen Bildqualität durch zwei geblindete Radiologen.

Ergebnisse Es wurden signifikant höhere Bewertungen für GRASP hinsichtlich der morphologischen Bildqualität, der Qualität der Perfusionskarten und der AlFs vergeben $(p<0.01)$. Der durchschnittliche rPF der GRASP-Perfusion in normaler Rektumwand lag bei $31.78 \pm 7.39 \mathrm{ml} / 100 \mathrm{ml} / \mathrm{min}$ im Vergleich zu $127.22 \pm 121.8 \mathrm{ml} / 100 \mathrm{ml} / \mathrm{min}$ unter Verwendung der TWIST. TWIST und GRASP zeigten jeweils signifikant höhere Perfusionswerte in Tumoren als in normaler Rektumwand $(\mathrm{p}<0.01)$.

Schlussfolgerungen Unsere Daten deuten darauf hin, dass mittels GRASP reproduzierbare Perfusionswerte mit im Vergleich zur TWIST geringerer Varianz generiert werden können, die sich eignen, um zwischen gesunder und tumoröser Rektumwand zu unterscheiden. Darüber hinaus ermöglicht GRASP eine verbesserte morphologische Beurteilung des Rektums mit hoher zeitlicher Auflösung.

\section{RK 102.6 Quantifizierung des Pankreasfettgehalts im MRT als proton-density fat fraction (PDFF) bei Probanden mit Prädiabetes, Diabetes und gesunden Kontrollen in einer Kohorte aus der Allgemeinbevölkerung}

\footnotetext{
Autoren Heber $S^{1}$, Hetterich $\mathrm{H}^{2}$, Lorbeer $\mathrm{R}^{2}$, Bayerl $\mathrm{C}^{2}$, Machann $\mathrm{J}^{1}$, Auweter $S^{2}$, Storz $C^{1}$, Schlett $C^{3}$, Nikolaou $K^{1}$, Reiser $M^{2}$, Peters $A^{4}$, Bamberg $\mathrm{F}^{1}$

Institut 1 Universitätsklinikum Tübingen, Abteilung für Diagnostische und Interventionelle Radiologie, Tübingen; 2 Klinikum der Universität München, Institut für Klinische Radiologie, München; 3 Universitätsklinikum Heidelberg, Diagnostische und Interventionelle Radiologie, Heidelberg; 4 Helmholtz Zentrum München, Institute of Epidemiology II, Neuherberg DOI 10.1055/s-0037-1600210
}

Zielsetzung Bestimmung des MRT-basierten Pankreasfettgehalts als protondensity fat fraction (PDFFpanc) in einer bevölkerungsbezogenen, prospektiven Kohortenstudie bestehend aus Probanden mit Prädiabetes, Diabetes und gesunden Kontrollen sowie Untersuchung der Assoziation mit etablierten kardiovaskulären Risikofaktoren und anderen Fettkompartimenten.

Material und Methodik Probanden ohne etablierte kardiovaskuläre Erkrankung jedoch bekanntem Prädiabetes, Diabetes sowie gesunde Kontrollen wur- den mittels 3T Multi-echo DIXON zur Quantifizierung von Leber- und Pankreasfett (PDFFhepatic und PDFFpanc; time-to-repetition [TR] $8.90 \mathrm{~ms}, 6$ Echos à $1.23 \mathrm{~ms}$ [TE]) sowie 2-Punkt DIXON zur Quantifizierung von viszeralem [VAT] und subkutanem [SAT] Fett untersucht. Die PDFFpanc wurde als Mittelwert im Pankreaskopf, -körper und -schwanz durch unabhängige Befunder bestimmt. Multi- und univariate Analysemodelle wurden verwendet.

Ergebnisse Insgesamt wurden 385 Probanden in die Analyse eingeschlossen (medianes Alter 57 Jahre, $58.2 \%$ männlich) und die Prävalenz von Prädiabetes und Diabetes betrug ca. $24.7 \%$ und $13.8 \%$. Die mediane PDFFpanc war $5.2 \%$ [IQR 3.3 - 9.4] und zeigte einen signifikanten Anstieg von gesunden Kontrollen zu Prädiabetikern und Diabetikern (PDFFpanc $4.9 \% 3.1-7.4$ vs. $6.2 \%$ [3.5 - 12] vs. 8.6\% [4.3 - 17.5]; pTrend <0.001). Nach Adjustierung für Alter, Geschlecht und BMI waren die beobachteten Unterschiede rückläufig. Während in der univariaten Analyse SAT, VAT, BMI und PDFFhepatic mit PDFFpanc assoziiert waren, zeigte in der multivariaten Analyse nur VAT eine unabhängige Korrelation mit PDFFpanc.

Schlussfolgerungen Probanden mit Prädiabetes und Diabetes haben einen signifikant höheren Pankreasfettgehalt als gesunde Kontrollen. Diese Assoziation ist allerdings stark beeinflusst durch das Alter, männliche Geschlecht, sowie die Menge an VAT.

\section{WISS 302.8 Bildqualität in der thorakoabdominellen Staging-Computertomografie - Vergleich zwischen Single Energy mit automatischer Reduktion der Röhrenspannung und Dual Energy}

Autoren Klapsch $\mathrm{F}^{1}$, Lurz $\mathrm{M}^{2}$, Wüst $\mathrm{W}^{2}$, Wiesmüller $\mathrm{M}^{2}$, Heiß $\mathrm{R}^{2}$, Uder $\mathrm{M}^{2}$, May $\mathrm{M}^{2}$

Institut 1 Universitätsklinik Erlangen, Radiologie, Erlangen; 2 Universität

Erlangen, Radiologisches Institut, Erlangen

DOI 10.1055/s-0037-1600211

Zielsetzung In der thorakoabdominellen Staging-CT ist die anatomisch gesteuerte Reduktion der Röhrenspannung ein etabliertes Verfahren. Neben verschiedenen Nachverarbeitungsmöglichkeiten versprechen Dual Energy Aufnahmen auch in den Mischbildern Vorteile im Verhältnis von Bildkontrast und -rauschen. Ziel dieser Studie war es diese beiden Methoden bezüglich Strahlendosis und Bildqualität zu vergleichen.

Material und Methodik Dual Energy Aufnahmen des Thorax und Abdomen wurden bei 100 Patienten in einer portalvenösen Kontrastmittelphase mit anatomischer Röhrenstrommodulation an eine Dual Source Gerät der 3. Generation angefertigt (70/Sn150 kV, 560/140 ref.mAs) und Mischbilder mit einem Wichtungsfaktor von 0,7 berechnet $(5 / 5 \mathrm{~mm})$. Zum Vergleich wurden 95 Datensätze mit anatomisch gesteuerter Reduktion der Röhrenspannung (120 ref. kV, 180 ref. mAs) vom selben Gerät mit identischer Referenzdosis (32 cm Acryl Phantom) retrospektiv ausgewertet. Die Bildqualität wurde objektiv als Kontrast-zu-Rauschen Verhältnis (CNR) und subjektiv gemäß der European Guidelines evaluiert. Die Strahlendosis wurde als volumetrischer CT-Dosisindex (CTDlvol) verglichen.

Ergebnisse Der Unterschied in der Strahlendosis zwischen den Dual Energy Untersuchungen (8,0, Spannweite 4,5-13,6 mGy) und dem Referenzkollektiv (8,2, Spannweite 4,1-15,6 mGy) war statistisch nicht signifikant $(p=0,132)$. Alle Aufnahmen wurden als gut oder exzellent für diagnostische Zwecke bewertet. Das CNR war in den großen thorakalen Gefäßen $(p=0,245)$ und für die hypodensen Leberläsionen ( $p=0,322)$ vergleichbar zum Referenzkollektiv, in den Oberbauchorgane sogar signifikant erhöht $(p<0,01)$.

Schlussfolgerungen Aus Dual Energy Untersuchungen rekonstruierte Mischbilder bieten bei vergleichbarer Patientendosis und diagnostischer Bildqualität Vorteile im CNR der Oberbauchorgane. Aufgrund der erhöhten Flexibilität durch die vielfältigen Nachverarbeitungsmöglichkeiten sollten, wenn verfügbar, in der klinischen Routine Dual Energy Protokolle etabliert werden. 


\section{WISS 302.4 Multiparametrische [18F]-FDG PET/MR Enterografie zur Evaluation der Entzündungsaktivität bei Morbus Crohn - Welchen Wert haben die unterschiedlichen Biomarker?}

\author{
Autoren $\mathrm{Li} \mathrm{Y}^{1}$, Beiderwellen $\mathrm{K}^{1}$, Nensa $\mathrm{F}^{1}$, Herrmann $\mathrm{K}^{2}$, Umutlu $\mathrm{L}^{1}$, \\ Langhorst $]^{3}$ \\ Institut 1 Uniklinikum Essen, Institut für diagnostische und Interventionelle \\ Radiologie und Neuroradiologie, Essen; 2 Uniklinikum Essen, Institut für \\ Nuklearmedizin, Essen; 3 Kliniken Essen-Mitte, Zentrum für Integrative \\ Gastroenterologie, Essen \\ DOI 10.1055/s-0037-1600212
}

Zielsetzung Das Ziel dieser prospektiven Studie war es, die diagnostische Genauigkeit einzelner Biomarker der integrierten PET/MR Enterografie bei Patienten mit Morbus Crohn zu evaluieren.

Material und Methodik 50 Patienten unterzogen sich einer [18F]-FDG PET/ MR Enterografie an einem integrierten PET/MRT System. Als Referenzstandard diente die Koloskopie. Zur Analyse wurden die zu untersuchenden Darmanteilen in 3 ileokolische Segmente aufgeteilt. Die Entzündungsaktivität wurde anhand der Simplified Endoscopic Activity Score for Crohn's Disease (SES-CD) bewertet und zusätzlich in 3 Grade eingeteilt. 14 qualitative und quantitative PET/MR Parameter wurden erfasst. Für die statistische Auswertung wurden der Chi-Quadrat-Test für die qualitativen Parameter, der Mann-Whitney U Test für die quantitativen Parameter sowie der Korrelationstest nach Spearman für die Korrelationen zwischen Parametern, dem Entzündungsschweregrad und SES-CD angewandt.

Ergebnisse Insgesamt wurden 309 Segmente analysiert. Kamm-Zeichen und geschichtete Kontrastmittel-Wandanreicherung wiesen die beste diagnostische Genauigkeit über $90 \%$ bei der Entzündungsdetektion auf. Die höchste diagnostische Genauigkeit der quantitativen Parameter wies die Analyse der Wanddicke (AUC der ROC =0,92) mit einem cut-off Wert von 4,5 mm (Sens. $=81 \%$, Spez.=93\%) auf, während die geringste Genauigkeit für den $A D C$-Wert $(A U C=0,42)$ gezeigt werden konnte. Zur Detektion von hochgradiger Entzündung lag die AUC zwischen 0,92 und 0,96 bei 4 der 7 quantitativen Parameter, geführt mit der höchsten Genauigkeit für die Wanddicke $(0,96)$ und gefolgt vom relativen SUVmax-Ratio $(0,94)$. Die Wanddicke war der einzige Parameter, der mit dem Entzündungsschweregrad sowie SEC-CD mäßig korrelierte, da die Korrelationen bezüglich der anderen Parameter niedrig oder sehr niedrig waren.

Schlussfolgerungen Die aus der integrierten PET/MR Enterografie extrahierten MR-Parameter und metabolischen PET-Parameter führen zu einer hohen diagnostischen Genauigkeit zur Detektion von intestinalen Entzündungen.

\section{WISS 302.3 Bildgebung des Pankreaskarzinoms mittels Dual-layer Spektral-CT}

Autoren Lohöfer $F^{1}$, Kaissis $G^{1}$, Noël $P^{1}$, Friess $H^{2}$, Ceyhan $G^{2}$, Weichert $W^{3}$, Rummeny $\mathrm{E}^{1}$, Braren $\mathrm{R}^{1}$

Institut 1 TU München, Institut für diagnostische und Interventionen Radiologie, München; 2 TU München, Klinik und Poliklinik für Chirurgie, München; 3 TU München, Institut für Pathologie, München DOI 10.1055/s-0037-1600213

Zielsetzung Ziel der Studie ist die Evaluation der Vorteile der Dual-layer Spektral-CT beim Pankreaskarzinom im Vergleich zur konventionellen kontrastverstärkten CT.

Material und Methodik Patienten mit Erstdiagnose eines Pankreaskarzinoms ( $n=10$ ) wurden mit einem Dual-layer Spektral-CT untersucht. (IQon Spectral CT, Philips Healthcare, USA). Die Bildaquisition erfolgte in arterieller und venöser Kontrastmittelphase. Virtuelle monoenergetische Bilder (Mono) bei 40keV und $70 \mathrm{keV}$, sowie lod-Maps wurden berechnet und verglichen. Bildgebung und Histopathologie wurden korreliert.

Ergebnisse Die Mono 40keV Bilder waren den 70keV Bildern bei der Darstellung der Tumorausdehnung überlegen. Die Mono 40keV Bilder zeigten stärke- re Unterschiede in den Hounsfield Einheiten (HU) zwischen Tumor und gesundem Gewebe (67,4\% bei $40 \mathrm{keV}$ vs. $59,1 \% 70 \mathrm{keV})$. Die absoluten lod-Werte betrugen $0,80,4 \mathrm{mg} / \mathrm{ml}$ im Tumor und $2,20,4 \mathrm{mg} / \mathrm{ml}$ im gesunden Gewebe. Schlussfolgerungen Die Dual-layer Spektral-CT ermöglicht eine exaktere Erfassung der Tumorgrenzen sowie eine Quantifizierung der intratumoralen Jodaufnahme im Gewebe. Diese können potentiell zur verbesserten Therapieplanung und Patientenstratifizierung eingesetzt werden.

\section{WISS 206.3 Die klinische Wertigkeit der Multidetektor-Computertomografie bei klinisch obskuren Infektfoki}

Autoren Luerken $L^{1}$, Schreyer $A^{1}$, Stroszczynski $C^{1}$, Schleder $S^{1}$ Institut 1 Universitätsklinikum Regensburg, Röntgendiagnostik, Regensburg DOI 10.1055/s-0037-1600214

Zielsetzung Die Datenlage bezüglich der klinischen Wertigkeit der MDCT zur Diagnostik unklarer Infektfoki ist bisher nicht ausreichend. Ziel dieser Studie war es zu evaluieren, wie häufig eine MDCT, welche zur Infektfokussuche durchgeführt wurde, zu einer Diagnose führte und wie häufig diese Diagnose einen direkten Einfluss auf die Therapie hatte. Zusätzlich sollte eine Subgruppenanalyse analysieren, ob bestimmte Subgruppen von Patienten besonders häufig vom Ergebnis der MDCT im Sinne einer Therapieanpassung profitieren.

Material und Methodik Alle MDCT-Untersuchungen, die zur Infektfokussuche in einem Universitätskrankenhaus 2010 durchgeführt wurden, wurden per Volltext-Datenbank-Abfrage identifiziert. Die Ergebnisse der MDCT und ihr Einfluss auf die Therapie der Patienten sowie Informationen zum Patientenkollektiv wurden den Röntgenbefunden sowie den Arztbriefen der Patienten entnommen $(n=525)$.

Ergebnisse Bei insgesamt 26,3\% der untersuchten Patienten konnte eine Verdachtsdiagnose bestätigt werden und bei 33,3\% der Patienten wurde eine neue Diagnose gefunden. Die Ergebnisse der MDCT hatten bei 32\% der Patienten einen unmittelbaren Einfluss auf die weitere Therapie. Bei kürzlich voroperierten Patienten führte die MDCT häufiger zu einer Therapieanpassung $(36 \%)$ als bei nicht voroperierten Patienten $(31 \%)(p=0,289)$. Eine MDCT Abdomen/Becken führte häufiger zu einer Anpassung der Therapie der Patienten (42\%) als eine MDCT des Thorax (31\%) oder eine kombinierte MDCT des Thorax und Abdomen/Becken (29\%) $(p=0,013)$. Für immunsupprimierte Patienten ließ sich ebenfalls nachweisen, dass die MDCT häufiger zu einer Therapieumstellung führte (39\%) als bei nicht immunsuprimierten Patienten (31\%) $(p=0,089)$.

Schlussfolgerungen Die MDCT ist eine klinisch wertvolle Komponente bei der multimodalen Diagnostik von unklaren Infektfoki. Dies scheint insbesondere für Patienten zu gelten, bei denen der Infektfokus im Abdomen und Becken vermutet wird, sowie für Patienten, die immunsupprimiert sind oder kurz vor der MDCT operiert wurden.

\section{WISS 302.5 Spektrale Photon-Counting Computertomografie: Anwendung für die virtuelle Koloskopie ohne Abführen}

Autoren Münzel $D^{1}$, Bar-Ness $D^{2}$, Roessl $E^{3}$, Fingerle $A^{4}$, Daerr $H^{3}$, Pfeiffer $F^{5}$, Proksa $\mathrm{R}^{3}$, Rummeny $\mathrm{E}^{4}$, Douek $\mathrm{P}^{2}$, Noel $\mathrm{P}^{4}$

Institut 1 Klinikum rechts der Isar der TUM, Radiologie, München; 2 Louis Pradel University Hospital, Bron, France, Department of Interventional Radiology and Cardio-vascular and Thoracic Diagnostic Imaging, Lyon; 3 Philips GmbH Innovative Technologies, Hamburg; 4 Klinikum rechts der Isar der TUM, Institut für diagnostische und interventionelle Radiologie, München; 5 Technische Universität München, Lehrstuhl für Biomedizinische Physik, Garching DOI 10.1055/s-0037-1600215

Zielsetzung Es soll das klinische Potential der spektralen Photon-Counting Computertomografie (SPCCT) für die virtuelle Koloskopie mit elektronischem 
Abführen des iodmarkierten Stuhls untersucht werden, wobei die charakteristische K-Kante von Gadolinium zur Unterscheidbarkeit zwischen den Kontrastmitteln lod (oral) und Gadolinium (intravenös) einbezogen wird.

Material und Methodik Ein speziell hergestelltes Colonphantom wurde mit einem nicht-ionischen iodhaltigen Kontrastmittel gefüllt. Eine mit Gadolinium gefüllte Kapsel, die einen Kontrastmittel-anreichernden Polypen repräsentierte, wurde an der Wand des Colonphantoms platziert. Das Colonphantom wurde mit einem präklinischen SPCCT Scanner gescannt. Die vollständige spektrale Information aus den verschiedenen Photonenmesskammern wurde für die Materialzerlegung verwendet, und es wurden separate lod- und Gadoliniumkarten berechnet. Quantitative Messungen zur Bestimmung der absoluten Konzentrationen von lod und Gadolinium wurden im Lumen des Colonphantoms und im Polyp durchgeführt.

Ergebnisse In der konventionellen CT Darstellung wurden die Hounsfield Einheiten (HE) beider Kontrastmittel mit 110 HE bestimmt. Die Kontrastmittelkarten zeigten eine klare Unterscheidbarkeit der beiden Kontratsmittel: Gadolinium war nur im Polyp und lod nur im Lumen des Colonphantoms nachweisbar. Die quantitativen Messungen ergaben sehr genau die jeweiligen Konzentrationen der eingesetzten Kontrastmittel.

Schlussfolgerungen Die virtuelle Koloskopie mittels SPCCT kann durch die eindeutige Bestimmung von lod und Gadolinium ein elektronisches Abführen mittels lodmarkierung des Stuhls möglich machen, mit einer klaren Unterscheidbarkeit von kontrastmittelaufnehmenden Polypen nach i.v.-Gadoliniumgabe. Dies ermöglicht eine verbesserte diagnostische Performance der virtuellen Koloskopie ohne laxative Vorbereitung.

\section{WISS 302.1 Spektrale Photon-Counting Computertomografie für Mehrphasen-Leberdiagnostik mit zwei Kontrastmitteln}

Autoren Münzel $D^{1}$, Daerr $H^{2}$, Proksa $R^{2}$, Fingerle $A^{3}$, Douek $\mathrm{P}^{4}$, Pfeiffer $F^{5}$ Rummeny $\mathrm{E}^{3}$, Noel $\mathrm{P}^{3}$

Institut 1 Klinikum rechts der Isar der TUM, Radiologie, München; 2 Philips GmbH Innovative Technologies, Hamburg; 3 Klinikum rechts der Isar der

TUM, Institut für diagnostische und interventionelle Radiologie, München; 4 Louis Pradel University Hospital, Bron, France, Department of Interventional Radiology and Cardio-vascular and Thoracic Diagnostic Imaging, Lyon; 5 Technische Universität München, Lehrstuhl für Biomedizinische Physik, Garching

DOI 10.1055/s-0037-1600216

Zielsetzung Wir präsentieren einen neues Untersuchungsprotokoll für Leberdiagnostik für zwei Kontrastmittel (KM) und spektrale Photon-Counting Computertomografie (SPCCT) mit nur einem Scan.

Material und Methodik Wir stellen ein spezielles KM-Injektionsprotokoll für zwei intravenöse KM vor, mit dem zu einem Zeitpunkt die Verteilung von KM 1 (lod) und KM 2 (Gadolinium) dargestellt werden kann. Die Bildakquisitionen wurden für einen SPCCT Scanner simuliert. Wir haben vier Leberläsionen (Hämangiom, Hepatozelluläres Karzinom, Zyste, Metastase) mit typischem arteriellen und portalvenösen KM-Verhalten in verschiedenen Größen (5$20 \mathrm{~mm}$ ) untersucht. Die SPCCT Bildgebung wurde für den Zeitpunkt simuliert, an dem KM 1 eine portalvenöse Verteilung zeigt und KM 2 in arterieller Phase gezeigt ist. Mittels Materialzerlegung wurden quantitative lod- und Gadoliniumkarten berechnet. Ein multidimensionaler Klassifizierungsalgorithmus für eine automatische Detektion der verschiedenen Leberläsionen wurde eingeführt.

Ergebnisse Mittels SPCCT und einem angepassten Injektionsprotokoll für zwei Kontrastmittel ist es möglich, kontrastverstärkte Bilder in arterieller Phase und portalvenöser Phase zu einem einzigen Scanzeitpunkt zu erhalten. In unserer Studie waren alle vier Leberläsionen anhand ihres charakteristischen Verhaltens in den arteriellen und portalvenösen Kontrastmittelkarten klar zu unterscheiden. Eine automatisierte Detektion und Klassifikation von Leberläsionen mittels multidimensionaler Analyse ist möglich.
Schlussfolgerungen Die spektrale Information des SPCCT-Detektors macht es möglich, die Verteilung von lod und Gadolinium in einem Bild darzustellen und visualisiert dabei simultan das charakteristische KM-Verhalten von Leberläsionen in arterieller und portalvenöser KM-Phase in einem einzigen SPCCT Scan. Die simultane Darstellung der drei Phasen (nativ, arteriell, portalvenös) in nur einem Scan ermöglicht eine automatisierte optimierte Detektion von Leberläsionen und einer signifikante Reduktion der Strahlendosis.

\section{WISS 302.7 Ultraschnelle abdominelle MR Bildgebung mit einem neuartigen 60-Kanal Empfängerspulensystem}

Autoren Othman $\mathrm{A}^{1}$, Weiss $\mathrm{J}^{2}$, Taron $\mathrm{J}^{1}$, Nikolaou $\mathrm{K}^{1}$, Martirosian $\mathrm{P}^{1}$, Notohamiprodjo $\mathrm{M}^{1}$

Institut 1 Uniklinik Tübingen, Radiologie, Tübingen; 2 Universitätsklinikum Tübingen, Klinik für Diagnostische und Interventionelle Radiologie, Tübingen DOI 10.1055/s-0037-1600217

Zielsetzung Ziel der Studie ist die Evaluation eines neuartigen 60-Kanal Empfängerspulensystems hinsichtlich der Bildqualität ultraschneller abdomineller Sequenzen im Vergleich zu einem herkömmlichen Empfängerspulensystem.

Material und Methodik Alle Untersuchungen wurden an einem 3T MRT (Magnetom Skyra, Siemens Healthineers) durchgeführt. 3D-T1-GRE Sequenzen mit unterschiedlichen Parallel Acquisition Technique (PAT-) Faktoren (CAIPIRINHA: 0,2,3,2 × 2,3 ×2,2 ×3,3 ×3) wurden an einem Wasserphantom und 5 gesunden Probanden mit einem neuartigen 60-Kanal (30-Kanal anterior+posterior) Empfängerspulensystem und einem herkömmlichen 30-Kanal (18-Kanal-body+12-Kanal-spine) System akquiriert. Für die Phantomdaten wurde das SNR bestimmt. Subjektive Bildqualitätskriterien wurden von 2 Radiologen erhoben (5-Punkte Likertskala; 5 = exzellente Bildqualität). In einem weiteren Schritt wurden die herkömmlichen kontrastmittelverstärkten 3D-T1-GRE Sequenzen mit PAT $=2$ sowie beschleunigte Sequenzen mit PAT $=2 \times 2$ und $3 \times 2$ in 17 Patienten mit beiden Spulensystemen in randomisierter Abfolge durchgeführt (60-Kanal, $n=8 ; 30-K a n a l, n=9)$. Die Bildqualität beider Gruppen wurde verglichen.

Ergebnisse Im Vergleich zum 30-Kanalsystem führte das 60-Kanalsystem zu einem Anstieg des SNR für alle Sequenzen außer PAT $=0$. Der relative SNRAnstieg variierte zwischen $2.21 \%(P A T=2)$ und $22.69 \%(P A T=3 \times 2)$. Die subjektive Bildqualität der Probandendaten war signifikant höher für das 60-Kanalsystem für alle Sequenzen ( $p \leq .046)$ außer für PAT $=0$ ( $p \geq .180)$. In Patienten wurden ähnliche Ergebnisse hinsichtlich der Bildqualität beobachtet mit vergleichbarer Bildqualität der Spulensysteme mit niedrigen PAT-Faktoren (PAT $=2 ; p \geq .069$ ) und einer signifikant höheren Bildqualität für das 60-Kanalsystem bei höheren PAT-Faktoren $(2 \times 2$ und $3 \times 2$; $p \leq 0.036)$.

Schlussfolgerungen Das 60-Kanalspulensystem führt zu einer Erhöhung der Bildqualität und ermöglicht ultraschnelle abdominelle Sequenzen mit hohen PAT-Faktoren und einer suffizienten Bildqualität.

\section{WISS 103.1 Schnelle kontinuierliche multiarterielle MRT der Leber unter freier Atmung}

Autoren Othman $A^{1}$, Weiss $\mathrm{J}^{1}$, Nickel $\mathrm{M}^{2}$, Martirosian $\mathrm{P}^{1}$, Ruff $\mathrm{C}^{1}$, Kolb $\mathrm{M}^{1}$, Nikolaou $\mathrm{K}^{1}$, Notohamiprodjo $\mathrm{M}^{1}$

Institut 1 Uniklinik Tübingen, Radiologie, Tübingen; 2 Siemens Healthineers, MR Application Predevelopment, Erlangen

DOI 10.1055/s-0037-1600218

Zielsetzung Ziel der Studie ist die Evaluation der multiarteriellen Leber MRT unter freier Atmung mittels einer selbstnavigierten 3D T1 GRE Sequenz mit Compressed Sensing (CS).

Material und Methodik 40 Patienten wurden einer kontrastmittelverstärkten MRT der Leber mit Gadobutrol (Bayer Healthcare) unterzogen. Je 20 Patienten wurden an einem 1.5 T bzw. 3 T MRT untersucht (Magnetom Aera und Skyra, Siemens Healthineers). Die multiarterielle MRT der Leber wurde mit einer schnellen selbstnavigierten T1 Volume Interpolated Breath-hold Examination 
(VIBE) Sequenz mit CS und automatischer Atemnavigation in freier Atmung akquiriert (Beginn mit KM-Applikation, Dauer $64 \mathrm{~s}$, örtliche Auflösung $1,2 \times 1,2 \times 3 \mathrm{~mm} 3$, zeitliche Auflösung $8 \mathrm{~s}$ ). Zwei Radiologen wählten und evaluierten die hepatisch-arteriell dominante (HAD) Phase auf einer 5-Punkte Likert Skala $(5=$ exzellent) hinsichtlich Bildqualität, Artefaktkompensation, Kontrastierung der abdominellen Aorta und der Leberarterien, Detektierbarkeit von Läsionen und diagnostischer Sicherheit. Signalintensität (SI) und SNR wurden in der Leber und in der abdominellen Aorta gemessen.

Ergebnisse Die HAD Phase variierte zwischen den Patienten (3. - 7. Phase). Bildqualität und Artefaktkompensation wurden als gut bewertet ( $M d=4,3$ 5), ohne signifikante Unterschiede zwischen 1.5 $T$ und $3 T(p \geq 0.089)$. Die Detektierbarkeit von Läsionen, arterielle Kontrastierung und diagnostische Sicherheit wurden als exzellent bewertet (Md=5, 3-5), ohne signifikante Unterschiede zwischen beiden Feldstärken $(p \geq 0.165)$. SI und Leber SNR bei Untersuchungen in $3 \mathrm{~T}$ waren signifikant höher als bei $1.5 \mathrm{~T}(\mathrm{p} \leq 0.015)$. Das SNR in der Aorta abdominalis war in beiden Feldstärken hoch, ohne signifikante Unterschiede (p.=637).

Schlussfolgerungen Die Akquisition von multiarterieller MRT der HAD Phase in freier Atmung mittels einer selbstnavigierten CS T1 VIBE Sequenz ermöglicht gute Bildqualität, exzellente arterielle Kontrastierung und eine exzellente Artefaktkompensation bei $1.5 \mathrm{~T}$ und 3T.

\section{WISS 302.2 Spektral-Detektor-CT (SDCT) zur Verbesserung der Untersuchersicherheit bei der Beurteilung von Leberläsionen}

Autoren Persigehl $\mathrm{T}^{1}$, Slebocki $\mathrm{K}^{1}$, Große Hokamp N ${ }^{1}$, Rau $\mathrm{R}^{1}$, Pahn $\mathrm{G}^{2}$ Maintz $\mathrm{D}^{1}$, Persigehl $\mathrm{T}^{1}$

Institut 1 Uniklinik Köln, Radiologie, Köln; 2 Philips Healthcare, Hamburg DOI 10.1055/s-0037-1600219

Zielsetzung Evaluation von Spektral-Detektor-CT (SDCT) Daten zur Verbesserung der Untersuchersicherheit bei der Beurteilung von fokalen Leberläsionen. Material und Methodik Retrospektiv wurden 47 Patienten mit fokalen Leberläsionen identifiziert, die an einem neuen Spektral-Detektor-CT (SDCT; IQon, Philips Healthcare) untersucht wurden und eine Referenz zu Bestätigung der Diagnose aufwiesen (Histologie, MRT oder CEUS, vorheriges CT $\geq 6$ Monate). Es erfolgte die Darstellung von konventionellen (konv), virtuell monochromatischen Rekonstruktionen (vME mit $40 \mathrm{keV}$ ) und lod-Kartierungen. Die Bildanalyse erfolgte durch zwei Radiologen im Konsensus in Bezug auf die Läsionsabgrenzbarkeit und subjektive Sicherheit bei der Diagnosestellung anhand einer 5-Punkt Skala (Likert-Skala). Die statistische Analyse erfolgte mittels T-Test und ANOVA.

Ergebnisse Im Mittel wurden 2,1 Leberläsionen pro ausgewerteten Patienten eingeschlossen, hierbei wurden Leberläsionen $\leq 10 \mathrm{~mm}(45,8 \%)$ und $>11 \mathrm{~mm}$ (54,2\%) differenziert. Die Diagnosen umfassten Leberzysten, Hämangiome, Lebermetastasen und weitere sonstige wie fokale Mehrverfettungen. Insbesondere zeigte sich in Bezug auf die Untersuchersicherheit bei der Diagnosestellung von kleinen Leberläsionen ( $\leq 10 \mathrm{~mm}$ ) ein Vorteil unter Zunahme der weiteren verfügbaren spektralen CT-Informationen (konv Score: 2,25/40 vME Score: 2,08/Konv + vME + lod-Map Score: 1,91; $\mathrm{p}=0,039)$.

Schlussfolgerungen Die neue Spektral-Detektor-CT (SDCT) Technologie ermöglicht eine uneingeschränkte retrospektive Rekonstruktion von weiteren spektralen CT-Informationen ohne eine "a priori" Selektion von Patienten wie u. a. beim Dual-Source CT (DSCT) erforderlich. Diese zusätzlichen spektralen CT-Informationen (vME und lod-Map) ermöglichen eine Erhöhung der Untersuchersicherheit bei der Diagnosestellung insb. bei kleinen Leberläsionen $\leq 10 \mathrm{~mm}$ um ca. $17 \%$, so dass zukünftig teilweise auf eine weitere Bildbebung zur Diagnoseverifikation verzichtet werden könnte.

\section{WISS 206.1 Prognostischer Wert der hepatozellulären MRT mit leberspezifischem Kontrastmittel bei Patienten mit primär sklerosierender Cholangitis}

Autoren Ringe $\mathrm{K}^{1}$, Schulze $\mathrm{J}^{1}$, Lenzen $\mathrm{H}^{2}$, Hinrichs $\mathrm{J}^{1}$, Manns $\mathrm{M}^{2}$, Wacker $\mathrm{F}^{1}$ Institut 1 Medizinische Hochschule Hannover, Institut für Diagnostische und Interventionelle Radiologie, Hannover; 2 Medizinische Hochschule Hannover, Klinik für Gastroenterologie, Hepatologie und Endokrinologie, Hannover DOI 10.1055/s-0037-1600220

Zielsetzung Prognostische Wertigkeit der hepatozellulären Phase (HZP) in der MRT mit leberspezifischem Kontrastmittel bei Patienten mit primär sklerosierender Cholangitis (PSC) im Hinblick auf das klinische Outcome im Verlauf.

Material und Methodik 111 Patienten (83 m, 28w; mittl. Alter 45J) mit gesicherter PSC wurden in diese prospektive, durch die Ethikkommission genehmigte Studie, eingeschlossen. Das MRT Protokoll beinhaltete u.a. T1w VIBE Sequenzen vor, sowie durchschnittlich 20 min (HZP1) bzw. 120 min (HZP2) nach Injektion von Gd-EOB-DTPA $(0.1 \mathrm{ml} / \mathrm{kg})$. Signalintensitäts- (SI) Messungen erfolgten durch die Platzierung von ROls in den einzelnen Lebersegmenten. Das mittlere relative Enhancement (RE) in der HZP1 bzw. HZP2 wurde berechnet ((SIHZP)-(SIvor))/(Slvor) und mit Labor-Surrogatparametern (AP, Bilirubin, INR, Albumin) für das klinische Outcome (Spearman) sowie harten klinischen Endpunkten (Tod, Tumor, Lebertransplantation (LTx)) korreliert (logistische Regression).

Ergebnisse Im mittleren Follow-up von 603 Tagen nach der MRT wurden klinische Endpunkte von 21 Patienten erreicht ( $\operatorname{Tod} n=7$; Tumor $n=6$; LTx $\mathrm{n}=8)$ ). Das RE korrelierte signifikant mit den o.g. Surrogatparametern und klinischen Endpunkten, die stärksten Korrelationen waren in der frühen HZP1 nachweisbar: AP $(p<0.0001 ; r=-0.636)$, Bilirubin $(p<0.0001 ; r=-0.646)$, Albumin $(p<0.0001 ; r=0.538)$, INR $(p<0.0001, r=-0.456)$, sowie den Ereignissen Tod $(p=0.027)$, Tumor $(p=0.032)$ und LTx $(p=0.02)$.

Schlussfolgerungen Das RE in der HZP bei Patienten mit PSC korreliert mit den Leberparametern AP, Bilirubin, INR und Albumin und mit den klinischen Endpunkten Tod, Tumor und Lebertransplantation. Das RE kann als möglicher prognostischer MR-Parameter für die Krankheitsprogression dienen. Dies gilt es in Langzeitstudien zu untersuchen.

\section{RK 102.5 Biopsie-kontrollierter, zeitsynchroner Vergleich von Dual-Energy-CT und Ultraschall zur Quantifizierung von Leberverfettung}

Autoren Rogalla $\mathrm{P}^{1}$, Guenette $\mathrm{G}^{2}$, Rattansingh $\mathrm{A}^{3}$, Roest $\mathrm{M}^{3}$, Mao $\mathrm{K}^{4}$, Hoppel B ${ }^{2}$

Institut 1 University of Toronto, Medical Imaging, Toronto; 2 Toshiba, Forschung, Toronto; 3 UHN, Radiologie, Toronto; 4 UHN, Pathologie, Toronto DOI 10.1055/s-0037-1600221

Zielsetzung Ziel der Studie war herauszufinden, wie gut die Dual-Energy-CT mit monochromatischer Bildrekonstruktion und Ultraschall (US) mit normalisierter lokaler Varianzanalyse (NLV) geeignet sind, eine Leberverfettung nichtinvasiv zu quantifizieren.

Material und Methodik 41 Patienten mit vermuteter Leberverfettung erhielten eine klinisch indizierte Leberbiopsie zur Quantifizierung. Alle Biopsien wurden auf dem CT-Tisch (Toshiba Aquilion Vision) ultraschallgesteuert durchgeführt (Aplio 500). Vor der Nadelnavigation in den rechten Leberlappen wurde im US eine normalisierte lokale Varianzanalye durchgefuehrt. Mit der Nadel in situ erfolgte die Aufnahme eines Dual-Energy-Volumens der Leber $(16 \mathrm{~cm})$. Zwei Stanzbiopsien (Supercore, 18G) wurden entnommen und in Formalin fixiert. Monochromatische Serien wurden von 35 bis $130 \mathrm{keV}$ in $5 \mathrm{keV}$-Schritten rekonstruiert, die jeweiligen Dichtewerte (HE) der Leber in 2 ROls ermittelt und in einer Intensitätskurve DE-CT-I0 aufgetragen (curve-fit). Die NLV im US wurde in einer $3 \mathrm{~cm}$ ROI (jeweils mit 12.000 Messpunkten) im Areal der Biopsie durchgeführt. Alle Werte wurden mit dem pathologischen Ergebnis (Verfettungsgrad) korreliert. 
Ergebnisse Alle Biopsien konnten ohne Komplikationen durchgeführt werden. Der Mittelwert/SD des Verfettungsgrades in der Stanzbiopsie, NLV, DE-CT-I0 und $\mathrm{HE}$ gemessen auf den akquirierten $80 \mathrm{kV}$ und $135 \mathrm{kV}$-Serien waren jeweils $0.57 / 0.72,1.16 / 0.13,54.94 / 12.04,45.8 / 11.0$ und 52.84/11.24. Die Korrelationen/(two-tailed) p-Wert von DE-CT-I0 versus Biopsie, NLV versus Biopsie, und NLV versus DE-CT-I0 lagen jeweils bei $r 2=0.76 / p<0.01, r 2=0.59$ / $\mathrm{p}<0.01$ and $\mathrm{r} 2=0.61, \mathrm{p}<0.01$.

Schlussfolgerungen Sowohl Ultraschall als auch DE-CT erlauben die Vorhersage des Grades der Leberverfettung mit akzeptabler Genaugkeit, wobei die CT mit monochromatischer Bildrekonstruktion dem Ultrachall mit NLV gering überlegen ist.

\section{WISS 103.2 Klinischer Nutzen der diffusionsgewichteten Leberbildgebung mit extrapoliertem hohen b-Wert}

Autoren Roll $W^{1}$, Beyer $F^{2}$, Allkemper $T^{3}$, Tombach $B^{4}$, Bremer $C^{2}$ Institut 1 Uniklinikum Münster, Klinik für Nuklearmedizin, Münster; 2 Klinik für Radiologie, St. Franziskus Hospital, Münster; 3 Institut für klinische Radiologie, Uniklinikum Münster, Münster; 4 Röntgen und Strahlenklinik, Klinikum Osnabrück, Osnabrück

DOI 10.1055/s-0037-1600222

Zielsetzung Ziel dieser Studie ist die Untersuchung der Wertigkeit der diffusionsgewichteten Bildgebung (DWI) mit berechnetem hohen b-Wert implementiert in ein Standard-MRT-Leber-Protokoll.

Material und Methodik In der vorliegenden Studie beurteilten drei Auswerter MR-Leber-Datensätze inklusive (mit DWI) bzw. exklusive der DWI (ohne DWI). Die DWI umfasste dabei Sequenzen mit gemessenen (50/400/800 s/mm2), sowie mit berechnetem hohem b-Wert (1400 s/mm2) und der ADC-Map. Die Wertigkeit der zusätzlichen DWI mit extrapoliertem b-Wert wurde durch Evaluation von Detektionsraten, sowie Sensitivität, Spezifität, Genauigkeit und Sicherheit der Dignitätsentscheidung, und der Geschwindigkeit der Auswertung beurteilt. Darüber hinaus erfolgte eine Untersuchung auf zusätzliche Vorteile des berechneten hohen b-Wertes für die Läsions-Charakterisierung. Die ADC-Werte wurden auf die Möglichkeit einer Einordnung in die verschiedenen Tumorentitäten hin untersucht.

Ergebnisse Mit zusätzlicher DWI ergaben sich signifikant höhere Detektionsraten, insbesondere für kleinere Läsionen, bei gleichzeitig signifikant niedrigerer Auswertezeit für alle drei Auswerter. Die Entscheidung über die Dignität wurde mit DWI mit einer höheren Genauigkeit, Sicherheit und Sensitivität bei gleichbleibender Spezifität getroffen. Bei den ADC-Werten zeigten sich signifikant höhere Werte für benigne Läsionen im Vergleich zu malignen Läsionen. Benigne Läsionen mit teilweiser Diffusionsrestriktion zeigten ähnliche ADCWerte wie maligne Läsionen. Insbesondere bei diesen Leberläsionen zeigte der extrapolierte b-Wert einen Vorteil in der Charakterisierung. Die Wertigkeit des extrapolierten b-Wertes wurde höher eingestuft, je erfahrener der Auswerter in DWI war.

Schlussfolgerungen Die Implementierung einer DWI mit extrapoliertem hohen b-Wert in ein klassisches MR-Leberprotokoll ermöglicht eine effektivere und sicherere Detektion sowie eine bessere Charakterisierung fokaler Leberläsionen.

\section{WISS 206.4 Biphasische MSCT-Angiografie zur Diagnostik und Therapieplanung der nicht-okklusiven mesetenteriellen Ischämie (NOMI). Kann die MSCT die i. a.-DSA ersetzen?}

Autoren Schülke $C^{1}$, Kammerer $S^{2}$, Berkemeyer $S^{3}$, Heindel $W^{1}$, Köhler $M^{1}$, Buerke $\mathrm{B}^{1}$

Institut 1 Westfälische Wilhelms-Universität Münster, Institut für klinische Radiologie, Münster; 2 Westfälische Wilhelms-Universität Münster/GoetheUniversität Frankfurt, Institut für Klinische Radiologie/Institut für
Neuroradiologie, Münster; 3 Referenzzentrum Mammografie Münster/

Hochschule für Gesundheit, Münster

DOI 10.1055/s-0037-1600223

Zielsetzung Ziel der Arbeit ist die Evaulation der modernen Mehrzeilen-Computertomografie (MSCT) im Vergleich zur digitalen Subtraktionsangiografie (DSA) in der Diagnostik und Therapieplanung der NOMI.

Material und Methodik Retrospektive Studie an 30 Patienten (20 männlich, 10 weiblich, mittleres Alter 64,6 +/- 14,2 Jahre), bei denen aufeinander folgend bei V.a. auf eine NOMI ein biphasisches MSCT des Abdomens und eine DSA der Mesenterialgefäße durchgefuhrt wurden. Auswertung der MSCT durch zwei Radiologen (R1 mit 8 Jahren und R2 mit 4 Jahren Erfahrung in abdominaler MSCT) in Hinblick auf das Vorliegen einer NOMI. Zusätzliche qualitative und quantitative Bestimmung der Gefäßdurchmesser und des Kontrastierungsgrades der A. mesenterica superior (AMS). Weitere Auswertungskriterien umfassten sekundäre Darmischämiezeichen, u.a. eine vermindert Darmkontrastierung, ein Darmwandödem oder eine Pneumatosis intestinalis. Die MSCT-Diagnose und die sekundären Zeichen wurden gegenüber dem Goldstandard aus DSA-Diagnose und dem klinischen Verlauf statistisch ausgewertet.

Ergebnisse Bei 28 Patienten bestätigte die DSA-NOMI-Diagnose. In 2 MSCTVerdachtsfällen wurden in der DSA nicht bestätigt; 2 DSA-NOMI-Diagnosen wurden im MSCT nicht erkannt. Im Mann-Whitney-Test zeigte sich zwischen MSCT und DSA kein Unterschied in der NOMI-Diagnosestellung für R1 $(p=0,09)$ und eine geringe Unterlegenheit für $R 2(p=0,02)$. Für R1 ergaben sich die besten Korrelationen für den qualitativen Gefäßdurchmesser $(r=-0,39 ; p=0,03)$ und Gefäßkontrast $(r=0,45 ; p=0,01)$. Zu den quantitativen Gefäßdurchmessern bestand ebenfalls eine Korrelation (mittlere AMS $r=0,48 ; p=0,01$ und distale AMS $r=0,66 ; p<0,01)$. Die sekundären Darmischämiezeichen wiesen keine Korrelation auf.

Schlussfolgerungen Die MSCT ist bei Evaluation durch erfahrene Auswertung der DSA in der Diagnosestellung einer NOMI gleichwertig und kann kann die weitere Therapieentscheidung unmittelbar beeinflussen. Daher stellt sie insbesondere in Abteilungen ohne DSA-Möglichkeit - eine Alternative zur invasiven DSA dar.

\section{RK 102.4 Bestimmung des Leberfibrosegrades mittels Gd-EOB-DTPA-gestützter MRT}

Autoren Verloh $\mathrm{N}^{1}$, Haimerl $\mathrm{M}^{1}$, Utpatel $\mathrm{K}^{2}$, Stroszczynski $\mathrm{C}^{1}$, Wiggermann $\mathrm{P}^{1}$ Institut 1 Universitätsklinikum Regensburg, Institut für Röntgendiagnostik, Regensburg; 2 Institut für Pathologie, Institut für Röntgendiagnostik, Regensburg

DOI 10.1055/s-0037-1600224

Zielsetzung Ziel dieser Studie ist die Differenzierung der unterschiedlicher Leberfibrosegrade mithilfe der Gd-EOB-DTPA-gestützten MRT.

Material und Methodik 98 Patienten mit histopathologischer Untersuchung des Leberparenchyms unterzogen sich eine 3T-MRT Untersuchung der Leber mit leberspezifischem Kontrastmittel (Gd-EOB-DTPA). T1-gewichtete VIBE-Sequenzen der Leber mit Fettunterdrückung wurden ausgewertet, um die relative Signaländerung (RE) zwischen nativer und hepatobiliärer Phase (20 min) zu evaluieren. Zur Bestimmung des Fibrosegrades wurde der Ishak-score (1 6) verwendet und die Patienten wurden in 5 Patientengruppen eingeteilt: keine Leberfibrose (KLF; Ishak=0, $n=17$ ), geringgradige Leberfibrose (GLF; Ishak =1, $n=20)$, fortgeschrittene Leberfibrose (FLF; Ishak=2/3, $n=24)$, schwerwiegende Leberfibrose (SLF; Ishak $=4 / 5, n=17$ ) und Leberzirrhose (LC; Ishak=6, $\mathrm{n}=20$ ).

Ergebnisse Es zeigte sich hinsichtlich der RE ein signifikanter Unterschied zwischen Patienten ohne Leberfibrose $(R E=1.13 \pm 0.18)$ und Patienten mit Leberfibrose/Zirrhose ( $R E=0.62 \pm 0.29 ; p<0.001)$. Mithilfe der RE konnte eine signifikante Unterscheidung für alle Grade der Leberfibrose (GLF, FLF, SLF und LC) erreicht werden. Mit einer Sensitivität von $\geq 82 \%$ und einer Spezifität von 
$\geq 87 \%$ können die einzelnen Grade der Leberfibrose/Zirrhose, mithilfe der relativen Signaländerung unterschieden werden.

Schlussfolgerungen Die Bestimmung der Aufnahme von Gd-EOB-DTPA in die Hepatozyten stellt eine gute, bildbasierte Methode dar die unterschiedlichen Leberfibrose/Zirrhosegrade zu differenzieren.

\section{WISS 103.3 4D MRT-Bildgebung der Leber: Kontinuierliche Beurteilung der KM-Dynamik unter freier Atmung}

Autoren Weiß $\mathrm{J}^{1}$, Othman $\mathrm{A}^{2}$, Martirosian $\mathrm{P}^{2}$, Nickel $\mathrm{M}^{3}$, Taron $\mathrm{J}^{2}$, Kolb $\mathrm{M}^{2}$, Ruff $C^{2}$, Nikolaou $K^{2}$, Notohamiprodjo $M^{2}$

Institut 1 Uni Klinik Tübingen, Radiologie, Tübingen; 2 Radiologische Klinik, Diagnostische und Interventionelle Radiologie, Tübingen; 3 Siemens

Healthcare, MRT, Erlangen

DOI 10.1055/s-0037-1600225

Zielsetzung Beurteilung der klinischen Anwendung einer VIBE-Sequenz für die kontinuierliche KM-verstärkte MRT-Bildgebung der Leber unter freier Atmung mittels compressed sensing (CS) und retrospektivem self-gating (SG).

Material und Methodik Bei 20 Patienten wurde mit einer neuen VIBE-Sequenz (VIBESG-CS) eine Gadobutrol-verstärkte Leber-MRT bei 1,5T durchgeführt. Diese Sequenz erlaubt eine kontinuierliche Datenakquisition unter freier Atmung durch Implementierung eines CS Rekonstruktionsalgorithmus und atemabhängigen SG. Die Datenakquisition wurde zeitgleich mit der KM-Applikation gestartet und für 128 Sekunden durchgeführt. Anschließend wurden von den Rohdaten 16 aufeinanderfolgende Sequenzen (zeitl. Auflösung $8 \mathrm{~s}$; räuml. $1,2 \times 1,2 \times 3 \mathrm{~mm}$ ) rekonstruiert. Die native, arterielle, portal-venöse und venöse Phase mit der subjektiv besten Bildqualität wurde definiert und zu einer historischen konventionellen multiphasischen Leber-MRT mit Atemstopp (BH) der selben Patienten als Referenzstandard verglichen (VIBEBHSTD). Die Bildqualität wurde qualitativ (Qualität, Schärfe, Abgrenzbarkeit von Läsionen, Artefakte; 2 unabhängige Reader; 5-Punktskala, 5 =exzellent) und quantitative (Coefficient-of-Variation (CV), Signalintensität (SI) der Leber) untersucht.

Ergebnisse Die Bildqualität, die Abgrenzbarkeit der Läsionen und Bewegungsartefakte zeigten keinen signifikanten Unterschied zwischen den Sequenzen $(p \geq 0.07)$. Die Bildschärfe wurde in der arteriellen, portal-venösen und venösen Phase der VIBESG-CS signifikant höher gewertet als in der VIBEBH-STD $(p \leq 0.03)$. VIBESG-CS zeigte jedoch ein signifikantes Auftreten von Rekonstruktionsartefakten welche in der VIBEBH-STD nicht nachweisbar waren $(p=0.001)$. Die SI der Leber war in der VIBEBH-STD signifikant höher als in der VIBESG-CS $(p=0.002)$ während der CV keine signifikanten Unterschied zwischen den Sequenzen zeigte $(p=0,1)$.

Schlussfolgerungen VIBESG-CS kann für die kontinuierliche KM-verstärkte Leber-MRT unter freier Atmung angewendet werden und erlaubt einer vergleichbare Bildqualität wie VIBEBH-STD.

\section{WISS 103.4 Assessment of Gadoxetic Acid-enhanced MRI phases for LI-RADS categorization and non- invasive grading of hepatocellular carcinoma}

Authors Wutschke $\mathrm{M}^{1}$, Franiel $\mathrm{T}^{2}$, Teichgräber $\mathrm{U}^{1}$, Katenkamp $\mathrm{K}^{3}$, Lehmann $\mathrm{T}^{4}$

Institute 1 Universitätsklinikum Jena, Institut für diagnostische und interventionelle Radiologie, Jena; 2 Universitätsklinikum Jena, Institut für Diagnostische und Interventionelle Radiologie, Jena; 3 Universitätsklinikum Jena, Pathologisches Institut, Jena; 4 Universitätsklinikum Jena, Institut fuer Medizinische Statistik, Informatik und Dokumentation, Jena

DOI 10.1055/s-0037-1600226

Zielsetzung This retrospective study evaluated the impact of transitional and hepatobiliary phase on LI-RADS classification and non-invasive grading in patients with hepatocellular carcinoma (HCC).
Material und Methodik 61 patients with 61 histologically confirmed HCCs underwent MRI with gadoxetic acid. In all phases (arterial [AP], portal venous [PVP], transitional [TP], hepatobiliary [HP]) signal intensities (SI) of HCC and adjacent liver parenchyma were measured. For quantitative assessment ratio between SI of HCC and SI of normal liver were calculated. LI-RADS classification (LR-1 to LR-5) were done in A) LI-RADS major criterions with assessment of washout criterion only in PVP, B) same as $A+$ ancillary feature „transitional phase hypointensity“ and C) same as B + ancillary feature "hepatobiliary phase hypointensity". Histopathological HCC grading results were correlated with the $\mathrm{SI} \mathrm{HCC} \mathrm{ratio} \mathrm{(=} \mathrm{SI} \mathrm{mass} \mathrm{HP/SI} \mathrm{mass} \mathrm{AP).}$

Ergebnisse None HCC was graded as LR-1 or LR-2. The inclusion of the feature „transitional phase hypointensity“ led to a non-significant upgrading of 5/9 HCC from LR-3 to LR-4 ( $p=0.062)$. The addition of the feature "hepatobiliary phase hypointensity“ led to a statistical significant upgrading of $8 / 9$ HCC from LR-3 to LR-4 $(p=0.008)$. None HCC with initial LR-4 was upgraded. None lesions were downgraded. The HCC SI ratios between G1 vs. G2/G3 tumors were statistically significant different $(p=0.008)$.

Schlussfolgerungen The addition of transitional and hepatobiliary gadoxetic acid-enhanced MRI phases improves LI-RADS categorization. Quantitative assessment of HCC SI ratio enables non-invasive grading.

Herzdiagnostik/Gefäßdiagnostik

\section{WISS 102.3 Die koronare CT-Angiografie mittels Dual-Source-CT der dritten Generation: Auswirkungen auf die Bildqualität und die Strahlendosis}

Autoren Apfaltrer $\mathrm{P}^{1}$, Szolar $\mathrm{D}^{2}$, Wurzinger $\mathrm{E}^{2}$, Dutschke $\mathrm{A}^{3}$, Loewe $\mathrm{C}^{4}$, Ringl $\mathrm{H}^{1}$, Sorantin $\mathrm{E}^{5}$, Apfaltrer $\mathrm{G}^{6}$

Institut 1 Universitätsklinik für Radiologie und Nuklearmedizin, Medizinische Universität Wien, Klinische Abteilung für Allgemeine Radiologie und Kinderradiologie, Wien; 2 Diagnostikum Graz-Südwest, Graz;

3 Universitätsklinik Heidelberg, Diagnostische und Interventionelle Radiologie, Heidelberg; 4 Universitätsklinik für Radiologie und Nuklearmedizin,

Medizinische Universität Wien, Kardiovaskuläre und Interventionelle Radiologie, Wien; 5 Universitätsklinik für Radiologie, Medizinische Universität Graz, Klinische Abteilung für Kinderradiologie, Graz; 6 Universitätsklinik für Radiologie, Medizinische Universität Graz, Klinische Abteilung für

Kinderradiologie

DOI 10.1055/s-0037-1600227

Zielsetzung Ziel der Studie war die Evaluierung der Strahlendosis und der Bildqualität der CT-Angiografie der Koronararterien (cCTA) mittels eines Dual-Source-CT (DSCT) der dritten Generation im Vergleich zu der 64-Zeilen Single-Source-cCTA.

Material und Methodik 140 Patienten (73 männlich, Alter $62 \pm 11$ Jahre) mit niedriger bis mittlerer Vortestwahrscheinlichkeit für eine KHK wurden retrospektiv in diese Studie eingeschlossen. Die CCTA erfolgte entweder auf einem DSCT der dritten Generation mittels prospektiver EKG-Triggerung $(n=70)$ (Gruppe 1) oder mittels retrospektiven EKG-Gating auf einem 64 64-Zeilen Single-Source CT $(n=70)$ (Gruppe 2). Kontrast-zu-Rausch und Signal-zuRausch-Verhältnis wurden in der Aorta und den Koronararterien gemessen. Die subjektive Bildqualität (IQ) wurde mittels einer Fünf-Punkte-Likert-Skala von 2 Radiologen bewertet. Die Effektive Dosis (ED) wurde mittels spezifischer Umrechnungsfaktoren geschätzt.

Ergebnisse Das Kontrast-zu-Rausch-Verhältnis der Gruppe 1 war signifikant höher als in Gruppe 2 (alle Messpunkte: $p<0,001$ ). Das Signal-zu-RauschVerhältnis der Gruppe 1 war ebenfalls signifikant höher als in Gruppe 2 $(p<0,05)$, außer für die distale linke Ramus circumflexus $(p>0,05)$. Die subjektive IQ Gruppe 1 wurde signifikant besser bewertet als für in Gruppe 2, (Medianwert [25.-75-Perzentil.]: 1 [1 - 2] vs. 2 [2 - 3], $(p<0,001)$. Die mittlere ED betrug 1,55 [1,09-1,88] Millisievert (mSv) in Gruppe 1 vs. 12,29 [11,63- 
$14,36] \mathrm{mSv}$ in Gruppe $2(\mathrm{p}<0,001)$, was einer Strahlungsdosisreduktion von $87,4 \%$ entsprach.

Schlussfolgerungen Die cCTA mittels eines DSCT der dritten Generation führt zu einer signifikant verbesserten IQ bei deutlicher Strahlendosisreduktion.

\section{WISS 318.6 Nephroprotektive Methoden zur Evaluation von Gefäßstenosen bei Patienten mit chronischer Niereninsuffizienz: eine Vergleichsstudie zwischen der kontrastmittelfreien QISS-MRA und der invasiven CO2-DSA}

Autoren Arendt $C^{1}$, Leithner $D^{2}$. Wichmann $J^{2}$ Czwikla $R^{2}$ Vogl $T^{2}$, GruberRouh $\mathrm{T}^{2}$

Institut 1 Institut für Diagnostische und Interventionelle Radiologie, Universitätsklinikum Frankfurt, Zentrum der Radiologie, Frankfurt am Main; 2 Universitätsklinikum Frankfurt, Diagnostische und Interventionelle Radiologie, Frankfurt am Main DOI 10.1055/s-0037-1600228

Zielsetzung Kontrastmittelfreie MR-Angiografie und invasive DSA mit CO2Gas sind vielversprechende Verfahren in der Diagnostik der pAVK bei Patienten mit chronischem Nierenversagen. Zielsetzung war der Vergleich beider Methoden hinsichtlich der diagnostischen Genauigkeit für die Detektion von signifikanten Gefäßstenosen und der subjektiven Bildqualität.

Material und Methodik 11 konsekutive Patienten mit erniedrigtem eGFRBlutwert $\left(23,9 \pm 7,9 \mathrm{ml} / \mathrm{min} / 1,73 \mathrm{~m}^{2}\right)$ wurden retrospektiv in die Studie aufgenommen. Die DSA-Technik mit CO2-Gas wurde als Referenzstandard herangezogen. Zwei Beobachter bewerteten die Becken-und Beinarterien unter Zuhilfenahme eines 21-Segment-Modelles gefäß- und nicht patientenbasiert in zufälliger Reihenfolge. Hierbei erfolgte eine Bewertung hinsichtlich der Identifikation signifikanter ( $\geq 50 \%$ ) Gefäßstenosen und eine subjektive Beurteilung der Bildqualität anhand einer Likert-Skala (1: diagnostisch nicht verwertbar; 5: exzellente Qualität).

Ergebnisse Die Prävalenz signifikanter Stenosen lag bei 25,9\%. Die Ergebnisse der diagnostischen Genauigkeit (MRA gegenüber DSA; $n=112$ ) ergab Folgendes (p-Werte <0,001): Sensitivität 86,2\% \& 89,7\%; Spezifizität 90,4\% \& 95,8\%; diagnostische Genauigkeit: $89.3 \%$ \& 94.2\%; ICC: 0.96 \& 0.96. Die Medianwerte der subjektiven Bildqualität bei der QISS-MRA $(n=220)$ waren wie folgt: arterielle Signalintensität bewertet vom 1. Beobachter: 5 (gesamte Gefäßachse), 4 (Becken), 5 (Oberschenkel), 5 (Wade), und vom 2. Beobachter: 4,4,4,4 (ICC: 0,79$)$; venöse Überlagerung: 5,4,4,5 \& 5,4,5,5 (0,77); Bewegungsartefakte: 5,4,5,5 \& 5,4,5,5 (0,73); EKG-Artefakte mit 3,3,4,3 \& 4,4,4,3. Die Röntgendichte des CO2-Gases in der DSA ( $n=145)$ betrug $3,3,4,3$ und 4,4,4,3 (ICC $0,74)$.

Schlussfolgerungen Bei Patienten mit pAVK und chronischer Niereninsuffizienz stellt sich die kontrastmittelfreie QISS-MRA im 3-Tesla-Gerät als ebenbürtiges Verfahren gegenüber der invasiven CO2-DSA bei ähnlich hoher diagnostischer Genauigkeit zur Detektion von signifikanten Gefäßstenosen und bei subjektiv besserer Bildqualität dar.

\section{WISS 102.6 Systolische Akquisition bei High-Pitch CT- Angiografien zur TAVI-Planung - Wie gut gelingt das und wann besser nicht?}

Autoren Baumann $\mathrm{A}^{1}$, Winter $\mathrm{K}^{1}$, Garthe $\mathrm{T}^{1}$, Thäns $\mathrm{N}^{1}$, Bock $J^{1}$, Schindler $\mathrm{R}^{1}$, Bischoff $B^{1}$, Trumm $C^{1}$, Reiser $M^{2}$, Schwarz $F^{3}$

Institut 1 Institut für Klinische Radiologie, LMU München, Campus Großhadern, Radiologie, München; 2 Institut für Klinische Radiologie, LMU München, Radiologie, München; 3 Klinik für Diagnostische Radiologie und Neuroradiologie, Radiologie, Augsburg

DOI 10.1055/s-0037-1600229

Zielsetzung Bei der CT-Angiografie zur Planung einer TranskatheterAortenklappenimplantation(TAVI) hat sich in den letzten Jahren die systolische
Akquisition durchgesetzt. Ziel dieser Studie ist es, die Erfolgsrate systolischen Timings bei CTA-Protokollen mit hohem Pitchfaktor zu bestimmen und Patienten-seitige Risikofaktoren zu identifizieren.

Material und Methodik 225 Patienten erhielten im Zeitraum 10/2015-04/ 2016 eine High-Pitch CTA von Thorax und Abdomen zur TAVI-Planung mit systolischem Akquisitionsfenster des Herzens (Dual-Source CT der 2. Generation). Für diese Studie wurde die tatsächliche Herzphase durch Analyse des Öffnungszustandes von Aorten- und Mitralklappe bestimmt und mit dem automatisch registrierten Scanzeitpunkt des Annulus innerhalb des RR-Intervalls korreliert. Die minimale, maximale und mittlere Herzfrequenz (HF), Herzfrequenzvariabilität (HFV), das Vorliegen von Vorhofflimmern (VHF) und Extrasystolen wurden mittels multivariater und ROC-Kurven-Analyse auf ihren prädiktiven Wert für eine nicht-systolische Akquisition getestet.

Ergebnisse 50 Patienten wurden wegen nicht archivierter EKGs ausgeschlossen. Bei 103 (59\%) von 175 Patienten gelang die Akquisition in Systole. Bei systolischen Scans wurde der Annulus bei $31 \pm 9 \%$ und bei nicht-systolischen Scans bei $58 \pm 26 \%$ des RR-Intervalls abgebildet. Die Unterschiede für HF (68 \pm 10 vs. $76 \pm 14 / \mathrm{min}, \mathrm{p}<0.001)$ und HFV $(19 \pm 23$ vs. $35 \pm 24 / \mathrm{min}, \mathrm{p}<0.001)$ waren hochsignifikant. Patienten mit nicht-systolischen Scans wiesen signifikant häufiger VHF auf (14/103 vs. 27/72, p<0.001, Relatives Risiko: 2.74). HF und HFV hatten einen hohen prädiktiven Wert für nicht-systolische Scans (HF: $A \cup C=0.71$, bester cutoff: $>74 / \mathrm{min}$; HRV: $A \cup C=0.69$, bester cutoff: $>30 /$ $\min )$.

Schlussfolgerungen Bei unselektionierten Patienten vor TAVI gelingt die systolische Akquisition mittels High-Pitch-CTA nur bei ca. $60 \%$. Eine hohe mittlere HF, eine hohe HFV und VHF sind positive Prädiktoren für einen falschen Akquisitionszeitpunkt. Bei diesen Patienten sollte eine retrospektives EKG-Gating des Herzens erwogen werden.

\section{WISS 316.5 4D-Fluss-MRT der Pulmonalarterien: Validierung und Fehlerquantifizierung an einem 3T-MRT mit digitaler Breitband-Technik}

Autoren Berlin $\mathrm{C}^{1}$, Oechtering $\mathrm{T}^{1}$, Sieren $\mathrm{M}^{1}$, Drömann $\mathrm{D}^{2}$, Barkhausen $]^{1}$, Frydrychowicz $\mathrm{A}^{1}$

Institut 1 UKSH Lübeck, Klinik für Radiologie und Nuklearmedizin, Lübeck; 2 UKSH Lübeck, Medizinische Klinik III, Pulmologie, Lübeck

DOI 10.1055/s-0037-1600230

Zielsetzung 4D-Fluss-MRT lässt neben der nicht-invasiven Diagnose der pulmonalen Hypertonie einen Informationsgewinn aus den hämodynamischen Erkenntnissen erwarten. Ziel war die bisher noch ausstehende Validierung der Sequenz anhand des Schlagvolumens (SV) und der maximalen Flussgeschwindigkeit (Vmax) im Truncus pulmonalis (TP) an einem System mit digitaler Breitbandtechnik, welches ein verbessertes Signal-zu-Rausch-Verhältnis verspricht.

Material und Methodik Die zentralen Pulmonalarterien 23 gesunder Probanden (14w, $38 \pm 18 \mathrm{~J})$ wurden mit einer 4D-Fluss-Sequenz und 2D Phasenkontrast (2DPC)-Messungen an 3T (Philips Ingenia) untersucht. SV und Vmax in den Pulmonalarterien wurden mit GTFlow (GyroTools) gemessen, zwischen 4D und 2DPC, mit dem bSSFP-basierten rechtsventrikulären SV (RVSV) und der internen Konsistenzprüfung (conservation of mass, COM) verglichen. Messungen wurden an einem statischen Phantom zur Fehlerquantifizierung wiederholt. Die statistische Auswertung beinhaltete Bland-Altman-Analyse und t-Test.

Ergebnisse Die Ergebnisse der 4D-Fluss-MRT stimmten mit der 2DPC gut überein (mittlerer Fehler SV: 5,7 $\pm 10,6 \mathrm{ml}, \mathrm{p}<0,05$; Vmax: 3,9 $\pm 12,5 \mathrm{~cm} / \mathrm{s}$, $\mathrm{p}=\mathrm{n} . \mathrm{s}$.) und zeigten eine Verbesserung nach Phantomkorrektur (SV 4D: $90,5 \pm 25,3 \mathrm{ml}, 2 \mathrm{D}: 87,4 \pm 19,0 \mathrm{ml}$; mittlerer Fehler $=3,1 \pm 12,3 \mathrm{ml}$; Vmax 4D: $93,3 \pm 16,3,2 \mathrm{D}: 86,4 \pm 14,9 \mathrm{~cm} / \mathrm{s}$, mittlerer Fehler $=1,3 \pm 14,5 \mathrm{~cm} / \mathrm{s} ; \mathrm{p}=\mathrm{n} . \mathrm{s}$.). Die Phantomkorrektur verminderte den mittleren Fehler zwischen 4D-FlussMRT SV und RVSV von 5,5 $\pm 17,2 \mathrm{ml}$ auf $-0,1 \pm 20 \mathrm{ml}$ ( $\mathrm{p}=\mathrm{n}$. s.). Die COM-Analyse wies einen geringen mittleren Fehler von 2,2 $\pm 15,6 \mathrm{ml}(p=n$. s.) auf. 
Schlussfolgerungen Quantitative 4D-Flussmessungen der Pulmonalarterien sind mit klinischen MR-Routineverfahren gut vergleichbar. Fehlerverbesserung durch Phantomkorrektur und Streubreite im Bland-Altman-Fenster können mit unvollständig unterdrückten Phasenoffsets und Unterschieden in zeitlicher und örtlicher Auflösung erklärt werden. Temperaturschwankungen der Gradientenspulen können weitere, schlecht zu kontrollierende Fehlerquellen sein.

\section{RK/WISS 402.6 Experimental feasibility of dual contrast agent based spectral photon-counting computed tomografy for detection of endoleaks following EVAR}

Authors Dangelmaier J $]^{1}$, Bar-Ness $D^{2}$, Münzel $D^{3}$, Daerr $\mathrm{H}^{4}$, Pfeiffer $\mathrm{F}^{5}$, Proksa $\mathrm{R}^{4}$, Rummeny $\mathrm{E}^{3}$, Douek $\mathrm{P}^{2}$, Noël $\mathrm{P}^{3}$

Institute 1 Klinikum rechts der Isar der Technischen Universität München, Institut für diagnostische und interventionelle Radiologie, München; 2 Louis Pradel University Hospital, Department of Interventional Radiology and

Cardio-vascular and Thoracic Diagnostic Imaging, Bron; 3 Klinikum Rechts der Isar der Technischen Universität München, Diagnostische und Interventionelle Radiologie, München; 4 Philips GmbH, Innovative Technologies, Research Laboratories, Hamburg; 5 Technische Universität München, Lehrstuhl für Biomedizinische Physik, Physik-Department \&amp; Institut für Medizintechnik DOI 10.1055/s-0037-1600231

Zielsetzung After endovascular aortic repair (EVAR) discrimination of leaking contrast media and calcifications of similar attenuation within the aneurysm sac is challenging and often requires multiple $\mathrm{CT}$ acquisitions. Dual contrast agent based spectral photon-counting CT may provide reliable detection of endoleaks with a single $\mathrm{CT}$ acquisition.

Material und Methodik To experimentally evaluate the potential of spectral photon-counting computed tomografy (SPCCT), an aortic phantom was inhouse designed and produced. A centrally located stent lined compartment was filled with water as well as iodine and gadolinium mimicking enhanced blood within the aortic lumen. Representing the endoleak, adjacent compartments contained either one of the contrast agents or calcium chlorine to mimic calcifications. After data acquisition with a modified clinical base, small field-of-view SPCCT prototype scanner with multi-energy bins, material decomposition was performed to generate iodine, gadolinium and calcium maps.

Ergebnisse In a conventional CT slice, Hounsfield Units of the different compartments were similar ranging from about $145 \mathrm{HU}$ up $170 \mathrm{HU}$. Material specific maps clearly differentiate the distributions, within the compartments filled with iodine, gadolinium or calcium.

Schlussfolgerungen A single dual contrast agent based spectral photoncounting CT scan may replace conventional CT scans in native, arterial and delayed phase to detect endoleaks of different flow rates without interference from existing calcifications after endovascular aortic repair. It is a unique feature of our method to capture different contrast media dynamics in a single scan. Additionally, a significant reduction of radiation exposure is enabled.

\section{WISS 102.9 Statine beeinflussen die Zusammensetzung und reduzieren die Änderungsvariabilität von Koronarplaques: Subanalyse einer randomisierten, kontrollierten Studie von Atorvastatin in HIV Patienten}

\author{
Autoren Foldyna $\mathrm{B}^{1}$, Lo $\mathrm{J}^{2}$, Grinspoon $\mathrm{S}^{2}$, Lu $\mathrm{M}^{3}$, Hoffmann $\mathrm{U}^{4}$ \\ Institut 1 Massachusetts General Hospital, Radiology - Cardiac MR PET CT \\ Program, Boston; 2 Massachusetts General Hospital - Harvard Medical School, \\ Department of Medicine, Endocrine Division, Program in Nutritional \\ Metabolism, Boston; 3 Massachusetts General Hospital - Harvard Medical \\ School, Department of Radiology, Cardiac PET MR CT Program, Bosoton;
}

4 Massachusetts General Hospital - Harvard Medical School, Department of Radiology, Cardiac PET MR CT Program, Boston

DOI 10.1055/s-0037-1600232

Zielsetzung Koronare CT Angiografie (CTA)-basierte Analyse der Atorvastatineffekte auf die Zusammensetzung und Volumenänderung von koronaren Plaques als Teil einer placebo-kontrollierten randomisierten Studie in Patienten mit HIV. Der Hauptfokus richtet sich auf die vermutete Reduktion der fettigen und fibrotischen Anteile zugunsten der kalzifizierten Anteile sowie auf eine Analyse der Variabilität der Volumenveränderungen während des Beobachtungszeitraumes.

Material und Methodik Vierzig Patienten mit subklinscher Atherosklerose $(\mathrm{LDL}<130 \mathrm{mg} / \mathrm{dl})$ und unter HIV Therapie wurden in eine Atorvastatin(40 mg/d, p.o.) oder eine Placebogruppe randomisiert. Eine koronare CTA wurde zu Studienbeginn sowie nach 12 Monaten durchgeführt. Die Plaquevolumetrie und die Gewebeklassifizierung erfolgten semiautomatisch für jeden Koronarplaque einzeln bei zuvor festgelegten Dichtegrenzwerten (Fett $<39 \mathrm{HU}$, fibrotisch $40-129 \mathrm{HU}$, kalzifiziert $>130 \mathrm{HU}$ ).

Ergebnisse Im Gegensatz zu der Placebogruppe mit 20 Patienten und 37 Plaques, führte Atorvastatin in den 17 Patienten mit 33 Plaques (Statingruppe) zu einer relativen Reduktion der fettigen und fibrotischen Anteile mit einem Trend zum reduzierten Anstieg der kalzifizierten Komponenten. Mediane Volumenäderungen (fett/fibrotisch/kalzifiziert) waren $+24.5 \% /+15.9 \% /+28.7 \%$ unter Placebo und $-31.6 \% /-7.4 \% /+6.7 \%$ unter Atorvastatin (p für Differenzen der Änderungen 0.006/0.026/0.076). Die Kalziumdichte änderte sich weder unter Placebo noch unter Atorvastatin signifikant $(p=0.70)$. Statine reduzierten die Variabilität der Volumenänderung im Vergleich zum Placebo um 76\%. Schlussfolgerungen Im Vergleich zum Placebo reduzierten Statine die fettigen und fibrotischen Anteile der koronaren Plaques in HIV Patienten. Die verkalkten Anteile stiegen in beiden Gruppen ohne signifikante Änderung der Kalziumdichte an, mit einem Trend zu einer größeren Zunahme des Volumens unter Placebo. Im Vergleich zum Placebo, reduzierten Statine die hohe natürliche Variablitiät der Volumenänderung von koronaren Plaques.

\section{WISS 102.7 Analyse der Strahlenexposition unterschiedlicher CT-Angiografieprotokolle für die TAVI-Planung}

Autoren Fries $\mathrm{P}^{1}$, Legler $\mathrm{K}^{1}$, Dabew $\mathrm{R}^{1}$, Altmeyer $\mathrm{K}^{1}$, Seidel $\mathrm{R}^{1}$, Scheller $\mathrm{B}^{2}$, Stroeder $]^{1}$, Bücker $A^{1}$

Institut 1 Universitätsklinikum des Saarlandes, Klinik für Diagnostische und Interventionelle Radiologie, Homburg; 2 Universitätsklinikum des Saarlandes, Klinik für Innere Medizin III - Kardiologie, Angiologie und internistische

Intensivmedizin, Homburg

DOI 10.1055/s-0037-1600233

Zielsetzung Ziel dieser retrospektiven Untersuchung war die Analyse der Strahlenexposition unterschiedlicher CT-Angiografieprotokolle im Rahmen klinischer TAVI-Planungen.

Material und Methodik Bei 136 Patienten wurden unter klinischen Bedingungen drei unterschiedliche CTA Protokolle mit singulärem KM-Bolus mittels EKG-Synchronisation und automatischer Expositionskontrolle (CAREdose4D, Siemens) zur TAVI Planung durchgeführt (Protokoll 1: CTA von Thorax/Abdomen/Becken mittels retrospektivem EKG-Gating $(N=46) /$ Protokoll 2: CTA Thorax mit retrospektivem EKG-Gating + CTA Abdomen/Becken ohne EKG-Synchronisation ( $\mathrm{N}=39$ )/Protokoll 3: duale High-Pitch CTA Thorax/Abdomen/Becken mit prospektiver EKG-Triggerung in Systole und Diastole $(\mathrm{N}=51)$. Protokoll 1 und 2 wurden mit einem 128-Zeilen CT (Siemens Edge) und Protokoll 3 mit einem $2 \times 192$ Zeilen Dual-Source CT (Siemens Force) durchgeführt. Das Dosis-Längen-Produkt (DLP) wurde retrospektiv erfasst und die korrespondierende effektive Dosis (E) für die jeweiligen Untersuchungen berechnet $(E=D L P x k a p p a(k a p p a=$ Konversionsfaktor $))$. Die statistische Analyse erfolgte mittels ANOVA inklusive Bonferroni-Korrektur. 
Ergebnisse Für Protokoll 1 wurden die höchsten Dosiswerte ermittelt (MW+/SA: $\left.\mathrm{DLP}\left(\mathrm{mGy}{ }^{*} \mathrm{~cm}\right)=1871+/-987 ; \mathrm{E}(\mathrm{mSv})=28,3+/-15,0\right)$. Protokoll 2 geht mit deutlich geringen Dosiswerten einher $\left(\mathrm{DLP}\left(\mathrm{mGy}{ }^{*} \mathrm{~cm}\right)=1115+/-689,3 ; \mathrm{E}(\mathrm{mSv})\right.$ $=16,4+\mid-10,0)$ entsprechend einer Dosisreduktion von $42 \%$ gegenüber Protokoll 1. Die niedrigsten Dosiswerte ergaben sich für Protokoll $3\left(\mathrm{DLP}\left(\mathrm{mGy}^{*} \mathrm{~cm}\right)\right.$ $=306,3+/-115,1 ; E(m S V)=4,6+/-1,8)$ entsprechend einer Dosisreduktion von $72 \%$ bzw. $84 \%$ gegenüber Protokoll 1 und 2 . Im paarweisen Vergleich ergab die Bonferroni-Analyse jeweils statistisch signifikante Unterschiede zwischen den Untersuchungstechniken $(p<0,0001)$.

Schlussfolgerungen Die Anwendung von high-pitch CTA Protokollen mit prospektiver EKG-Triggerung mittels Dual-Source CT-Scanner der 3. Generation erlauben eine signifikante Reduktion der Strahlenexposition bei der TAVI Planung gegenüber Protokollen mit retrospektivem EKG-Gating.

\section{WISS 102.5 Falsch positive Verkalkungen und Strahlendosis beim koronaren Calciumscoring mit iterativer Rekonstruktion und rauschbasierter Dosisfindung}

Autoren Garmer $\mathrm{M}^{1}$, Lehrenfeld $\mathrm{C}^{2}$, Metz $\mathrm{F}^{1}$, Klein-Wiele $\mathrm{O}^{1}$, Brandts $\mathrm{B}^{3}$, Grönemeyer $D^{1}$

Institut 1 Institut für Mikrotherapie, Radiologie, Bochum; 2 Universität Witten/Herdecke; 3 Augusta Krankenanstalten/Universität Witten Herdecke, Kardiologie, Bochum

DOI 10.1055/s-0037-1600234

Zielsetzung Bestimmung des Effektes der iterativen Rekonstruktion beim koronaren Calciumscoring (CAC) auf falsch-positive Läsionen und die Strahlendosis unter Verwendung einer rauschbasierten Dosisfindung. Die semiautomatische software-basierte Quantifizierung von Kalzifikationen kann durch ein vermindertes Signal-Rausch-Verhältnis beeinträchtigt werden. Rauschbasierte Grenzwerte wurden in der Vergangenheit vorgeschlagen um falsch-positive Läsionen in dosisreduzierten Protokollen zu reduzieren.

Material und Methodik Retrospektive Untersuchung von 388 Patientenpaaren mit gleichem Geschlecht, Alter \pm 5 Jahre und BMI \pm 2 Punkte. Das CAC Scoring erfolgte mittels eines 320-Zeilen CT mit Standarddosis und gefilterter Rückprojektion (FBP) oder mit reduzierter Dosis und adaptive-iterative-dose-reduction in 3D (AIDR 3D). Die individuelle Dosismodulation basierte auf einem Rauschgrenzwert. Strahlendosis, Bildqualität und Ausmaß der falsch-positiven Verkalkungen wurden erfasst.

Ergebnisse AIDR 3D versus FBP zeigte eine reduzierte Strahlendosis (Median mSv 0,85 versus 1,04; $p<0,001$ ), weniger Rauschen (Median SD 14,71 versus 18,07; $p<0,001$ ) und höheres Signal-Rausch-Verhältnis (Median 4,01 versus 3,14; $p<0,001$ ). Bei den 388 mittels AIDR 3D untersuchten Patienten fanden sich ein geringes Ausmaß an falsch-positiven Kalzifikationen bei 302 Patienten, ein mäßiges Ausmaß in 76 Patienten und ein hohes Ausmaß bei 10 Patienten, während bei mittels FBP untersuchten Patienten die korrespondierenden Werte 79, 175 und 134 betrugen $(p<0.001)$.

Schlussfolgerungen CAC scoring mittels iterativer Rekonstruktion und rauschbasierter Dosisfindung ist eine geeignete Technik zur Reduktion der Strahlendosis und Verbesserung der Bildqualität bei gleichzeitiger Vermeidunng falsch-positiver Kalifikationen.

\section{WISS 102.4 Strahlenexposition des koronaren Calciums-Scorings und der koronaren CT-Angiografie mit einem 3. Generations Dual-Source CT in einem unselektionierten Patientenkollektiv}

\author{
Autoren Gassenmaier $\mathrm{T}^{1}$, Kunz $\mathrm{A}^{1}$, Kosmala $\mathrm{A}^{1}$, Bley $\mathrm{T}^{1}$, Petritsch $\mathrm{B}^{1}$ \\ Institut 1 Universitätsklinikum Würzburg, Institut für diagnostische und \\ interventionelle Radiologie, Würzburg \\ DOI 10.1055/s-0037-1600235
}

Zielsetzung Beurteilung der Dosis beim koronaren Calcium-Scoring (CCS) und der CT-Koronarangiografie (cCTA), sowie Bestimmung dosisbeeinflussender Parameter bei Untersuchung mit einem 3. Generations Dual-Source-CT in einem unselektionierten Patientenkollektiv.

Material und Methodik Bei 159 konsekutiven Patienten wurden 137 CCSs und 147 cCTAs durchgeführt. Anterior-posteriorer (AP) und lateraler Thoraxdiameter wurden bestimmt und die Summe diesen sowie der effektive Durchmesser berechnet. Die effektive Dosis wurde durch Multiplikation des Dosis-Längen-Produktes mit einem Umrechnungsfaktor von 0,014 mSv/ mGy* ${ }^{*}$ geschätzt. Um individuelle Unterschiede im Patientenhabitus zu berücksichtigen, wurden größenspezifische Dosisabschätzungen (size-specific dose estimates, SSDE) aus den (TDIs berechnet.

Ergebnisse Der mittlere Body-Mass-Index (BMI) betrug $27,9 \mathrm{~kg} / \mathrm{m}^{2} \pm 6,3$ $\left(17,4-50,4 \mathrm{~kg} / \mathrm{m}^{2}\right) .52$ Patienten wurden mittels prospektiv EKG-getriggerter High-Pitch-Spirale, 55 Patienten mittels prospektiv EKG-getriggerter Sequenz und 40 Patienten mittels retrospektiv EKG-getriggerter Spirale untersucht. Die mediane effektive Dosis betrug für das CCS 0,54 mSv (Interquartilsabstand [IQR] 0,42-0,64 mSv), für die High-Pitch-Spirale 0,80 mSv (0,60-0,98 mSv), für die prospektive Sequenz 1,06 mSv $(0,80-2,39 \mathrm{mSv})$ und für die retrospektive Spirale 7,35 mSv (4,08-9,82 mSv) (alle $p<0,001)$. Die medianen SSDEs ergaben für das CCS 2,2 mGy (IQR 2,0-2,7 mGy), für die High-PitchSpirale 3,6 mGy (2,9-4,7 mGy), für die prospektive Sequenz 8,3 mGy (5,514,2 mGy) und für die retrospektive Spirale 35,8 mGy (23,6 - 45,0 mGy) (alle $p<0,001)$. Hohe Korrelationen mit der effektiven Dosis wurden für Summe aus AP und lateralem Diameter $(r=0,80)$, effektivem Durchmesser $(r=0,79)$ und BMI $(r=0,78)(p<0,001)$ beobachtet.

Schlussfolgerungen Im Vergleich zur High-Pitch-Spirale werden bei der prospektiven Sequenz bzw. der retrospektiven Spirale etwa 2- bzw. 10-fach höhere SSDEs erreicht. Neben dem Protokoll ist der Patientenhabitus der entscheidende Einflussfaktor auf die effektive Dosis.

\section{WISS 318.2 Intermodaler Vergleich der Augenlinsendosis im interventionellen und nicht- interventionellen Setting zur Darstellung der intrakraniellen Gefäße}

Autoren Guberina $\mathrm{N}^{1}$, Forsting $\mathrm{M}^{1}$, Ringelstein $\mathrm{A}^{1}$

Institut 1 Institute of Diagnostic and Interventional Radiology and Neuroradiology, University Hospital Essen, Germany, Institute of Diagnostic and Interventional Radiology and Neuroradiology, University Hospital Essen, Germany, Essen

DOI 10.1055/s-0037-1600236

Zielsetzung Das Ziel der vorliegenden Studie ist der intermodale Vergleich der Augenlinsendosis für Patienten während interventioneller und nicht-interventioneller Techniken zur Darstellung der intrakraniellen Gefäße.

Material und Methodik Wir führten Phantommessungen mit LiF TLDs (Thermolumineszenz Dosimeter Typ-100) durch, und untersuchten die Augenlinsendosis für ein feminines, anthropomorphes Phantom (ATOM 702-D). Die interventionellen Techniken umfassten 2-Ebenen DSA und 3D-Ebenen Rotationsangiografie am Allura Xper FD20 Angiografiesystem (Phillips Healthcare). Die nicht interventionellen Techniken beinhalteten die CT Angiografie (CTA) mit und ohne Linsenprotektor; CTA mit und ohne vorherige Akquisition 
einer nativen, kraniellen CT Untersuchung ( $\mathrm{nCT}$ ). Die Augenlinsendosis wurde anhand der Empfehlungen der ICRP 103 kalkuliert.

Ergebnisse Im nicht-interventionellen Setting wurden folgende Augenlinsen Dosiswerte ermittelt: (I) CTA 3.9 mGy; (II) CTA mit Linsenprotektor 2.3mGy; (III) CTA mit vorheriger Akquisition einer nCT 31.9mGy; (IV) CTA mit $+n C T$ (nCT mit Gantryangulierung) 16.1mGy; (V) CTA mit nCT und Linsenprotektor 23mGy. Die Augenlinsendosis im interventionellen Setting war wie folgt verteilt: (VI) 3D-Ebenen Rotationsangiografie 1mGy; (VII) 2-Ebenen DSA p/a +lat.4.5mGy; (VIII) 3D-Ebenen Rotationsangiografie und 2-Ebenen DSA $5.5 \mathrm{mGy}$.

Schlussfolgerungen Die vorliegenden Ergebnisse reflektieren, dass die Augenlinsendosis für Patienten während einer 3D-Ebenen Rotationsangiografie signifikant niedriger ist als bei der klassischen 2-Ebenen DSA. Gantryangulierung und Linsenprotektoren vermögen es die Augenlinsendosis bis $50 \% \mathrm{zu}$ senken. Die 3D-Ebenen Rotationsangiografie, die Gantryangulierung und Linsenprotektoren sind ein wichtiges neuroradiologisches Tool, mit der man die Strahlendosis für Patienten in der diagnostischen und interventionellen Darstellung der zerebralen Gefäße und von Gefäßerkrankungen weiter reduzieren kann.

\section{RK/WISS 107.7 Myokardiale Kontraktilitätsanalyse mittels MRT-basiertem Feature Tracking bei rechtsventrikulärer Ausflusstrakttachykardie, Brugada Syndrom und arrhythmogener rechtsventrikulärer Kardiomyopathie}

Autoren Heermann $\mathrm{P}^{1}$, Heindel $\mathrm{W}^{2}$, Schülke $\mathrm{C}^{2}$

Institut 1 Universitätsklinikum Münster UKM, Institut für Klinische Radiologie, Münster; 2 Universitätsklinikum Münster, Institut für Klinische Radiologie, Münster

DOI 10.1055/s-0037-1600237

Zielsetzung Ziel der Arbeit war es - basierend auf einer regionalen myokardialen Bewegungsanalyse mittels Feature Tracking (FT) - eine Differenzierung unterschiedlicher rechtsventrikulärer Tachyarrhythmien (rechtsventrikuläre Ausflusstrakttachykardie [RVOVT], Brugada Syndrom [BrS] und arrhythmogene rechtsventrikuläre Kardiomyopathie [ARVC]) untereinander und gegenüber herzgesunden Probanden (HP) vorzunehmen.

Material und Methodik Retrospektive Auswertung von 65 MR Datensätzen (16 ARVC, 26 RVOVT, 9 BrS, 14 HP) durch zwei verblindete Radiologen. Bestimmung der myokardialen Kontraktilität mittels FT mit Augenmerk auf die Parameter Strain (\%) und Strainrate (s-1). Die Parameter wurden im rechtsventrikulären Ausflusstrakt (RVOT), linken Ventrikel (LV) und rechten Ventrikel (RV) in jeweils radialer und longitudinaler Orientierung erfasst. Ergänzend wurden die Parameter biventrikulär zirkumferentiell in basalen, mittventrikulären und apikalen Kurzachsenschnitten erhoben.

Ergebnisse Der RV longitudinale und RV radiale basale Strain bei ARVC (-12.9 $\pm 4.2 ; 11.4 \pm 5.1)$ sind signifikant niedriger als bei RVOVT $(-18.0 \pm 2.5$, $\mathrm{p} \leq 0.005 ; 16.4 \pm 5.2, \mathrm{p} \leq 0.05)$. Parallel ist der RVOT radiale Strain bei ARVC $(33.8 \pm 22.7)$ signifikant niedriger $(p \leq 0.05)$ gegenüber RVOVT $(54.3 \pm 14.5)$. Für BrS ergibt sich für den RV basalen und mittventrikulären radialen Strain $(13.3 \pm 6.1 ; 11.8 \pm 2.9)$ eine signifikante Reduktion gegenüber HP $(21.0 \pm 6.9$, $\mathrm{p} \leq 0.05 ; 20.1 \pm 6.6, \mathrm{p} \leq 0.005)$; dies für den mittventrikulären Strain (12.0 \pm 2.7 vs. $20.1 \pm 6.6, p \leq 0.05)$ sogar bei erhaltener RVEF ( $45 \% ; n=6)$. Synergistisch war der LV basale radiale Strain trotz gleicher LVEF bei BrS $(24.1 \pm 5.5)$ signifikant $(p \leq 0.05)$ eingeschränkt gegenüber HP $(32.7 \pm 7)$.

Schlussfolgerungen MR-basiertes FT ermöglicht die Differenzierung von ARVC- und RVOVT-Patienten untereinander sowie die Differenzierung von ARVC- und BrS-Patienten gegenüber HP. Der RVOT ist dabei eine anatomische Schlüsselregion. Bei ARVC und BrS ist der strain sogar bei noch normal globaler LVEF und RVEF auffällig erniedrigt.
WISS 102.2 Nutzen monoenergetischer Rekonstruktionen eines neuen Spectral-Detector-CTs für die Bildgebung von Koronarstents: erste in-vitro Ergebnisse

Autoren Hickethier $T^{1}$, Baeßler $B^{1}$, Kroeger $J^{1}$, Doerner $J^{1}$, Pahn $G^{2}$,

Maintz $D^{1}$, Michels $G^{3}$, Bunck $A^{1}$

Institut 1 Uniklinik Köln, Institut für Diagnostische und Interventionelle

Radiologie, Köln; 2 Philips Deutschland GmbH, Clinical Science CT, Hamburg;

3 Uniklinik Köln, Klinik III für Innere Medizin, Köln

DOI 10.1055/s-0037-1600238

Zielsetzung Die Beurteilbarkeit von Koronarstents im CT hat sich zwar kontinuierlich verbessert, ist jedoch weiterhin artefaktbedingt oft schwierig. Diese Studie untersucht systematisch den Einfluss von unterschiedlichen virtuell monoenergetischen Bildern (MonoE) eines neuen Spectral-Detector-CTs (SDCT) auf die Lumensichtbarkeit von verschiedenen Koronarstents.

Material und Methodik 10 unterschiedliche Koronarstents (Diameter $3 \mathrm{~mm}$ ) wurden in Gefäßphantomen mittels SDCT (IQon, Philips) gescannt (120 kV/ $125 \mathrm{mAs}$ ). Für jeden Stent wurden spezifische konventionelle Bilder mittels statistisch-iterierter Rekonstruktion (IR) und MonoE-Datensätze (60, 70, 80, $90,100,150 \mathrm{keV})$ generiert. Jede Bildserie wurde hinsichtlich etablierter Bildqualitätskriterien beurteilt: Bildrauschen (Standardabweichung einer standardisierten ROI), Dichteabweichung (Dichteunterschied gestentetes Lumens vs. ungestentetes Lumen) und einsehbarer Lumendiameter (Durchmesser des artefaktfreien Stentlumens).

Ergebnisse Das Bildrauschen war bei allen MonoE signifikant geringer als bei IR (IR 13,41, MonoE $60 \mathrm{keV}$ 11,62, $70 \mathrm{keV} \mathrm{11,67,} 80 \mathrm{keV}$ 11,69, $90 \mathrm{keV}$ 11,71, $100 \mathrm{keV}$ 11,75, $150 \mathrm{keV}$ 11,80 HU SD; $\mathrm{p}<0,01)$. Die Dichteunterschiede zwischen gestentetem und ungestentetem Lumen waren in höherenergetischen MonoE signifikant geringer als bei IR (IR 148,18, MonoE $60 \mathrm{keV}$ $154,13 p=, 036,70 \mathrm{keV} 143,43 p=, 109,80 \mathrm{keV} 137,25 p=, 052,90 \mathrm{keV}$ $133,02 p=, 043,100 \mathrm{keV} 130,12 p=, 039,150 \mathrm{keV}$ 123,99 HU $p=, 035)$. Der einsehbare Lumendiameter war in höherenergetischen MonoE signifikant gröBer als bei IR (IR 0,65, MonoE $60 \mathrm{keV} 0,68 \mathrm{p}=, 542,70 \mathrm{keV} 0,71 \mathrm{p}=, 053,80$ $\operatorname{keV} 0,74 p<, 01,90 \mathrm{keV} 0,77 p<, 01,100 \mathrm{keV} 0,82 \mathrm{p}<, 01,150 \mathrm{keV} \mathrm{0,87mm}$ $\mathrm{p}<, 01)$.

Schlussfolgerungen Etablierte Bildqualitätskriterien für die Beurteilung von Koronarstents können durch die Nutzung von monoenergetischen Bildern eines neuen Spectral-Detector-CTs signifikant verbessert werden.

\section{WISS 316.3 C-Arm CT (CACT) bei Patienten mit chronisch thromboembolischer pulmonaler Hypertonie (CTEPH) und positivem V/Q SPECT/CT: Evaluation der diagnostischen Zusatzinformation}

Autoren Hinrichs J ${ }^{1}$, Werncke $\mathrm{T}^{1}$, Hoepker $\mathrm{M}^{2}$, Olsson $\mathrm{K}^{2}$, Wacker $\mathrm{F}^{1}$, Brunkhorst $\mathrm{T}^{3}$, Sohns $\mathrm{J}^{3}$, Meyer $\mathrm{B}^{1}$

Institut $1 \mathrm{MHH}$, Institut für Diagnostische und Interventionelle Radiologie, Hannover; 2 MHH, Klinik für Pneumologie, Hannover; 3 MHH, Klinik für Nuklearmedizin, Hannover

DOI 10.1055/s-0037-1600239

Zielsetzung Beurteilung des potentiellen zusätzlichen Nutzens der Kontrastmittel-verstärkten C-Arm CT in einem Patientenkollektiv mit CTEPH und positivem Ventilations-/Perfusions-Mismatch (V/Q) in der SPECT/CT.

Material und Methodik Siebzehn CTEPH-Patienten (11 M, $73 \pm 11$ Jahre) mit SPECT/CT, Rechtsherzkatheteruntersuchung (RHK) und CACT wurden eingeschlossen. Jeweils zwei Leser haben die SPECT/CT-Aufnahmen und zwei andere die CACT-Aufnahmen hinsichtlich CTEPH-typischer Befunde und deren Lokalisation (segmental/sub-segmental) ausgewertet. Die Übereinstimmung der Befunde zwischen den Lesern und zwischen den untersuchten Modalitäten (Kondensbefund) wurde berechnet (Cohen's Kappa). 
Ergebnisse Insgesamt wurden 306 Pulmonalarteriensegmente in beiden Modalitäten untersucht. Die SPECT/CT hat im Konsensbefund 128/306 (41\%) Arteriensegmente ohne und 178/306 (59\%) mit CTEPH-typischen V/Q Mismatch bewertet. Die CACT hat im Konsensbefund 57/306 (19\%) Arteriensegmente ohne und 249/306 (81\%) mit CTEPH-typischen Befunden bewertet. Die Übereinstimmung zwischen den Lesern war gut für beide Modalitäten (SPECT/CT $\mathrm{k}=0,73$; CACT $\mathrm{K}=0,77$ ). Die Übereinstimmung der Konsensbefunde zwischen beiden Modalitäten war ausreichend $(\kappa=0,28)$. Diskrepante Befunde zwischen SPECT/CT und CACT sind durch eine höhere Detektionsrate von sub-segmentalen Befunden in der CACT zu erklären, welche in der SPECT/ CT als normal bewertet wurden (79 sub-segmentale Befunde).

Schlussfolgerungen Im Vergleich zur CACT unterschätzt die SPECT/CT das Ausmaß und die Komplexität der vaskulären Befunde bei Patienten mit CTEPH.

\section{RK/WISS 107.6 Epikardiales Fettvolumen, LV-Strain und T1-Mapping bei Hypertonikern mit erhaltener Ejektionsfraktion}

Autoren Homsi $R^{1}$, Sprinkart $A^{1}$, Kuetting $D^{1}$, Meier-Schroers $M^{1}$, Luetkens $J^{1}$, Fischer $S^{1}$, Marx $C^{1}$, Dabir $D^{1}$, Gieseke $J^{2}$, Schild $H^{1}$, Thomas $D^{1}$

Institut 1 Universitätsklinik Bonn, Radiologie, Bonn; 2 Philips Healthcare, Hamburg

DOI 10.1055/s-0037-1600240

Zielsetzung MRT zur Bestimmung des epikardialen Fettvolumens (EFV), des links-ventrikulären (LV) Strains und der LV myokardialen T1-Relaxationszeiten als Hinweis für eine Myokardfibrose (T1) bei Hypertonikern mit sowie solchen ohne stabiler koronare Herzerkrankung (KHK).

Material und Methodik 98 Hypertoniker mit und ohne KHK, jedoch ohne Nachweis eines stattgehabten ischämischen Myokardschadens (58 Männer; $63 \pm 11$ Jahre; BMI $29 \pm 6 \mathrm{~kg} / \mathrm{m}^{2}$ ) sowie 25 gesunde Studienteilnehmer wurden mittels 1.5 Tesla MRT untersucht. Das EFV $\left(\mathrm{ml} / \mathrm{m}^{2}\right)$ wurde mittels einer 3DDixon Sequenz und T1 (ms) mittels einem 3(3)3(3)5 MOLLI-Schema akquiriert. Zur Bestimmung der LV Kontraktilität wurde der LV Strain (\%) sowohl longitudinal (LS) im 4-Kammerblick als auch zirkumferentiell (CS) in der kurzen Herzachse bestimmt.

Ergebnisse Hypertoniker zeigten, unabhängig vom Vorliegen einer KHK, signifikant $(p<0.05)$ höhere EFV und T1-Werte sowie niedrigere CS und LS Werte als Gesunde: - Kontrollpatienten ( $n=25)$ : EFV 58 \pm 21, T1 $967 \pm 17$, LS $-25 \pm 5$, CS $-29 \pm 5$ - Hypertoniker (alle) $(n=98)$ : EFV $71 \pm 25$, T1 991 \pm 46 , LS: $-21 \pm 4$, CS: $-25 \pm 6$ - Hypertoniker ohne KHK $(n=56)$ : EFV $71 \pm 27$, T1 $992 \pm 48$, LS $-21 \pm 5$, CS $-25 \pm 6$ - Hypertoniker mit KHK $(n=42)$ : EFV 71 \pm 24 , T1 $987 \pm 39$, LS $-21 \pm 4$, CS $-26 \pm 5$ Es gab keine signifikanten Unterschiede zwischen Hypertonikern mit und ohne KHK. Es gab auch keine signifikanten Unterschiede nach Gruppierung der Hypertoniker in 0-, 1-, 2- oder 3Gefäß-KHK.

Schlussfolgerungen Die Studie konnte bei Hypertonikern ohne KHK eine erhöhte EFV und Zeichen der myokardialen Fibrose sowie eine eingeschränkte LV Kontraktilität trotz erhaltener Ejektionsfraktion nachweisen. Eine zusätzliche stabile KHK hat in diesem Krankengut keinen weiteren Einfluss auf die gemessenen Parameter.
WISS 318.4 Quantifizierung arteriosklerotischer Gefäßwandverkalkungen zur Prognoseabschätzung vor endovaskulärer Revaskularisation infrapoplitealer Arterien

Autoren Klaus $\mathrm{T}^{1}$, Roller $\mathrm{F}^{1}$, Alejandre-Lafont $\mathrm{E}^{1}$, Breithecker $\mathrm{A}^{1}$, Krombach $\mathrm{G}^{1}$, Schneider $\mathrm{C}^{1}$

Institut 1 Universitätsklinikum Giessen, Diagnostische und Interventionelle Radiologie, Giessen

DOI 10.1055/s-0037-1600241

Zielsetzung Evaluation des Zusammenhanges zwischen mittels Computertomografie (CT) erhobenen ausgewählten Merkmalen von Gefäßwandverkalkungen infrapoplitealer Arterien und technischem Revaskularisationserfolg sowie klinischem Outcome bei Patienten mit kritischer Beinischämie.

Material und Methodik Die prospektive Studie wurde von der lokalen Ethikkommission genehmigt. 71 konsekutive Patienten mit kritischer Beinischämie (Fontaine Stadien III und IV) erhielten eine native CT des symptomatischen Unterschenkels (US) direkt vor oder nach der Angiografie. Bei 103 Läsionen (51 Patienten; mittleres Alter 75; 41 männlich; 12 Stadium III, 39 Stadium IV) erfolgte ein Revaskularisationsversuch. Die in der Angiografie diagnostizierten Läsionen (Verschlüsse und hochgradige Kettenstenosen) wurden mit der CT verglichen. Ausgewählte Charakteristika der Verkalkungen je Läsion (Volumen, Masse, Agatston-Score (AS), Exzentrizität, Kalk-Längen-Ratio und Auftreten fokaler Kalzifikationen am Beginn und/oder Ende der Läsion) wurden mit dem Revaskularisationsergebnis (Revaskularisation ja/nein) korreliert. Ferner wurde der Gesamt-AS aller Kalzifikationen des US mit dem klinischen Outcome (Endpunkte: Rehospitalisation, Amputation, Tod) nach 6 und 12 Monaten korreliert.

Ergebnisse Ein technischer Revaskularisationserfolg konnte bei 90 von 103 Läsionen (87\%) erzielt werden. Der durchschnittliche Läsions-AS und Gesamt-AS des US betrug 1044 bzw. 6322. Ein hoher Läsions-AS korrelierte signifikant $(p<0.05)$ mit einem technischen Revaskularisationsmisserfolg. 13 Patienten (26\%) erreichten die klinischen Endpunkte nach 6 Monaten und weitere 7 Patienten (14\%) nach 12 Monaten. Ein hoher Gesamt-AS korrelierte signifikant $(p<0.01)$ mit der Rehospitalisation nach 12 Monaten. Das durchschnittliche Dosislängenprodukt der CT betrug 74 mGy* cm.

Schlussfolgerungen Eine native CT zur Evaluation der Gefäßwandverkalkungen kann bei Patienten mit kritischer Beinischämie zur Prognoseabschätzung vor interventioneller Revaskularisation beitragen.

\section{RK 411.4 4D-Fluss-MRT zur nichtinvasiven 4D- Blutdruckkartierung bei Patienten mit syndromalen Aneurysmen der Aortenwurzel}

Autoren Leidenberger $\mathrm{T}^{1}$, Gordron $\mathrm{Y}^{1}$, Farag $\mathrm{M}^{2}$, Weis $\mathrm{C}^{1}$, Müller-Eschner $\mathrm{M}^{1}$, Delles $\mathrm{M}^{3}$, Kallenbach $\mathrm{K}^{2}$, Dillmann $\mathrm{R}^{4}$, Kauczor $\mathrm{H}^{1}$, Beller $\mathrm{C}^{2}$, Rengier $\mathrm{F}^{1}$ Institut 1 Universitätsklinikum Heidelberg, Diagnostische und Interventionelle Radiologie, Heidelberg; 2 Universitätsklinikum Heidelberg, Klinik für Herzchirurgie, Heidelberg; 3 Karlsruher Institut für Technologie, Institut für Anthropomatik und Robotik, Karlsruhe; 4 Karlsruher Institut für Technologie, Institut für Anthropomatik und Robotik, Karlsruhe DOI 10.1055/s-0037-1600242

Zielsetzung Aneurysmen der Aortenwurzel treten vermehrt bei Patienten mit Erkrankungen des Bindegewebes auf. Zielstellung dieser Arbeit war die Evaluierung der räumlichen und zeitlichen Blutdruckveränderungen bei Patienten mit syndromalen Aneurysmen der Aortenwurzel im Vergleich zu einer Kontrollgruppe gleichen Alters und Geschlechts durch die nichtinvasive Blutdruckkartierung mittels 4D-Fluss-MRT.

Material und Methodik 4D-Flussmessungen der thorakalen Aorta wurden an einem 1,5T MRT bei 11 Patienten mit syndromalen Aneurysmen der Aortenwurzel (Durchschnittsalter 34 Jahre, 4 weiblich, 7 männlich) und einer Kontrollgruppe von 11 Probanden (Durchschnittsalter 33,6 Jahre) durchgeführt. Auf der Navier-Stokes Gleichung basierende Blutdruckkartierungen wurden 
mithilfe validierter Algorithmen und intern entwickelter Bildverarbeitungssoftware erstellt. Die Blutdruckamplituden während eines Herzzyklus wurden an acht verschiedenen anatomischen Positionen erfasst; P1, Sinus Valsalvae, P2/ P3, proximale/distale Aorta ascendens, P4, Aortenbogen; P5-P8, abstandsgleiche Ebenen der Aorta descendens. Zur Erhebung der Interobserver-Variabilität wurde die Analyse von zwei Radiologen durchgeführt.

Ergebnisse Die durchschnittlichen Blutdruckamplituden (in $\mathrm{mm} \mathrm{Hg} / \mathrm{cm}$ ) für Patienten/Kontrollgruppe: P1 141,3/224,0 ( $p=0,0004), \quad P 2 \quad 122,0 / 255,8$ $(p=0,07)$, P3 126,1/132,4 ( $p=0,69)$, P4 122,3/123,3 ( $p=0,95)$, P5 105,5/ $163,9(p=0,14), P 6131,0 / 148,1(p=0,21), P 7139,7 / 159,6(p=0,18), P 8$ $152,1 / 227,7(p=0,09)$. Es zeigte sich eine hohe Interobserver-Übereinstimmung mit einem Korrelationskoeffizienten von 0,954-0,996 $(p<0,001)$.

Schlussfolgerungen Die 4D-Fluss-MRT zur nichtinvasiven 4D-Blutdruckkartierung zeigte bei Patienten mit syndromalen Aneurysmen der Aortenwurzel einen signifikanten Abfall der Blutdruckamplitude in der Aortenwurzel, proximalen Aorta ascendens und distalen Aorta descendens. Diese Technik könnte zu einem umfassenderen Verständnis der zugrundeliegenden pathophysiologischen Prozesse und einer Verbesserung der therapeutischen Ansätze beitragen.

\section{WISS 316.2 Virtuelle monoenergetische Rekonstruktionen und Jodperfusionskarten verbessern die diagnostische Genauigkeit der Dual-Energy CT Pulmonalangiografie mit suboptimalen Kontrastverhältnissen}

Autoren Leithner $D^{1}$, Wichmann $J^{2}$, Vogl $T^{1}$, Trommer $J^{1}$, Martin $S^{1}$, Scholtz $\mathrm{J}^{1}$, Bodelle $\mathrm{B}^{1}$, De Cecco $\mathrm{C}^{3}$, Duguay $\mathrm{T}^{3}$, Nance Jr. $\mathrm{J}^{3}$, Schoepf $\mathrm{J}^{3}$, Albrecht $\mathrm{M}^{1}$

Institut 1 Universitätsklinikum Frankfurt am Main, Institut für Diagnostische und Interventionelle Radiologie, Frankfurt am Main; 2 Universitätsklinikum

Frankfurt, Institut für Diagnostische und Interventionelle Radiologie, Frankfurt am Main; 3 Medical University of South Carolina, Department of Radiology and Radiological Science, Charleston

DOI 10.1055/s-0037-1600243

Zielsetzung Ziel war, die Auswirkung von rauschoptimierten monoenergetischen Rekonstruktionen (Mono+) und Jodperfusionskarten (DECTPM) auf die diagnostische Genauigkeit und Sicherheit in der Erkennung von Lungenembolien (LAE) in der Dual-Energy-CT Pulmonalangiografie (DE-CTPA) bei suboptimalen Kontrastverhältnissen herauszufinden.

Material und Methodik 68 Patienten mit suboptimalen Kontrastverhältnissen in der DE-CTPA wurden retrospektiv eingeschlossen. Linear gemischte (M_0.6), Mono+ Serien bei $40 \mathrm{keV}$ und DECT-PM wurden rekonstruiert. ROIMessungen wurden im Truncus pulmonalis durchgeführt zur Objektivierung des schlechten Kontrastes und Berechnung des Kontrast-zu-Rausch Verhältnisses (CNR). Vier Radiologen bewerteten unabhängig voneinander das Vorhandensein einer Lungenembolie und ihre diagnostische Sicherheit mithilfe von 5Punkt-Skalen unter Verwendung dreier Protokolle: Protokoll 1 - M_0.6; Protokoll 2 - M_0.6 und DECT-PM; Protokoll 2 - M_0.6, DECT-PM und Mono+ Serien. Eine Receiver operating characteristic (ROC) Analyse wurde durchgeführt.

Ergebnisse Eine zentrale LAE lag in 14, eine segmentale in 29 Patienten vor. Mono+ Bilder zeigten eine höhere objektive Bildqualität als M_0.6 Serien $(P<0,001)$. Die diagnostische Genauigkeit für das Auffinden einer segmentalen LAE war überlegen bei Protokoll 3 (92,6\%) im Vergleich zu Protokoll 1 und 2 (69,1\% und 86,8\%). Protokoll 3 resultierte in einer signifikant größeren Area under the curve (AUC) für die Diagnose einer segmentalen LAE (0.991, $P \leq 0,033)$ im Vergleich zu Protokoll 1 und $2(0,897$ und 0,951$)$, und bot die höchste diagnostische Sicherheit $(\mathrm{P}<0,001)$.

Schlussfolgerungen Ein Rekonstruktionsprotokoll mit $40 \mathrm{keV}$ Mono+ Serien und DECT-PM verbessert die diagnostische Genauigkeit und Sicherheit in der
Diagnose einer segmentalen LAE im Vergleich zu linear gemischten M_0.6 Bildern in der DE-CTPA mit suboptimalen Kontrastverhältnissen.

\section{WISS 318.1 90-kVp CT-Angiografie der Carotis und intrazerebral in Kombination mit ADMIRE: Auswirkungen auf Strahlendosis, Bildqualität und diagnostische Genauigkeit in der Erkennung von Carotisstenosen}

Autoren Leithner $D^{1}$, Wichmann $J^{1}$, Martin $S^{1}$, Lenga $L^{1}$, Albrecht $\mathrm{M}^{1}$, Beeres $\mathrm{M}^{1}$, Vogl $\mathrm{T}^{1}$, Scholtz $\mathrm{J}^{1}$

Institut 1 Universitätsklinikum Frankfurt am Main, Institut für Diagnostische und Interventionelle Radiologie, Frankfurt am Main

DOI 10.1055/s-0037-1600244

Zielsetzung Ziel war es, Strahlendosis, Bildqualität, Artefakte und Erkennung von Carotisstenosen der niedrig-Röhrenspannung 90-kVp Bildakquisition kombiniert mit „Advanced modeled iterative reconstruction algorithm" (ADMIRE) in der CT Angiografie der Carotis und intrazerebral (CCTA) zu evaluieren.

Material und Methodik 43 Patienten, die sich einer Dual-Energy CCTA an einem 192-Zeilen Scanner der 3. Generation unterzogen haben, wurden retrospektiv evaluiert. 90-kVp und linear gemischte 120-kVp Bilder wurden verglichen. Jodsignal und Rauschen der ACC auf Schulter- und Bifurkationshöhe, $\mathrm{ACl}$ im Carotissiphon, ACM und Basilararterie wurden gemessen um das Kontrast-zu-Rausch Verhältnis (CNR) zu errechnen. Auf jeder Höhe wurde die subjektive Bildqualität im Sinne von Artefakten und Eignung zur Diagnose einer Carotisstenose von drei unabhängigen Radiologen mittels 5-Punkt-Skalen bewertet. Erkennung und Einstufung von Carotisstenosen wurden durchgeführt. Interobserver Reliabilität wurde mithilfe des Intraclass correlation coefficient (ICC), Strahlendosis als Dosislängenprodukt (DLP) ausgedrückt.

Ergebnisse Jodsignal und Rauschen waren in allen Arterien höher bei 90-kVp, verglichen mit 120-kVp $(P<0,001)$, resultierend in einer signifikant gesteigerten $\mathrm{CNR}$ bei $90-\mathrm{kVp}$ ( $\mathrm{ACl}, 38,2$ vs. 22,4 $\mathrm{HU}$; $\mathrm{P}<0,001$ ). Artefakte und Eignung waren in beiden Serien exzellent, ohne Nachweis signifikanter Unterschiede. Die höchste Artefaktlast fand sich auf Schulterebene, ohne signifikante Unterschiede (90-kVp, 4,9; 120-kVp, 4,8). Die Detektion von Carotisstenosen war in beiden Serien ausgezeichnet, ohne signifikanten Unterschied (Stenosen in 32 von 129 Segmenten; ICC, 0,94). Das DLP wurde durch die 90-kVp Bildakquisition um 40,3\% reduziert (110,6 vs. 185,4 mGy.cm, $\mathrm{P}<0,001)$.

Schlussfolgerungen $90-k V p$ CCTA in Kombination mit ADMIRE resultiert in gesteigerter CNR, exzellenter Eignung zur Diagnose von Carotisstenosen, geringen Artefakten und signifikanter Reduktion der Strahlendosis, während Erkennung und Einstufung von Carotisstenosen ausgezeichnet bleiben.

\section{RK/WISS 103.5 Myokardiale Strainanalyse mittels Feature-Tracking bei akuter Myokarditis: Diagnostische Wertigkeit und Assoziation mit myokardialer Inflammation}

Autoren Luetkens $J^{1}$, Schlesinger-Irsch $U^{1}$, Kütting $D^{1}$, Dabir $D^{1}$, Homsi $R^{1}$, Doerner $J^{1}$, Schmeel $F^{1}$, Sprinkart $A^{1}$, Naehle $C^{1}$, Schild $H^{1}$, Thomas $D^{1}$ Institut 1 Uniklinik Bonn, Radiologie, Bonn

DOI 10.1055/s-0037-1600245

Zielsetzung Die multiparametrische kardiale Magnetresonanztomografie (MRT) kann inflammatorische Herzmuskelveränderungen bei Patienten mit dem Verdacht auf eine akute Myokarditis detektieren. Funktionelle Marker, wie die myokardiale Strain-Analyse, können die diagnostische Wertigkeit der kardialen MRT möglicherweise weiter erhöhen und eine zusätzliche diagnostische Zielgröße darstellen.

Material und Methodik Bei 48 Patienten mit dem Verdacht auf eine akute Myokarditis und 35 Kontrollpatienten wurde eine kardiale MRT durchgeführt. Der globale longitudinale, circumferentielle und radiale Strain wurde mittels Feature-Tracking aus den Cine-SSFP-Daten ermittelt. Als weitere Inflamma- 
tionsparameter wurden die nativen T1 und T2 Relaxationszeiten, die T2-Signalintensitätsratio, die „early gadolinium enhancement ratio“ und das „late gadolinium enhancement" bestimmt.

Ergebnisse Patienten mit dem Verdacht auf eine akute Myokarditis hatten im Vergleich zu den gesunden Kontrollen signifikant erniedrigte Strainparameter (longitudinaler Strain: $-19,5 \pm 4,4 \%$ vs. $-23,6 \pm 3,1 \%$, circumferentieller Strain: $-23,0 \pm 5,8 \%$ vs. $-27,4 \pm 3,4 \%$, radialer Strain: $28.9 \pm 8.5 \%$ vs. $32.4 \pm 7.4 \%$; jeweils $p<0,05)$. Der longitudinale Strain korrelierte als einziger Strain-Parameter signifikant mit allen akquirierten, entzündlichen MRT-Parametern (jeweils mit $\mathrm{p}<0,05)$. Bezüglich der diagnostischen Wertigkeit der einzelnen StrainParameter hinsichtlich der Diagnosestellung der Myokarditis zeigte der longitudinale Strain die höchste „area under curve“ (AUC) (Iongitudinaler Strain: 0,79, circumferentieller Strain: 0,75, radialer Strain: 0,62).

Schlussfolgerungen Die Analyse des myokardialen Strains mittels Feature-Tracking erlaubt der kardialen MRT zusätzliche Parameter der myokardiale Dysfunktion zu bestimmen, welche die Diagnosestellung einer akuten Myokarditis erleichtern können. Insbesondere der longitudinale Strain liefert wichtige diagnostische Informationen und zeigte den stärksten Zusammenhang mit entzündlichen MRT-Parametern.

\section{RK/WISS 402.5 Endoleaks nach endovaskulärer Aortenreparatur: Verbesserte Detektion mit rauschoptimierter virtueller monoenergetischer Dual-Energy-CT}

Autoren Martin $S^{1}$, Wichmann $J^{1}$, Leithner $D^{1}$, Lenga $L^{1}$, Scholtz $J^{1}$, Vogl $T^{1}$, Albrecht $\mathrm{M}^{1}$

Institut 1 Zentrum der Radiologie, Institut für Diagnostische und Interventionelle Radiologie, Frankfurt

DOI 10.1055/s-0037-1600246

Zielsetzung Ziel der Studie ist es, die Bildqualität eines rauschoptimierten Algorithmus zur Rekonstruktion virtueller monoenergetischer Bilder (VMI+) in Dual-Energy CT Angiografien (DE-CTA) zu untersuchen, sowie die Diagnosesicherheit bezüglich der Detektion von Endoleaks nach endovaskulärer Aortenreparatur (EVAR) zu evaluieren.

Material und Methodik 75 Patienten (42 Männer, 66,2 $\pm 11,7$ Jahre) wurden nach EVAR mittels DE-CTA untersucht. Die arteriellen DECT Datensätze wurden standardmäßig mit linear gewichteten M_0.5, VMl+ und herkömmlichen monoenergetischen Bildern (VMI) von 40 bis $100 \mathrm{keV}$ in 10-keV-Intervallen rekonstruiert. Das Kontrast-zu-Rausch-Verhältnis (CNR) wurden für die Aorta descendens, die lliakalarterien und innerhalb der Leckage bei Patienten mit Endoleaks berechnet. Die Diagnosesicherheit für die Detektion von Endoleaks der objektiv besten Serien jeder Rekonstruktionstechnik wurde von drei Radiologen ausgewertet.

Ergebnisse 34 von 75 Patienten zeigten Endoleaks in DE-CTA Untersuchungen. Die quantitativen Bildparameter zeigten die höchsten Werte bei 40-keV VMI+ Rekonstruktionen (CNR, 21,3 $\pm 11,1$ ), verglichen mit M_0.5 (CNR, 10,9 $\pm 5,5$ ) und allen VMI-Serien, die die höchsten Werten bei $70 \mathrm{keV}$ (CNR, 13,5 $\pm 6,6 ; P<0,001$ ) aufwiesen. Sensitivität und Spezifität für den Nachweis von Endoleaks waren bei 40-keV VMI+ (98\% bzw. 89\%) gegenüber 70-keV VMI (96\% bzw. 81\%) und M_0.5 Rekonstruktionen (97\% bzw. 82\%). Die ROC-Analyse ergab eine Fläche unter der Kurve (AUC) von 0,992 für 40-keV VMI+, der im Vergleich zu 70-keV VMI $(0,914)$ und M_0.5 $(0,916)$ signifikant höher war $(P \leq 0,039)$.

Schlussfolgerungen Rauschoptimierte VMl+ Rekonstruktionen bei $40 \mathrm{keV}$ verbessern die Diagnosesicherheit für die Erkennung und den Ausschluss von Endoleaks nach EVAR im Vergleich zu standardmäßigen linear-gewichteten und herkömmlichen VMI-Rekonstruktionen in der DE-CTA.
RK/WISS 107.5 Herzfrequenzabhängigkeit beim kardialen T1-mapping: Eine Analyse der „modified Look-Locker inversion recovery“ (MOLLI)-Sequenz in einem Phantommodel bei verschiedenen Herzfrequenzen

Autoren Nadjiri $]^{1}$, Rasper $M^{1}$, Sträter $A^{1}$, Rummeny $E^{1}$, Huber $A^{1}$ Institut 1 Klinikum rechts der Isar der TU München, Institut für diagnostische und interventionelle Radiologie, München

DOI 10.1055/s-0037-1600247

Zielsetzung Mit einer neueren Technik, dem T1 mapping, konnte gezeigt werden, dass die Erfassung der myokardialen Perfusion auch ohne Kontrastmittel denkbar ist. Dies konnte jedoch nur für die sogenannte „shortened modified Look-Locker inversion recovery“ (ShMOLLI)-Sequenz gezeigt werden, da diese Sequenz als herzfrequenzunabhängig gilt. Zwar ist die standard (MOLLI)-Sequenz möglicherweise präziser und weltweit leichter zugänglich, jedoch gilt sie auch als stark herzfrequenzabhängig. Diese Arbeit untersucht die Herzfrequenzabhängigkeit der MOLLI-Sequenz mit modifizierten Auslesemuster, welches weniger abhängig von der Herzfrequenz sein soll. Ziel dieser Arbeit war es zu evaluieren, ob sich die MOLLI-Sequenz theoretisch für eine native Myokardperfusionsmessung eignet.

Material und Methodik In einem Modell wurden Phantome mit unterschiedlichen Verdünnungen von Dimeglumingadopentetat untersucht. Diese verschiedenen Phantome wurden mit verschiedenen simulierten Herzfrequenzen mit einer MOLLI-Sequence mit einem Auslesemuster von $5 \mathrm{~s}-(3 \mathrm{~s})-3 \mathrm{~s}$ im Vergleich zum 5b-(3b)-3-Muster gescannt. Exemplarisch wurde bei einem Patienten vor und während der Adenosin-Belastung eine T1-Mapping-Untersuchung mit dem Auslesemuster $5 \mathrm{~s}-(3 \mathrm{~s})-3 \mathrm{~s}$ durchgeführt.

Ergebnisse Alle Scans mit der MOLLI 5b-(3b)-3b-Sequence zeigten eine inverse Korrelation zwischen der gemessenen Relaxationszeit und der simulierten Herzfrequenz. Zudem nahm die negative Steigung der Regressionsgraden mit ansteigenden T1-Relaxationszeiten weiter ab. Im Gegensatz dazu konnte bei der MOLLI 5 s-(3s)-3s-Sequenz weder ein Trend für die Korrelationen der gemessenen T1-Relaxationszeiten noch für die Herzfrequenz gezeigt werden. Am Testpatienten zeigte sich eine Steigerung der T1-Relaxationszeit von $1254 \mathrm{~ms}$ auf $1316 \mathrm{~ms}(5 \%$; $\mathrm{p}=0,041)$.

Schlussfolgerungen Die MOLLI-Sequenz erscheint theoretisch geeignet um T1-Relaxationszeiten bei unterschiedlichen Herzfrequenzen zu vergleichen. Die Ergebnisse des Beispielpatienten zeigen, dass unter Belastung die myokardiale Durchblutung zunimmt.

\section{WISS 211.3 Transpedale MR-Lymphangiografie des zentralen Lymphsystems - Eine neue Technik zur Planung lymphatischer Interventionen bei Patienten mit chylösen Leckagen Bewerber für den Young Investigator Award}

Autoren Pieper $\mathrm{C}^{1}$

Institut 1 Universitätsklinikum Bonn, Radiologische Klinik, Bonn DOI 10.1055/s-0037-1600248

Zielsetzung Evaluation des klinischen Nutzens transpedaler MR-Lymphangiografien (tMRL) zur Planung lymphatischer Interventionen bei Patienten mit chylösen Leckagen.

Material und Methodik Eine tMRL wurde bei 25 Patienten (m/w: 13/12, mittleres Alter 54 Jahre) mit chylösen Leckagen vor lymphatischer Intervention durchgeführt. Indikationen waren: traumatischer/nicht-traumatischer Chylothorax $n=6 / 8$, traumatischer/nicht-traumatischer Chylaszites $n=3 / 3$, kombierter Chylothorax/Chylaszites $n=5$. Die tMRL erfolgte am 1.5T Scanner mit T1-gewichteter Bildgebung nach pedaler intrakutaner Injektion von gadoliniumhaltigem Kontrastmittel (KM) unter Lokalanästhesie. Die Daten wurden evaluiert hinsichtlich der Darstellung einer Leckagestelle, eines möglichen 
Zugangsweges für eine Lymphgefäßembolisation und anatomischer Varianten.

Ergebnisse Die KM-Injektion wurde von allen Patienten gut toleriert. Komplikationen traten nicht auf. Eine Sedierung war in einem Fall nötig. Bei 20/25 Patienten konnten kontrastierte zentrale Lymphbahnen abgegrenzt werden (Gründe für Versagen: unruhiger Patient trotz Sedierung $n=1$, Lymphom mit Lymphödem $n=1$, technische Gründe $n=3$ ). Eine Leckage konnte bei 13/20 Patienten sicher identifiziert werden. In 5 Fällen (Chylaszites) trat das KM intraabdominell aus und erreichte nicht die Cisterna chyli (CC). Bei den verbleibenden 15 Patienten konnte ein Zugangsweg zur Ductus thoracicus (DT) Embolisation in 14/15 Fällen identifiziert werden (CC bei $12 / 15$, DT bei 14/15 [9 durchgängige, 5 diskontinuierliche Kontrastierung]). 8/14 Patienten hatten einen einzelnen, 6/14 einen gedoppelten DT. Ein DT mündete im rechten Venenwinkel. Insgesamt lieferten 20/25 tMRLs klinisch hilfreiche Informationen zur Interventionsplanung.

\section{WISS 318.7 Transpedale MR-Lymphangiografie des zentralen Lymphsystems - Eine neue Technik zur Planung lymphatischer Interventionen bei Patienten mit chylösen Leckagen}

\author{
Autoren Pieper $\mathrm{C}^{1}$, Schild $\mathrm{H}^{1}$ \\ Institut 1 Universitätsklinikum Bonn, Radiologische Klinik, Bonn \\ DOI 10.1055/s-0037-1600249
}

Zielsetzung Evaluation des klinischen Nutzens transpedaler MR-Lymphangiografien (tMRL) zur Planung lymphatischer Interventionen bei Patienten mit chylösen Leckagen.

Material und Methodik Eine tMRL wurde bei 25 Patienten (m/w: 13/12, mittleres Alter 54 Jahre) mit chylösen Leckagen vor lymphatischer Intervention durchgeführt. Indikationen waren: traumatischer/nicht-traumatischer Chylothorax $n=6 / 8$, traumatischer/nicht-traumatischer Chylaszites $n=3 / 3$, kombierter Chylothorax/Chylaszites $n=5$. Die tMRL erfolgte am 1.5T Scanner mit T1-gewichteter Bildgebung nach pedaler intrakutaner Injektion von gadoliniumhaltigem Kontrastmittel (KM) unter Lokalanästhesie. Die Daten wurden evaluiert hinsichtlich der Darstellung einer Leckagestelle, eines möglichen Zugangsweges für eine Lymphgefäßembolisation und anatomischer Varianten.

Ergebnisse Die KM-Injektion wurde von allen Patienten gut toleriert. Komplikationen traten nicht auf. Eine Sedierung war in einem Fall nötig. Bei 20/25 Patienten konnten kontrastierte zentrale Lymphbahnen abgegrenzt werden (Gründe für Versagen: unruhiger Patient trotz Sedierung $n=1$, Lymphom mit Lymphödem $n=1$, technische Gründe $n=3$ ). Eine Leckage konnte bei 13/20 Patienten sicher identifiziert werden. In 5 Fällen (Chylaszites) trat das KM intraabdominell aus und erreichte nicht die Cisterna chyli (CC). Bei den verbleibenden 15 Patienten konnte ein Zugangsweg zur Ductus thoracicus (DT) Embolisation in 14/15 Fällen identifiziert werden (CC bei $12 / 15$, DT bei 14/15 [9 durchgängige, 5 diskontinuierliche Kontrastierung]). 8/14 Patienten hatten einen einzelnen, 6/14 einen gedoppelten DT. Ein DT mündete im rechten Venenwinkel. Insgesamt lieferten 20/25 tMRLs klinisch hilfreiche Informationen zur Interventionsplanung.

Schlussfolgerungen Die tMRL ist ein neues und wertvolles diagnostisches Werkzeug bei Patienten mit chylösen Leckagen, bei denen eine lymphatische Intervention geplant ist und liefert Informationen über die Anatomie der zentralen Lymphbahnen. Die Leckagestelle kann aber nicht in allen Fällen sicher identifiziert werden.
WISS 318.3 Der Einfluss von in der MRT-detektierten, eingebluteten Karotisplaques auf das erstmalige oder wiederholte Auftreten zerebrovaskulärer Ereignisse: eine Individuen-basierte Metaanalyse (Big Data)

Autoren Schindler $A^{1}$, Schinner $R^{1}$, Altaf $N^{2}$, Kooi $M^{3}$, Moody $A^{4}$, Poppert $H^{5}$, Reiser $\mathrm{M}^{1}$, Auer $\mathrm{D}^{2}$, Saam $\mathrm{T}^{1}$

Institut 1 Klinikum der LMU München, Institut für klinische Radiologie,

München; 2 The University of Nottingham, Radiological Sciences,

Nottingham; 3 Maastricht University, Department of Radiology, Maastricht; 4 University of Toronto, Department of medical imaging, Toronto; 5 TU

München, Neurologische Klinik, München

DOI 10.1055/s-0037-1600250

Zielsetzung Obwohl Studien zeigten konnten, dass mittels MRT diagnostizierte, eingeblutete Karotisplaques mit ischämischen Symptomen assoziiert sind, ist für eine genauere Risikoabschätzung Data-Sharing publizierter Studien notwendig. Ziel dieser Studie war es, den Einfluss von Plaqueeinblutungen (PE) und deren Assoziation mit ischämischen Ereignissen in einer auf individuellen Patientendaten basierenden Metaanalyse zu untersuchen.

Material und Methodik Individuelle Patientendaten von 772 Individuen aus 7 Studienkohorten (77,6\% symptomatische Patienten; Prävalenz von PE zu Baseline: 46,8\%), welche die Assoziation zwischen eingebluteten Karotisplaques und ischämischen Ereignissen untersuchten, wurden gesammelt. Der klinische Verlauf von der Detektion einer PE bis zur ersten Behandlung oder der letzten erhältlichen Verlaufskontrolle wurden dafür verwendet, das Risiko eines neu aufgetretenen Schlaganfalls oder neu aufgetretener Ischämiesymptomatik (Schlaganfall, TIA, Amaurosis fugax) nach 3, 12 und 24 Monaten in einer Survival-Analyse zu bestimmen.

Ergebnisse Über einen Beobachtungszeitraum von 1205 Patientenjahren ereigneten sich 70 Schlaganfälle (annualisierte Ereignisrate; AER=5,81\%) und insgesamt 131 Ereignisse ( $A E R=10,9 \%$ ). Eine PE zur Baseline erhöhte das Risiko eines Schlaganfalls in symptomatischen, asymptomatischen und allen Individuen auf das 7,1-, 3,5- und 6,1-fache. Entsprechend erhöhte sich das Risiko für eine neue Ischämiesymptomatik auf das 4,8-, 5,8- und 5,3-fache. Das kumulative Risiko für einen Schlaganfall nach 3, 12 und 24 Monaten betrug abhängig vom Vorliegen bzw. Fehlen einer PE 6,3\%, 13,7\% und $24,4 \%$, bzw. $0,5 \%, 0,9 \%$ und $2,5 \%$.

Schlussfolgerungen Diese individuen-basierte Metaanalyse mit Big-Data-Ansatz ermöglicht im Gegensatz zu bisher veröffentlichten Studien eine genauere Abschätzung des Risikos von PE unter Berücksichtigung der einzelnen ischämischen Entitäten. Diese Risikoschätzer haben einen hohen Wert für die Planung von Interventionsstudien, die das Ziel einer optimalen Schlaganfalltherapie verfolgen.

\section{RK 411.5 Beschleunigte 4D-MR- Phasenkontrastflussmessung zur Analyse flussdynamischer Prozesse im Truncus coeliacus und der Arteria mesenterica superior}

Autoren Siedek $\mathrm{F}^{1}$, Giese $\mathrm{D}^{2}$, Weiss $\mathrm{K}^{2}$, Brinkmann $\mathrm{S}^{3}$, Schröder $\mathrm{W}^{3}$, Maintz $D^{4}$, Haneder $\mathrm{S}^{4}$

Institut 1 Uniklinik Köln, Radiologie, Köln; 2 Uniklinik Köln - Institut für Diagnostische und Interventionelle Radiologie, MRI-Physics, Köln; 3 Uniklinik Köln - Klinik für Allgemein-, Viszeral- und Tumorchirurgie, Köln; 4 Uniklinik Köln - Institut für Diagnostische und Interventionelle Radiologie, Köln DOI 10.1055/s-0037-1600251

Zielsetzung Die 4D-Fluss-MRT erlaubt zeitaufgelöste Aufnahmen des Blutflusses zur Visualisierung und Quantifizierung innerhalb eines 3D-Volumens ohne die Gabe von Kontrastmittel. Dies ermöglicht die nicht-invasive Beurteilung zahlreicher Flusscharakteristika wie dem Peak Flow (PF), der Peak Velocity (PV), der Average velocity (AV) oder dem Stroke Volume (SV). Das Studienziel war der Vergleich dieser Flussparameter in einem komplexen Gefäßterrito- 
rium, dem Truncus coeliacus (TC) und der Art. mesenterica sup. (AMS) zwischen gesunden Probanden (PRO) und Patienten mit vorbekannten abgangsnahen niedriggradigen Stenosen (PAT).

Material und Methodik Die Datensätze (3,0T) von 23 Probanden und 6 Patienten wurden mittels GT-Flow ausgewertet und der PF, die PV und AV und das SV im TC und der AMS (jeweils $5 \mathrm{~mm}$ distal der Aorta [AO]) und in der AO (10 mm proximal des TC/zwischen dem TC und der AMS/10 mm distal der AMS) bestimmt. Zweiseitige T-tests wurden zur statistischen Auswertung durchgeführt (Signifikanzlevel bei $\mathrm{p}<0,05$ ).

Ergebnisse Die Flussgeschwindigkeiten waren im TC der PAT signifikant höher [AV $(p=0,02) ; P V(p=0,001)]$. In der AMS der PAT war die maximale Flussgeschwindigkeit signifikant erhöht [PV $(p=0,004)]$. Im TC und der AMS fand sich kein signifikanter Unterschied des Flussvolumens [PF; SV] in den PAT im Vergleich zu den PRO. In der AO wiesen die PAT auf allen drei Höhen ein signifikant höheres maximales Flussvolumen [PF $(p=0,0004 / 0,001 / 0,0008)$ ] und durchschnittliche Flussgeschwindigkeit $[A V(p=7 \times 10-5 / 9,8 \times 10-5 /$ 0,0002)] auf.

Schlussfolgerungen Mithilfe der beschleunigten 4D-Fluss-MRT konnten signifikante Unterschiede im Flussprofil bei erhaltenem Flussvolumen zwischen gesunden Probanden und Patienten mit einer niedriggradigen TC/AMS-Stenose nachgewiesen werden. Somit stellt sich eine nicht-invasive Technik zur Beurteilung komplexer Flusscharakteristika auch in kleineren Gefäßen wie dem TC und der AMS dar. Ein zukünftiges klinisches Einsatzfeld ist die präoperative Evaluation vor Ösophagoektomie.

\section{WISS 316.4 Vergleich hämodynamischer Parameter von Probanden und Patienten mit pulmonaler Hypertonie mittels 4D- und 2D-Fluss MRT}

Autoren Sieren $\mathrm{M}^{1}$, Berlin $\mathrm{C}^{1}$, Oechtering $\mathrm{T}^{1}$, Hunold $\mathrm{P}^{1}$, Drömann $\mathrm{D}^{2}$, Barkhausen $\mathrm{J}^{1}$, Frydrychowicz $\mathrm{A}^{1}$

Institut 1 UKSH, Klinik für Radiologie und Nuklearmedizin, Lübeck; 2 UKSH, Medizinische Klinik III, Pneumologie/Infektiologie, Lübeck

DOI 10.1055/s-0037-1600252

Zielsetzung Die nicht-invasive Diagnostik der pulmonalen Hypertonie $(\mathrm{PH})$ mit 2D- und 4D-Fluss MRT rückt zunehmend in den klinischen Fokus. Ziel der Studie war es, beide Techniken zu vergleichen und an bereits in 2D-etablierten Parametern an Patienten mit PH und gesunden Probanden zu testen.

Material und Methodik 11 Patienten mit PH (4 weibl., Alter: $63 \pm 15$ Jahre), 15 alterskorrelierte $(9 \mathrm{w}, 56 \pm 11 \mathrm{~J}$.) und 20 junge Probanden $(13 \mathrm{w}, 23 \pm 2 \mathrm{~J}$.) wurden an 3T (Philips Ingenia) mit 2D- und 4D-Phasenkontrast-Sequenzen untersucht. Im Truncus pulmonalis (TP) wurden mit GTFlow (GyroTools, CH) folgende Parameter ausgewertet: Schlagvolumen (SV [ml/s]), die Maxima der Geschwindigkeit (Vmax $[\mathrm{cm} / \mathrm{s}])$ und des Flusses (Qmax $[\mathrm{ml} / \mathrm{s}])$ und die Zeit zum jeweiligen Maximum (tVmax, tQmax, [ms]). Strain [\%] wurde als Variabilität der max. und min. Fläche des Gefäßquerschnitts (Amin, Amax, [mm2]) berechnet. In 4D wurden Blutflussmuster visualisiert. Die Statistik umfasste t-Test und Bland-Altman-Analyse.

Ergebnisse Die 4D-Fluss MRT zeigte gute Übereinstimmung mit den in 2D in der Studienpopulation gemessenen Werten mit einer Tendenz zu höheren Werten, exemplarisch des SV $(98,6 \pm 20,8$ vs. 91,9 $\pm 18,7 ; p=<0,001)$ und tQmax (143,4 $\pm 33,9$ vs. 132,5 $\pm 30 ; \mathrm{p}=<0,001)$. Zur Identifizierung eines $\mathrm{PH}$ erreichten Amin, Amax und Strain in 2D und 4D statistisch signifikante Unterschiede zwischen Patienten und alterskorrelierten Probanden (jeweils $\mathrm{p}<0,05)$. Dabei zeigte sich eine Erhöhung der Mittelwerte bei Patienten von Amin um 92,8\%/71,6\% (2D/4D), Amax um 62,2\%/57,9\% (2D/4D) und eine Verminderung des Strain um 48,3\%/27,9\% (2D/4D) gegenüber Probanden. Ein für PH indikativer Vortex im TP konnte bei 9 von 11 Patienten und 2 Probanden nachgewiesen werden (Spezifität/Sensitivität von 95,7\%/81,8\% für die Detektion einer $\mathrm{PH}$ ).

Schlussfolgerungen Die Studie belegt die verlässliche Messung diagnoserelevanter Parameter und die mögliche Unterscheidung von Patienten und Probanden mit der 4D-Fluss MRT. Es gelang Trends verschiedener Parameter für die Diagnose der PH für zukünftige Studien aufzuzeigen.

\section{RK/WISS 107.8 Inzidenz, Lokalisation und Ausprägung von Myokardvernarbungen bei ambitionierten Triathleten im Kardio-MRT}

Autoren Stáreková $\mathrm{J}^{1}$, Tahir $\mathrm{E}^{1}$, Avanesov $\mathrm{M}^{1}$, Patten-Hamel M², Münch $\mathrm{J}^{2}$, Weinrich $J^{1}$, Bohnen $S^{2}$, Radunski $U^{2}$, Müllerleile $K^{2}$, von Stritzky $A^{3}$, Adam $G^{1}$, Lund $G^{1}$

Institut 1 UKE, Klinik und Poliklinik für Diagnostische und Interventionelle Radiologie und Nuklearmedizin, Hamburg; 2 UHZ, Allgemeine und

Interventionelle Kardiologie, Hamburg; 3 UHZ, Allgemeine und

interventionelle Kardiologie, Hamburg

DOI 10.1055/s-0037-1600253

Zielsetzung Langjähriges intensives körperliches Training führt zur funktionellen Anpassung des Herzens mit typischen morphologischen Veränderungen. Im Einzelfall können Myokardvernarbungen auftreten, die mittels KardioMRT (CMR) identifiziert werden können. Ziel der Studie war es bei ambitionierten Triathleten die Inzidenz, Lokalisation und Ausprägung von Myokardvernarbungen mittels CMR zu untersuchen.

Material und Methodik 51 männliche $(43 \pm 9$ J.) und 25 weibliche $(44 \pm 9$ J.) Triathleten wurden mittels 1,5 T MRT (Achieva, Phillips) untersucht. Das CMRProtokoll bestand aus EKG-getriggerten Cine-SSFP-Sequenzen in AtemanhalteTechnik sowie Late-Gadolinium-Enhancement (LGE) in kurzen und langen Achsen. Mittels Spiroergometrie wurden die Parameter der kardiovaskulären Ausdauer objektiviert. Zur LGE-Quantifizierung wurde eine Schwellenwertmethode (5SD) benutzt.

Ergebnisse Die Spiroergometrie zeigte eine hohe maximal erreichbare Leistung (428 \pm 118 Watt Männer; $253 \pm 66$ Watt Frauen) und eine hohe maximale Sauerstoffaufnahme (VO2max $56 \pm 7 \mathrm{ml} / \mathrm{kg} / \mathrm{min}$ Männer; $46 \pm 6 \mathrm{ml} / \mathrm{kg} / \mathrm{min}$ Frauen) für beide Gruppen. Bei 9 der 51 männlichen Triathleten (18\%) fand sich ein LGE, während kein weiblicher Triathlet ein LGE aufwies. Bei allen LGE+ Triathleten fand sich eine nicht-ischämische, mittmyokardial gelegene Vernarbung. Die Größe der Vernarbung betrug 2,2 $\pm 2,1 \%$ (Range 0,5\%-6,7\%) des LV-Myokards. Bei allen LGE+ Triathleten lag die Vernarbung in der basalen bis mittleren Hinter- und Seitenwand. Bei einem Triathleten mit großem LGE wurden koronarangiografisch relevante Koronarstenosen ausgeschlossen. Hinsichtlich der Ejektionsfraktion zeigte sich bei LGE+ und LGE- Triathleten kein Unterschied (EF $65 \pm 5 \%$ vs. $62 \pm 5 \%, P=n s$ ).

Schlussfolgerungen Myokardiale Vernarbungen finden sich bei $18 \%$ der männlichen, aber nicht bei weiblichen ambitionierten Triathleten. Die Myokardnarben sind typischer Weise in der inferolateralen Wand lokalisiert und weisen ein nicht-ischämisches Muster auf, was auf eine abgelaufene Myokarditis oder einen sport-induzierten Myokardschaden hinweist.

\section{RK/WISS 107.3 Unterschiede der myokardialen Gewebecharakterisierung mittels kardialer Magnetresonanztomografie bei Prädiabetikern, Diabetikern und einem Kontrollkollektiv: Ergebnisse der KORA MRT Studie}

Autoren Storz $\mathrm{C}^{1}$, Hetterich $\mathrm{H}^{2}$, Lorbeer $\mathrm{R}^{3}$, Auweter $\mathrm{S}^{3}$, Rathmann $\mathrm{W}^{4}$, Schlett $C^{5}$, Peters $A^{6}$, Nikolaou $K^{7}$, Schulz-Menger $]^{8}$, Bamberg $F^{7}$ Institut 1 Universitätsklinikum Tübingen, Interventionelle und diagnostische Radiologie, Tübingen; 2 Ludwig-Maximilans Universität München, Klinische Radiologie, München; 3 Ludwig-Maximilians Universität München, Klinische Radiologie, München; 4 Deutsches Diabetes-Zentrum, Institut für Biometrie und Epidemiologie, Düsseldorf; 5 Universitätsklinikum Heidelberg, Diagnostische und Interventionelle Radiologie, Heidelberg; 6 Helmholtz Zentrum München, Deutsches Forschungszentrum für Gesundheit und Umwelt (GmbH) Institute of Epidemiology II, München; 7 Universitätsklinikum 
Tübingen, Interventionelle und Diagnostische Radiologie, Tübingen; 8 Charité Berlin - Experimental and Clinical Research Center (ECRC), Kardiologie, Berlin DOI 10.1055/s-0037-1600254

Zielsetzung Ziel dieser Kohortenstudie war es, Unterschiede der myokardialen Gewebeeigenschaften zwischen Probanden mit Prädiabetes, Diabetes und einer Kontrollgruppe mittels kardialer Magnetresonanztomografie (cMRT) zu bestimmen.

Material und Methodik Gesunde Teilnehmer sowie Probanden mit Prädiabetes und Diabetes (laut WHO Kriterien) aus einer bevölkerungsbasierten Kohortenstudie ohne kardiovaskuläre Vorerkrankungen wurden mittels Kontrastmittel-verstärkter cMRT (3 Tesla) untersucht. Erhobene Parameter umfassten die linksventrikuläre Funktion und Morphologie, Late Gadolinum Enhancement (LGE), sowie T1-Mapping und Ableitung des extrazellulären Volumens (EZV) mittels modifizierter Look-Locker Inversion Recovery Sequenz (MOLLI). Weiterhin wurde die diffuse myokardiale Fibrose und das myokardiale Zellvolumen (ZV) bestimmt. Die Gruppen wurden uni- und multivariat verglichen.

Ergebnisse In der Studienpopulation (insgesamt 343 Teilnehmer, mittleres Alter: 56,19,2 Jahren, 57\% männlich) wurden 14\% als Diabetiker, $23 \%$ als Prädiabetiker und $64 \%$ als Kontrollprobanden eingeteilt. Die cMRT ergab einen signifikant erhöhten LV-Remodeling-Index (LV Masse/LV enddiastolisches Volumen) bei Prädiabetikern und Diabetikern, unabhängig von BMI, Hypertonie, Alter und Geschlecht (1,17; 95\%-Cl: 1,11 bis 1,23 und 1,28; $95 \%-\mathrm{Cl}: 1,20$ bis $1,36$; jeweils $p<0,002)$. Weiterhin war im Vergleich zur gesunden Kontrollgruppe das EZV bei Probanden mit Prädiabetes oder Diabetes signifikant reduziert $(23,1 \pm 2,4 \%$ und $22,8 \pm 3,0 \%$, jeweils $p<0.007)$. Dagegen war das ZV bei Prädiabetikern und Diabetikern signifikant erhöht $(109,1 \pm 23,8 \mathrm{ml}$ und $114,9 \pm 32,3 \mathrm{ml}$ vs. $96,5 \pm 26,9 \mathrm{ml}$, jeweils $\mathrm{p}<0.03$ ).

Schlussfolgerungen In einer westlich europäischen Bevölkerungsstichprobe zeigt sich, dass Prädiabetiker und Diabetiker höhere LV-Remodeling-Indizes und ZV im Vergleich zur gesunden Kontrollgruppe aufweisen, wohingegen die Entwicklung einer diffusen Fibrose weniger dominant imponiert.

\section{WISS 316.1 Durchführbarkeit eines Niedrigdosis- Protokolls mit reduzierter Kontrastmittelmenge für eine CT-Angiografie der Pulmonalarterien}

\author{
Autoren Suntharalingam $S^{1}$, Mikat $C^{1}$, Erfanian $Y^{1}$, Nassenstein $K^{1}$ \\ Institut 1 Universitätsklinikum Essen, Institut für Diagnostische und \\ Interventionelle Radiologie und Neuroradiologie, Essen \\ DOI 10.1055/s-0037-1600255
}

Zielsetzung Evaluation der Bildqualität und der Strahlendosis eines Niedrigdosis-Protokolls mit reduzierter Kontrastmittelmenge für eine CT-Angiografie der Pulmonalarterien.

Material und Methodik 60 Patienten mit Verdacht auf Lungenarterienembolie wurden mit zwei verschiedenen Untersuchungsprotokollen untersucht (jeweils $n=30$, Gruppe $A, 100 \mathrm{kVp}$, ref. mAs 150, $60 \mathrm{ml} \mathrm{KM}$; Gruppe B, 80 kVp, ref. mAs 115, 25 ml Kontrastmittel). Bildqualität und Strahlendosis der beiden Gruppen wurden ermittelt und verglichen.

Ergebnisse Signalintensitäten in der Hauptlungenarterie (351,4 vs. 291,8; $p=0,08)$, linke Pulmonalarterie $(339,2$ vs. 311,$6 ; p=0,42)$, rechte Pulmonalarterie $(340,8$ vs. 309,$9 ; p=0,37)$, Lappenarterie $(368,9$ vs. 343,$0 ; p=0,49)$, Segmentarterie $(347,6$ vs. 323,$4 ; p=0,50)$ und Subsegmentarterie $(334,4$ vs. 330,$4 ; p=0,91$ ) waren geringfügig, jedoch nicht signifikant höher in Gruppe A. Signal-Rausch-Verhältnis und Kontrast-Rausch-Verhältnis waren vergleichbar zwischen beiden Gruppen (alles p > 0.05). Die subjektive Bildanalyse ergab keine signifikanten Unterschiede zwischen beiden Gruppen. Alle Untersuchungen wurden als "gut bis exzellent" oder als „diagnostisch ausreichend“ gewertet. Verglichen mit Gruppe A war die Strahlendosis in Gruppe B um $71,8 \%$ reduziert ( 2,4 vs. $0,7 \mathrm{mSv}$; $\mathrm{p}<0,001)$.

Schlussfolgerungen Ein Niedrigdosis-Protokoll mit $80 \mathrm{kV}$ und $25 \mathrm{ml}$ Kontrastmittel kann eine zur Diagnostik geeignete Bildqualität liefern und dabei die Strahlendosis im Vergleich zu einem Standard-Protokoll um 71,8\% reduzieren.

\section{RK/WISS 103.6 Quantitatives T1- und T2-Mapping CMR zur Differenzierung von akutem und chronischem Myokardinfarkt}

Autoren Tahir $\mathrm{E}^{1}$, Sinn $\mathrm{M}^{1}$, Avanesov $\mathrm{M}^{1}$, Bohnen $\mathrm{S}^{2}$, Müllerleile $\mathrm{K}^{2}$, Radunksi $\mathrm{U}^{2}$, Stehning $\mathrm{C}^{3}$, Säring $\mathrm{D}^{4}$, Starekova $\mathrm{J}^{1}$, Schnackenburg $\mathrm{B}^{3}$, Adam $G^{1}$, Lund $G^{1}$

Institut 1 Universitätsklinikum Eppendorf-Hamburg, Diagnostische und Interventionelle Radiologie, Hamburg; 2 Universitäres Herzzentrum Hamburg, Allgemeine und Interventionelle Kardiologie, Hamburg; 3 Philips Research,

Philips, Hamburg; 4 FH Wedel, Medizinische und Industrielle Bildverarbeitung, Wedel

DOI 10.1055/s-0037-1600256

Zielsetzung Die quantitative Gewebecharakterisierung mittels den neuen Kardio-MRT-Techniken (CMR) des T1 und T2 Mapping können wichtige Information zur Differenzierung von akutem und chronischem Myokardinfarkt (MI) liefern. Wir haben die klinische Anwendung der neuen Mapping-Techniquen im Vergleich zur konventionellen T2-gewichteten CMR zur Diskriminierung von akutem und chronischem Ml untersucht.

Material und Methodik 67 Patienten mit akutem reperfundierten MI wurden prospektiv rekrutiert. CMR wurde $8 \pm 5$ Tage nach erstmaligem MI (Basisuntersuchung) und nach $6 \pm 1,4$ Monaten durchgeführt. Alle Aufnahmen wurden in der Enddiastole und in der LV-Kurzachse durchgeführt. Die T1-Relaxationszeiten wurden mit der modifizierten Look-Locker Inversion Recovery Sequenz bestimmt. Zwei erfahrene Auswerter haben unabhängig voneinander ROIs in das infarzierte Myokard platziert. LGE diente als Refenrenzstandard. Zusätzlich wurde ein T2w-Ratio generiert: T2w-Ratio = MW SI-Infarkt/MW SI-Gesund.

Ergebnisse Natives T1-Mapping zeigte eine nahezu perfekte diagnostische Leistung zur Differenzierung von akutem und chronischem MI (AUC von 0,984). Diese war signifikant größer als bei der Anwendung von T2w-Ratio (AUC von 0,906; $P<0,05$ ) und T2 (AUC 0,903; $P<0,05$ ). Die extrazelluläre Volumenfraktion hatte eine niedrige Diskriminierungsleistung mit AUC von 0,655 und signifikant kleiner als die anderen Techniquen $(P<0.001)$. Ein Cutoff von $\geq 1138$ ms für Natives T1 zeigte eine Sensitivität und Spezifität von entsprechend $96 \%$ und $100 \%$. Die übrigen Cutoffs waren wie folgt: $\geq 3,3$ für T2w-Ratio, $\geq 69$ ms für T2 und $\geq 39 \%$ für ECV.

Schlussfolgerungen Natives T1-Mapping ist der beste Diskriminator zwischen akutem und chronischem MI und sollte vorzugsweise als ein quantitativer Parameter zur Differenzierung zwischen dem akuten und chronischen Stadium von Myokardinfarkten verwendet werden. Ein wichtiger Einsatzbereich könnte die Indentifikation von Ursprungsläsionen bei Patienten mit Mehrgefäßerkrankung sein, die sich mit kürzlich diagnostiziertem MI vorstellen.

\section{WISS 318.5 Bildqualität und diagnostische Genauigkeit von autonom durch Lumen Care erstellten 3D-MIP Bildern in CT-Angiografien der unteren Extremität}

Autoren Tjiong $\mathrm{R}^{1}$, Seyfer $\mathrm{P}^{2}$, Mahnken $\mathrm{A}^{1}$

Institut 1 UKGM Marburg, Radiologie, Marburg; 2 UKGM, Radiologie,

Marburg

DOI 10.1055/s-0037-1600257

Zielsetzung Die CT-Angiografie ist ein schnelles und wenig invasives Verfahren zur Diagnose der peripheren arteriellen Verschlusskrankheit. Zur schnellen Übersicht über das Ausmaß und die Lokalisation von Okklusionen und Stenosen werden häufig 3D- und MIP-Reformationen, teils mit (semiautomatischer) Knochenentfernung erstellt. Dieser, meist von medizintechnischen Assistenten durchgeführte Schritt ist zeitaufwändig und fehleranfällig. Ziel dieser Studie war es Bildqualität und diagnostische Genauigkeit von autonom durch Lumen Care (Siemens) erstellten 3D-MIP Bildern zu evaluieren.

Material und Methodik Insgesamt 134 Unterschenkel von 69 Patienten (45 Männer; Durchschnittsalter: 71,9 \pm 12,2 Jahre) wurden inkludiert. Die Unterschenkelarterien wurden in fünf Segmente unterteilt: A. pop. ab dem P3- 
Segment, Truncus tibiofibularis, ATA, ATP und AF. Die Beurteilung der Bilder wurde von zwei Radiologen unabhängig voneinander und ohne Kenntnis der Diagnose durchgeführt. Bildqualität wurde mittels folgender Skala evaluiert ( 0 = Keine Artefakte, 1 =Artefakte ohne Einschränkung der diagnostischen Aussagekraft, 2 = 1 Segment nicht beurteilbar, 3 = 2 Segmente nicht beurteilbar, 4=3 oder mehr Segmente nicht beurteilbar). Verglichen wurden durch Lumen Care erstellte Reformationen mit axialen Bildern, welche als Referenz dienten.

Ergebnisse 618 Segmente wurden beurteilt.57 Segmente wurden exkludiert. 109 Beine konnten ohne Einschränkungen befundet werden (Note 1 u. 2; $81,3 \%$ ), bei 8 Beinen konnte ein Segment nicht beurteilt werden. 5 Bilder erhielten die Note 3 und 12 Bilder die Note 4. Für die Diagnose von hochgradigen Stenosen (> 70\%) und Okklusionen erreichten wir eine diagnostische Genauigkeit, Sensitivität und Spezifizität von respektive 91\%, 94\% und 89\%. Interobserver-Übereinstimmung war mit einem $\mathrm{k}$ von 0.859 sehr gut.

Schlussfolgerungen Lumen Care Bilder bieten eine schnelle Übersicht über Lokalisation und Ausmaß von Stenosen mit einer relativ hohen Aussagekraft, geringen Artefakten und sehr guter Interobserver-Übereinstimmung.

\section{RK/WISS 402.4 Dynamische 3D-MR-Angiografie bei Patienten mit Aortendissektion als Risikofaktor für eine chronische Expansion}

\author{
Autoren Trojan $\mathrm{M}^{1}$, Müller-Eschner $\mathrm{M}^{1}$, Kotelis $\mathrm{D}^{2}$, MD Partovi $\mathrm{S}^{3}$, \\ Karmonik $C^{4}$, Fink $C^{5}$, von Tengg-Koblink $H^{6}$, Ruhparwar $A^{7}$, Böckler $D^{2}$, \\ Kauczor $\mathrm{H}^{1}$, Rengier $\mathrm{F}^{1}$ \\ Institut 1 Universitätsklinikum Heidelberg, Diagnostische und \\ Interventionelle Radiologie, Heidelberg; 2 Universitätsklinikum Heidelberg, \\ Gefäßchirurgie, Heidelberg; 3 University Hospitals Case Medical Center \\ Cleveland, Radiology, Cleveland, Ohio USA; 4 Houston Methodist, \\ Neurosurgery, Houston, Texas USA; 5 AKH Celle, Radiologie, Celle; \\ 6 Universitätsspital Bern, Inselspital, Diagnostische, Interventionelle und \\ pädiatrische Radiologie, Bern, Schweiz; 7 Universitätsklinikum Heidelberg, \\ Herzchirurgie, Heidelberg
}

DOI 10.1055/s-0037-1600258

Zielsetzung Analyse der dynamischen kontrastmittelverstärkten MR-Angiografie bei Patienten mit chronisch expandierender oder stabiler Aortendissektion und Evaluation als potentieller Risikofaktor für eine chronische aortale Expansion.

Material und Methodik Bei 20 Patienten mit Aortendissektion wurde eine dynamische 3D-MR-Angiografie der Aorta an einem 1,5T MRT und Nachsorge-CTs (Mittelwert Nachsorge 6,1 Jahre) durchgeführt. 7 Patienten (Mittelwert 65,3 Jahre) zeigten eine chronische Expansion, 13 Patienten (Mittelwert 64,3 Jahre) wiesen einen stabilen Aortendurchmesser auf. Die Auswertung der MRAngiografien erfolgte durch Platzieren von ROls zum einen zentral in das Lumen des nicht-dissezierten Aortenbogens und zum anderen in das falsche Lumen auf Höhe des thorakoabdominellen Übergangs. Aus den sich daraus ergebenden Zeit-Intensitäts-Kurven wurden folgende Parameter berechnet: Halbwertsbreite (FWHM), die Steigung im Anstieg und im Abfallen sowie die Zeitverzögerung zum Erreichen der maximalen Intensität zwischen falschem Lumen und nicht-disseziertem Aortenbogen.

Ergebnisse Folgende Werte ergaben sich für die expandierende/stabile Gruppe: FWHM 0,19 $\pm 0,02 / 0,41 \pm 0,17(p=0,009)$, Steigung im Anstieg 10,97 $\pm 3,11 / 7,64 \pm 2,68 \quad(p=0,038)$, Steigung im Abfallen $-6,55 \pm 0,87 /-1,97$ $\pm 2,67(p=0,013)$, Zeitverzögerung der maximalen Intensität im falschen Lumen $1,32 \pm 0,25 / 1,42 \pm 0,24(p=0,027)$.

Schlussfolgerungen Die zeitlich aufgelöste 3D-MR-Angiografie zeigte signifikante Unterschiede der Kontrastmitteldynamik zwischen Patienten mit expandierender und stabiler Aortendissektion als Ausdruck eines beschleunigten Ein- uns Ausströmens in das falsche Lumen. Die Technik könnte dabei helfen, das Verständnis der komplexen Pathophysiologie der chronischen Expansion weiter zu verbessern und besitzt das Potential als Risikofaktor für eine aortale Expansion.

\section{WISS 211.1 Kosteneffizienz von CT-Angiografie bei akutem Brustschmerz im Vergleich zu konservativer Behandlung in 2.156 Patienten Bewerber für den Young Investigator Award}

Autoren Wichmann j $]^{1}$

Institut 1 Universitätsklinikum Frankfurt am Main, Institut für Diagnostische und Interventionelle Radiologie, Frankfurt am Main

DOI 10.1055/s-0037-1600259

Zielsetzung Das Ziel dieser Studie war der Vergleich der Kosteneffizienz und klinischen Effektivität von Koronar-CT-Angiografie (CCTA) und konservativer Behandlung in der klinischen Versorgung von Patienten mit akutem Brustschmerz.

Material und Methodik Wir führten eine retrospektive single-center Analyse in 2.156 Patienten durch welche sich in der Notaufnahme (ZNA) mit akutem Brustschmerz vorgestellt hatten. Patientenkohorten, welche hinsichtlich Charakteristika und Vortestwahrscheinlichkeit für koronare Herzkrankheit (CAD) gematcht wurden und sich einer CCTA als primäre Bildgebung $(n=1.139)$ oder einem traditionellen Behandlungsprotokoll $(n=1.017)$ unterzogen hatten, wurden analysiert. Kosten-relevante Faktoren der ZNA-Besuche, Verwendung von weiteren klinischen Tests und kumulative Kosten pro Patient wurden verglichen.

Ergebnisse Es wurden keine signifikanten Differenzen zwischen den Gruppen hinsichtlich Alter, Geschlecht, Rasse, Body-Mass-Index oder KHK-Risikofaktoren beobachtet (alle $P>0,08$ ). Darüberhinaus wurden keine signifikanten Differenzen hinsichtlich der Diagnose einer KHK, Lungenembolie oder Aortendissektion beobachtet (alle P>0,11). Zeit bis zur Entlassung (4,5 vs. 7 Stunden), Hospitalisierungsrate (12,6\% vs. 54,2\%), Länge der Krankenhausaufenthalte (48 vs. 72 Stunden) und Re-Hospitalisierungsraten innerhalb von 30 Tagen $(3,5 \%$ vs. $14,6 \%$ ) waren signifikant niedriger (alle $P<0,001)$ in mit CCTA untersuchten Patienten. Die CCTA-Gruppe zeigte geringere Raten an zusätzlichen klinischen Tests (z. B. Stressbildgebung) und invasive Koronarangiografie (4,9\% vs. 22,7\%; $\mathrm{P}<0,001)$ sowie kumulative Kosten pro Patient (11.783\$ vs. $18.996 \$, P<0,001)$.

Schlussfolgerungen In dieser großen single-center Study war die Verwendung von CCTA als primäre Bildgebungsuntersuchung in Patienten mit akutem Brustschmerz assoziiert mit kürzeren ZNA- und Krankenhausaufenthalten, niedrigeren Re-Hospitalisierungsraten und geringeren kumulativen Kosten pro Patient, hauptsächlich aufgrund von niedrigeren Raten an zusätzlichen klinischen Tests.

\section{WISS 102.8 Zusammenhang zwischen Komplikationen in der Schwangerschaft und späterer koronarer Atherosklerose in afroamerikanischen Frauen: eine Koronar-CT-Angiografie Studie}

Autoren Wichmann $\mathrm{J}^{1}$, Nunez $\mathrm{J}^{2}$, Vliegenthart $\mathrm{R}^{3}$, Otani $\mathrm{K}^{4}$, Schoepf $\mathrm{U}^{2}$, De Cecco $C^{2}$, Vogl $T^{1}$, Wenger $N^{5}$

Institut 1 Universitätsklinikum Frankfurt am Main, Institut für Diagnostische und Interventionelle Radiologie, Frankfurt am Main; 2 Medical University of South Carolina, Department of Radiology and Radiological Science,

Charleston, SC, USA; 3 University Medical Center Groningen, Department of Radiology, Groningen, Niederlande; 4 Siemens Japan K. K., Imaging \&amp;

Therapy Systems Division, Healthcare Sector, Japan, Tokio; 5 Emory University School of Medicine, Division of Cardiology, Department of Medicine, Atlanta, GA, USA

DOI 10.1055/s-0037-1600260

Zielsetzung Das Ziel dieser Studie war der Vergleich der Prävalenz und des Ausmaßes von koronarer Atherosklerose detektiert mittels Koronar-CT-Angio- 
grafie (CCTA) in afroamerikanischen Frauen mit und ohne eine Anamnese von früheren Komplikationen in der Schwangerschaft.

Material und Methodik Wir analysierten retrospektiv die Patientencharakteristika und CCTA Ergebnisse in verschiedenen Gruppen von afroamerikanischen Frauen mit einer Anamnese von vorzeitiger Geburt ( $n=154)$, Präeklampsie $(n=137)$ oder Gestationsdiabetes $(n=148)$ sowie einer gematchten Kontrollgruppe von afroamerikanischen Frauen welche ohne Komplikationen entbunden hatten $(n=445)$. Univariate und multivariate Analysen wurden durchgeführt, um mögliche Prädiktoren von koronarer Atherosklerose zu evaluieren.

Ergebnisse Das durchschnittliche Alter bei Geburt und CCTA, die Anzahl an Schwangerschaften, der Body-Mass-Index, eine Raucheranamnese und die Prävalenz von hypertensiver Herzkrankheit oder Hyperlipidämie waren nichtsignifikant ähnlich zwischen den Gruppen (alle $P>0,2$ ). Alle Gruppen mit früheren Schwangerschaftskomplikationen zeigten höhere Raten an jeglicher ( $\geq 20 \%$ Lumeneinengung) und obstruktiver ( $\geq 50 \%$ Lumeneinengung) koronarer Atherosklerose (vorzeitige Geburt: 29,2\% und 9,1\%; Präeklampsie: 29,2\% und 7,3\%; Gestationsdiabetes: $47,3 \%$ und 15,5\%) im Vergleich zur Kontrollgruppe (23,8\% und 5,4\%). Nach der Berücksichtigung von möglichen Störfaktoren während der multivariaten Analyse verblieb nur Gestationsdiabetes als reliabler Prädiktor von jeglicher (OR 3,26; $95 \%$ Konfidenzintervalle 2,03 - 5,22; $\mathrm{P}<0,001)$ und obstruktiver koronarer Atherosklerose (OR 3,00; $95 \%$ Konfidenzintervalle 1,55-5,80; $\mathrm{P}<0,001)$ detektiert via CCTA.

Schlussfolgerungen Afroamerikanische Frauen mit einer Anamnese von Komplikationen in der Schwangerschaft zeigten eine höhere Prävalenz von koronarer Atherosklerose detektiert mittels CCTA, wohingegen in unserer Studie nur ein früherer Gestationsdiabetes ein unabhängiger Prädiktor von jeglicher und auch obstruktiver koronarer Atherosklerose in der CCTA war.

\section{WISS 102.1 Kosteneffizienz von CT-Angiografie bei akutem Brustschmerz im Vergleich zu konservativer Behandlung in 2.156 Patienten}

Autoren Wichmann $\mathrm{J}^{1}$, Otani $\mathrm{K}^{2}$, Tesche $\mathrm{C}^{3}$, Baumann $\mathrm{S}^{4}$, De Cecco $\mathrm{C}^{3}$, Schoepf $U^{3}$, Vogl $\mathrm{T}^{1}$, Carr $\mathrm{C}^{5}$

Institut 1 Universitätsklinikum Frankfurt am Main, Institut für Diagnostische und Interventionelle Radiologie, Frankfurt am Main; 2 Siemens K.K. Japan, Imaging \&amp; Therapy Systems Division, Healthcare Sector, Tokio, Japan; 3 Medical University of South Carolina, Department of Radiology and Radiological Science, Charleston, SC, USA; 4 Universitätsmedizin Mannheim, I. Medizinische Klinik - Kardiologie, Mannheim; 5 Medical University of South Carolina, Division of Emergency Medicine, Charleston, SC, USA

DOI 10.1055/s-0037-1600261

Zielsetzung Das Ziel dieser Studie war der Vergleich der Kosteneffizienz und klinischen Effektivität von Koronar-CT-Angiografie (CCTA) und konservativer Behandlung in der klinischen Versorgung von Patienten mit akutem Brustschmerz.

Material und Methodik Wir führten eine retrospektive single-center Analyse in 2.156 Patienten durch welche sich in der Notaufnahme (ZNA) mit akutem Brustschmerz vorgestellt hatten. Patientenkohorten, welche hinsichtlich Charakteristika und Vortestwahrscheinlichkeit für koronare Herzkrankheit (CAD) gematcht wurden und sich einer CCTA als primäre Bildgebung $(n=1.139)$ oder einem traditionellen Behandlungsprotokoll $(n=1.017)$ unterzogen hatten, wurden analysiert. Kosten-relevante Faktoren der ZNA-Besuche, Verwendung von weiteren klinischen Tests und kumulative Kosten pro Patient wurden verglichen.

Ergebnisse Es wurden keine signifikanten Differenzen zwischen den Gruppen hinsichtlich Alter, Geschlecht, Rasse, Body-Mass-Index oder KHK-Risikofaktoren beobachtet (alle $\mathrm{P}>0,08$ ). Darüberhinaus wurden keine signifikanten Differenzen hinsichtlich der Diagnose einer KHK, Lungenembolie oder Aortendissektion beobachtet (alle P $>0,11$ ). Zeit bis zur Entlassung (4,5 vs. 7 Stunden), Hospitalisierungsrate (12,6\% vs. 54,2\%), Länge der Krankenhausauf- tenthalte (48 vs. 72 Stunden) und Re-Hospitalisierungsraten innerhalb von 30 Tagen ( $3,5 \%$ vs. $14,6 \%$ ) waren signifikant niedriger (alle $\mathrm{P}<0,001$ ) in mit CCTA untersuchten Patienten. Die CCTA-Gruppe zeigte geringere Raten an zusätzlichen klinischen Tests (z. B. Stressbildgebung) und invasive Koronarangiografie ( $4,9 \%$ vs. $22,7 \%$; $\mathrm{P}<0,001)$ sowie kumulative Kosten pro Patient (11.783\$ vs. 18.996\$, $\mathrm{P}<0,001)$.

Schlussfolgerungen In dieser großen single-center Study war die Verwendung von CCTA als primäre Bildgebungsuntersuchung in Patienten mit akutem Brustschmerz assoziiert mit kürzeren ZNA- und Krankenhausaufenthalten, niedrigeren Re-Hospitalisierungsraten und geringeren kumulativen Kosten pro Patient, hauptsächlich aufgrund von niedrigeren Raten an zusätzlichen klinischen Tests.

\section{RK/WISS 107.4 Herz-MRT: Interscanner- Reproduzierbarkeit des linksventrikulären T1- Mappings}

Autoren Wiesmüller $M^{1}$, Wüst $W^{2}$, May $M^{2}$, Heiß $R^{2}$, Uder $M^{2}$ Institut 1 Radiologisches Institut Erlangen, Erlangen; 2 Radiologisches Institut Erlangen DOI 10.1055/s-0037-1600262

Zielsetzung Ziel dieser Studie war es die Interscanner-Reproduzierbarkeit von T1 Relaxationszeiten unter Verwendung einer kommerziellen Software zu evaluieren.

Material und Methodik Insgesamt wurden 26 gesunde Probanden (14 Frauen und 12 Männer, Durchschnittsalter: $26 \pm 3$ Jahre) ohne kardiale Vorerkrankungen oder kardiale Risikofaktoren an drei baugleichen 1.5 T MRT-Geräten untersucht. Alle MRT-Geräte waren mit einer identischen, kommerziell erhältlichen Software für T1-Mapping mit automatisierter Inline-Bewegungskorrektur ausgestattet. Für den Vergleich zwischen den verschiedenen MRT-Geräten wurden pro Patient jeweils drei Langachsen- und drei Kurzachsenschnitte an korrespondierenden Schichtpositionen angefertigt. Die Bildauswertung der einzelnen Herzsegmente gemäß des AHA Modell erfolgte durch Einzeichnen einer midventrikulären Region of Interest. Für die statistische Auswertung wurde eine Varianzanalyse durchgeführt.

Ergebnisse Insgesamt wurden 102 Segmente pro Proband ausgewertet. Davon entfielen pro Untersuchung 16 Segmente auf die Kurzachsen- und 18 Segmente auf die Langachsenschnitte. Die durchschnittliche T1-Relaxationszeit in allen ausgewerteten Segmenten für alle Probanden lag bei $995 \pm 38$ ms. In der jeweiligen Segmentanalyse zwischen den MRT Geräten für die Kurzachsen- und Langachsenschnitte ergab sich kein signifikanter Unterschied zwischen den Segmenten außer für Segment $13 \mathrm{im}$ Kurzachsenschnitt $(p=0.02)$ und Segment $10 \mathrm{im}$ Langachsenschnitt $(p=0.001)$.

Schlussfolgerungen Unter Verwendung baugleicher MRT-Geräte können mittels dezidierter Mappingsoftware verlässlich reproduzierbare T1 Relaxationszeiten gemessen werden.

\section{RK/WISS 103.4 Myokardialer Strain mittels MR- basiertem Feature Tracking: Machbarkeit und Assoziation zu Risiko-Faktoren in einer Bevölkerungsstichprobe ohne kardiovaskuläre Erkrankungen}

Autoren Zitzelsberger $\mathrm{T}^{1}$, Hetterich $\mathrm{H}^{2}$, Lorbeer $\mathrm{R}^{3}$, Scholz $\mathrm{A}^{1}$, Auweter $\mathrm{S}^{3}$, Bamberg $F^{1}$, Peters $A^{4}$, Reiser $M^{3}$, Schlett $C^{5}$

Institut 1 Universitätsklinik Tübingen, Diagnostische und interventionelle Radiologie, Tübingen; 2 LMU München, Insitut für Radiologie, München; 3 LMU München, Institut für Radiologie, München; 4 Helmholtz Zentrum 
München, Institute of Epidemiology II, Neuherberg; 5 Universitätsklinikum Heidelberg, Diagnostische und Interventionelle Radiologie, Heidelberg DOI 10.1055/s-0037-1600263

Zielsetzung Die Strainanalyse ist eine vielversprechende Methode für die Detektion von subtilen Veränderungen der LV-Funktion. Daher ermittelten wir die Machbarkeit von Strain-Analyse mittels MR Feature Tracking und untersuchten die Assoziation von Strain zu möglichen Risikofaktoren in einer Bevölkerungsstichprobe ohne kardiovaskuläre Erkrankungen.

Material und Methodik Personen ohne Apoplex, KHK oder PAVK wurden in diese Teilstudie der KORA-Kohorte eingeschlossen und erhielten ein 3T Ganzkörper-MRT, welches u.a. CINE SSFP (TR/TE 29.97/1.46 ms, ST 8 mm, 25 Phasen) sowie LGE Sequenzen beinhaltete. Bei allen Personen ohne Fibroseoder Narbenareale wurde mittels eines semiautomatischen Segmentierungsalgorhythmus (CVI 42, Circle Canada) der globale maximale systolische Strain ermittelt. Lineare Regressionsmodelle wurden genutzt zur Untersuchung der Assoziationen.

Ergebnisse Von insgesamt 379 Probanden konnten 360 analysiert werden; häufigster Ausschlussgrund waren Triggerartefakte. Intra- und Interreader Reproduzierbarkeit war hoch (ICC: $00.79-0.93$ bzw. 0.59-0.73). In der verbliebenen Kohorte $(56.2 \pm 9.2$ Jahre, $57 \%$ ふै) lag der maximale systolische Strain bei radial $40.1 \pm 8.2 \%$, circumferentiell $19.9 \pm 2.7 \%$ und longitudinal $19.8 \pm 3.2 \%$. Männer zeigten in allen Strainorientierungen erniedrigte Werte (alle $\mathrm{p}<0.001$ ). Die Assoziation von Strain zu Alter variierte zwischen den Strainorientierungen; in voll adjustierten Modellen war ein höheres Alter mit höherem radialen und circumferentiellen Strain assoziiert $(p=0.01$ und $p=0.04)$, jedoch nicht für longitudinal $(p=0.5)$. Ferner zeigten eine erhöhte WHR und erhöhte HbA1c Werte in volladjustierten Modellen eine unabhängige Korrelation mit erniedrigtem radialem und circumferentiellem Strain ( $p=0.003$ bzw. $p \leq 0.01$ ).

Schlussfolgerungen MR Feature Tracking ist in populationsbasierten Studien möglich. In einer Kohorte ohne kardiovaskuläre Vorerkrankungen, variiert der globale maximale systolische Strain mit Geschlecht und Alter und ist unabhängig mit Risikofaktoren aus dem metabolischen Formenkreis assoziiert.

Interventionelle Radiologie

WISS 301.3 Frühes Therapieansprechen mittels DWMRT vs. PET/CT zur Prädiktion des Gesamtüberlebens von Patienten nach Radioembolisation von Lebermetastasen

Autoren Barabasch $A^{1}$, Ciritsis $A^{1}$, Heinzel $A^{2}$, Krämer $N^{1}$, Kuhl $C^{1}$

Institut 1 Universitätsklinikum der RWTH Aachen, Diagnostische und Interventionelle Radiologie, Aachen; 2 Universitätsklinikum der RWTH Aachen, Nuklearmedizin, Aachen

DOI 10.1055/s-0037-1600264

Zielsetzung Sowohl die Diffusions-gewichtete MRT (DW-MRT) als auch die PET/CT sind zum Abschätzen des Therapieansprechens bereits sehr früh (4 6 Wochen (Wo)) nach Radioembolisation (RE) geeignet. Wir untersuchten, ob der Nachweis eines frühen Ansprechens mit dem Gesamtüberleben korreliert, und ob diese Informationen über die schon über übliche onkologische Parameter (ECOG Performance Status, extrahepatische Metastasierung und Lebertumorlast) abzuleitende Information hinausgehen.

Material und Methodik Prospektive, intraindividuelle Vergleichsstudie von 36 Patienten (mittleres Alter $60 \pm 10$ J; 25 Frauen), die 6 Wo vor und 4-6 Wo nach RE eine 18FDG-PET/CT und eine DW-MRT zur Behandlung von Lebermetastasen (20 Kolorektal-, 14 Mammakarzinome, 2 andere) erhielten. Ein Anstieg des ADC bzw. ein Abfall des SUVmax von mehr als 30\% wurde als Therapieansprechen definiert (ADC- bzw. SUV-responder). Mittels Kaplan-Meier-Kurven und log-rank-test wurde das Gesamtüberleben verglichen. Der Einfluss etablierter Überlebensprädiktoren, wie ECOG-status (0 vs. 1), hepatische Tumorlast $(<25 \%$ vs. $>25 \%$ ) und Vorhandensein bzw. Fehlen von extra- hepatischen Metastasen wurde mittels multivariater Cox-Regressionsanalyse ermittelt.

Ergebnisse Das mediane Gesamtüberleben aller Patienten lag bei 37 Wo. Auf Basis der PET/CT wurden 18/36 Patienten (50\%) als PET-responder, auf Basis der MRT 24/36 Patienten (67\%) als ADC-responder eingestuft. Die PET-Responder zeigten kein signifikant längeres Überleben als die PET-non-responder (45 Wo vs. 34 Wo; $p=0,64)$. Die ADC-Responder zeigten dagegen ein signifikant längeres Überleben als die ADC-non-responder (53 Wo vs. 24 Wo; $p<0,04$ ). In der multivariaten Analyse korrelierte das Therapieansprechen auf Basis der DWMRT als einziger unabhängiger Faktor mit dem Überleben $(p<0,03)$.

Schlussfolgerungen Der Nachweis eines frühen Ansprechen 4-6 Wo nach RE mittels DWI-MRT liefert prognostisch relevante Informationen, die genauer sind und über das hinausgehen, was mittels PET/CT oder etablierter onkologischer Parameter ermittelt werden kann.

WISS 202.3 LASER-Atherektomie bei komplexen hochgradig stenosierten oder verschlossenen Beckenund Beinarterien: Ergebnisse aus der prospektiven europäischen Multicenter-Excimer-Laser-

\section{Registerstudie}

Autoren Bürckenmeyer $\mathrm{F}^{1}$, Aschenbach $\mathrm{R}^{1}$, Diamantis $\mathrm{I}^{1}$, Teichgräber $\mathrm{U}^{1}$ Institut 1 IDIR Uni Jena, Allgemeine und interventionelle Radiologie, Jena DOI 10.1055/s-0037-1600265

Zielsetzung Evaluierung der Sicherheit und primären Erfolgsrate beim Einsatz des Excimer-LASER-Atherektomiesystems bei komplexen hochgradig stenosierten oder verschlossenen Becken- und Beinarterien bei PAVK-Patienten.

Material und Methodik Zwischen 10/2013 und 05/2016 wurden von 14 teilnehmenden Zentren 293 Patienten (Alter: 70,5 \pm 10,4 Jahre; $204 \mathrm{~m}$ : $89 \mathrm{w}$ ) in die prospektive europäische Multicenter-Excimer-Laser-Registerstudie eingeschlossen. Patientencharakteristik: Rutherford-Stadium 3,68 $\pm 0,92$, mittlere Läsionslänge $11,4 \pm 10,6 \mathrm{~cm}$, Stenosegrad $94,5 \% \pm 9,6 \%$, Occlusionsrate 56,7\%, Kalzifikation: $43 \%$ schwer, 45,4\% moderat, 11,6\% gering. Bei 89 Patienten wurde ein Follow-Up (nach 6 und 12 Monaten) mit Reinterventions(en. Target lesion revascularistation, TLR) und Komplikationsraten erfasst. Ergebnisse Bei 96,9\% der Patienten ( $n=284$ ) war die Revaskularisation der Zielläsion erfolgreich. Die Reststenoserate lag bei 8,5 $\pm 14,5 \%$. Bei 97,2\% der Patienten ( $n=285)$ erfolge eine additive PTA (58,4\% mit Paclitaxel-beschichteten DCB, 41,6\% POBA) und bei 34,1\% ( $n=100)$ eine zusätzliche Stentimplantation. Es traten keine schweren periprozeduralen Laser-assoziierten unerwünschten Ereignisse auf.

Schlussfolgerungen LASER-Atherektomie ist eine sichere und zuverlässige Methode zur Rekanalisation von komplexen peripheren arteriellen Verschlüssen oder hochgradigen Stenosen in Kombination mit PTA und Stentimplantation und geringer Reinterventionsrate der Zielläsion nach 1 Jahr und stellt eine Alternative zur Bypass-Chirurgie dar.

\section{WISS 211.7 Transjugulärer intrahepatischer portosystemischer Shunt (TIPS): Viatorr- versus Bare- Stent. Eine prospektive, nicht-interventionelle Studie unter „real life“" Bedingungen Bewerber für den Young Investigator Award}

Autoren Byrtus J'

Institut 1 Uniklinik Köln, Köln

DOI 10.1055/s-0037-1600266

Zielsetzung Der prospektive Vergleich von beschichteten Stents (Viatorr (V)) versus unbeschichteten "bare-stents" (BS) unter "real-life" Bedingungen.

Material und Methodik Insgesamt wurden 412 Patienten aus zwei verschiedenen Zentren in dieser Registerstudie ausgewertet. Die Entscheidung zugunsten des einen oder des anderen Stents wurde anhand verschiedener klinischer Kriterien durch den Interventionalisten getroffen (z.B. Indikation, 
Alter, Schweregrad der Erkrankung, Risiko einer hepatischen Enzephalopathie). Postinterventionelle Antikoagulation erfolgte nach individuellem Bedürfnis. Beobachtungszeit war bis zu 80 Monaten. Ein Shuntversagen wurde mittels Ultraschall und klinischer Untersuchung festgestellt und durch TIPS-Revision bestätigt.

Ergebnisse 227 Patienten wurden mit einem Viatorr Stent versorgt, 185 mit einem "bare-stent". Die Gruppen unterschieden sich signifikant in Bezug auf Alter (V: mean 55 Jahre (J.) $(20-81)$ vs. BS: mean 63J. $(20-86) ; p<0,001)$, Child-Pugh-Score (V: $8(5-13)$ vs. BS: $9(5-12)$; $p=0,036)$ und Indikation (V: hauptsächlich Varizenblutung; BS: hauptsächlich Aszites). Keine signifikanten Unterschiede fanden sich hinsichtlich Geschlecht (V: weiblich 33\% vs. BS: 35\%; $p=0,677)$ und INR (V: 1,2 $(0,2-16,1)$ vs. bare: $1,2(0,9-3,2) ; p=0,533)$. Unter der angewandten Patientenselektion und der individuellen Antikoagulation waren die Shuntinsuffizienzraten der beiden Stents vergleichbar. Die multivariate Analyse zeigte ein ähnliches Gesamtüberleben der beiden Gruppen (HR: 0,69, 95\%Cl: 0,44-1,06, $\mathrm{p}=0,09$ ).

Schlussfolgerungen Unter den Vorgaben dieser "real-life" Studie (Patientenselektion \& Antikoagulation) sind Verschluss- und Überlebensraten von beschichteten und unbeschichteten Stent-Typen vergleichbar.

\section{WISS 101.1 Transjugulärer intrahepatischer portosystemischer Shunt (TIPS): Viatorr- versus Bare- Stent. Eine prospektive, nicht-interventionelle Studie unter „real life“ Bedingungen}

Autoren Byrtus J', Trebicka J ${ }^{2}$, Rössle $\mathrm{M}^{3}$, Bettinger $\mathrm{D}^{3}$, Dörner J $J^{4}$, Maintz $\mathrm{D}^{4}$ Institut 1 Uniklinik Köln, Köln; 2 Uniklinik Bonn, Gastroenterologie, Bonn; 3 Uniklinik Freiburg, Gastroenterologie, Freiburg; 4 Uniklinik Köln, Radiologie, Köln

DOI 10.1055/s-0037-1600267

Zielsetzung Der prospektive Vergleich von beschichteten Stents (Viatorr (V)) versus unbeschichteten "bare-stents" (BS) unter "real-life" Bedingungen.

Material und Methodik Insgesamt wurden 412 Patienten aus zwei verschiedenen Zentren in dieser Registerstudie ausgewertet. Die Entscheidung zugunsten des einen oder des anderen Stents wurde anhand verschiedener klinischer Kriterien durch den Interventionalisten getroffen (z. B. Indikation, Alter, Schweregrad der Erkrankung, Risiko einer hepatischen Enzephalopathie). Postinterventionelle Antikoagulation erfolgte nach individuellem Bedürfnis. Beobachtungszeit war bis zu 80 Monaten. Ein Shuntversagen wurde mittels Ultraschall und klinischer Untersuchung festgestellt und durch TIPS-Revision bestätigt.

Ergebnisse 227 Patienten wurden mit einem Viatorr Stent versorgt, 185 mit einem "bare-stent". Die Gruppen unterschieden sich signifikant in Bezug auf Alter (V: mean 55 Jahre (J.) $(20-81)$ vs. BS: mean 63 J. $(20-86)$; $p<0,001)$, Child-Pugh-Score (V: $8(5-13)$ vs. BS: $9(5-12) ; p=0,036)$ und Indikation (V: hauptsächlich Varizenblutung; BS: hauptsächlich Aszites). Keine signifikanten Unterschiede fanden sich hinsichtlich Geschlecht (V: weiblich $33 \%$ vs. BS: $35 \%$; $\mathrm{p}=0,677)$ und INR (V: $1,2(0,2-16,1)$ vs. bare: $1,2(0,9-3,2) ; \mathrm{p}=0,533)$. Unter der angewandten Patientenselektion und der individuellen Antikoagulation waren die Shuntinsuffizienzraten der beiden Stents vergleichbar. Die multivariate Analyse zeigte ein ähnliches Gesamtüberleben der beiden Gruppen (HR: 0,69, 95\%Cl: 0,44-1,06, p=0,09).

Schlussfolgerungen Unter den Vorgaben dieser "real-life" Studie (Patientenselektion \& Antikoagulation) sind Verschluss- und Überlebensraten von beschichteten und unbeschichteten Stent-Typen vergleichbar.

\section{WISS 301.4 Detektion des hepatozellulären Karzinoms bei Leberzirrhose mittels digitaler Volumentomografie im Rahmen der interventionellen intraarteriellen Tumortherapie}

\author{
Autoren Diamantis ${ }^{1}$, Aschenbach $\mathrm{R}^{1}$, Lehmkuhl L ${ }^{1}$, Teichgräber $\mathrm{U}^{1}$ \\ Institut 1 Universitätsklinikum Jena, Institut für Diagnostische und \\ Intervetionelle Radiologie, Jena \\ DOI 10.1055/s-0037-1600268
}

Zielsetzung Evaluation der Detektionsrate hepatozellulärer Karzinome (HCC) bei Leberzirrhose mittels einer arteriell-monophasischen digitalen Volumentomografie (CBCT) in der Angiografie im Vergleich zur kontrastmittelgestützten mehrphasigen Computertomografie (CE-CT) bzw. zur kontrastmittelgestützten mehrphasigen Magnetresonanztomografie (CE-MRT).

Material und Methodik Die retrospektive monozentrische Studie schließt 28 an einem HCC erkrankte Patienten ein, welche im Rahmen einer interventionellen intraarteriellen Therapie ein $\mathrm{CBCT}$ mit dem Ziel einer optimierten Interventionsplanung erhalten haben. Die Akquisition des CBCT erfolgte dabei nach maschineller Kontrastmittelapplikation aus der A. hepatica propria. Es erfolgte die Bewertung der Sichtbarkeit jeder Läsion im Vergleich zur vorangegangenen CE-CT bzW. CE-MRT Voruntersuchung anhand einer fünfstufigen Skala (1: Sehr gut beurteilbar, 2: Gut beurteilbar, 3: Eingeschränkt beurteilbar, 4: Nicht sicher beurteilbar 5: Nicht beurteilbar). Das Zeitintervall zwischen der diagnostischen Bildgebung und der periinterventionell durchgeführten CBCT betrug dabei durchschnittlich $18+$ - 7 Tage.

Ergebnisse Insgesamt lagen 55 Läsionen vor, von denen 22 mittels CE-MRT und 33 mittels CE-CT als HCC-typisch eingestuft wurden. Insgesamt 48 der 55 Läsionen wurden im CBCT detektiert. Von den 33 in der CE-MRT detektierten Läsionen wurden im CBCT 26 Läsionen diagnostiziert ( $p=0.58)$, wobei eine relevante Anzahl falsch negativer Befunde im CBCT resultierte. In der CBCT konnten alle in der CE-CT detektierten Läsionen diagnostiziert werden. Die Beurteilungssicherheit unterschied sich signifikant zwischen CBCT und CE-CT/ CE-MRT ( $p=0.04$, mittlerer Rang 1.49 bzw. 1.51).

Schlussfolgerungen Die Detektionsrate für HCC typische Herdbefunde in der CBCT entspricht der in der CE-CT, es ist jedoch bei alleiniger Anwendung der $\mathrm{CBCT}$ mit falsch negativen Befunden im Vergleich zur CE-MRT zu rechnen.

\section{WISS 310.6 DSM Triple-TACE zur Behandlung des irresektablen, intrahepatischen cholangiozellulären Karzinoms}

Autoren Goerg $\mathrm{F}^{1}$, Bruners $\mathrm{P}^{2}$, Kuhl $\mathrm{C}^{2}$

Institut 1 Uniklinik Aachen, Radiologie, Aachen; 2 Uniklinik Aachen, Klinik für diagnostische und interventionelle Radiologie, Aachen

DOI 10.1055/s-0037-1600269

Zielsetzung Evaluation der Wirksamkeit der transarteriellen Multi-Chemoembolisation unter Verwendung von degradierbaren Stärkepartikeln (DSM-TACE) bei Patienten mit irresektablem intrahepatischem CCC.

Material und Methodik Single-Center Studie bei 19 Patienten (mittleres Alter: 63 Jahre) mit irresektablem CCC, welche unter systemischer Chemotherapie progredient waren. 10 Patienten waren voroperiert, hiervon 7 mittels Hemihepatektomie oder Trisektorektomie, 3 mittels Segmentektomie. Insgesamt wurden 57 Sitzungen einer Multichemo-DSM-TACE in jeweils 4-wöchigem Intervall (im Mittel 3 Sitzungen pro Patient) durchgeführt. Per Mikrokatheter wurde zunächst Cisplatin perfusorgesteuert mit $1 \mathrm{ml} / \mathrm{min}$ in die A. hepatica dextra und/oder sinistra infundiert. Danach wurden degradierbare $50 \mu \mathrm{m}$ Stärkepartikel (EmboCept S, PharmaCept GmbH, Berlin) mit Mitomycin $C$ und Doxorubicin gemischt und manuell unter intermittierender Durchleuchtung zur Flusskontrolle appliziert. Patienten mit biliodigestiver Anastomose erhielten eine aggressive Antibiotikaprophylaxe. Wir untersuchten, wie gut mittels TACE eine lokale Kontrolle (definiert als Complete Response [CR], Partial Response [PR, oder Stable Disease [SD]) erreicht werden konnte. 
Ergebnisse Zwei Patienten sind lost-to-follow-up; ein Patient mit bilobärem CCC wurde transplantiert. Von den zur Analyse verfügbaren 16 Patienten zeigte keiner einen intrahepatischen Progress, mithin erfüllten alle 16 die Kriterien einer erfolgreichen lokalen Kontrolle (100\%). Bei 2 (12.5\%) wurde eine komplette Remission (CR) erreicht; einer dieser Patienten ist seit $>52$ Monaten rezidiv-frei. Bei 9 Patienten (56.3\%) wurde eine partielle Remission (PR) erreicht, 4 davon mit nahezu vollständiger Reduktion der intrahepatischen Tumorlast. Bei den übrigen 5 Patienten (31.3\%) wurde eine stable disease (SD) erreicht.

Schlussfolgerungen Triple-TACE mit degradierbaren Stärkepartikeln ist eine einfach durchzuführende und hoch wirksame Lokaltherapie für Patienten mit therapierefraktärem inoperablem intrahepatischem CCC.

\section{WISS 101.6 Klinischer Stellenwert einer postinterventionellen Kontrastdarstellung von CT- gesteuerten Drainageanlagen}

\author{
Autoren Goessmann $\mathrm{H}^{1}$, Uller $\mathrm{W}^{1}$, Bayer $\mathrm{L}^{1}$, Teusch $\mathrm{V}^{1}$, Poschenrieder $\mathrm{F}^{1}$, \\ Dendl $L^{1}$, Stroszczynski $C^{1}$, Schreyer $A^{1}$ \\ Institut 1 Universitaetsklinikum Regensburg, Institut fuer \\ Roentgendiagnostik, Regensburg \\ DOI 10.1055/s-0037-1600270
}

Zielsetzung Ziel dieser Arbeit war die Evaluation der zusätzlichen postinterventionellen Kontrolle von computertomografisch eingebrachten Verhaltdrainagen mit verdünntem Kontrastmittel bezüglich relevanter Zusatzinformationen.

Material und Methodik Alle CT-assistierten Drainagen im Zeitraum von 1.1.14 bis 30.9.16 in einem Haus der Maximalversorgung wurden in dieser retrospektiven Studie über das RIS identifiziert. Es erfolgte eine Subgruppierung in unmittelbar voroperierte und nicht voroperierte Patienten. Alle Drainagen, bei denen eine Kontrastmittelgabe über die neu eingebrachte Drainage erfolgte wurden evaluiert, in wieweit durch die Kontrastierung zusätzliche relevante Aussagen zur Genese des Verhalts (z.B. Anastomoseninsuffizienz) oder zusätzliche Kofaktoren (z. B. Fistelstraßen in Hohlorgane bei Pankreatitis) diagnostiziert werden konnten und ob sich daraus eine unmittelbare Therapieänderung ergab.

Ergebnisse Von 499 Drainagenanlagen bei 352 Patienten (123 weiblich, Durchschnittsalter 60 Jahre, Range 11-87 Jahre) wurden 197 im Rahmen der Drainageanlage mit Kontrastmittel dargestellt. Hierbei ergab sich in 51 Fällen (26\%) eine relevante Zusatzinformation zur Genese des Verhaltes oder zu Kofaktoren. Eine unmittelbare Therapieänderung ergab sich in 19 Fällen (9\%). Bei dem Patientenkollektiv, das unmittelbar vor Drainagenanlage operiert wurde (303 Drainagen mit 120 Kontrastierungen) ergaben sich in 32 Fällen (27\%) Zusatzinformationen und in 13 Fällen (11\%) unmittelbare Therapieänderungen. Dieses Patientenkollektiv unterschied sich hierbei nicht signifikant von der nicht voroperierten Gruppe (196 Drainagen mit 77 Kontrastierungen) mit 19 (25\%) Zusatzinformationen und 5 (6\%) unmittelbare Therapieänderungen.

Schlussfolgerungen Die kontrastmittelgestützte Darstellung von computertomografisch eingebrachten Verhaltdrainagen konnte in dieser Studie in nahezu 26\% der Fälle wertvolle Zusatzinformationen über die Genese der Verhaltformation liefern und führte in über $9 \%$ zu einer unmittelbaren Änderung des Therapieregiems.

\section{WISS 309.4 Endovaskuläre Therapie aorto-iliaker Aneurysmata: initiale Single Center Erfahrung mit einer neuartigen iliakalen Bifurkationsprothese}

Autoren Goltz J ${ }^{1}$, Anton $\mathrm{S}^{1}$, Wiedner $\mathrm{M}^{2}$, Kleemann $\mathrm{M}^{2}$, Barkhausen J', Stahlberg $E^{1}$

Institut 1 Universitätsklinikum Schleswig-Holstein, Campus Lübeck, Klinik für Radiologie und Nuklearmedizin, Lübeck; 2 Universitätsklinikum Schleswig-

Holstein, Campus Lübeck, Klinik für Chirurgie, Lübeck

DOI 10.1055/s-0037-1600271

Zielsetzung Evaluation des technischen Erfolgs, der Sicherheit, Komplikationen und Reinterventionsrate nach Implantation einer neuartigen iliakalen Bifurkationsprothese (IBP) zur Behandlung aorto-iliakaler Aneurysmata.

Material und Methodik Zwischen 5/2014 und 5/2016 wurden 16 Patienten (74+/-6 Jahre) mit isoliertem Aneurysma der A. iliaca communis (AIC, $n=1$ ) und aorto-iliakalen Aneurysmata $(n=15)$ durch Implantation einer IBP $\left(E-l l i a c^{\circledR}\right.$, Jotec, Deutschland) behandelt. Primäre Endpunkte waren technischer Erfolg (kein Typ I oder III Endoleak der iliakalen Aneurysmata mit erhaltener Perfusion der ipsilateralen A. iliaca int.), 30-Tage-Überlebensrate und periprozedurale Komplikationen. Sekundäre Endpunkte umfassten kurzfristige Offenheitsraten der IBP und ischämische pelvine Komplikationen.

Ergebnisse Die Durchmesser der aortalen und iliakalen Aneurysmata maßen 56+/-7 bzw. 33+/-8 mm. Der technische Erfolg der Prozedur sowie die 30Tage-Überlebensrate betrugen 100\%. Die kontralaterale All wurde bei 11/16 Patienten $(68,8 \%)$ embolisiert, das Ostium anschließend überstentet. Periprozedurale Komplikationen wurden bei 2/16 Patienten beobachtet $(12,5 \%)$ : Aneurysma spurium der A. brachialis $(n=2)$, Makrohämturie $(n=1)$. Der Seitarm der IBP war nach einem mittleren Nachbeobachtungszeitraum von 165 Tagen bei 15/16 Patienten (93,8\%) offen: ein Seitarm mit Typ Ib Endoleak musste embolisiert worden. Ein Prothesenbeinverschluss (Tag 19) wurde operativ thrombektomiert. Endoleaks (Typ II) ohne Sackexpansion wurden bei Aneurysmata der Aorta $(n=8)$, AIC $(n=2)$ und All $(n=2)$ beobachtet. Schlussfolgerungen Die Behandlung iliakaler Aneurysmata mit der neuartigen Bifurkationsprothese ist sicher und ermöglicht die Aneurysmaausschaltung unter Erhalt der Beckenversorgung mit hoher technischer Erfolgsrate und hoher Offenheitsrate des iliakalen Seitarms. Langzeitergebnisse werden benötigt, um die Beständigkeit dieser frühen Ergebnisse zu bestätigen.

\section{WISS 310.5 Transarterielle Chemoembolisation} (TACE) mit degradierbaren Stärkemikrosphären (DSM) und Anthrazyklinen in Patienten mit lokal fortgeschrittenem HCC: Toxizität und Wirksamkeit

Autoren Gross $A^{1}$, Albrecht $T^{2}$

Institut 1 Vivantes Klinikum Neukölln, Radiologie und interventionelle

Therapie, Berlin; 2 Vivantes Klinikum Neukölln, Radiologie und

Interventionelle Therapie, Berlin

DOI 10.1055/s-0037-1600272

Zielsetzung TACE von HCCs mit degradierbaren Stärkemikrosphären (DSM) wird vorwiegend in multifokalen und lokal fortgeschrittenen Stadien angewendet, in denen eine superselektive TACE nicht erwünscht ist, sondern eine weniger selektive Behandlung angestrebt wird. Potentieller Vorteil der DSMTACE ist ihre bessere Verträglichkeit aufgrund kürzerer Ischämiezeiten, Daten zu Toxizität und Wirksamkeit liegen jedoch kaum vor. Wir untersuchten 29 mit DSM-TACE behandelte HCC-Patienten retrospektiv hinsichtlich Anwendungssicherheit und Therapieansprechen.

Material und Methodik 29 HCC-Patienten in BCLC-Stadien A-D (3xA, 17xB, $8 \times C, 1 \times D)$ wurden mit $\geq 3$ Embolisationen mit Doxo-/Epirubicin und DSM (Embocept S, Pharmacept) behandelt. Sechs HCCs waren unilokulär, 23 multilokulär (18 bilobär). Die mittlere Läsionsgröße betrug $7 \mathrm{~cm}(0,8-20 \mathrm{~cm})$. Zwei Patienten wiesen einen Pfortadereinbruch auf. Sechs Patienten wurden selektiv, 10 lobär und 13 bilobär chemoembolisiert. Das Therapieansprechen wurde alle 3-4 Monate mittels CE-CT oder MRT beurteilt. 
Ergebnisse Insgesamt wurden 139 DSM-TACE durchgeführt (3-12/Patient, durchschnittlich 4,8). Es traten keine interventionsbedingten schweren Komplikationen oder Todesfälle auf. Unerwünschte Ereignisse waren: Analgetikasensible Schmerzen (23\%), vorübergehende Übelkeit (14\%) oder Erbrechen (4\%) sowie Post-Embolisationssyndrom (4\%). Vorübergehende und meist geringe Laborwertveränderungen waren: Knochenmarkstoxizität (8\%) sowie Anstieg von INR (10\%), Kreatinin (7\%) und Bilirubin (39\%). Das Therapieansprechen war: komplette Remission in 2, partielle Remission in 17, stabile Erkrankung in 4 und Krankheitsprogression in 2 Fällen. Die mediane Überlebenszeit betrug insgesamt 10,8 Monate: 19,1 Monate für BCLC-Stadien A+B und 6,7 Monate für BCLC-Stadien C+D.

Schlussfolgerungen DSM-TACE des HCC ist ein sicheres Therapieverfahren selbst in fortgeschrittenen Krankheitsstadien. Therapieansprechen und Überlebensrate in der vorliegenden Patientenkohorte mit lokal fortgeschrittener Erkrankung waren vielversprechend.

\section{WISS 101.3 Retrospektiver Vergleich der CT- gesteuerten Knochenbiopsie mit einem Handbohrer und einem elektrischen Bohrer}

Autoren Harth $\mathrm{M}^{1}$, Burck $\mathrm{I}^{2}$, Eichler $\mathrm{K}^{3}$, Leberer $\mathrm{L}^{3}$, Gruber $\mathrm{T}^{3}$, Mohamed $\mathrm{N}^{3}$, Vogl $T^{3}$

Institut 1 , Frankfurt a.M.; 2 Institut für interventionelle und diagnostische Radiologie, Radiologie Universität Frankfurt a. M., Frankfurt a.M.; 3 Institut für interventionelle und diagnostische Radiologie, Radiologie Universität Frankfurt a. M., Frankfurt

DOI 10.1055/s-0037-1600273

Zielsetzung Beurteilung eines Batterie betriebenen Bohrer (Vidacare OnControl Bone Marrow system) beim Durchführen einer CT-gesteuerten Knochenbiopsie in verschiedenen anatomischen Regionen, als Alternative zum konventionellen Handbohrer (Safe-cut biopsie system).

Material und Methodik Von 12/2006 bis 2/2016 wurden bei 83 Patienten eine CT-gesteuerte Knochenpunktion mit einem Batterie-betriebenen Bohrers durchgeführt. Diese wurden mit 171 Punktionen mit einem konventionellen Handbohrer verglichen. Erfasst und verglichen wurden die benötigte Zeit, die Dicke des korikalen, sowie des ggf. sklerotischen Knochens, die Anzahl der Scans, die Strahlenbelastung (gemessen in CTD/vol*mGy) und das Ergebnis der Biopsie.

Ergebnisse Das Bohren des Zugangs im Knochen wurde bei dem Batteriebetriebenen Bohrer ohne Probleme bei 81 von 83 Patienten und bei dem Handbohrer ohne jegliche Probleme durchgeführt. Die durchschnittliche Eingriffszeit betrug 10 Minuten für den Batterie-betriebenen Bohrer und 8 Minuten für den Handbohrer. Der Vergleich der Scananzahl war nicht signifikant $(p=0,34)$. Die Strahlendosis betrug 136,2 CTD/vol*mGy bei dem Handbohrer und 107,3 CTD/vol*mGy bei dem Batterie-betriebenen Bohrer, dieser Unterschied war signifikant $(p=0,0098)$. Die mittlere Dicke des kortikalen Knochens betrug 3,61 mm für den Batterie-betriebenen Bohrer und 1,62 mm für den Handbohrer, dieser Unterschied war auch signifikant $(p<0,0001)$. Dieses Ergebnis korreliert mit dem mittleren Alter der Patienten, welches bei 55,4 Jahren bei der Verwendung des Batterie-betriebenen Bohrer und bei 63,2 Jahren bei Verwendung des Handbohrers lag.

Schlussfolgerungen Eine Verwendung des Batterie-betriebenen Bohrer reduziert die Strahlendosis und erweitert das Anwendungsgebiet, weil der Radiologe bei Verwendung des Batterie-betriebenen Bohrers in der Lage war jüngere Patienten mit dickerer Knochen-Kortikalis zu punktieren.

\section{WISS 309.3 Sicherheit und Effektivität perkutaner Nahtsysteme bei der Behandlung von aortoiliakalen Pathologien}

Autoren Henning $\mathrm{P}^{1}$, Wagenhofer $\mathrm{K}^{2}$, Siemens $\mathrm{P}^{2}$, Daum $\mathrm{H}^{3}$, Burmeister $\mathrm{H}^{2}$, Gross-Fengels $\mathrm{W}^{2}$

Institut 1 Asklepios Klinikum Harburg, Diagnostische und Interventionelle Radiologie, Hamburg; 2 Asklepios Klinikum Harburg, Diagnostische und interventionelle Radiologie, Hamburg; 3 Asklepios Klinikum Harburg, Gefäßchirurgie und endovaskuläre Chirurgie, Hamburg

DOI 10.1055/s-0037-1600274

Zielsetzung Untersuchung des technischen Erfolgs und der Sicherheit eines nahtbasierten perkutanen vaskulären Verschlusssystems (Perclose ProGlide, PG) bei der interventionellen Behandlung von aortoiliakalen Pathologien. Schwerpunkte bildeten die Identifizierung von Risikofaktoren und Ergebnisse aus Langzeitbeobachtungen.

Material und Methodik In einer retrospektiven Studie wurden 79 Patienten bzw. 118 Leisten analysiert die mit insgesamt 313 PG in sog. „preclose“ Technik behandelt wurden, überwiegend im Rahmen einer „endovascular aortic repair“ (83,5\%) mit Zugangsgrößen zwischen 10 - 20F. Hierzu erfolgte u.a. eine systematische Erfassung dokumentierter Risikofaktoren, Interventionsberichte und qualitative Auswertung archivierter Bildmodalitäten.

Ergebnisse Der technische Erfolg für PG lag bei 94\% und die Gesamtkomplikationsrate bei 6,8\% (2,5\% Major-Kompl.). Als Komplikation traten auf: Aneurysma spurium, Dissektion, Gefäßstenose, Thrombosen, relevante Nachblutungen und Leistenschmerzen. Der Nachbeobachtungszeitraum lag bei 11 Mon. (max. 40 Mon.) mit einer hohen apparativen Abdeckung von 97,5\% (CT 93,7\%). Hierbei fand sich eine Frühkomplikation (0,8\%, 1/118). Technische Versager oder behandlungswürdige Komplikationen konnten nicht festgestellt werden. Die Analyse vaskulärer Risikofaktoren wie Ateriosklerose, Gefäßdurchmesser, Zugangsgröße und sekundärer Einflussgrößen wie u.a. Adipositas, Diabetes oder pAVK zeigten keine signifikante $(p>0,05)$ Assoziation bezüglich technischen Versagern oder Komplikationsauftreten. Bei Verwendung von zwei PG vs. drei PG konnte eine statistisch signifikante Häufung von Komplikationen ( $p=0,001)$ festgestellt werden.

Schlussfolgerungen PG bietet kurz- und langfristig ein effizientes und sicheres Verfahren für den interventionellen Verschluss von großlumigen arteriellen Leistenzugängen. Relevante, den PG-Einsatz limitierende Risikofaktoren konnten nicht identifiziert werden. Es fanden sich Hinweise die einen primären Einsatz von drei vs. zwei PG zugunsten des Komplikationsauftretens favorisieren.

\section{WISS 202.6 Dual layer micromesh Karotisstent bei Tandemläsionen im Rahmen der akuten Schlaganfallbehandlung: eine Machbarkeitsstudie}

Autoren Hopf-Jensen $S^{1}$, Preiß $M^{1}$, Vatankhah $D^{2}$, Müller-Hülsbeck $\mathrm{P}^{1}$ Institut 1 Diakonissenkrankenhaus Flensburg, Institut für diagnostische und interventionelle Radiologie/Neuroradiologie, Flensburg;

2 Diakonissenkrankenhaus Flensburg, Neurologische Klinik, Flensburg DOI 10.1055/s-0037-1600275

Zielsetzung Relevanz und Machbarkeit der endovaskulären Rekanalisation und Analyse der stentgeschützten Angioplastie der A. carotis interna mittels dual layer micromesh Roadsaver TM Stent (Terumo) während der mechanischen Thrombektomie.

Material und Methodik Innerhalb 24 Monaten (10/2014-10/2016) wurden 30 Pat. (m:27, w:31, Ø 69,2 Jahre, range 44 - 90) mit Tandemläsion auf dem Boden a) atherosklerotischer Stenosen oder Verschlüsse der $\mathrm{ACl}(\mathrm{n}=24,80 \%)$, b) okkludierenden Dissektionen der $A$. carotis interna $(n=6,20 \%)$ endovaskulär behandelt. Analyse der epidemiologischen Daten (mRS, NIHSS, Risikofaktoren), der Intervention (Technik, Zeit, Stentlage) und des Outcomes. Ergebnisse In allen Fällen wurde eine vollständige Rekanalisation erreicht ( $\mathrm{TICl}$ 3). 27 Pat. (93\%) verbesserten sich im NIHSS bei Aufnahme ( $\varnothing 14, \pm 2,2$, range 
$12-18$ ) bis zur Entlassung ( $\varnothing 4, \pm 1,9$, range $0-7$ ), auf der mRS von 4.8 $( \pm 1.78$, range $3-5$,$) auf 2.6( \pm 1.3$, range $0-4$,$) und nach 90$ Tagen auf 2.1 (range $0-2, \pm 0.5) .2(6,6 \%)$ Patienten verstarben postinterventionell an den folgenden eines malignen Mediainfarktes, 1 (3,3\%) Pat. infolge eines Myokardinfarktes.

Schlussfolgerungen Der dual layer micromesh RoadsaverTM Stent ist im Rahmen der endovaskulärer Behandlung von Tandemläsionen effektiv einsetzbar.

\section{WISS 202.5 Analyse des Dual layer Micromesh RoadsaverTM Stents zur Behandlung hochgradiger Karotisstenosen - eine Single Center Verlaufsstudie}

Autoren Hopf-Jensen $S^{1}$, Leißner $M^{1}$, Marques $L^{1}$, Preiß $M^{1}$, MüllerHülsbeck $\mathrm{P}^{1}$

Institut 1 Diakonissenkrankenhaus Flensburg, Institut für diagnostische und interventionelle Radiologie/Neuroradiologie, Flensburg

DOI 10.1055/s-0037-1600276

Zielsetzung Analyse der Effektivität, technischer Details und des Handlings. Untersuchungen zur 30-Tage Morbidität/Mortalität und im mittelfristigen Verlauf.

Material und Methodik Von Jan. 2015- Okt. 2016 wurden 70 Patienten mit dem RoadsaverTM Stent ohne Protektionssystem behandelt. Analyse der epidemiologischen Daten, des technischen und klinischen Erfolgs, sowie des Einflusses des dual layer micromesh Stentdesigns auf anatomische Besonderheiten der Gefäße (Kinking, Coiling, Elongation, Straight). Das klinische Outcome wurde nach 30 Tagen und im mittelfristigen Verlauf untersucht ( $\varnothing$ 6,2 Monate).

Ergebnisse Bei den 62 sympt. (88,6\%) und den 8 asympt. Patienten (11,4\%) betrug das Durchschnittsalter 72 Jahre (SD \pm 10 ). Periinterventionell traten keine klinischen Komplikationen auf. Bei 61 Pat. (87,1\%) änderte sich nach Stentimplantation die Gefäßanatomie nicht, bei 5 Pat. (7,2\%) kam zu einer geringen Änderung der Anatomie, bei 4 Pat. (5,7\%) trat ein kurzfristiger asympt. Vasospasmus auf. Die 30 Tage Morbidität/Mortalität betrug 2,8\% (1 TIA und 1 Hyperperfusionssyndrom). Im mittelfristige Verlauf wurden bei 2 Pat.(2,8\%) asympt. In-Stentstenosen detektiert (> 70\% nach NASCET)

Schlussfolgerungen Das micromesh dual layer Stentsystem erscheint sicher und effektiv in der Behandlung der ACl Stenosen. Geringe Änderungen der Gefäßanatomie erscheinen klinisch nicht relevant. In-Stentstenosen spielen in den mittelfristigen Verlaufsuntersuchungen keine wesentliche Rolle.

\section{WISS 101.4 Dysphagie bei benigner Ösophagusstenose: Diagnose und Behandlung durch einen retrograden perkutanen radiologischen Gastrostomiezugang}

Autoren Hundt $\mathrm{W}^{1}$, Steinbach $\mathrm{S}^{2}$, Wiegand $\mathrm{S}^{3}$, Mahnken $\mathrm{A}^{1}$ Institut 1 Philipps Universität Marburg, Klinik für diagnostische und interventionelle Radiologie, Marburg; 2 Philipps Universität Marburg, Klinik für Phoniatrie, Marburg; 3 Philipps Universität Marburg, HNO Kinik, Marburg DOI 10.1055/s-0037-1600277

Zielsetzung Prospektive Evaluierung des Therapieerfolges einer Ballondilatation in der Behandlung von benignen Anastomosenstrikturen nach einer chirurgischen und oder Strahlentherapie unter Verwendung eines retrograden perkutanen radiologischen Gastrostomiezugang.

Material und Methodik Von Januar 2012 bis Juni 201620 Therapien einer Ballondilatation bei 12 Patienten mit benigner Striktur nach einer chirurgischen und oder Strahlentherapie unter Verwendung eines retrograden perkutanen radiologischen Gastrostomiezugang wurden durchgeführt. Die technische Durchführbarkeit, klinische Erfolgsrate, Komplikationen und Restenoserate wurden untersucht. Weitere Paramenter waren Alter, Geschlecht, das chirurgischen Verfahren und die Radiochemotherapie. Die technisch interventionellen Parameter waren die Ballongröße, Zeit der Stenosensondierung und Passage der Ösophagus über den gastralen Zugang, Lokalisation und
Länge der Striktur und die Zeit bis zum Auftreten der Stenose sowie die Zeit bis zum Auftreten einer Restenose nach Dilatation.

Ergebnisse Bei allen Patienten war es möglich die Stenose über diesen Zugang zu passieren. Klinisch erfolgreich war die Dilatation bei allen 12 Patienten (100\%), nach einer Dilatation ( $n=6)$ oder zwei bis drei $(n=14)$ Ballondilatationen. Im klinischen Verlauf trat eine Restenose in 6 Patienten wieder auf die erneute Redilatationen notwendig machten. Keine Komplikation.

Schlussfolgerungen Der retrograde perkutane radiologische Gastrostomiezugang ist eine gute Möglichkeit in der Behandlung von benignen Ösophagusstenosen, die nicht durch einen antegraden Zugang passiert werden können. Die klinische Erfolgsrade beider Techniken ist identisch.

\section{WISS 301.5 Bildmorphologische Unterschiede nach irreversibler Elektroporation und Radiofrequenzablation im Follow-up mittels Mehrphasen-CT}

Autoren Isfort $\mathrm{P}^{1}$, Pedersoli $\mathrm{F}^{1}$, Liebl $\mathrm{M}^{1}$, Zimmermann $\mathrm{M}^{1}$, SchulzeHagen $\mathrm{M}^{1}$, Scheck J ${ }^{1}$, Kuhl $\mathrm{C}^{1}$, Bruners $\mathrm{P}^{1}$

Institut 1 Universitätsklinikum RWTH Aachen, Klinik für Diagnostische und Interventionelle Radiologie, Aachen

DOI 10.1055/s-0037-1600278

Zielsetzung Vergleich der bildmorphologischen und volumetrischen Veränderung von Ablationsarealen im Mehrphasen-Spiral-CT Follow-up von Patienten, die bei primären und sekundären Lebertumoren mittels Irreversibeler Elektroporation (IRE) oder Radiofrequenzablation (RFA) behandelt wurden.

Material und Methodik 39 Ablationsareale (19 RFA, 20 IRE) bei 38 Patienten wurden mittels Mehrphasen-Spiral-CT hinsichtlich Veränderungen des Ablationsvolumens und der Kontrastmittelaufnahme im Randbereich des Ablationsareals untersucht. Die Follow-up Zeitpunkte gliederten sich in 4 Zeitintervalle: Tag 0, Tag 1-7, Tag 8-55, Tag 55-250. Der Volumenveränderung der Ablationsareale ab Tag 0 wurde errechnet und hinsichtlich der Faktoren Geschlecht, Alter, Ablationstechnik (RFA/IRE) und Z.n. Verabreichung platinhaltiger Chemotherapeutika untersucht.

Ergebnisse Die Volumenregredienz der Ablationsareale war signifikant ( $p<0,05$ für alle Zeitintervalle) größer nach IRE im Vergleich zu RFA. Nach RFA war die Volumenreduktion der Ablationsareale signifikant geringer bei Patienten mit Z.n. platinhaltiger Chemotherapie in der Vorgeschichte im Vergleich zur Kontrollgruppe, was nicht zutraf bei den mittels IRE behandelten Patienten. Patientenalter und Geschlecht hatten keinen Einfluss auf das Ablationsvolumen. Eine Kontrastmittelmehranreicherung im Randbereich zeigte sich in der arteriellen Phase nach IRE und RFA gleichermaßen - in der venösen Phase signifikant häufiger nach IRE.

Schlussfolgerungen In unserer Analyse zeigte sich eine langsamere und insgesamt geringere Volumenregredienz der Ablationsareale nach RFA im Vergleich zur IRE mit persistent größeren Ablationsarealen nach RFA im längerfristigen Verlauf. Eine stattgehabte platinhaltige Chemotherapie verringert die Größenregredienz nach RFA, jedoch nicht nach IRE, möglicherweise bedingt durch den unterschiedlichen Modus des Zelltodes. In Anbetracht der randständigen Perfusion der Ablationsareale könnte eine additive TACE auch im Rahmen einer IRE sinnstiftend sein.

\section{RK 301.6 Die Rolle der MRT in der postinterventionellen Bildgebung nach Mikrowellenablation der Lunge}

Autoren Kaltenbach $B^{1}$, Roman $A^{2}$, Vogl $T^{1}$, Nour-Eldin $N^{1}$

Institut 1 Goethe Uni Frankfurt, Radiologie, Frankfurt; 2 Uniklinik Frankfurt, Radiologie, Frankfurt

DOI 10.1055/s-0037-1600279

Zielsetzung Evaluation der diagnostischen Aussagekraft einer MRT der Lunge nach Behandlung von Lungenmetastasen mittels Mikrowellenablation. 
Material und Methodik Bei 77 Patienten wurden 24 Stunden nach Mikrowellenablation pulmonaler Metastasen eine konstrastmittelverstärkte MRT der Lunge sowie eine $\mathrm{CT}$ durchgeführt. Die folgenden Frühkomplikationen wurden mit beiden Modalitäten bewertet: Pneumothorax, Pleuraerguss, Blutung, Weichteilverletzung. Zusätzlich wurden die Größe der Ablationszone, die Sichtbarkeit des behandelten Indextumors sowie der Sicherheitssaum bestimmt und der diagnostischer Wert hinsichtlich Rezidivrisiko mittels ROCKurven evaluiert. CT-Kontrolluntersuchungen wurden nach 3,6,12 und 24 Monaten durchgeführt.

Ergebnisse Im Vergleich zum Goldstandard CT betrug die Sensitivität und Spezifität der MRT bei der Erkennung eines Pneumothorax 60,6\% bzw. 87\%. Alle Pneumothoraces mit einer Saumbreite $>1 \mathrm{~cm}$ konnten mit der MRT detektiert werden. Blutung, Pleuraerguss und Weichteilverletzungen konnten allesamt zuverlässig mit der MRT beurteilt werden. Bei sichtbarem behandeltem Indextumor innerhalb der Ablationszone war es möglich den Sicherheitsabstand zum Ablationsrand direkt zu messen. Daraus folgte eine AUC für die T1 von 0,763 und für die CT von 0,782. Der Schwellenwert mit der höchsten Sensitivität für die T1 betrug <0,25 cm (85\%) und für die CT <0,55 cm (90\%). Bei nicht sichtbarem Indextumor innerhalb der Ablationszone wurde der Sicherheitssaum durch Subtraktion des Diameters der Metastase mit dem Diameter der Ablationszone abgeschätzt. Daraus folgte eine leicht schlechtere prognostische Vorhersagbarkeit für die T1 (AUC: 0,706; Schwellenwert: $<0,27 \mathrm{~cm}$; Sensitivität: 82,17\%) und die CT (AUC: 0,719; Schwellenwert: $0,62 \mathrm{~cm}$; Sensitivität: $83,3 \%)$.

Schlussfolgerungen In der postinterventionellen Beurteilung von Mikrowellenablationen von Lungenmetastasen kann die MRT eine sinnvolle Ergänzung sein. Die Möglichkeit einer genaueren Evaluation des Ablationserfolges kann die Entscheidung zur Re-Intervention erleichtern.

\section{WISS 101.2 Transjuguläre Nierenbiopsie bei Kontraindikation zur perkutanen Punktion}

Autoren Kettenbach J

Institut 1 Universitätsklinikum St. Pölten, Klinisches Institut für Medizinische Radiologie, Diagnostik, Intervention, St. Pölten

DOI 10.1055/s-0037-1600280

Zielsetzung Evaluierung der transjugulären Nierenbiopsie (TJNBx) bei Risikopatienten mit Kontraindikationen zur perkutanen Punktion.

Material und Methodik Retrospektiv wurden 57 Patienten (72\% männlich, Durchschnittsalter 58 (15-82) Jahre) nach Durchführung einer TJNBx beurteilt. Alle Punktionen wurden transjugulär über eine 9F-Schleuse mit einem 19-Gauge, 70-cm-langen Nierenbiopsiesystem (QuickCore, Cook) durchgeführt. Spezifische Indikationen, Sampling-Effektivität und Komplikationsraten wurden ausgewertet.

Ergebnisse Die TJNBx war technisch in 65 (98\%) der Patienten erfolgreich, dabei wurde bei 58 (89\%) der Patienten Nierengewebe gewonnen, bei 51 von 58 (87\%) Patienten in diagnostischer Qualität. Spezifische Indikationen für den transjugulären Zugang waren orale Antikoagulation (42\%), Thrombozytopenie (37\%), Adipositas (4\%), Einzelniere (2\%) oder andere Faktoren (15\%). Im Mittel wurden von 3,2 (Bereich, 0 - 11) Biopsiezylinder mit einer Gesamtzahl von 8,6 Glomeruli (Bereich 0 - 41) gewonnen; davon 7.2 Glomeruli (Bereich 0 - 35) für die Lichtmikroskopie, 0.2 (Bereich $0-2$ ) für die Elektronenmikroskopie und 1.7 (Bereich 0 - 6) für die Immunfluoreszenzanalyse. Histologisch wurde in 14 (26\%) Proben eine Glomerulonephritis, in 10 (18\%) Proben eine vaskuläre Nephropathie, in 7 (12\%) Proben eine tubuläre Nekrose, eine CAST-Nephropathie in 3 (5\%) Proben, sowie eine Amyloidose in 2 (4\% 14\%) Proben nachgewiesen. Das histologische Ergebnis hatte in 50 (75\%) der Patienten direkte Auswirkungen auf das Patientenmanagement. In 12 (18\%) der Eingriffe kam es zu einer Kapselperforation, in 2 (4\%) der Fälle zu einer Makrohämaturie, beide Komplikationen erforderten keine weitere Therapie.

Schlussfolgerungen Die TJNBx erleichtert die histologische Diagnostik bei Risikopatienten und leistet damit einen wichtigen Beitrag zum Patienten- management. Die TJNBx sollte bei Vorliegen einer Kontraindikation für eine perkutane Punktion frühzeitig indiziert werden. Obwohl technisch anspruchsvoller als ein perkutaner Zugang ist die TJNBx ein risikoarmer Eingriff.

\section{WISS 310.1 Validierung des Vorhersagemodells SNACOR nach transarterieller Chemoembolisation von hepatozellulären Karzinomen}

Autoren Kloeckner $R^{1}$, Pitton $M^{1}$, Mähringer-Kunz $A^{1}$, Düber $C^{1}$, Schmidtmann $\mathrm{I}^{2}$, Koch $\mathrm{S}^{3}$, Galle $\mathrm{P}^{4}$, Weinmann $\mathrm{A}^{4}$

Institut 1 Johannes Gutenberg-Universität Mainz, Klinik und Poliklinik für Diagnostische und Interventionelle Radiologie, Mainz; 2 Johannes GutenbergUniversität Mainz, Institut für Medizinische Statistik, Biometrie, Epidemiologie und Informatik, Mainz; 3 Johannes Gutenberg-Universität Mainz, Clinical Registry Unit (CRU), Mainz; 4 Johannes Gutenberg-Universität Mainz, Innere Medizin, Mainz

DOI 10.1055/s-0037-1600281

Zielsetzung Transarterielle Chemoembolisation (TACE) ist die Standardtherapie des hepatozellulären Karzinoms (HCC) im intermediären Stadium nach BCLC. Der optimale Zeitpunkt für einen Therapiewechsel ist jedoch häufig unklar. Den Radiologen diesbezüglich in seiner Entscheidungsfindung zu unterstützen und diese objektiver zu gestalten, ist die Aufgabe des klinischen Vorhersagemodells SNACOR. Eine externe Validierung ist bislang noch nicht erfolgt. Das Ziel dieser Arbeit war es daher, diese durchzuführen und SNACOR auf seinen prädiktiven Wert hin zu überprüfen.

Material und Methodik 933 HCC-Patienten wurden mittels TACE zwischen $01 / 2000$ und 09/2015 in unserem Institut behandelt. Alle zur Berechnung von SNACOR benötigten Variablen wurden vor der ersten (Tumorgröße und -anzahl, AFP, Child-Pugh) oder zweiten TACE (radiologisches Tumoransprechen) erfasst. Gesamtüberleben (OS), Harrell's C-Index und integrierter Brier score (IBS) wurden berechnet. Eine Cox-Regression diente dazu, unabhängige Prädiktoren des OS zu identifizieren.

Ergebnisse 251 Patienten konnten endgültig eingeschlossen werden. Das mediane OS in der Gruppe mit niedrigem, mittlerem und hohem Risiko betrug 24,9, 17,9 und 7,7 Monate. Harrell's C-Index lag bei 0.615, IBS war 0.132. Unabhängige Prädiktoren des OS waren das Alpha-Fetoprotein vor der ersten und das radiologische Tumoransprechen vor der zweiten TACE ( $p=0,020$ und 0,002). Child-Pugh sowie Tumorgröße und -anzahl vor der ersten TACE waren nicht prädiktiv ( $p=0,056,0,071$ und 0,090$)$.

Schlussfolgerungen SNACOR war nicht in der Lage HCC-Patienten nach der ersten TACE ausreichend gut hinsichtlich Ihrer Prognose zu differenzieren. Folglich kann auf der Basis von SNACOR alleine nicht entschieden werden ob die TACE-Therapie fortgesetzt werden sollte oder nicht.

\section{WISS 202.4 Meta-Analyse zum Vergleich von Paclitaxel-beschichteten (DCB) versus unbeschichteten Ballonkatheter (POBA) zur Behandlung von okklusiven oder stenosierenden Läsion in der femoropoplitealen Arterie}

Autoren Klumb $\mathrm{C}^{1}$, Eckardt $\mathrm{N}^{1}$, Aschenbach $\mathrm{R}^{1}$, Teichgräber $\mathrm{U}^{1}$ Institut 1 Universitätsklinikum Jena, Institut für Diagnostische und Interventionelle Radiologie, Jena

DOI 10.1055/s-0037-1600282

Zielsetzung Paclitaxel-beschichtete Ballonkatheter versprechen eine verbesserte Offenheitsrate nach perkutaner transluminaler Angioplastie. Es existieren eine Vielzahl unterschiedlicher Produkte. Diese Meta-Analyse untersucht, ob ein sogenannter Klasseneffekt für die beschichteten Ballonkatheter existiert.

Material und Methodik Haupteinschlusskriterien für die Meta-Analyse waren: 1) randomisiert-kontrollierte Studien; 2) Vergleich DCB vs. POBA; 3) Behandlung von de-novo femoropoplitealen Stenosen/Okklusionen; 4) Ergebnisse in 
einem peer-review Journal publiziert; 5) Studienprotokoll publiziert oder online in einem Studienregister einsehbar. Endpunkte: 1) Freiheit von Reintervention (FfTLR), 2) primäre (pP) und 3) sekundäre Offenheit (sP), 4) $A B I$, 5) Rutherford, 6) Lebensqualität (QOL), 7) Sicherheit der DCB (Tod, Amputation, Thrombose).

Ergebnisse 9 geeignete Studien wurden identifiziert. 1552 Patienten wurden rekrutiert und 5 verschiedene DCB Produkte untersucht. 5 Studien haben Ergebnisse nach 12 Monaten berichtet, weitere 4 Studien auch nach 24 Monaten. Nach 12 Monaten wurden eine höhere FfTLR (RR $1.29[1.13,1.47])$ und höhere PP (RR 1.55 [1.25, 1.91]) nach DCB-Behandlung im Vergleich zu POBA berichtet. Die Anzahl der notwendigen Behandlungen (en. number needed to treat NNT) zur Verhinderung einer Reintervention (TLR) nach 12 Monaten reicht von 3 bis 33. Auch nach 24 Monaten waren FfTLR (RR 1.47 [1.19, 1.82]) und $\mathrm{pP}$ (RR $1.56[1.30,1.86])$ nach DCB-Behandlung höher - die NNT zur Vermeidung einer TLR reicht von 3 bis 8. Keine klinischen Unterschiede (Rutherford, ABI, QoL) und kein erhöhtes Sicherheitsrisiko nach DCB-Behandlung wurden beobachtet.

Schlussfolgerungen Die klinische Wirksamkeit und Sicherheit von Paclitaxelbeschichteten Ballonkathetern für die Inhibition der Neointimalen-Hyperplasie nach PTA kann als gesichert bezeichnet werden. Im indirekten Vergleich zeigen sich deutliche Wirksamkeitsunterschiede der verfügbaren DCB - ein Klasseneffekt kann somit nicht bestätigt werden.

\section{WISS 310.7 Evaluation des Einflusses einer TACE auf die quantitative Leberfunktion mittels 13C- Methacetin-Atemtest (MBT) in Patienten mit HCC}

\author{
Autoren Kunz $J^{1}$, Götze $\mathrm{O}^{2}$, Kleinbach $\mathrm{S}^{2}$, Herz $\mathrm{S}^{1}$, Bley $\mathrm{T}^{1}$, Geier $\mathrm{A}^{2}$, \\ Kickuth $\mathrm{R}^{1}$ \\ Institut 1 Universitätsklinikum Würburg, Institut für diagnostische u. \\ interventionelle Radiologie, Würzburg; 2 Universitätsklinikum Würzburg, \\ Medizinische Klinik und Poliklinik II, Würzburg \\ DOI 10.1055/s-0037-1600283
}

Zielsetzung Der Methacetin-Atemtest (MBT) ist ein nichtinvasiver Test zur Evaluation der hepatischen Funktionsreserve. Das Ziel dieser Studie war es, den Effekt einer transarteriellen Chemoembolisation (TACE) auf die Leberfunktion zu quantifizieren.

Material und Methodik Der MBT wurde bei 41 Patienten mit unresezierbarem HCC (alle BCLC B davon 35 Child Pugh Stadium A und 6 Stadium B, Alter 64.7 \pm 3.1 , BMI $28.9 \pm 3.5 \mathrm{~kg} / \mathrm{m} 2$, MELD $9.52 \pm 2.6$ ) prä- und postinterventionell (Tag 0, 1 und 3) durchgeführt. Für jede Testdurchführung wurden $75 \mathrm{mg}$ 13C-Methacetin p.o. appliziert und anschließend das endexspiratorische 13C/12C-Verhältnis im Atem über 60 Minuten gemessen und die Ergebnisse als maximales 13C/12C-Isotopenverhältnis (DOBmax[\%o]) und Isotopenwiederfindungsrate (PDRmax [\%/h]) analysiert. Für die TACE wurde ein Gemisch aus $12 \mathrm{ml}$ Lipiodol, $6 \mathrm{ml}$ Kontrastmittel und $50 \mathrm{mg}$ Epirubicin via Mikrokatheter appliziert, bis zur Tumorsättigung. Post-TACE wurde eine native Computertomografie (CT) zur Kontrolle durchgeführt. Mittels manueller Lebersegmentierung wurde das embolisierte Volumen u. Lebergesamtvolumen bestimmt. Zusätzliche Dichtemessungen wurden zur Differenzierung des voll- und teilembolisierten Lebervolumens herangezogen. Die Daten wurden mit einem gemischten linearen Modell und Korrelationsanalyse ausgewertet.

Ergebnisse An Tag 0 zeigte sich eine PDRmax [\%/h] von 18.5 \pm 1.5 , welche über folgenden $72 \mathrm{~h}$ signifikant abfiel (Tag 1:14.3 \pm 1.0 und Tag 3: $14.0 \pm 1.3$ ). Ebenso fiel die DOBmax [\%o] von $12.1 \pm 1.0$ an Tag 0 signifikant ab (Tag 1: 9.3 \pm 0.7 und Tag 3: $9.06 \pm 0.9$ ). Eine statistisch signifikante Korrelation mit dem prozentual embolisierten Lebervolumen konnte nicht gezeigt werden. Schlussfolgerungen Eine TACE geht mit einer deutlichen Reduzierung der hepatischen Funktion einher. Das Ausmaß der Reduzierung korreliert jedoch nicht mit dem embolisierten Lebervolumen.

\section{RK 301.4 Persönlichkeitsprofil und Erfahrung von Interventionsradiologen auf das Ergebnis CT- gestützter perkutaner Lungenbiopsien}

Autoren Kühn J $]^{1}$, Schäfer $S^{2}$, Quadrat $A^{2}$, Grabe $H^{3}$, Mensel B ${ }^{2}$, Schulze $L^{3}$ Ittermann $\mathrm{T}^{4}$, Hosten $\mathrm{N}^{5}$, Kromrey $\mathrm{M}^{2}$

Institut 1 Universitätsmedizin, Institu für Diagnostische Radiologie und Neuroradiologie, Greifswald; 2 Universitätsmedizin Greifswald, Institut für Diagnostische Radiologie und Neuroradiologie, Greifswald;

3 Universitätsmedizin Greifswald, Institut für Psychiatrie und Psychotherapie, Greifswald; 4 Universitätsmedizin Greifswald, Institut für Community Medicine, Greifswald; 5 Universitätsmedizin Greifswald, Institut für Diagnostische Radiologie und Neuroradiologie, Greifswald DOI 10.1055/s-0037-1600284

Zielsetzung Die Untersuchung des Einflusses des individuellen Persönlichkeitsprofiles sowie der Berufserfahrung von Interventionsradiologen auf die Technische Erfolgsrate und die Rate der Komplikationen standardisiert durchgeführter CT-gestützter perkutaner Biopsien pulmonaler Herdbefunde.

Material und Methodik Zwischen 2006 - 2014 führten 38 Interventionsradiologen 1056 perkutane Lungenbiopsien unter standardisierten Bedingungen durch. Für jede Intervention wurde die Technische Erfolgsrate und die Rate der Komplikationen, in Kenntnis des klinischen Verlaufes und des Biopsieergebnises, ermittelt. Untersuchungen von Radiologen, die weniger als 20 Untersuchungen durchgeführt haben und bei denen weitere Variablen fehlten, wurden ausgeschlossen. Das Persönlichkeitsprofil der Interventionsradiologen wurde mittels des NEO-FFI-30 zur Erhebung der „Big Five“ Neurotizismus, Extraversion, Offenheit, Verträglichkeit und Gewissenhaftigkeit sowie der Skala für Eigenschaftsangst („trait“) aus dem STAI-Fragebogen erhoben und adjustiert für bekannte Prädiktoren der Lungenbiopsie dem Ergebnis der Lungenbiopsien gegenübergestellt. Zusätzlich wurden die Jahre der Berufserfahrung des Interventionalisten in die Analysen mit eingeschlossen.

Ergebnisse Die Technische Erfolgsrate betrug zwischen 75,0-95,0\%, $\mathrm{p}=0,471 ; 13$ Interventionalisten; 421 Biopsien. Die Rate der Komplikationen schwankte zwischen 44,8-85,7\%, p=0,112 (Majorkomplikationen: 15,640,9\%, $p=0,601$; Minorkomplikationen: 31,6-71,4\%, $p=0,056)$. Die untersuchten Persönlichkeitsmerkmale hatten keinen Einfluss auf den Ausgang der Biopsie. Ebenfalls zeigten die Erfahrung der Radiologen keine signifikanten Unterschiede in der Technischen Erfolgsrate $(p=0,112)$ und der Komplikationsrate $(p=0,490)$.

Schlussfolgerungen Unter Verwendung eines standardisierten Prozedere für die Durchführung von Lungenbiopsien spielen Persönlichkeitsmerkmale und die Berufserfahrung von Interventionsradiologen eine untergeordnete Rolle für den Biopsieerfolg.

\section{WISS 301.6 Der Einfluss von OP-Clips auf die Mikrowellenablation in der Leber - eine systematische in-vitro Untersuchung}

Autoren Liebl $\mathrm{M}^{1}$, Zimmermann $\mathrm{M}^{2}$, Schulze-Hagen $\mathrm{M}^{3}$, Isfort $\mathrm{P}^{3}$, Bruners $\mathrm{P}^{3}$, Kuhl $\mathrm{C}^{3}$

Institut 1 Uniklinikum Aachen, Radiologie, Aachen; 2 Uniklinik Aachen, Radiologie, Aachen; 3 Uniklinik Aachen, Radiologie

DOI 10.1055/s-0037-1600285

Zielsetzung Die Mikrowellenablation (MWA) ist ein etabliertes Verfahren zur Behandlung von nicht resektablen malignen Lebertumoren. Materialien mit hoher elektrischer Leitfähigkeit, wie OP-Clips, können sich unter Einfluss von Mikrowellen stark erhitzen und möglicherweise das Ablationsareal beeinflussen. Wir haben die Erhitzung von OP-Clips im Mikrowellenablationsareal untersucht und den Einfluss auf die Größe des Ablationsareals evaluiert.

Material und Methodik Es wurden 18 Mikrowellenablationen in Rinderlebern durchgeführt, wobei 7,5 mm neben der Ablationssonde ein Titan-OP-Clip im Leberparenchym platziert wurde. Die Ablation erfolgte mit einem AMICAMikrowellen-Gerät von Mermaid Medical mit jeweils 60 Watt für 3 Minuten. 
Während der Ablation wurde die Temperatur sekündlich an vier Positionen gemessen. Unmittelbar am Clip sowie $15 \mathrm{~mm}$ von der Ablationssonde (bzw. 7,5 mm jenseits des Clips), und jeweils 7,5 mm und $15 \mathrm{~mm}$ von der Sonde entfernt auf der Gegenseite vom Clip. Nach Ablauf der Ablation wurde die Rinderleber seziert und die Form und die Größe des Ablationsareals dokumentiert. Die dokumentierten Temperaturdaten wurden mittels Student's T-Test ausgewertet.

Ergebnisse Die Temperatur an Stelle des Titan-Clips war signifikant höher im Vergleich zu der Position mit gleicher Entfernung zur Ablationssonde auf der Gegenseite (ohne Clip) während der gesamten Zeit der Ablation mit einem maximalen Temperaturunterschied von 17 Grad Celsius am Ende der Ablation $(p=0,009)$. Es konnten keine signifikanten Veränderungen der Form oder Größe des Ablationsareals festgestellt werden.

Schlussfolgerungen Titan-OP-Clips im Mikrowellenablationsareal werden signifikant heißer als das umliegende Gewebe, jedoch hat dies keinen Einfluss auf die Form oder Größe des Ablationsareals.

\section{WISS 310.2 Validierung der Vorhersagemodelle STATE und START in der TACE-Therapie bei Patienten mit hepatozellulärem Karzinom}

Autoren Maehringer-Kunz $A^{1}$, Kloeckner $R^{1}$, Pitton $M^{1}$, Düber $C^{1}$, Schmidtmann $\mathrm{I}^{2}$, Galle $\mathrm{P}^{3}$, Koch $\mathrm{S}^{4}$, Weinmann $\mathrm{A}^{3}$

Institut 1 Johannes Gutenberg-Universität Mainz, Klinik und Poliklinik für Diagnostische und Interventionelle Radiologie, Mainz; 2 Johannes GutenbergUniversität Mainz, Institut für Medizinische Statistik, Biometrie, Epidemiologie und Informatik, Mainz; 3 Johannes Gutenberg-Universität Mainz, Innere Medizin, Mainz; 4 Johannes Gutenberg-Universität Mainz, Clinical Registry Unit (CRU, Mainz

DOI 10.1055/s-0037-1600286

Zielsetzung TACE ist die Standardtherapie bei inoperablen Patienten mit HCC im intermediären Stadium. Doch nicht jeder Patient profitiert gleichermaßen von einer TACE. Der STATE-Score soll eine Hilfestellung geben, welcher Patient von einer ersten TACE profitiert. Die sequentielle Anwendung von STATE-Score + ART-Score (=START-Strategie) soll helfen die Frage zu beantworten, bis zu welchem Punkt eine Fortführung einer bereits begonnenen TACE-Therapie sinnvoll ist. Ziel dieser Studie ist die externe Validierung von STATE-Score und START-Strategie.

Material und Methodik Von 2000-2015 erhielten 933 Patienten mit HCC in unserem Institut eine TACE. Alle Variablen zur Berechnung des STATE-Scores und zur Implementierung der START-Strategie, wurden erhoben (STATE: Albumin, „Up-to-seven”, CRP) (ART: AST-Anstieg, Child-Pugh-Anstieg, radiologisches Tumoransprechen). Das Gesamtüberleben (OS) wurde berechnet und eine multivariate Analyse durchgeführt. Die Validierung von STATE und START wurde mithilfe des Harrell's C-Index und des integrierten Brier Scores (IBS) durchgeführt.

Ergebnisse Für 228 Patienten konnte der STATE-Score berechnet werden. Ein niedriger STATE-Score (hohes Risiko) führte zu einem medianen OS von 14,3 Monaten, ein hoher STATE-Score (niedriges Risiko) zu 20,2 Monaten. Harrell's $C$ betrug 0,558 und IBS 0,133 . Für den STATE-Score waren Prädiktoren für das OS „up-to-seven“ ( $p=0,006)$ und Albumin $(p=0,022)$. CRP war nicht prädiktiv $(p=0,367)$. Für 207 Patienten konnte zusätzlich der ART-Score berechnet werden. Die Kombination von STATE-Score und ART-Score führte zu einem Harrell's C von 0,58 und IBS von 0.132 .

Schlussfolgerungen Der STATE-Score unterstützt in der Entscheidungsfindung, ob ein Patient von einer ersten TACE profitiert. Die zusätzliche sequentielle Verwendung des ART-Scores (=START-Strategie) kann in der Entscheidung helfen, ob die TACE weitergeführt werden sollte. Im Ergebnis sind jedoch weder STATE-Score noch START-Strategie ausreichend prädiktiv um ausschließlich hierauf basierend klinische Entscheidungen zu treffen.

\section{WISS 101.5 Schmerzmanagement bei Uterusarterienembolisation: Vergleich der periinterventionellen Blockade des Plexus hypogastricus mit der Periduralanästhesie}

Autoren Malouhi $\mathrm{A}^{1}$

Institut 1 Universitätsklinikum Jena, Institut für Diagnostische und

Intervetionelle Radiologie, Jena

DOI 10.1055/s-0037-1600287

Zielsetzung Verschiedene Verfahren führen zu einer Verbesserung der Schmerzempfindung während und nach Uterusarterienembolisation (UAE) bei Uterus myomatosus.. Es soll evaluiert werden, ob die periprozedural durchgeführte Plexus hypogastricus Blockade (PHB) auch einen anhaltend analgetischen Effekt im Vergleich zur Anästhesie mittels Periduralkatheter (PDK) aufweist.

Material und Methodik In einer prospektiven Beobachtungsstudie wurden 50 Patientinnen konsekutiv (1:1) in zwei Gruppen eingeteilt. Die Kontrollgruppe mit 25 Pat. bekam präoperativ einen PDK und die Studiengruppe mit 25 Pat. erhielt eine PHB. Die Dosierung des PDK erfolgte mit 3,0 ml Bupivacain 0,25\% sowie dem nachfolgenden titrierendem Aufspritzen mittels 8-10 ml Ropivacain $0,15 \%$ und zusätzl. $10 \mu \mathrm{g}$ Sufentanil. Die PHB erfolgt über eine fluoroskopisch-gestützte perkutane Punktion mit einer 20G Chiba Nadel und Injektion von $10 \mathrm{ml}$ Bupivacain 0,25\% unterhalb der Aortenbifurkation auf Höhe der LWK 5 umittelbar vor der UAE. Es wurden Fragebögen über Schmerzempfinden an die Patientinnen verteilt, die diese vor und 6, 12, 18, 24, 30 Std sowie 6 Monaten post UAE ausfüllen sollten.

Ergebnisse Verschiedene Verfahren führen zu einer Verbesserung der Schmerzempfindung während und nach Uterusarterienembolisation (UAE) bei Uterus myomatosus.. Es soll evaluiert werden, ob die periprozedural durchgeführte Plexus hypogastricus Blockade (PHB) auch einen anhaltend analgetischen Effekt im Vergleich zur Anästhesie mittels Periduralkatheter (PDK) aufweist.

Schlussfolgerungen Eine signifikante Verbesserung der Schmerzqualität nach 30 Stunden postoperativ wurde bei Patientinnen festgestellt, die eine PHB erhielten. Diese Daten verstärken die These, dass diese interventionelle Analgesietechnik zu einer signifikanten Schmerzreduzierung für den schmerzintensiven Zeitraum der ersten 24 Std. post UAE führt und auf eine PDK verzichtet werden kann.

\section{RK 301.7 Evaluation der 2D-Perfusions-Angiografie bei Patienten mit chronischer thromboembolischer pulmonaler Hypertonie (CTEPH) vor und nach pulmonaler Ballonangioplastie (BPA)}

Autoren Maschke $\mathrm{S}^{1}$, Meyer $\mathrm{B}^{1}$, Hoeper $\mathrm{M}^{2}$, Olsson $\mathrm{K}^{2}$, Wacker $\mathrm{F}^{1}$, Hinrichs ${ }^{1}$ Institut 1 Medizinische Hochschule Hannover, Radiologie, Hannover;

2 Medizinische Hochschule Hannover, Pneumologie, Hannover DOI 10.1055/s-0037-1600288

Zielsetzung Evaluation der Durchführbarkeit der 2D-Perfusions-Angiografie zur Quantifizierung von Perfusionsunterschieden vor und nach BPA.

Material und Methodik 30 BPA-Sitzungen bei 16 Patienten (12w; 68.4 [77; 54.5] Jahre) mit insgesamt 99 intervenierten Pulmonalarteriensegmenten wurden mittels einer Nachverarbeitungstechnik zur Quantifizierung der vaskulären Flussverhältnisse anhand von digitalen Subtraktionsangiografien (DSA) evaluiert. Vor und nach BPA wurden DSA-Serien mit manueller Kontrastmittelapplikation, in gleicher Projektion und mit vergleichbarer Katheterpositon aufgenommen. An diesen wurde mittels korrespondierenden proximalen Referenz-ROls (arterieller Einstrom) in der Pulmonalarterie und einem distalen ZielROI im Lungenparenchym eine 2D-Perfusionsanalyse durchgeführt. Aus den Zeit-Dichte-Kurven wurden Time-to-peak (TTP), Peak-density (PD) und Areaunder-the-curve (AUC) sowie Quotienten aus Referenz- und Parenchym-ROI (TTPParenchym/TTPEinstrom; PDParenchym/PDEinstrom; AUCParenchym/ 
AUCEinstrom) vor und nach BPA berechnet. Die Differenzen der genannten Parameter wurden mit den Änderungen im Pulmonary-Flow-Grade-Score, einem visuell-basierten Score zur Einschätzung der Lungenperfusion, korreliert (paarweise Korrelation).

Ergebnisse Der Pulmonary-Flow-Grade-Score stieg signifikant (1 vs. 3; $\mathrm{p}<0,0001)$. Entsprechend verkürzte sich das Verhältnis TTPParenchym/ TTPEinstrom um etwa $12 \%$ (4,2 vs. 3,7 s; $p<0,0001)$, erhöhte sich die PDParenchym/PDEinstrom um etwa $46 \%(0,18$ vs. 0,$39 ; p<0,0001)$ und vergrößerte sich die AUCParenchym/AUCEinstrom um etwa $36 \%$ (0,16 vs. 0,44 ; $\mathrm{p}<0,0001)$. In der paarweisen Korrelation zeigte sich ein signifikanter Zusammenhang zwischen den genannten Parametern und dem Pulmonary-FlowGrade-Score $(p<0.0001)$.

Schlussfolgerungen Die 2D-Perfusions-Angiografie erlaubt eine quantitative Analyse der Lungenparenchymdurchblutung vor und nach BPA und hat das Potential das Therapiemonitoring der BPA zu verbessern.

\section{WISS 309.2 Beeinflussung der Nierenfunktion durch das Überstenten von akzessorischen Nierenarterien im Rahmen der endovaskulären Versorgung von Bauchaortenaneurysmen (EVAR)}

\author{
Autoren Maurer $\mathrm{K}^{1}$, Lürken $\mathrm{L}^{2}$, Verloh $\mathrm{N}^{2}$, Stroszczynski $\mathrm{C}^{2}$, Pfister $\mathrm{K}^{3}$, \\ Wohlgemuth $W^{2}$, Müller-Wille $R^{2}$ \\ Institut 1 Universitätsklinikum Regensburg, Radiologie, Regensburg; \\ 2 Uniklinikum Regensburg, Radiologie, Regensburg; 3 Uniklinikum \\ Regensburg, Gefäßchirurgie, Regensburg \\ DOI 10.1055/s-0037-1600289
}

Zielsetzung Ziel war die Beurteilung des Einflusses der Überstentung von akzessorischen Nierenarterien auf die postoperative Nierenfunktion im Rahmen der endovaskulären Aneurysmaversorgung.

Material und Methodik Bei 120 Patienten (104 Männer, 16 Frauen; Durchschnittsalter, $70 \pm 8$ Jahre) wurden im Rahmen einer endovaskulären Aneurysmaversorgung eine oder mehrere akzessorische Nierenarterien durch die Überlagerung des Stentgrafts verschlossen. Grundlage der Analyse sind präund postoperative CT-Angiografien. Wir untersuchten retrospektiv die Auswirkung dieser Überstentung auf die postoperative Nierenfunktion (Serumkreatininspiegel, Nierenversagen nach AKIN-Definition). Es wurden nur Patienten mit normaler Nierenfunktion vor EVAR eingeschlossen.

Ergebnisse Bei 20 von 120 Patienten (16,7\%) wurde eine akzessorische Nierenarterie durch Überstentung verschlossen. In der Kontrollgruppe (100 Patienten) stieg der Serumkreatininspiegel von $0,87 \mathrm{mg} / \mathrm{dl}$ auf $0,96 \mathrm{mg} / \mathrm{dl}$ an. In der Gruppe der Patienten mit überstenteten akzessorischen Nierenarterien stieg das Serumkreatinin im Mittel von 0,89 mg/dl auf $1,12 \mathrm{mg} / \mathrm{dl}$ an. In dieser Gruppe trat tendenziell häufiger ein akutes Nierenversagen auf (15\% vs. $25 \%$, $\mathrm{p}=0,32$ ).

Schlussfolgerungen Das Überstenten von akzessorischen Nierenarterien führt tendenziell zu einer Verschlechterung der Nierenfunktion unmittelbar nach EVAR. Die Unterschiede waren in unserem Kollektiv jedoch statistisch nicht signifikant.

\section{WISS 309.1 Radiologisch-interventionelle Therapie von Endoleaks nach endovaskulärer Versorgung infrarenaler Bauchaortenaneurysmata}

\author{
Autoren Natho $\mathrm{O}^{1}$, Wagenhofer $\mathrm{K}^{1}$, Siemens $\mathrm{P}^{1}$, Burmeister $\mathrm{H}^{1}$, Daum $\mathrm{H}^{2}$, \\ Gross-Fengels W3 \\ Institut 1 Asklepios Klinikum Harburg, Diagnostische und Interventionelle \\ Radiologie, Hamburg; 2 Asklepios Klinikum Harburg, Gefäßchirurgie und \\ endovaskuläre Chirurgie, Hamburg; 3 Diagnostische und Interventionelle \\ Radiologie, Asklepios Klinikum Harburg, Hamburg \\ DOI 10.1055/s-0037-1600290
}

Zielsetzung Endovaskuläre Therapie von Bauchaortenaneurysmata (BAA) und interdisziplinäres Management persistierender Endoleaks (pEL) als relevante
Komplikation der EVAR (endovascular aortic repair) bei BAA wurden retrospektiv analysiert. Leitlinienkonform sollten pEL in max. 15\% der Fälle auftreten. Material und Methodik Vom 1.1.2011-30.6.2016 wurden 336 Patienten (Pat.) mit BAA interventionell-radiologisch mittels EVAR therapiert. Das Durchschnittsalter der Pat. betrug 75,1 $\pm 8,6$ Jahre (306 Männer, 30 Frauen). Alle Pat. $(n=336)$ erhielten CT-Angiografie und/oder Kontrastmittelsonografie. Die Behandlung postinterventionell aufgetretener Endoleaks (EL) wurde analysiert.

Ergebnisse Bei 113 von 336 Pat. wurden im Verlauf EL diagnostiziert (33,6\%). Bei 69 Pat. erfolgte keine Intervention bei nicht relevantem EL, Spontanremission oder Tod des Pat. Bei 44 Pat. (13,1\%) lagen therapiebedürftige pEL vor (Patientenalter 76,6 \pm 6,6 Jahre; 43 Männer, 1 Frau). Bei 44 Pat. wurden 55 pEL diagnostiziert (16 Fälle pEL I, 31 Fälle pEL II, 7 Fälle pEL III, 1 Fall pEL IV; bei 8 Pat. mehrere pEL-Typen). Es erfolgten 93 interventionell-radiologische (z.T. chirurgisch/operativ ergänzte) Eingriffe. Bei 36/44 Pat. (81,8\%) verlief die interdisziplinäre Therapie erfolgreich (interventionell-radiologisch 23/44; 52,3\%). Die komplette Ausschaltung des pEL wurde bei 29 Pat. (65,9\%; davon interventionell-radiologisch $17 / 29 ; 58,6 \%$ ), eine Schrumpfung des Aneurysmasacks bei 2 Pat. (4,6\%), ein nicht mehr therapierelevantes pEL bei 5 Pat. $(11,3 \%)$ und in 7 Fällen (15,9\%) eine Befundkonstanz erreicht. Ein Pat. $(2,3 \%)$ mit progredientem pEL lehnte die weitere Therapie ab. Intensivtherapiepflichtige Komplikationen traten nicht auf. Die postinterventionelle Mortalität lag nach 4 Wochen bei $0 \%$.

Schlussfolgerungen Die radiologisch-interventionelle Therapie des pEL ist ein effektives und sicheres Verfahren. In einigen Fällen ist eine Kombination mit chirurgischen Verfahren erforderlich. Die Therapieentscheidung sollte im interdisziplinären Konsens erfolgen.

\section{WISS 301.1 Residuelle Radioaktivität von Mikrokathetern nach Y-90 Radioembolisation mit Glas- und Harzmikrosphären}

Autoren Pieper $C^{1}$, Haslerud $T^{2}$, Sabet $A^{2}$, Schild $H^{1}$, Ezziddin $S^{2}$, Meyer $C^{1}$ Institut 1 Universitätsklinikum Bonn, Radiologische Klinik, Bonn;

2 Universitätsklinikum Bonn, Klinik für Nuklearmedizin, Bonn DOI 10.1055/s-0037-1600291

Zielsetzung Bestimmung der Menge und der Verteilung residueller Radioaktivität von Mikrokathetern nach Radioembolisation (RE).

Material und Methodik Die Radioaktivität von Mikrokathetern wurde gemessen unmittelbar nach 15 Harz- und 10 Glas-Res mit applizierten Aktivitäten von 0,8 bis 2,2 GBq (Mittelwert 1,51 GBq) bei Harz-REs und 0,5 bis 5,7GBq (Mittelwert 1.86GBq) bei Glas-REs. Nach der Mikrosphärenapplikation wurden die Mikrokatheter in Gruppen von jeweils fünf Kathetern mit unterschiedlichen Flüssigkeitsvolumina gespült (Gruppe A: Harz mit $10 \mathrm{ml}$ Spülvolumen, B: Harz mit 50 ml, C: Harz mit 100 ml, D: Glas mit 100 ml, E: Glas mit 200 ml; insgesamt 25 Katheter). Die Mikrokatheter wurden anschließend aus dem Führungskatheter entfernt und in zwei Segmente zerschnitten (proximales $1 / 3$ ( $45 \mathrm{~cm}$ inklusive Hub) und distale 2/3). Die residuelle Aktivität wurde in einem kalibrierten Aktivimeter gemessen und verglichen zwischen Spülvolumina, Mikrosphärentypen und Kathetersegmenten.

Ergebnisse Die gemessene residuelle Aktivität war niedrig $(<0,1 \%$ und $<1 \%$ der injizierten Aktivität bei Harz- bzw. Glas). Die applizierte therapeutische Aktivität korrelierte nicht mit der residuellen Aktivität der Katheter. Die Menge des Spülvolumens beeinflusste die residuelle Aktivität ebenfalls nicht. Die gemessene Aktivität war nach Harz-RE signifikant niedriger als nach Glas-RE (proximal: Mittelwert $0,5 \mathrm{MBq}[0,1-1,9 \mathrm{MBq}]$ vs. $12,4 \mathrm{MBq}[1,8-33,4 \mathrm{MBq}]$, $\mathrm{p}<0,0001$; distal: $0,04 \mathrm{MBq}[0,0-0,2 \mathrm{MBq}]$ vs. $0,4 \mathrm{MBq}[0,01-1,7 \mathrm{MBq}]$, $p=0,008)$. Signifikant mehr Radioaktivität wurde im proximalen Kathetersegment vs. dem distalen retiniert (Harz: $p=0,007$; Glas: $p=0,005$ ).

Schlussfolgerungen Die residuelle Radioaktivität von Mikrokathetern nach RE ist generell niedrig, wird aber nicht von der Menge des Spülvolumens nach Mikrosphärenapplikation beeinflusst. Nach Glas-RE lassen sich deutlich höhere 
Aktivitäten beobachten als nach Harz-RE. Im proximalen Kathetersement wird besonders viel Aktivität retiniert, so dass dieses vorsichtig gehandhabt werden sollte.

\section{RK 101.6 MR-Diffusionsbildgebung vor und nach Anlage eines transjugulären intrahepatischen portosystemischen Shunts (TIPS) - Erste Erfahrungen mit einer Intravoxel Incoherent Motion (IVIM) Auswertung}

Autoren Pieper $C^{1}$, Sprinkart $A^{1}$, Thomas $D^{1}$, Meyer $C^{1}$, Block $W^{1}$, Schild $H^{1}$, Kukuk $G^{1}$, Mürtz $P^{1}$

Institut 1 Universitätsklinikum Bonn, Radiologische Klinik, Bonn DOI 10.1055/s-0037-1600292

Zielsetzung Untersuchung von Änderungen der Intravoxel Incoherent Motion (IVIM) Parameter nach TIPS-Anlage bei Zirrhosepatienten.

Material und Methodik 16 Patienten (13 m, mittleres Alter 53 Jahre) mit Leberzirrhose und portaler Hypertension erhielten Leber-MRTs an einem 1.5T Scanner inklusive Diffusionsbildgebung ( $\mathrm{b} 0=0$, b1 $=50$, b2 $=800 \mathrm{~s} / \mathrm{mm} 2$ ) vor und nach TIPS-Anlage. Der Apparent Diffusion Coefficient $\operatorname{ADC}(0,800)$, der geschätzte Diffusionskoeffizient D' und die Perfusionsfraktion f' wurden voxelbasiert berechnet. Bei jedem Patienten wurden 3 Regions-of-Interest (ROIs) vor und nach TIPS untersucht (rechter Leberlappen ventral/dorsal, linker Leberlappen). Als Subgruppen wurden die ROls zusätzlich anhand kontrastverstärkter Sequenzen eingeteilt in ROls mit erhaltener bzw. fehlender Kontrastierung portalvenöser Gefäßäste nach TIPS.

Ergebnisse Alle TIPS-Anlagen waren erfolgreich. Nach TIPS-Anlage stieg f* insgesamt signifikant von 9,3 $\pm 3,8 \%$ auf $11,0 \pm 5,0 \%(p<0,028)$ an, während sich $\operatorname{ADC}(0,800)$ und $D^{\prime}$ nicht änderten $(1263,9 \pm 195,8$ vs. $1274,2 \pm 167,8$; $\mathrm{p}=0,461$ bzw. $1151,1 \pm 174,9$ vs. $1133,7 \pm 152,6 ; \mathrm{p}=0,490$ ) (ADC und $\mathrm{D}^{\prime}$ in $10-6 \mathrm{~mm} 2 / \mathrm{s}$ ). Nach TIPS wurde in $8 / 48$ ROls eine fehlende Kontrastierung der kleinen Pfortaderäste beobachtet (2 rechts ventral, 5 rechts dorsal, 1 links). Nach TIPS stieg $f^{\prime}$ 'signifikant in ROls mit offenen Pfortaderästen von $9,0 \pm 3,6 \%$ auf $12,1 \pm 4,7 \%$ ( $p<0,00002$ ). Demgegenüber fiel $f^{\prime}$ in ROls mit fehlender Kontrastierung der Pfortaderäste von $11,1 \pm 4,5 \%$ auf $5,3 \pm 1,8 \%$ $(p=0,003)$. In beiden Gruppen änderten sich $\operatorname{ADC}(0,800)$ und D' nach TIPS nicht.

Schlussfolgerungen Nach TIPS-Anlage steigt die IVIM-Perfusionsfraktion in Leberregionen mit offenen Pfortaderästen an. Dies ist ein Hinweis auf einen verbesserten mikrovaskulären Blutfluss im Lebergewebe nach Dekompression der Pfortader. In Regionen mit fehlender Kontrastierung der Pfortaderäste (hämodynamisch bedingt oder durch thrombotische Obstruktion), kann eine Abnahme der Gewebeperfusion als verminderte IVIM-Perfusionsfraktion gemessen werden.

\section{WISS 310.9 Radioembolisation von nicht operablen hepatozellulären Karzinomen}

Autoren Pieper $C^{1}$, Wilhelm $\mathrm{K}^{1}$, Lampe $\mathrm{N}^{1}$, Matuschek $\mathrm{E}^{1}$, Maschke $\mathrm{T}^{1}$, Ahmadzadehfar $\mathrm{H}^{2}$, Willinek $\mathrm{W}^{1}$, Schild $\mathrm{H}^{1}$, Meyer $\mathrm{C}^{1}$

Institut 1 Universitätsklinikum Bonn, Radiologische Klinik, Bonn;

2 Universitätsklinikum Bonn, Klinik für Nuklearmedizin, Bonn

DOI 10.1055/s-0037-1600293

Zielsetzung Bestimmung der Wertigkeit der Radioembolisation zur Behandlung von nicht operablen hepatozellulären Karzinomen (HCC).

Material und Methodik Daten der Patienten mit nicht operablen HCCs, die eine Radioembolisation erhalten haben, wurden retrospektiv erhoben. Biochemische sowie klinische Toxizitäten, das morphologische Tumoransprechen, die Zeit bis zur Progression und das Gesamtüberleben wurden analysiert. Die Daten wurden anhand klinischer und prozeduraler Parameter stratifiziert und statistisch ausgewertet.

Ergebnisse 115 Patienten (89 männlich, mittleres Alter 69.3 Jahre) erhielten 158 Radioembolisationen (119 Harz-, 39 Glasmikrosphären) [Barcelona Clinic
Liver Cancer (BCLC) A: 6,1\%, B: 33,9\%, C: 60,0\%]. Das mediane klinische Follow-up betrug 178 [26-2505] Tage. Klinische Toxizitäten 3. Grades oder höher traten nicht auf. Die objektive Ansprechrate betrug 35,6\%; die Tumorkontrollrate 76,7\%. Über alle Patienten gemittelt betrug die mediane Zeit bis zur Progression der behandelten Leberareale 120 [26 - 1361] Tage. 108/115 Patienten verstarben während der Nachbeobachtungszeit (medianes Gesamtüberleben nach erster Radioembolisation: 252 (9-2484) Tage; BCLC A: 1585 Tage, B: 373 Tage, C: 183 Tage). Die multivariate Analyse ergab, dass ein Eastern Cooperative Oncology Group (ECOG) Status < 1 und das Fehlen von Aszites vor der Radioembolisation sowie ein morphologisches Therapieansprechen ein längeres Gesamtüberleben vorhersagen. Bei BCLC C Patienten wurden als Prädiktoren eines längeren Überlebens identifiziert: geringe hepatische Tumorlast, Fehlen von Aszites, niedrige Baseline Gamma-Glutamyl-Transferase (GGT) sowie die wiederholte Durchführung einer Radioembolisation bei erneut progredienten Tumoren.

Schlussfolgerungen Die Radioembolisation ist eine sichere und effektive Therapieoption für sorgfältig ausgewählte HCC-Patienten und ist mit einer niedrigen Komplikationsrate verbunden. Ein niedriger ECOG-Status und das Fehlen von Aszites vor Therapie sind positive Prognosefaktoren.

\section{WISS 202.7 Percutaneous creation of an arteriovenous fistula (pAVF) for hemodialysis access}

Authors Radosa $\mathrm{C}^{1}$, Weiss $\mathrm{N}^{2}$, Hofmockel $\mathrm{T}^{1}$, Radosa $]^{3}$, Laniado $\mathrm{M}^{1}$, Hoffmann $\mathrm{R}^{1}$

Institute 1 Universitätsklinikum Dresden, Radiologie, Dresden; 2 Universitätsklinikum Dresden, Universitäts GefäßCentrum und Medizinische Klinik III, Dresden; 3 Universitätsklinikum des Saarlandes, Klinik für Frauenheilkunde, Geburtshilfe und Reproduktionsmedizin, Homburg DOI 10.1055/s-0037-1600294

Zielsetzung Standard for vascular access for hemodialyis is surgical creation of a radio-cephalic fistula. If not suitable, a brachiocephalic or -basilic fistula may be created, which, however, is associated with a higher complication rate. Recently, an endovascular approach for ulnar-ulnar fistula creation (EndoAVF) had been developed, which may be an alternative to surgical upper arm dialysis fistula. We aimed to study feasibility, technical success, early complications and outcome of this novel treatment option.

Material und Methodik Between July 2015 and February 2016 eight patients were included in this prospective observational study. Indications for pAVF were confirmed by a multidisciplinary vascular board upon contraindication for cimino fistula creation. Patients were included after a pretherapeutic ultrasound showing patent brachial and ulnar arteries and veins of adequate size, perforating veins between deep and superficial veins in the cubital area, and no ipsilateral central venous stenosis. Patient characteristics, technical success, duration of intervention, total patient radiation dose, complication rates, time to maturation of pAVF and clinical effectiveness at 6 month were assessed. Ergebnisse From eight patients included in the study, creation of pAVF was successful in all cases. Median duration of intervention was 60 min (range: 40 - 80) and decreased over time from 80 minutes to 40 - 50 minutes. There was one minor intraoperative complication and no postoperative complications. Median time to pAVF maturation were 63 days (range: 26 - 137 d). One patient was lost to follow-up after the first monitoring. Due to the remaining seven patients hemodialysis was started without problems. Patency after fourmonths was $100 \%$.

Schlussfolgerungen EndoAVF seems to be feasible and safe for the creation of arteriovenous fistula - showing fast maturation. Bigger sample sizes and longer follow-ups are needed to prove the comparability to surgical dialysis access creation. 


\section{RK 321.3 Aneurysmen und Pseudoaneurysmen viszeraler Arterien: Erfahrungen in der interventionellen Therapie an einem Haus der Maximalversorgung}

Autoren Ruhnke $\mathrm{H}^{1}$, Kröncke $\mathrm{T}^{1}$

Institut 1 Klinikum Augsburg, Institut für Diagnostische und Interventionelle Radiologie und Neuroradiologie, Augsburg

DOI 10.1055/s-0037-1600295

Zielsetzung Evaluation der Erfahrungen in der interventionellen Therapie von inzidentellen und symptomatischen Aneurysmen des viszeralen Gefäße in der elektiven sowie der Notfallsituation.

Material und Methodik Zwischen 2011 und 2015 wurden 43 Aneurysmen von 38 Patienten in 48 Interventionen behandelt. Betrachtet wurden die Charakteristika der Aneurysmen (echtes vs. Pseudoaneurysma, Größe, Genese, Lokalisation, Blutungsstatus, Symptomatik), die präinterventionelle Diagnostik und die postinterventionelle Erfolgskontrolle sowie die Intervention selbst hinsichtlich technischem Erfolg, verwendeten Materialien und interventionsassoziierten Komplikationen.

Ergebnisse Behandelt wurden 24 echte Aneurysmen (max. Durchmesser 22 +/- $18 \mathrm{~mm}(11-67 \mathrm{~mm})$ ) sowie 19 Pseudoaneurysmen (max. Durchmesser 9 $+/-33 \mathrm{~mm}(3-150 \mathrm{~mm}))$. Am häufigsten wurden die A. lienalis $(n=14)$ sowie die $A$. renalis dextra et sinistra $(n=18)$ behandelt. Die Genese war am häufigsten atherosklerotisch (44\%) oder iatrogen postoperativ (21\%). 18/48 Interventionen erfolgten aufgrund einer aktiven oder stattgehabten Blutung, Pseudoaneurysmen waren signifikant häufiger aktiv blutend (63\% vs. $25 \%$, $p=0,012)$. Eine tendenzielle Differenz ergab sich hinsichtlich der Symptomatik zugunsten der Pseudoaneurysmen (57,9\% vs. 37,5\%, p =0,424). Die Mehrzahl der Aneurysmen wurde mittels CT diagnostiziert (77\%). 41/48 Behandlungen (85\%) waren primär technisch erfolgreich. Die Behandlung wies eine Komplikationrate von $10 \%(n=5)$ auf, in 4 Fällen minor, in 1 Fall eine major Komplikation. Kein Patient litt postinterventionell an dauerhaften therapiebedingten Folgeschäden. Vornehmlich wurden die Aneurysmen mithilfe von Coils und ggf. zusätzlichen Embolisaten (Flüssigembolisat, Vascular Plug) (36/48, $75 \%)$ versorgt.

Schlussfolgerungen Die interventionelle Behandlung von Aneurysmen der viszeralen Arterien ist eine sichere und erfolgreiche Therapie. Bei vergleichbarer Morbidität und Mortalität profitieren insbesondere multimorbide Patienten vom minimalinvasiven Vorgehen.

\section{RK 321.2 Endovaskuläre Therapie von akuten postoperativen Blutungen der Viszeralarterien mittels Stentgrafts: Effektivität und Komplikationen}

\author{
Autoren Schaarschmidt $B^{1}$, Boos D ${ }^{1}$, Kröpil $P^{1}$, Kröpil $P^{2}$, Lanzman $P^{1}$, \\ Fürst $\mathrm{P}^{1}$, Thomas $\mathrm{P}^{1}$ \\ Institut 1 Heinrich-Heine-Universität Düsseldorf, Medizinische Fakultät, \\ Institut für Diagnostische und Interventionelle Radiologie, Düsseldorf; \\ 2 Heinrich-Heine-Universität Düsseldorf, Medizinische Fakultät, Klinik für \\ Allgemein-, Viszeral- und Kinderchirurgie, Düsseldorf \\ DOI 10.1055/s-0037-1600296
}

Zielsetzung Eine der schwerwiegendsten Komplikationen nach Pankreas-, Leber- oder Gallenwegschirurgie ist die sogenannte Sentinel-Blutung, bei denen austretendes Pankreassekret oder Gallenflüssigkeit zu einer Arrosion von Viszeralarterien und akut lebensbedrohlichen Blutungen führt. Die Therapie dieser Blutungen ist herausfordernd, da die Blutversorgung der Oberbauchorgane erhalten werden muss. Daher war eine Evaluation der Effektivität der endovaskulären Therapie von Sentinelblutungen mittels Stentgrafts Ziel dieser Studie.

Material und Methodik Insgesamt wurden in diese retrospektive Studie 29 Patienten (7 weiblich, 22 männlich, Durchschnittsalter 66,4 Jahre) eingeschlossen, bei denen zwischen 2009 und 2016 eine postoperative Blutung im Oberbauch mittels Stentgraft (Gore Viabahn ${ }^{\circledR}$ ) versorgt werden sollte.
Der technischen Erfolg, interventionsassoziierte Komplikationen und das Kurzzeitüberlebens (30 Tage) wurden analysiert

Ergebnisse Bei $83 \%$ der Patienten $(n=24)$ gelang es, die Gefäßprothese zu platzieren, die Blutung zu unterbinden und den Blutfluss wiederherzustellen. Die chirurgische Konversionsrate betrug 17\% ( $n=5)$. Periinterventionelle Komplikationen traten bei sechs Patienten (21\%) auf (reversible Vasospasmen $\mathrm{n}=4$; reversibler Verschluss der Gefäßprothese $\mathrm{n}=1$; Aneurysmaruptur $n=1)$. Stent-assoziierte Komplikationen traten in $38 \%$ der Fälle $(n=11)$ auf (Nachblutungen, $n=7$; Verschluss des Stentgrafts, $n=4$ ). Zugangsweg-assoziierte Komplikationen bei transbrachialem Zugangsweg zeigten sich bei drei Patienten (10\%). Sieben Patienten verstarben im kurzfristigen Verlauf, entweder aufgrund einer nicht beherrschbaren abdominalen Rezidivblutung $(n=3)$, einer Sepsis $(n=3)$ oder eines kardialen Ereignisses $(n=1)$.

Schlussfolgerungen Die endovaskuläre Therapie von postoperativ auftretenden, akut lebensbedrohlichen Oberbauchblutungen mittels Stentgrafts ist eine primär lebensrettende Therapieoption mit akzeptablem technischem Erfolg. Problematisch ist jedoch die hohe Komplikationsrate, die durch die Komorbiditäten der Patienten begünstigt wird.

\section{WISS 202.2 Behandlung von Stenosen und Okklusionen der Arteria femoralis superficialis (AFS) mit dem MIMICS 3D-Stent: Single-center Ergebnisse einer prospektiven Studie}

Autoren Scheer $F^{1}$, Luedtke $C^{2}$, Andresen $R^{1}$, Wissgott $C^{1}$

Institut 1 Westküstenklinikum Heide, Akademisches Lehrkrankenhaus der Universitäten Kiel, Lübeck und Hamburg, Institut für Diagnostische und Interventionelle Radiologie/Neuroradiologie Heide, Heide; 2 Klinikum Nordfriesland Husum, Radiologisches Zentrum, Husum DOI 10.1055/s-0037-1600297

Zielsetzung Der limitierende Faktor bei der Behandlung von Läsionen der AFS ist die hohe Restenoserate nach Angioplastie. Ziel dieser prospektiven Studie ist die Evaluierung der Effektivität und Sicherheit des neuartigen MIMICS 3DStents bei der Behandlung von Stenosen und Okklusionen der AFS.

Material und Methodik Es wurden 22 Patienten in die Studie eingeschlossen mittleres Alter 69,4 \pm 8,4 Jahre. Bei allen Patienten wurden im Anschluss an die Angioplastie die helikal konfigurierten MIMICS 3D-Stents implantiert. Im Mittel wurden 1,2 $\pm 0,4$ Stents mit einer mittleren Stentlänge von $86,5 \mathrm{~mm}$ \pm 19 ,8 mm und einem mittleren Durchmesser von $6,1 \mathrm{~mm} \pm 0,5 \mathrm{~mm}$ implantiert. Die mittlere Läsionslänge betrug $80,5 \mathrm{~mm} \pm 32,1 \mathrm{~mm}$. Der durchschnittliche Stenosegrad betrug 93,1 $\pm 8,5 \%$. Präprocedural sowie nach 1 Monat und 12 Monate postinterventionell erfolgte die Bestimmung des Knöchel-ArmIndex (KAI) und die Evaluierung des Rutherford Stadiums und zusätzlich eine farbkodierte Duplexsonografie (FKDS).

Ergebnisse Die technische Erfolgsrate betrug 100\% (22/22). In allen Fällen konnte mittels Implantation eines MIMICS 3D-Stent eine Reststenose < 30\% erzielt werden. Klinisch zeigte sich eine Steigerung des Knöchel-Arm-Index von 0,61 $\pm 0,07$ auf 0,93 $\pm 0,09$ nach 1 Monat und 0,83 $\pm 0,14$ nach 12 Monaten. Außerdem zeigte sich bei allen Patienten eine Reduktion des RutherfordStadiums um mindestens eine Kategorie im 12 monatigen Beobachtungszeitraum. Es traten keine peri- oder postinterventionellen Komplikationen auf. Im gesamten Nachbeobachtungszeitraum gab es 2 Reokklusion (9\%), die klinisch keine Reintervention benötigten.

Schlussfolgerungen Die Implantation eines helikalen MIMICS 3D-Stents ist eine sichere und effektive Alternative zur Behandlung von Stenosen oder Okklusionen der Arteria femoralis superficialis. 


\section{WISS 301.2 Prognostische Wertigkeit der Diffusionswichtung vor Radioembolisation kolorektaler Lebermetastasen}

Autoren Schmeel $\mathrm{F}^{1}$, Simon $\mathrm{B}^{1}$, Luetkens $]^{1}$, Träber $\mathrm{F}^{1}$, Schmeel $\mathrm{L}^{1}$, Meyer $\mathrm{C}^{1}$, Schild $\mathrm{H}^{1}$, Hadizadeh $\mathrm{D}^{1}$

Institut 1 Universitätsklinikum Bonn, Radiologische Klinik, Bonn

DOI 10.1055/s-0037-1600298

Zielsetzung Funktionelle Bildgebungsmethoden wie die MRT-Diffusionswichtung (DWI) können ein Therapieansprechen oftmals vor morphologisch fassbaren Änderungen aufzeigen. Zur Therapieplanung lokal-interventioneller Behandlungsverfahren ist es wünschenswert, die Erfolgsaussichten bereits vor Therapiebeginn abschätzen zu können. Ziel dieser Studie war es daher zu prüfen, ob sich die DWI mittels quantitativer Analyse des Diffusionskoeffizienten (ADC) zur Vorhersage des progressionsfreien (PFS) und Gesamtüberlebens (OS) von Patienten mit kolorektalen Lebermetastasen vor Radioembolisation (RE) eignet.

Material und Methodik 46 Patienten erhielten $20 \pm 17$ Tage vor RE mit 90YMikrosphären eine MRT mit DWI (1.5T, Philips Intera). Prätherapeutische mittlere ADC-Werte $(b=0,50,800)$ wurden innerhalb solider Tumoranteile in den 3 größten Lebermetastasen im Behandlungsareal gemessen und gemittelt. Mit ROC-Analyse wurde ein ADC-Schwellenwert zur Prädiktion eines verlängerten OS definiert, dessen prognostische Wertigkeit für das PFS und OS mittels Kaplan-Meier- und multivariater Cox-Regressionsanalyse untersucht wurde. Weitere untersuchte Prognosefaktoren waren Alter, Geschlecht, ECOG Score, Bilirubin, hepatische Tumorlast und das Vorhandensein extrahepatischer Metastasen.

Ergebnisse Das mediane PFS und OS waren 4 bzw. 8 Monate. Patienten mit einem prätherapeutischen $A D C<935 \mathrm{~mm}^{2} / \mathrm{s} \times 10^{-6}$ wiesen ein kürzeres PFS (3 vs. 5 Monate, $p=0.022$ ) und OS ( 6 vs. 14 Monate, $p=0.02$ ) als Patienten mit höheren Werten auf. Eine hepatische Tumorlast $\geq 25 \%$ war mit einem kürzeren PFS als auch OS assoziiert, wohingegen ECOG Score $>1$ lediglich auf OS einen signifikanten Einfluss hatte. In der multivariaten Analyse verblieb ein niedriger ADC als einziger signifikanter und unabhängiger Risikofaktor zur Prädiktion eines schlechteren OS ( $p=0.036)$.

Schlussfolgerungen Mittels DWI kann bereits vor Radioembolisation deren Therapieerfolg abgeschätzt werden. Dies unterstützt eine evidenzbasierte Patientenselektion zur Vermeidung von Nebenwirkungen und frustraner Therapieversuche.

\section{WISS 310.3 Lokale Tumorkontrolle des HCC nach transarterieller Lipiodolembolisation gefolgt von einer Mikrowellenablation}

Autoren Seidel $R^{1}$, Maßmann $A^{1}$, Fries $P^{1}$, Mehrmann $M^{1}$, Bücker $A^{1}$ Institut 1 Uniklinik Saarland, Klinik für diagnostische uns interventionelle Radiologie, Homburg DOI 10.1055/s-0037-1600299

Zielsetzung Studienziel war die retrospektive Untersuchung des lokalen Tumoransprechens bei Patienten mit HCC nach transarterieller LipiodolEmbolisation und anschließender Mikrowellenablation.

Material und Methodik 48 Patienten mit 70 HCC Läsionen (mittlere Tumorgröße 23 mm, SD $8 \mathrm{~mm}$ ) wurden retrospektiv eingeschlossen. Alle Patienten erhielten eine superselektive Embolisation mittels Lipiodol gefolgt von einer $\mathrm{CT}$ gesteuerten perkutanen Mikrowellenablation durch einen $2,45 \mathrm{GHz}$ Generator (80 bis 120 Watt Energieleistung) und wassergekühlter Sonde (Acculis, Angiodynamics, USA). Alle Patienten erhielten vor der Therapie native und kontrastunterstützte MRT- oder CT Untersuchungen. Die postinterventionellen Kontrollen erfolgten durch CT 1 Tag postinterventionell und durch MRT nach 1, 3, 6 und mehr Monaten. Als erfolgreiche lokale Therapie wurde eine komplette Ablationsnekrose gewertet, die deutlich über den Tumorrand hinaus reichte und kein weiteres Tumorwachstum über mindestens 6 Monate aufwies. Die Daten wurden retrospektiv an einer PACS Station durch zwei Radiologen im Konsens ausgewertet.

Ergebnisse Bei 47 Patienten (98\%) wurde eine komplette Ablation in der frühen Nachsorgeuntersuchung gesehen. Der Patient mit initial inkompletter Ablation zeigte auch im Verlauf einen Progress. Ein weiterer initial als komplett erfasst eingestufter Patient zeigte einen Rezidivverdacht, erhielt eine selektive Chemoembolisation und zeigte keinen residuellen Tumor im Verlauf. Die Komplettablationsrate pro Patient betrug 96\% und 97\% pro Läsion.

Schlussfolgerungen Die Studie zeigt eine hohe lokale Tumorkontrolle bei Patienten mit HCC vergleichbar mit chirurgischen Ergebnissen. Insbesondere die gewebesparende Komponente macht die Methode attraktiv für Patienten mit eingeschränkter Leberfunktion. Die Kombination aus gleichzeitiger Tumorembolisation und Markierung ist ein wichtiger synergistischer Kofaktor für die effiziente Mikrowellenablation.

\section{WISS 209.10 Strahlenexposition während Uterusmyomembolisation (UME): Ein Störgrößen- kontrollierter Vergleich zwischen einem hochmodernen Angiografiesystem und einem konventionellen Angiografiesystem}

Autoren Sommer $\mathrm{C}^{1}$, Voigt $\mathrm{W}^{2}$, Klapp Oliger $\mathrm{M}^{1}$, Schlett $\mathrm{C}^{3}$, Thomas $\mathrm{K}^{1}$, Erpenbach $S^{1}$, Hatopp $A^{1}$, Kurz $P^{1}$, Richter $G^{1}$

Institut 1 Klinikum Stuttgart, Diagnostische und Interventionen Radiologie, Stuttgart; 2 Siemens Healthineers, Erlangen, Germany, Strategy and Innovation, Erlangen; 3 Universitätsklinikum Heidelberg, Diagnostische und Interventionen Radiologie, Heidelberg

DOI 10.1055/s-0037-1600300

Zielsetzung Vergleich der Strahlenexposition zwischen einem hochmodernen und einem konventionellen Angiografiesystem bei Patienten, die sich einer Uterusmyomembolisation (UME) unterziehen

Material und Methodik Zwischen Januar 2009 und Dezember 2014 unterzogen sich insgesamt 245 Patienten einer UME. Einschlusskriterien für diese Retrospektive Studie beinhalteten: erstmalige transarterielle Embolisation aufgrund symptomatischer Myome, bilaterale Embolisation entsprechend eines standardisierten Behandlungsplanes, Prozeduren mittels hochmodernem (Gruppe1) oder konventionellem (Gruppe2) Angiografiesystem sowie bilateraler technischer Erfolg mit adequatem Embolisationsendpunkt nach Injektion größen-kalibrierter biokompatibler Mikrosphären. Studienendpunkte beinhalteten Strahlenexposition, Major-Komplikationsrate und Devaskularisation des dominanten Myoms. Die Kontrolle klinischer and prozeduraler Störgrößen erfolgte mittels Propensity Score Matching

Ergebnisse Die Einschlusskriterien wurden von 27 (Gruppe1) bzw. 177 (Gruppe2) Patienten erfüllt. Nach Durchführung des Propensity Score Matching ergaben sich keine signifikanten Unterschiede zwischen Gruppe1 $(n=25)$ and Gruppe2 ( $n=50)$ in Bezug auf Body-Mass-Index, Volumen des dominanten Myoms, Uterusvolumen, Fluoroskopiezeit, Menge an Embolisat sowie Prozeduren je Radiologe (jeweils $p \geq 0.24$ ). Das Dosisflächenprodukt war significant geringer in Gruppe1 verglichen mit Gruppe2 (1233,8 cGycm2 vs. 3572,9 cGycm2; P<0.001), während die Major-Komplikationsrate und der Grad der Devaskularisation vergleichbar für beide Studiengruppen waren ( $0 \%$ vs. $0 \%$ and 1,0 vs. 1,0; jeweils $P>0.99$ ). Außerdem zeigten die MRT Kontrollen keine signifikanten Unterschiede in Bezug auf Schrumpfung von sowohl Uterus als auch dominantes Myom

Schlussfolgerungen Mittels hochmodernem Angiografiesystem kann eine signifikante Reduktion der Strahlenexposition bei Patienten, die sich einer UME unterziehen, erreicht werden ohne negativen Einfluss auf Komplikationsrate sowie Devaskularisation und Schrumpfung des dominanten Myoms 
WISS 202.1 Bestimmung von Gefäßdurchmessern: Vergleich der semiautomatischen Auswertung von CTAngiografien, kalibrierten extravaskulären Markern und Messkathetern

\author{
Autoren Stahlberg $\mathrm{E}^{1}$, Planert $\mathrm{M}^{2}$, Panagiotopoulos $\mathrm{N}^{3}$, Horn $\mathrm{M}^{4}$, \\ Barkhausen J $J^{5}$, Goltz $J^{5}$ \\ Institut 1 UKSH Campus Lübeck, Klink für Radiologie und Nuklearmedizin, \\ Lübeck; 2 UKSH Campus Lübeck, Klinik für Radiologie und Nuklearmedizin, \\ Lübeck; 3 UKSH Campus Lübeck, Klinik für Radiologie und Nuklearmedizin, \\ Lübeck; 4 UKSH Campus Lübeck, Klinik für Chirurgie, Lübeck; 5 UKSH \\ Campus Lübeck, Klinik für Radiologie und Nuklearmedizin, Lübeck \\ DOI 10.1055/s-0037-1600301
}

Zielsetzung Vergleich der Messgenauigkeit der semiautomatischen Auswertung von CT-Angiografien (CTA) und extravaskulären Markern während der digitalen Subtraktionsangiografie (DSA) mit einem kalibrierten intravaskulären Messkatheter als Referenzstandard.

Material und Methodik Bei 33 konsekutiven Patienten (28 männlich, mittleres Alter $72 \pm 11$ ) wurde ein Messkatheter während der DSA der femoro-poplitealen Achse genutzt. Gleichzeitig wurden ein skaliertes röntgendichtes Klebeband am lateralen Oberschenkel und ein röntgendichtes Lineal auf den Angiografietisch verwendet. Die Gefäßdurchmesser wurden mittels Kalibrierung der verschiedenen Messinstrumente während der DSA und anhand der CTA (SyngoVia, Siemens Healthcare, Erlangen, Deutschland) bestimmt, wobei adipöse $(n=21)$ und nicht-adipöse $(n=12)$ Patienten differenziert wurden. Nach Testung der Normalverteilung, wurde der t-Test für unabhängige Stichproben für den Vergleich der Methoden und der Gruppen durchgeführt $(\mathrm{p}<0,05)$.

Ergebnisse Verglichen mit dem Messkatheter zeigte das Klebeband eine Überschätzung von $1,2 \pm 4,1 \%(p=0,045)$. Die CTA führte zu einer geringen $3.2 \pm 9,7 \%(p=0,048)$, das Lineal zu einer deutlichen Unterschätzung 21,3 $\pm 9.4 \%(p=0,001)$ der Gefäßdurchmesser. Unter Verwendung des Lineals wurden in der proximalen Arteria femoralis superficialis die größten (25\%) und im P2-Segment der Arteria poplitea die geringsten Differenzen gefunden (15\%, $p=0,001)$. Die Unterschiede waren bei adipösen $(23,3 \pm 6,6 \%)$ deutlich größer als bei normgewichtigen $(7,3 \pm 3,1 \%)$ Patienten $(p=0,003)$. Die anderen Messfahren zeigten keine gewichtsabhängigen Unterschiede.

Schlussfolgerungen Im Vergleich zum Messkatheter ermöglichen sowohl das Klebeband am lateralen Oberschenkel als auch die semiautomatische CTA Auswertungen eine exakte Bestimmung der Gefäßdurchmesser. Die Verwendung eines Röntgenlineals führt insbesondere in der proximalen AFS und bei adipösen Patienten zu erheblichen, klinisch relevanten Messfehlern.

\section{RK 321.4 Endovaskuläre Behandlung der Lungenarterienembolie}

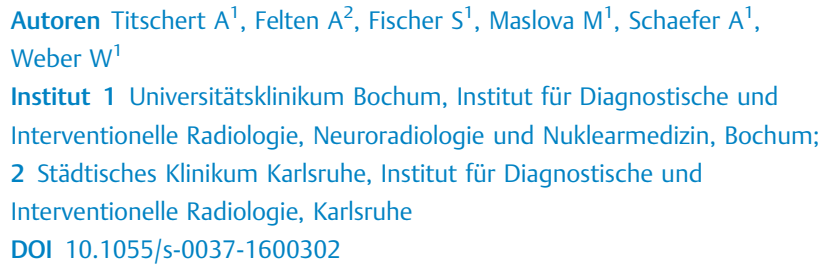

Zielsetzung Prüfung der interventionell-radiologischen Behandlung von Hochrisiko-Patienten mit akuter Lungenarterienembolie durch Aspirationsthrombektomie als Alternative zur Standardtherapie der i.v.-Thrombolyse und der Chirurgischen Embolektomie (Trendelenburg-Operation).

Material und Methodik Bei sechs Hochrisiko-Patienten mit akuter Lungenarterienembolie und Kontraindikationen zur i.v.-Thrombolyse wurde eine lokale Aspirationsthrombektomie durchgeführt unter Verwendung des IndigoKathetersystems (CAT8 Aspirationskatheter und SEP8 Führungsdraht) der Firma Penumbra.
Ergebnisse Bei allen behandelten Patienten konnte eine Senkung des pulmonalarteriellen Drucks, eine Reduktion der Katecholamindosis, eine Normalisierung der Herzfrequenz $(<100 / \mathrm{min})$ und des systolischen Blutdruckes (>100 mm Hg), ein Anstieg der peripheren Sauerstoffsättigung (paO2 >96\%) sowie eine Reduktion des inspiratorischen Sauerstoffanteils (FiO2) am Respirator erreicht werden. Ausschlaggebend für die Interventionsführung sind die o. g. klinischen, therapeutischen und lokalen Parameter, nicht die Bildmorphologie.

Schlussfolgerungen Die interventionell-radiologische Behandlung von Hochrisiko-Patienten mit akuter Lungenarterienembolie durch Aspirationsthrombektomie stellt eine wirksame und risikoarme Alternative bzw. Ergänzung zur Standardtherapie der i. v.-Thrombolyse und der Trendelenburg-Operation dar, insbesondere bei Vorliegen von Lysekontraindikationen. Dies trifft speziell auf (Neuro-) Chirurgische Patienten zu oder in Fällen des Nichtansprechens auf die i.v.-Thrombolyse; die Chirurgische Embolektomie hingegen ist mit hohen Mortalitätsraten behaftet. In der Literatur existieren lediglich kleine Beobachtungsstudien und Fallberichte. Weitere klinische Studien sind nötig, derzeit ist noch keine gesicherte Datenlage erhältlich.

\section{WISS 301.8 CT-gesteuerte Mikrowellenablation von Leberläsionen mit subkapsulärer Lage und/oder transpleuralem Zugangsweg}

\section{Autoren Vo Chieu $V^{1}$, Werncke $T^{1}$, Hensen $B^{1}$, Wacker $F^{1}$, Ringe $K^{1}$}

Institut 1 Medizinische Hochschule Hannover, Institut für Diagnostische und Interventionelle Radiologie, Hannover

DOI 10.1055/s-0037-1600303

Zielsetzung Durchführbarkeit und Ergebnisse der CT-gesteuerten Mikrowellenablation (MWA) von Leberläsionen in subkapsulärer Lage und/oder mit transpleuralem Zugangsweg, im Vergleich zur Behandlung „unkomplizierter“ Läsionen.

Material und Methodik 94 Patienten mit 174 abladierten Leberläsionen ( $\mathrm{n}=97 \mathrm{HCC}$ oder CCA, $\mathrm{n}=77$ Metastasen) wurden in diese retrospektive Studie eingeschlossen. 125 Läsionen (med. DM $17 \mathrm{~mm}$ ) mit subkapsulärer Lage $(n=91)$ und/oder bei denen ein transpleuraler Zugang erfolgte $(n=53)$, wurden identifiziert (Risikogruppe). Komplikationen und Lokalrezidivrate wurden mittels univariater Analyse, logistischer Regression und Kaplan-Meier-Kurven unter Berücksichtigung von Risikofaktoren (Patienten- und Tumoreigenschaften, Ablationsparameter) analysiert und mit den Ergebnissen einer Kontrollgruppe ( $n=49$; med. DM $19 \mathrm{~mm}$ ) verglichen.

Ergebnisse In der Risikogruppe traten Komplikationen in 31 Fällen (24,8\%) auf (Pneumothorax $n=20$, Hämatom $n=11$ ), wobei in fünf Fällen eine Thoraxdrainagenanlage erforderlich war. In der Kontrollgruppe traten Komplikationen signifikant seltener auf $(8,2 \% ; n=4$ (Hämatom); $p=0,001)$. In beiden Gruppen waren die meisten Komplikationen ohne klinische Konsequenz (Kategorie A/B nach der SIR-Klassifikation). Als Risikofaktor für Komplikationen wurden transpleuraler Zugangsweg $(p<0,001)$, Ablationszeit $(p=0,018)$ und Anzahl der Punktionen $(p=0,018)$ identifiziert. In Bezug auf die Lokalrezidivrate (medianes Follow-up 265 Tage) gab es keinen signifikanten Unterschied zwischen beiden Gruppen (10,2 vs. $13,6 \%, \mathrm{p}=0,49$ ).

Schlussfolgerungen Die CT-gesteuerte MWA von subkapsulären Läsionen bzw. unter Verwendung eines transpleuralen Zugangsweges erscheint sicher und effektiv. Komplikationen mit klinischer Konsequenz sind selten und im Hinblick auf das Auftreten eines Lokalrezidivs gibt es keinen signifikanten Unterschied im Vergleich zur Ablation „unkomplizierter“ Läsionen. 


\section{WISS 301.7 Ablationszonengeometrie von Leberherden nach Mikrowellenablation - Evaluation zweier Systeme unter Verwendung einer semi- automatischen Segmentierungssoftware}

Autoren Vo Chieu $\mathrm{V}^{1}$, Wacker $\mathrm{F}^{1}$, Rieder $\mathrm{C}^{2}$, Schumann $\mathrm{C}^{2}$, Ballhausen $\mathrm{H}^{2}$, Ringe $\mathrm{K}^{1}$

Institut 1 Medizinische Hochschule Hannover, Institut für Diagnostische und Interventionelle Radiologie, Hannover; 2 Fraunhofer-Institut für Bildgestützte Medizin MEVIS, Bremen

DOI 10.1055/s-0037-1600304

Zielsetzung 1. Vergleich der Ablationszonengeometrie nach CT-gesteuerter Mikrowellenablation (MWA) von Leberherden mittels zwei unterschiedlicher Ablationssysteme; 2. Evaluation einer semi-automatischen Segmentierungssoftware zum Vergleich der Ablationszonen.

Material und Methodik Die CT-Interventionen von 27 Patienten mit 40 Herden (HCC $n=17$, CCA $n=3$, Metastasen $n=20$ ) wurden retrospektiv analysiert. Jeweils 20 Herde wurden mit einem 2,45 GHz (System 1) bzw. einem $915 \mathrm{MHz}$ (System 2) MWA-System abladiert. Die Segmentierung der Ablationszonen und Berechnung des Elliptizitätsindex wurde unter Verwendung einer semi-automatischen Software durchgeführt (SAFIR; Software Assistant for Interventional Radiology). Zur Validierung der Softwareberechnungen wurden die Ergebnisse (Maximal- und Minimaldurchmesser, Elliptizitätsindex) mit denen manueller Messungen verglichen (Pearson-Korrelation, Mann-WhitneyU-Test).

Ergebnisse Die manuell gemessenen, maximalen transversalen Durchmesser betrugen im Mittel $32 \mathrm{~mm}$ (System 1) und $27 \mathrm{~mm}$ (System 2). Manuelle und semi-automatische Messungen korrelierten sehr gut für den maximalen $(r=0,83, p<0,0001)$ und mäßig für den minimalen transversalen Durchmes$\operatorname{ser}(r=0,46, p=0,003)$. Die mit System 1 erzeugten Ablationszonen hatten im Vergleich zu System 2 einen signifikant niedrigeren Elliptizitätsindex $(1,17$ $\pm 0,23$ vs. $1,86 \pm 0,51, p<0,0001$ ) in der nicht-automatischen Analyse, wobei ein Elliptizitätsindex von 1 eine vollkommen sphärische Ablationszone anzeigte. Diese Ergebnisse korrelierten gut mit denen der Softwareauswertung $(r=0,79, p<0,0001)$.

Schlussfolgerungen Das 2,45 GHz Mikrowellen-Ablationssystem erzielte im Vergleich zum $915 \mathrm{MHz}$-System eine deutlich sphärischere Ablationszone. Die Messungen der Ablationszonengeometrie mittels SAFIR korrelieren gut mit den manuellen Messungen. Zusatzinformationen, welche durch die Software geliefert werden, könnten im Rahmen der Planung und Durchführung komplexer Ablationen hilfreich sein, was es gilt in prospektiven Studien zu prüfen.

\section{WISS 310.8 Chemosaturation mittels perkutaner hepatischer Perfusion von Melphalan: Ergebnisse nach der Behandlung von Patienten mit nicht-resektablen isolierten hepatischen Metastasen bei okulärem Melanom}

Autoren Vogl $T^{1}$, Koch $S^{2}$, Gebauer $B^{3}$, Willinek $W^{4}$, Engelke $C^{5}$, Brüning $R^{6}$, Enk $A^{7}$, Wacker $F^{8}$

Institut 1 Uniklinik Frankfurt, Institut für Diagnostische und Interventionelle Radiologie, Frankfurt a.M.; 2 Uniklinik Frankfurt, Institut für Diagnostische und Interventionelle Radiologie, Frankfurt; 3 Charite Berlin, Institut für Radiologie, Berlin; 4 Brüderkrankenhaus Trier, Institut für Radiologie, Trier;

5 Evangelisches Krankenhaus, Institut für Radiologie, Göttingen; 6 Asklepios Kliniken, Institut für Radiologie, Hamburg; 7 Uniklinik Heidelberg, Institut für Radiologie, Heidelberg; 8 Med. Hochschule Hannover, Institut für

Diagnostische und Interventionelle Radiologie, Hannover

DOI 10.1055/s-0037-1600305

Zielsetzung Retrospektive Evaluation der palliativen Behandlung von nichtresektablen Lebermetastasen bei okulärem Melanom mittels perkutaner he- patischer Perfusion (PHP; Hepatic CHEMOSAT ${ }^{\circledR}$ Delivery System; Delcath Systems Inc., USA).

Material und Methodik Zwischen 2012 und 2015 erhielten 18 Patienten mit hepatischen Metastasen bei okulärem Melanom ein bis drei Sitzungen Chemosaturationstherapie. 17 Patienten wurden mittels RECIST Kriterien ausgewertet. Eine Überlebenszeitanalyse wurde durchgeführt und unerwünschte Ereignisse dokumentiert.

Ergebnisse Die Chemosaturation zeigte sich gut verträglich bei der Mehrzahl der Patienten. Nach der Therapie entwickelten sieben Patienten eine Leukopenie, sechs eine Thrombopenie, jeweils 2 Patienten zeigten eine Neutropenie, eine Infektion sowie Fieber. Von den 17 Patienten, die mittels RECIST-Kriterien ausgewertet wurden, zeigten 9 (53\%) eine partielle Remission (PR), eine stabiles Krankheitsbild (SD) wurde bei sechs Patienten (35\%) beobachtet und bei zwei (12\%) kam es zu einem Fortschreiten der Erkrankung (PD). Drei Patienten erhielten eine weitere Sitzung. Nach dem zweiten Zyklus veränderte sich das Tumoransprechen aller Patienten von SD zu PR. 4 Patienten hatten insgesamt 3 Sitzungen. Das Behandlungsansprechen der anderen 3 Patienten war PR in allen 3 Sitzungen. Das Gesamtüberleben nach der ersten Therapiesitzung beträgt 4 bis 29,8 Monate (medianes Gesamtüberleben 9,6 Monate). Die mediane Überlebenszeit bei den beiden PD Patienten betrug 3,6 Monate. Ein Patient verstarb 1,7 Monate nach der Therapie.

Schlussfolgerungen Chemosaturation-PHP zeigt sich als eine sichere und effektive Behandlungsoption für Patienten mit nicht-resektablen hepatischen Metastasen bei okulärem Melanom.

\section{WISS 310.4 Prospektive randomisierte FAST II-Studie: Tumoransprechen beim hepatozellulären Karzinom (HCC) nach transarterieller Chemoembolisation (TACE) mit zwei verschiedenen Protokollen mittels MRT}

Autoren Vogl $\mathrm{T}^{1}$, Langenbach $\mathrm{M}^{2}$, Gruber-Rouh $\mathrm{T}^{2}$, Hammerstingl $\mathrm{R}^{2}$

Institut 1 Uniklinik Frankfurt, Institut für Diagnostische und Interventionelle Radiologie, Frankfurt a.M.; 2 Uniklinik Frankfurt, Institut für Diagnostische und Interventionelle Radiologie, Frankfurt

DOI 10.1055/s-0037-1600306

Zielsetzung Vergleich von zwei verschiedenen Embolisationsprotokollen für die transarterielle Chemoembolisation (TACE) bei hepatozellulärem Karzinom (HCC) bezüglich Tumorvolumen, RECIST/mRECIST-Kriterien und dem apparenten Diffusionskoeffizienten (ADC) in einer randomisierten, doppelverblindeten Studie.

Material und Methodik Insgesamt wurden 49 Patienten (32 Männer, 17 Frauen; Durchschnitt: 70 Jahre; Bereich: 43 - 84) mit HCC in die Studie eingeschlossen. Das Behandlungsprotokoll war wie folgt: drei TACE-Sitzungen in 4-wöchigem Abstand mit MRT vor der TACE und 4 Wochen nach der letzten TACE-Sitzung. Die Patienten wurden randomisiert auf zwei verschiedene Gruppen verteilt: Gruppe 1 mit Lipiodol allein und Gruppe 2 mit Lipiodol in Kombination mit Embocept. Tumorvolumen, RECIST und mRECIST wurden nach jedem Scan evaluiert, ADC nur für die erste und letzte MRT.

Ergebnisse Eine durchschnittliche Reduktion des Tumorvolumens von 2,15\% wurde dokumentiert in Gruppe 1 vs. 1,39\% in Gruppe 2 ( $p=0,3849$ ), und eine Reduktion des Durchmessers von 11,8\% (Gruppe 1) vs. 18,32\% (Gruppe 2) $(p=0,72)$. Bezüglich der RECIST/mRECIST-Kriterien zeigte Gruppe 1 ein stabiles Krankheitsbild (SD) in 18 Fällen, ein Fortschreiten der Tumorerkrankung $(P D)$ in 4 Fällen und partielle Remission (PR) in einem Fall. Gruppe 2 zeigte PR $(n=4)$, SD $(n=20)$ und PD $(n=2)$. Während der Behandlung stieg der ADCKoeffizient durchschnittlich an auf $878,46 \mathrm{~mm}^{2} / \mathrm{s}$ in Gruppe 1 vs. $578,91 \mathrm{~mm}^{2}$ in Gruppe 2 ( $p=0,4336)$.

Schlussfolgerungen Bei der lokalen Tumorkontrolle wurde kein signifikanter Unterschied zwischen Gruppe 1 und 2 festgestellt. Eine Korrelation der Tumorvolumenreduktion nach TACE und ein Anstieg der ADC-Werte wurden dokumentiert. Damit könnte derADC ein individueller Prädiktor für den Erfolg der TACE-Behandlung sein. 


\section{RK 301.5 Mikrowellenablation von Lungenmetastasen mit konstanter Energiekontrolle zum Erreichen einer sphärischen Ablationszone: Retrospektive Evaluation}

Autoren Vogl $T^{1}$, Basten $\mathrm{L}^{1}$, Ackermann $\mathrm{H}^{2}$, Nour-Eldin $\mathrm{N}^{1}$, $\mathrm{Nag}^{-1}$ Institut 1 Uniklinik Frankfurt, Institut für Diagnostische und Interventionelle Radiologie, Frankfurt a.M.; 2 Universität Frankfurt, Institut für Medizinische Statistik, Frankfurt a.M.

DOI 10.1055/s-0037-1600307

Zielsetzung Evaluation eines neuen Mikrowellenablationssystems mit konstanter Energiekontrolle (Thermosphere ${ }^{\top \mathrm{M}}$ ) zur Therapie von Lungenmetastasen zum Erzielen einer lokalen Ortskontrolle.

Material und Methodik Zwischen 05/14 und 11/15 wurden in dieser retrospektiven Studie 45 Patienten (23 Männer, 22 Frauen; Durchschnittsalter 58,6 Jahre; Bereich 27 - 75) in 45 Sitzungen mittels Mikrowellenablation (MWA) mit Thermosphere ${ }^{\mathrm{TM}}$ Technologie behandelt. Die Bewertung der sphärischen Morphologie der Ablationszone erfolgte anhand der CT-Bilder 24 Stunden nach der Intervention nach folgendem Schema: Für jeden einzelnen transversalen und longitudinalen Anschnitt der Ablationszone wurden Länge, Breite und deren Verhältnis ausgemessen. Vom Mittelwert dieser Seitenverhältnisse wurde pro Ablationszone die Abweichung vom Zielwert 1.0 (entsprechend einer Kugel) berechnet. Die gleichen Parameter wurden retrospektiv mittels konvenntioneller Niederfrequenz-(NF)-MWA von 45 Sitzungen evaluiert. Die Ergebnisse beider Gruppen wurden mit Wilcoxon-Mann-Whitney-Test miteinander verglichen.

Ergebnisse Thermosphere Interventionen waren in 97,8\% der Fälle erfolgreich, NF-MWA in $100 \%$. Insgesamt betrug die Komplikationsrate $22,2 \%$ mit Thermosphere und 31,1\% mit NF-MWA: leichte Komplikationen 17,8\% und $20,0 \%$, schwerwiegende Komplikationen $4,4 \%$ und $11,1 \%(p=0,474)$. Die durchschnittliche Interventionsdauer betrug 7,98 min (Median: 8,0 min) für Thermosphere und 9,59 min (Median: 10,0 min) für NF-MWA mit einem signifikanten Unterschied $(p=0,005)$ Für die morphologische Evaluation der Ablationszone ergab sich für die Abweichung von 1,0 einen Medianwert von 0,194 (Bereich 0,078-0,500) für Thermosphere-Interventionen und 0,384 (Bereich: 0,218-0,610) für NF-MWA mit einem signifikanten Unterschied $(\mathrm{p}<0,0001)$.

Schlussfolgerungen Die MWA bei oligonodulären Lungenläsionen mittels Thermosphere erzielt eine deutliche sphärische Ablationszone in signifikant kürzerer Ablationszeit und eine geringere Komplikationsrate bei hoher Ortskontrolle.

\section{Kinderradiologie}

\section{RK/WISS 320.2 Evaluation der Strahlenexposition thorakaler CT-Untersuchungen im Kindesalter unter Verwendung eines Dual-Source CT der 3. Generation}

\footnotetext{
Autoren Kremp $\mathrm{K}^{1}$, Seidel $\mathrm{R}^{1}$, Pfeifer $\mathrm{J}^{2}$, Abdul-Khaliq $\mathrm{H}^{2}$, Krenn $\mathrm{T}^{3}$, Altmeyer $K^{1}$, Bücker $A^{1}$, Fries $P^{1}$

Institut 1 Universitätsklinikum des Saarlandes, Klinik für Diagnostische und Interventionelle Radiologie, Homburg; 2 Universitätsklinikum des Saarlandes, Klinik für Kinderkardiologie, Homburg; 3 Universitätsklinikum des Saarlandes, Klinik für pädiatrische Onkologie und Hämatologie, Homburg

DOI 10.1055/s-0037-1600308

Zielsetzung Ziel dieser Untersuchung war die retrospektive Analyse der Strahlenexposition bei CT-Untersuchungen des Thorax im Kindesalter unter Verwendung eines Dual-Source CT der 3. Generation (Siemens Force).

Material und Methodik Bei 56 pädiatrischen Patienten (Alter(MW+/-SA): 7,66 $+/-6,8 \mathrm{~J} / 0-17,97 \mathrm{~J})$ wurden $73 \mathrm{CT}$-Untersuchungen des Thorax bei unterschiedlichen klinischen Indikationen durchgeführt. Die CT wurden in HighPitch-Technik (Kollimation: $192 \times 0,6$; Pitch: 2,8) mit automatischer Exposi-
}

tionskontrolle (CAREdose4D, Siemens) und zum Teil mit $100 \mathrm{kV}$ Zinnfilter (100 kV Sn) akquiriert. Mit dem Dosis-Längen-Produkt (DLP) wurde retrospektiv die korrespondierende effektive Dosis $(\mathrm{E})$ berechnet $(\mathrm{E}=$ DLPxkappa (kappa: Alter- und kV-abhängige Konversionsfaktoren).

Ergebnisse Die Untersuchungen erfolgten aufgrund kardiovaskulärer $(\mathrm{N}=38)$ und nicht kardiovaskulärer Indikationen, u. a. Entzündungen ( $N=15)$, Tumoren $(N=13)$ und angeborener Lungenerkrankungen $(N=7)$. Die durchschnittliche Strahlenexposition für alle Untersuchungen betrug $E($ alle $)=0,85+/-0,56 \mathrm{mSv}$ (Bereich: 0,04-2,97 mSv). Für die kardiovaskulären CT war die Dosis signifikant niedriger als für die nicht-kardiovaskulären $C T(E($ kardiovask $)=0,70+1$ $0,33 \mathrm{mSv} ; \mathrm{E}$ (non-kardiovask)=1,13+/-0,63 mSv; $\mathrm{P}<0,001)$. Dies beruht auf den signifikant niedrigeren Röhrenspannungen bei kardiovaskulären CT (Median: $70 \mathrm{kV}$; Bereich:70 - 80 kV) gegenüber nicht-kardiovaskulären CT (median: $100 \mathrm{kV}$, Bereich: $70-120 \mathrm{kV}, \mathrm{P}<0,001)$. Bei nativen $\mathrm{CT}$ kann durch ein $100 \mathrm{kV}$ Sn Protokoll $(\mathrm{E}(100 \mathrm{kV} \mathrm{SN})=0,16+/-0,02 \mathrm{mSv})$ die Strahlenexposition signifikant gegenüber konventionellen High-Pitch Protokollen gesenkt werden ( $E(C T-T h$ nativ $)=1,3+/-0,7 \mathrm{mSv}, \mathrm{p}<0,0001)$.

Schlussfolgerungen Mit einem Dual-Source CT der 3. Generation können mittels High-Pitch Protokollen und automatischer Expositionskontrolle CT des Thorax im Kindesalter mit einer Strahlenexposition $<1 \mathrm{mSv}$ für ein breites klinisches Spektrum durchgeführt werden. Niedrige kV-Stufen und Protokolle mit 100 kV Zinnfilter zeigen dabei besonders niedrige Dosiswerte.

\section{RK/WISS 404.6 Einfluss von intranasal verabreichter Muttermilch bei Frühgeborenen mit hochgradigen intraventrikulären Blutungen - erste sonografische Ergebnisse}

Autoren Körber $F^{1}$, Wengenroth $L^{2}$, Oberthuer $A^{2}$, Mehler $K^{2}$, Kribs $A^{2}$, Keller $\mathrm{T}^{2}$

Institut 1 Universitätsklinik Köln, Institut für Diagnostische und Interventionelle Radiologie - Schwerpunkt Kinderradiologie, Köln; 2 Uniklinik Köln, Klinik und Poliklinik f. Kinder- u. Jugendmedizin, Schwerpunkt Neonatologie und Pädiatrische Intensivstation, Köln

DOI 10.1055/s-0037-1600309

Zielsetzung Hochgradige intraventrikuläre Blutungen (IVH) bei Frühgeborenen (FG) haben eine hohe Mortalität und Morbidität. Experimentelle Studien zeigen, dass die intranasale Applikation von epidermalem Wachstumsfaktor (EGF) effiziente neuroprotektive Effekte bei neonatalen Hirnschädigungen hat. Muttermilch, die beim Stillen physiologischerweise den Nasen-Rachenraum durchfließt, enthält neben EGF in hoher Konzentration Neurotrophine und immunologisch wirksame Zellen. Eine effiziente Zellmigration über die Lamina cribrosa wurde experimentell nachgewiesen.

Material und Methodik Von 31 FG mit einer IVH Grad III-IV wurden 16 Kinder mit intranasal verabreichten Tropfen Milch der eigenen Mutter behandelt (Interventionsgruppe - IG: $9 \mathrm{ml}, 7$ wbl, mittleres Gestationsalter 24,9 SSW, Geburtsgewicht $770 \mathrm{~g}$ ). 15 FG mit gleich schweren Blutungen erhielten zwar Muttermilch, nicht jedoch intranasal (Kontrollgruppe - KG: $11 \mathrm{ml}, 4 \mathrm{wbl}$, 24,4 SSW, 684 g). Die Schädelsonografien von Geburt bis Entlassung wurden retrospektiv von 2 erfahrenen Untersuchern bezüglich der Schwere der Liquorzirkulationsstörungen und der Marklagerschäden geblindet ausgewertet.

Ergebnisse Die Inzidenz progressiver Liquorzirkulationsstörungen stellte sich in der IG mit $71 \%$ niedriger dar als in der KG ( $91 \%)$, ebenso die Rate erforderlicher chirurgischer Entlastungs-Operationen (50\% versus $67 \%$ ). Insbesondere war die Zahl relevanter periventrikulärer Marklagerdefekte bei Entlassung in der IG niedriger (21\% versus 58\%). 6 Kinder der IG zeigten einen für das Ausmaß der Blutung ungewöhnlich guten Verlauf. Eine statistische Signifikanz wurde bei der kleinen Fallzahl nicht erreicht.

Schlussfolgerungen In Zusammenschau mit dem Nachweis von Neurotrophinen und aktiven Immunzellen in Muttermilch ergibt sich die Hypothese, dass die frühe intranasale Applikation einen unterstützenden Effekt auf die neurologische Entwicklung von FG hat. Weitere Untersuchungen an größeren 
Kollektiven und die Korrelation der Morphologie mit dem klinisch-neurologischen Outcome werden erforderlich sein.

\section{RK/WISS 414.4 Einsatz der Scherwellen Elastografie (SWE) zur Beurteilung intrakranieller Druckverhältnisse bei gesunden Säuglingen und Säuglingen mit Hydrocephalus - eine Machbarkeitsstudie}

Autoren Meiser $\mathrm{N}^{1}$, Panek $\mathrm{A}^{2}$, Treppels-Kottek $\mathrm{S}^{2}$, Orlikowsky $\mathrm{T}^{2}$, Kuhl $\mathrm{C}^{1}$, Schrading $S^{1}$

Institut 1 Uniklinik RWTH Aachen, Klinik für Diagnostische und Interventionelle Radiologie, Aachen; 2 Uniklinik RWTH Aachen, Klinik für Kinder- und Jugendmedizin, Aachen

DOI 10.1055/s-0037-1600310

Zielsetzung Die Beurteilung der Gewebesteifigkeit mittels der SchwerwellenElastografie (SWE) hat sich an verschiedenen Organsystemen in der klinischen Routine fest etabliert. Ziel der Studie war es, erste Erfahrung zu den mittels SWE ermittelten Elastizitätsverhältnisse im Hirnparenchym bei gesunden Säuglingen und Säuglingen mit Hydrozephalus und erhöhtem Hirndruck zu gewinnen.

Material und Methodik Es wurden 70 gesunde und 25 Säuglinge mit Hydrozephalus und bekanntem erhöhtem Hirndruck systematisch mittels SWE untersucht. Die SWE wurde von zwei, in der Anwendung der SWE erfahrenen, Radiologen unter Verwendung eines $10.5 \mathrm{MHz}$-Linearschallkopfes (Aixplorer, Supersonic) in standardisierter Technik durchgeführt. Bei jedem Säugling wurden beidseits paraventrikulär ROI-basiert je 3 SWE-Messungen akquiriert und der Mittelwert der SWEmax-Werte gebildet. Die SWEmax-Mittelwerte der gesunden und der symptomatischen Säuglinge wurden mittels des Student's t-Test verglichen.

Ergebnisse Bei 62 der 70 (89\%) gesunden Säuglinge konnte die SWE technisch erfolgreich durchgeführt werden. Der SWEmax-Werte Mittelwert lag bei den gesunden Säuglingen bei 16,2 kPA, Median 16 kPA, Standardabweichung 3,4 kPA. Bei 24/25 (96\%) Säuglingen mit Hydrozephalus konnte die SWE erfolgreich durchgeführt werden. Der Mittelwert der SWEmax-Werte lag bei diesen bei 26,4 kPA, Median 30 kPA, Standardabweichung 8,5 kPA. Der Unterschied der SWEmax-Werte der gesunden Säuglingen und der mit Hydrozephalus war statistisch signifikant $(p=0,013)$.

Schlussfolgerungen Die SWE des Hirnparenchyms ist technisch machbar und liefert reproduzierbare Ergebnisse. Die mittels der SWE erhobenen Elastizitätswerte bei Säuglingen mit Hydrozephalus sind signifikant höher sind als bei gesunden Säuglingen. Wenn sich diese ersten Ergebnisse in größeren Studien reproduzieren lassen, wäre die SWE eine nützliche Methode zur ergänzenden Diagnostik und Monitoring von Kindern mit nachgewiesenem oder vermuteten Hirndruck.

\section{RK/WISS 414.8 Objektive Bildqualität der kranialen MRT bei Früh- und Neugeborenen mit und ohne Anwendung des MR-kompatiblen Inkubators}

\footnotetext{
Autoren Müller $\mathrm{F}^{1}$, Proquitté $\mathrm{H}^{2}$, Renz $\mathrm{D}^{1}$, Mentzel $\mathrm{H}^{1}$

Institut 1 Universitätsklinikum Jena, Sektion Pädiatrische Radiologie, Institut für Diagnostische und Interventionelle Radiologie, Jena; 2 Universitätsklinikum Jena, Sektion Neonatologie und Pädiatrische Intensivmedizin, Klinik für Kinderund Jugendmedizin, Jena

DOI 10.1055/s-0037-1600311

Zielsetzung Die Untersuchung im MR-kompatiblen Inkubator (MRcl) gestattet unkompliziert und sicher eine MRT auch bei Früh- und Neugeborenen. In dieser Studie sollte die objektive Bildqualität cranialer MRT (cMRT) unter Einsatz des MRcl im Vergleich zur cMRT ohne MRcl untersucht werden.

Material und Methodik 100 cMRT bei 1,5 T (Siemens) von 79 Patienten ( $45 \mathrm{~m}, 34 \mathrm{w}$; medianes korrigiertes postnatales Alter 1,00 Wo. 8 Wo.) wurden
}

retrospektiv analysiert. Einschlusskriterien waren korrigiertes Alter von max. 26 Lebenswochen und Vorhandensein der Sequenzen: TSE PD tra, TSE T2 tra, TSE T2 sag, SE T1 tra. Zur objektiven Einschätzung der Bildqualität wurde das Signal-To-Noise-Ratio (SNR) im Thalamus und im parietalen Marklager sowie das Contrast-To-Noise-Ratio (CNR) berechnet. Die Untersuchungen wurden in zwei Gruppen eingeteilt: Untersuchungen mit $\mathrm{MRcl}(n=76)$ und ohne MRcl $(n=24)$. Diese wurden hinsichtlich der SNRs und CNRs verglichen. Die Untersuchungen ohne MRcl erfolgten vorwiegend in Narkose, die mit MRcl mehrheitlich nach Füttern oder in Sedierung.

Ergebnisse Das statistische Regressionsmodell zeigte signifikante Unterschiede in den geschätzten Mittelwerten der SNRs und CNRs zugunsten der cMRT mit MRcl. Die Mittelwerte der SNRs im Marklager lagen in TSE PD tra mit MRcl bei 214,0 $\pm 15,1$ und ohne MRcl bei 145,5 $\pm 19,0$ ( $p=0,019)$, in TSE T2 tra mit MRcl bei $156,2 \pm 13,0$ und ohne MRcl bei $98,9 \pm 17,3$ ( $p=0,029)$, in TSE T2 sag mit MRcl bei 121,9 $\pm 11,2$ und ohne MRcl bei 36,9 $\pm 15,9(p<0,001)$. Die Mittelwerte der SNRs im Thalamus waren in TSE T2 sag signifikant höher mit MRcl $(87,5 \pm 8,3)$ als ohne MRcl $(37,9 \pm 11,7 ; p<0,005)$. Die Differenzen der Mittelwerte der CNRs lagen in TSE T2 sag mit MRcl um 37,1 \pm 8,9 höher als ohne MRcl $(p<0,001)$ und in TSE T2 tra mit MRcl um 25,8 \pm 8,3 höher als ohne $\operatorname{MRcl}(p=0,002)$. In SE T1 tra traten keine signifikanten Unterschiede auf. Schlussfolgerungen Die craniale MRT von Früh- und Neugeborenen im MRkompatiblen Inkubator gestattet eine Verbesserung der objektiven Bildqualität.

\section{RK/WISS 310.4 Synoviale Bildgebung mit intravoxel incoherent motion - MRT bei Patienten mit juveniler idiopathischer Arthritis}

Autoren Neubauer $\mathrm{H}^{1}$, Fabian $\mathrm{H}^{1}$, Sauer $\mathrm{A}^{1}$, Holl-Wieden $\mathrm{A}^{2}$, Köstler $\mathrm{H}^{1}$ Institut 1 Uniklinikum Würzburg, Institut für Diagnostische und Interventionelle Radiologie, Würzburg; 2 Uniklinikum Würzburg DOI 10.1055/s-0037-1600312

Zielsetzung Wir untersuchten, ob die "intravoxel incoherent motion" (IVIM) MRT zur Darstellung der synovialen Mikroperfusion am Kniegelenk bei Patienten mit juveniler idiopathischer Arthritis (JIA) diagnostisch einsetzbar ist. Material und Methodik 12 Patienten (10 Mädchen, medianes Alter 11 Jahre) mit diagnostizierter oder klinisch vermuteter JIA erhielten in der Routine-MRT bei 1,5 T neben der kontrastverstärkten T1-Bildgebung (ce-T1w) eine Diffusionsmessung (RESOLVE, b-Werte 0, 200, 400 und $800 \mathrm{~s} / \mathrm{mm} 2$ ). Die IVIMParameter tissue diffusion coefficient (D) und die Perfusionsfraktion (f) wurden berechnet. Diffusionsbilder $b=800$ und die $f$-Karten wurden durch zwei unabhängige und verblindete Untersucher retrospektiv mit der ce-T1w hinsichtlich Synovialitis und diagnostischer Sicherheit verglichen.

Ergebnisse 8 (67\%) Patienten zeigten eine floride Synovialitis in ce-T1w. Die Auswertung der f-Karten im Vergleich zu ce-T1w erbrachte vollständig konsistente Beurteilungen beider Untersucher. Anhand der Diffusionsbilder konnten dagegen zwei Patienten nicht sicher zugeordnet werden. Der Median der Perfusionsfraktion $f$ wurde als $6,7 \pm 2,0 \%$ für entzündete Synovia, $2,1 \pm 1,2 \%$ für Gelenkerguss, 5,0 $\pm 1,0 \%$ für Muskelgewebe und 10,6 $\pm 5,7 \%$ für reaktive popliteale Lymphknoten gemessen. Der between-measurements coefficient of variation (CV) betrug 7,0\% für D (Synovia), 11,5\% für D (Erguss), 30,6\% für $f$ (Synovia) und 32\% für $\mathrm{f}$ (Erguss).

Schlussfolgerungen IVIM-MRT ermöglicht in der klinischen Routinediagnostik kombinierte Diffusions-/Perfusionsbildgebung des Kniegelenks ohne i.v. Kontrastmittelgabe und visualisiert anhand der Perfusionsfraktion zuverlässig synoviale Hyperperfusion bei Arthritis-Patienten. Die Abgrenzbarkeit Synovia vs. Erguss wird durch die f-Karten im Vergleich zur Diffusionsbildgebung verbessert. 


\section{RK/WISS 404.4 Vergleich der MRT-Charakteristika molekularer Subgruppen bei kindlichen supratentoriellen Ependymomen unter Berücksichtigung der neuen WHO-Klassifikation}

\author{
Autoren Nowak J' \\ Institut 1 Universitätsklinikum Würzburg, Radiologie, Würzburg \\ DOI 10.1055/s-0037-1600313
}

Zielsetzung Die neue WHO-Klassifikation teilt ZNS-Tumoren erstmals auch nach Ihrer Molekulargenetik ein. Hierbei kommen supratentorielle Ependymome (ST-EPN) mit C11orf95-RELA Fusionen in über 70\% der betroffenen Patienten vor. Zudem wurde gezeigt dass CDKN2A Deletionen mit einer besonders schlechten Prognose korrelieren. In der vorliegenden Studie werden die MRT-Charakteristika von pädiatrischen ST-EPN mit RELA-Fusion sowie mit/ohne CDKN2A Deletion erstmals systematisch analysiert und den übrigen ST-EPN gegenübergestellt.

Material und Methodik Die Studienpopulation umfasst 57 pädiatrische STEPN. Sämtliche Fälle unterlagen einem zentralen Review bezüglich mRNA Analyse auf RELA-Fusionen, Immunhistochemie für p65RelA und Genomanalysen mit molecular inversion profiling. Ein Paarvergleich 25 verschiedener MRTParameter wurde durchgeführt zwischen i) RELA-fusionierten/übrigen ST-EPN, ii) ST-EPN mit/ohne CDKN2A Deletion, und iii) RELA-fusionierten ST-EPN mit/ ohne CDKN2A Deletion.

Ergebnisse Zellreiche hemisphärische Tumoren mit zystischen Anteilen und Nekrosen wurden in sämtlichen Subgruppen von ST-EPN gefunden. Es fanden sich signifikante Unterschiede zwischen RELA-fusionierten und den übrigen ST-EPN bezüglich Perifokalödem, Zysten, T1 Signalintensität, Signalhomogenität (T1/T2) und Gd-Anreicherung. Tumoren mit CDKN2A Deletionen wurden in einem höheren Patientenalter diagnostiziert, zudem fielen Unterschiede bezüglich intratumoraler Hämorrhagien und Diffusionsrestriktion in dieser Subgruppe auf. Innerhalb der ST-EPN mit RELA-Fusion fanden sich signifikante Unterschiede bezüglich Diagnosealter und Hämorrhagien, abhängig vom CDKN2A Deletionsstatus.

Schlussfolgerungen Die durchgeführte Studie beschreibt erstmals im Detail die MRT-Morphologie von ST-EPN in Abhängigkeit von der molekularen Subgruppierung. Eine sichere Vorhersage des Genotyps von ST-EPN bei Kindern scheint mit dem MRT im Einzelfall nicht möglich zu sein. Weitere Studien sind nötig um die Rolle der MRT-Bildgebung für das Therapiemonitoring der Subgruppen zu überprüfen.

\section{WISS 305.4 T1-Signalintensität des Nucleus dentatus und des Globus pallidus nach mehrfachen MR- Kontrastmittel-Applikationen bei Kindern und Jugendlichen: Vergleich von Gadopentetat- Dimeglumin und Gadobutrol}

Autoren Renz $D^{1}$, Kümpel $S^{1}$, Böttcher $\rfloor^{2}$, Waginger $M^{1}$, Pfeil $A^{3}$, Streitparth $\mathrm{F}^{4}$, Teichgräber $\mathrm{U}^{5}$, Mentzel $\mathrm{H}^{1}$

Institut 1 Universitätsklinikum Jena, Institut für Diagnostische und Interventionelle Radiologie/Kinderradiologie, Jena; 2 SRH Wald-Klinikum Gera, Institut für Diagnostische und Interventionelle Radiologie, Gera;

3 Universitätsklinikum Jena, Klinikum für Innere Medizin III, Jena; 4 Charité Universitätsmedizin Berlin, Klinik für Radiologie, Berlin; 5 Universitätsklinikum Jena, Institut für Diagnostische und Interventionelle Radiologie, Jena DOI 10.1055/s-0037-1600314

Zielsetzung Für Erwachsene gibt es zahlreiche Evaluationen, ob mehrfache intravenöse Applikationen von MR-Kontrastmitteln zu Gadolinium-Ablagerungen in tiefen Hirnkernen führen können. Im Gegensatz dazu sind Ergebnisse diesbezüglich für Kinder und Jugendliche rar. Das Ziel dieser Studie war daher ein Vergleich des linearen Kontrastmittels Gadopentetat-Dimeglumin und des makrozyklischen Wirkstoffs Gadobutrol in einem pädiatrischen Kollektiv mit der Fragestellung, ob mehrfache Gaben Auswirkungen auf die T1-Signalintensität in tiefen Hirnkernen haben.

Material und Methodik Eingeschlossen wurden 24 Patienten (mittleres Alter 7,3 $\pm 6,5$ Jahre; 10 Jungen, 14 Mädchen) mit mindestens dreifacher Applikation von Gadopentetat-Dimeglumin und 20 Patienten (9,9 $\pm 5,5$ Jahre; 12 Jungen, 8 Mädchen) mit mindestens drei Gaben von Gadobutrol. Als wichtiges Ausschlusskriterium fungierten eine Radiatio des Kopfes und eine Chemotherapie. Die T1-Signalintensität von Nucleus dentatus, Pons, Globus pallidus und Thalamus wurde mittels manuell eingezeichneter „Regions of interest“ bestimmt und das Verhältnis von Nucleus dentatus zu Pons (DNP) und Globus pallidus zu Thalamus (GPT) berechnet.

Ergebnisse Für Gadobutrol zeigte sich kein signifikanter Unterschied der T1Ratios DNP und GPT zwischen dem initialen MRT und nach dreifacher KMApplikation: initialer Mittelwert DNP 0,98 $\pm 0,08$ und GPT 1,02 $\pm 0,04$, Mittelwert nach drei KM-Gaben DNP 0,99 $\pm 0,05$ und GPT 1,02 $\pm 0,08$ (t-Tests für verbundene Stichproben $p>0,05$ ). Für Gadopentetat-Dimeglumin zeigte sich jedoch ein signifikanter T1-Signalanstieg des Nucleus dentatus und Globus pallidus im Verlauf: initialer Mittelwert DNP 0,99 $\pm 0,06$ und GPT 1,02 $\pm 0,04$, Mittelwert nach drei KM-Gaben DNP $1,05 \pm 0,06$ und GPT $1,04 \pm 0,04$ ( $p=0,003$ und $p=0,004)$.

Schlussfolgerungen Ähnlich wie bei Erwachsenen führen mehrfache Applikationen des linearen Kontrastmittels Gadopentetat-Dimeglumin zu einem T1Signalanstieg des Nucleus dentatus und des Globus pallidus bei Kindern und Jugendlichen, im Gegensatz zu dem makrozyklischen Kontrastmittel Gadobutrol.

\section{RK/WISS 404.5 Zerebrale Mikroblutungen und kortikale Siderose bei pädiatrischen Patienten mit Down-Syndrom}

Autoren Schöppe $F^{1}$, Stöcklein $S^{1}$, Reiser $M^{1}$, Rossi $A^{2}$, Ertl-Wagner $B^{1}$ Institut 1 Klinikum der Universität München, Institut für Klinische Radiologie, München; 2 Istituto Giannina Gaslini, Abteilung für Neuroradiologie, Genua (Italien)

DOI 10.1055/s-0037-1600315

Zielsetzung Patienten mit Down-Syndrom (DS) sind Träger einer dritten Kopie des Amyloid-Precursor-Protein-Gens, welches auf Chromosom 21 lokalisiert ist. Aufgrund erhöhter Amyloid-Level sind diese Patienten prädisponiert dafür, eine zerebrale Amyloidangiopathie (CAA) zu entwickeln. Ziel unserer Studie war es, herauszufinden, ob die MRT-basierten Biomarker der CAA bereits bei pädiatrischen Patienten mit DS nachweisbar sind. Hierbei wurden sowohl die Häufigkeit als auch die geografische Verteilung von zerebralen Mikroblutungen (CMB) und von kortikaler superfizieller Siderose (cSS) ausgewertet. Material und Methodik 12 Patienten mit DS (mittleres Alter =5,0 Jahre, 6 weiblich) and 12 altersgematche Patienten der Kontrollgruppe ohne DS (mittleres Alter $=4,8$ Jahre, 6 weiblich) wurden retrospektiv in diese Fall-KontrollStudie eingeschlossen. Die Häufigkeit sowie die Verteilung der CMB in den einzelnen Hirnregionen wurden erfasst. CMB und cSS wurden im Rahmen eines Konsensus-Readings durch zwei Radiologinnen mit neuroradiologischer Vorerfahrung anhand einer modifizierten Skala (Microbleed Anatomical Rating Scale) anhand von T2* oder SWI-Sequenzen evaluiert.

Ergebnisse DS-Patienten wiesen eine signifikant höhere Anzahl an CMB auf $(N=11,92, S D=13,31)$ als die Patienten der Kontrollgruppe ohne DS ( $N=2,0$, SD 2,7, p = 0,019). Insgesamt war in beiden Gruppen die mediane Anzahl von CMB in den Lobärregionen am höchsten. Bei den pädiatrischen Patienten mit DS war eine cSS signifikant häufiger vorhanden als in der Kontrollgruppe (42 vs. $25 \%$, respektive). Bei den drei DS-Patienten mit Verlaufskontrolle zeigte sich eine Zunahme der CMB sowie eine höhere Anzahl an Subregionen, die von cSS betroffen waren.

Schlussfolgerungen Anhand unserer Studie konnten wir zeigen, dass CMB und cSS bereits bei pädiatrischen Patienten mit DS nachweisbar sind. Der Einsatz von blutungssensitiven MRT-Sequenzen (T2* oder SWI) erlaubt die in 
vivo-Detektion von cSS und CMB und könnte damit zur Identifikation von Frühzeichen einer CAA bei pädiatrischen Patienten mit DS beitragen.

\section{RK/WISS 414.3 Diffusionsgewichtete MRT und FDG PET-CT in der Diagnostik des mediastinalen Lymphoms bei Kindern: Erlaubt der Apparent-Diffusion- Coefficient (ADC) die Dignitätsbestimmung mediastinaler Raumforderungen?}

Autoren Stoffels $\mathrm{M}^{1}$, Herrmann $\mathrm{J}^{1}$, Groth $\mathrm{M}^{1}$, Berliner $\mathrm{C}^{1}$, Klutmann $\mathrm{S}^{1}$, Adam $\mathrm{G}^{1}$, Regier $\mathrm{M}^{1}$

Institut 1 Universitätsklinikum Hamburg Eppendorf, Klinik und Poliklinik für Diagnostische und Interventionelle Radiologie und Nuklearmedizin, Hamburg DOI 10.1055/s-0037-1600316

Zielsetzung Evaluation des Stellenwertes der diffusionsgewichteten echoplanaren Bildgebung (DWI) mit ADC-mapping zur Differenzierung benigner und maligner mediastinaler Raumforderungen und Bestimmung der Stoffwechselaktivität des mediastinalen Lymphoms bei pädiatrischen Patienten in Korrelation mit der Glukoseutilisation des Tumors in der 18F-FDG PET-CT.

Material und Methodik 16 Patienten im Alter von 14,1 44 Jahren und gesichertem Hodgkin- und Non-Hodgkin-Lymphom wurden jeweils vor Therapiebeginn mittels DWI-MRT und 18F-FDG PET-CT untersucht. Die Gesamtzahl aller untersuchter mediastinaler Lymphknoten betrug 104. Die DWI erfolgte an einem 3T-MRT mit folgenden Parametern: TR/TE 2000/66 ms; FOV $425 \mathrm{~mm}$; Schichtdicke $4 \mathrm{~mm}$; b-Werte 0, 25, 50, 75, 150, 500 und 900. Durch das Platzieren einer den gesamten Tumor umgebenden Region-of-interest (ROI) wurden der minimale (ADCmin), mittlere (ADCmean) und maximale ADC (ADCmax) sowie der minimale und mittlere SUV (SUVmean und SUVmax) durch 2 unabhängige Radiologen bestimmt. Die Ergebnisse von DWI und 18F-FDG PET-CT wurden basierend auf der separaten Analyse jedes einzelnen Lymphknotens statistisch mittels Pearson-Korrelationskoeffizient verglichen.

Ergebnisse Der Vergleich zwischen ADCmin und SUVmax zeigte eine statistisch signifikante inverse Korrelation $(r=0,91 ; p=0,02)$, die Gegenüberstellung von $A D C m e a n$ und SUVmean wies jedoch keine Signifikanz auf $(r=0,37 ; p=0,51)$. Insgesamt zeigten maligne Lymphknoten beim HodgkinLymphom eine höhere Traceraufnahme im 18F-FDG PET-CT und einen niedrigeren mittleren ADC-Wert als beim Non-Hodgkin-Lymphom.

Schlussfolgerungen In unserem Patientenkollektiv zeigten die minimalen ADC-Werte einen höheren Tumormetabolismus an, welches durch die korrespondierende 18F-FDG PET-CT bestätigt wurde, und erlauben somit eine Unterscheidung benigner und maligner Raumforderungen. Bei Kindern mit mediastinaler Lymphommanifestation sollte die DWI mit ADC-Messung als ein zusätzliches, strahlungsfreies diagnostisches Werkzeug in der Erst- sowie Verlaufsdiagnostik Verwendung finden.

\section{RK/WISS 414.7 MR-Lungenperfusion bei 12-jährigen Kindern nach operierter Zwerchfellhernie}

Autoren Weis $\mathrm{M}^{1}$, Schaible $\mathrm{T}^{2}$, Neff $\mathrm{K}^{1}$, Schoenberg $\mathrm{S}^{1}$, Henzler $\mathrm{C}^{1}$ Institut 1 Universitätsmedizin Mannheim, Institut für Klinische Radiologie und Nuklearmedizin, Mannheim; 2 Universitätsmedizin Mannheim, Klinik für Neonatologie, Mannheim

DOI 10.1055/s-0037-1600317

Zielsetzung Im Alter von zwei Jahren zeigen Kinder nach operierter Zwerchfellhernie $(C D H)$ ein ipsilaterales Perfusionsdefizit. Diese Studie soll die Frage beantworten, ob im Alter von 12 Jahren weiterhin ein Perfusionsdefizit nachweisbar ist.

Material und Methodik Zehn Patienten im Alter von 12 Jahren $(11,7 \pm 1,2$ Jahre) wurden nach operierter linksseitiger $\mathrm{CDH}$ mittels dynamischer Kontrastmittel gestützter Perfusions-MRT (3Tesla, Magnetom TimTrio, Siemens Healthineers $\mathrm{GmbH}$, Erlangen, Deutschland) untersucht. Der pulmonale Blutfluss (PBF), das pulmonale Blutvolumen (PBV) und die mittlere Transitzeit (MTT) wurden auf Basis einer pixelweisen Dekonvolution (UMM perfusion, OsiriX plug-in) errechnet. Die mittleren Perfusionswerte der ipsi- und kontralateralen Lunge wurden mittels gepaartem t-Test verglichen.

Ergebnisse Der PBF ist ipsilateral signifikant gegenüber der kontralateralen Seite vermindert $(45,5 \pm 26,3 \mathrm{ml} / 100 \mathrm{ml} / \mathrm{min}$ vs. $72,2 \pm 29,0 \mathrm{ml} / 100 \mathrm{ml} / \mathrm{min}$; $\mathrm{p}=0,0019)$. Das PBV ist ipsilateral ebenso geringer als kontralateral $(3,4$ $\pm 2,2 \mathrm{ml} / 100 \mathrm{ml}$ vs. $5,2 \pm 1,9 \mathrm{ml} / 100 \mathrm{ml} ; \mathrm{p}=0,0053)$. Bei der MTT zeigen sich keine Seitenunterschiede $(p=0,20)$.

Schlussfolgerungen Mittels Perfusions-MRT kann im Alter von 12 Jahren weiterhin ein Perfusionsdefizit der ipsilateralen Lunge nach $\mathrm{CDH}$ nachgewiesen werden. Somit kann mithilfe der Perfusions-MRT das fortbestehende funktionelle Defizit der pulmonalen Gefäße visualisiert und quantifiziert werden. Eine Verlaufskontrolle scheint demnach sinnvoll und sollte im Rahmen einer intensivierten Nachsorge erfolgen.

\section{RK/WISS 320.5 Bestimmung von effektiver Dosis in Fluoroskopie-gesteuerten pädiatrischen abdominalen Interventionen mittels Rando-Alderson Phantomen}

Autoren Wildgruber $\mathrm{M}^{1}$, Müller-Wille $\mathrm{R}^{2}$, Goessmann $\mathrm{H}^{3}$, Uller $\mathrm{W}^{4}$, Wohlgemuth $\mathrm{W}^{4}$

Institut 1 Universitätsklinikum Münster, Institut für Klinische Radiologie, Münster; 2 Institut für Rötngendiagnostik, Universitäsklinikum Regensburg, Regensburg; 3 Institut für Rötngendiagnostik, Universitätsklinikum Regensburg, Regensburg; 4 Institut für Röntgendiagnostik,

Universitätsklinikum Regensburg, Regensburg

DOI 10.1055/s-0037-1600318

Zielsetzung Ziel der Studie ist die detaillierte Berechnung der effektiven Dosis für pediatrische interventionelle Eingriffe der Leber in einem Phantommodel vor und nach der Adjustierung von dosisrelevanten Aufnahmeparametern.

Material und Methodik Die einzelnen Organdosen wurden in drei anthropomorphen Rando-Alderson-Phantomen gemessen, die Kinder verschiedenen Alters/Gewichtes repräsentieren (Neugeborenes $3.5 \mathrm{Kg}$, Kleinkind $10 \mathrm{Kg}$, Kind $19 \mathrm{Kg}$ ). Kollimiert wurde auf die Region des Oberbauches, um typische abdominelle Interventionen wie PTCD Anlagen zu repräsentieren. Fluoroskopie Akquisitionen wie auch Digitale Subtraktionsangiografien wurden im anteriorposterior Strahlengang aufgenommen. Die Aufnahmen wurden an einer State of The Art Angiografie Anlage mit digitalem Flachdetektorsystem durchgeführt. Die effektive Dosis wurde direkt mittels multiplen, in die Phantome integrierten Thermolumineszenz Dosimetern gemessen. Im Anschluss an die Phantommessungen wurden die Dosiswerte (Hauteintrittsdosis sowie Dosisflächenprodukt) in einem pädiatrischen Patientenkollektiv vor und nach Anpassung des Dosisparameter bestimmt.

Ergebnisse Die effektive Dosis lag für alle Phantome unter $0.1 \mathrm{mSv}$ pro Minute Fluorskopie und unter $1 \mathrm{mSv}$ pro Minute DSA, bei einer Bildrate von 2/s. Die Absenkung der Detektoreintrittsdosis ermöglichte im Phantommodel eine Absenkung der effektiven Dosis von 12 bis $27 \%$ für die Fluoroskopie, sowie 22 bis $63 \%$ für die Akquisition von DSA Serien. Gleichermaßen konnten die Organdosen speziell für strahlensensible Organ um über $50 \%$ gesenkt werden. In der pädiatrischen Patientenpopulation konnte gleichermaßen die Hauteintrittsdosis sowie das Dosisflächenprodukt signifikant reduziert werden.

Schlussfolgerungen Die Adjustierung, respektive weitere Absenkung von Parametereinstellung, die vom Hersteller für pädiatrische Interventionen vorgegeben werden, ermöglichen eine relevante Senkung der effektiven Dosis im Phantommodel und bietet gleichermaßen erhebliches Einsparpotential für pädiatrische Interventionen im klinischen Alltag. 


\section{RK/WISS 414.5 Vergleich nicht-invasiver Methoden zur Verlaufsbeurteilung der Eisenbeladung der Leber bei transfusionspflichtigen Kindern und Jugendlichen}

Autoren Wurschi $\mathrm{G}^{1}$, Kentouche $\mathrm{K}^{2}$, Krumbein $\mathrm{I}^{3}$, Herrmann $\mathrm{K}^{4}$, Mentzel $\mathrm{H}^{5}$ Institut 1 Universitätsklinikum Jena, Institut für Diagnostische und Interventionelle Radiologie, Abt. Kinderradiologie, Jena; 2 Klinik für Kinderund Jugendmedizin; Universitätsklinikum Jena, Abt. f. Hämostaseologie \&amp; Immunologie, Jena; 3 Institut für Diagnostische und Interventionelle Radiologie, Universitätsklinikum Jena, Jena; 4 Institut für Diagnostische und Interventionelle Radiologie, Universitätsklinikum Jena, AG Medizinische Physik, Jena; 5 Institut für Diagnostische und Interventionelle Radiologie,

Universitätsklinikum Jena, Abt. f. Kinderradiologie, Jena

DOI 10.1055/s-0037-1600319

Zielsetzung Nach vermehrten Bluttransfusionen können Organe durch eine chronische Eisenüberladung $(\mathrm{IO})$ beeinträchtigt werden, bspw. Fibrosierung im Lebergewebe. Gerade junge Patienten sollten diesbezüglich einfach überwacht werden können, um Sekundärschäden frühzeitig erkennen und behandeln zu können.

Material und Methodik Es wurden 43 Patienten (MW 11,5J $\pm 6,2 \mathrm{~J}$ SD) vor sowie nach (100T, 365T) erhaltener Stammzelltransplantation mittels Ultraschall-Elastografie (UE) (iU22, Philipps, C5-1 MHz) und anhand der T2*-MRRelaxationszeit (1,5T, Avanto, Siemens) mit etablierten Serumparametern zur Leberfunktion (LF) und Eisenstoffwechsel, wie Serum-Ferritin, verglichen. In der weiteren Beurteilung wurde der cut-off für Hyperferritinämien bei $1 \mathrm{mg} / \mathrm{l}$ festgesetzt, um Einflüsse von Akute-Phase-Reaktionen zu minimieren.

Ergebnisse Eine signifikante Korrelation zwischen T2* und Serum-Ferritin ließ sich feststellen ( $r=.61, p=, 001, N=74)$, wobei die Varianz der Messungen im MRT deutlich geringer ausfiel. Beide Methoden wiesen eine vergleichbare Spezifität in ROC-Analysen auf ( $A \cup C=, 932, r 2=, 029, N=74$ ). Ein Ausschluss von Patientendaten bei einem CRP > 7,5 mg/dl erhöhte die Korrelation zu T2* um das 1,34-fache ( $p=, 001, N=63)$. Zur Beurteilung der LF wurde aus etablierten Serum-Parametern ein qualitativer Score ermittelt. Demnach erhöhte Parameter gingen im Mittel mit 1,79-fach erhöhten Ferritin-Werten sowie 1,4fach erhöhten UE-Parametern bzw. 1,26-fach erhöhtem R2* $(=1 / T 2 *)$ einher. Schlussfolgerungen Da T2* gut mit Ferritin-Werten korrelierte, ist eine zukünftige MR-gestützte Überwachung denkbar. Obwohl eine erhöhte Steifheit der Leber in der UE zwar mit erhöhten Serum-Parametern einherging, ließ sich dies nicht primär einer IO zuordnen. Im Vergleich ist dieses Verfahren zwar durchaus sensitiv für eine Fibrosierung, jedoch unspezifisch hinsichtlich der Ursache, wie möglicher IO. Eine Kontrolle per UE könnte daher nur helfen, Folgeschäden früh zu erkennen.

\section{Kontrastmittel}

\section{WISS 305.8 Sind sog. „breakthrough“ Reaktionen (BTR) nach Prämedikation bei Kontrastmittelallergie eine Kontraindikation für Kontrastmittel?}

\footnotetext{
Autoren Böhm I ${ }^{1}$, Nairz $\mathrm{K}^{1}$, Endrich $\mathrm{O}^{2}$, Hasembank $\mathrm{S}^{1}$, Keller $\mathrm{P}^{1}$, Heverhagen $]^{1}$

Institut 1 Inselspital, Universität Bern, Universitätsinstitut für Diagnostische, Interventionelle und Pädiatrische Radiologie, Bern; 2 Inselspital, Universität

Bern, Operatives Medizincontrolling, Bern

DOI 10.1055/s-0037-1600320

Zielsetzung Allergische Reaktionen trotz Prämedikation, sog. breakthroughReaktionen (BTR), gelten als Kontraindikation für Kontrastmittel (KM). Ziel unserer Untersuchung war es, anhand einer kausalen Analyse der in einem Grossklinikum aufgetretenen BTR eine Strategie zu entwickeln, um diese Reaktionen zukünftig weitgehend zu vermeiden.

Material und Methodik RIS-/KIS-Daten von Patienten, die von 2000 - 2016 untersucht und antiallergisch prämediziert wurden, analysierten wir retro-
}

spektiv. Da der Begriff breakthrough-Reaktion in der Routine nicht etabliert ist, suchten wir zur Identifikation nach geeigneten Umschreibungen bzw. aussagekräftigen Stichwort-Kombinationen.

Ergebnisse Wir identifizierten 39 BTR-Patienten. Bei 31 Fällen löste ein jodiertes KM die Reaktion aus, bei 8 ein Gd-haltiges. Die BTR trat meistens $(89,7 \%)$ einmalig auf. Ursächlich waren: symptomorientierte Prämedikationen, die angesichts des Schweregrads (z. B. Anaphylaxie) zu gering dosiert waren $(56,4 \%)$ und nicht symptomorientierte Prämedikationen (33,3\%) sowie unbekannte Ursachen (10,3\%). 28 Patienten stellten sich nach der BTR erneut zur Untersuchung vor: 35,7\% bekamen kein KM (native Bildgebung), 39,3\% erhielten eine korrekt dosierte und symptomorientierte Prämedikation und 32,1\% ein alternatives KM aus der gleichen chemischen Gruppe ohne Prämedikation (Mehrfachnennung). Bei den Nachfolgeuntersuchungen traten keine KMReaktionen auf. Aus diesem Datenmaterial leiten sich folgende Strategien zur BTR-Minimierung ab: 1) KM-Wechsel; 2) symptomorientierte Prämedikation (geeignete Medikamente und Dosen), wenn ein KM-Wechsel nicht möglich sein sollte.

Schlussfolgerungen BTR sind keine Kontraindikationen für KM, sondern lassen sich durch einen KM-Wechsel bzw. eine adäquate Prämedikation vermeiden. Kenntnisse der pathologischen Hintergründe sowie die exakte Dokumentation des verabreichten KM und der klinischen Symptome der Initialreaktion sind für ein effektives Management, eine BTR-Minimierung und damit für die KM-Sicherheit bei Risikopatienten essentiell.

\section{WISS 305.1 No Signal Intensity Increase in the Dentate Nucleus on Unenhanced T1-weighted MR Images after more than 20 Serial Injections of Macrocyclic Gadolinium-Based Contrast Agents}

Authors Haase $\mathrm{R}^{1}$, Kieslich $\mathrm{P}^{2}$, Weberling $\mathrm{L}^{3}$, Kickingereder $\mathrm{P}^{3}$, Wick $\mathrm{W}^{4}$, Schlemmer $\mathrm{H}^{5}$, Bendszus $\mathrm{M}^{3}$, Radbruch $\mathrm{A}^{3}$

Institute 1 Deutsches Krebsforschungszentrum, Radiologie, Heidelberg; 2 Universität Mannheim, Psychologie, Mannheim; 3 Universitätsklinikum Heidelberg, Neuroradiologie, Heidelberg; 4 Universitätsklinikum Heidelberg, Neurologie, Heidelberg; 5 Deutsches Krebsforschungszentrum (DKFZ), Radiologie, Heidelberg DOI 10.1055/s-0037-1600321

Zielsetzung To determine the effect of more than twenty serial injections of macrocyclic gadolinium-based contrast agents (GBCAs) on the signal intensity (SI) of the dentate nucleus (DN) on unenhanced T1-weighted MR images.

Material und Methodik In this retrospective, institutional review board-approved study, 33 patients who underwent at least 20 consecutive MRI examinations (plus an additional MRI for reference) with the exclusive use of macrocyclic GBCAs (gadoterate meglumine and gadobutrol) were analyzed. SI ratio differences were calculated for DN-to-pons (DNP) and DN-to-middle cerebellar peduncle (DNMCP) ratios by subtracting the SI ratio at the first MRI from the SI ratio at the last MRI scan. One-sample t-tests were employed to examine if the SI ratio differences differed from 0 and Bayes factors were calculated to quantify the strength of evidence for each test.

Ergebnisse Patients underwent a mean of 23.03 \pm 4.20 GBCA administrations (mean accumulated dose: $491.21 \pm 87.04 \mathrm{ml}$ of a $0.5 \mathrm{M}$ GBCA solution) with an average of $12.09 \pm 2.16$ weeks between every administration. Both ratio differences did not differ significantly from 0 (DNP: $-0.0032 \pm 0.0154$, $\mathrm{p}=0.248$; DNMCP: $-0.0011 \pm 0.0093, \mathrm{p}=0.521$ ) and one-sided Bayes factors provided substantial to strong evidence against an $\mathrm{SI}$ ratio increase (Bayes factor for DNP=0.09, for DNMCP=0.12).

Schlussfolgerungen Our study indicates that 20 or more serial injections of macrocyclic GBCAs administered with on average three months between each injection are not associated with an SI increase in the DN. 


\section{WISS 305.6 Akute transiente Dyspnoe: Einfluss der Scanzeit in der arteriellen Phase der Primovist ${ }^{\circledR}$. verstärkten Leber-MRT}

Autoren Kromrey $\mathrm{M}^{1}$, Schorn $\mathrm{F}^{1}$, Liedtke $\mathrm{K}^{1}$, Ittermann $\mathrm{T}^{2}$, Kühn J ${ }^{1}$ Institut 1 Universitätsmedizin Greifswald, Institut für Diagnostische Radiologie und Neuroradiologie, Greifswald; 2 Universitätsmedizin Greifswald, Institut für Community Medicine, Greifswald

DOI 10.1055/s-0037-1600322

Zielsetzung Untersuchung des Einflusses der Scanzeit auf das Auftreten von schwerwiegenden transienten Bewegungsartefakten (transient severe motion artifacts, TSM) aufgrund einer akuten transienten Dyspnoe in der arteriellen Phase der Primovist ${ }^{\circledR}$-verstärkten Magnetresonanztomografie der Leber.

Material und Methodik Zwischen 2013 - 2016 erhielten 345 Patienten (155 Frauen, mittleres Alter $60.8 \pm 14.2$ Jahre) eine kontrastmittelgestützte MRT der Leber mit standardisierter Bolusinjektion von $10 \mathrm{ml}$ Gadoxetate (Primovist $\left.^{\circledR}\right)$. Zwei Untersucher evaluierten retrospektiv die Bilder in der arteriellen Phase hinsichtlich des Auftretens von TSM. Das Ausmaß der Artefakte wurde nach einer 4-Punkteskala bewertet $(0=$ keine Bewegungsartefakte; $1=$ leichte Artefakte; 2 = ausgeprägte Artefakte; 3 = schwerwiegende Artefakte/nicht diagnostisch). Anhand des Zeitpunkts zwischen Kontrastmittelinjektion und der ersten Aufnahme wurde die arterielle Phase in 3 Stadien eingeteilt (frühe, reine und späte arterielle Phase) und mit Auftreten und Graduierung der TSM korreliert.

Ergebnisse TSM-Artefakte traten in 49,0\% (169/345) der Patienten auf. Mit $23,2 \%$ zeigten die meisten Patienten einen Bewegungsgrad von $1(n=80)$, $18,6 \%$ hatten einen Grad von $2(n=64)$ und 7,3\% einen Grad von $3(n=25)$. Auftreten $(p=0.021)$ und Schwere der TSM $(p=0.009)$ waren signifikant assoziiert mit dem Stadium der arteriellen Phase. Ein höheres Vorkommen von TSM-Artefakten wurde in der reinen arteriellen Phase (60,5\%), verglichen mit der früh- $(51,7 \%)$ und der spätarteriellen Phase $(42,2 \%)$, detektiert. Weiterhin zeigten sich schwere TSM (Grad 3) hauptsächlich in der reinen arteriellen Phase. Das Auftreten von TSM war signifikant assoziiert mit dem BMI $(p=0.001)$, aber nicht mit Geschlecht $(p=0.189)$, Aszites $(p=0.072)$ oder Alter $(p=0.956)$.

Schlussfolgerungen Die reine arterielle Phase in der Primovist ${ }^{\circledR}$-verstärkten MRT prädisponiert zu einem gehäuften Auftreten von TSM-Artefakten, was ein mögliches Problem für die Auswertung in der diagnostischer Bildgebung darstellt.

\section{WISS 305.5 Repeated intravenous administration of gadobutrol does not lead to increased signal intensity on unenhanced T1-weighted images - a voxel-based whole brain analysis}

Authors Langner $\mathrm{S}^{1}$, Kromrey $\mathrm{M}^{1}$, Kühn $\mathrm{J}^{1}$, Grothe $\mathrm{M}^{2}$, Domin $\mathrm{M}^{3}$ Institute 1 Universitätsmedizin Greifswald, Institut für Diagnostische Radiologie und Neuroradiologie, Greifswald; 2 Universitätsmedizin Greifswald, Klinik für Neurologie, Greifswald; 3 Universitätsmedizin Greifswald, Institut für Diagnostische Radiologie und Neuroradiologie, Funktionelle Bildgebung, Greifswald

DOI 10.1055/s-0037-1600323

Zielsetzung To identify a possible association between repeated intravenous administration of gadobutrol and increased signal intensity in the gray and white matter using voxel-based whole brain analysis.

Material und Methodik Patients $(n=217)$ with clinically isolated syndrome underwent baseline brain magnetic resonance imaging and at least one annual follow-up examination with intravenous administration of $0.1 \mathrm{mmol} / \mathrm{kg}$ body weight of gadobutrol. Patients were assigned to different groups according to the number of follow-up examinations performed. Using the DARTEL normalization process, tissue templates for gray matter, white matter, and CSF were calculated for each group, as were gray matter-to-CSF and white matterto-CSF ratios. Voxel-based whole brain analysis was used to calculate signal intensity of gray matter for each voxel in each group. The paired t-test was applied to test differences to baseline MRI for significance.

Ergebnisse No significant changes in signal intensity of gray and white matter were observed after up to five gadobutrol administrations. There was also no significant change in gray matter-to-CSF and gray matter-to-CSF ratios after repeated administration of gadobutrol.

Schlussfolgerungen Voxel-based whole brain analysis did not demonstrate increased signal intensity of GM and WM on unenhanced T1-weighted images after repeated gadobutrol administration.

\section{WISS 305.2 Evaluation einer möglichen langfristigen cerebralen Anreicherung des leberspezifischen MRT- Kontrastmittels Gd-EOB - eine prospektive Querschnittstudie}

Autoren Posch $\mathrm{H}^{1}$, Kahn $\mathrm{J}^{2}$, Steffen $\mathrm{I}^{2}$, Geisel $\mathrm{D}^{2}$, Bauknecht $\mathrm{C}^{3}$, Liebig $\mathrm{T}^{2}$, Denecke $T^{1}$

Institut 1 Charité - Universitätsmedizin Berlin, Campus Virchow-Klinikum, Klinik für Radiologie, Berlin; 2 Charité - Universitätsmedizin Berlin, Campus Virchow-Klinikum, Klinik für Radiologie, Berlin; 3 Charité - Universitätsmedizin Berlin, Campus Virchow-Klinikum, Institut für Radiologie, Berlin

DOI 10.1055/s-0037-1600324

Zielsetzung Ziel der Studie war die MRT-gestützte Evaluierung einer möglichen Erhöhung der Signalintensität (SI) in definierten Hirnregionen als Folge der wiederholten Gabe des leberspezifischen MRT-Kontrastmittels (KM) Gadolinium-Ethoxybenzyl (Gd-EOB, Primovist ${ }^{\circledR}$, Bayer). Untersucht wurden dabei Nucleus dentatus (DN) und Globus pallidus (GP) in Relation zu den Referenzregionen Kleinhirnstiel (MCP), Pons (P) und Thalamus $(\mathrm{TH})$ in der nativen T1wSequenz.

Material und Methodik Im Rahmen dieser prospektiven Querschnittstudie erhielten ausgewählte Patienten einer Studiengruppe $(A, n=91)$ sowie einer Kontrollgruppe (B, $n=52)$ eine cMRT-Untersuchung $(1,5 T)$ in nativer T1w-SESequenz. Die Patienten der Studiengruppe hatten Gd-EOB jeweils zwischen 1 - 37 Mal erhalten, wobei die vergangene Verabreichung von anderen linearen MRT-KM oder mehr als zwei Dosen makrozyklischer MRT-KM als Ausschlusskriterien galten. (Die Studienkohorte wurde entsprechend der Anzahl an Gd-EOB-Gaben in 3 Gruppen unterteilt: A1 <5, A2 5-10, A3 > 10.) Die Kontrollgruppe beschränkte sich auf Patienten ohne jeglichen vorherigen MRT-KM-Kontakt.

Ergebnisse Die ermittelten SI-Quotienten aus DN/MCP und DN/P zeigten signifikante Abweichungen zwischen den Patienten der Kontrollgruppe und denjenigen Patienten, die bereits > 10 Gd-EOB-Dosen erhalten hatten. Nach 5 - 10 Gaben Gd-EOB erreichten die SI-Veränderungen im DN Werte nahe der Signifikanz, Patienten mit < $5 \mathrm{KM}$-Gaben wiesen hingegen keine Unterschiede zu Patienten der Kontrollgruppe auf. Die Quotienten aus GP/TH waren bei der Gesamtheit der Studienpatienten im Vergleich zur Kontrollgruppe ebenfalls signifikant erhöht; es zeigte sich jedoch kein signifikanter Anstieg der Signalintensität zwischen den Untergruppen A1, A2 und A3.

Schlussfolgerungen Die erhobenen Daten bestätigen einen signifikanten Zusammenhang zwischen der Anzahl erfolgter Gd-EOB-Gaben und erkennbaren SI-Erhöhungen des Nucleus dentatus in der nativen T1w-MRT-Sequenz und weisen damit auf eine mögliche langfristige cerebrale Anreicherung dieses Kontrastmittels hin. 


\section{WISS 305.3 Gadoliniumablagerungen in neuronalen Strukturen nach Applikation von makrozyklischem Gadolinium? Eine fallbasierte, pädiatrische Kontrollstudie}

Autoren Rademacher $C^{1}$, Tibussek $D^{2}$, Caspers $\left.\right|^{3}$, Turowski $B^{4}, S C h a p e r J^{5}$, Antoch $\mathrm{C}^{4}$, Klee $\mathrm{D}^{4}$

Institut 1 Heinrich-Heine-Universität Düsseldorf, Kinderradiologie, Düsseldorf; 2 Medizinische Fakultät, Heinrich-Heine-Universität Düsseldorf, Klinik für Allgemeine Pädiatrie, Neonatologie und Kinderkardiologie, Düsseldorf; 3 Medizinische Fakultät, Heinrich-Heine-Universität Düsseldorf; Forschungszentrum Jülich, Institut für Diagnostische und Interventionelle Radiologie, Institut für Neurowissenschaften und Medizin (INM-1), Düsseldorf; 4 Medizinische Fakultät, Heinrich-Heine-Universität Düsseldorf, Institut für Diagnostische und Interventionelle Radiologie, Düsseldorf; 5 Medizinische Fakultät, Heinrich-Heine-Universität Düsseldorf, Institut für Diagnostische und Interventionelle Radiologie, Düsseldorf

DOI 10.1055/s-0037-1600325

Zielsetzung Gadoliniumablagerungen im Gehirn nach wiederholter Applikation von linearem, gadoliniumhaltigem Kontrastmittel sind ein relevantes Thema. Makrozyklische Präparate lagern sich nach Studienlage bei Erwachsenen geringer in den neuronalen Strukturen ab. Ziel unserer Studie ist es zu klären, ob wir Hinweise auf Gadoliniumablagerungen in den neuronalen Strukturen von Kindern nach wiederholter Gabe von makrozyklischen, gadoliniunumhaltigen Kontrastmitteln (GBCAs) nachweisen können.

Material und Methodik In der retrospektiven, fallkontrollierten Studie wurden Kinder (Fälle) untersucht, die mindestens 9 Applikationen von GBCA (ProHance ${ }^{\circledR}$ und Dotarem ${ }^{\circledR}$ ) erhalten haben. Für jeden Fall wurde ein Kind (Kontrolle) mit unauffälliger, kranieller MR-Bildgebung ausgewählt. Die Signalintensität in der nativen $\mathrm{T} 1$ Sequenz wurde in folgenden Regionen (ROIs) gemessen: Nucleus dentatus (DN), Pons, Substantia nigra, Pulvinar thalami and Globus pallidus (GP). Mittels gepaartem t-Test wurden SI und SI ratios (DN/ Pons, GP/Thalamus) in beiden Gruppen verglichen. Die Pearson Korrelation zwischen relativen Signalveränderungen und der Anzahl an GBCA Applikationen und an der totalen GBCA Dosis wurde berechnet.

Ergebnisse 24 Fälle erfüllten (mittleres Alter 8,25 Jahre, Jungen, 15 Mädchen) die Einschlusskriterien. Sie erhielten eine mittlere Anzahl von $14.2(9-24)$ an makrozyklischen GBCA Applikationen. Zwischen Fällen und Kontrollen wurden keine signifikanten Differenzen der SI für die einzelnen ROls sowie für die ND/ Pons und die GP/Pulvinar-Quotienten berechnet.Es ist keine Korrelation zwischen der Anzahl der makrozyklischen GBCA Applikationen oder der Gesamtmenge an appliziertem makrozyklischem GBCA und der Signalveränderung für jede ROI.

Schlussfolgerungen Nach repetitiven intravenösen Gaben von makrozyklischen GBCAs im Kindesalter können keine Signalveränderungen in den T1 gewichteten Sequenzen detektiert werden. Dies weist auf eine hohe Sicherheit der makrozyklischen GBCAs bzgl. der möglichen Ablagerung von Gadolinium in den neuronalen Strukturen hin.

\section{WISS 305.7 Transiente schwere Atemartefakte bei Dinatriumgadoxetat-gestützter MRT: Inzidenz und Risikoevaluation an einer europäischen Klinik}

\author{
Autoren Well $\mathrm{L}^{1}$, Rausch $\mathrm{V}^{2}$, Gerhard $\mathrm{A}^{2}$, Henes $\mathrm{O}^{2}$, Bannas $\mathrm{P}^{2}$ \\ Institut 1 Universitätsklinikum Hamburg-Eppendorf, Klinik und Poliklinik für \\ diagnostische und interventionelle Radiologie, Hamburg; 2 Klinik und \\ Poliklinik für diagnostische und interventionelle Radiologie und \\ Nuklearmedizin, Hamburg \\ DOI 10.1055/s-0037-1600326
}

Zielsetzung Das Auftreten kontrastmittelinduzierter, transienter schwerer Atemartefakte (TSA) nach Applikation von Dinatriumgadoxetat wurde mit einer Inzidenz von 5 bis $18 \%$ beschrieben. Da bisherige Studien aus den USA und Japan stammen, war es unser Ziel die Inzidenz der TSA an einem europäis- chen Institut zu ermitteln, potentielle Risikofaktoren zu bestimmen und unsere mit bisherigen Studienergebnissen zu vergleichen.

Material und Methodik Zwei alters- und geschlechtsabgestimmte Gruppen wurden retrospektiv aus dem Kollektiv der in unserer Klinik angefertigten MRT-Untersuchungen bestimmt. (Dinatriumgadoxetat $n=89$; Gadobenat Dimeglumin $n=89$ ). Zwei gegenüber dem verwendeten Kontrastmittel geblindete Radiologen bewerteten unabhängig die atemabhängigen Bewegungsartefakte in nativen und dynamischen Kontrastmittelphasen auf einer 4-Punkte Skala. Werte von $\geq 3$ wurden als schwere Artefakte angesehen. Wenn zudem Atemartefakte in allen anderen Untersuchungsphasen mit Werten $<3$ bewertet wurden, erfolgte eine Einstufung der Artefakte als TSA. Eine Korrelation von potentiellen Risikofaktoren und TSA erfolgte mittels logistischer Regression.

Ergebnisse Nach Injektion von Dinatriumgadoxetat zeigten sich signifikant mehr atemabhängige Bewegungsartefakte in den arteriellen $(2.2 \pm 0.9)$ Kontrastmittelphasen als in allen anderen Phasen $(1.6 \pm 0.7)(p<0.05)$. Die Inzidenz der TSA nach Gabe von Dinatriumgadoxetat $(n=19 ; 21.1 \%)$ war signifikant höher als nach Gabe von Gadobenat Dimeglumin $(n=1 ; 1.1 \%)$ $(p<0.001)$. Die Inzidenz der TSA in unserer Klinik ist vergleichbar mit der einiger, aber nicht aller veröffentlichten Studien. Es konnte keine signifikante Korrelation von TSA und den erhobenen potentiellen Risikofaktoren ermittelt werden (alle $\mathrm{p}>0.05$ ).

Schlussfolgerungen Wir konnten eine hohe Inzidenz von TSA an einer europäischen Institution demonstrieren und die Bedeutung eines Diagnose limitierenden Phänomens untermauern, welches bisher nur in den USA oder Japan beschrieben wurde. Eine Korrelation von TSA mit potentiellen Risikofaktoren fanden wir nicht.

\section{Kopf/Hals-Diagnostik}

\section{RK 202.3 Potential eines PC-augmentierten kombinatorischen Verfahrens zur Erhöhung der Treffsicherheit in der KM-gestützten CT- Rezidivdiagnostik von Kopf-/Halstumoren: Eine retrospektive Patientenstudie}

\author{
Autoren Bruhn $\mathrm{H}^{1}$, Dietz $\mathrm{C}^{1}$, Cho $\mathrm{C}^{1}$, Teichgräber $\mathrm{U}^{1}$, Bruhn $\mathrm{H}^{1}$ \\ Institut 1 Friedrich-Schiller Universität, Institut für Diagnostosche und \\ Interventionelle Radiologie, Jena
}

DOI 10.1055/s-0037-1600327

Zielsetzung Ziel unserer Studie war es zu prüfen, ob lokale Tumorrezidive von Kopf-Hals-Malignomen in Gegenwart von posttherapeutischen Gewebeveränderungen anhand eines systematischen objektivierenden statistisch-kombinatorischen computergestützten Verfahrens besser erkannt werden können.

Material und Methodik Es wurden retrospektiv 154 konsekutive Fälle über 4 Jahre (93 mit histologisch gesichertem Rezidivtumor, 61 ohne) nach R0-Resektion von Kopf-Hals-Tumoren (Primärtumor in $>99 \%$ der Fälle Plattenepithelkarzinom; ein adenoid-zystisches Karzinom) in die Studie eingeschlossen. Die Untersuchungen wurden mit 2 MD-CT (64-Zeiler, GE Healthcare) standardmäßig in einem Untersuchungsfeld vom Aortenbogen bis oberhalb des Kieferköpfchens durchgeführt. Die Kontrastmittelapplikation (Solutrast 300) erfolgte gleichförmig iv in einem geteilten Bolus $(70 / 40 \mathrm{ml})$ mit einem Fluss von $3 \mathrm{ml} / \mathrm{s}$ und einer Startverzögerungszeit von $72 \mathrm{~s}$. Alle CT-Aufnahmen wurden auf das Vorliegen von sieben morphologischen Beurteilungskriterien (Gewebsvermehrung, Randunschärfe, zentrale Hypodensität, Inhomogenität, Infiltration, Knochendestruktion, Kontrastmittelanreicherung) geprüft. Für jede CT-Aufnahme erfolgte eine Einschätzung über die zugrunde liegende Dignität durch die kombinatorische Betrachtung aller Kriterien unter gleichzeitiger Gewichtung derselben und dem anschließenden Vergleich mit einem ermittelten Schwellenwert. Das Vorgehen nutzt das Modell der binär logistischen Regression auf statistischer Basis. 
Ergebnisse Mit dem vorgestellten diagnostischen Verfahren erhielten wir in unserer Studie eine Sensitivität von 90,3\% und eine Spezifität von $80,3 \%$ sowie einen positiven und negativen Vorhersagewert von $87,5 \%$ bzw. $84,5 \%$. Damit konnten um 5 - 10\% höhere Sensitivitäts- und Spezifitätswerte sowie um 4 $14 \%$ höhere Werte für den positiven und negativen Vorhersagewert im Vergleich zur bisherigen einfachen radiologischen Standardauswertung erzielt werden.

Schlussfolgerungen Das in dieser Studie vorgestellte Verfahren zeigt ein vielversprechendes Potential.

\section{WISS 211.9 70-kVp CT-Untersuchung des Halses zur Lymphomabklärung und Verlaufskontrolle: Beurteilung der Bildqualität und Strahlendosis Bewerber für den Young Investigator Award}

Autoren Bucher $\mathrm{A}^{1}$

Institut 1 Uniklinik Frankfurt, Institut für Diagnostische und Interventionelle Radiologie, Frankfurt

DOI 10.1055/s-0037-1600328

Zielsetzung Bisherige Studien belegen einen verbesserten Kontrast der Halsweichteile in $70 \mathrm{kV}$ und $90 \mathrm{kV}$ Untersuchungen bei deutlich eingeschränkter Bildqualität im unteren Halsbereich. In dieser Studie vergleichen wir die Bildqualität und Strahlendosis von 2 Hals-CT Niedrigdosisprotokollen im Rahmen der Lymphomabklärung- und Verlaufskontrolle.

Material und Methodik Kontrastmittelgestützte Hals-CT Untersuchungen von 40 Patienten (13 weiblich, Alter: 19-57Jahre) wurden in zwei gleich großen Gruppen ausgewertet. CTs wurden auf einem Dual-Source CT der dritten Generation mit $70 \mathrm{kV}$ (Referenz-Röhrenstrom $457 \mathrm{mAs}$ ) oder $90 \mathrm{kV}$ (Referenz-Röhrenstrom $197 \mathrm{mAs}$ ) aquiriert und in mittlerer Admire Stufe rekonstruiert. Bildqualität und Artefaktlast der oberen, mittleren und unteren Halsabschnitte wurden von zwei Radiologen bewertet. Bildrauschen und Dichte-Werte wurden vaskulär, muskulär und im Fettgewebe bestimmt und das Kontrast-zu-Rausch Verhältnis (KRV) berechnet. DLP und CTDI und die effektive Dosis wurden erhoben $(k=0.0059)$. Mann-Whitney $U$ Test und Cohen Kappa-Statistik wurden verwendet.

Ergebnisse Die Weichteilstrukturen waren in allen 3 Halsabschnitten in beiden Gruppen sehr gut beurteilbar (Oben: $p=1.000$; Mitte: $p=0.208$; Unten: $p=0.277)$ mit signifikant besseren Bewertungen bei 70-kV $(p=0.021)$. Bei 70 kV war die Halswirbelsäule in allen Fällen vollständig beurteilbar. Die Untersucher-Übereinstimmung war exzellent $(k=0.81, p<0.001)$. KRV war in $70 \mathrm{kV}$ in allen drei Halsabschnitten verbessert $(70 \mathrm{kV}: 42.0 \pm 13.1 ; 90 \mathrm{kV}$ : $22.7 \pm 11.1 ; P=0,010)$. CTDI war bei $70 \mathrm{kV}$ um ca. $30 \%$ reduziert $(4,3 \pm 0.6)$ im Vergleich zu $90 \mathrm{kV}(6,1 \pm 0.6, \mathrm{P}<0,001)$ die effektive Strahlendosis um ca. $50 \%$ (70 kV: $0.6 \pm 0.1 \mathrm{mSv} ; 90 \mathrm{kV}: 1.2 \pm 0.1 \mathrm{mSv} ; \mathrm{p}<0.001)$.

Schlussfolgerungen Die aktuelle CT-Scannergeneration erlaubt eine Reduzierung der Röntgenröhrenspannung bis zu 70-kVp in kontrastmittelunterstützten CT-Halsuntersuchungen mit gesteigertem Weichteilkontrast ohne Limitation der Beurteilung der unteren Halsabschnitte und erlaubt eine deutliche Strahlenreduktion im klinischen Alltag.

\section{RK 202.4 Multiparametrische Bildgebung bei Plattenepithelkarzinomen der Kopf-Hals-Region}

Autoren Leifels $L^{1}$, Stumpp $\mathrm{P}^{1}$, Purz $\mathrm{S}^{2}$, Kahn $\mathrm{T}^{1}$, Surov $\mathrm{A}^{1}$

Institut 1 Klinik und Poliklinik für Diagnostische und Interventionelle Radiologie, Leipzig; 2 Klinik und Poliklinik für Nuklearmedizin, Leipzig DOI 10.1055/s-0037-1600329

Zielsetzung Analyse möglicher Zusammenhänge zwischen DCE, PET und DWI beim Plattenepithelkarzinom der Kopf-Hals-Region.

Material und Methodik Bei 34 Patienten mit histologisch gesicherten primären Plattenepithelkarzinomen der Kopf-Hals-Region wurde eine simultane 18F-FDG-PET/MRT mit Diffusionsbildgebung (DWI) und T1-gewichteter dynamischer kontrastmittelgestützter MRT-Sequenz (T1w-DCE) durchgeführt. Für alle Tumoren wurden folgende Parameter erhoben: SUVmax, ADCmean, ADCmax und ADCmin, Ktrans, Kep und Ve.

Ergebnisse Im Gesamtkollektiv korrelierten sowohl ADC und ADC-abhängige Parameter als auch SUV und SUV-abhängige Parameter gut miteinander. Es zeigte sich eine inverse Korrelation von SUVmax mit rADCmin (ADCmin/ADCmean) $(r=-0,34, p=0,047)$ sowie eine Tendenz bzgl. der Korrelation von SUVmax mit $\operatorname{ADCmin}(p=0,052)$ bzw. mit Ktrans $(p=0,065)$. Ktrans korrelierte stark mit den anderen Perfusionsparametern. SUVmax/ADCmean zeigte ebenfalls eine Tendenz der Korrelation mit ADCmin $(p=0,052)$. SUVmax/ADCmean, Ve sowie SUVmax/Kep waren in der Gruppe der T3/4-Tumoren signifikant höher als in der Gruppe der T1/2-Tumoren. Kep war tendenziell niedriger in T3/4 Tumoren. Es ergaben sich keine signifikanten Unterschiede bzgl der Parameter zwischen N0/1- und N2/3-Tumoren sowie zwischen M0- und M1Tumoren. Kep $(p=0.072)$ war im Trend höher und SUVmax/Kep $(p=0.095)$ war im Trend niedriger in N2/3-Tumoren.

Schlussfolgerungen Unsere Arbeit dokumentiert komplexe Zusammenhänge zwischen Glukosestoffwechsel, Zellularität und Vaskularisation bei HNSCC. Multiparametrische Bildgebung liefert neue Erkenntnisse zur Tumorcharakterisierung bei HNSCC.

\section{RK 201.5 How we do it: CT-Angiografie mit direkter arterieller Kontrastmittel-Applikation vor Unter- und Oberkieferrekonstruktion mit einem vaskularisierten Fibulatransplantat}

Autoren Loberg $C^{1}$, Modabber $A^{2}$, Ayyoub $N^{2}$, Möhlhenrich $S^{2}$, Lethaus $B^{2}$ Institut 1 UK Aachen, Klinik für diagnostische und Interventionelle Radiologie, Aachen; 2 Uniklinik RWTH Aachen, Mund-Kiefer- und Gesichtschirurgie, Aachen

DOI 10.1055/s-0037-1600330

Zielsetzung Die Rekonstruktion von Ober- und Unterkieferdefekten ist ein komplexer operativer Eingriff mit dem Ziel Funktion und Ästhetik wieder herzustellen. Unter anderem werden Transplantate bestehend aus Fibula mit einem gestielten Gefäß-Haut-Lappen verwendet. Bei i.v. Kontrastmittelgabe lassen sich häufig die Perforatorgefäße der A. fibularis nicht identifizieren mit der Konsequenz eines relativ ausgedehnten operativen Zugangsweg. Ziel: Erlaubt die CTA mit direkter arterieller Applikation des Kontrastmittel eine verbesserte Identifikation der Perforatoren der A. fibularis und eine exaktere Planung der Präparation der Lappenplastik?

Material und Methodik 25 Patienten wurden mittels direkter arterieller CTA untersucht. Die Untersuchung erfolgte nach retrograder Punktion der AFS rechts und Einbringen einer 4F Schleuse und eines $4 \mathrm{~F}$ Pigtail Angiografiekathteter der oberhalb der Aortenbifurkation platziert wurde. Im Anschluss erfolgte eine Dual-Energy CT der arteriellen Becken-Bein Strombahn. Anhand von VRT Rekonstruktionen wurden die Perforatoren der A. fibularis identifiziert und die Distanz von der Konvexität, ausgehend vom Malleolus lateralis bis zum Perforatorgefäß vermessen. Die Auswertung erfolgte durch 2 Radiologen mit 4 und 16 Jahren Erfahrung. Zudem erfolgte ein Vergleich zu CTA Aufnahmen mit intravenöser KM Gabe.

Ergebnisse 2 CTs waren aufgrund von Bewegungsartefakten nicht auswertbar. Bei 23 Patienten ließen sich die Perforatorgefäße der A. fibularis eindeutig identifizieren. In der CT mit i.v. KM Gabe betrug die Identifikationsrate 33\%. Die präoperativ durchgeführte Messung korrelierte in $89 \%$ mit der intraoperativ identifizierten Position des Perforatorgefäß. Die Interobserver-Übereinstimmung betrug $k=0.92$

Schlussfolgerungen Die CTA mit direkter intraarterieller Kontrastmittelgabe stellt mit großer Präzision die Unterschenkelperforatorgefäße bezüglich der Lokalisation dar und ist der CTA mit i.v. KM Gabe deutlich überlegen. 


\section{RK 202.2 Bildgebung von Kopf-Hals-Tumoren mittels Dual-layer Spektral-CT}

Autoren Lohöfer $F^{1}$, Kaissis $G^{1}$, Schwarz $M^{1}$, Koerdt $S^{2}$, Noël $P^{1}$, Weichert $W^{1}$, Muecke $T^{2}$, Rummeny $E^{1}$, Braren $R^{1}$

Institut 1 TU München, Institut für diagnostische und Interventionen

Radiologie, München; 2 TU München, Klinik und Poliklinik für Mund- Kiefer-

Gesichtschirurgie

DOI 10.1055/s-0037-1600331

Zielsetzung Ziel der Studie ist die Evaluation der Vorteile der Dual-layer Spektral-CT bei Kopf-Hals-Tumoren im Vergleich zur konventionellen kontrastverstärkten $\mathrm{CT}$.

Material und Methodik Patienten mit Erstdiagnose oder Rezidiv eines KopfHals-Tumors wurden mit einem Dual-layer Spektral-CT untersucht. (IQon Spectral CT, Philips Healthcare, USA). Die Bildaquisition erfolgte in der venösen Kontrastmittelphase. Virtuelle $40 \mathrm{keV}$ und $70 \mathrm{keV}$ monoenergetische Bilder (Mono) und lod-Maps wurden berechnet und verglichen. CT-Bildgebung und Histopathologie wurden korreliert.

Ergebnisse Die Mono 40keV Bilder waren 70keV Bildern bei der Darstellung der Tumorausdehnung subjektiv überlegen. Die Mono $40 \mathrm{keV}$ Bilder zeigten relativ größere Unterschiede in den Hounsfield Einheiten (HU) zwischen Tumor und peritumoralem Gewebe (53\% bei $40 \mathrm{keV}$ vs. $42 \%$ in der konventionellen $\mathrm{CT}$ ). Die absoluten lod-Werte betrugen $2.50 .74 \mathrm{mg} / \mathrm{ml} \mathrm{im}$ Tumor und $1.00 .38 \mathrm{mg} / \mathrm{ml}$ im gesunden Gewebe. Die Tumorgrenzen korrelierten gut zwischen Spectral-CT und Histopathologie.

Schlussfolgerungen Die Dual-layer Spektral-CT ermöglicht eine verbesserte Detektion von Kopf-Hals-Tumoren und deren Rezidiven. Zudem erlaubt sie eine Quantifizierung der absoluten Jodaufnahme. Diese Ergebnisse ermöglichen potentiell eine präzisere OP-Planung und eine Etablierung der Jodaufnahme als Biomarker.

\section{RK 201.4 Strategien zur MRT-Bildgebung bei neuartigen Cochlea-Implantaten mit selbstausrichtenden Magneten - 1,5 vs. 3T; hohe Bandbreite vs. WARP Metall-Artefakt-Reduktion - eine Phantom Studie}

Autoren Pomschar $A^{1}$, Schöppe $F^{2}$, Ertl-Wagner $B^{2}$

Institut 1 Klinikum der Universität München, Institut für klinische Radiologie, München; 2 Ludwig-Maximilians-Universität München, Institut für klinische Radiologie, München

DOI 10.1055/s-0037-1600332

Zielsetzung Durch Magnete in Cochlea-Implantaten (CI) verursachte Suszeptibilitätsartefakte schränken die MRT Bildgebung des Gehirns massiv ein. Neuartige Cls sind MRT kompatibel bis 3T, ohne dass der Magnet chirurgisch entfernt werden muss. Sie verwenden einen selbstausrichtenden Magneten, der sich an dem äußeren Magnetfeld ausrichtet und so einer Demagnetisierung entgeht. Sie verursachen jedoch sehr große Artefakte mit zentraler Signalauslöschung und umgebenden Verzerrungen. Informationen zur Reduzierung dieser Artefakte sind nur spärlich verfügbar. Unser Ziel war es, anhand eines Phamtoms Strategien zur optimierten Bildgebung zu entwickeln.

Material und Methodik Wir untersuchten ein 3T kompatibles $\mathrm{Cl}$ mit Magnet (Synchrony, MED-EL) in einem Wasser gefüllten Phantom an einem 1,5 und 3T MRT (Aera und Skyra, SIEMENS) mit T1 und T2 gewichteten Sequenzen. Hierbei verglichen wir unterschiedlich hohe Bandbreiten (BB;niedrig, mittel, hoch) mit der WARP Metall-Artefakt-Reduktion mit VAT (view angle tilting). Die maximale Eindringtiefe der Signalauslöschung wurde vermessen. Verzerrung und Bildqualität wurden auf einer 5 Punkte Likert-Skala bewertet.

Ergebnisse Reduktion der Eindringtiefe: 1,5T war $3 T$ überlegen, um 21,4\% $\pm 7,7$; WARP+VAT zeigte eine Reduktion um $12 \% \pm 6,5$; bei $1,5 \mathrm{~T}$ zeigten T1 Aufnahmen eine Reduktion um 8,3\% $\pm 3,3$, bei $3 T$ jedoch eine leichte Zunahme um 2,9\% \$3,5; mit höherer BB zeigte sich eine Reduktion von 7,5 cm auf 7 bzw. 6,8. Reduktion der Verzerrungen: durch WARP mit VAT deutlich um 1.8 \pm 0.5 Punkte; sowie durch erhöhte BB von $4 \pm 1.1$ vs. $2.9 \pm 1.1$ vs. $2.5 \pm 1.2$ Punkte. WARP+VAT führt zu einer leichten Reduktion der Schärfe, dieser Effekt ist bei höheren BB geringer.

Schlussfolgerungen Aus diesen vorläufigen Phantom Messungen schließen wir, dass 1,5T mit mittlerer Bandbreite und aktivierter WARP Option mit VAT die besten Ergebnisse liefert. Die Untersuchung spezifischer Areale im Gehirn ist jedoch auch bei $3 T$ möglich, solange sie eine gewisse Mindestentfernung vom Implantat aufweisen.

\section{RK 202.6 Strukturierte Befunde von Videofluoroskopien des Schluckaktes: Verbesserung der Befundqualität und wertvoller Beitrag zur klinischen Entscheidungsfindung}

Autoren Schöppe $F^{1}$ Sommer $W^{1}$, Haack $M^{2}$, Havel $M^{2}$, Rheinwald $M^{3}$, Wechtenbruch $\mathrm{J}^{2}$, Fischer $\mathrm{M}^{4}$, Reiser $\mathrm{M}^{1}$, Sommer $\mathrm{N}^{1}$

Institut 1 Klinikum der Universität München, Institut für Klinische Radiologie, München; 2 Klinikum der Universität München, Klinik und Poliklinik für HalsNasen-Ohren-Heilkunde, München; 3 Klinikum der Universität München, Klinik und Poliklinik für Orthopädie, Physikalische Medizin und Rehabilitation (OPMR), München; 4 Klinikum der Universität München, Institut für Didaktik und Ausbildungsforschung in der Medizin, München

DOI 10.1055/s-0037-1600333

Zielsetzung Ziel der Studie war die Evaluation von strukturierten Befunden (SB) im Vergleich zu Freitext-Befunden (FTB) von Videofluoroskopien des Schluckaktes (VF) durch potenzielle klinische Zuweiser.

Material und Methodik Für diese Studie wurden 52 Befunde (FTB und SB) zu VF von 26 Patienten akquiriert. Die SB wurden anhand einer Befundvorlage, die mithilfe einer Online-Software zur Erstellung von Entscheidungsbäumen mit automatischer Textausgabe erstellt wurde, generiert. Diese Vorlage beinhaltete sowohl detaillierte Angaben zu den verschiedenen Phasen des Schluckaktes als auch die Einteilung nach der etablierten Penetration-Aspiration-Skala nach Rosenbek. Alle Befunde wurden retrospektiv durch potenzielle klinische Zuweiser hinsichtlich ihres Inhalts, des Aufwandes für die Extraktion relevanter Informationen, ihrem Beitrag zur klinischen Entscheidungsfindung (10-Punkte Likert-Skala ( 0 = Stimme überhaupt nicht zu, $10=$ Stimme vollständig zu) und der Gesamtqualität des Befundes (5-Punkte-Skala, $0=$ unzureichend, $5=$ sehr gut) bewertet.

Ergebnisse Insgesamt wurden N=104 Online-Fragebögen durch zwei HNOÄrztinnen und zwei Sprachtherapeutinnen ausgefüllt. Die SB enthielten signifikant mehr Details als FTB zu den Phasen des Schluckaktes (mediane Wertung: 10 vs. $5 ; \mathrm{p}<0.001)$ und zu Penetration and Aspiration (10 vs. 5; $\mathrm{p}<0.001)$. Der Aufwand für die Extraktion der relevanten Informationen war bei den FTB signifikant höher als bei SB (10 vs. 4; $p<0.001)$. SB haben die Fragestellung nach Einschätzung der Zuweiser signifikant besser beantwortet (9.6 vs. 8.4; $p<0.001$ ) und einen besseren Beitrag zur Entscheidungsfindung über das weitere klinische Management geleistet (10 vs. 8 ; $p<0.001$ ). Signifikant mehr SB (96\%) wurden als Befunde mit hoher oder sehr hoher Qualität bewertet als FTB $(73 \%, p<0.001)$.

Schlussfolgerungen SB von VF sind inhaltlich vollständiger und vereinfachen die Extraktion der relevanten Informationen. Durch eine verbesserte Befundqualität könnten SB damit einen besseren Beitrag als FTB zur klinischen Entscheidungsfindung leisten. 
Mammadiagnostik

\section{WISS 401.10 Radiomics in der Mamma-MRT: semantische Kriterien sind mit therapeutisch relevanten Brustkrebsgruppen assoziiert}

Autoren Baltzer $\mathrm{P}^{1}$, Leone $\mathrm{F}^{1}$, Kapetas $\mathrm{P}^{1}$, Woitek $\mathrm{R}^{1}$, Bernathova $\mathrm{M}^{1}$, Pinker $\mathrm{K}^{1}$, Helbich $\mathrm{T}^{1}$, Clauser $\mathrm{P}^{1}$

Institut 1 Universitätsklinik für Radiologie und Nuklearmedizin,

Allgemeinradiologie, Wien

DOI 10.1055/s-0037-1600334

Zielsetzung Identifikation semantischer Kriterien in der MRT der Mamma (MRM) und ihrer Assoziation mit klinisch relevanten Brustkrebsgruppen.

Material und Methodik In diese prospektive Beobachtungsstudie wurden konsekutive Patientinnen mit der Diagnose invasives Mammakarzinom eingeschlossen, welche sich einer multiparametrischen (KM-Dynamik mit einer zeitlichen Auflösung von 14 Sekunden, DTI, T2w) 3T MRT der Mamma unterzogen. Zwei erfahrene Fachärzte beurteilten folgende semantische Bildkriterien: T2w Signalintensität; Größe, Zahl und Erscheinungsbild der Läsionen nach BI-RADS (Mass, Non-mass, beides); Vorhandensein von Blooming, perifokalem Ödem, zuführenden Gefäßen; suspektester Kurventyp in der Kontrastmitteldynamik (I-III). Östrogenrezeptorstatus (ÖR), HER-2neuund MIB-1/ ki67 wurden entsprechend internationaler Richtlinien erfasst. Ein multivariater, 10-fach kreuzvalidierter data mining approach wurde auf die erfassten semantischen Kriterien angewandt um dichotomisiert ÖR, HER-2neu und MIB-1/ki67 Status zu prädizieren.

Ergebnisse 77 Patientinnen (mittleres Alter: 56a, min/max 29/85a) mit 77 Indexläsionen (55 Mass und 22 mass mit assoziierter Non-mass) wurden inkludiert. Die T2w Signalintensität war in 90,9\% der Läsionen mit erhöhtem Proliferationsindex MIB-1/ki67 hoch $(P=0,001)$. 97,4\% der Läsionen ohne perifokales Ödem waren ÖR positiv $(P<0,001)$. Die Abwesenheit von Nonmass Komponenten zeigte sich spezifisch (92,7\%) für HER-2neu Negativität $(P=0,005)$. Alle übrigen Kriterien trugen nicht unabhängig zur Differenzierung der genannten Tumortypen bei.

Schlussfolgerungen Semantische Kriterien in der MRM zeigten einen diagostischen Nutzen zur nichtinvasiven charakterisierung diagnostisch relevanter Brustkrebsgruppen.

\section{WISS 401.6 MRT der Mamma zur Evaluierung von BI-RADS 4 Mikrokalk-Läsionen}

Autoren Bennani-Baiti $\mathrm{B}^{1}$, Dietzel $\mathrm{M}^{2}$, Baltzer $\mathrm{P}^{3}$

Institut 1 Medizinische Universität Wien, Department of Biomedical Imaging and Image-guided Therapy, Wien; 2 University of Erlangen-Nürnberg, Department of Radiology, Erlangen; 3 Medizinische Universität Wien, Department of Biomedical Imagind and Image-guided Therapy, Wien DOI 10.1055/s-0037-1600335

Zielsetzung Ungefähr ein Drittel aller Mammografien weisen Mikrokalk auf. Da Mikrokalk mit einer nicht unwesentlichen Malignitätsrate behaftet ist, werden solche Läsionen derzeit primär bioptisch abgeklärt, zumeist mit einem benignen Ergebnis. Diese retrospektive Studie evaluiert die statistischen Kennzahlen der MRT der Mamma (MRM) in der Abklärung von BI-RADS 4 Mikrokalk-Läsionen und ob die MRM im Anschluss an die Mammografie geeignet ist Brustkrebs in BI-RADS 4 Mikrokalk-Läsionen auszuschließen.

Material und Methodik Eingeschlossen in diese retrospektive Studie wurden konsekutive Patientinnen (36 Monate) mit BI-RADS 4 mammografisch detektiertem Mikrokalk, der mittels MRM abgeklärt wurde. Referenzstandard war die Histopathologie. MRM wurden unter Verwendung eines einheitlichen Messprotokolls nach EUSOBI Richtlinien (1,5T, nach i.v. Gabe von 0,1 mmol/kg KG Gd-DTPA) gemessen. 2 Radiologen bewerteten die MRM im Konsensus gemäß BI-RADS. Die Daten wurden dichotomisiert (maligne vs. benigne) und die statistischen Kennzahlen danach berechnet.
Ergebnisse 248 Patientinnen mit 107 malignen und 141 benignen MikrokalkLäsionen wurden in die Studie eingeschlossen. Die Prävalenzraten von Brustkrebs betrugen 18.3\% (23/126 BI-RADS 4a), 41.7\% (25/60 BI-RADS 4b) und 95\% (59/62 BI-RADS 4c). Es gab 103 richtig-positive, 116 richtig-negative, 25 falsch-positive, und 4 falsch-negative (ein invasives Karzinom, drei DCIS; 2 BIRADS 4c, je1BI-RADS 4a und 4b in der Mammografie) MRM-Ergebnisse, die eine Sensitivität, Spezifität, sowie einen positiven und negativen Vorhersagewert von $96.3 \%$ (95\%-Cl $90.7-99.0 \%$ ), 82.3\% (95\%-Cl 75.0-88.2\%), 80.5\% (95\%-Cl $72.5-87.0 \%)$ and $96.7 \%$ (95\%-Cl $91.7-99.1 \%)$ ergaben.

Schlussfolgerungen Die MRM ist ein präzises Verfahren zur weiteren Abklärung von BI-RADS 4 Mikrokalk. Die MRM hat das Potential unnötige Biposien in weniger suspektem Mikrokalk zu vermeiden.

\section{WISS 401.7 Lokalrezidiv beim Mammakarzinom - Mit welchen morphologischen Eigenschaften müssen wir in der MR-Mammografie rechnen?}

Autoren Knaudt J ${ }^{1}$, Sutton $\mathrm{E}^{2}$, Zhao $\mathrm{Q}^{2}$, Morris $\mathrm{E}^{2}$

Institut 1 Universitätsmedizin Mannheim, medizinische Fakultät Universität Heidelberg, Institut für klinische Radiologie und Nuklearmedizin, Mannheim; 2 Memorial Sloan Kettering Cancer Center, Department of Radiology, Breast and Imaging Center, New York, USA

DOI 10.1055/s-0037-1600336

Zielsetzung Kenntnisse der morphologischen Eigenschaften lokaler Rezidive in der MR-Mammografie (MRM) sind essenziell, um eine frühe Diagnose und optimale Therapieplanung zu gewährleisten. In dieser Studie wurden die Bildeigenschaften lokaler Rezidive des Mammakarzinoms nach brusterhaltender Therapie (BET) in der MRM untersucht.

Material und Methodik 71 Patientinnen mit histologisch gesichertem lokalem Rezidiv nach BET und diagnostsicher MRM wurden retrospektiv eingeschlossen. Es erfolgte die Re-Evaluation der MRM durch zwei Reader im Konsensus. Rezidive wurden nach den aktuellen MR BI-RADS Kriterien einschließlich Lokalisation, Morphologie und Verteilung klassifiziert und mit Patientenund Tumorparametern korreliert.

Ergebnisse 71 Frauen (Medianes Alter 50,4 Jahre, range 36,2-81,4 Jahre) wurden inkludiert. Zwischen Erstdiagnose und Rezidiv lagen im Median 36,4 Monate (range 3,3-168,8 Monate). Die Mehrheit der Rezidive war unifokal (44/71, 61,9\%) und im selben Quadranten wie der primäre Tumor lokalisiert (59/71, 83,0\%). Typische morphologische Charakteristika waren: Herdbefunde (mass like lesions) 47/71 (66,2\%) mit runder/ovaler Form 34/47 (72,3\%) und umschriebenem Rand 25/47 (53,2\%), homogener Konstrastmittelaufnahme 39/71 (64,7\%) und isointensem T2-Signal 57/71 (80,2\%). In 63/71 $(88,7 \%)$ stimmten die Histologie von Primarius und Rezidiv überein. Die MRM Charakteristika früher Rezidive (<2 Jahre) unterschieden sich nicht signifikant von denen späterer Rezidive ( $>2$ Jahre) $(p>0,05)$.

Schlussfolgerungen Lokalrezidive nach BET des Mammakarzinomes sind häufig im Bereich der Lumpektomie lokalisiert und zeigen nur selten typische Malignitätskriterien. Dominierende Charakteristika in der MRM sind unifokale Herdbefunde mit runder/ovaler Form, umschriebenem Rand, homogener KM Aufnahme und isointensem T2-Signal.

\section{WISS 401.2 Biologisch-typisierte invasive Mammakarzinome: Detektionshäufigkeiten durch Screening und im Intervall}

Autoren Polzer $\mathrm{P}^{1}$, Bokhof $\mathrm{B}^{2}$, Heindel $\mathrm{W}^{1}$, Weigel $\mathrm{S}^{1}$

Institut 1 Universitätsklinikum Münster, Institut für Klinische Radiologie, Münster; 2 Universitätsklinikum Münster, Referenzzentrum Mammografie, Münster

DOI 10.1055/s-0037-1600337

Zielsetzung Beim Mammakarzinom wird der Therapieansatz und die Prognose wesentlich durch die Tumorbiologie beeinflusst. Wir analysierten die mittels Mammografie-Screening entdeckten Karzinome nach immunhistochemischer 
Subtypisierung - Hormonrezeptorstatus (Östrogen- und/oder Progesteron) (HR) und HER2-Expression - und verglichen sie mit den im nachfolgenden 2-jährigen Screening-Intervall diagnostizierten Tumoren.

Material und Methodik Bei 434 diagnostizierten invasiven Karzinomen unter 53.376 Teilnehmerinnen (TN) einer Screening-Einheit (1/2006-12/2010) konnten folgende Subtypengruppen gebildet werden: a) HR+ HER2-, b) HR+ HER2+, c) HR- HER2+ oder d) HR- HER2- (triple negativ). Detektionsraten (DR) pro 1.000 untersuchte Frauen wurden pro Modus (Screening, Intervall) und als deren Summe (2-jahres-Inzidenz) aller TN und getrennt nach Altersgruppen ( $50-59$ vs. $60-69$ Jahre) berechnet.

Ergebnisse Die 2-Jahres-Inzidenz invasiver Karzinome aller TN lag bei 8,34\%。 mit folgender Subtypenverteilung: a) $71,7 \%$; b) $13,9 \%$; c) $4,9 \%$; d) $7,0 \%$; unbekannt 2,5\%. Die DR aller Subtypen war im Screening höher als im Intervall (a) $4,96 \%$ vs. $1,01 \%$; b) $0,92 \%$ vs. $0,24 \%$; c) $0,36 \%$ vs. $0,06 \%$; d) $0,39 \%$ vs. $0,19 \%$ ). Die Summe der DR der HER2+ und triple-negativen Karzinome betrug für das Screening 1,67\%, für das Intervall 0,49\%. Die 2-Jahres-Inzidenz invasiver Karzinome der 60-69-Jährigen lag höher als die der 50 - 59-Jährigen ( $11,22 \%$ vs. 6,18\%). Auch für die Altersgruppen $50-59$ Jahre bzw. 60 - 69 Jahre wies die Summe der DR der HER2+ und triple-negativen Karzinome höhere Raten im Screening als im Intervall auf (1,28\% vs. $0.43 \%$ bzw. $2,19 \%$ vs. $0,57 \%$ ).

Schlussfolgerungen Die aggressiveren HER2+ und triple-negativen Karzinome umfassen 26\% der 2-Jahres-Inzidenz der invasiven Mammakarzinome unter Screening-TN. Ihre Detektionsraten sind im Screening zwischen 50 - 59 bzw. 60 - 69 Jahren etwa dreimal höher als im Intervall.

\section{WISS 401.1 Früherkennung des Mammakarzinoms: Welchen Einfluss hat die mammografische Drüsengewebs-Dichte tatsächlich bei mammografisch okkulten Karzinomen?}

Autoren Schrading $\mathrm{S}^{1}$, Keulers $\mathrm{A}^{1}$, Dirrichs $\mathrm{T}^{1}$, Strobel $\mathrm{K}^{1}$, Kuhl $\mathrm{C}^{1}$

Institut 1 Klinik für Diagnostische und Interventionelle Radiologie der RWTH Aaachen, Aachen

DOI 10.1055/s-0037-1600338

Zielsetzung Es ist gut belegt, dass die Dichte des Drüsengewebes die Detektion von Karzinomen in der Mammografie erschwert. Daten zur falsch-negativ Quote der Mammografie im Früherkennungs-Kollektiv basieren im Wesentlichen auf der Erfassung von tastbaren (Intervall-)Karzinomen. Ziel unserer Studie war es zu klären, welche Drüsengewebs-Dichte bei asymptomatischen Frauen vorliegt, deren Karzinom nicht mittels Früherkennungs-Mammografie diagnostiziert wurde, sondern mittels Ultraschall oder MRT.

Material und Methodik Zwischen 2013-2016 wurden 352 mammografisch okkulte Karzinome bei 321 asymptomatischen Frauen detektiert und analysiert. Alle Mammografien wurden als digitale Vollfeld-Mammografie (bis 2013) oder 3D-Mammografie (DBT plus C-view; ab 2013) durchgeführt und doppelbefundet. 87/352 (25\%) der Malignome waren DCIS, 265/352 (75\%) invasive Karzinome (mittlere bzw. mediane Größe 16 bzw. 13 mm). 105 (30\%) Karzinome wurden primär durch die Früherkennungs-Sonografie, 247 (70\%) durch die Früherkennungs-MRT detektiert. Es erfolgte die Analyse der mammografischen Drüsengewebedichte (ACR A-D) im Areal des sonografisch oder MRtomografisch detektierten Karzinoms.

Ergebnisse Die lokale Drüsengewebsdichte im Bereich des Karzinoms wurde als ACR-A in 28/352 (8\%), als ACR-B in 90/352 (26\%), als ACR-C in 143/352 (44\%), und als ACR-D in 91/352 (26\%) eingestuft - entsprechend einer Verteilung von nicht-dicht (ACR A/B) zu dicht (ACR C/D) von 34\% (118/352) zu $66 \%(234 / 352)$. Dabei unterschied sich die Verteilung nicht für mammografisch okkulte DCIS oder okkulte invasive Karzinome.

Schlussfolgerungen Im Bereich von mammografisch okkulten DCIS bzw. invasiven Karzinomen findet sich zwar häufiger mitteldichtes oder sehr dichtes Drüsengewebe als es der Verteilung im gesamten Früherkennungs-Kollektiv entsprechen würde. Allerdings bleibt zu beachten, dass ein Drittel aller mammografisch okkulten Karzinome bei Frauen mit nicht-dichter Brust gefunden werden. Dies sollte bei der Beratung von Frauen zur Früherkennung berücksichtigt werden.

\section{WISS 401.9 Suche nach einem bildgebenden Biomarker für eine Tamoxifen-Resistenz: Korrelation des Hintergrund-Anreicherns (BPE) in der Mamma- MRT mit dem CYP2D 6 Genotyp bei Patientinnen unter Tamoxifen-Therapie}

Autoren Schrading $S^{1}$, Keulers $A^{1}$, Dirrichs $T^{1}$, Arnemann $J^{2}$, Kuhl $C^{1}$ Institut 1 Klinik für Diagnostische und Interventionelle Radiologie der RWTH Aaachen, Aachen; 2 Labor Dr. Wisplinghoff, Köln

DOI 10.1055/s-0037-1600339

Zielsetzung Tamoxifen ist ein Prodrug, das durch CYP2D6 in das aktive Endoxifen metabolisiert wird. Tamoxifen-Resistenzen werden mutmaßlich durch Mutationen in CYP2D 6-Allelen verursacht. Eine Gen-Testung hat sich bislang nicht durchgesetzt, da Ergebnisse mit dem klinischen Outcome nicht korrelieren. Es ist bekannt, dass Tamoxifen zu einer Reduktion des BPE in der MRT führt. Unsere Hypothese ist, dass die Stärke des BPE in der MRT als Biomarker für eine (reduzierte) Wirksamkeit von Tamoxifen fungieren könnte. Ziel war es, das BPE mit Veränderungen im Genotyp CYP2D 6 bei Frauen unter Tamoxifen zu korrelieren.

Material und Methodik Prospektive Studie zwischen 5/2014-7/2015 bei 100 Frauen unter mind. 3-monatiger Therapie mit Tamoxifen. Das BPE in der MRT wurde entsprechend den MR-ACR-Kategorien mit ACR1 (kein BPE) bis ACR4 (starkes BPE) kategorisiert. Zum Zeitpunkt der MRT wurden folgende CYP2D 6 Allele bestimmt und mit dem Grad der BPE korreliert: CYP2D 6*4, Stop-codon; CYP2D 6*5, Deletion; CYP2D 6*10, reduzierte Enzymaktivität.

Ergebnisse 91/100 Frauen zeigten kein BPE (ACR1), 8/100 minimales (ACR2) und 1/100 moderates (ACR3). Keiner der 9 Frauen mit relevantem BPE (ACR2/3) wies einen pathogenen CYP2D 6 Genotyp auf: 6 hatten einen Wildtyp CYP2D 6, 3 eine heterozygote Mutation ohne Einfluss auf den TamoxifenMetabolismus. Von den 91 Frauen ohne BPE (ACR1) wiesen 67 einen Wildtyp, 22 geringe heterozygote Genotyp Variationen mit einzelnen oder kombinierten Mutationen von CYP2D 6*4, *5 und *10 auf. 2/91 Frauen ohne BPE (ACR1) hatten eine pathogene Mutation in CYP2D6. Entsprechend fand sich keine Korrelation zwischen dem CYP2D6 Genotyp und der Stärke des BPE $(r=0.42)$

Schlussfolgerungen Die fehlende Korrelation zwischen BPE und CYP2D 6 Genotyp kann dadurch bedingt sein, dass das BPE nicht geeignet ist, um Tamoxifen-Resistenzen zu identifizieren. Da keine Korrelation zwischen Genotyp und klin. Outcome besteht, könnten die Ergebnisse auch ein weiterer Hinweis sein, dass der CYP2D 6 Genotyp für eine apparente Tamoxifen-Resistenz nicht entscheidend ist

\section{WISS 401.8 Upgrade-Raten nach MR-geführter Vakuum-Biopsie (MR-g VAB)}

Autoren Schrading $S^{1}$, Dirrichs $T^{1}$, Schneider $H^{1}$, Kuhl $C^{1}$

Institut 1 Klinik für Diagnostische und Interventionelle Radiologie der RWTH Aaachen, Aachen

DOI 10.1055/s-0037-1600340

Zielsetzung Bei Nachweis von B3-Läsionen in der VAB ist eine operative Nachresektion erforderlich, da in bis zu 50\% der Fälle ein „upgrade“ besteht, d.h, dass im operativen Resektat nicht nur eine B3, sondern eine behandlungspflichtige Läsion (DCIS oder invasives Karzinom) gefunden wird. Ein „upgrade" kann zudem bei der VAB Diagnose eines DCIS (zu einem invasiven Karzinom) beobachtet werden. Bei der MR-g VAB werden in unserer Klinik mindestens 24 9G-Stanzzylinder pro Läsion entnommen. Ziel war es zu klären, wie nach MR-g VAB solcher größeren Biopsie-Volumina die Upgrade-Raten sind. 
Material und Methodik Zwischen 2009-2016 wurde die MR-g VAB bei 1213 Frauen mit insgesamt 1887 Zielläsionen in standardisierter Technik mit einen 9G-System durchgeführt. Bei allen Frauen mit einer B3-Läsion (ADH, FEA, LIN, Papillom mit Atypien) oder DCIS in der MR-g VAB erfolgte die operative Resektion. Die Upgraderate nach B3- oder DCIS-Diagnose sowie Komplikationen (z. B. Blutungen) nach MR-g VAB wurden ermittelt.

Ergebnisse Bei der MR-g VAB wurden im Mittel 38 (24-60) 9G-Proben entnommen. Histologisch ergab sich bei $41 \%(783 / 1887)$ Läsionen ein maligner (352 DCIS, 431 invasive Karzinome); bei 40\% (735/1887) ein benigner Befund und bei 19\% (369/1887) eine B3-Läsion mit: ADH in 28\% (103/369), FEA 21\% (78/369), LIN 15\% (56/369) und 36\% (132/369) Papillome mit Atypien. Unter den 352 DCIS zeigte sich bei der nachfolgenden Resektion ein „upgrade" in ein (mikro-)invasives Karzinom in 3 Fällen (3/352; 0.8\%). Unter den 369 B3-Läsionen fand sich bei der nachfolgenden Resektion ein „upgrade“ in ein DCIS in 3 Fällen (3/369; 0.8\%), ein „upgrade“ in ein invasives Karzinom in keinem Fall. In allen drei Fällen entsprach die B3-Läsion einer ADH. Es wurden keine behandlungsbedürftigen Komplikationen beobachtet.

Schlussfolgerungen Nach MR-g VAB mit Asservierung größerer GewebeMengen und Nachweis einer ADH oder eines DCIS sind Upgrade-Raten extrem niedrig. Operative Nach-Resektionen sind nach solchen MR-geführten Eingriffen unserer Erfahrung nach nicht mehr indiziert.

\section{WISS 401.5 Galaktografie mit Tomosythese - Renaissance einer Methode?}

Autoren Schulz-Wendtland $\mathrm{R}^{1}$, Uder $\mathrm{M}^{2}$, Müller-Schimpfle $\mathrm{M}^{3}$ Institut 1 Radiologisches Institut, Gynäkologische Radiologie, Erlangen;

2 Universitätsklinikum Erlangen, Radiologisches Institut, Erlangen; 3 Klinikum Frankfurt-Hoechst, Klinik für Radiologie, Frankfurt-Hoechst

DOI 10.1055/s-0037-1600341

Zielsetzung Ziel unserer Untersuchung war ein Vergleich der duktusorientierten Sonografie mit der kontrastmittelunterstützten Galaktografie in Tomosynthesetechnik und den daraus generierten synthetischen digitalen 2D-VollfeldMammografien.

Material und Methodik In der Zeit vom 01.10.2014 bis 31.03.2016 führten wir (Radiologisches Institut des Universitätsklinikums Erlangen und der Klinik für Radiologie - Klinikum Frankfurt Hoechst) bei insgesamt 100 Patientinnen sowohl eine duktusorientierte Sonografie, eine kontrastmittelunterstützte Galaktografie mithilfe der Tomosynthese in 3D- als auch den daraus generierten synthetischen digitalen 2D-Vollfeld-Mammografien durch. Das mittlere Alter der Patientinnen betrug 54,2 Jahre (minimal 21 Jahre, maximal 83 Jahre). Die Auswertung der 3 Untersuchungsmodalitäten erfolgte durch 3 in der komplementären Mammadiagnostik erfahrene Untersucher (1, 5 und 15 Jahre) und wurde mit der endgültigen Histologie korreliert.

Ergebnisse Über alle drei Untersucher resultierte für den duktusorientierten, hochfrequenten Ultraschall eine Sensitivität von 79,7\%, einer Spezifität von $58,3 \%$, einem $p p \bigvee$ von $77,3 \%$ und einem npV von $61,8 \%$; demgegenüber für die synthetischen digitalen 2D-digitalen Vollfeld-Mammografien von $80,0 \%$, $60,0 \%, 78,8 \%, 61,8 \%$ und der 3D-digitalen Tomosynthese von 90,9\%, 73,9\%, $92,1 \%$ und $73,9 \%$. Die Genauigkeit der einzelnen Methoden ergab für den duktusorientierten Ultraschall einen Wert von 0,72 , die synthetische digitale 2D-digitalen Vollfeld-Mammografie von 0,73 und der 3D-digitalen Tomosynthese von 0,87 .

Schlussfolgerungen Erstmaliger Einsatz der Tomosynthese in der Galaktografie mit deutlich besseren Ergebnissen im Vergleich zum duktusorientierten hochfrequenten Ultraschall - dies kann zu einer Renaissance dieser Methode und Erweiterung des Spektrums der Tomosynthese in der komplementären Mammadiagnostik führen.

\section{WISS 401.3 Digitales Mammografie-Screening: Einfluss der regelmäßigen Teilnahme auf die Detektion des ductalen Carcinoma in situ}

Autoren Weigel $\mathrm{S}^{1}$, Heindel $\mathrm{W}^{1}$, Heidinger $\mathrm{O}^{2}$, Heidrich $\mathrm{J}^{2}$, Khil $\mathrm{L}^{2}$, Hense $\mathrm{H}^{3}$ Institut 1 Universitätsklinikum Münster, Institut für Klinische Radiologie und Referenzzentrum Mammografie, Münster; 2 Landeskrebsregister Nordrhein Westfalen, Münster; 3 Institut für Epidemiologie und Sozialmedizin,

Universität Münster, Münster

DOI 10.1055/s-0037-1600342

Zielsetzung Bestimmung der Detektionsraten des ductalen Carcinoma in situ (DCIS) und Vergleich der Anteile der Kernmalignitätsgrade zwischen der Implementierungsrunde (IR) und der ersten bzw. zweiten Folgerunde (FR1, FR2) bei einem zweijährigen Screeningintervall.

Material und Methodik Eingeschlossen wurden 713.981 Untersuchungen der IR (50 - 69 Jahre, 2005 - 2008), 516.151 der FR1 (52 - 69 Jahre, 2007 - 2010) und 393.938 der FR2 (54-69 Jahre, 2009-2012). Die totale DCIS-Detektionsrate pro 100 gescreenter Frauen (DetR\%) sowie die Unterteilung nach den Kernmalignitätsgraden (Grade) gering, intermediär und hoch wurden pro Runde ermittelt. Zur Prüfung von Unterschieden der Anteile der Grade an der totalen DCIS-DetR\% wurden logistische Regressionsmodelle verwendet. Statistische Signifikanz wurde bei $p<0,05$ angenommen.

Ergebnisse Die totale DCIS-DetR\% der IR betrug 0,14\% (Anteil Grade: gering 18,8\%, intermediär 38,6\%, hoch 42,7\%), die der FR1 0,11\% (Anteil Grade: gering $11,0 \%$, intermediär 38,3\%, hoch 50,7\%), der FR2 0,12\% (Anteil Grade: gering $13,6 \%$, intermediär 35,5\%, hoch 50,9\%). Der DCIS-Anteil vom geringen Grad war in der FR1 (OR: 0,53; 95\%KI: 0,38-0,72; $\mathrm{p}<0,001$ ) bzw. FR2 (OR: $0,71 ; 0,51-0,97 ; p=0,036)$ signifikant niedriger als in der IR. Der Anteil des DCIS vom hohen Grad lag in der FR1 (OR: 1,38, 95\%KI; 1,11-1,71; $p=0,003$ ) bzw. FR2 (OR: 1,31; 95\% KI: 1,11-1,65; p=0,022) signifikant höher als in der IR. Hinsichtlich des DCIS vom intermediären Grad ergaben sich keine signifikanten Unterschiede.

Schlussfolgerungen Das DCIS vom geringen Kernmalignitätsgrad wird unter allen Differenzierungsgraden in der Implementierungsrunde und in den Folgerunden am seltensten diagnostiziert, vom hohen Kernmalignitätsgrad am häufigsten. Im Vergleich zur prävalenten Erstrundenuntersuchung führt die regelmäßige Teilnahme im zweijährigen Intervall zu einer signifikanten Abnahme des DCIS-Anteils vom geringen Kernmalignitätsgrad sowie zu einer signifikanten Zunahme des DCIS-Anteils vom hohen Kernmalignitätsgrad, der biologisch relevantesten Form.

\section{WISS 401.4 KM-gestütztes cone-beam Brust-CT: Erste Ergebnisse zur Detektion des Mammakarzinoms in dichtem Drüsengewebe}

Autoren Wienbeck $S^{1}$, von Fintel $E^{2}$, Stahnke $V^{1}$, Lotz $J^{1}$, Fischer $U^{3}$ Institut 1 Universitätsmedizin Göttingen, Institut für Diagnostische und Interventionelle Radiologie, Göttingen; 2 Universitätsmedizin Göttingen, Institut für Daignostische und Interventionelle Radiologie, Göttingen;

3 Diagnostisches Brustzentrum Göttingen, Göttingen

DOI 10.1055/s-0037-1600343

Zielsetzung Es wird der Stellenwert des cone-beam Brust-CT (CBBCT) in Nativtechnik im Vergleich zu einem KM-gestütztem cone-beam Brust-CT (CECBBCT) zur Detektion des Mammakarzinoms in dichtem Drüsengewebe evaluiert.

Material und Methodik In dieser prospektiven, klinischen Studie, wurde bei Frauen mit einem ACR-Dichtetyp c oder d und einem BI-RADS 4 oder 5 Befund in der digitalen Vollfeldmammografie und/oder Ultraschall der Brust ein ergänzendes CBBCT (Fa. Koning Corp., Rochester, USA) in Nativtechnik und nach KM-Applikation angefertigt. Das Patientenalter, die Brustgewebsdichte nach $A C R$, die Befundgröße und das histopathologische Ergebnis nach erfolgter Biopsie wurden evaluiert. Eine relative Änderung der mittleren Dichtewerte in Houndsfield Einheiten (HE) zwischen der Tumormasse und dem umgeb- 
enden Drüsengewebe wurden für alle Fälle aufgezeichnet. Hierfür wurden jeweils drei Messungen des Tumors und des umgebenden Drüsenparenchyms anhand der koronaren Schichten vor und nach KM-Applikation vorgenommen. Ergebnisse In dieser laufenden Studie wurde von Januar bis August 2016 ein CBBCT und CE-CBBCT bei 30 Frauen mit 49 Herdbefunden (12 benigne, 18 semimaligne (B3-Befunde), 29 invasive Karzinome) durchgeführt. Die bisherige Untersuchungen zeigen eine zuverlässige Detektion von Karzinomen mittels CE-CBBCT. Die Erkennbarkeit ist besser im Vergleich zu einem CBBCT in Nativtechnik und der Mammografie. Nach KM-Applikation konnte für benigne Läsionen ein Anstieg der Dichtewerte von im Median 38,9 +/- 3,5 HE, für B3Befunde von 42,8 +/2,5 HE und für invasive Karzinome von 66,2 +/- 3,2 HE gesehen werden.

Schlussfolgerungen Die ersten Ergebnisse mit einem KM-gestütztem CBBCT CBBCT zeigen eine bessere Detektion des Mammakarzinoms für Frauen mit ACR-Dichtetypen c oder d im Vergleich zu Mammografie und CBBCT. Maligne und semimaligne Befunde zeigen einen höheren Anstieg der Dichtewerte im Vergleich zu benignen Befunden. Diese Quantifizierung kann die Erkennung und Diagnose von Brustkrebs unterstützen.

Molekulare Bildgebung

\section{WISS 204.5 Kathepsine als Marker für die molekulare Fluoreszenzbildgebung der Riesenzellarteriitis}

Autoren Both $\mathrm{M}^{1}$, Humbert $\mathrm{J}^{1}$, Müller $\mathrm{A}^{2}$, Duwendag $D^{3}$, Holl-Ulrich $\mathrm{K}^{4}$, Heneweer $C^{5}$, Meyer $P^{1}$, Fritzer $E^{6}$, Oltmann Schröder $]^{7}$, Glüer $C^{1}$, Jansen $O^{1}$, Tiwari $S^{1}$

Institut 1 Universitätsklinikum Schleswig-Holstein, Campus Kiel, Klinik für Radiologie und Neuroradiologie, Kiel; 2 Universitätsklinikum SchleswigHolstein, Campus Lübeck, Klinik für Rheumatologie, Lübeck;

3 Universitätsklinikum Schleswig-Holstein, Campus Kiel, Klinik für Ophthalmologie, Kiel; 4 Universitätsklinikum Schleswig-Holstein, Campus Lübeck, Institut für Pathologie, Lübeck; 5 Universitätsklinikum Köln, Institut für Diagnostische und Interventionelle Radiologie, Köln;

6 Universitätsklinikum Schleswig-Holstein, Campus Kiel, Institut für Medizinische Informatik und Statistik, Kiel; 7 Universitätsklinikum Schleswig Holstein, Campus Kiel, Klinik für Innere Medizin I, Kiel

DOI 10.1055/s-0037-1600344

Zielsetzung Ziel dieser Studie war die experimentelle Etablierung eines exvivo-Modells zur spezifischen bildgebenden Diagnostik der Riesenzellarteriitis mittels fluoreszenzoptischer Visualisierung krankheitstypischer enzymatischer Prozesse.

Material und Methodik Biopsate der Arteria temporalis superficialis von Patienten ( $n=91$, Durchschnittsalter: 74 Jahre) mit dem klinischen Verdacht auf eine aktive Riesenzellarteriitis wurden anteilig immunhistochemisch auf die Expression von Kathepsin K und Kathepsin L sowie auf die enzymatische Aktivität von Kathepsin K untersucht. Zudem wurden entsprechende Gefäßbiopsate in vitro mit einem Kathepsin-aktivierbaren fluoreszenzoptischen Kontrastmittel inkubiert und im Nahinfrarotbereich mittels Fluoreszenztomografie untersucht. Es erfolgte der Abgleich mit der klinischen Diagnostik (American College of Rheumatology-Kriterien) und der ergänzend erhobenen Serologie (CRP), der präoperativ durchgeführten MRT sowie dem Routine-Histologiebefund.

Ergebnisse Die abschließende Evaluation der $\mathrm{n}=91$ Patienten erbrachte $\mathrm{n}=18$ eindeutig negative Fälle, $\mathrm{n}=45$ positive Fälle einer Riesenzellarteriitis und $\mathrm{n}=\mathbf{2 8}$ nicht eindeutige Befunde. Die Expression der Kathepsine $\mathrm{K}$ und $\mathrm{L}$ war bei positiven Befunden einer Riesenzellarteriitis in den histologischen Befunden signifikant höher $(p<0,001)$ als bei Patienten mit Normalbefunden, auch die für Kathepsin $\mathrm{K}$ gemessene Enzymaktivität $(p<0,001)$. In der experimentellen in vitro-Fluoreszenzdiagnostik konnte eine signifkant höhere Signalgebung $(p<0,01)$ bei den Biopsaten mit Riesenzellarteriitis nachgewiesen werden.
Schlussfolgerungen Mit den vorliegenden Untersuchungen wurde in vitro eine umfassende Grundlage für die translationale Idee einer möglichen künftigen optischen Bildgebung der Riesenzellarteriitis geschaffen. Kathepsine sind als Marker für eine derartige fluoreszenbasierte Diagnostik funktionell geeignet.

\section{WISS 201.5 In vitro und in vivo Bildgebung des ErbB/ Her2 Rezeptors}

Autoren Hundt $\mathrm{W}^{1}$, Nadine Tischer $\mathrm{N}^{2}$, Béhé $\mathrm{M}^{2}$, Alfke $\mathrm{H}^{1}$

Institut 1 Philipps Universität Marburg, Klinik für diagnostische und

interventionelle Radiologie, Marburg; 2 Philipps Universität Marburg, Klinik für Nuklearmedizin, Marburg

DOI 10.1055/s-0037-1600345

Zielsetzung In vitro und in vivo Bildgebung des ErbB/Her2 Rezeptors mit Herceptin und den F(ab')2 und F(ab')- Fragmenten des Herceptins

Material und Methodik Herceptin und die F(ab')2 - und F(ab')-Fragmente des Herzeptins wurden an Cy5.5-NHS gekoppelt. In den in vitro Untersuchungen wurden ErbB/Her2-positive SKOV3 Zellen mit Herceptin, dem $F\left(a b^{\prime}\right) 2$ oder dem F(ab)' Fragment bis zu 24 inkubiert und eine Fluoreszenzmikroskopie durchgeführt. In den in vivo Untersuchungen wurde Mäusen mit Her2-positiven Tumoren markierte Herceptin Antikörper, und die markierten $F\left(a b^{\prime}\right) 2$ - und $F\left(a b^{\prime}\right)$-Fragmente injiziert und eine in vivo NIR-Fluoreszenz Bildgebung durchgeführt.

Ergebnisse Bei den in vitro Untersuchungen konnte ein spezifisches Fluoreszenzsignal innerhalb der Zellen nachgewiesen werden, hinweisend auf eine effiziente zelluläre Internalisation des Rezeptorligantenkomplexes. In den in vivo Experimenten konnten die ErbB2-positiven Tumoren erfolgreich mit dem farbstoffmarkierten Liganden Cy5.5-Herceptin und Cy5.5-F(ab') dargestellt werden. Beim Cy5.5-F(ab')2 Fragment konnte jedoch nur eine schwacher Kontrast zwischen Tumor und umgebendem Gewebe nachgewiesen werden. Möglicherweise bedingt durch die schlechteren pharmacokinetischen Eigenschaften des $\mathrm{F}(\mathrm{ab}$ ')2 Fragmentes.

Schlussfolgerungen Die in vitro und in vivo Bildgebung des ErbB/Her2 Receptors mit markiertem Herceptin und dessen F(ab')2 - und F(ab')-Fragmente ist möglich. Die mögliche klinische Anwendung könnte in der nicht invasiven Diagnostik von ErbB/Her2neu-positiven Mammatumoren liegen. Weitere Anwendungen dieser Fluoreszenzbildgebung mit Herceptin Derivaten sind die intraoperative oder lapraskopische Identifikation von abdominellen Metastasen of ErbB/Her2neu-positiver Ovarial-, Cervical-, Colon oder Mammakarzinomen mit endoskopischen Fluoreszenzbildgebungstechniken.

\section{WISS 201.2 Multi-patch MPI zur Ganzkörperbildgebung der Maus unter Einsatz eines lang zirkulierenden Blood-Pool-Tracers}

Autoren Jung $C^{1}$, Salamon $]^{2}$, Swargulski $P^{3}$, Kaul $M^{2}$, Hofmann $M^{3}$, Gdaniec $\mathrm{N}^{3}$, Adam $\mathrm{G}^{2}$, Knopp $\mathrm{T}^{3}$, Ittrich $\mathrm{H}^{4}$

Institut 1 Interventionelle und Diagnostische Radiologie, Uniklinik Hamburg Eppendorf, Hamburg; 2 Interventionelle und Diagnostische Radiologie und Nuklearmedizin, Uniklinik Hamburg Eppendorf, Hamburg; 3 Interventionelle und Diagnostische Radiologie und Nuklearmedizin, Experimentelle biomedizinische Bildgebung, Uniklinik Hamburg Eppendorf, Hamburg; 4 Interventionelle und Diagnostische Radiologie und Nukleramedizin, Uniklinik Hamburg Eppendorf, Hamburg DOI 10.1055/s-0037-1600346

Zielsetzung Eine Limitation der Magnetic Particle Bildgebung (MPI) stellt aktuell die abnehmende räumliche Auflösung bei Vergrößerung des Field of views (FOV) dar. Ziel war es, das FOV mittels Multi-patch MPI und dem Einsatz eines lang zirkulierenden Blood Pool Tracers LS-008 zu vergrößern, um eine Ganzkörperbildgebung der Maus in vivo in einer hohen räumlichen Auflösung zu etablieren. 
Material und Methodik 10 Minuten nach der iv Injektion von $60 \mu \mathrm{LS}-008$ (Lode-Spin) erfolgten die MPI Messungen an FVB Mäusen $(n=4)$ bei einer Drive-Field Amplitude von 14mT. Zum Vergleich der räumlichen Auflösung wurden zwei Gradientenstärken eingesetzt: bei 1,5 T/m wurden drei unterschiedliche Patches (FOV 37,3 $\times 37,3 \times 18,6 \mathrm{~mm} 3$ ) und bei $2,5 \mathrm{~T} / \mathrm{m}$ sechs Patches (FOV $22,4 \times 22,4 \times 11,2 \mathrm{~mm} 3$ ) benötigt, um die gesamte Maus abzubilden. Für die anatomische Hintergrundinformation wurden MRT Untersuchungen (T2w 2D Turbo-Spinecho-Sequenzen) an einem 7T ClinScan (Bruker) durchgeführt. Zur Fusionierung der MPI und MRT Daten wurden Fiducial Marker eingesetzt. Die Bildrekonstruktion erfolgte mittels einer eigens entwickelten Software basierend auf der Programmiersprache Julia.

Ergebnisse Die kombinierten MPI/MRT Messungen konnten erfolgreich durchgeführt werden. Die Bildrekonstruktionen der unterschiedlichen Patches zur Darstellung der gesamten Maus in vivo zeigten keine Artefakte an den einzelnen Bildrändern. Im Vergleich zu 1,5T/m konnte mit der höheren Gradientenstärke von 2,5T/m eine bessere räumliche Auflösung erzielt werden. Hier konnten neben der Darstellung der Vena cava inferior und des Herzens, eine detailliertere Abbildung der Halsgefäße, der thorakalen Aorta und der Nierengefäße und eine Detektion der Lebergefäße erzielt werden.

Schlussfolgerungen Die Ganzkörperbildgebung mittels Multi-Patch MPI konnte in dieser Studie zum ersten Mal in vivo erfolgreich eingesetzt werden. Der lang zirkulierende Blood-Pool Tracer ermöglicht die Bildgebung ohne Bewegungsartefakte, die bei einem Tracer mit kurzer Halbwertszeit und schneller Leberaufnahme auftreten würden.

\section{WISS 204.6 Evaluation praktischer Hindernisse in der Beurteilung der 68Ga-PSMA PET/CT bei 55 Patienten: Physiologische Tracerverteilung und inzidenteller Uptake}

Autoren Kirchner $J^{1}$, Schaarschmidt $B^{1}$, Sawicki $L^{1}$, Heusch $\mathrm{P}^{1}$, Hautzel $\mathrm{H}^{2}$, Antoch $\mathrm{G}^{1}$, Buchbender $\mathrm{C}^{1}$

Institut 1 Heinrich-Heine-Universität Düsseldorf, Medizinische Fakultät, Institut für Diagnostische und Interventionelle Radiologie, Düsseldorf;

2 Heinrich-Heine-Universität Düsseldorf, Medizinische Fakultät, Klinik für Nuklearmedizin, Düsseldorf

DOI 10.1055/s-0037-1600347

Zielsetzung Das Ziel der vorliegenden Arbeit war die Evaluation der physiologischen 68Ga-PSMA Verteilung sowie eines fokalen oder diffusen 68Ga-PSMA Uptakes in nicht-Prostatakarzinom assoziierten Prozessen.

Material und Methodik Es wurde eine retrospektive Analyse von 55 Patienten durchgeführt, die eine 68Ga-PSMA PET/CT Untersuchung bei Prostatakarzinom (49) oder Nierenzellkarzinom (6) erhalten hatten. Die 68Ga-PSMA PET/ CT wurde durch zwei unabhängige Radiologen bezüglich eines physiologischen sowie eines inzidentellen 68Ga-PSMA Uptakes ausgewertet. In den Organen und Läsionen mit 68Ga-PSMA Uptake wurden die SUVmax und SUVmean gemessen. Als Referenzstandard dienten radiologische, klinische und histopathologische Nachsorgeuntersuchungen.

Ergebnisse Ein homogener 68Ga-PSMA Uptake zeigte sich bei allen Patienten in den Tränendrüsen (SUVmax 15.7 \pm 7.2 ), Parotiden (SUVmax 24.4 \pm 8.1 ), Submandibulardrüsen (SUVmax 26.7 \pm 7.1 ), Stimmbändern (SUVmax 8.4 \pm 3 ), Nieren (SUVmax $66.4 \pm 25.4$ ) sowie im Waldeyer'schen Rachenring (SUVmax 10.4 \pm 4.3 ) und in der Leber (SUVmax 8.2 \pm 2.5 ), Milz (SUVmax 10.9 \pm 3.9 ) sowie dem Pars descendens duodeni (SUVmax 17.6 \pm 8.9 ). Bei 36/55 Patienten $(65 \%)$ zeigte sich ein homogener Uptake im Colon descendens (SUVmax $10.6 \pm 9.2$ ) und bei 20/55 Patienten (36\%) im Rektum (SUVmax 3.7 \pm 1.1 ). Ein 68Ga-PSMA Uptake der Schilddrüse (SUVmax $4.5 \pm 1.2$ ) zeigte sich bei $22 \%$ der Patienten sowie bei $21 \%$ der Patienten in der Synovia der Knie (SUVmax $2.9 \pm 0.2$ ). Darüber hinaus wurde bei einem Patienten ein 68Ga-PSMA Uptake des rechten Os iliums aufgrund einer fibrösen Dysplasie nachgewiesen (SUV$\max 7.7)$.
Schlussfolgerungen Ein regelmäßiger 68Ga-PSMA Uptake zeigt sich in den Tränen- und Speicheldrüsen sowie Stimmbändern, Waldeyer'schen Rachenring, Leber, Milz, Nieren und verschiedenen Teilen des Magen-Darm-Traktes. Darüber wurde ein unspezifischer Traceruptake der Schilddrüse und der Synovia der Knie nachgewiesen. Ein inzidenteller Uptake, wie bei der fibrösen Dysplasie, findet sich auch bei nicht-Prostatakarzinom assoziierten Prozessen.

\section{WISS 204.3 Diagnostischer Mehrwert der 68Ga- DOTATATE PET-CT in der Beurteilung transossärer Meningeome}

Autoren Kunz $W^{1}$, Jungblut $L^{1}$, Kazmierczak $\mathrm{P}^{1}$, Rominger $\mathrm{A}^{2}$, Albert $\mathrm{N}^{2}$, Reiser $\mathrm{M}^{1}$, Cyran $\mathrm{C}^{1}$

Institut 1 Klinikum der Universität München, Institut für Klinische Radiologie, München; 2 Klinikum der Universität München, Klinik und Poliklinik für

Nuklearmedizin, München

DOI 10.1055/s-0037-1600348

Zielsetzung Untersuchung der diagnostischen Genauigkeit der 68Ga-DOTATATE PET-CT und der MRT zur Detektion und Darstellung transossärer Meningeome.

Material und Methodik Aus 325 konsekutiven Patienten mit einer 68Ga-DOTATATE PET-CT Untersuchung des Schädels zur Beurteilung eines Meningeoms wurden Patienten nach folgenden Einschlusskriterien ausgewählt: (1) MRT Bildgebung innerhalb 1 Monats der entsprechenden prä- oder postoperativen PET-CT, und (2) histologisch gesichertem Ein- oder Ausschluss einer knöchernen Infiltration. Die Bilddaten wurden von 2 erfahrenen, verblindeten Readern bezüglich knöcherner Infiltration, Tracer Uptake und Tumorvolumen analysiert. Chi-Quadrat-, Mann-Whitney-U- und McNemar's Tests sowie Receiver Operating Characteristics wurden zur statistischen Auswertung angewendet.

Ergebnisse Zweiundachtzig Patienten erfüllten die Einschlusskriterien. Transossäre Meningiome ( $n=67$ ) zeigten größere Tumorvolumina (Median $12.8 \mathrm{ml}$ vs. $3.3 \mathrm{ml}, \mathrm{p}<0.001$ ) und höheren Tracer Uptake (Median SUVmax: 14.2 vs. 7.6, $p=0.011$; Median SUVmean: 4.3 vs. 2.7, $p=0.001$ ) als extraossäre Meningeome. Die 68Ga-DOTATATE PET-CT zeigte eine höhere Sensitivität ( $98.5 \%$ vs. $53.7 \%$ ) bei nahezu ebenso hoher Spezifität ( $86.7 \%$ vs. $93.3 \%$ ) in der Detektion der ossären Infiltration verglichen mit der MRT ( $<<0.001)$. In ROC-Analysen erlaubte die qualitative PET-CT eine genauere Klassifikation der ossären Infiltration als die MRT (Area under the curve: 0.932 vs. 0.773). PET-CT- und MRTbasierte Volumetrierung ergaben eine gute Übereinstimmung für extraossäre Meningiome $(p=0.132)$ und für den extraossären Anteil transossärer Meningiome $(p=0.636)$. Dahingegen erzielte die PET-CT für den intraossären Anteil signifikant größere Volumina $(p<0.001)$.

Schlussfolgerungen Die 68Ga-DOTATATE PET-CT bietet gegenüber der MRT einen signifikanten diagnostischen Mehrwert zur Detektion und Darstellung transossärer Meningeome. Die 68Ga-DOTATATE PET-CT hat das Potential die präoperative Planung und das postoperative Management von Meningeompatienten zu verbessern.

\section{WISS 201.6 Bestimmung der Herzfunktion in einem Mausmodell zum Myokardinfarkt mittels optoakustischer Bildgebung}

Autoren Lohöfer $\mathrm{F}^{1}$, Lin $\mathrm{H}^{2}$, Déan-Ben $\mathrm{X}^{2}$, Kimm $\mathrm{M}^{1}$, Haas $\mathrm{H}^{1}$, Meier $\mathrm{R}^{1}$, Razansky $\mathrm{D}^{2}$, Wildgruber $\mathrm{M}^{3}$

Institut 1 TU München, Institut für diagnostische und Interventionen

Radiologie, München; 2 Helmholtz Zentrum München, Institute of Biological and Medical Imaging, München; 3 Universitätsklinikum Münster, Institut für klinische Radiologie, Münster

DOI 10.1055/s-0037-1600349

Zielsetzung Ziel der Studie ist es die Kontrast-verstärkte dreidimensionale optoakustische Bildgebung ohne kardiales Gating für funktionelle Untersuchungen des murinen Myokardinfarktes zu etablieren. 
Material und Methodik Es wurde eine neue dreidimensionale optoakustische Bildgebung mit hoher volumetrischer Bildfrequenz $(50 \mathrm{~Hz})$ verwendet. Kardiale Perfusionsparameter wurden in Echtzeit mit dem optischen Kontrastmittel Indocyanin Green (ICG) gemessen. Als Mausmodell des akuten Myokardinfarktes wurde die operative Infarktinduktion durch Ligatur der LAD mittels transparentem Fadenmaterial gewählt, das für die Artefakt-freie optoakustische Bildgebung optimiert wurde. DiePulmonary-transit-time wurden zwischen Mäusen mit und ohne Herzinfarkt verglichen. Als Referenz für die Quantifizierung der Herzfunktion wurde die MRT eingesetzt.

Ergebnisse Mit der optoakustischen Bildgebung konnten die Pulmonary Transit Time mittels beat-by-beat Analyse bestimmt werden. Bei den Herzen mit Infarkt $(n=9)$ zeigte sich eine signifikant höhere Pulmonary Transit Time ( $2.35 \mathrm{~s}$ [2.07 s-2.4 s] versus $1.25 \mathrm{~s}$ [1.20 s-1.32 s], $\mathrm{p}=0.0195)$ im Vergleich zu gesunden Kontrolltieren. Die MRT zeigte, dass die Pulmonary Transit Time mit der Infarktgröße sowie einer Abnahme der Ejektionsfraktion (EF) ansteigt. Schlussfolgerungen Die optoakustische Bildgebung ist in der Lage Herzanatomie und Funktion sowohl mit hoher Zeit-, als auch Ortsauflösung darzustellen. Die optoakustische Charakterisierung der Herzdynamik kann neue Einblicke in die Pathophysiologie nach myokardialer Ischämie ermöglichen.

\section{WISS 201.3 Time lapse MRT: Single cell tracking in der experimentellen autoimmunen Enzephalomyelitis}

\author{
Autoren Masthoff $\mathrm{M}^{1}$, Gran $\mathrm{S}^{2}$, Zhang $\mathrm{X}^{3}$, Wachsmuth $\mathrm{L}^{1}$, Becker $\mathrm{A}^{1}$, \\ Bietenbeck $M^{1}$, Heindel $W^{1}$, Sorokin $L^{3}$, Roth $\rfloor^{2}$, Eisenblätter $M^{1}$, \\ Wildgruber $\mathrm{M}^{1}$, Faber $\mathrm{C}^{1}$ \\ Institut 1 Universitätsklinikum Münster, Institut für klinische Radiologie, \\ Translational Research Imaging Center, Münster; 2 Universität Münster, \\ Institut für Immunologie, Münster; 3 Universität Münster, Institut für \\ Physiologische Chemie und Pathobiochemie, Münster \\ DOI 10.1055/s-0037-1600350
}

Zielsetzung Time lapse MRT ist eine innovative Technologie mit erheblichem Potential für cell tracking Studien. Über die repetitive Bildakquisition soll nicht nur ein einzeitiger, statischer Bildeindruck, sondern eine dynamische Abbildung markierter Leukozyten ermöglicht werden. Ziel dieser Studie war die Optimierung der zeitlichen Auflösung von time lapse MRT zur Anwendung in einem Modell muriner autoimmuner Enzephalomyelitis (EAE).

Material und Methodik Die Induktion der EAE erfolgte in C57BL/6 J Mäusen via i. v. Injektion des Myelin Oligodendrozyten Glykoproteins. Eisenoxid Nanopartikel (Resovist ${ }^{\circledR}, 1,3 \mathrm{ml}$ pro kg/KG) wurden $24 \mathrm{~h}$ vor der MRT-Untersuchung des Gehirns intravenös injiziert. Diese wurde an einem 9,4T Kleintier-MRT bei EAE Mäusen in unterschiedlichen Krankheitsstadien (symptomatisch $n=8$, präsymptomatisch $n=2)$ bzw. gesunden Kontrolltieren $(n=3)$ mit einer T2* Multigradienten-Echo Sequenz und folgenden Parametern durchgeführt: TR: $649 \mathrm{~ms}$, TE: $8.0 \mathrm{~ms}$, FA: $60^{\circ}$, Mittelungen: 4, Schichten: 38, Schichtdicke: $300 \mu \mathrm{m}$, zeitliche Auflösung: $8 \mathrm{~min} 12 \mathrm{~s}$ (einzelner time frame) bzw. $2 \mathrm{~h}$ 44 min bei 20 time frames. Nach der MRT Untersuchung wurden die Mäuse geopfert und das Gehirn histologisch mittels der Berliner-Blau Eisenfärbung und der Mac-3 Makrophagenfärbung aufbereitet. Die MR-Datensätze wurden in Bezug auf die Detektion hypointenser Spots (Events) manuell ausgewertet. Ergebnisse Die zeitliche Auflösung von time lapse MRT betrug für eine vollständige Akquisition des Gehirns 8 min pro time frame. Die Technik ermöglichte die Verfolgung zirkulierender, mit Eisenpartikeln beladener Leukozyten in gesunden Tieren sowie Tieren mit EAE. Die Anzahl detektierter Events zeigte eine signifikante Veränderung zwischen EAE- und Kontrolltieren.

Schlussfolgerungen Time lapse MRT ermöglicht single cell tracking im Tiermodell. Am Beispiel der EAE konnte die Möglichkeit zur Detektion einer veränderten Immunzelldynamik bei inflammatorischen Erkrankungen aufgezeigt werden.

\section{WISS 204.2 ADC Histogramm-Analyse beim primären Zervixkarzinom}

Autoren Meyer $\mathrm{H}^{1}$, Exner $\mathrm{M}^{2}$, Bremicker $\mathrm{K}^{2}$, Höhn $\mathrm{A}^{3}$, Schob $\mathrm{S}^{4}$, Surov $\mathrm{A}^{2}$ Institut 1 Universitätsklinikum Leipzig, Klinik und Poliklinik für Diagnostische und Interventionelle Radiologie, Leipzig; 2 Klinik und Poliklinik für Diagnostische und Interventionelle Radiologie, Leipzig; 3 Institut für Pathologie, Leipzig; 4 Klinik und Poliklinik für Diagnostische und Interventionelle Radiologie, Neuroradiologie, Leipzig DOI 10.1055/s-0037-1600351

Zielsetzung Die Apparent diffusion coefficient (ADC) Histogramm Analyse ist ein aufkommendes neues Verfahren, um die Heterogenität von Läsionen darzustellen. Es ist jedoch unklar, ob damit histopathologische Parameter reflektiert werden. Deshalb ist das Ziel dieser Studie mögliche Assoziationen zwischen verschiedenen ADC-Fraktionen und der Histopathologie bei Zervixkarzinomen zu untersuchen.

Material und Methodik 18 Patientinnen (mittleres Alter 55,4 Jahre) mit histologisch gesichertem Plattenepithelkarzinom der Cervix uteri wurden prospektiv in diese Studie eingeschlossen. DWI wurde mit den b-Werten b0 und b1000 s/mm2 akquiriert. Es erfolgte eine Histogramm Analyse der eingezeichneten ROI auf der ADC-Karte mit Ermittlung folgender Parameter: ADCPerzentilen (P10, P25, P75 und P90), ADCmin, ADCmean, ADCmax, ADCMode, Median ADC, Kurtosis, Skewness und Entropy. Klinisch-pathologische Stadien und die histopathologischen Parameter KI67-Index und p53-Wert wurden auch erhoben.

Ergebnisse Die Korrelationsanalyse ermittelte eine statistisch signifikante Korrelation zwischen der Entropy und dem p53-Wert ( $r=-0,472, p=0,048)$. G1 $/ 2$ Tumoren hatten signifikant niedrigere $A D C m i n$ Werte im Vergleich zu G3 Läsionen $(0,40 \pm 0,19$ vs. $0,65 \pm 0,24 \times 10-3 \mathrm{~mm} 2 \mathrm{~s}-1, \mathrm{p}=0.035)$. Die Entropy war als einziger Parameter statistisch signifikant höher in T3/4 Tumoren gegenüber T2-Tumoren. Nodal positive Tumoren zeigten statistisch signifikant niedrigere folgende Parameter: ADCmin, ADCmean, median ADC, Mode, P10, P25, P75 und P90.

Schlussfolgerungen Diese Studie zeigte verschiedene Assoziationen zwischen der Histopathologie und ADC Histogramm-Parametern in Plattenepithelkarzinomen der Cervix uteri. Diese Zusammenhänge könnten nicht mithilfe einer konventionellen DWI-Analyse erhoben werden.

\section{WISS 204.4 Bildgebung der Großgefäßvaskulitis im kontrastgehobenen FDG PET/CT - Welche Bildgebungsparameter eignen sich zur Aktivitätsbeurteilung in Zusammenschau mit den Entzündungswerten?}

Autoren Olthof $\mathrm{S}^{1}$, Krumm $\mathrm{P}^{1}$, Jenes $\mathrm{J}^{2}$, Xenitidis $\mathrm{T}^{2}$, Nikolaou $\mathrm{K}^{1}$, la Fougère $C^{3}$, Pfannenberg $C^{1}$, Schwenzer $N^{1}$

Institut 1 Eberhard Karls Universität Tübingen, Diagnostische und Interventionelle Radiologie, Tübingen; 2 Eberhard Karls Universität Tübingen, Innere Medizin II - Onkologie, Hämatologie, Klinische Immunologie, Rheumatologie und Pulmologie, Tübingen; 3 Eberhard Karls Universität Tübingen, Nuklearmedizin und Klinische Molekulare Bildgebung, Tübingen DOI 10.1055/s-0037-1600352

Zielsetzung Evaluierung visueller und quantitativer Bildgebungsparameter im kontrastangehobenen FDG PET/CT bei unbehandelten Großgefäßvaskulitis-Patienten hinsichtlich Interreader Übereinstimmung und Korrelation mit Inflammationsmarkern.

Material und Methodik Durchführung einer retrospektiven Analyse von 17 Großgefäßvaskulitis-Patienten, die vor Therapieeinleitung ein kontrastangehobenes FDG PET/CT erhielten und über Entzündungswerte (CRP, BSG) verfügten. Zwei Radiologen werteten verblindet 7 Gefäßregionen pro Patient aus (A. carotis, subclavia, axillaris, Aorta thorakalis und abdominalis, Ilakal- und Beckengefäßachse). Die visuelle PET Analyse erfolgte nach Meller1 (Werte 0 3 je nach FDG-Aufnahme im Gefäß im Verhältnis zur FDG-Aufnahme der 
Leber) bzw. für das CT nach Hata2 (Werte 1 - 5, 1: Äste des Aortenbogens, 2: Aorta thorakalis, 3: Aorta descendens und abdominalis 4: Aorta abdominalis 5: Aorta thorakalis und abdominalis). Die quantitative PET-Evaluierung umfasste Quotienten aus SUVmax oder SUVavg des betroffenen Gefäßabschnitts zur FDG-Aufnahme in Leber, rechten Vorhof und Vena jugularis interna. Die quantitative CT Evaluierung wurde anhand der mittleren Gefäßwanddicke zum Gefäßdiameter durchgeführt. 1Meller et al., EJNMMI. 2003, 2Hata et al., Int J Cardiol. 1996

Ergebnisse CRP-Werte korrelierten mit dem visuellen PET bei beiden Radiologen ( $\rho$ jeweils 0,640 und 0,541). Die höchste Korrelation zu Entzündungswerten wies das quantitative PET bei Verwendung der Leber als Referenzgewebe auf (BSG $\rho$ 0,681 und 0,652; CRP $\rho 0,708$ und 0,738). Zwischen Entzündungsparametern und visueller bzw. quantitativer CT-Auswertung ergaben sich keine Korrelationen. Die Interreader Übereinstimmung variierte von $\mathrm{K}$ 0,341 (quantitative CT) bis zu K 0,826 (höchster SUVavg Gefäß/höchster SUVavg Leber).

Schlussfolgerungen Aufgrund der hohen Interreader Übereinstimmung und der starken Korrelation zu den Entzündungswerten stellt das quantitative PET eine zuverlässige Methode zur Aktivitätsbeurteilung der Großgefäßvaskulitis im Gegensatz zur CT-Bildgebung dar.

\section{WISS 204.1 Facettengelenkstropismus und -sagittalisierung: Risikofaktoren für die Entwicklung molekularer Bandscheibendegeneration bei gesunden Probanden}

Autoren Schleich $C^{1}$, Tschischka $A^{1}$, Bittersohl B ${ }^{2}$, Aissa J ${ }^{1}$, Boos J $]^{1}$, Sawicki $L^{1}$, Caspers $]^{1}$, Müller-Lutz $A^{1}$, Antoch $G^{1}$

Institut 1 Heinrich-Heine-Universität Düsseldorf, Medizinische Fakultät, Institut für Diagnostische und Interventionelle Radiologie, Düsseldorf;

2 Universitätsklinikum Düsseldorf, Klinik für Orthopädie, Düsseldorf DOI $10.1055 / \mathrm{s}-0037-1600353$

Zielsetzung Zur Beurteilung der Glykosaminoglykangehalts (GAG) lumbaler Bandscheiben (BS) bei gesunden Probanden mit Facettengelenkstropismus (FT) und -sagittalisierung (FS) mittels Glycosaminoglycan Chemical Exchange Saturation Transfer (gagCEST) Bildgebung.

Material und Methodik 75 BS der Lendwirbelsäule von 25 jungen, gesunden Probanden ohne Vorerkrankung der Lendenwirbelsäule (13 weiblich, 12 männlich, mittleres Alter: 28,0 $\pm 4,4$ Jahre, zwischen 21 - 35 Jahre) wurden in einem 3T-MRT untersucht. Ausrichtung der Facettengelenke wurden im Hinblick auf Tropismus und Steilstellung für L3/4, L4/5 und L5/S1 mit Standard-T2-gewichteten Bildern beurteilt. Molekulare Bildgebung mittels gagCEST wurde zur Bestimmung des GAG-Gehalts des Nucleus pulposus (NP) und Anulus fibrosus (AF) verwendet.

Ergebnisse Signifikant höhere gagCEST Werte von NP wurden bei Probanden ohne FT und normaler Facettengelenksorientierung im Vergleich zu Probanden mit FT und FS $>45^{\circ}(p<0,0001)$ gefunden. Die gagCEST Werte waren signifikant höher bei Probanden ohne FT im Vergleich zu Probanden mit mittelschwerer oder schwerer FT (moderate FT: $p<0,0001$, schwere FT: $\mathrm{p}=0,0033$ ). Probanden mit normaler Facettengelenksorientierung zeigten signifikant höhere gagCEST Werte im Vergleich zu denen mit sagittaler Facettengelenksausrichtung $>45^{\circ}(\mathrm{p}<0,001)$. Wir fanden eine signifikante, negative Korrelation zwischen gagCEST Werten und der sagittaler Facettengelenksausrichtung (rho $=-0,459 ; \mathrm{p}<0,0001$ ).

Schlussfolgerungen Die molekulare Bildgebung mit gagCEST zeigte niedrigere GAG-Werte im NP in Bandscheiben mit FT und FS, was darauf hinweist, dass Tropismus und sagittale Ausrichtung der Facettengelenke Risikofaktoren für die Entwicklung von frühen biochemischen Veränderungen der lumbalen BS darstellen.

\section{WISS 201.4 CD 38 spezifische Nanobodies für die in vivo Bildgebung von Lymphomen}

Autoren Schriewer $\mathrm{L}^{1}$, Bannas $\mathrm{P}^{1}$, Koch-Nolte $\mathrm{F}^{2}$, Adam $\mathrm{G}^{1}$, Kunick $\mathrm{V}^{1}$, Fumey $\mathrm{W}^{1}$, Schütze $\mathrm{K}^{1}$

Institut 1 Universitätsklinikum Hamburg-Eppendorf, Klinik für interventionelle und diagnostische Radiologie und Nuklearmedizin, Hamburg; 2 Universitätsklinikum Hamburg-Eppendorf, Institut für Immunologie, Hamburg

DOI 10.1055/s-0037-1600354

Zielsetzung CD 38 ist ein transmembranes Ektoenzym exprimiert auf Plasmazellen und über exprimiert auf hämatopoetischen Tumoren wie z.B. dem Multiplen Myelom und Burkitt Lymphom. CD 38 repräsentiert somit ein vielversprechendes Zielmolekül für die molekulare Bildgebung von hämatopoetischen Tumoren. Das Ziel unserer Studie ist es CD38-spezifische Einzeldomänenantikörper (Nanobodies), gewonnen aus Schwere-Ketten-Antikörpern von Lamas, für die in vivo Bildgebung von hämatopoetischen Tumoren zu entwickeln.

Material und Methodik Wir haben mehrere CD 38-spezifsche Nanobodies aus immunisierten Lamas gewonnen. Die Bindungsspezifität, Affinität und Epitopkartierung wurden in vitro mittels Durchflusszytometrie analysiert. Ausgewählte Nanobodies wurden aufgereinigt und an AF680 konjugiert. Qualitätskontrollen erfolgten mittels SDS-Page, Coomassie Färbung und Fluoreszenzimaging der Gele. Für die in vivo Bildgebung von CD 38 positiven und negativen Tumoren im Mausmodell, wurden mit Luziferase transduzierte, menschliche und murine Tumorzellen s.c. injiziert. Fluorophore-konjugierte Nanobodies wurden nach Detektion von subkutanen Tumoren intravenös injiziert. Die Bildgebung erfolgte vía Biolumineszenz und Nah-Infrarot-Fluoreszenzoptischer (NIRF) Bildgebung. Ex vivo Analysen entnommener Tumor erfolgte mittels Mikroskopie und Durchflusszytometrie.

Ergebnisse Wir konnten 22 Nanobody-Familien selektieren, die 3 unterschiedliche Epitope auf CD 38 erkennen. CD 38 positive Tumoren konnten in vivo mit hoher Spezifität nach intravenöser Injektion mittels Fluorophor konjugierter Nanobodies detektiert werden.

Schlussfolgerungen Unsere CD38 spezifischen Nanobodies erscheinen als vielversprechendes Werkzeug für die Charakterisierung und Detektion von CD38 überexprimierenden hämatopoetischen Tumoren in vitro und in vivo.

\section{WISS 201.1 Bioluminescence imaging for monitoring therapeutic response of CD 38-specific nanobody-Fc fusion proteins in a lymphoma model}

Authors Schriewer $L^{1}$, Bannas $P^{1}$, Koch-Nolte $F^{2}$, Schütze $K^{1}$, Fumey $W^{1}$, Adam $G^{1}$

Institute 1 Universitätsklinikum Hamburg-Eppendorf, Klinik für interventionelle und diagnostische Radiologie und Nuklearmedizin, Hamburg; 2 Universitätsklinikum Hamburg-Eppendorf, Institut für Immunologie, Hamburg DOI 10.1055/s-0037-1600355

Zielsetzung CD38 is overexpressed by lymphomas and other hematological tumors and represents a promising target for immunotherapy. We aimed to develop CD38-specific nanobody-Fc fusion proteins for imaging and treatment of hematological tumors and to test them in a bioluminescent lymphoma model.

Material und Methodik We generated CD 38-specific nanobodies (15kDa) by phage-display technology from immunized llamas. Specific binding, affinity and epitope specificity of selected nanobodies were analyzed by flow cytometry. Nanobodies were fused to the Fc-domains of human IgG1. The capacity of $\mathrm{Nb}-\mathrm{Fc}$ fusion proteins $(60 \mathrm{kDa})$ to induce complement-dependent cytotoxicity (CDC) of human lymphoma cell lines and bone marrow cells of multiple myeloma patients were analyzed by flow cytometry. In vivo effects of Nb-Fc fusion proteins on tumor growth were assessed in SCID-mice injected with luciferase-transduced human Burkitt lymphoma cells. Therapeutic response 
was determined via bioluminescence imaging of disseminated tumors (IVIS200,PerkinElmer) and survival curves.

Ergebnisse We selected 22 families of CD 38-specific nanobodies. Crossblockade analyses indicate that most of these bind to 3 non-overlapping epitopes. While single $\mathrm{Nb}-\mathrm{Fc}$ fusion proteins showed little if any capacity to induce $\mathrm{CDC}$, the combination of two $\mathrm{Nb}$-Fc-fusion proteins recognizing distinct epitopes showed very potent $C D C$ toward $C D$ 38-expressing cell lines as well as toward primary CD 38+ tumor cells from bone marrow samples of myeloma patients. In vivo bioluminescence imaging of luciferase-transduced CD 38+ Burkitt lymphoma cells confirmed the therapeutic efficacy of combinations of two Fcfusion proteins recognizing distinct epitopes. Such combinations significantly reduced tumor growth and significantly prolonged survival of treated mice compared to controls.

Schlussfolgerungen Bioluminescence imaging is a reliable tool for monitoring diffuse tumor growth and therapy response. CD38-specific nanobody-Fc fusion proteins hold promise as therapeutics for CD 38-expressing lymphomas.

\section{Muskuloskelettale Radiologie}

\section{WISS 313.3 Computertomografie nach spinaler Instrumentierung: Evaluation zweier iterativer Algorithmen zur Metallartefaktreduktion}

\author{
Autoren Aissa J $]^{1}$, Thomas $C^{1}$, Sawicki $L^{1}$, Caspers J ${ }^{1}$, Kröpil $P^{1}$, Antoch $G^{1}$ \\ Boos $]^{1}$ \\ Institut 1 Universitätsklinikum Düsseldorf, Institut für Diagnostische und \\ Interventionelle Radiologie, Düsseldorf \\ DOI 10.1055/s-0037-1600356
}

Zielsetzung Ziel dieser Studie war die Evaluation zweier iterativer Algorithmen zur Metallartefaktreduktion in der postoperativen Computertomografie (CT) von Patienten nach spinalen Instrumentierungen.

Material und Methodik 24 Patienten (13 männlich, 11 weiblich, 60,1 \pm 16 ,6 Jahre) mit postoperativen spinalen CTs wurden retrospektiv eingeschlossen. Die CT Bilder wurden mit gefilterter Rückprojektion (FBP) und mit zwei dezidierten iterativen Metallartefaktreduktions-Algorithmen rekonstruiert (MARAlgo 1:spinale Instrumentierungen, MAR-Algo2: große orthopädische Metallimplantate). Zur objektiven Bestimmung der Bildqualität wurden Frequenzen von Dichteänderungen ermittelt. Die subjective Bildqualität wurde anhand der Beurteilbarkeit anatomischer Strukturen auf einer 5-Punkt-Skala bewertet (0: Struktur nicht beurteilbar, 5: Struktur gut abgrenzbar mit hoher diagnostischer Sicherheit).

Ergebnisse Beide iterativen Algorithmen reduzierten Artefakte im Vergleich zur FBP in der objektiven Auswertung signifikant (MAR-Algo1: 59527,2 $\pm 70182,6$; MAR-Algo2: $89593,3 \pm 41590,5$, FBP: $123273,4 \pm 104734,9$, $\mathrm{p}<0,001)$. In der subjektiven Auswertung verbesserten beide Algorithmen die Beurteilbarkeit von knöchernen Strukturen (Median MAR-Algo1: 3; inter quartile range (IQR): 3-4; MAR-Algo2: 4; IQR: 4-5) und Weichteilen (MARAlgo1: 3; IQR: 1,5-3; MAR-Algo2: 4; IQR: 3,5-4) verglichen mit FBP signifikant (knöcherne Strukturen: 2; IQR: 1-3; Weichteile: 2; IQR: 0,5-2) $(p<0,001)$. Dabei führte MAR-Algo2 objektiv und subjektiv zu einer stärkeren Artefaktreduktion und besseren Beurteilbarkeit von knöchernen und Weichteilstrukturen als MAR-Algo1 ( $p<0,001)$.

Schlussfolgerungen Beide hier untersuchten iterativen Metallartefaktreduktions-Algorithmen verbessern essentiell die Bildqualität und reduzieren Artefakte in der CT von Patienten mit spinaler Instrumentierung verglichen zur FBP. Der eigentlich auf große orthophädische Implantate abgestimmte Algorithmus ist dabei offensichtlich noch effektiver als der auf spinale Instrumentation ausgerichtete Algorithmus.

\section{WISS 313.9 Zementaugmentation zur Behandlung von Insuffizienzfrakturen des Os sacrum mittels Ballonsakroplastie (BSP), Radiofrequenzsakroplastie (RFS), Vertebrosakroplastie (VSP) oder Zementsakroplastie (ZSP)}

Autoren Andresen $\mathrm{R}^{1}$, Radmer $\mathrm{S}^{2}$, Andresen $\mathrm{J}^{3}$, Schober $\mathrm{H}^{4}$ Institut 1 Westküstenklinikum Heide, Akademisches Lehrkrankenhaus der Universitäten Kiel, Lübeck und Hamburg, Institut für Diagnostische und Interventionelle Radiologie/Neuroradiologie, Heide; 2 Zentrum für Bewegungsheilkunde, Facharztpraxis für Orthopädie, Berlin; 3 SigmundFreud-Privatuniversität, Wien; 4 Klinikum Südstadt Rostock, Akademisches Lehrkrankenhaus der Universität Rostock, Klinik für Innere Medizin I, Rostock DOI 10.1055/s-0037-1600357

Zielsetzung In dieser Studie sollte die Machbarkeit und das klinische Outcome für die unterschiedlichen Behandlungsverfahren überprüft werden. Material und Methodik Bei 80 Patienten mit insgesamt 122 Os sacrum Frakturen erfolgte die Zementaugmentation CT gesteuert mittels BSP, RFS, VSP oder ZSP. Zementleckagen wurden mit einem CT am 1. post operativen Tag detektiert. Die Schmerzintensität wurde prä interventionem, am 2. Tag sowie 6, 12 und 18 Monate post interventionem mittels VAS bestimmt. Die Ergebnisse wurden auf Signifikanz mittels gepaarter Wilcoxon-Rangsummentests und Mann-Whitney U-Tests überprüft.

Ergebnisse Bei allen Patienten ließ sich die BSP, RFS, VSP und ZSP technisch gut durchführen. Pro Fraktur wurden im Durchschnitt 6,4 ml in der BSP Gruppe, $6,2 \mathrm{ml}$ in der RFS Gruppe, 5,8 $\mathrm{ml}$ in der VSP Gruppe und $6 \mathrm{ml}$ in der ZSP Gruppe eingebracht. Eine Leckage konnte für die BSP Gruppe mit 38 Frakturen und RFS Gruppe mit 37 Frakturen ausgeschlossen werden, in VSP Gruppe fanden sich bei 22 Frakturen 5 Leckagen (23\%) und in der ZSP 25 Frakturen 4 Leckagen (16\%). Der Mittelwert für Schmerzen lag nach VAS vor Intervention bei 8,6 in der BSP-Gruppe, bei 8,8 in der RFS-Gruppe, bei 8,6 in der VSPGruppe, bei 8,7 in der ZSP-Gruppe. Am 2. postoperativen Tag zeigte sich eine signifikante Schmerzreduktion $(p<0,0005)$ mit einem Durchschnittswert von 2,5 für alle Gruppen. Nach $6(12 ; 18)$ Monaten lagen diese mit $2,2(2,3 ; 2,0)$ für die BSP-Gruppe, $2,3(2,2 ; 2,0)$ für die RFS-Gruppe, $2,4(2,2 ; 2,0)$ für die VSP-Gruppe und $2,4(2,3 ; 2,2)$ für die ZSP-Gruppe stabil. Hinsichtlich Schmerzen zeigten sich Überschreitungswahrscheinlichkeiten von $p>0,9$ unter allen Verfahren im Verlauf, so dass alle Behandlungsmethoden zu keinen Ergebnisunterschieden führten.

Schlussfolgerungen Die BSP, RFS, VSP und ZSP sind interventionelle, minimalinvasive Verfahren welche mittelfristig eine gleich gute Schmerzreduktion erreichen. Bei der VSP und ZSP muss man mit einer höheren Zementleckagerate rechnen, so dass die BSP und RFS die deutlich sichereren Verfahren darstellen.

\section{WISS 313.2 Intra-individueller Vergleich zwischen Ultra-Low-Dose und Standard-Dosis CT der Lumbalwirbelsäule bei $150 \mathrm{kV}$ mit Zinn-Filter: CT Untersuchungen unterhalb der Dosis eines konventionellen Röntgenbildes}

Autoren Bucher $A^{1}$, Scholtz $\rfloor^{1}$, Kohle $C^{1}$, Albrecht $M^{1}$, Kaup $M^{1}$, Vogl $T^{1}$ Bodelle $B^{1}$

Institut 1 Uniklinik Frankfurt, Institut für Diagnostische und Interventionelle Radiologie, Frankfurt DOI 10.1055/s-0037-1600358

Zielsetzung Da für CT Untersuchungen der Lumbalwirbelsäule (LWS) bei äquivalenter Strahlendosis zum konventionellen Röntgenbild eine bessere Beurteilbarkeit gezeigt wurde, verfolgten wir die Zielsetzung einer systematischen Auswertung der Bildqualität aus Sub-Millisievert CT-Protokollen zur Knochenbeurteilung. 
Material und Methodik 216 Bildserien aus 36 postmortalen-CT Untersuchungen auf einem Dritt-Generation Dual-Source CT wurden ausgewertet. Bei $150 \mathrm{kV}$ (mit Zinn-Filter) wurde pro Patient eine Referenzserie (RS, $350 \mathrm{mAs}$ ) und 3 Niedrigdosis-Protokolle (ND; $60 \mathrm{mAs}$ [ND60], $30 \mathrm{mAs}$ [ND30] und $15 \mathrm{mAs}$ [ND 15]) akquiriert. Rekonstruktionen erfolgten in gefilterter Rückprojektion (FBP) und Iterativer ADMIRE Rekonstruktion (IR). Der Abdominaldurchmesser wurde auf Höhe L4, das Bildrauschen auf selber Höhe im Musculus Psoas dextra gemessen. Subjektive Bilqualität der Wirbelkörper und der Facettengelenke wurden von 2 unabhängigen Untersuchern auf einer 5-Punkt Skala bewertet. Friedman-Test und Cohens Kappa-Statistik wurden zur Analyse benutzt.

Ergebnisse Der durchschnittliche Abdominaldurchmesser betrug frontal 27,6 $\pm 9,4 \mathrm{~cm}$, sagittal 19,6 $\pm 6,2 \mathrm{~cm}$.DLP wurde in ND 15 auf ca. $7 \%$ von RS reduziert ( $p<0.001$; RS: $198,4 \pm 98,1 \mathrm{mGycm}$, ND 60: 48,4 $\pm 23,1 \mathrm{mGycm}$, ND 30: $24,6 \pm 15,5 \mathrm{mGycm}$, ND 15: $15.5 \pm 12,1 \mathrm{mGycm})$. Das Bildrauschen war zwischen RS mit FBP $(15,8 \pm 3,9)$ und ND60 mit IR $(14,7 \pm 2,6)$ vergleichbar $(p=0,41)$, in den übrigen ND Serien mit IR erhöht (ND30: 19,5 $\pm 4,2$; ND 15: $20,9 \pm 9,7 p<0,001)$. Alle Serien wurden als diagnostisch beurteilt. Die gemittelte subjektive Bildqualität wurde in RS als exzellent bewertet (5[5-5]), ND 60 (4[4 - 5]) und ND30 (4[4 - 4]) zeigten eine sehr gute mediane Bildqualität, ND15 eine gute mediane Bildqualität (3[3-3]; p-overall<0.001). Die Untersucher-Übereinstimmung war exzellent $(k=0,80 ; p<0,001)$.

Schlussfolgerungen CT-Bildgebung der LWS kann bei $20-3 \%$ der äquivalenten Strahlendosis einer konventionellen 3mSv LWS-Röntgenaufnahme in zwei Ebenen zur Knochenbeurteilung eine ausreichende bis exzellente Bildqualität liefern.

\section{WISS 313.8 Evaluation der suszeptibilitäts- gewichteten Magnetresonanztomografie zur Beurteilung von Wirbelkörperfrakturen}

\author{
Autoren Böker $\mathrm{S}^{1}$, Adams $\mathrm{L}^{1}$, Hamm B ${ }^{1}$, Makowski $\mathrm{M}^{1}$ \\ Institut 1 Radiologie Charité, Berlin \\ DOI 10.1055/s-0037-1600359
}

Zielsetzung Evaluation von suszeptibilitätsgewichteter Magnetresonanztomografie (SWMR) zur Beurteilung von Wirbelkörperfrakturen im Vergleich zur Computertomografie (CT) und Projektionsradiografie als Referenzstandard. Material und Methodik Von 42 Patienten mit Wirbelkörperfrakturen wurde eine Magnetresonaztomografie (MRT) der Wirbelsäule mit einer suszeptibilitätsgewichteten und T1-gewichtete Sequenz sowie eine T2- und/oder TIRMgewichteten Sequenzen durchgeführt. 99 Frakturen konnten detektiert werden, wovon es sich bei 49 um frische und bei 50 um ältere handelte. Als Referenzstandard lagen bei 61 der Frakturen eine CT und bei allen anderen eine projektionsradiografische Aufnahme vor. Die Hinterkantenbeteiligung sowie die Sichtbarkeit einer Frakturline und einer Kortikalisstufe wurden in den unterschiedlichen Sequenzen, CT und Projektionsradiografie bewertet. Außerdem erfolgte die Messung der Hinterkantenverlagerung.

Ergebnisse Die Sensitivität der SWMR in Bezug auf die Hinterkantenverlagerung und die Sichtbarkeit der Frakturlinie und der Kortikalisstufe war höher als in T1-, T2- und TIRM-gewichteter MRT. Im Vergleich zur CT konnten insbesondere die Frakturline und die Kortikalisstufe bei der SWMR besser erkannt werden als in den übrigen MRT-Wichtungen (Sensitivität Frakturline: SWMR 83\%, T1w 16\%, T2w 20\%, TIRM 0\%; Sensitivität Kortikalisstufe: SWMR $91 \%$, T1w 63\%, T2w 50\%, TIRM 36\%), die Spezifitäten der einzelnen Wichtungen unterschied sich nur gering (96\%-100\%). Für die Verlagerung der Hinterkante nach dorsal zeigte sich zwischen CT und SWMR eine stärkere Korrelation als zwischen CT und den anderen MRT-Wichtungen (SWMR R2=0,93; T1w $R 2=0,82 ; T 2 W R 2=0,67 ;$ TIRM R2 =0,32).

Schlussfolgerungen Die SWMR ist eine verlässliche Methode zur Beurteilung der Hinterkantenverlagerungen sowie zur Detektion von Frakturline und Kortikalisstufe bei Wirbelkörperfrakturen mit einer höheren Sensitivität der
Detektion und einer besseren Korrelation des Ausmaßes der Hinterkantenverlagerung als T1-, T2- und TIRM- gewichtete MRT.

\section{WISS 313.1 Auf der Suche nach Referenzwerten: Scherwellenelastografie (SWE) asymptomatischer Achilles-Sehnen: Ein Vergleich zwischen professionellen Athleten und der Normalbevölkerung}

Autoren Dirrichs $\mathrm{T}^{1}$, Kuhl $\mathrm{C}^{2}$, Quack $\mathrm{V}^{3}$, Hamm N $\mathrm{N}^{2}$, Schrading $\mathrm{S}^{2}$ Institut 1 Uniklinik RWTH Aachen, Klinik für Diagnostische und Interventionelle Radiologie, Aachen; 2 Klinik für Diagnostische und Interventionelle Radiologie, Uniklinik RWTH Aachen, Aachen; 3 Klinik für Orthopädie, Uniklinik RWTH Aachen, Aachen

DOI 10.1055/s-0037-1600360

Zielsetzung Die Scherwellenelastografie (SWE) hat sich als nützliches Verfahren zur Beurteilung der Sehnen-Elastizität, insb. bei Tendinopathien, bewährt. Erkrankte Sehnen sind intraindividuell weicher als gesunde, weisen also niedrigere SWE-Werte auf. Wenig ist jedoch bisher über Referenzwerte und interindividuelle Unterschiede zwischen versch. Personengruppen bekannt. Ziel der klinischen Studie war daher ein Vergleich zwischen der Achilles-Sehnenhärte gesunder Profi-Athleten und gesunder Nicht-Athleten, um anhand möglicher Referenzwerte die Früherkennung von Sehnen-Pathologien zu verbessern.

Material und Methodik Prospektive Studie mit 70 asympt. Probanden, 35 (50\%) davon Profi-Athleten (5 Lauf-Einheiten/Woche) und 35 (50\%) NichtAthleten. Es wurden 140 Achilles-Sehnen nach standardisiertem, multi-modalem Ultraschall (US)-Protokoll (B-mode US, Power Doppler (PD) und SWE), mittels hochauflösendem $15 \mathrm{MHz}$ Linearschallkopf (Aixplorer, Supersonic) untersucht. Semi-quantitative Analysen der SWE-Farbkarten und quantitative ROI-basierte Analysen der Sehnen-Elastizität wurden durchgeführt. Die SWEMittelwerte wurden mittels student's t-test verglichen.

Ergebnisse Der mittlere SWE-Wert betrug $183.8 \mathrm{kPa}( \pm 98 \mathrm{kPa})$ bei Athleten und $91.3 \mathrm{kPa}( \pm 30.5 \mathrm{kPa})$ bei Nicht-Athleten $(\mathrm{p}<0.001)$. Zwischen rechter und linker Seite waren intraindividuell keine signifikanten Unterschiede feststellbar. Semi-quantitativ (SWE-Farbkarten) wurden Athleten-Sehnen in 1/70 $(1,4 \%)$ als „weich”, in $12 / 70(17,1 \%)$ als „intermediär“ und in $57 / 70(81,5 \%)$ als "hart" eingestuft. Nicht-Athleten-Sehnen wurden in 35/70 (50\%) als "weich”, in 26/70 (37,1\%) als „intermediär“ und in 9/70 (12,9\%) als „hart“ eingestuft.

Schlussfolgerungen Die Achilles-Sehnen-Härte differiert signifikant zwischen Athleten und Nicht-Athleten. Athleten weisen höhere SWE-Werte auf, haben also härtere Sehnen. Solche interindividuellen Unterschiede müssen bei der Bewertung der Sehnenhärte, insb. bei sympt. Personen, berücksichtigt werden. Ein intraindiv. Seitenvergleich ist besonders wichtig.

\section{RK 325.1 Vergleich der diagnostischen Genauigkeit der integrierten 18F-FDG PET/MRT im Vergleich zur MRT zur Detektion lokaler Rezidive bei Weichteilsarkomen}

Autoren Erfanian $Y^{1}$, Grüneisen $J^{1}$, Kirchner $\int^{2}$, Pöppel $T^{3}$, Herrmann $K^{4}$, Umutlu $L^{1}$

Institut 1 Unikilinkum Essen, Institut für Diagnostische und Interventionelle Radiologie und Neuroradiologie, Essen; 2 Unikilinkum Düsseldorf, Institut für Diagnostische und Interventionelle Radiologie und Neuroradiologie,

Düsseldorf; 3 Unikilinkum Essen, Klinik für Nuklearmedizin, Essen; 4 Unikilinkum Essen, Klinik für Nuklearmedizin, Essen DOI 10.1055/s-0037-1600361

Zielsetzung Beurteilung der diagnostischen Genauigkeit zwischen 18F-FDG PET/MRT und der alleinigen Verwendung von MRT zur Identifizierung von lokalen Weichteilsarkom-Rezidiven nach vorheriger und vollständig operativer Entfernung des Pimärtumors. 
Material und Methodik 41 Patienten mit klinischem Verdacht auf ein Weichteilsarkom-Rezidiv wurden mittels 18F-FDG PET/MRT untersucht. Zwei erfahrene Radiologen beurteilten in einem fünfwöchigen Abstand die MRT- und PET/MRT-Daten Rezidivdiagnostik von lokalen Weichteilsarkomen. Zusätzlich wurde die diagnostische Sicherheit für jede ausgewertete Tumordetektion auf einer Skala von 1 bis 3 festgelegt, sowie ein McNemar-Test zur Differenzierung beider Analysen und ein Wilcoxon signed-rank Test zur Identifizierung dieser durchgeführt. Als Referenzstandard galten gleichermaßen ein histopathologischer Nachweis sowie mehrere Verlaufsuntersuchungen.

Ergebnisse 27/41 der Patienten zeigten ein Tumorrezidiv auf. Die kalkulierte Sensitivität, Spezifität, der positive und negative prädiktive Wert, sowie die diagnostische Genauigkeit für lokale Tumorrezidive betrugen für die MRT $81 \%$, $85 \%, 91 \%, 70 \%, 82 \%$ und $96 \%, 75 \%, 89 \%, 91 \%, 90 \%$ für die PET/MRT ( $p>0.05$ ). Ebenfalls wies die PET/MRT eine signifikant höhere diagnostische Sicherheit im Hinblick auf die Detektion der malignen Läsionen auf.

Schlussfolgerungen Die Ergebnisse zeigen die exzellente Aussagekraft der 18F-FDG PET/MRT zur Evaluation möglicher Weichteilsarkom-Rezidive nach chirugischer Resektion der Primärtumore auf. Ferner konnte eine überlegene Detektionsgenauigkeit der PET/MRT im Vergleich zur Standard-MR-Verlaufskontrolle nachgewiesen werden.

\section{WISS 307.4 Morphologische Bildgebung von Meniskus- und Knorpelschäden des Kniegelenkes mit Ultrahochfeld-MRT bei 7Tesla - ein Vergleich mit 3-Tesla Bildgebung mit arthroskopischer Korrelation}

Autoren Friebe $B^{1}$, Richter $M^{1}$, Penzlin $S^{1}$, Stärke $C^{2}$, Godenschweger $F^{3}$, Ricke $\mathrm{J}^{1}$, Kropf $\mathrm{S}^{4}$, Fischbach $\mathrm{F}^{1}$, Speck $\mathrm{O}^{5}$

Institut 1 Universitätsklinik Magdeburg, Radiologie und Nuklearmedizin, Magdeburg; 2 Universitätsklinik Magdeburg, Orthopädie, Magdeburg;

3 Institut für experimentelle Physik, Biomedizinische Magnetresonanz, Magdeburg; 4 Universitätsklinik Magdeburg, Institut für Biometrie und medizinische Informatik, Magdeburg; 5 Institut für experimentelle Physik und Leibniz Institut für Neurobiologie, Biomedizinische Magnetresonanz,

Magdeburg

DOI 10.1055/s-0037-1600362

Zielsetzung Zur Bestimmung der diagnostischen Genauigkeit der Ultrahochfeld-MRT bei 7Tesla (7T-MRT) für Meniskus- und Knorpelschäden im Vergleich zur klinischen Routine-MRT bei 3Tesla (3T-MRT).

Material und Methodik 41 Patienten mit vermutetem Meniskusschaden oder leichter Arthrose (Kellgren-Lawrence 0 - 2) wurden sowohl mit einem 7T-MRT als auch mit einem 3T-MRT untersucht. Das Untersuchungsprotokoll bei beiden Feldstärken bestand aus einer fettgesättigten PD-gewichteten und einer T1-gewichteten Turbo-Spin-Echo-Sequenz (TSE) mit einer höheren Auflösung bei 7Tesla. Die Bilder wurden in Bezug auf die Feldstärke und die Patientendaten verblindet von drei Readern mit unterschiedlichem Erfahrungsstand in muskuloskelettaler Radiologie (3 Jahre, 6 Jahre und 10 Jahre) nach einem modifizierten Ganzorgan-MRT-Score des Kniegelenkes (WORMS) beurteilt. Bei 12 Patienten lagen Arthroskopiebefunde als Goldstandard vor.

Ergebnisse Es wurde eine multifaktorielle Analyse gemischter Modelle durchgeführt. Der mittlere akkumulierte diagnostische Score war bei 7T-MRT im Vergleich zum 3T-MRT signifikant näher am Goldstandard bei Patienten, bei denen der Goldstandard verfügbar war ( $p<0,001)$. In allen 41 Patienten wurden durch das 7T-MRT signifikant schwerere Schäden des Knorpels und des Meniskus detektiert $(p<0,001)$. Kein Unterschied bestand in Bezug auf die Interreader-Variabilität. Die Bildaquisitionszeit war vergleichbar.

Schlussfolgerungen Morphologische Bildgebung von Knorpel- und Meniskusschäden mit Ultrahochfeld-MRT bei 7Tesla mit T1- und PD-gewichteten TSESequenzen hat eine signifikant höhere diagnostische Genauigkeit als bei 3 Tesla und kann mit höherer Auflösung bei vergleichbarer Aquisitionszeit durchgeführt werden. 7T-MRT zeigte signifikant schwerere Knorpel- als auch Meniskusschäden.
WISS 307.7 Zusammenhang zwischen quantitativer Qualität des regenerativen Knorpelgewebes und subchondraler Knochenstruktur, gemessen mit 3T MRT, nach autologer Chondrozyten-Transplantation mit Spongiosaplastik

Autoren Gersing $A^{1}$, Feuerriegel $G^{1}$, Holwein $D^{2}$, Suchowierski $A^{2}$, Karampinos $P^{1}$, Baum $P^{1}$, Schwaiger $D^{1}$, Imhoff $P^{2}$, Kirschke $P^{3}$, Rummeny $P^{1}$, Jungmann $\mathrm{P}^{1}$

Institut 1 Technische Universität München, Klinikum rechts der Isar, Institut für diagnostische und interventionelle Radiologie, München; 2 Technische Universität München, Klinikum rechts der Isar, Abteilung für Sportorthopädie, München; 3 Technische Universität München, Klinikum rechts der Isar, Abteilung für diagnostische und interventionelle Neuroradiologie, München DOI 10.1055/s-0037-1600363

Zielsetzung Ziel war es zu untersuchen, ob nach Matrix-gestützter autologer Chondrozyten-Transplantation (MACT) mit Spongiosaplastik ein Zusammenhang zwischen der molekularen Zusammensetzung des Regeneratgewebes des Gelenkknorpels und der Qualität des subchondralen trabekulären Knochens besteht.

Material und Methodik Beide Knie von 25 Patienten (Alter $25.5 \pm 7.8$ Jahre; 10 weiblich) wurden 2 Jahre nach einseitiger MACT mit subchondraler Spongiosaplastik im Bereich der Femurcondylen oder der Patella mittels 3T-MRT untersucht. Zur Evaluation der Ultrastruktur des Gelenkknorpels einschließlich des Regeneratgewebes mittels quantitativen T2 Relaxationszeit-Messungen wurden sagittale T2 MSME Spin-echo Sequenzen akquiriert. Dreidimensionale phase-cycled balanced steady state free-precision (bSSFP) -Sequenzen wurden für die Quantifizierung der subchondralen Trabekelstruktur verwendet. T2Werte des Knorpels im operierten Kompartiment wurden als erhöht betrachtet, wenn sie größer waren als die T2-Werte des entsprechenden Kompartimentes am kontralateralen Knie. Die Werte wurden mittels abhängiger und unabhängiger t-Tests verglichen.

Ergebnisse In mit MACT behandelten Kompartimenten waren die Knorpel-T2Werte signifikant höher als in den jeweiligen gesunden Kompartimenten am kontralateralen Knie (Mittelwert \pm Standardabweichung $41.1 \pm 1.2$ ms vs. 38.8 $\pm 1.6 \mathrm{~ms}, \mathrm{P}=0.035$ ). Die trabekuläre Dicke und die Knochenfraktion in MAClKompartimenten waren signifikant höher als in den jeweiligen kontralateralen Kompartimenten $(\mathrm{P}<0.001)$. In MACT-Kompartimenten mit im Seitenvergleich erhöhten Knorpel-T2-Werten ( $n=17 ; 0.37 \pm 0.05 \mathrm{~mm})$ war die trabekuläre Dicke signifikant höher als in MACl-Kompartimenten ohne erhöhte T2Werte $(n=8 ; 0.27 \pm 0.05 \mathrm{~mm} ; \mathrm{P}=0.042)$.

Schlussfolgerungen Nach MACT mit Spongiosaplastik zeigten Patienten mit erhöhten T2-Werten im Knorpeltransplantat eine Verdickung der subchondralen trabekulären Strukturen. Dies suggeriert synergistische Effekte zwischen der Reifung des Knorpels und des subchondralen Knochens nach osteochondraler Transplantation.

\section{WISS 307.6 Degeneration der Knorpelmatrix im Kniegelenk, gemessen mit 3T-MRT T2-Relaxationszeit, hängt mit Vorhandensein und Schweregrad des Diabetes mellitus Typ 2 zusammen: Daten der Osteoarthritis Initiative}

Autoren Gersing $A^{1}$, Chanchek $D^{2}$, Schwaiger $D^{1}$, Zarnowski ${ }^{2}$, Joseph $D^{2}$, Nevitt $\mathrm{P}^{3}$, McCulloch $\mathrm{P}^{3}$, Link $\mathrm{P}^{2}$

Institut 1 Technische Universität München, Klinikum rechts der Isar, Institut für diagnostische und interventionelle Radiologie, München; 2 University of California, San Francisco, Department of Radiology and Biomedical Imaging, 
San Francisco, CA, USA; 3 University of California, San Francisco, Department of Epidemiology and Biostatistics, San Francisco, CA, USA

DOI 10.1055/s-0037-1600364

Zielsetzung Ziel war es, den Zusammenhang zwischen molekularer Degeneration des Kniegelenkknorpels, gemessen mit quantitativer 3T-MRT T2-Relaxationszeitanalyse, und Vorhandensein und Schweregrad des Typ-2-Diabetes mellitus (DM) zu untersuchen.

Material und Methodik Patienten mit DM ( $n=100$, Alter 60.6 $\pm 8.5 y$; 57 Frauen) und erhöhtem Risiko für Arthrose oder milder radiografischer Gonarthrose (Kellgren-Lawrence-Score $(\mathrm{KL}) \leq 2$ ) wurden mit 200 Patienten ohne DM verglichen, kontrolliert für Alter, Geschlecht, BMI und KL. Als Subgruppe wurde das Vorhandensein von diabetischer Nephropathie oder Retinopathie ( $n=28)$ als „schwerer DM“ definiert. Im MRT wurden strukturelle Gelenkläsionen mittels Whole-Organ Magnetic Resonance Imaging Score (WORMS) erfasst. T2-Werte wurden gemessen gemittelt in allen Knorpelkompartimenten, in der Patella und der medialen Tibia sowie in der tiefen, knochenseitigen Knorpelschicht in diesen Regionen. Multivariable lineare Regressionsmodelle wurden verwendet um Zusammenhänge mit DM zu berechnen.

Ergebnisse Patienten mit DM hatten signifikant höhere Knorpel-T2-Werte in der Patella als Patienten ohne DM (mittlere Differenz 1.1msec [95\% Konfidenzintervall ( $\mathrm{Cl})$ 0.6, 1.5]; $\mathrm{P}=0.023$ ). Knorpel-T2-Werte der knochennahen Knorpelschicht der medialen Tibia und Patella waren bei Patienten mit DM ebenfalls signifikant erhöht (mittl. Diff. $1.2 \mathrm{msec}[95 \% \mathrm{Cl} 0.5,1.9], \mathrm{P}=0.003$, und $1.1 \mathrm{msec}[95 \% \mathrm{Cl} 0.2,2.0], \mathrm{P}=0.022$ ). Patienten mit schwerem DM wiesen in allen Kompartimenten signifikant höhere T2-Werte auf als Patienten ohne DM (Knochennaher Knorpel, gemittelt: mittl. Diff. 2.2 [95\% Cl 0.3, 3.4], $\mathrm{P}=0.044$ ). Hinsichtlich der strukturellen Gelenkläsionen (WORMS für Knorpel, Menisken, Knochenmark) wurden keine signifikanten Unterschiede gefunden $(P>0.05)$.

Schlussfolgerungen Patienten mit DM zeigten signifikant höhere Knorpel-T2Werte als Patienten ohne DM; mit den deutlichsten Unterschieden bei Patienten mit schwerem DM. Der Gelenkknorpel von Patienten mit DM scheint somit einer ausgeprägteren molekularen Degeneration zu unterliegen.

\section{WISS 313.5 Reduktion von Metallartefakten in Spectral-Detector-CT Untersuchungen der Wirbelsäule durch Verwendung von Metalreduktionsalgorithmen und virtuell monoenergetischen Rekonstruktionen}

Autoren Große Hokamp $N^{1}$, Neuhaus $V^{1}$, Nuran $A^{1}$, Laukamp $K^{1}$, Simon L ${ }^{1}$, Anastasios $\mathrm{M}^{1}$, Maintz $\mathrm{D}^{1}$, Jan $\mathrm{B}^{1}$

Institut 1 Uniklinik Köln, Institut für Diagnostische und Interventionelle Radiologie, Köln

DOI 10.1055/s-0037-1600365

Zielsetzung Vergleich des Ausmaßes von Artefakten durch Spondylodesematerial zwischen konventionellen, iterativen Rekonstruktionen ( $\mathrm{Cl}$, iDose), virtuell monoenergetischen Rekonstruktionen (MonoE) und mittels Metallartefaktreduktionsalgorithmen (OMAR) rekonstruierten SDCT-Untersuchungen.

Material und Methodik 25 Patienten mit einliegender Spondylodese und SDCT-Untersuchung (IQon, Philips, Amsterdam; 120kvP, Dosismodulation $100-400 \mathrm{mAs}$ ) wurden retrospektiv in diese Untersuchung eingeschlossen und unter Verwendung von $\mathrm{Cl}$, MonoE (40-200 keV; 10keV Stufen) und OMAR rekonstruiert. Es erfolgte eine objektive, ROI-basierte Bildanalyse der Schwächung (HU), des Rauschens (SD Schwächung) und des Kontrast-zuRausch-Verhältnissen (CNR) in folgenden Regionen: Auslöschungsartefakt, autochthoner Rückenmuskulatur, Spinalkanal und subkutanem Fett an der ventralen Bauchwand.

Ergebnisse OMAR und hoch-keV-MonoE zeigen gegenüber $\mathrm{Cl}$ eine signifikante Artefaktreduktion gemessen an der Schwächungszunahme des hypodensen Artefakts (CI/OMAR und MonoE-200keV: $-396,5 \pm 214,9 /-115,2 \pm 96,4$ und $-48,1 \pm 180,0 ; p \leq 0,01)$. Hierbei war OMAR in $20 / 25$ und MonoE-200keV in 5/25 Fällen überlegen. Auch die Artefakte gemessen an Rauschen in a) Spi- nalkanal, b) autochthoner Rückenmuskulatur und c) subkutanem Fett an der ventralen Bauchwand wurde durch den Einsatz von OMAR und hoch-keV-vMEI gegenüber Cl signifikant reduziert (CI/OMAR und MonoE-200keV: a) 103,4/ 72,6 und 60,9 ; $p \leq 0,01$; b) $55,1 / 19,6$ und 31,7 ; $p \leq 0,05$; c) $27,0 / 17,6$ und 20,2; $p \leq 0,05)$.

Schlussfolgerungen Sowohl OMAR als auch hoch-keV-MonoE reduzieren die durch Spondylodese verursachten Artefakte signifikant, wobei OMAR und hoch-keV-MonoE in unterschiedlichen Patienten jeweils überlegen sind. Eine weitere Aufschlüsselung bezüglich operativem Zugangsweg, Implantatmaterial oder Wirbelsäulenregion und insbesondere die Kombination von OMAR und MonoE sollten weiter untersucht werden.

\section{WISS 307.3 3T-MRT zur Verlaufsbeurteilung nach hüftgelenkserhaltender Knorpeltherapie - Vergleich zwischen autologer Chondrozytentransplantation und Mikrofrakturierung}

Autoren Haubold $\mathrm{J}^{1}$, Theysohn $\mathrm{J}^{1}$, Geis $\mathrm{C}^{1}$, Körsmeier $\mathrm{K}^{2}$, Landgraeber $\mathrm{S}^{3}$, Kraff $\mathrm{O}^{4}$, Lazik-Palm $\mathrm{A}^{1}$

Institut 1 Uniklinik Essen, Institut für Diagnostische und Interventionelle Radiologie und Neuroradiologie, Essen; 2 Facharztklinik Essen, Orthopädie, Essen; 3 Uniklinik Essen, Institut für Orthopädie, Essen; 4 Universität Duisburg-Essen, Erwin L. Hahn Institut für Magnetresonanz, Essen DOI 10.1055/s-0037-1600366

Zielsetzung MR-morphologische und klinische Beurteilung der Knorpelregeneration nach autologer Chondrozytentransplantation(ACT) bzw. Mikrofrakturierung(MF) in Patienten mit acetabulärem Knorpelschaden.

Material und Methodik Die Hüften von Patienten mit Knorpeldefekten, die aus einem CAM-Impingement resultieren, wurden 6 und 12 Monate nach ACT $(n=20)$ und $\operatorname{MF}(n=16)$ unter Verwendung von hochauflösenden 3T PDwSequenzen(PD SPACE $0,8 \mathrm{~mm}$ isotrop, PD TSE sagittal $0,5 \times 0,5 \times 2,5 \mathrm{~mm}$ und coronar $0,8 \times 0,8 \times 2,5 \mathrm{~mm}$ ) untersucht. Das Knorpelreparaturgewebe wurde mittels eines angepassten MOCART-Scores hinsichtlich der Integrität, der Oberflächen- und Signalintensität des Reparaturgewebes sowie der umgebenden Pathologien bis zu einem Maximum von 85 Punkten bewertet. Die MOCART-Scores wurden mittels One-way-Anova mit Bonferroni-Korrektur und die Differenzen der MOCART-Scores zwischen 6 und 12 Monaten mittels Student's t-test verglichen. Die klinische Beurteilung erfolgte anhand standardisierter Fragebögen(WOMAC und NAHS).

Ergebnisse Sechs und zwölf Monate nach MF waren die Eigenschaften des Reparaturgewebes mit einem mittleren MOCART-Score von 51 bzw. 49 Punkten nahezu stabil. Im Gegensatz dazu erhöhte sich der MOCART-Score nach ACT von durchschnittlich 58 Punkten nach 6 Monaten auf einen Mittelwert von 66 Punkten nach 12 Monaten. Dies war vor allem auf eine fortschreitende Defektfüllung(35\% der Punktezunahme) zurückzuführen. Die Absolutwerte nach 12 Monaten $(p<0,01)$ sowie die Anstiege der MOCART-Scores zwischen dem 6- und 12-Monats-Follow-up $(p<0,001)$ waren nach ACT signifikant höher als nach der MF. Klinisch zeigte sich im Jahresverlauf kein signifikanter Unterschied zwischen der ACT- und der MF-Gruppe.

Schlussfolgerungen Mittels hochaufgelösten PDw 3T-MRT-Sequenzen konnten wir eine signifikant bessere Knorpelregeneration im 1-Jahres-Verlauf nach ACT vs. MF zeigen. Nach ACT war auch nach mehr als 6 Monaten eine fortschreitende Knorpeldefektfüllung zu beobachten, wohingegen nach MF die Knorpelregeneration bereits nach 6 Monaten stagnierte. 


\section{WISS 313.7 Detektionsrate von Wirbelkörperfrakturen und Identifikation frischer Wirbelkörperfrakturen mittels CT}

\author{
Autoren Hedderich $D^{1}$, Maegerlein $C^{1}$, Zimmer $C^{1}$, Kirschke $J^{1}$ \\ Institut 1 Klinikum rechts der Isar der TU München, Abteilung für \\ Neuroradiologie, München \\ DOI 10.1055/s-0037-1600367
}

Zielsetzung Ziel der Studie ist, die Wirbelkörperfrakturdetektionsraten durch CT und MRT alleine sowie die Möglichkeit zur Identifikation einer frischen Wirbelkörperfraktur in der CT zu untersuchen.

Material und Methodik Es erfolgte eine retrospektive, unabhängige Analyse der Wirbelsäulen-CT bzw. -STIR-MRT von 104 Patienten durch zwei Radiologen mit vier bzw. sieben Jahren Erfahrung. Neben morphologischen Kriterien wurden die Wahrscheinlichkeiten für das Vorliegen einer (frischen) Fraktur auf einer 5-Punkte-Skala bewertet. Als Goldstandard diente eine Konsensbewertung beider Modalitäten inklusive vorhandener Verlaufsbildgebung. Die statistische Analyse wurde mit binär logistischer Regression (unter Adjustierung für die Analyse mehrerer Wirbelkörper pro Patient) und ROC-Kurven durchgeführt, die AUC, Sensitivität und Spezifität wurden berechnet.

Ergebnisse Es zeigten sich 230 Frakturen in 351 analysierten Wirbelkörpern (173 Kompressions-, 27 Berstungs-, 19 Distraktions- und 11 Kontusionsfrakturen). 192 Frakturen wurden als frisch identifiziert, 47 als pathologisch. Die Frakturdetektion durch die CT alleine hatte eine Sensitivität und Spezifität von 0.93 und 0.88 ( $A \cup C=0.964$ ), durch die STIR-MRT alleine 0.96 und 0.70 $(A \cup C=0.867)$. Die Identifikation frischer Frakturen durch die CT alleine zeigte eine Sensitivität von 0.72 und eine Spezifität von 0.98 (AUC=0.920). Das CTmorphologische Zeichen einer gedoppelten trabekulären Kompaktierung alleine zeigte eine Spezifität von 0.95 für frische Frakturen. Die Interreaderreliabilität war sehr gut für die CT-/MRT-Frakturdetektion und Identifizierung frischer Frakturen (Cronbach's alpha =0.964, 0.949 und 0.859).

Schlussfolgerungen Die Identifizierung frischer Wirbelkörperfrakturen in der CT ist mit guter Sensitivität und hoher Spezifität möglich. Das Vorliegen einer typischen doppelten trabekulären Kompaktierung kann als beweisend für eine frische Fraktur angesehen werden, sodass in solchen Fällen auf eine zusätzliche MRT zur Einordnung des Frakturalters verzichtet werden kann.

\section{WISS 307.8 MRT-basierte, semi-quantitative Analyse des Kniegelenks eignet sich zur Vorhersage der Implantation von Knie-Totalendoprothesen}

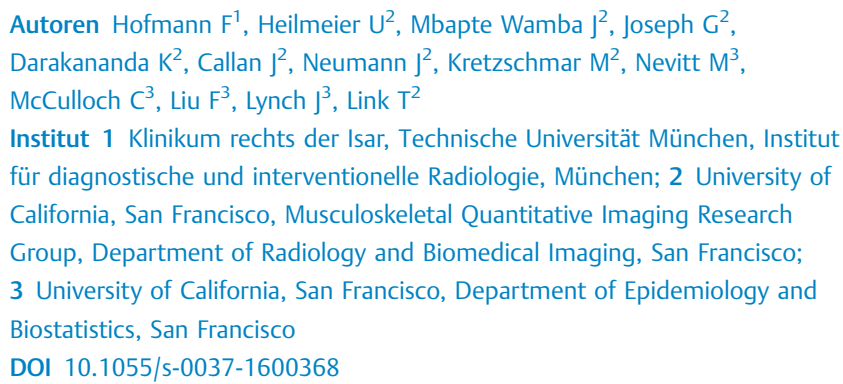

Zielsetzung Ziel dieser Fall-Kontroll-Studie war zu untersuchen, ob morphologische Parameter des semi-quantitativen, MRT-basierten Whole-Organ Magnetic Resonance Imaging Scores (WORMS) die operative Einbringung von Knie-Totalendoprothesen (K-TEP) 4 - 7 Jahre später vorhersagen können. Material und Methodik Aus der Osteoarthritis Initiative (OAI) wurden 309 Patienten ausgewählt, darunter 81 Patienten, bei denen 4 - 7 Jahre nach Studieneinschluss eine operative, rechtsseitige K-TEP erfolgte. Diesen wurden mittels Gruppen-Matching 228 Kontrollpatienten zugeordnet. Die Ausgangs3T-MRT-Aufnahmen von allen 309 Patienten wurden von einem Radiologen hinsichtlich morphologischer Knieveraenderungen mittels des WORMS semiquantitativ evaluiert. Zur statistischen Analyse kamen konditionale, logistische Regressionsmodelle zum Einsatz.
Ergebnisse Patienten mit und ohne erfolgter K-TEP waren hinsichtlich ihres Ausgangsalters, BMIs, Geschlechts, Kellgren-Lawrence-Scores und ihres $\mathrm{Ar}$ throse-Risikoprofils vergleichbar (Alter: $63,3 \pm 8,0$ vs. 63,7 $\pm 8,1$; BMI: 29,0 $\pm 4,5$ vs. $29,4 \pm 4,6$; $27 \%$ männlich; $P>0,05$ ). Zwischen Studieneinschluss und Implantation der K-TEP lagen im Schnitt $2018 \pm 325$ Tage. Es zeigte sich, dass ein Gelenkerguss im Ausgangs-MRT signifikant mit einer um 145\% erhöhten Odds Ratio (OR) für die Implantation einer K-TEP 4 - 7 Jahre später assoziiert war $(P=0,003)$, während Knochenmarksödeme im medialen Femur mit einer $65 \%$ erhöhten $O R$ einhergingen $(P=0,000)$. Für Knorpelläsionen fanden sich je nach Kompartment $14-37 \%$ erhöhte OR für eine spätere, operative K-TEP (Medialer Femur: $O R=1,37$ (Cl 95\% 1,21-1,56), $P=0,000$; Patella: $\mathrm{OR}=1,29(\mathrm{Cl} 95 \% 1,10-1,50), \mathrm{P}=0,001$; Laterale Tibia: $\mathrm{OR}=1,14(\mathrm{Cl} 95 \%$ $1,00-1,31), P=0,057)$.

Schlussfolgerungen Die WORMS-basierte Auswertung von Knie-MRTs liefert klinisch wertvolle Informationen über das patientenindividuelle Risiko einer künftigen Knie-TEP. Insbesondere das Vorhandensein eines Gelenkergusses, eines Knochenmarködems im medialen Femur oder von Knorpelläsionen erweisen sich als besonders bedeutsam.

\section{WISS 307.9 Einfluss anthropometrischer Parameter auf die Nervenquerschnittsflächen peripherer Nerven: Messen wir tatsächlich richtig?}

Autoren Josip $\mathrm{E}^{1}$, Gruber $\mathrm{H}^{1}$, Skalla $\mathrm{E}^{1}$, Leonhard $\mathrm{G}^{1}$, Plaikner $\mathrm{M}^{1}$, Loizides $\mathrm{A}^{2}$ Institut 1 Medizinische Universität Innsbruck, Department Radiologie,

Innsbruck; 2 Medizinische Universität Innsbruck, Deparment Radiologie, Innsbruck

DOI 10.1055/s-0037-1600369

Zielsetzung Die sonografische Beurteilung peripherer Nerven beruht besonders bei der Qualifikation von Kompressionssyndromen in erster Linie auf der Nervenquerschnittsfläche. Was bisher nicht berücksichtigt wurde, sind geschlechtliche und anthropometrische Parameter: wir vermuten, dass individuell unterschiedliche anthropometrische Parameter (z. B. Adipositas) Dimensionsunterschiede neuraler Strukturen nach sich ziehen können, was indirekt die sonografischen neuronalen Messungen und damit auch die Diagnose neuropathischer Veränderungen grundlegend beeinflussen sollte.

Material und Methodik In dieser prospektiven Studie wurden 50 gesunde Probanden untersucht. Es wurden sowohl anthropometrische als auch sonografische Messungen durchgeführt: Alter, Geschlecht, Größe und Gewicht wurden erhoben. Zudem wurde der BMI, Fettanteil, Taillenumfang, Taille zu Hüfte und Taille zu Statur-Verhältnis, Oberarm- und Unterarm- sowie Oberschenkel- und Unterschenkel-Umfang und -längen evaluiert. Die sonografischen Querschnittsflächenmessungen des N. medianus, N. ulnaris, N. tibialis und $N$. peronaeus wurden an jeweils anatomisch vordefinierten Stellen durchgeführt.

Ergebnisse Mittels einer Zählstatistik sowie einer Regressionsanalyse zeigen sich verlässliche Abhängigkeiten der gesunden Nervenquerschnitte von o.g. Grundparametern: es zeigen sich Geschlechts- sowie BMI-Abhängigkeiten, ebenso lassen sich berücksichtigungswerte Abhängigkeiten vom Körperfettanteil bzw. von der Probandenstatur nachweisen.

Schlussfolgerungen Die im Rahmen dieser Studie erhobenen Parameter ergeben anthropometrische Konstellationen, welche bei der sonografischen Beurteilung z. B. von Kompressionsneuropathien berücksichtigt werden sollten; durch diese Berücksichtigung sollten sich i.R. einer veränderten Vortestwahrscheinlichkeit eine Verbesserung der Validität sonografischer Messwerte ergeben. 


\section{RK 115.1 Komplementäre Metallartefaktreduktion von Hüftvollprothesen durch monoenergetische Rekonstruktionen und O-MAR in der Dual-Layer Computertomografie}

Autoren Laukamp $\mathrm{K}^{1}$, Lennartz $\mathrm{S}^{2}$, Neuhaus $\mathrm{V}^{2}$, Große Hokamp $\mathrm{N}^{2}$, Abdullayev $\mathrm{N}^{2}$, Maintz $\mathrm{D}^{2}$, Borggrefe $\mathrm{J}^{2}$

Institut 1 Uniklinik Köln, Radiologie, 48153; 2 UKK Radiologie, Köln DOI 10.1055/s-0037-1600370

Zielsetzung Vergleich der Metallartefakte von Hüftvollprothesen in der DualLayer CT (DLCT) zwischen a) konventionellen gefilterten Rückprojektionen (FBP), b) O-MAR Rekonstruktionen und c) monoenergetischen Rekonstruktionen (MER).

Material und Methodik DLCT (IQON, Philips, Amsterdam, NL) von 27 Patienten mit Hüftvollprothesen. Protokoll: Dosismodulation 100-400 mAs, 120 kVp, 2 mm-Schichtdicke, Pitch 0,671, 0,33 s Rotationsdauer. Bildrekonstruktionen mit O-MAR, FBP und MER (60 - $200 \mathrm{keV}$; Intervall $20 \mathrm{keV}$ ). Erfassung der maximalen Artefaktdicke sowie ROI basierte Auswertung der Dichte (HU), des Rauschens (SD HU-Messwerte) und des Kontrast-zu-Rausch-Verhältnisses (CNR) in folgenden Regionen: Artefakt, Muskulatur, Knochen, kleines Becken. Ergebnisse MER (200 keV) sowie O-MAR zeigen eine deutliche Artefaktreduktion zur FBP: a) Metallartefaktbreite (Mittelwertï, \pm SD): $F B P 29,9 \pm 6,8 \mathrm{~mm}$, MER $17,6 \pm 13,6 \mathrm{~mm}(p<0,01)$, O-MAR $16,5 \pm 14,9 \mathrm{~mm}(p<0,0001)$; b) HU Auslöschungsartefakt: FBP $-412,1 \pm 204,5$, MER $-279,7 \pm 283,7 \quad(p<0.01)$, O-MAR $-116,74 \pm 105,6(p<0.0001)$ c) Bildrauschen: FBP 117,4 $\pm 74,2$, MER $105,4 \pm 69,2$ ( $p>0,05)$, O-MAR 64,4 $\pm 44,5$, $(p<0,05)$. In $n=6$ war bei der Artefaktreduktion MER (200 keV, $\mathrm{p}<0.05)$ und in $\mathrm{n}=15$ Untersuchungen O-MAR überlegen $(p<0.01)$. In $n=6$ Fällen waren MER $(200 \mathrm{keV})$ und O-MAR gleichwertig. O-MAR reduziert das Rauschen über Blase (CNR: O-MAR $6,8 \pm 3,8$, MER $4,1 \pm 3,4, p<0,05$ ) und Muskulatur (CNR: O-MAR $10,1 \pm 4,8$, MER $5,7 \pm 3,6, p<0,05)$ stärker als MER. Hingegen zeigte der Knochen bei $200 \mathrm{keV}$ MER im Vergleich zu O-MAR weniger Bildrauschen $(\mathrm{p}<0.001)$.

Schlussfolgerungen O-MAR und MER (200 keV) ermöglichen im Vergleich zur FBP jeweils eine signifikante Artefaktreduktion von Hüftvollprothesen. In Abhängigkeit des Implantates ist zum Teil O-MAR und zum Teil MER deutlich vorteilhafter. Daher erscheint die Kombination dieser Methoden zur Artefaktreduktion sinnvoll. O-MAR reduziert die Artefakte deutlicher im kleinen Becken. MER ermöglicht ein reduziertes Bildrauschen im Bereich des Knochens.

\section{RK 325.2 Unterscheidung zwischen Enchondromen und niedriggradigen Chondrosakomen mittels MR-basierter 3D-Texturanalyse}

Autoren Lisson $C^{1}$, Lisson $C^{2}$, Mayer-Steinacker $R^{3}$, Schultheiss $M^{4}$, von Baer $A^{5}$, Barth $T^{6}$, Baumhauer $M^{7}$, Meier $R^{2}$, Beer $M^{2}$, Schmidt $S^{2}$ Institut 1 Uniklinik Ulm, Radiologie, Ulm; 2 Uniklinik Ulm, Klinik für Diagnostische und Interventionelle Radiologie, Ulm; 3 Uniklinik Ulm, Klinik für Innere Medizin III, Ulm; 4 Uniklinik Ulm, Klinik für Unfall-, Hand-, Plastische und Wiederherstellungschirurgie; 5 , Klinik für Unfall-, Hand-, Plastische und Wiederherstellungschirurgie, Ulm; 6 Uniklinik Ulm, Institut für Pathologie, Ulm; 7 Mint Medical, Heidelberg DOI 10.1055/s-0037-1600371

Zielsetzung Die Differenzierung zwischen low-grade Chondrosarkomen (CS) und Enchondromen (EC) stellt sowohl radiologisch wie auch histopathologisch eine Herausforderung dar. Eine Unterscheidung ist jedoch umso wichtiger, da völlig unterschiedliche therapeutischer Ansätze beider Diagnosen bestehen: ECs erfordern keine Operation, wohingegen im Fall eines CS eine chirurgische Resektion unter kurativer Intention erforderlich ist. Unsere Studie soll die diagnostische Genauigkeit der MRT-basierten 3D-Texturanalyse zur Unterscheidung zwischen G1-CS und EC untersuchen.

Material und Methodik Die Daten von 22 Patienten wurden retrospektiv ausgewertet. 11 mit histologisch gesichertem CS und 11 mit EC, wobei diese
Diagnose entweder auf einer Histologie oder auf einem mindestens 5-jährigen, konstanten Kontrollintervall basiert. Die Texturanalyse erfolgte mit der Software MintLesion (Mint Medical), einem Spinoff des Deutschen Krebsforschungszentrums (DKFZ). Die erstellten 2D- und volumetrischen Messungen sowie die multiparametrische Texturparameter (Kurtosis, Entropie, Schiefe/ Skewness, MPP, UPP) wurden mittels Mann-Whitney-U-Test sowie ROC-Analyse statistisch ausgewertet und die anhand des Youden-Index optimale CutOff-Werte ermittelt.

Ergebnisse Signifikante Unterschiede zwischen CS und EC zeigten sich bei 6 von 20 Texturparametern $(p<0,05)$. Die AUC-Werte für diese 6 Parameter betrugen im Fall der kontrastverstärkten T1w 0,876 für die Kurtosis bzw. 0,826 für die Skewness. In der nativen T1w ergaben sich Werte von 0,851 für die Entropie, 0,793 für die MPP und 0,822 für die UPP, in der T2-STIR lag der Wert für die MPP bei 0,802 . Die höchste Diskriminierungskraft hatte die Kurtosis in der kontrastgestützten T1w mit einem Cut-off von $\geq 3,15$, um ein CS zu identifizieren (Sensitivität 82\%, Spezifität 91\%, Genauigkeit 86\%).

Schlussfolgerungen Die MRT-basierte 3D-Texturanalyse hat das Potenzial, G1-Chondrosarkome von Enchondromen mit hoher Sensitivität und Spezifität zu unterscheiden.

\section{WISS 307.5 Functional in-situ assessment of human articular cartilage using MRI: An entire-knee joint loading device}

Authors Nebelung $\mathrm{S}^{1}$, Post $\mathrm{M}^{1}$, Kuhl $\mathrm{C}^{1}$, Truhn $\mathrm{D}^{1}$

Institute 1 Universitätsklinikum Aachen, Klinik für Diagnostische und Interventionelle Radiologie, Aachen

DOI 10.1055/s-0037-1600372

Zielsetzung The response to loading of human articular cartilage as assessed by Magnetic Resonance Imaging (MRI) remains to be defined in relation to histology and biomechanics. Thus, an MRI-compatible entire-knee joint loading device for the functional in-situ assessment of cartilage was developed and validated.

Material und Methodik A formalin-fixed human knee was scanned by CT in its native configuration and digitally processed to create femoral and tibial bone models, which were covered by artificial articular cartilage in its native configuration using cartilage-mimicking polyvinyl-siloxane. At the medial femoral condyle a defect of $8 \mathrm{~mm}$ diameter was created within the artificial cartilage layer, into which native cartilage samples of similar dimensions were placed. Displacement-controlled quasi-static uni-axial loading to $2.5 \mathrm{~mm}$ $(\delta 2.5)$ and $5.0 \mathrm{~mm}(\delta 5.0)$ of tibia (mobile) versus femur (immobile)was performed to study the response to loading of macroscopically intact human articular cartilage samples $(n=5)$ by serial imaging using PDW TSE sequences and T2W multi-spin echo sequences on a clinical 3.0T MRI system (Achieva, Philips). Biomechanics and histology served as the standards-of-reference.

Ergebnisse All samples were histologically intact (Mankin score, 1.8 \pm 1.3 ) and biomechanically homogeneous (Young's Modulus, $0.4 \pm 0.1 \mathrm{MPa}$ ). They could be visualized in their entirety and loading-induced significant decreases in sample height ( $\delta 0: 2.9 \pm 0.3 \mathrm{~mm} ; \delta 2.5: 2.6 \pm 0.3 \mathrm{~mm} ; \delta 5.0: 2.0 \pm 0.2 \mathrm{~mm}$; $\mathrm{p}<0.001$ ) and decay of $\mathrm{T} 2$ signal intensity indicative of tissue pressurization were found.

Schlussfolgerungen The response-to-loading assessment of cartilage by MRI in a close-to-physiological experimental setting and with reference to histology and biomechanics is possible. Articular cartilage may be functionally evaluated beyond mere static analysis in a basic research context. 


\section{WISS 307.2 T1p-mapping von humanem Knorpelgewebe unter sequentieller Be- und Entlastung}

Autoren Nebelung $\mathrm{S}^{1}$, Kuhl $\mathrm{C}^{1}$, Truhn $\mathrm{D}^{1}$

Institut 1 Universitätsklinikum Aachen, Klinik für Diagnostische und

Interventionelle Radiologie, Aachen

DOI 10.1055/s-0037-1600373

Zielsetzung Ziel ist die Untersuchung von Be- und Entlastungs-induzierten Veränderungen innerhalb histologisch intakten humanen Knorpelgewebes mittels T1 $\rho$-mappings.

Material und Methodik Im Rahmen von Kniegelenkersatz-Operationen wurden makroskopisch intakte Knorpel-Knochen-Stücke des lateralen Femurkondylus (Outerbridge Grad 0; $\mathrm{n}=18$ ) gewonnen und nach Standard präpariert. Die Belastung des Gewebes erfolgte durch Weg-gesteuerte quasi-statische Indentation mit einem zylindrischen Stempel (strain 20\%). Jeweils 3-faches sequentielles T1p-mapping erfolgte unter Belastung und nachfolgender vollständiger Entlastung unter Nutzung einer modifizierten Prostataspule und eines 3T-MR-Scanners (Achieva, Philips). Nach Erhalt der räumlich aufgelösten T1p-Parameterkarten und manueller Segmentierung wurden die absoluten und relativen Änderungen in indentierten und nicht-indentierten Probenarealen und verschiedenen Gewebetiefen erfasst. Histologie stellte den Referenzstandard dar. Der statistische Vergleich der relativen Änderungen erfolgte mittels gepaartem Student-t-Test.

Ergebnisse Histologisch zeigten sich alle Proben intakt. Unter Belastung ließen sich konsistente und deutliche T1p-Erhöhungen feststellen, die im indentierten Areal deutlicher waren als daneben (Belastung t1: 9,5 $\pm 7,8$ vs. 4,2 $\pm 5,8 \%$ [p < 0,001] $\mathrm{M} \pm \mathrm{SD}$ ) und im Zeitverlauf zunahmen (Belastung $\mathrm{t3}: 14,1$ $\pm 8,1$ vs. $7,7 \pm 5,9 \%[p<0,001])$. Ausgeprägte bzw. geringe Änderungen ließen sich für die Übergangs- bzw. tiefe Knorpelzone feststellen. Unter Entlastung ließen sich weniger konsistente Änderungen im gesamten Gewebe nachweisen.

Schlussfolgerungen Die Beurteilung von Belastungs-induzierten Veränderungen mittels quantitativem T1 $\rho$-mapping ist ein vielversprechendes Tool für die nicht-invasive funktionelle Beurteilung von Knorpelgewebe und erlaubt Aussagen über Anpassungsvorgänge im Gewebe als Funktion der Belastung und Zeit. Zukünftige Studien werden zeigen, ob die Detektion von Knorpelpathologien, insbesondere früher Degenerationsstadien, dergestalt möglich ist.

\section{WISS 313.4 Metallartefaktreduktion durch virtuell monoenergetische Rekonstruktionen der Spektraldetektor-Computertomografie}

Autoren Neuhaus $V^{1}$, Abdullayev $N^{1}$, Große Hokamp N ${ }^{1}$, Rau $\mathrm{R}^{1}$, Mpotsaris $A^{1}$, Maintz $D^{1}$, Borggrefe $J^{1}$

Institut 1 Uniklinik Köln, Institut für Diagnostische und Interventionelle Radiologie, Köln

DOI 10.1055/s-0037-1600374

Zielsetzung Untersuchung der Artefaktreduktion durch virtuelle monoenergetische Rekonstruktionen (VMR) der Spektraldetektor-Computertomografie (SDCT).

Material und Methodik SDCT von 35 Patienten mit orthopädischen Implantaten. Protokoll: Dosismodulation 100-400 mAs, 120 kVp, $1-2 \mathrm{~mm}$ Schichtdicke, Pitch 0,671, 0,33 s Rotationsdauer. Reguläre CT-Rekonstruktionen und VMR (64keV, 70keV, 105keV, 140keV, 200keV und subjektiv optimierter keV). Auswertung durch drei geblindete Radiologen. Subjektive Auswertung Ausmaß der Artefakte und diagnostische Wertigkeit mit 5-Punkte Likert-Skalen sowie objektive Auswertung durch Quantifizierung der HU-Dichte und Breite der maximalen Artefakte.

Ergebnisse 21 Patienten mit Implantaten der Wirbelsäule, 8 im Becken und 6 im Bereich der Extremitäten. Im Vergleich zu niedrigen keV-Werten (40keV) und konventionellen Rekonstruktionen zeigte sich bei VMR mit hohen keVWerten ein signifikanter Anstieg der diagnostischen Wertigkeit sowie eine signifikante Reduktion der Artefakte (jeweils $\Delta p \leq 0.0001 ; 105 \mathrm{keV}, 140 \mathrm{keV}$,
$200 \mathrm{keV}$ und individuell adjustierte Kiloelektronenvolt). In der subjektiven radiologischen Beurteilung zeigte sich eine signifikante Verbesserung der Bewertung (Likert-Skala \pm SD) von 4,3 $( \pm 0,83)$ auf $2,3( \pm 1,02)$ bzw. von 4,3 $( \pm 0,66)$ auf $2,6( \pm 2,57)$. Die durchschnittlich ausgewählten, optimalen Kiloelektronenvolt betrugen 139,2 $\pm 39,4 \mathrm{keV}$. Die Dichte der Artefakte ließ sich von $-374,6 \pm 251,9 \mathrm{HU}$ (VMR $70 \mathrm{keV}$ ) auf $-12,5 \pm 258,4 \mathrm{HU}$ (optimaler keV) reduzieren $(p \leq 0.0001)$, die maximale Artefaktbreite von $14,5 \pm 8,74 \mathrm{~mm}$ (70 $\mathrm{keV}$ ) auf 6,4 $\pm 10,76 \mathrm{~mm}$ (optimaler keV).

Schlussfolgerungen SDCT mit VMR im hohen keV Bereich erlauben bei orthopädischen Implantaten im Vergleich zur konventionellen CT eine signifikante Verbesserung der Bildqualität und Artefaktreduktion. Die optimale Bildqualität zeigte sich im Mittel bei $140 \mathrm{keV}$. In 20\% der Fälle zeigte sich bei subjektiver visueller Bestimmung der keV eine weitere Verbesserung der Bildqualität.

\section{RK 115.2 Pilotevaluation einer 2-phasigen 18F-Fluoride PET/MRT der Sakroiliakalgelenke bei Patienten mit M. Bechterew}

Autoren Sawicki $\mathrm{L}^{1}$, Lütje $\mathrm{S}^{2}$, Baraliakos $\mathrm{X}^{3}$, Kirchner J ${ }^{1}$, Ruhlmann $\mathrm{V}^{2}$, Umutlu $\mathrm{L}^{4}$, Quick $\mathrm{H}^{5}$, Antoch $\mathrm{G}^{1}$, Buchbender $\mathrm{C}^{1}$

Institut 1 Universitätsklinikum Düsseldorf, Institut für Diagnostische und Interventionelle Radiologie, Düsseldorf; 2 Universitätsklinikum Essen, Klinik für Nuklearmedizin, Essen; 3 Ruhr-Universität Bochum, Rheumazentrum Herne, Herne; 4 Universitätsklinikum Essen, Institut für Diagnostische und Interventionelle Radiologie und Neuroradiologie, Essen; 5 Universitätsklinikum Essen, Medizinische Physik, Essen

DOI 10.1055/s-0037-1600375

Zielsetzung Eine vermehrte Traceraufnahme in der Blutpoolphase einer 18F-F PET zeigt regionale Hyperämie an, während sie in der Mineralisationsphase osteoblastische Aktivität widerspiegelt. Ziel der Arbeit war die erstmalige Evaluation einer zweiphasigen 18F-F PET/MRT bei M. Bechterew und eines möglichen Zusammenhangs zwischen regionaler Hyperämie und osteoblastischer Aktivität bei verschiedenen Bechterewläsionen.

Material und Methodik Bei 13 Patienten mit M. Bechterew wurde eine 18F-F PET/MRT der Sakroiliakalgelenke durchgeführt. Die Blutpoolphase-PET wurde 6 und die Mineralisationsphase-PET 40 Minuten nach 18F-F Injektion akquiriert. Alle PET/MRT Bilder wurden bezüglich Bildqualität (DIQ) beurteilt. Die Sakroiliakalgelenke wurden in Quadranten (SQ) geteilt und hinsichtlich Knochenmarködem, Erosion, Knochenmarkverfettung, Sklerosierung, Ankylosierung und gesteigertem 18F-F Uptake ausgewertet.

Ergebnisse Die DIQ der MRT und beider PET-Datensätze war hoch, wobei die DIQ der Mineralisationsphase-PET höher als die der Blutpoolphase-PET war $(p<0.001)$. Von 104 SQ zeigten 63\% eine Knochenmarkverfettung, 42\% ein Knochenmarködem, 27\% Erosionen, 26\% Sklerosierung und 11\% Ankylosierung. SQ bei denen nur ein Knochemarködem vorlag, wiesen zu 91\% einen gesteigerten 18F-F Uptake in der Mineralisationsphase-PET/MRT und zu 64\% in der Blutpoolphase-PET/MRT auf. SQ mit ausschließlich Knochenmarkverfettung, Erosion, Sklerosierung oder Ankylosierung zeigten keinen gesteigerten $18 \mathrm{~F}-\mathrm{F}$ Uptake. SQ bei denen ein Knochemarködem in Kombination mit anderen Bechterewläsionen vorlag, zeigten signifikant häufiger $(p<0.001)$ einen gesteigertem 18F-F Uptake in der Blutpoolphase-PET/MRT und (48\%) und der Mineralisationsphase-PET/MRT (86\%) als SQ ohne Knochenmarködem (10\% bzw. 17\%).

Schlussfolgerungen Eine zweiphasige 18F-F PET/MRT der Sakroiliakalgelenke gelingt mit hoher Bildqualität. Insbesondere bei inflammatorischen Bechterewläsionen mit Knochenmarködem besteht ein Zusammenhang zwischen regionaler Hyperämie und osteoblastischer Aktivität. 


\section{WISS 313.6 Dosisreduktion bei CT-basierter Knochendichtemessung und Mikrostruktur-Analyse: Effekte der Niedrigdosis-Simulation und des Sparse- Samplings}

Autoren Schwaiger $B^{1}$, Mei $K^{1}$, Kopp $F^{1}$, Bippus $R^{2}$, Gersing $A^{1}$, Rummeny $E^{1}$, Kirschke $J^{3}$, Noël $P^{1}$, Baum $T^{1}$

Institut 1 Klinikum rechts der Isar der TU München, Institut für diagnostische und interventionelle Radiologie, München; 2 Philips Hamburg Research

Laboratory, Hamburg; 3 Klinikum rechts der Isar der TU München, Abteilung für Diagnostische und Interventionelle Neuroradiologie, München DOI 10.1055/s-0037-1600376

Zielsetzung Es sollte untersucht werden, ob anhand von Knochendichte-Messungen (BMD) und Mikrostruktur-Analysen des trabekulären Knochens nach simulierter Dosisreduktion und Sparse-Sampling in Kombination mit statistischer iterativer Rekonstruktion (SIR) weiterhin zwischen Patienten mit und ohne Wirbelkörperfrakturen unterschieden werden kann.

Material und Methodik In Thorax-/Abdomen CTs, die in der klinischen Routine bei 12 Patienten mit osteoporotischen Wirbelkörperfrakturen sowie 12 Patienten ohne Frakturen, paarweise zugeordnet nach Alter und Geschlecht, angefertigt worden waren, wurden durch virtuell reduzierte Röhrenstrom (10, 25 und $50 \%$ des ursprünglichen Röhrenstroms) und durch Sparse-Sampling (10, 25 und 50\% der ursprünglichen Projektionen) niedrigere Strahlendosen simuliert. BMD und Mikrostruktur-Parameter (u.a. Knochenvolumenfraktion und trabekuläre Dicke) wurden für BWK10 bis LWK5 aus den mittels SIR rekonstruierten Bilddaten berechnet.

Ergebnisse Die effektive Dosis der Original-Untersuchung war $10 \mathrm{mSv}$; und jeweils 5, 3, 1 mSv für die 50, 25 und 10\% Sparse-Samplings sowie virtuell reduzierten Röhrenstrom. Sämtliche BMD-Messungen basierend auf den verschiedenen virtuellen Dosisreduktionen und Sparse-Samplings waren signifikant niedriger in Patienten mit Frakturen verglichen mit Patienten ohne Frakturen (Spannweite: $89-110$ vs. $125-187 \mathrm{mg} / \mathrm{ml}$; jeweils $\mathrm{P}<0.003$ ). Analog dazu waren die Knochenvolumenfraktion ( $0.31-0.47$ vs. $0.50-0.52$, jeweils $\mathrm{P}<0.004)$ und trabekuläre Dicke $(1.1-1.3$ vs. $1.7-2.4 \mathrm{~mm}$; jeweils $\mathrm{P}<0.011)$ in Patienten mit Frakturen durchweg signifikant niedriger.

Schlussfolgerungen Nach simulierter Dosisreduktion und Sparse-Sampling von CT-Untersuchungen unterschieden sich BMD-Messungen und Mikrostruktur-Analysen in Patienten mit und ohne Wirbelkörperfrakturen weiterhin signifikant voneinander. Damit erscheint CT-basierte Osteoporose-Diagnostik auch anhand von Niedrigdosis-Protokollen möglich.

\section{WISS 307.1 Quantitatives MR-mapping des Response to Loading von humanem Knorpelgewebe in Abhängigkeit vom histologischen Degenerationsgrad}

\author{
Autoren Truhn $D^{1}$, Kuhl $C^{1}$, Nebelung $S^{1}$ \\ Institut 1 Universitätsklinikum Aachen, Klinik für Diagnostische und \\ Interventionelle Radiologie, Aachen \\ DOI 10.1055/s-0037-1600377
}

Zielsetzung Untersuchung der Belastungs-induzierten Veränderungen in humanem Knorpelgewebe mittels quantitativen MR-Parameter-mappings in Referenz zum histologischen Degenerationsgrad.

Material und Methodik Im Rahmen von Knie-TEP-Operationen wurden Knorpel-Knochen-Stücke unterschiedlicher makroskopischer Degeneration (Outerbridge $0-2)$ des lateralen Femurkondylus präpariert $(n=59)$. Die Belastung des Gewebes erfolgte durch weggesteuerte quasi-statische Indentation mit einem zylindrischen Stempel in einer MR-kompatiblen Apparatur. Vor und nach Indentation (um jeweils $0,4 \mathrm{~mm}$ [ $\delta 1 / 2]$ bzw. 0,8 $\mathrm{mm}[\delta 1]$ ) wurden folgende Parameterkarten akquiriert: T1, T1 $\rho, \mathrm{T} 2$ und T2*. Nach manueller Segmentierung der Knorpelproben wurden relative Änderungen der MR-Parameter $(\Delta)$ erfasst. Die histologische Degeneration der Proben wurde nach Mankin bestimmt und eine dichotome Einteilung in gering vs. moderat degeneriert vorgenommen (Mankin Summe 0-4 vs. Mankin Summe 5-8). Die Statistik erfolgte mittels Mann-Whitney-Test sowie die Korrelationsanalyse nach Pearson.

Ergebnisse Histologisch zeigte sich eine gleichmäßige Verteilung der Degenerationsgrade (Mankin Summe: 4,1 $\pm 1,9$ ). Unter Indentation ließen sich für $\mathrm{T} 1$ und $\mathrm{T}^{*} / \mathrm{T} 1 \rho$ und $\mathrm{T} 2$ konsistente Parameterwertreduktionen $(\Delta<0) /$-erhöhungen $(\Delta>0)$ feststellen. Lediglich für T1p konnte eine Abhängigkeit des $\Delta \mathrm{T} 1 \rho$ vom histologischen Degenerationsgrad festgestellt werden (Spearmans's $r=0,35[\delta 1 / 2]$ bzw. 0,31 [ $\delta 1]$; jeweils $p<0,05)$. $\Delta T 1 \rho$ war signifikant unterschiedlich zwischen gering und moderat degenerierten Proben $(p<0,01$ für $\delta 1 / 2$ und $\delta 1)$. Für $T 1, T 2$ und $T 2 *$ ließen sich keine signifikanten Degenerations-assoziierten Änderungen unter Belastung feststellen.

Schlussfolgerungen Die nicht-invasive funktionelle Beurteilung Belastungs-induzierter Veränderungen von humanem Knorpel mittels T1p-mapping ist mit dem histologischen Degenerationsgrad assoziiert und bietet die Perspektive einer feineren Graduierung und früheren Detektion von knorpeldegenerativen Veränderungen.

\section{Neuroradiologie}

\section{WISS 404.2 Diagnostische Genauigkeit verschiedener Perfusionsalgorithmen zur Detektion zerebraler Vasospasmen}

Autoren Afat $\mathrm{S}^{1}$, Brockmann $\mathrm{C}^{1}$, Nikoubashman $\mathrm{O}^{1}$, Müller $\mathrm{M}^{1}$, Haberland $\mathrm{U}^{2}$, Brockmann $\mathrm{M}^{3}$, Nikolaou $\mathrm{K}^{4}$, Wiesmann $\mathrm{M}^{1}$, Othman $\mathrm{A}^{4}$ Institut 1 Universitätsklinikum Aachen, Klinik für Diagnostische und Interventionelle Neuroradiologie, Aachen; 2 Siemens Healthcare, Forchheim; 3 Universitätsklinikum Mainz, Klinik und Poliklinik für Neuroradiologie, Mainz; 4 Universitätsklinikum Tübingen, Diagnostische und Interventionelle

Radiologie, Tübingen

DOI 10.1055/s-0037-1600378

Zielsetzung Ziel der Arbeit ist die Evaluation der diagnostischen Genauigkeit zweier unterschiedlicher Perfusionsalgorithmen zur Detektion zerebraler Vasospamen im Vergleich zur digitalen Substraktionsangiografie (DSA).

Material und Methodik Es wurden Daten von 25 Patienten mit Subarachnoidalblutung und V.a. zerebrale Vasospasmen eingeschlossen. Kraniales natives CT, CT-Perfusion und DSA-Untersuchung wurden innerhalb von 6 Stunden durchgeführt. Perfusionskarten wurden aus den Daten sowohl im maximum Slope-Algorithmus (MS) als auch im Dekonvolutionsalgorithmus (DC) erstellt. Die generierten Farbkarten aus den CT Perfusionsdaten wurden von 2 Neuroradiologen bezüglich der schwere und des Vorhandenseins von Vasospasmen evaluiert (3-point Likert Skala $0=$ keine Vasospasmen, $1=<50 \%$ Vasospasmen und $2 \Rightarrow 50 \%$ Vasospasmen). Ein dritter Neuroradiologe evaluierte die Daten aus der DSA bezüglich der Schwere und des Vorhandenseins von Vasospasmen. MS und DC CT-Perfusionsdaten wurden hinsichtlich der diagnostischen Genauigkeit zur Detektion von zerebralen Vasospamen mit der DSA als Standard verglichen. Korrelationsanalysen der Ergebnisse der CT-Perfusionsdaten und der DSA wurden durchgeführt. Ebenso wurde das Agreement zwischen MS und DC CT Perfusionsdaten und das inter-reader Agreement ermittelt. Ergebnisse Die DC Daten zeigen eine signifikant höhere diagnostische Genauigkeit als die MS Daten (DC: AUC =0.821; MS: AUC:.755; p =0.014) mit höherer Sensitivität als die MS Daten (DC: Sensitivität $=0.724$; MS: Sensitivität $=0.590$ ). Die DC Daten zeigen im Vergleich zu den MS Daten eine höhere Korrelation mit der DSA (DC: $R=0.664$; MS: $R=0.565 ; p=0.007$ ). Ergebnisse der MS- und DC Daten zeigen eine beachtliche Übereinstimmung (Kappa $=0.732$ ). Die Interrater-Reliabilität war nahezu eine perfekte Übereinstimmung für beide Algorithmen (Kappa $\geq 0.980$ ).

Schlussfolgerungen Die Ergebnisse dieser Studie zeigen, dass die DC Perfusionskarten eine signifikant höhere diagnostische Genauigkeit aufweisen im Vergleich zu den MS Perfusionsdaten zur Detektion zerebraler Vasospasmen. 


\section{WISS 306.4 Der Einfluss von Urbanicity auf Stressverarbeitungsmechanismen - eine fMRT-Studie}

Autoren Al-Bayati $\mathrm{M}^{1}$, Gruber $\mathrm{O}^{2}$, Kraemer B ${ }^{2}$, Zilles $\mathrm{D}^{3}$ Institut 1 Universitätsklinikum Essen, Institut für Diagnostische und Interventionelle Radiologie und Neuroradiologie, Essen; 2 Universitätsklinikum Heidelberg, Zentrum für psychosoziale Medizin, Heidelberg;

3 Universitätsmedizin Göttingen, Klinik für Psychiatrie und Psychotherapie, Göttingen

DOI 10.1055/s-0037-1600379

Zielsetzung Der Anteil der Weltbevölkerung, welcher in Städten lebt, nimmt kontinuierlich zu. Neben Vorteilen, zeigt sich durch Leben in der Stadt (Urbanicity) auch eine erhöhte Prävalenz an psychiatrischen Erkrankungen (Depressionen, Schizophrenie). Eine These besagt, dass dies an vermehrtem Stress im Stadtleben liegt. Analog zu einer anderen Arbeitsgruppe (Lederbogen et al., 2011) haben wir in einer fMRT-Untersuchung (T2*-Sequenzen, BOLD-Signal) 42 Probanden einem stresserzeugenden Paradigma unterzogen und untersucht inwiefern die Aktivität in stressassoziierten Hirnarealen (ex. Amygdala; anteriorer zingulärer Kortex) in Abhängigkeit von der Größe des aktuellen Wohnorts und von der Größe der Wohnorte in der Kindheit variieren.

Material und Methodik Im MRT führten die Probanden ein stresserzeugendes Paradigma durch (Montreal Imaging Stress Task): Hierzu mussten sie Rechenaufgaben ohne und mit Stressinduktion lösen. Zur Stressinduktion wurden verbales und visuelles Feedback (Zeitdruck, Vergleich der Rechenergebnisse mit anderen Probanden) eingesetzt. Vor und nach Betreten des Scanners wurden Blutdruck und subjektiv empfundener Stress erhoben. Während des Scans wurde kontinuierlich die Herzfrequenz erfasst. Datenverarbeitung und Auswertung erfolgten mit SPM8.

Ergebnisse Es zeigte sich, dass die Intensität der Aktivierung in der Amygdala von der Größe des Wohnorts abhängt $(t=3.56, p<0.001)$. Außerdem zeigten Städter eine vermehrte Aktivierung im Hypothalamus. Die als Beleg der Stressinduktion erhobenen physiologischen Parameter zeigten nach Stressinduktion einen signifikanten Anstieg ( $p<0.001)$.

Schlussfolgerungen Es konnte gezeigt werden, dass die Aktivität der Amygdala im Stressexperiment abhängig von der Größe des Wohnorts ist. Weitere Hirnareale zeigten bei Städtern ebenfalls eine Mehraktivierung. Es wird nun spannend zu beobachten sein, ob weitere Untersuchungen eine Brücke zwischen (1) Urbanicity, (2) hirnbildgebend nachweisbar veränderter neuronaler Verarbeitung und (3) Pathogenese psychiatrischer Erkrankungen schlagen können.

\section{WISS 317.2 Differenzierung der Zusammensetzung von Thromben in der Spektral-Detector Computertomografie}

\author{
Autoren Borggrefe $\mathrm{J}^{1}$, Kottlors $\mathrm{J}^{1}$, Mirza $\mathrm{M}^{2}$, Maus $\mathrm{V}^{1}$, Kabbasch $\mathrm{C}^{1}$, \\ Neuhaus $\mathrm{V}^{1}$, Abdullayev $\mathrm{N}^{1}$, Maintz $\mathrm{D}^{1}$, Mpotsaris $\mathrm{A}^{1}$ \\ Institut 1 Uniklinik Köln, Institut für Diagnostische und Interventionelle \\ Radiologie, Köln; 2 Neuravi Ltd., Clinical Research, Galway, Ireland \\ DOI 10.1055/s-0037-1600380
}

Zielsetzung Untersuchung der Differenzierbarkeit von Thromben mit variierendem Anteil an roten Blutkörperchen (RBC) in der Spektral-Detector Computertomografie (SDCT).

Material und Methodik Je fünf ovine Thromben mit $3 \quad(n=15)$ unterschiedlichen, histologisch gesichterten Anteilen an RBC (0\%-RBC (Fibrin), 36\%-RBC, 99\%-RBC, Neuravi-Ltd., Ireland). Drei Versuchsreihen (V1-V3): V1) DLCT nativ in Plastikröhrchen mit NaCl, V2) 5 Minuten nach Exposition mit 1:50 Dilution von Accupaque-350 (GE-Healthcare, USA) und V3) nach drei Tagen mit KM-Exposition. Auswertung der Thrombusdichte in konventionellen, virtuell monoenergetischen und virtuell nativen Rekonstruktionen (VNC) der SDCT durch drei geblindete Untersucher ausgewertet.
Ergebnisse In V1 ließen sich die drei Thrombustypen in der konventionellen CT differenzieren (Fibrin 23.6 \pm 1.1 , 36\%-RBC 34.9 \pm 1.6 , 99\%-RBC $46.7 \pm 1.6$ (Mittelwert $\mathrm{HU} \pm \mathrm{SD})$ ). Hingegen konnten die Thromben mittels konventionellen Rekonstruktionen in der frühen Phase nach KM-Exposition (V2) nicht differenziert werden (Fibrin 108.5 \pm 7.8, 36\%-RBC 105.3 \pm 3.5 , 99\%-RBC 104.8 \pm 3.8 ). Nach prolongierter KM-Exposition (V3) zeigten die Fibrinthromben eine zunehmende Dichte (Fibrin 163.6 \pm 3.6 , 36\%-RBC 138.3 \pm 4.1 , 99\%-RBC 109.6 \pm 5.4 , je $\Delta p<0.01 \mathrm{zu} \vee 1$ ). Es bestand eine lineare Korrelation zwischen dem RBC-Anteil und der nativ gemessenen Dichte sowie hierzu invers eine lineare Korrelation zwischen dem Fibrin-Anteil und der Dichte nach Kontrastmittelexposition. Im nativen Scan und der VNC (V2/V3) zeigten die Thrombustypen keine Überlappung der gemessenen Dichte (je $\Delta p<0.0001$ ). Schlussfolgerungen SDCT erlaubt die Differenzierung histologisch definierter Blutgerinnsel vor und nach Kontrastmittelexposition. Hierbei korrelieren native Dichte und VNC-Dichte der Thromben mit dem RBC-Anteil. Hingegen korreliert der prozentuale Fibrinanteil mit der Kontrastmittelaufnahme der Thromben nach prolongierter Exposition. Mit diesen kombinierten Bildinformationen könnte zukünftig die Thrombuszusammensetzung in der CT-Schlaganfalldiagnostik bestimmt werden.

\section{WISS 306.1 MRI based diagnosis of schwannomatosis}

Authors Bäumer $\mathrm{P}^{1}$, Farschtschi $\mathrm{S}^{2}$, Godel $\mathrm{T}^{3}$, Schlemmer $\mathrm{H}^{4}$, Bendszus $\mathrm{M}^{5}$, Mautner $\mathrm{V}^{2}$

Institute 1 DKFZ - Deutsches Krebsforschungszentrum, Radiologie, Heidelberg; 2 Universitätsklinikum, Neurologie, Hamburg-Eppendorf; 3 Universitätsklinikum, <input type ="text">, Heidelberg; 4 DKFZ, Radiologie, Heidelberg; 5 Universitätsklinikum, Neuroradiologie, Heidelberg DOI 10.1055/s-0037-1600381

Zielsetzung Schwannomatosis is the third major entity of the neurofibromatoses with an incidence of 1:25.000. Dominant symptom is the development of a chronic pain syndrome. This study examined the imaging correlate of schwannomatosis using high resolution MR Neurografy (MRN).

Material und Methodik 17 patients with schwannomatosis (w, years) were clinically examined by neurological examination, routine nerve conduction measurements, standardized quantitative sensory testing (QST), laser evoked potentials (LEP), and whole body magnetic resonance imaging (wbMRI). Additionally, high-resolution magnetic resonance neurografy (MRN) at 3.0T of at least one entire extremity (at least two extremities in 13 patients) was performed to examine peripheral nerves with high resolution using a fat-saturated, T2-weighted sequences at $0.27 \times 0.27 \mathrm{~mm} 2$. Twenty healthy controls and 20 patients with inflammatory or metabolic neuropathy served as controls.

Ergebnisse No neurological deficits were observed in our patients. WbMRI showed localized masses associated to peripheral nerves. Routine nerve conduction measurements showed no pathological alterations. MRN showed multiple ubiquitous intrafascicular microlesions of varying sizes in all patients. The pattern of lesions was distinct from the control groups and from any other peripheral neuropathy previously observed except for neurofibromatosis 2 (Bäumer et al. J Neurol 2013).

Schlussfolgerungen Intraneural fascicular microlesions and multiple schwannomas are distinct disease features of schwannomatosis. These imaging correlates in MRN are pathognomonic for one of the neurofibromatoses - together with clinical history, a definitive diagnosis of schwannomatosis can be made even if few to none major schwannomas are observed on wbMRI. 


\section{WISS 306.2 Unterschiedliche Veränderungen der funktionellen Konnektivität von zwei Teilregionen des rechten dorsolateralen präfrontalen Cortex (dIPFC) bei Morbus Parkinson}

\author{
Autoren Caspers $]^{1}$, Mathys $C^{1}$, Hoffstaedter $F^{2}$, Südmeyer $M^{3}$, Cieslik $E^{2}$, \\ Rubbert $C^{1}$, Sigl $B^{1}$, Turowski $B^{1}$, Schnitzler $A^{4}$, Eickhoff $S^{2}$ \\ Institut 1 Universitätsklinikum Düsseldorf, Institut für Diagnostische und \\ Interventionelle Radiologie, Düsseldorf; 2 Heinrich-Heine-Universität \\ Düsseldorf, Institut für Klinische Neurowissenschaften und Medizinische \\ Psychologie, Düsseldorf; 3 Ernst von Bergmann Klinikum Potsdam, Klinik für \\ Neurologie, Potsdam; 4 Universitätsklinikum, Zentrum für \\ Bewegungsstörungen und Neuromodulation der Klinik für Neurologie, \\ Düsseldorf
}

DOI 10.1055/s-0037-1600382

Zielsetzung Das idiopathische Parkinsonsyndrom (IPS) geht mit verschiedenen Defiziten exekutiver Funktionen (EF) einher. Der rechte dorsolaterale präfrontale Cortex (dIPFC) ist eine Schlüsselregion für EF. Kürzlich wurde eine Unterteilung des rechten dIPFC in einen hinteren und vorderen Teil mittels konnektivitätsbasierter Parzellierung aufgedeckt. Die aktuelle Studie untersucht, ob diese Teilregionen unterschiedliche Veränderungen der funktionellen Konnektivität (FK) bei IPS aufweisen.

Material und Methodik Resting-state fMRT (3T Siemens Trio, TR $=2,2 \mathrm{~s}$, $\mathrm{TE}=30 \mathrm{~ms}, \quad$ flip-Winkel $=90^{\circ}, \quad$ Voxelgröße $=3,1 \mathrm{~mm}^{3}, \quad$ Akquisitionszeit $=$ 11 min) wurde bei 39 IPS Patienten und 44 Kontrollen (gematcht für Alter, Geschlecht, Bewegung) erhoben. Patienten wurden unter ihrer regulären dopaminergen Medikation (ON) und nach $12 \mathrm{~h}$ Entzug (OFF) gescannt. fMRT Daten wurden mit SPM12 vorverarbeitet (Bewegungskorrektur, Normalisierung, $5 \mathrm{~mm}$ Glättung) und die seed-basierte FK der beiden dIPFC Teilregionen zwischen Patienten und Kontrollen verglichen ( $p<0,05$ cluster-level FWE Korrektur).

Ergebnisse Nur der hintere rechte dIPFC zeigte FK Änderungen bei IPS, der vordere rechte dIPFC wies keine Veränderungen auf. Der hintere dIPFC zeigte verminderte FK mit dem bilateralen Precuneus und linken prämotorischen Cortex im OFF. Im ON normalisierte sich die FK mit dem linken Prämotorcortex, während die Abkopplung vom Precuneus bestehen blieb. Zusätzlich zeigte sich eine erhöhte FK zwischen hinterem dIPFC und dem bilateralen dorsomedialen präfrontalen Cortex (dmPFC).

Schlussfolgerungen Der hierarchisch organisierte rechte dIPFC zeigt unterschiedliche Beeinträchtigungen bei IPS. Der hintere Anteil für basale EF ist stärker betroffen als der vordere Teil für abstraktere EF. Die Integration räumlich-visueller Stimuli zur Handlungskontrolle ist durch die Entkopplung des hinteren dIPFC mutmaßlich gestört. Diese Störung wird nur teilweise durch dopaminerge Medikation gebessert, aber möglicherweise durch eine verbesserte Performanzkontrolle im Rahmen der Rekrutierung des dmPFC kompensiert.

\section{WISS 404.3 Prädiktiver Wert der CT-Perfusions- bildgebung bei akuter Kleinhirnischämie für die Entstehung eines malignen Kleinhirnödems}

Autoren Fabritius $\mathrm{M}^{1}$, Schuler $\mathrm{F}^{1}$, Höhne $\mathrm{C}^{2}$, Reiser $\mathrm{M}^{1}$, Ertl-Wagner $\mathrm{B}^{1}$, Sommer $\mathrm{W}^{1}$, Thierfelder $\mathrm{K}^{1}$, Kunz $\mathrm{W}^{1}$

Institut 1 Ludwig-Maximilians-Universität, Klinikum der Universität München, Institut für klinische Radiologie, München; 2 Ludwig-Maximilians-Universität, Klinikum der Universität München, Neurologische Klinik und Poliklinik, München

DOI 10.1055/s-0037-1600383

Zielsetzung Das maligne Kleinhirnödem (malignant cerebellar edema, MCE) ist eine lebensbedrohliche Komplikation des ischämischen Kleinhirninfarkts. Ziel der Studie war die Identifikation von bildgebenden Prädiktoren in der multimodalen CT inklusive Ganzhirn-CT-Perfusion (CTP).
Material und Methodik Aus einem konsekutiven Kollektiv von 3254 Patienten mit multimodaler CT und Verdacht auf akuten Schlaganfall wurden Patienten mit (1) Kleinhirn-Perfusionsdefizit und (2) Follow-up-gesichertem Kleinhirninfarkt ausgewählt. Im Follow-up wurde das Auftreten eines Ödems anhand einer etablierten 10-Punkte-Skala bestimmt, wobei ein Wert $\geq 4$ als MCE gewertet wurde. Mithilfe des posterior circulation Acute Stroke Prognosis Early CT Score (pc-ASPECTS) wurden ischämische Veränderungen in der nativen CCT (NCCT), der CT-Angiografie (CTA), sowie in CTP Parameterkarten (cerebral blood flow, CBF; cerebral blood volume, CBV; mean transit time, MTT; time to drain, TTD) beurteilt. CBF, CBV, MTT und TTD Defizitvolumina wurden gemessen. Zur statistischen Analyse wurden Chi-Quadrat-Tests, Mann-Whitney-U-Tests und Receiver Operating Characteristic (ROC) Kurven verwendet. Ergebnisse Siebenundvierzig Patienten erfüllten die Einschlusskriterien, wovon 38 Patienten (80.9\%) als MCE- und 9 (19.1\%) als MCE+ klassifiziert wurden. MCE+ Patienten zeigten im Vergleich zu MCE- Patienten größere CBF, CBV, MTT und TTD Defizitvolumina (jeweils $\mathrm{p}<0.001$ ) und niedrigere mittlere pc-ASPECTS in der CTP (CBF: 5 vs. 8 ; CBV: 8 vs. 9; MTT: 5 vs. 8 ; TTD: 5 vs. 8 ; jeweils $p<0.001)$, während die mittleren pc-ASPECTS in der NCCT keinen signifikanten Unterschied aufwiesen (9 vs. 10, $\mathrm{p}=0.097$ ). In der ROC-Analyse erzielten CBF (0.977) und CBV Defizitvolumina (0.952) sowie der pc-ASPECTS der CBF-Karte (0.934) die größten areas under the curve. Die pc-ASPECTS in der NCCT (0.681) und der CTA (0.712) erlaubten keine sichere MCE Klassifikation.

Schlussfolgerungen Die CT-Perfusionsbildgebung der akuten Kleinhirnischämie ermöglicht eine Prädiktion des MCE.

\section{WISS 205.3 Die perioptische leptomeningeale Kontrastmittelaufnahme in der postkontrast T2-FLAIR als neuer Bildmarker bei der Retrobulbärneuritis}

Autoren Förster $A^{1}$, Pino-Lopez $L^{2}$, Wenz $H^{1}$, Schlichtenbrede $F^{2}$, Groden $C^{1}$ Institut 1 Universitätsmedizin Mannheim, Abteilung für Neuroradiologie, Mannheim; 2 Universitätsmedizin Mannheim, Universitätsaugenklinik, Mannheim

DOI 10.1055/s-0037-1600384

Zielsetzung Die T2-FLAIR nach Kontrastmittelgabe ist sehr sensitiv für den Nachweis einer leptomeningealen Kontrastmittelanreicherung. In dieser Studie untersuchten wir die Inzidenz einer perioptischen leptomeningealen Kontrastmittelaufnahme in der T2-FLAIR bei der Retrobulbärneuritis (RBN).

Material und Methodik In einer prospektiven Studie wurde bei 29 Patienten mit RBN (medianes Alter 24 (IQR 24,5 - 37.5) Jahre, 31 (72,4\%) Frauen) ein erweitertes MRT-Protokoll (Magnetom Avanto, Siemens, Erlangen, Deutschland) durchgeführt inklusive T2-FLAIR in axialer (FOV $230 \times 169 \mathrm{~mm}$, matrix $256 \times 164 \mathrm{~mm}$, Schichtzahl 24, Schichtdicke $3 \mathrm{~mm}$, TR $9000 \mathrm{~ms}$, TE $89 \mathrm{~ms}$, TI $2500 \mathrm{~ms}$ ) und koronarer (FOV $230 \times 169 \mathrm{~mm}$, matrix $256 \times 168 \mathrm{~mm}$, Schichtzahl 24, Schichtdicke 3 mm, TR 9000 ms, TE 89 ms, TI 2500 ms) Schichtführung vor und nach Kontrastmittelgabe. Das MRT wurde ausführlich analysiert mit besonderem Schwerpunkt auf Veränderungen in der T2 sowie einer Kontrastmittelaufnahme in der postkontrast T1 sowie T2-FLAIR.

Ergebnisse Eine perioptische leptomeningeale Kontrastmittelaufnahme in der T2-FLAIR fand sich bei 18 (62,0\%) Patienten mit RBN. Bei 2 (6,9\%) Patienten war sie sogar der einzige pathologische Befund. Patienten mit Nachweis einer perioptischen leptomeningealen Kontrastmittelaufnahme in der T2-FLAIR wiesen häufiger chronisch-entzündliche Läsionen im Gehirn (12/18 (66,7\%) vs. $3 / 11(27,3 \%) ; p=0,039)$ sowie oligoklonale Banden im Liquor $(8 / 11(73,3 \%)$ vs. $1 / 6(16,7 \%) ; p=0,018)$ auf. Bei $13(44,8 \%)$ Patienten fand sich zudem in der T2 ein auffälliger Befund im N. opticus (Schwellung, Signalanhebung). In der postkontrast T1 fand sich eine Kontrastmittelaufnahme im N. opticus bei 17 (58,6\%) Patienten.

Schlussfolgerungen Eine perioptische leptomeningeale Kontrastmittelaufnahme in der T2-FLAIR tritt bei der RBN häufig auf und ist bei einzelnen Patienten der einzige pathologische Befund im MRT. Mittels dieses Bildmarkers ist die 
Darstellung einer ablaufenden leptomeningealen Entzündung möglich, die die RBN begleitet und ihr möglicherweise sogar vorausgeht.

\section{WISS 205.8 Prävalenz einer neurovaskulären Kompression der rostralen ventrolateralen Medulla oblongata in einer populationsbasierten MR-Studie}

\author{
Autoren Grothe $F^{1}$, Grothe $S^{1}$, Fleck $S^{2}$, Rathmann $E^{1}$, Langner $S^{1}$ \\ Institut 1 Universitätsmedizin Greifswald, Institut für Diagnostische \\ Radiologie und Neuroradiologie, Greifswald; 2 Universitätsmedizin Greifswald, \\ Klinik für Neurochirurgie, Greifswald \\ DOI 10.1055/s-0037-1600385
}

Zielsetzung Eine Kompression der rostralen ventrolateralen Medulla (RVLM) durch eine Gefäßschlinge soll zu einem Anstieg des Blutdrucks führen. Deren Bedeutung als Ursache für eine essentielle Hypertonie wird kontrovers diskutiert. Die Prävalenz dieser neurovaskulären Kompression wurde bisher nur an kleinen Studienkollektiven untersucht. Ziel dieser Arbeit war es, die Prävalenz und das Ausmaß einer neurovaskulären Kompression der RVLM bei gesunden Probanden in einer populationsbasierten MR-Studie zu untersuchen.

Material und Methodik Untersucht wurden 3079 gesunde Freiwillige im Rahmen der SHIP (Study of Health in Pomerania)-Studie. Bei allen Probanden wurde eine Ganzkörper-MRT mit 1,5 T durchgeführt, die T1-gewichtete MPR-Sequenzen mit $1 \mathrm{~mm}$ isotopen Voxeln und eine TOF-MR Angiografie beinhaltete. Auftreten und Schweregrad einer neurovaskulären Kompression wurde auf einer 3-Punkt-Skala bewertet und das betreffende Gefäß wurde identifiziert.

Ergebnisse 249 gesunde Freiwillige zeigten einen neurovaskulären Kontakt und 138 Probanden eine Kompression. Dabei zeigten 70 Probanden (50,7\%) eine leichte und 12 Probanden (8,7\%) eine schwere Kompression. Die meist betroffenen Gefäße waren die A. vertebralis $(43,6 \%)$ und die A. cerebelli posterior inferior (29,2\%) oder eine Kombination beider Arterien (25,3\%).

Schlussfolgerungen Eine neurovaskuläre Kompression der RVLM in einer populationsbasierten MR-Studie ist häufiger als bisher berichtet. Zur Einschätzung der klinischen Relevanz als mögliche Ursache einer Hypertonie ist eine Korrelation mit weiteren klinischen Daten erforderlich.

\section{WISS 404.5 Time is brain - Unterschiedliche Effektstärken eines Paradigmas der endovaskulären Schlaganfalltherapie in Abhängigkeit von der Okklusionsstelle}

Autoren Hedderich $D^{1}$, Zimmer $C^{1}$, Kleine $J^{2}$, Kaesmacher J Institut 1 Klinikum rechts der Isar der TU München, Abteilung für Neuroradiologie, München; 2 Vivantes Klinikum Neukölln, Klinische und Interventionelle Neuroradiologie, Berlin

DOI 10.1055/s-0037-1600386

Zielsetzung Eine vollständige und zeitnahe Reperfusion ist das Schlüsselelement einer erfolgreichen endovaskulären Schlaganfalltherapie. Die Zeitabhängigkeit ist nicht linear und ihre Effektstärke wird durch unterschiedliche Kovariablen moduliert (z. B. Grad der Kollateralisierung). Ziel der Studie war es, die Zeitabhängigkeit zwischen Mediaverschlüssen mit und ohne Involvierung der striatokapsulären Region als nicht kollateralisiertem Gewebe zu untersuchen. Material und Methodik Aus 325 konsekutiven, endovaskulär behandelten, isolierten Mediaverschlüssen wurde eine bezüglich der Reperfusionszeit gematchte Fall-Kontroll-Gruppe von Fällen mit und ohne Involvierung der striatokapsulären Region extrahiert. Mittels Interaktionsmodellen und aufgeteilten Gruppenanalysen wurde die Zeitabhängigkeit der Rekanalisation und deren Einfluss auf das neurologische Outcome analysiert.

Ergebnisse Der präinterventionelle NIHSS zeigte für beide Patientengruppen eine Assoziation zum Outcome. Lediglich für die Patienten mit striatokapsulärem Infarkt war die Rekanalisationszeit statistisch signifikant $(p=0.001)$ mit dem klinischen Outcome assoziiert, nicht für Patienten ohne striatokapsulären Infarkt. Die Effektmodellierung ergab unterschiedliche Regressionskoeffizien- ten für Patienten mit und ohne striatokapsulärem Infarkt ((-.014 \pm 0.004 bzw. $-0.004 \pm 0.003)$. Die ROC-Analyse zur Vorhersage des klinischen Outcomes zeigte eine AUC von 0.741 bzw. 0.569 für Patienten mit bzw. ohne striatokapsulärem Infarkt.

Schlussfolgerungen Die Effektstärken zur Vorhersage des klinischen Outcomes, im Speziellen des Parameters Zeit bis zur erfolgten Reperfusion unterscheiden sich zwischen Patienten mit und ohne striatokapsulärem Infarkt. Nicht kollateralisiertes Gewebe in der striatokapsulären Region zeigt möglicherweise eine stärker lineare Zeitabhängigkeit als das kollateralisierte, periphere Media-Territorium. Diese Zusammenhänge sind klinisch relevant, da in der endarteriell versorgten striatokapsulären Region klinisch wichtige Strukturen und Bahnen lokalisiert sind.

\section{WISS 317.5 Normvarianten der basalen Hirnvenen als Blutungsquelle bei der angiografisch negativen perimesencephalen und präpontinen Subarachnoidalblutung: Evaluation der Wertigkeit von SWI Sequenzen}

Autoren Hopf-Jensen $S^{1}$, Lohse $A^{1}$, Lehrke $D^{1}$, Preiß $M^{1}$, Börm $P^{2}$, MüllerHülsbeck $\mathrm{P}^{1}$

Institut 1 Diakonissenkrankenhaus Flensburg, Institut für diagnostische und interventionelle Radiologie/Neuroradiologie, Flensburg;

2 Diakonissenkrankenhaus Flensburg, Neurochirurgische Klinik, Flensburg DOI 10.1055/s-0037-1600387

Zielsetzung Mit 10\% aller spontanen SAB bleiben Ursache und Pathophysiologie der perimesencephalen (pm) und präpontinen (pp) SAB ohne angiografischen Nachweis einer Blutungsquelle noch weitestgehend unklar. Die Studie dient der Analyse und Evaluation der Wertigkeit der SWI-Sequenzen zur Detektion von Blutungsquellen.

Material und Methodik Von 2007 - 2016 haben wir 1200 Pat. mit SAB analysiert, von denen 17 Pat. eine pm/pp SAB $(\varnothing 52,3$ Jahre, $\pm 12,4)$ aufwiesen nach gleichzeitig initialer negativer Bildgebung. Als Kontrollgruppe dienten 12 Pat. mit aneurysmatischer (an) SAB (Ø 56,7 Jahre, $\pm 7,3$ ). Analyse der klinischen Parameter und der tiefen Hirnvenen in den SWI Sequenzen.

Ergebnisse In den SWI Sequenzen zeigen sich bei der pm/pp SAB häufiger venöse Normvarianten mit atypischen Drainagewegen der Vena basalis (Watanabe Typ C: $26,5 \%$ vs. 8,3\%, p <0,05). Bei der pm/pp SAB finden sich häufiger lokuläre Mikroblutungen mit engem Lagebezug zur Vena peduncularis und Vena pontomesencephalica anterior ( $25 \%$ vs. $0 \%, p<0,05)$. Pat. mit pm/pp $\mathrm{SAB}$ waren bei Aufnahme klinisch besser $(\mathrm{HH} \varnothing 1,7, \pm 0,87$ vs. $\varnothing 3,2 \pm 1,2$; $p<0,05$ ), bei ähnlicher Blutverteilung in der CCT (Fisher $\varnothing 3,1, \pm 0,8$, vs. $\varnothing$ $3,4 \pm 0,8 ; p>0,05)$. In der pm/pp SAB Gruppe traten weniger Vasospasmen auf ( 31 vs. $50 \%$ ), die benigner verlaufen ( $0 \%$ vs. $41,6 \%$ Infarktdemarkierung) und es sind weniger VP- Shunt Versorgung notwendig (6 vs. 33\%). Die GOS (Glasgow Outcome Scale) lag in pm/pp Gruppe bei $\varnothing 1,2$ (SD $\pm 0,56)$, in der aneurysmatischen Gruppe bei $\varnothing 2,82$ (SD $\pm 0,75$ ).

Schlussfolgerungen Bei der pm/pp SAB finden sich häufiger Normvarianten der Vena basalis (Rosenthal) im Vergleich zur aneurysmatischen Blutung. Der Lagebezug umschriebener Suszeptibilitätsartefakte zu perimesencephalen Venen unterstreicht die venöse Genese dieser Blutungen. Komplikationen wie Hydrozephalus, Rezidivblutungen oder klinisch relevante Vasospasmen treten seltener auf. Die Analyse der basalen Hirnvenen, als mögliche Blutungsquelle der pm/pp SAB ist mittels SWI Sequenz effektiv möglich. 


\section{WISS 404.4 Peri-iktale zerebrale Perfusionsveränderungen als wichtige Differentialdiagnose zum akuten ischämischen Schlaganfall}

Autoren Huhndorf $\mathrm{M}^{1}$, Austein $\mathrm{F}^{1}$, Jansen $\mathrm{O}^{1}$ Institut 1 UKSH Kiel, Klinik für Radiologie und Neuroradiologie, Kiel DOI 10.1055/s-0037-1600388

Zielsetzung Die klinische Differenzierung zwischen Schlaganfall und post-iktalem Geschehen ist in der akuten und subakuten Phase nicht immer eindeutig, da post-iktale neurologische Defizite mit einer Schlaganfallssymptomatik identisch sein können. Wenn das Krankheitsbild nicht sicher erkannt wird, können inadäquate Akuttherapien ein Risiko darstellen. Die erweiterte zerebrale Bildgebung mittels CT-Perfusion und CT-Angiografie bei Patienten mit Verdacht auf einen akuten ischämischen Schlaganfall ist etabliert und wird in vielen Akutkliniken zur individuellen Risikoabschätzung und Therapieentscheidung v. a. außerhalb des $6 \mathrm{~h}$ Zeitfensters eingesetzt.

Material und Methodik In einer retrospektiven Analyse wurden die CT-Perfusionsmuster von 30 Patienten mit post-iktalem Status ausgewertet. Alle Patienten hatten in der Akutdiagnostik bei Verdacht auf Schlaganfall eine CTP und CTA erhalten. Im weiteren Verlauf wurde bei allen Patienten die Diagnose eines stattgehabten Krampfanfalls gestellt. Ein Gefäßverschluss oder eine vorgeschaltete Stenose wurde in der CT-Angiografie jeweils nicht nachgewiesen. Ergebnisse Neben der in der Literatur bereits beschriebenen post-iktalen Hyperperfusion, konnte in 6 Fällen eine reversible Hypoperfusion dokumentiert werden. In Zusammenschau mit der unauffälligen CT-Angiografie, der followup-Bildgebung und dem weiteren klinischen Verlauf konnten die Perfusionsveränderungen jeweils sicher als post-iktal gewertet werden.

Schlussfolgerungen Die zerebrovaskuläre Dysfunktion im Rahmen eines iktalen Geschehens ist eine wichtige Differentialdiagnose zum akuten Schlaganfall und äußert sich nicht nur in der häufig beschriebenen Hyperperfusion, sondern kann auch in einer reversiblen Hypoperfusion resultieren. Die Perfusionsveränderungen entsprechen typischerweise nicht klassischen Gefäßterritorien und es wird in der CTA kein Gefäßverschluss nachgewiesen. Die Kenntnisse der post-iktalen Perfusionsmuster ist wichtig für die Differentialdiagnose zum akuten ischämischen Schlaganfall.

\section{WISS 205.7 Prognostische Information aus präoperativen MR-Daten von Patienten mit Glioblastomen: eine Radiomics-Studie}

Autoren Ingrisch $\mathrm{M}^{1}$, Schneider $\mathrm{M}^{1}$, Nörenberg $\mathrm{D}^{1}$, Negrao de Figueiredo $\mathrm{G}^{1}$, Maier-Hein $\mathrm{K}^{2}$, Suchorska $\mathrm{B}^{3}$, Reiser $\mathrm{M}^{1}$, Tonn $\mathrm{J}^{3}$, Ertl-Wagner $\mathrm{B}^{1}$

Institut 1 Klinikum der Universität München, Institut für Klinische Radiologie, München; 2 Deutsches Krebsforschungszentrum, Medizinische und

Biologische Informatik, Heidelberg; 3 Klinikum der Universität München, Neurochirurgische Klinik und Poliklinik, München

DOI 10.1055/s-0037-1600389

Zielsetzung Die Kombination von maschinellem Lernen mit radiologischen Bilddaten eröffnet vielversprechende Perspektiven und ermöglicht, zusätzliche Information aus Bilddaten zugänglich zu machen. In der vorliegenden Studie wird untersucht, ob ein Radiomics-Ansatz prognostische Informationen aus präoperativen, kontrastverstärkten MRT-Daten von einheitlich behandelten Patienten mit Glioblastomen extrahieren kann.

Material und Methodik In dieser retrospektiven Studie wurden präoperative, kontrastverstärkte T1-gewichtete MR-Daten von 66 Patienten aus einer vorhergehenden prospektiven Studie analysiert. Tumoren wurden manuell segmentiert und 208 quantitative Bildeigenschaften (,features'), die Form, Signalintensität und Tumortextur charakterisieren, wurden erhoben. Auf diesen Daten wurde ein sogenannter ,random survival forest" trainiert; dabei handelt es sich um ein prognostisches Modell, welches das individuelle Risiko jedes Patienten vorhersagt. Das prognostische Modell wurde mit 10-facher Kreuzvalidierung berechnet. Die Assoziation des vorhergesagten Risikos mit dem Ge- samtüberleben wurde über den ,concordance index‘, Kaplan-Meier-Analyse und ein univariates Cox-Proportional-Hazards-Modell evaluiert.

Ergebnisse Im Mittel überlebten die Patienten 14 Monate (Spanne von 0,8 bis 85 Monate). Das 10-fach kreuzvalidierte statistische Modell erreichte einen concordance-index von 0,66; in der Kaplan-Meier-Analyse waren die Patientengruppen mit niedrigem und hohem vorhergesagten Risiko deutlich getrennt $(p=5 e-5)$. Ein niedriges vorhergesagtes Risiko ist ein günstiger prognostischer Faktor (hazard ratio $=1.03$, 95\% C.I. 1.005-1.056, $p=0.022$ )

Schlussfolgerungen In dieser Studie konnte gezeigt werden, dass präoperative MR-Aufnahmen von Patienten mit GBM prognostische Information enthalten, die erst durch eine Radiomics-basierte Analyse zugänglich wird. Die Aussagekraft kann vermutlich noch weiter gesteigert werden, indem zusätzliche Bildkontraste wie etwa FLAIR in die Auswertung integriert werden.

\section{WISS 317.8 Zeitlich aufgelöste Flachdetektor- Angiografie (4D/DSA) der lentikulostriären Arterien}

Autoren Kammerer $\mathrm{S}^{1}$, Berkefeld $\mathrm{J}^{1}$, Müller-Eschner $\mathrm{M}^{1}$, Tritt $\mathrm{S}^{1}$ Institut 1 Uniklinikum Frankfurt, Institut für Neuroradiologie, Frankfurt DOI 10.1055/s-0037-1600390

Zielsetzung Schlaganfallpatienten mit M1-Verschluss haben oft Stammganglieninfarkte im Versorgungsgebiet lentikulostriärer Arterien. Ziel der Arbeit war die Darstellung lentikulostriärer Arterien und anderer aszendierender Perforatoren mit zeitlich aufgelöster Flachdetektor-CT-Angiografie zur Evaluation der Verlaufsvarianten und Versorgungsgebiete.

Material und Methodik Nachberechnung von zeitlich aufgelösten multiplanaren Rekonstruktionen aus 18 vorhandenen 4D-DSA-Datensätzen, die bei vorhandener rechtfertigender Indikation zur Durchführung einer 3DRotationsangiografie unter Injektion von $21 \mathrm{ml}$ KM an einer biplanen Flachdetektor-Angiografieanlage (Siemens Axiom Artis Zee) mit 12 s Scanzeit akquiriert wurden.

Ergebnisse Die lentikulostriären Arterien sind in allen Fällen sehr gut beurteilbar und zeigen eine große Variationsbreite bezüglich Ursprungsort und Zahl der Gefäßursprünge. Ursprungsort eines Truncus, aus dem die lentikulostriären Arterien entspringen sind in $n=5$ Fällen das M1-Segment, in $n=4$ das A1-Segment und in $\mathrm{n}=1$ die distale $\mathrm{ACl}$. In den übrigen Fällen zeigen sich alternativ separat abgehende Arterien meist aus dem M1- und vereinzelt aus dem A1-Segment. Anastomosen lassen sich nur vereinzelt $(n=2)$ bei M1-Verschlüssen abgrenzen. Die perforierenden Arterien ziehen von caudal und lateral in die Stammganglien hinein, während die Venen cranial und medial entspringen und im Verlauf Anschluss an Ventrikelvenen finden.

Schlussfolgerungen Die 4D DSA ermöglicht eine durch etablierte Methoden nicht vergleichbar hochauflösende, überlagerungsfreie Darstellung der aszendierenden, Basalganglien und innere Kapsel versorgenden Gefäße. Meist entspringen die Perforatoren aus dem M1-Segment, wir konnten jedoch auch Versorgungstypen mit Abgang aus dem A1-Segment und der $\mathrm{ACl}$ nachweisen. Die Darstellung der Perforatoren kann erklären, warum Stammganglieninfarkte beim M1-Verschluss häufig, jedoch nicht in allen Fällen auftreten und zudem dazu beitragen, Gefäßschädigungen bei intrakraniellen Angioplastien oder Stentimplantationen zu vermeiden. 


\section{WISS 306.5 The Deep Blue im MRT - Eine komparative 3D pCASL und IVIM-MRT Studie zur Adaptation der cerebralen Perfusion bei Apnoetauchern}

Autoren Keil $V^{1}$, Eichhorn $L^{2}$, Mädler $B^{3}$, Gieseke $J^{1}$, Müller $A^{1}$, Block $W^{1}$, Träber $F^{1}$, Sprinkart $M^{1}$, Scheef $L^{1}$, Schneider $C^{4}$, Hadizadeh $D^{1}$, Hattingen $E^{1}$, Schild $\mathrm{H}^{1}$

Institut 1 UK Bonn, Radiologie, Bonn; 2 UK Bonn, Anästhesiologie, Bonn; 3 Philips Healthcare, Clinical Science, Hamburg; 4 UK Bonn, Neurologie, Bonn DOI 10.1055/s-0037-1600391

Zielsetzung Apnoetauchen ist Trendsport. Das Gehirn scheint dabei schadlos Apnoephasen von teils mehr als $10 \mathrm{Min}$. zu tolerieren. Über die physiologischen Anpassungsprozesse zur Versorgung des Gehirns ist nur wenig bekannt. Diese Studie evaluiert 1. die Anpassung der Hirnperfusion bei Apnoe mittels 3D pseudokontinuierlichem Arterial Spinlabeling (3D pCASL; cerebraler Blutfluss, rCBF) und der intravoxel-incoherent motion (IVIM) Perfusionsfraktion (PF) und 2. Unterschiede zwischen den beiden innovativen Methoden zur Perfusionsmessung.

Material und Methodik 14 männl. Taucher (20 - 64 J., 76 Mon. Erfahrung gemittelt) führten 2 Apnoephasen à 5 oder 7,5 Minuten unter kontinuierlicher Pulsoxymetrie und EKG im 3T MRT durch. Es wurden je 5 Sequenzen à 2,5 Min. mit beiden Methoden akquiriert (1 Ausgangswert, 2 bei Apnoe, 2 nach Apnoe. 3D pCASL: 1,8 Sek. label duration, 2 Sek. post-label delay, voxel $3,75 \times 4 \times 6 \mathrm{~mm}$; IVIM: 32 b-Werte bis $2000 \mathrm{~s} / \mathrm{mm} 2$, voxel $4 \times 4 \times 6 \mathrm{~mm}$; je 16 Schichten). rCBF und bi-exponentielle PF Karten wurden erstellt. rCBF (ml/ $100 \mathrm{~g} /$ Min.) und PF (in \%) wurden ROI-basiert (Kortex/Basalganglien/Centrum semiovale bds.) miteinander und gegen die Zeit sowie mit Herzfrequenz und Blutsauerstoff korreliert.

Ergebnisse Unter Apnoe kam es zu einer Zunahme des rCBF bis auf max. $320 \%$ des Ausgangswertes; rCBF korrelierte signifikant mit dem Abfall des Sauerstoffsättigung $(r=0,74-0,99 ; P<0,001 ; n=14)$, und fiel in der Erholungsphase auf $50-100 \%$ des Ausgangswertes. Eine Korrelation von rCBF mit der Herzfrequenz und PF war schwach $(r<0,25 ; \mathrm{P}>0,05)$. Die PF stieg nach initialem Abfall während der ersten 2,5 Min. auf bis zu 146\% des Ausgangswerts, um bei Erholung auf den Ausgangswert abzufallen. PF korrelierte schwächer mit dem Sauerstoffabfall $(r=0,13-0,73)$.

Schlussfolgerungen 3D pCASL ermöglicht den Nachweis eines mit SpO2 korrelierenden rCBF Anstiegs unter Apnoe. Die divergente Dynamik von PF u. rCBF unter Apnoe impliziert eine eingeschränkte Vergleichbarkeit und möglicherweise unterschiedliche physiologische Hintergründe der Signale.

\section{WISS 205.6 Medulloblastome des Erwachsenen - Eine Multicenter-Studie zur Identifikation genetischer Subtypen anhand bildgebender Marker im MRT}

Autoren Keil $\mathrm{V}^{1}$, Warmuth-Metz $\mathrm{M}^{2}$, Reh $\mathrm{C}^{1}$, Enkirch J ${ }^{1}$, Pietsch $\mathrm{T}^{3}$, Schild $\mathrm{H}^{1}$, Hattingen $E^{1}$, Hau $P^{4}$

Institut 1 UK Bonn, Radiologie, Bonn; 2 Uniklinikum Würzburg, Institut für Diagnostische und Interventionelle Neuroradiologie, Würzburg; 3 UK Bonn, Neuropathologie, Bonn; 4 Universitätsklinikum Regensburg, Wilhelm SanderTherapieeinheit NeuroOnkologie und Klinik und Poliklinik für Neurologie, Regensburg DOI 10.1055/s-0037-1600392

Zielsetzung Medulloblastome (WHO IV $)$ treten äußerst selten im Erwachsenenalter auf. Es existieren prognoserelevante genetische Subtypen. Bei Kindern scheinen diese Subtypen aufgrund bildgebender MRT-Marker, z.B. Tumorlage, teils vorhersagbar zu sein. Diese Studie hat als Subanalyse der NOA07-Studie zum Ziel erstmalig für ein Erwachsenenkollektiv radiologische Biomarker zur Bestimmung genetischer Medulloblastom-Subtypen zu identifizieren und diese mit Befunden bei Kindern zu vergleichen.

Material und Methodik 28 Erwachsene mit bestätigter Medulloblastomdiagnose wurden eingeschlossen ( $10 \mathrm{~W} ; 23$ - 53 J., 18 rekrutierende Zentren). Alle
Patienten erhielten ein MRT vor OP (1,5 o. 3T, axiale T2 TSE, FLAIR, native und KM-T1 SE). Durch 3 Neuroradiologen erfolgte die multiparametrische MRAnalyse nach vordefinierten Kriterien (Staging nach Chang, Signalmorphologie, Volumetrie, anatomische Lage). Das Gewebe wurde histologisch (klassischer, desmoplastisch/nodulärer, großzellig/anaplastischer Typ) und molekulargenetisch (SHH p53wt, SHH p53mut, WNT, non-WNT/non-SHH (Gruppe4)) aufgearbeitet. Genetische und radiologische Befunde wurden mit ANOVAs und Fischer Tests verglichen. Identifizierte Bildmarker wurden mit der einzig verfügbaren Studie bei Kindern verglichen (S Perreault et al., AJNR 2014).

Ergebnisse Es fanden sich ausschließlich klassische u. desmoplastisch/noduläre Medulloblastome sowie SHH, WNT und Gruppe 4-Subtypen. SHH, WNT und Gruppe 4 unterschieden sich signifikant in Kontakthäufigkeit zum 4. Ventrikel, Kontakt zur Rhombusformation, der Hydrocephalus- u. Hämorrhagiehäufigkeit u. dem T2w-Chang-Stadium ( $p=0,02 ; 0,03 ; 0,03 ; 0,04 ; 0,01$; alle bei Kindern nicht signifikant). Nuclear-ß-Catenin überexprimierende Tumore waren kleiner $(p=0.03)$. Klassische MR-Tumormorphologiekriterien, wie Signalhomogenität, ergaben keine signifikanten Ergebnisse.

Schlussfolgerungen Genetische Medulloblastom-Subtypen sind bei Erwachsenen möglicherweise anhand radiologischer MR-Biomarker differenzierbar und weisen Unterschiede zum pädiatrischen Kollektiv auf.

\section{WISS 306.3 Evaluation quantitativer MRT-Biomarker bei chronisch entzündlicher demyelinisierender Polyneuropathie (CIDP)}

Autoren Kronlage $\mathrm{M}^{1}$, Bäumer $\mathrm{P}^{1}$, Schwarz $\mathrm{D}^{1}$, Schwehr $\mathrm{V}^{1}$, Godel $\mathrm{T}^{1}$, Heiland $S^{1}$, Pitarokoili $\mathrm{K}^{2}$, Gold $\mathrm{R}^{2}$, Bendszus $\mathrm{M}^{1}$, Yoon $\mathrm{M}^{2}$

Institut 1 Universitätsklinikum Heidelberg, Neuroradiologie, Heidelberg; 2 St. Josef Hospital, Universitätsklinikum Bochum, Neurologie, Bochum DOI 10.1055/s-0037-1600393

Zielsetzung Die CIDP ist eine entzündliche Erkrankung des peripheren Nervensystems (PNS), deren Diagnostik bislang auf klinischen Befunden und Elektrophysiologie basiert. Die MRT des PNS mittels dedizierter Sequenzen (MRNeurografie, MRN) hat in den letzten Jahren zunehmende Bedeutung in der Diagnostik von Erkrankungen des PNS erlangt. Studienziel war, bildgebende Biomarker in hochauflösenden morphologischen und quantitativen (T2Relaxometrie, Diffusions-Tensor-Bildgebung (DTI)) MRT-Sequenzen an CIDPPatienten zu evaluieren.

Material und Methodik 18 Patienten mit CIDP und 18 gesunde Kontrollen wurden an einem 3T-MRT (Siemens TRIO) prospektiv untersucht. Über die obere und untere Extremität wurden hochauflösende fettgesättige T2-TSESequenzen (Morphologie), multiecho EPI-Sequenzen (T2-Relaxometrie, TE $10-120 \mathrm{~ms}$ ) sowie DTI-EPI-Sequenzen (b-Werte 0 und $1000 \mathrm{~s} / \mathrm{mm} 2,20$ Richtungen) akquiriert. Zudem wurden Nervus medianus und $\mathrm{N}$. tibialis elektrophysiologisch untersucht.

Ergebnisse Nervenquerschnittsfläche (CSA) und normalisiertes T2w-Signal (nT2) waren bei CIDP-Patienten signifikant erhöht. Die T2-Relaxometrie zeigte, dass die Erhöhung des T2w-Signals vor allem durch eine erhöhte apparente Protonenspindichte bei nicht signifikant verschiedener T2-Zeit verursacht wurde. Die fraktionale Anisotropie (FA) war in der Gruppe der CIDP-Patienten signifikant reduziert $(0,42$ vs. 0,52$)$, welches durch eine Zunahme der radialen Diffusivität bei nicht signifikant verschiedener axialer Diffusivität erklärt werden kann. CSA und FA wiesen eine hohe diagnostische Genauigkeit (ROCAnalyse: AUC 0,87 bzw. 0,90) sowie eine hohe Korrelation mit elektrophysiologischen Parametern (NLG und MSAP) auf. Im Gegensatz hierzu zeigte nT2 eine mäßige diagnostische Genauigkeit (AUC 0,80) und eine nur partielle Korrelation mit elektrophysiologischen Parametern.

Schlussfolgerungen CSA und FA sind bildgebende Biomarker mit hoher diagnostischer Genauigkeit und guter Korrelation mit etablierten elektrophysiologischen Parametern demyelinisierender Schaedigung bei CIDP. 


\section{WISS 404.11 Evaluation der Kosten-Effektivität der endovaskulären Schlaganfalltherapie basierend auf einer Meta-Analyse der randomisierten klinischen Studien ESCAPE, EXTEND-IA, MR CLEAN, REVASCAT und SWIFT PRIME}

Autoren Kunz $W^{1}$, Hunink $M^{2}$, Beyer $S^{1}$, Meinel $F^{1}$, Reiser $M^{1}$, $S o m$, Thierfelder $\mathrm{K}^{1}$

Institut 1 Klinikum der Universität München, Institut für Klinische Radiologie, München; 2 Erasmus University Medical Center, Departments of Radiology and Epidemiology, Rotterdam

DOI 10.1055/s-0037-1600394

Zielsetzung Die endovaskuläre Therapie zusätzlich zur intravenösen Thrombolyse (EVT+IVT) ist effektiver als die alleinige IVT bei der akuten Mediaischämie durch proximalen Gefäßverschluss im Zeitfenster von 6 Stunden. Wir evaluierten die Kosten-Effektivität der EVT+IVT insgesamt und abhängig von National Institutes of Health Stroke Scale (NIHSS) Score, Zeit seit Symptombeginn, Alberta Stroke Program Early CT Score (ASPECTS) und Verschlusslokalisation.

Material und Methodik Ein Entscheidungsmodell basierend auf Markov-Simulationen errechnete Lebenszeitkosten und qualitätskorrigierte Lebensjahre (QALY) beider Strategien aus der Perspektive des Gesundheitswesens. Die Input Parameter basierten auf aktueller Literatur, inklusive einer Meta-Analyse der Outcome-Daten aus 5 randomisierten klinischen Studien (ESCAPE, EXTEND-IA, MR CLEAN, REVASCAT, SWIFT PRIME). Probabilistische Sensitivitätsanalysen (PSA) anhand von Monte-Carlo-Simulationen wurden verwendet um die Ungewissheit der Modellergebnisse zu schätzen. Net Monetary Benefits (NMB), inkrementelle Kosten (IC), inkrementelle Effektivität (IE) and inkrementelle Kosten-Effektivitäts-Relationen (ICER) wurden aus PSAs abgeleitet. Die Willingness-to-Pay (WTP) wurde mit $\$ 50.000 /$ QALY festgelegt.

Ergebnisse Insgesamt war die EVT+IVT kosten-effektiv verglichen mit IVT (IC: $\$ 4.938$, IE: 1,59 QALYs, ICER: $\$ 3.110 /$ QALY) in $100 \%$ der Simulationen. Bei allen Patienten erzielte EVT+IVT zusätzliche QALYs (Reichweite: 0,47-2,12) und die mittleren ICERs lagen unterhalb der WTP. In Subgruppen mit ASPECTS $\leq 5$ oder mit M2 Verschlüssen zeigten sich jedoch deutlich höhere ICERs (\$14.273/QALY und \$28.812/QALY) und eine suboptimale Akzeptabilität in den PSAs (75,5\% und 59,4\%). Alle anderen Subgruppen erzielten Akzeptabilitätsraten von $90-100 \%$.

Schlussfolgerungen EVT+IVT ist kosten-effektiv in nahezu allen PatientenSubgruppen. Bei Patienten mit ASPECTS $\leq 5$ oder M2 Verschlüssen bleibt die Kosten-Effektivität basierend auf der aktuellen Datengrundlage ungewiss.

\section{WISS 404.7 Crossed cerebellar diaschisis im akuten ischämischen Schlaganfall: Einfluss auf morphologisches Outcome, klinisches Outcome und assoziierte subakute Komplikationen}

Autoren Kunz $W^{1}$, Sommer $W^{1}$, Fabritius $M^{1}$, Schuler $F^{1}$, Huber $T^{1}$, Höhne $C^{2}$, Reiser $M^{1}$, Thierfelder $K^{1}$

Institut 1 Klinikum der Universität München, Institut für Klinische Radiologie, München; 2 Klinikum der Universität München, Neurologische Klinik und Poliklinik, München

DOI 10.1055/s-0037-1600395

Zielsetzung Crossed cerebellar diaschisis (CCD) ist das Phänomen einer Hypoperfusion der kontralateralen cerebellären Hemisphäre durch eine Ischämie der funktionell verknüpften supratentoriellen Regionen. Die klinische Relevanz der Detektion einer CCD im akuten Schlaganfall mittels Whole-Brain-CT-Perfusion (WB-CTP) wurde untersucht.

Material und Methodik Patienten mit Follow-up-gesichertem Infarkt durch einen proximalen Gefäßverschluss der vorderen Strombahn wurden aus einer Kohorte von 1644 konsekutiven Patienten mit WB-CTP Untersuchung ausgewählt. Zwei verblindete, erfahrene Reader beurteilten die hintere Schädelgru- be bezüglich Zeichen einer CCD (CCD+). Finales Infarktvolumen (FIV), modified Rankin Scale Scores bei Entlassung (discharge mRS) und nach 90 Tagen (90-day mRS) sowie das Auftreten von assoziierten subakuten Komplikationen wurden als Indikatoren des Outcomes erhoben. Lineare und logistische Regressionsanalysen wurden verwendet um Assoziationen mit CCD zu untersuchen.

Ergebnisse Insgesamt erfüllten 156 Patienten die Einschlusskriterien, davon wurden 102 als CCD+ (65.4\%) und 54 (34.6\%) als CCD- kategorisiert. In den Regressionsanalysen zeigte sich keine unabhängige Assoziation zwischen CCD und FIV $(\beta=-0.440, p=0.972$ ), discharge $m R S \leq 2$ (odds ratio $(O R)=1.897$, $p=0.320)$, oder 90-day $m R S \leq 2(O R=0.531, p=0.492)$. $C C D+$ Patienten hatten größere supratentorielle zerebrale Blutflussdefizitvolumina (Median: $164 \mathrm{ml}$ vs. $115 \mathrm{ml}$; = 0.001). In der Regressionsanalyse der subakuten Komplikationen zeigte sich eine signifikante Assoziation mit der Entwicklung von Parenchymhämatomen $(O R=4.793, p=0.035)$.

Schlussfolgerungen CCD ist ein häufiger Befund der WB-CTP beim akuten ischämischen Schlaganfall durch proximalen Gefäßverschluss der vorderen Strombahn. CCD zeigte eine Assoziation mit der Entwicklung von Parenchymhämatomen, hatte jedoch keinen unabhängigen Einfluss auf das morphologische oder klinische Patientenoutcome.

\section{WISS 404.1 Die Detektion CTA-okkulter Gefäßverschlüsse mittels Post-Processing der CT Perfusion begünstigt den Therapieerfolg der intravenösen Thrombolyse im akuten ischämischen Schlaganfall}

Autoren Kunz $W^{1}$, Fabritius $M^{1}$, Höhne $C^{2}$, Havla $L^{1}$, Reiser $M^{1}$, Sommer $W^{1}$, Thierfelder $\mathrm{K}^{1}$

Institut 1 Klinikum der Universität München, Institut für Klinische Radiologie, München; 2 Klinikum der Universität München, Neurologische Klinik und

Poliklinik, München

DOI 10.1055/s-0037-1600396

Zielsetzung Intrakranielle Gefäßverschlüsse stellen den therapeutischen Angriffspunkt der intravenösen Thrombolyse (IVT) im akuten ischämischen Schlaganfall dar. Wir untersuchten den prädiktiven Wert der Detektion CTAokkulter Gefäßverschlüsse mittels wavelet-basierter angiografischer Rekonstruktion (waveletCTA) der Whole-Brain-CT-Perfusion (WB-CTP) für den Therapieerfolg der IVT.

Material und Methodik Aus 1351 konsekutiven Patienten mit multiparametrischer CT inklusive WB-CTP wurden alle Patienten eingeschlossen mit: (1) signifikantem cerebral blood flow (CBF) Defizit, (2) fehlendem Gefäßverschluss in der CTA, und (3) Follow-up-gesichertem Infarkt. Das angiografische waveletCTA Signal wird über Voxel-basierte wavelet-Transformation von Zeit-Dichte-Kurven der WB-CTP generiert. Zwei verblindete Reader analysierten die waveletCTA. Das morphologische Therapieansprechen wurde durch den Quotienten aus finalem Infarktvolumen (FIV) und CBF Defizitvolumen repräsentiert. Das klinische Therapieansprechen wurde als Abnahme des modified Rankin Scale Scores $\geq 1$ von Aufnahme zu Entlassung ( $\Delta \mathrm{mRS}$ ) bzw. des National Institutes of Health Stroke Scale Scores $\geq 3$ von Aufnahme zu 24 Stunden später ( $\triangle$ NIHSS) definiert. Mittels linearer und logistischer Regressionsanalysen wurden unabhängige Assoziationen untersucht.

Ergebnisse Unter allen eingeschlossenen Patienten $(N=106)$ zeigten 50 (47\%) einen CTA-okkulten Gefäßverschluss in der waveletCTA. Bei der Gruppe der IVT-behandelten Patienten $(n=50)$ zeigte sich eine unabhängige Assoziation des CTA-okkulten Gefäßverschlusses mit günstigem morphologischen (beta $=-0.524, \quad p=0.015)$ und klinischen Therapieansprechen ( $\Delta \mathrm{mRS}$ : $\mathrm{OR}=9.018, \mathrm{p}=0.047 ; \Delta \mathrm{NIHSS}: \mathrm{OR}=5.810, \mathrm{p}=0.024)$. Dahingegen zeigte sich keine unabhängige Assoziation in der Gruppe der Patienten ohne IVT Therapie ( $n=56$; jeweils $p>0.05$ )

Schlussfolgerungen Die Detektion CTA-okkulter Gefäßverschlüsse mittels waveletCTA begünstigt den Therapieerfolg der IVT. Die waveletCTA hat das 
Potential die klinische Therapieentscheidung im akuten Schlaganfall zu unterstützen.

\section{WISS 205.2 Wavelet-basierte angiografische Rekonstruktion der Whole-Brain-CT-Perfusion für die Beurteilung der Cerebralen Venen- und Sinusthrombose}

Autoren Kunz $W^{1}$, Schuler $F^{1}$, Fabritius $M^{1}$, Havla $L^{1}$, Reiser $M^{1}$, Sommer $W^{1}$, Thierfelder $\mathrm{K}^{1}$

Institut 1 Klinikum der Universität München, Institut für Klinische Radiologie, München

DOI 10.1055/s-0037-1600397

Zielsetzung Untersuchung des diagnostischen Wertes der wavelet-basierten angiografischen Rekonstruktion (waveletCTA) der Whole-Brain-CT-Perfusion (WB-CTP) für die Beurteilung der Cerebralen Venen- und Sinusthrombose (CVST).

Material und Methodik Aus 2863 konsekutiven Patienten mit Schlaganfallverdacht und multiparametrischer CT inklusive arterieller CT-Angiografie (artCTA) und WB-CTP wurden Fälle mit gesicherter CVST $(n=10)$ und artCTA-basiertem Verdacht auf CVST $(n=6)$ ausgewählt. Das angiografische waveletCTA Signal wird über Voxel-basierte wavelet-Transformation von ZeitDichte-Kurven der WB-CTP Daten generiert. Zwei verblindete Reader analysierten die Bilddaten bezüglich CVST. Venöse CT-/MR-Angiografie diente als Referenzstandard. Die diagnostische Sicherheit bezüglich CVST und die Qualität der Darstellung von 13 venösen Strukturen wurden auf 5-Punkt-LikertSkalen bewertet. Die Thrombuslänge und die Ratio der CT Dichtewerte bzw. waveletCTA Signals von thrombosierten zu perfundierten venösen Strukturen ("Signalverhältnis") wurde gemessen.

Ergebnisse Die waveletCTA Rekonstruktion war bei allen Patienten erfolgreich. Bei gesicherter CVST demonstrierte die waveletCTA das Vorhandensein, die Lokalisation sowie das Ausmaß der Thrombose in 10/10 Fällen, während mittels artCTA nur 7/10 Fällen erkannt wurden. Bei Patienten mit artCTAbasiertem Verdacht, welcher sich im follow-up nicht bestätigte, konnten die Reader mittels waveletCTA diesen in 5/6 Fällen ausräumen. Das Reading der waveletCTA steigerte die diagnostische Sicherheit gegenüber der artCTA (4.4 vs. 3.6, $p=0.044)$. Die waveletCTA bietet ein signifikant höheres Signalverhältnis ( 146.2 vs. 2.6, $\mathrm{p}<0.001)$ und eine bessere Qualität in der Darstellung der venösen Strukturen (4.2 vs. 2.6, $\mathrm{p}<0.001$ ) verglichen mit der artCTA. Schlussfolgerungen Die waveletCTA erweitert die diagnostischen Möglichkeiten der WB-CTP um die Beurteilung der cerebralen Venen und Sinus. In Patienten die mittels WB-CTP untersucht wurden, ermöglicht dies eine CVST auszuschließen oder inzidentell zu detektieren.

\section{WISS 404.8 Balloon Guide Catheter oder Distal Access Catheter: Was ist die effektivste Rekanalisationstechnik bei akutem ischämischen Schlaganfall?}

Autoren Madjidyar J $]^{1}$, Pineda Vidal $\mathrm{L}^{1}$, Jansen $\mathrm{O}^{1}$

Institut 1 Universitätsklinikum Schleswig-Holstein, Campus Kiel, Klinik für Radiologie und Neuroradiologie, Kiel

DOI 10.1055/s-0037-1600398

Zielsetzung Die Thrombektomie mit Stentretriever (SR) ist die Therapie der Wahl bei Patienten mit akutem Schlaganfall und Verschluss großer Gefäße. In einer experimentellen Studie sollte unter standardisierten Bedingungen an einem Flussmodell untersucht werden, wie SR am effektivsten verwendet werden können. Zusätzlich sollte die Effektivität der direkten Aspiration im Vergleich zu SR evaluiert werden.

Material und Methodik Es wurden zwei Thrombusarten aus humanem Blut hergestellt: ein erythrozytenreicher und ein fibrinreicher. Diese wurden in die A.cerebri media eines transparenten, möglichst physiologischen Flussmodells aus Silikon eingeführt. Folgende Rekanalisationstechniken wurden unter direkter visueller Kontrolle untersucht: 1. SR (Trevo) und distale Aspiration mit 5F SOFIA Catheter (DAC), 2. SR (Trevo) und Aspiration mit 7F Cello Balloon Guide Catheter (BGC), 3. Direkte Aspiration mit 5F SOFIA Catheter (ADAPT). Das Auftreten distaler Embolien (DE) und von Embolien in neue Territorien (ENT) wurde ausgewertet.

Ergebnisse Es wurden jeweils 6 Versuche pro Technik und Thrombusart durchgeführt $(n=36)$. ADAPT zeigte die kürzeste Rekanalisationszeit (ADAPT $\mu=8,2$ min vs. $B G C+S R \mu=10,4$ min vs. $D A C+S R \mu=21,4 \mathrm{~min}$ ), insbesondere bei fibrinreichen Thromben ( $\mu=5,3 \mathrm{~min}$ ). Bei fibrinreichen Thromben war mittels BGC+SR oder ADAPT jeweils nur ein Thrombektomiemanöver notwendig, ansonsten im Mittel 1,5 Manöver. ADAPT zeigte bei erythrozytenreichen Thromben die geringste Rate an DE (2/6), BGC+SR bei fibrinreichen Thomben (1/6). ENT traten in 2/36 Versuchen auf: ADAPT bei erythrozytenreichem und BGC + SR bei fibrinreichem Thrombus.

Schlussfolgerungen In dieser experimentellen Studie war ADAPT die effektivste Rekanalisationstechnik. Nahezu ebenbürtig war BGC+SR mit Vorteilen bei der Rate an distalen Embolien bei Verschlüssen mit fibrinreichen Thromben. DAC+SR war die einzige Technik ohne Embolien in neue Territorien, aber ansonsten den anderen Techniken unterlegen. Die Effektivität der Thrombektomie hing zusätzlich von der Thrombusbechaffenheit ab.

\section{WISS 404.9 Angiografisches und klinisches Ergebnis nach mechanischer Thrombektomie bei Schlaganfallpatienten mit partieller Okklusion der Hirngefäße}

Autoren Maus $\mathrm{V}^{1}$, You $\mathrm{S}^{1}$, Kalkan $\mathrm{A}^{1}$, Borggrefe $\mathrm{J}^{1}$, Kabbasch $\mathrm{C}^{1}$, Dorn $\mathrm{F}^{2}$, Dohmen $C^{3}$, Liebig $T^{4}$, Fink $G^{3}$, Mpotsaris $A^{1}$

Institut 1 Uniklinik Köln, Diagnostische und Interventionelle Radiologie, Köln; 2 LMU, Abteilung für Neuroradiologie, Muenchen; 3 Uniklinik Köln, Zentrum für Neurologie und Psychatrie, Köln; 4 Charite, Institut für Neuroradiologie, Berlin DOI 10.1055/s-0037-1600399

Zielsetzung Analyse des Einflusses von partiell okkludierten zerebralen Gefäßen bei akuter Ischämie auf das angiografische und klinische Ergebnis nach mechanischer Thrombektomie.

Material und Methodik 503 Patienten mit einem gesicherten proximalen Verschluss der zerebralen Hirngefäße wurden konsekutiv mittels Thrombektomie zwischen Oktober 2010 und Juli 2016 behandelt und retrospektiv ausgewertet. Primärer Endpunkt war ein gutes klinisches Ergebnis nach 90 Tagen (mRS $\leq 2)$, sekundäre Endpunkte waren Interventionsdauer, Anzahl der Thrombektomiemanöver, erfolgreiche Reperfusion (mTIClï, ${ }^{32 b}$ ), Auftreten von Thrombembolien in neue Territorien (ENT) und postinterventionell symptomatischer intrakranieller Blutungen.

Ergebnisse 432 Patienten wurden nach Ausschluss von 71 Patienten mit Tandemokklusion ausgewertet. Das mittlere Alter betrug 69,7 $( \pm 13,7)$ Jahre. $51,6 \%$ der Patienten waren männlich. 49 Patienten (11,3\%) mit partieller Okklusion (AOL 1/2) wiesen initial einen medianen NIHSS von 11 (IQR 9,5) auf, die verbliebenen Patienten mit vollständiger Okklusion (AOL 0) einen NIHSS von 15 (IQR 10) ( $<$ 0.001). Die mediane Dauer von Leistenpunktion bis zur Reperfusion betrug 30 Minuten (IQR 37) bei AOL 1/2 im Vergleich zu 67 Minuten (IQR 66) bei AOL 0 ( $p<0.001)$; die mediane Dauer von Symptombeginn bis zur Reperfusion betrug 242 Minuten (IQR 90) gegenüber 283 Minuten (IQR 124) ( $<$ 0.02). Mit einer geringeren Anzahl an Thrombektomiemanövern $(1.7 \pm 2.2$ vs. $3.0 \pm 2.5)(p<0.0001)$ konnte bei $47 / 49$ Patienten (95.9\%) mit AOL $1 / 2$ eine erfolgreiche Reperfusion erzielt werden im Vergleich zu 298/381 (78.2\%) Patienten mit AOL 0 ( $p<0.005)$. Ein gutes klinisches Ergebnis nach 90 Tagen wurde in $81 \%$ der Fälle mit AOL $1 / 2$ erreicht gegenüber $29,1 \%$ in der Vergleichsgruppe ( $<<0.0001)$; die Mortalität betrug $6,4 \%$ vs. $28,5 \%(p<0.001)$. 
Schlussfolgerungen Die mechanische Thrombektomie bei partieller Okklusion zerebraler Gefäße im Rahmen einer akuter Ischämie geht einher mit einer annähernd dreifach höheren Chance eines guten klinischen Ergebnisses.

\section{WISS 317.4 Verbesserung der Bildqualität von Kopf- Hals-Angiografien durch virtuell monoenergetische Rekonstruktionen der Spektral-Detektor Computertomografie}

\author{
Autoren Neuhaus $V^{1}$, Abdullayev N ${ }^{1}$, Große Hokamp N ${ }^{1}$, Mpotsaris $\mathrm{A}^{1}$, \\ Maintz $\mathrm{D}^{1}$, Borggrefe J' \\ Institut 1 Uniklinik Köln, Institut für Diagnostische und Interventionelle \\ Radiologie, Köln
}

DOI 10.1055/s-0037-1600400

Zielsetzung Vergleich der Bildqualität und des Bildkontrastes zwischen virtuell monoenergetischen Rekonstruktionen (VMR) von Spektral-Detektor CT-Angiografien (SDCTA) und konventionellen CT-Rekonstruktionen ( $k R$ ).

Material und Methodik SDCTA des Kopfes von 30 Patienten. Scanprotokoll: Schichtdicke $1 \mathrm{~mm}$, pitch 1, $320 \mathrm{mAs}, 120 \mathrm{kVp}$. Bildrekonstruktion mit VMR (40keV-120keV, Intervall $5 \mathrm{keV}$ ) und kR mit jeweils Verwendung von iterativer Rekonstruktion iDose4, Level 3. ROI-basierte Bestimmung der HU-Dichte und Standardabweichung (SD, Bildrauschen) im Bereich der kontrastierten Arteria carotis interna (Pars cervicalis und Pars cavernosa), der Arteria vertebralis (V3Segment), der Arteria basilaris sowie der Arteria cerebri anterior, media und posterior. Referenzmessungen der Luft und des Musculus pterygoideus lateralis. Bestimmung des Kontrast-zu-Rausch Verhältnisses (CNR).

Ergebnisse Die Dichte und das Bildrauschen in den kontrastierten Arterien stieg im Vergleich zu den $\mathrm{kR}$ mit niedrigen keV-Werten kontinuierlich mit jedem absteigenden keV-Wert der VMR $(\Delta p<0,0001$ bei $40 \mathrm{keV}$, bei $40 \mathrm{keV}$ jeweils Anstieg um ca. Faktor 3 zu kR). Hingegen zeigte das Referenzgewebe (M. pterygoideus lateralis) bei VMR $40 \mathrm{keV}$ im Vergleich zur kR einen Dichteanstieg um den Faktor 1,33, das Bildrauschen in der Referenzmessung Luft nahm um den Faktor 2 zu. Somit zeigten VMR bei $40 \mathrm{keV}$ für große und kleine Gefäße im Vergleich zu kR im Mittel einen Anstieg des CNR um ca. 50\% $(p<0,02-p<0,002)$.

Schlussfolgerungen VMR der SDCT ermöglichen im Vergleich zu konventionellen CT-Rekonstruktionen mit iterativer Bildnachbearbeitung eine Erhöhung des CNR um ca. 50\%. Hierdurch zeigt sich eine Verbesserung der Bildqualität der kranialen CT-Angiografien, sogar auf Höhe der Schädelbasis. Zudem ist hierdurch eine entsprechende Reduktion der Kontrastmittelgabe möglich.

\section{WISS 317.7 Doppelresonante $(23 \mathrm{Na} / 1 \mathrm{H})$ vs. klinische 1 H Schädel-MRT Spule - Intraindividueller Vergleich der Bildqualität an gesunden Probanden}

Autoren Ong $\mathrm{M}^{1}$, Schmidt $\mathrm{A}^{1}$, Hausmann $\mathrm{D}^{1}$, Schönberg $\mathrm{S}^{1}$, Haneder $\mathrm{S}^{2}$ Institut 1 Universitätsmedizin Mannheim, Institut für klinische Radiologie und Nuklearmedizin, Mannheim; 2 Universitätsklinik Köln, Institut für

Diagnostische und Interventionelle Radiologie, Köln

DOI 10.1055/s-0037-1600401

Zielsetzung Prospektiver Vergleich der Bildqualität zwischen einer doppel-resonanten $(23 \mathrm{Na} / 1 \mathrm{H})$ im Vergleich zu einer klinisch genutzten $1 \mathrm{H}$ Kopfspule in gesunden Probanden.

Material und Methodik In der vorliegenden intraindividuellen Vergleichsstudie wurden 10 gesunde Probanden (6 weiblich; Altersdurchschnitt 25 Jahre) eingeschlossen. Es wurden zwei konsekutive cerebrale MRT-Untersuchungen mit einer doppelresonanten $(23 \mathrm{Na} / 1 \mathrm{H})$ Kopfspule, sowie mit einer 12-KanalProtonen-Spule am 3.0 Tesla MRT (TimTrio, Siemens Healthineers) durchgeführt. Die folgenden Sequenzen wurden akquiriert: T1-gewichtete MP-RAGE, axiale T2-TSE, T1 flash, T2 TIRM, DW-EPI. Die subjektive Bildqualität wurde durch zwei Radiologen anhand einer 5-Punkte Likert-Skala evaluiert ( 1 = schlecht Bildqualität; 5 = exzellente Bildqualität). Die objektive Bildqualität wurde anhand zirkulärer region-of-interests (ROI) Platzierungen in der weißen
Substanz auf allen Sequenzen verglichen. Für die statistische Auswertung wurde JMP 10.0 verwendet (SAS Institute Inc., Cary, North Carolina, USA). Die signal-to-noise ratio wurde mittels eines zweiseitigen Mann-Whitney-UTests verglichen.

Ergebnisse Die durchschnittliche Akquisitionszeit betrug 20 Minuten für die Protonen-Spule vs. 30 Minuten (inklusive 23Na Scan) für die doppelresonante $(23 \mathrm{Na} / 1 \mathrm{H})$ Kopfspule. Die Bildqualität wurde für beide Spulen als diagnostisch bewertet. Die subjektive Bildqualität war für beide Spulen exzellent. Die SNR zeigte keinen signifikanten Unterschied zwischen der doppelresonanten und der klinisch genutzten Kopfspule (alle Sequenzen $p>0,05$ ).

Schlussfolgerungen Bilddaten einer doppelresonanten Spule im Vergleich zu einer klinisch genutzten Protonenspule zeigten eine vergleichbare diagnostische Bildqualität. Die alleinige Nutzung einer doppelresonanten Spule würde die Akquisitionszeit deutlich verringern und den Patientenkomfort erhöhen. Dies könnte die klinische Implementierung von Natrium-MRTs erleichtern und die Lücke zwischen präklinischen und klinischen Studien schließen.

\section{WISS 317.6 Cerebrale Natrium (23Na)-Bildgebung in Migränepatienten}

Autoren Ong $\mathrm{M}^{1}$, Schmidt $\mathrm{A}^{1}$, Konstandin $\mathrm{S}^{2}$, Benrath $\mathrm{J}^{3}$, Eisele $\mathrm{P}^{4}$, Schad $\mathrm{L}^{5}$, Schönberg $S^{1}$, Haneder $S^{6}$

Institut 1 Universitätsmedizin Mannheim, Institut für klinische Radiologie und Nuklearmedizin, Mannheim; 2 Fraunhofer Institut, Bremen;

3 Universitätsmedizin Mannheim, Klinik für Anästhesiologie und Operative Intensivmedizin, Mannheim; 4 Universitätsmedizin Mannheim, Neurologische Klinik, Mannheim; 5 Universitätsmedizin Mannheim, Computer Assisted

Clinical Medicine, Mannheim; 6 Universitätsklinik Köln, Institut für

Diagnostische und Interventionelle Radiologie, Köln

DOI 10.1055/s-0037-1600402

Zielsetzung Evaluation der 23Na-Konzentrationen in Subgruppen von Patienten mit klinisch manifester Migräne.

Material und Methodik In dieser prospektiven Studie wurden 12 Patienten eingeschlossen (alle weiblich, Durchschnittsalter 34 J), die klinisch hinsichtlich einer Migräne evaluiert wurden und einen Fragebogen bzgl. Krankheitsbeginn, Dauer, Intensität, Häufigkeit der Attacken und begleitenden Aura ausgefüllt haben. Die Patienten erhielten eine cerebrale 23Na-MRT bei 3.0T. Die folgenden Sequenzen wurden mittels einer doppelresonanten $(23 \mathrm{Na} / 1 \mathrm{H})$ Kopfspule akquiriert: eine T1 MP-RAGE-Sequenz als anatomische Referenz, sowie eine 3D-Dichte-angepasste, radiale Gradienten-Echo-Sequenz für die 23Na-Bildgebung. Die 23Na-Sequenzen wurden anhand der MP-RAGE rekonstruiert, welches eine direkte Ko-Registrierung von region-of-interests (ROI) erlaubte. Die ROls wurden innerhalb folgender anatomischer Regionen platziert: Liquor, graue und weiße Substanz (GS/WS), Hirnstamm und Kleinhirn. Externe 23Na-Referenzphantome mit 5\% und 2\% Agar-Gel und einer 23Na-Konzentration von 154 und 50 Millimol wurden verwendet, um die 23Na-Gewebekonzentrationen zu berechnen. Der Kendall Tau und Wilcoxon-Rangsummentest wurden für die statistische Analyse verwendet.

Ergebnisse Signifikante Unterschiede in 23Na-Konzentrationen bei Patienten mit und ohne begleitende Aura konnten innerhalb der WS und anteriorem Liquor beobachtet werden $(p<0,05)$. Moderate bis gute Korrelationen konnten zwischen Zeitintervall zur letzten Migräneattacke und 23Na-Konzentrationen in anteriorem Liquor, sowie zwischen Krankheitsbeginn und 23Na-Konzentrationen in GS, posteriorem Liquor, Hirnstamm und Kleinhirn $(r \geq 0,4)$ beobachtet werden. Darüber hinaus zeigten Schmerzintensität und 23Na-Konzentrationen in GS, Liquor und Hirnstamm eine moderate Korrelation $(r=0,4-0,5)$.

Schlussfolgerungen Die cerebrale 23Na-Bildgebung hat das Potenzial zwischen verschiedenen Subgruppen einer Migräne zu differenzieren und Informationen über intraindividuelle Schmerzintensitäten zu offenbaren. 
WISS 306.6 Quantitative MRT-basierte 3DVolumetrie des Duralsacks und der Wirbelkörper verbessert die Diagnose der Duraektasie bei Patienten mit Marfan-Syndrom

\author{
Autoren Rengier $\mathrm{F}^{1}$, Naas $\mathrm{O}^{1}$, Norajitra $\mathrm{T}^{2}$, Messerli $\mathrm{M}^{3}$, Kallenbach $\mathrm{K}^{4}$, \\ Karck $\mathrm{M}^{4}$, Maier-Hein $\mathrm{K}^{2}$, Kauczor $\mathrm{H}^{1}$ \\ Institut 1 Universitätsklinikum Heidelberg, Diagnostische und \\ Interventionelle Radiologie, Heidelberg; 2 Deutsches \\ Krebsforschungszentrum (DKFZ), Medizinische und Biologische Informatik, \\ Heidelberg; 3 Universitätsspital Zürich, Nuklearmedizin, Zürich; \\ 4 Universitätsklinikum Heidelberg, Herzchirurgie, Heidelberg \\ DOI 10.1055/s-0037-1600403
}

Zielsetzung Evaluation einer MRT-basierten 3D-Volumetrie des Duralsacks und der Wirbelkörper für die Diagnose der Duraektasie bei Patienten mit Marfan-Syndrom im Vergleich zum Duralsack-Durchmesser-Quotienten als aktuellen klinischen Standard.

Material und Methodik Bei 188 in Abklärung für ein Marfan-Syndrom befindlichen Patienten in den Jahren 2012-2015 wurde eine single slab 3D T2W TSE Sequenz der lumbosakralen Wirbelsäule in einem 1,5 T MRT-Scanner akquiriert. Die räumliche Auflösung betrug $1 \times 1 \times 1 \mathrm{~mm}^{3}$. Eine Zufallsauswahl von 20 Patienten (durchschnittlich 32,0 Jahre, 7 Frauen) wurde eingeschlossen. Bei neun dieser Patienten wurde die Diagnose des Marfan-Syndroms nach den Kriterien der 2010 Revised Ghent Nosology bestätigt, die elf anderen Patienten dienten als Kontrollen. Der Duralsack und die Wirbelkörper L5 und S1 wurden mit der Software Medical Imaging Interaction Toolkit (MITK) segmentiert. Zum Vergleich mit dem aktuellen klinischen Standard wurden der anteroposteriore Durchmesser der Wirbelkörper und des Duralsacks auf halber Höhe der Wirbelkörper gemessen. Schließlich wurden die jeweiligen Quotienten zwischen Duralsack und Wirbelkörper berechnet.

Ergebnisse Die Duralsack-Volumina betrugen bei Marfan-Patienten durchschnittlich $11,0 \mathrm{ml}$ für $\mathrm{L} 5$ und $13,8 \mathrm{ml}$ für $\mathrm{S} 1$, verglichen mit $6,9 \mathrm{ml}$ für $\mathrm{L} 5$ und $5,0 \mathrm{ml}$ für $\mathrm{S} 1$ bei den Kontrollen (beide $\mathrm{p}<0,05$ ). Sensitivität, Spezifität, positiver prädiktiver Wert und negativer prädiktiver Wert für die Diagnose eines Marfan-Syndroms waren: für die 3D-Volumetrie mit 7,7/6,4 ml als Grenzwert 78\%/91\%/88\%/83\%, für die Kriterien nach Habermann et al. $78 \% / 45 \% / 54 \% / 71 \%$, und für die Kriterien nach Oosterhof et al. $67 \% / 73 \% /$ $67 \% / 73 \%$

Schlussfolgerungen Die MRT-basierte 3D-Volumetrie des Duralsacks und der Wirbelkörper zur Quantifizierung der Duraektasie ist machbar und verbesserte insbesondere die Spezifität der Diagnose eines Marfan-Syndroms im Vergleich zum aktuellen klinischen Standard veröffentlichter quantitativer Kriterien, die auf den Dursalsack-Durchmesser-Quotienten beruhen.

\section{WISS 317.1 Dual-layer Spektral-CT versus MRT bei der Differenzierung zwischen Hämorrhagie und Kontrastmittelextravasation nach mechanischer Rekanalisation}

Autoren Riederer $\mathrm{I}^{1}$, Sauter $\mathrm{A}^{2}$, Renz $\mathrm{M}^{3}$, Dangelmaier $\mathrm{J}^{3}$, Kirschke $\mathrm{J}^{4}$, Fingerle $A^{2}$, Rummeny $E^{3}$, Noel $P^{3}$, Muenzel $D^{3}$

Institut 1 Klinikum rechts der Isar, Technische Universität München, Diagnostische und Interventionelle Radiologie, München; 2 Klinikum rechts der Isar, Technische Universität München, Diagnostische und interventionelle Radiologie, München; 3 Klinikum rechts der Isar, Technische Universität München, Diagnostische und interventionelle Radiologie, München; 4 Klinikum rechts der Isar, Technische Universität München, Diagnostische und interventionelle Neuroradiologie, München

DOI 10.1055/s-0037-1600404

Zielsetzung Ziel dieser Studie ist, die Vorteile der Dual-layer Spektral-Computertomografie (CT) bei der Differenzierung zwischen intrazerebraler Hämorrhagie und Extravasation von jodhaltigem Kontrastmittel aufgrund einer ge- störten Blut-Hirn-Schranke in Schlaganfallpatienten nach mechanischer Rekanalisation zu evaluieren.

Material und Methodik Insgesamt wurden zwanzig Patienten, die eine mechanische Thrombektomie erhalten haben, an einem Dual-layer Spektral-CT (IQon spectral CT, Philips Healthcare, USA) $11+/-3 \mathrm{~h}$ nach Rekanalisation untersucht. Virtual non-contrast (VNC) Bilder und iodine overlay maps (IOM) wurden berechnet und mithilfe einer speziellen Software (Intellispace Portal, Philips Healthcare, USA) analysiert. Region of interests (ROIs) Analysen wurden innerhalb hyperdenser Areale im konventionellen CT, in den IOM und VNC Bilder durchgeführt. Als Goldstandard wurden die Magnetresonanztomografie (MRT)-Untersuchungen innerhalb des Routine-Follow up herangezogen. Sensitivität, Spezifität und positiver prädiktiver Wert für den Nachweis einer Hämorrhagie im Dual-layer Spektral-CT wurden berechnet.

Ergebnisse Insgesamt zeigten sich 20 hyperdense Areale in 12 Patienten im Dual-layer Spektral-CT. Im Vergleich zum Follow-up MRT, welches als Goldstandard herangezogen wurde, war es möglich, diese Hyperdensitäten korrekt in Hämorrhagie $(n=8)$, Kontrastmittelextravasation $(n=8)$, oder beides $(n=4)$ im Spektral-CT zu differenzieren. Bei der ROI Analyse zeigten sich HU-Werte von 64 +/- 2 („konventionelles CT”) und $51+/$-2 (VNC) für Hämorrhagie beziehungsweise $61+/-3$ (,konventionelles CT”) und $22+/-2$ (VNC) für lod. Schlussfolgerungen Die Dual-layer Spektral-CT erlaubt eine verbesserte Differenzierung zwischen intrazerebraler Hämorrhagie und Extravasation von jodhaltigem Kontrastmittel in Patienten mit gestörter Blut-Hirn-Schranke nach mechanischer Rekanalisation.

\section{WISS 205.1 Vorhersage eines schlechten funktionellen Outcomes nach aneurysmatischer Subarachnoidalblutung mittels Random Forests}

Autoren Rubbert $C^{1}$, May $\mathrm{R}^{1}$, Beseoglu $\mathrm{K}^{2}$, Turowski $\mathrm{B}^{1}$, Mathys $\mathrm{C}^{1}$, Caspers $]^{1}$ Institut 1 Universitätsklinikum Düsseldorf, Institut für Diagnostische und Interventionelle Radiologie, Düsseldorf; 2 Universitätsklinikum Düsseldorf, Klinik für Neurochirurgie, Düsseldorf

DOI 10.1055/s-0037-1600405

Zielsetzung Patienten nach aneurysmatischer Subarachnoidalblutung (SAB) leiden häufig unter einem schlechten funktionellen Outcome. Nur etwa 33\% der primär überlebenden Patienten kehren wieder in Vollzeit in ihren vormaligen Beruf zurück. Die zugrundeliegende Pathogenese ist nicht vollständig verstanden. Einer dysfunktionalen Mikrozirkulation wird dabei eine Schlüsselrolle zugeschrieben. Diese kann mit CT-Perfusionsuntersuchungen (CTP) indirekt quantifiziert werden. Basierend auf zum Aufnahmezeitpunkt einfach zu bestimmenden Eigenschaften sowie frühen CTP-Untersuchungen haben wir maschinelles Lernen eingesetzt um ein Modell zur Vorhersage eines schlechten funktionellen Outcomes zu trainieren.

Material und Methodik Von 614 konsekutiven SAB Patienten zwischen 2009-2015 wurden 351 eingeschlossen (aneurysmatische SAB, $\geq 1$ CTP innerhalb von $72 \mathrm{~h}$ nach Iktus und dokumentierte modified Rankin Scores (mRS) nach 6 Monaten). 67,4\% der Patienten waren weiblich. Das mittlere Alter betrug 53,9 +/-11,7 Jahre. Die Patienten wurde in einen Trainings- $(n=264)$ und einen Testdatensatz $(n=87)$ partitioniert. Aus den CTP Daten wurden der Maximal- und Mittelwert, deren Differenz und die Standardabweichung der bihemisphärischen mittleren Transitzeit (MTT) und des zerebralen Blutflusses (CBF) in das Modell aufgenommen. Zusätzlich wurden Alter, Geschlecht, der WFNS und der Fisher Grad integriert. Das Modell wurde mittels des Random Forest Algorithmus trainiert, um einen dichotomisierten mRS vorherzusagen $(\leq 2,>2)$. Die externe Validierung erfolgte anhand des Testdatensatzes.

Ergebnisse Das Modell sagt im Testdatensatz einen mRS $>2$ mit einer Spezifität von $81,8 \%$ und einer Sensitivität von $68,8 \%$ voraus. Die Fläche unter der ROC Kurve beträgt 0,82. Die Standardabweichung der MTT, WFNS und Alter tragen am stärksten zur Vorhersage bei.

Schlussfolgerungen Das funktionelle Outcome nach aneurysmatischer SAB kann mittels Random Forests gut anhand von CTP und klinischen Daten vor- 
hergesagt werden. Ein neue Erkenntnis ist die Relevanz der Standardabweichung der MTT.

\section{WISS 404.6 Der Kollateralstatus beim akuten Schlaganfall: Die Rolle des Capillary Index Score (CIS) in Korrelation zu Parametern der Perfusions-CT}

Autoren Schaudinn $A^{1}$, Friedrich $B^{2}$, Raviolo $\mathrm{M}^{3}$, Fritzsch $\mathrm{D}^{4}$, Quäschling $\mathrm{U}^{3}$, Hoffmann $\mathrm{K}^{3}$, Lobsien $\mathrm{D}^{5}$, Gawlitza $\mathrm{M}^{6}$

Institut 1 Universitätsklinikum Leipzig, Klinik für Diagnostische und Interventionelle Radiologie, Leipzig; 2 Klinikum rechts der Isar, Technische Universität München, Abteilung für Diagnostische und Interventionelle Neuroradiologie, München; 3 Universitätsklinikum Leipzig, Abteilung für Neuroradiologie, Leipzig; 4 Diakonissenkrankenhaus Leipzig, Abteilung für Radiologie, Leipzig; 5 Abteilung für Neuroradiologie, Klinik für Diagnostische und Interventionelle Radiologie, Leipzig; 6 Hôpital Maison Blanche, Centre Hospitalier Universitaire Reims, Service de Neuroradiologie, Reims DOI 10.1055/s-0037-1600406

Zielsetzung Der Capillary Index Score (CIS) gilt als angiografischer Marker zur Vorhersage des Patienten-Outcomes vor Thrombektomie. Ziel dieser Studie war es, die Beziehung zwischen CIS und Parametern der CT-Perfusion sowie des Rekanalisationsergebnisses zu überprüfen.

Material und Methodik 45 Patienten (25 weiblich, mittleres Alter $69(18-85)$ Jahre) mit akutem ischämischen Schlaganfall bei CT-angiografischem Verschluss der Arteria carotis interna $(n=11)$ bzw. Arteria cerebri media $(n=34)$ und nachfolgender mechanischer Rekanalisation mittels Stent-retriever, wurden retrospektiv analysiert. Auf Parameterkarten des Cerebral Blood Volume (CBV), Cerebral Blood Flow (CBF), Mean Transit Time (MTT) und Time to Peak (TTP) der präinterventionellen Perfusions-CTs wurden die Läsionsvolumina quantitativ und Perfusions-Alberta Stroke Programm Early CT-Scores (pASPECTS) semiquantitativ bestimmt. CIS wurde auf der initialen Übersichtsangiografie von zwei verblindeten Readern im Konsens bestimmt, der Thrombolysis In Cerebral Infarction (TICl)-Grad nach Thrombektomie auf der Abschluss-Übersichtsangiografie.

Ergebnisse Folgende CIS wurden ermittelt: CIS $0(n=13)$, CIS $1(n=12)$, CIS 2 $(n=10)$, CIS $3(n=10)$. Das CBV/MTT Mismatch lag im Median bei $74+/-22 \%$. CIS korrelierte signifikant mit CBF-pASPECTS $(\rho=0,66 ; P<0,0001)$ sowie MTTbzw. TTP-pASPECTS ( $\rho=0,47 ; P=0,001$ bzw. $\rho=0,42 ; P=0,004)$. Zudem korrelierten die Läsionsvolumina von CBF und TTP invers mit CIS $(\rho=-0,47$; $P=0,001$ und $\rho=-0,3 ; P=0,042$ ). Keine signifikante Korrelation mit CIS fand sich für CBV-, MTT-Läsionsvolumina und CBV-pASPECTS bzw. Mismatch-Volumina. Auch $\mathrm{TICl}$ und CIS waren nicht assoziiert. TICI $2 \mathrm{~b}-3$ wurde in $73 \%$ der Fälle erreicht.

Schlussfolgerungen CIS korreliert signifikant mit CBF-, TTP- und MTT-Paramatern, wobei sich insbesondere CBF-pASPECTS als robuster Prädiktor des CIS darstellt. Aussagen zur Größe des Infarktkerns und zur ischämischen Penumbra lassen sich anhand des CIS aber nicht treffen, weshalb er eine Perfusionbildgebung aus Sicht der Autoren nicht ersetzen kann.

\section{WISS 205.4 Limbische Enzephalitis, Fallstrick der MRT-Bildgebung}

Autoren Schievelkamp $A^{1}$, Jurcoane $A^{1}$, Mädler $B^{1}$, Rüber $T^{2}$, Ernst $L^{2}$, Elger $C^{2}$, Schild $\mathrm{H}^{1}$, Hattingen $\mathrm{E}^{1}$

Institut 1 Universitätsklinikum Bonn, Radiologische Klinik, Bonn;

2 Universitätsklinikum Bonn, Epileptologie, Bonn

DOI 10.1055/s-0037-1600407

Zielsetzung Ein MR-Kriterium der limbischen Enzephalitis (LE) ist das hyperintense T2-Signal der Amygdala, welches oft asymmetrisch erscheint. Diese Veränderungen sind visuell oft nur diskret erkennbar oder können ganz fehlen. Wir haben untersucht, ob diese Signalveränderungen bei LE messbar sind und ob eine objektivierbare Veränderung der T2-Relaxationszeit (qT2) zugrunde liegt.
Material und Methodik Gemessen wurden 24 Patienten mit der klinischen Diagnose einer LE und 21 altersadaptierte gesunde Kontrollpersonen (Ko) (3TScanner). Quantitative T2-Karten wurden aus temporal angulierten koronaren Sequenzen, mit steigenden Echozeiten von 15, 30, 45, 60, 75, 105, $120 \mathrm{~ms}$ und $2 \mathrm{~mm}$ Schichtdicke, errechnet. In beiden Amygdalae wurden die Signalintensitäten $(\mathrm{SI})$ der $\mathrm{T} 2$-gewichteten $(\mathrm{TE}=105)$ und der PD- gewichteten $(T E=15)$ Bilder sowie die qT2, anhand manuell platzierter ROls, bestimmt. Über einen Lateralitätsindex haben wir ermittelt, ob die Werte in der Amygdala asymmetrisch waren.

Ergebnisse Bei LE-Patienten und bei den Kontrollen ließen sich keine signifikanten Seitenunterschiede nachweisen. Bei LE war die qT2 signifikant höher (LE: li $97 \pm 8 \mathrm{~ms}$ (Mittelwert \pm SD), re $98 \pm 9 \mathrm{~ms}$ vs. Ko: li $90 \pm 5 \mathrm{~ms}$, re 89 $\pm 6 \mathrm{~ms} ; \mathrm{p}<0,01)$. Das PD-SI (TE = $15 \mathrm{~ms}$ ) war bei Vorliegen einer $\mathrm{LE}$ tendenziell niedriger als bei den Kontrollen. Die T2-SI (TE $=105 \mathrm{~ms})$ zeigte keine signifikanten Unterschiede.

Schlussfolgerungen Bei LE ist die qT2 erhöht, während das PD-SI sinkt. Beide, sich gegenläufig verhaltenden Größen, beeinflussen das T2-SI. Das erklärt, warum trotz pathologischer Veränderungen der Amygdala oft zwischen LEPatienten und Gesunden keine visuellen Signalunterschiede in T2-gewichteten Bildern zu sehen sind, zumal auch keine intraindividuelle Seitenasymmetrie besteht.

\section{WISS 205.5 Einfluss der AQP4 Expression auf DWI Parameter in Meningeomen}

Autoren Schob $S^{1}$, Surov $A^{2}$, Hoffmann $\mathrm{K}^{3}$, Wienke $A^{4}$, Meyer $\mathrm{H}^{5}$, Fiedler $\mathrm{E}^{6}$, Spielmann $\mathrm{R}^{5}$

Institut 1 Uniklinik Leipzig, Klinik und Poliklinik für diagnostische und interventionelle Radiologie, Abt. für Neuroradiologie, Leipzig; 2 Uniklinik Leipzig, Diagnostische und Interventionelle Radiologie, Leipzig; 3 Uniklinik Leipzig, Abteilung für Neuroradiologie, Leipzig; 4 Universität HalleWittenberg, Institut für Biometrie und Statistik, Halle a.d. Saale; 5 Uniklinik Halle-Wittenberg, Radiologie, Halle an der Saale; 6 Uniklinik Halle-Wittenberg, Institut für Dermatologie und Dermatopathologie

DOI 10.1055/s-0037-1600408

Zielsetzung Diffusionsbildgebung (DWI) quantifiziert die Bewegung von Wassermolekülen in biologischen Geweben. Zellulärer Wassertransport beruht u. a. auf Proteinen, die als Wasserkanäle fungieren (Aquaporine). Ziel der Arbeit war es, den Zusammenhang zwischen Aquaporin4 (AQP4) Expression und verschiedenen DWI Parametern in Grad I Meningeomen zu untersuchen.

Material und Methodik 23 Patienten mit Grad I Meningeomen wurden in die Studie eingeschlossen. DWI wurde mittels 3 b-Werten $(0,500,1000)$ bei $1,5 T$ durchgeführt. AQP4 Expression wurde immunhistochemisch evaluiert. Der Zusammenhang zwischen DWI-Parametern und AQP4 Expression wurde mittels Spearman-Rho Berechnung ermittelt.

Ergebnisse Die ermittelten Werte der DWI Parameter (Mittelwert \pm Standardabweichung, in $10-3 \mathrm{~mm} 2 \mathrm{~s}-1$ ) der untersuchten Meningeome waren wie folgt: ADCmin $0.67 \pm 0.16$, ADCmean $0.94 \pm 0.23, A D C m a x 1.29 \pm 0.50$, und $D$ $0.65 \pm 0.23$. Die durchschnittliche AQP4 Expression der Meningeome zeigte $2.02 \pm 0.75$ Punkte. AQP4 Expression und ADCmax zeigten einen statistisch signifikanten Zusammenhang $(r=0.508, p=0.013)$.

Schlussfolgerungen Ein klarer Zusammenhang zwischen AQP4 Expression und ADCmax in Grad I Meningeomen konnte in unserer Studie nachgewiesen werden. 


\section{WISS 317.3 Diagnostic accuracy for detection of intracranial hemorrhage using automatic tube current modulation and advanced modeled iterative reconstruction in unenhanced head single-energy and dual-energy CT}

\author{
Authors Scholtz $\mathrm{J}^{1}$, Wichmann $\mathrm{J}^{2}$, Bennett $\mathrm{D}^{2}$, Leithner $\mathrm{D}^{2}$, Albrecht $\mathrm{M}^{2}$, \\ Bucher $A^{2}$, Bauer $R^{3}$, Vogl $T^{2}$, Bodelle $B^{2}$ \\ Institute 1 Massachusetts General Hospital, Division of Cardiovascular \\ Imaging, Boston; 2 Universitätsklinikum Frankfurt, Institut für Diagnostische \\ und Interventionelle Radiologie, Frankfurt/Main; 3 Kantonspital St. Gallen, \\ Klinik für Radiologie und Nuklearmedizin, St. Gallen/Schweiz \\ DOI 10.1055/s-0037-1600409
}

Zielsetzung To determine diagnostic accuracy for detection of intracranial hemorrhage $(\mathrm{ICH})$, image quality, and radiation dose of low-dose single-energy (SE) and dual-energy (DE) unenhanced head computed tomografy (CT) on third-generation dual-source CT.

Material und Methodik 123 patients with suspected ICH were examined on a 192-slice dual-source CT. Standard-dose SE $(120-k V$,Group $A, n=36)$ and DE $(80-/ S n 150-k V p, G r o u p \quad B, n=30)$ images were compared with low-dose SE (Group $\mathrm{C}, \mathrm{n}=32$ ) and DE (Group $\mathrm{D}, \mathrm{n}=25$ ) using automated-tube-currentmodulation (ATCM). Advanced-modeled-iterative-reconstruction (ADMIRE) was used for all protocols. Detection of ICH was performed by three blinded readers. Quantitative and qualitative image quality was assessed. Interobserver agreement was calculated using Fleiss' Kappa. Radiation dose was assessed as dose-length product (DLP).

Ergebnisse Detection of ICH was excellent (sensitivity,94.9-100\%; specificity,94.7 -100\%) in all protocols $(p=1.00)$ with perfect interobserver agreement $(0.83-0.96)$. Qualitative ratings showed significantly better ratings for both standard-dose protocols regarding gray-matter-to-white-matter (GMWM) contrast $(p \leq 0.014)$, whereas highest GM-WM CNR was observed in low-dose DE-CT (all, $\mathrm{p} \geq 0.057$ ). Lowest posterior-fossa-artifact-index was measured for standard-dose DE-CT with significantly lower values compared to low-dose protocols (both $\mathrm{p} \leq 0.034$ ). Delineation of ventricular margins and subarachnoidal spaces sharpness were rated excellent in all protocols $(p \geq 0.096)$. Low-dose SE lowered radiation dose by $26 \%$ (DLP, 575.0 $\pm 72.3 \mathrm{mGy}^{*} \mathrm{~cm}$ vs. $\left.771.4 \pm 146.8 \mathrm{mGy}^{*} \mathrm{~cm}, \mathrm{p}<0.001\right)$ and by $24 \%$ in DE (DLP, $587.0 \pm 103.2 \mathrm{mGy}^{*} \mathrm{~cm}$ vs. $\left.770.6 \pm 90.2 \mathrm{mGy}^{*} \mathrm{~cm}, \mathrm{p}<0.001\right)$. No significant differences were observed between low-dose protocols $(p=1.00)$.

Schlussfolgerungen Low-dose unenhanced head SE and DE-CT using ATCM and ADMIRE provide excellent diagnostic accuracy for detection of ICH with good quantitative and qualitative image quality in third-generation dualsource CT while allowing for significant radiation dose reduction.

\section{WISS 404.10 Postinterventionelle Verschlüsse neuer doppelschichtiger Karotisstents bei der endovaskulären Behandlung akuter Tandemokklusionen}

Autoren Yilmaz $U^{1}$, Körner $H^{2}$, Mühl-Benninghaus $\mathrm{R}^{2}$, Simgen $\mathrm{A}^{2}$, Kraus $C^{2}$, Walter $\mathrm{S}^{3}$, Behnke $\mathrm{S}^{3}$, Fassbender $\mathrm{K}^{3}$, Reith $\mathrm{W}^{2}$, Unger $\mathrm{M}^{3}$

Institut 1 Universitätsklinikum des Saarlandes, Klinik für Diagnostische und Interventionelle Neuroradiologie, Homburg; 2 Universitätsklinikum des

Saarlandes, Klinik für Neuroradiologie, Homburg; 3 Universitätsklinikum des Saarlandes, Klinik für Neurologie, Homburg

DOI 10.1055/s-0037-1600410

Zielsetzung In der Vergangenheit wurde nachgewiesen, dass "closed-cell"Stents mit kleiner Zellgröße eine geringere Rate embolischer Komplikationen bei der Behandlung von Karotisstenosen aufweisen. Kürzlich wurde eine neue Generation von Karotisstents eingeführt, die die Zellgröße durch ein zusätzliches Netz als zweite Schicht weiter verkleinert. Erste prospektive Studien zeigten gute Ergebnisse bei der Benutzung dieser neuartigen Stents. Über den Nutzen der neuen Stents bei der endovaskulären Behandlung akuter Tandemokklusionen ist dagegen noch nichts bekannt. Ziel dieser retrospektiven Studie war es die Raten akuter postinterventioneller Stentverschlüsse der neuartigen Stents und der klassischen Karotisstents zu vergleichen.

Material und Methodik Aus unserem Register neuroendovaskulärer Interventionen wurden alle Patienten identifiziert, die aufgrund akuter Tandemokklusionen seit 2011 in unserer Klinik mittels mechanischer Thrombektomie und Stentimplantation behandelt wurden. Klinische und angiografische Daten wurden analysiert. Endpunkte waren das Auftreten akuter Stentverschlüsse und symptomatischer intrazerebraler Blutungen.

Ergebnisse Die Daten von 47 Patienten wurden retrospektiv eingeschlossen. 20 wurden mit neuartigen und 27 mit klassischen Karotisstents behandelt. Die neuartigen Stents hatten eine signifikant höhere Rate akuter postinterventioneller Stentverschlüsse ( $45 \%$ vs. 3,7\%, P=.001, OR 21.3, 95\% Cl $2.4-188.4$ ). Es gab keinen signifikanten Unterschied der Raten symptomatischer intrazerebraler Blutungen.

Schlussfolgerungen Die kürzlich eingeführten doppelschichtigen Karotisstents haben bei der Behandlung akuter Tandemokklusionen ein signifikant höheres Risiko für das Auftreten akuter Stentverschlüsse. In der Notfallbehandlung sollten daher klassische Karotisstents eingesetzt werden.

Notfalldiagnostik/Intensivmedizin

\section{WISS 403.2 Polytrauma CT Diagnostik: Signifikante Reduktion des Zeitbedarfs durch optimierte Lagerung bei erhaltener Bildqualität}

Autoren Hickethier $\mathrm{T}^{1}$, Mammadov $\mathrm{K}^{1}$, Baeßler $\mathrm{B}^{1}$, Maintz $\mathrm{D}^{1}$, Chang $\mathrm{D}^{1}$ Institut 1 Uniklinik Köln, Institut für Diagnostische und Interventionelle Radiologie, Köln

DOI 10.1055/s-0037-1601385

Zielsetzung Über die ideale Durchführung der CT-Bildgebung bei Polytraumata besteht weiterhin kein eindeutiger Konsens. Ziel dieser Studie war es, Zeitbedarf und Artefaktanfälligkeit der CT mit konventioneller und optimierter Patientenlagerung, wobei letztere die durchgehende Erfassung aller Untersuchungsregionen ermöglicht, zu vergleichen.

Material und Methodik Bei 200 Polytraumata wurde der Zeitbedarf der CT (bei 84 zusätzlich mit Kopf-Hals-Angiografie (hnCTA)) erfasst und die Artefakte in relevanten Regionen (Leber, Milz, Aorta, Lunge, Skelett) von 2 Ärzten auf einer 4-Punkte-Skala (von $1=$ keine Artefakte bis $4=$ diagnostisch relevante Artefakte) beurteilt. Bei 100 Patienten (inkl. 41 hnCTAs) wurden die Arme für die Bildgebung von Kopf und Hals neben dem Körper und anschließend für die Bildgebung des Körperstammes über den Kopf gelagert (Gruppe A). Bei den anderen 100 Patienten (inkl. 43 hnCTAs) wurden die Arme mit einem speziellen Kissen über dem Thorax verschränkt und in dieser Position alle Untersuchungsregionen akquiriert (Gruppe B).

Ergebnisse Der Zeitbedarf war mit hnCTA („B“ 6:55 \pm 1:54 min vs. „A“ 10:54 $\pm 2: 04 \mathrm{~min} ; \mathrm{p}<0.001)$ und ohne hnCTA (6:46 $\pm 1: 49$ min vs. 10:19 $\pm 2: 42 \mathrm{~min}$; $\mathrm{p}<0.001$ ) in Gruppe B signifikant geringer als in Gruppe A. In Gruppe B kam es häufiger zu Artefakten von Aorta, Leber und Milz (Mittelwert Aorta 1,27 $\pm 4,46$ vs. $1,07 \pm 2,93$; Leber $1,43 \pm 5,55$ vs. $1,09 \pm 3,21$; Milz $1,20 \pm 4,49$ vs. $1,05 \pm 2,19$; jeweils $p<0.001$ ). In Lunge und Skelett kam es nicht signifikant häufiger zu Artefakten und es kam insgesamt zu keiner nicht-diagnostischen Untersuchung. Random Forest- und logistische Regressions-Analysen zeigten, dass die Artefaktzunahme zu einem relevanten Anteil durch beeinflussbare Faktoren (Fremdmaterial/Lagerungsfehler) bedingt war.

Schlussfolgerungen Durch eine optimierte Lagerung ist bei Polytraumata die Erfassung aller Untersuchungsregionen ohne Umlagerung der Arme möglich, was eine signifikante Verkürzung der Untersuchungsdauer ohne relevante Verschlechterung der Bildqualität und somit eine schnellere Diagnose erlaubt. 


\section{WISS 403.3 Ist die inflationäre Entwicklung der Notfall-CT der Halswirbelsäule gerechtfertigt?}

Autoren Maiwald $\mathrm{B}^{1}$, Petersen $\mathrm{T}^{2}$, Kahn $\mathrm{T}^{1}$, Josten $\mathrm{C}^{3}$, Fakler $]^{3}$, Hoffmann $\mathrm{K}^{4}$, Stumpp $\mathrm{P}^{1}$

Institut 1 Universitätsklinikum Leipzig, Klinik und Poliklinik für Diagnostische und Interventionelle Radiologie, Leipzig; 2 Universitätsklinikum Leipzig, Klinik und Poliklinik für Diagnostische und Interventionelle Radiologie, Leipzig; 3 Universitätsklinikum Leipzig, Klinik für Orthopädie, Unfallchirurgie und Plastische Chirurgie, Leipzig; 4 Universitätsklinikum Leipzig, Abteilung für Neuroradiologie, Leipzig DOI 10.1055/s-0037-1601386

Zielsetzung Der Einsatz der Computertomografie (CT) zur Diagnostik von Verletzungen an der Halswirbelsäule (HWS) hat in den letzten Jahren deutlich zugenommen. Bei traumatologischen Fragestellungen wird diese häufig in Ergänzung zur craniellen CT (cCT) eingefordert. In der Routine geschieht dies auch bei jungen Patienten $(<60 \mathrm{~J}$.) und bei Symptomfreiheit. Ziel unserer Studie war es die Inzidenz der HWS-Frakturen zu evaluieren, um mögliche Empfehlungen bzgl. der kombinierten Bildgebung (cCT/CT-HWS) ableiten zu können.

Material und Methodik Retrospektive Auswertung von 545 CT-HWS unter traumatischer Fragestellung zw. Juni und September 2016. Erfasst wurden Alter, Geschlecht und Nachweis einer traumatischen Verletzung craniell oder zervikal.

Ergebnisse Von den insgesamt 545 durchgeführten CT-HWS entstanden 124 CT in einer Polytraumaspirale und 421 unter kombinierter Fragestellung mit cCT. 56,9\% der Patienten waren weiblich $(n=310)$. Das mittlere Alter betrug $69,9 \pm 19$ Jahre. In 72 aus 545 untersuchten Fällen zeigte sich eine intrakranielle Blutung (ICB) und bei 24 Patienten lag eine Schädelfraktur vor, wobei diese in 5 Fällen ohne ICB einherging. Aus dem Gesamtkollektiv ergab sich eine HWS-Fraktur bei ca. 2,4\% ( $n=13)$, wobei diese lediglich in einem Fall mit einer ICB kombiniert war. 4 Fälle der HWS-Frakturen wurden im Rahmen eines schweren Traumas (Polytrauma) dokumentiert. 2 Frakturen der HWS waren symptomlos, wobei es sich zum einen um eine Densfraktur Anderson und D‘Alonzo II, die als möglicherweise älter eingeschätzt wurde, und zum anderen um eine Querfraktur des HWK 7 und Abrissfraktur der Procc. spinosi HWK 2 und 4 handelte. Beide Patienten wurden konservativ therapiert. Das mittlere Alter der Patienten mit HWS-Fraktur betrug ca. 76,9 \pm 13,5 Jahre.

Schlussfolgerungen Die Inzidenz einer kombinierten craniocervikalen Verletzung war im Untersuchungskollektiv gering, trotz Einschluss auch von schweren Traumamechanismen (Polytraumata). Eine Empfehlung zur Standardkombination $\mathrm{CCT} / \mathrm{CT}$-HWS erscheint daher nicht generell indiziert

\section{WISS 403.5 Reduzierte z-Achsen Thorax- Computertomografie zur Diagnostik einer Lungenarterienembolie}

Autoren Maurer $\mathrm{M}^{1}$, Zinsser $\mathrm{D}^{2}$, Do $\mathrm{P}^{1}$, Weiß $\mathrm{J}^{1}$, Notohamiprodjo $\mathrm{M}^{1}$, Nikolaou $\mathrm{K}^{1}$, Bamberg $\mathrm{F}^{1}$, Othman $\mathrm{A}^{1}$

Institut 1 Uniklinik Tübingen, Diagnostische und Interventionelle Radiologie, Tübingen; 2 Universitätsklinikum Tübingen, Diagnostische und

Interventionelle Radiologie, Tübingen

DOI 10.1055/s-0037-1601387

Zielsetzung In dieser Studie sollen die diagnostische Genauigkeit und die benötigte effektive Dosis zur Diagnosestellung einer Lungenarterienembolie bei einer Computertomografie (CT) mit nachträglich reduziertem Scanbereich in der z-Achse mit der einer ursprünglichen CT-Untersuchung verglichen werden.

Material und Methodik 150 Patienten die eine Thorax-CT bei der Verdachtsdiagnose LAE erhalten haben wurden in die Studie eingeschlossen. Die vorhandenen Untersuchungen wurden mit einer reduzierten horizontalen Scanabdeckung von oberhalb des Aortenbogen bis unterhalb der Herzbasis rekonstruiert. Anschließend wurde der ursprüngliche Datensatz mit dem in der z-Achse reduzierten Datensatz in Hinblick auf die Verdachtsdiagnose einer LAE, relevante Nebendiagnosen und Zufallsbefunde verglichen. Mit einem Dosistrackingsystem wurden die effektive Dosis und die einzelnen Organdosen für beide Datensätze errechnet.

Ergebnisse 150 Patienten (w: 58; m: 92) wurden im Rahmen der vorliegenden Arbeit untersucht und statistisch ausgewertet. Es konnte gezeigt werden, dass sowohl bei reduziertem als auch bei unverändertem Scanbereich eine korrekte Diagnosefindung einer LAE (59 von 150) erfolgte. Alle in den ursprünglichen CT-Untersuchungen beschriebenen Nebenbefunde konnten auch im reduzierten Datensatz nachgewiesen werden. Zudem konnte bei den reduzierten Datensätzen, im Vergleich mit den ursprünglichen Untersuchungen eine Senkung der effektiven Dosis von 54,5\% gezeigt werden (Reduzierter Scanbereich: 1,4 $\pm 1,05$ vs. vollständiger Scanbereich: 2,57 $\pm 1.98 \mathrm{mSv} ; \mathrm{p} \leq 0.001)$. Hierbei zeigte sich der größte Dosisunterschied bei den oberen Bauchorganen: Gallenblase $89 \%$ (0,32 $\pm 0,02$ vs. $2.95 \pm 0,23 \mathrm{mSv}$; $p=0,001)$, Nieren $93 \%(0,32 \pm 0,03$ vs. $4,10 \pm 0,28 \mathrm{mSv} ; \mathrm{p}=0,001)$, Pankreas $76 \%(0,90 \pm 0,06$ vs. $3,79 \pm 0,26 \mathrm{mSv} ; \mathrm{p}=0,001)$ und Milz $80 \%(0,91 \pm 0,07$ vs. 4,64 $\pm 0,30 \mathrm{mSv} ; \mathrm{p}=0,001)$.

Schlussfolgerungen Computertomografische Untersuchungen mit reduziertem Scanbereich in der z-Achse zeigen eine signifikant reduzierte effektive Dosis bei gleicher diagnostischer Genauigkeit.

\section{WISS 403.4 Die Carotisdissketion beim polytraumatisierten Patienten}

Autoren Schleder $S^{1}$, Meier $R^{1}$, Dendl $L^{1}$, Stroszczynski $C^{1}$, Schreyer $A^{1}$ Institut 1 Universitätsklinikum Regensburg, Institut für Röntgendiagnostik, Regensburg DOI 10.1055/s-0037-1601388

Zielsetzung Ziel der Studie war es, die Häufigkeit und die klinische Relevanz von Dissektionen der Arteria carotis interna beim polytraumatisierten Patienten zu evaluieren, sowie deren Begleitverletzungen und den zugrundeliegenden Unfallmechanismus zu analysieren.

Material und Methodik Hierzu wurden über einen Zeitraum von 2 Jahren retrospektiv über das RIS (radiologisches Informationssystem) einer Klinik der Maximalversorgung alle Patienten identifiziert, die bei einem ISS (injury severity score) $\geq 16$ einer Computertomografie-Ganzkörperdiagnostik über den Schockraum zugewiesen wurden. Dieses Patientenkollektiv wurde anhand des Arztbriefes und des radiologischen Befundes detailliert auf das Verletzungsausmaß, den Unfallmechanismus, das Vorliegen einer Carotisdissektion sowie auf das klinische Outcome analysiert.

Ergebnisse Es wurden 12 Patienten (42\% weiblich, mittleres Alter 53, durchschnittlischer ISS 35) mit einer Dissektion der Karotisstrombahn identifiziert (58\% linksseitig, 42\% rechtsseitig). Bei zwei Dritteln (8 Patienten, 67\%) hiervon bestand begleitend ein höhergradiges Schädelhirntrauma und bei ebenso 8 Patienten (67\%) bestand gleichzeitig eine knöcherne Traumafolge an der Hals- oder oberen Brustwirbelsäule. Zudem erlitten 3 Patienten (25\%) eine traumatisch gedeckte Aortenruptur. Bei keinem Patienten lag bereits initial eine zerebrale Ischämie vor, stellte sich aber bei 2 Patienten (17\%) im Verlauf ein. Mit insgesamt 10 Fällen (83\%) war das Hochrasanztrauma im Strassenverkehr (8 mal mittels Personenkraftwagen, 67\% und 2 mal mittels Motorrad, $17 \%)$ der dominierende Unfallmechanismus.

Schlussfolgerungen Die Dissektion der Arteria carotis interna beim polytraumatisierten Patienten stellt ein schweres Krankheitsbild dar, das nur durch eine zielgerichtete radiologische Diagnostik erkannt werden kann. Sie tritt besonders bei schweren Traumen begleitend auf und stellt zudem eine Herausforderung an die intensivmedizinsche Therapie dar. 


\section{WISS 403.1 Routinemäßige \\ Ultraschallverlaufskontrollen bei schwer verletzten Patienten nach unauffälligem Ganzkörper-CT sind Zeitverschwendung}

\section{Autoren Schüer $\mathrm{M}^{1}$, Kahn $\mathrm{T}^{1}$, Josten $\mathrm{C}^{2}$, Fakler $\mathrm{J}^{2}$, Stumpp $\mathrm{P}^{1}$ Institut 1 Universitätsklinikum Leipzig, Klinik und Poliklinik für Diagnostische und Interventionelle Radiologie, Leipzig; 2 Universitätsklinikum Leipzig, Klinik für Orthopädie, Unfallchirurgie und Plastische Chirurgie, Leipzig DOI 10.1055/s-0037-1601389 \\ Zielsetzung Ziel der retrospektiven Studie war es, die diagnostische Signifi- kanz und klinische Relevanz von routinemäßig durchgeführten Ultra- schallverlaufskontrollen bei schwer verletzten Patienten nach vorangegange- nem Ganzkörper-CT zu untersuchen. \\ Material und Methodik In einem Zeitraum von 48 Monaten wurden 611 konsekutive, schwer verletzte Patienten ausgewählt, welche initial ein Ganz- körper-CT und sechs Stunden danach eine Ultraschallverlaufskontrolle erhalten hatten. Die Patienten wurden in zwei Kohorten aufgeteilt: Patienten mit $(A)$ und ohne (B) traumaassoziierte abdominelle Verletzungen im initialen CT. Beide Kohorten wurden dann weiter untersucht auf das Vorliegen von Befun- den in der Ultraschallverlaufskontrolle, die Unterschiede der Befunde zum CT und eine Änderung des therapeutischen Vorgehens. \\ Ergebnisse 150 Patienten wurden in Kohorte A und 461 in Kohorte B kate- gorisiert. Nur zwei Patienten (1.3\%) in Kohorte A zeigten neue Befunde im Ultraschall und einer dieser Patienten benötigte eine klinische Intervention. In Kohorte B zeigten 6 Patienten (1.3\%) minimale neue Befunde in Form von kleinen Mengen freier Flüssigkeit, von diesen benötigte keiner eine Interven- tion. Insgesamt wurde also das therapeutische Vorgehen nur bei einem von 611 Patienten (0.16\%) aufgrund des Ultraschallbefundes geändert. \\ Schlussfolgerungen Da es bei Patienten mit unauffälligem Ganzkörper-CT keine Befunde gab, die eine klinische Intervention nötig machten, profitiert diese Gruppe von Patienten nicht von routinemäßig durchgeführten Ultra- schallverlaufskontrollen. \\ WISS 403.6 Presepsin und Entzündungswerte korrelieren mit dem Auftreten und dem Schweregrad der Nicht-Okklusiven-Mesenterialen Ischämie (NOMI) nach kardiovaskulären Operationen}

Autoren Ströder J ${ }^{1}$, Bomberg $H^{2}$, Wagenpfeil $S^{3}$, Bücker $A^{1}$, Schäfers $H^{4}$, Katoh $\mathrm{M}^{5}$, Groesdonk $\mathrm{H}^{6}$, Minko $\mathrm{P}^{1}$

Institut 1 Universitätsklinikum des Saarlandes und Medizinische Fakultät der Universität des Saarlandes, Klinik für Diagnostische und Interventionelle Radiologie, Homburg; 2 Universitätsklinikum des Saarlandes und Medizinische Fakultät der Universität des Saarlandes, Klinik für Anästhesiologie,

Intensivmedizin und Schmerztherapie, Homburg; 3 Universitätsklinikum des

Saarlandes und Medizinische Fakultät der Universität des Saarlandes, Institut für Medizinische Biometrie, Epidemiologie und Medizinische Informatik, Homburg; 4 Universitätsklinikum des Saarlandes und Medizinische Fakultät der Universität des Saarlandes, Thorax- und Herz-Gefäß-Chirurgie, Homburg; 5 Helios Klinikum Krefeld, Klinik für Diagnostische und Interventionelle Radiologie, Krefeld; 6 Universitätsklinikum des Saarlandes und Medizinische Fakultät der Universität des Saarlandes, Klinik für Anästhesiologie,

Intensivmedizin und Schmerztherapie,

DOI 10.1055/s-0037-1601390

Zielsetzung Prospektive Evaluierung des Zusammenhangs zwischen Presepsin und anderen Entzündungswerten mit dem Auftreten und dem Schweregrad der NOMI.

Material und Methodik Zwischen 2/2011 und 3/2012 wurden 1272 Patienten, die eine kardiovaskuläre Operation erhielten in diese prospektive Studie eingeschlossen. Patienten, die klinisch unter dem Verdacht einer NOMI standen, erhielten eine Katheterangiografie der A. mesenterica superior. Die an- gefertigten Aufnahmen wurden auf Konsensusbasis von zwei erfahrenen Radiologen bewertet, wobei ein standardisiertes Punktesystem verwendet wurde (Homburger-NOMI-Score, bestehend aus drei Kategorien: Gefäßmorphologie, Kontrastmittelreflux in die Aorta und Zeit bis zur Kontrastierung der Pfortader). Hierdurch ist es zudem auch möglich die NOMI in eine milde und schwere Form einzuteilen. Die erhobenen Daten der Angiografie wurden sowohl mit den prä- und postoperativ erhobenen Entzündungswerten korreliert: C-reaktives Protein (CRP), Leukozytenzahl, Procalcitonin (PCT) und Presepsin als auch mit prognostischen Daten (Tod, akutes Nierenversagen) mittels logistischer Regression und ROC-Analyse korreliert.

Ergebnisse Milde NOMI trat bei 4,5\% und schwere NOMI bei 3,2\% der Patienten auf. Mediane postoperative Presepsinwerte waren signifikant höher in diesen Gruppen (milde NOMI: 793 pg/ml [interquartile range [IQR] 411 1048], schwere NOMI: 1485 pg/ml [IQR: 709-2142]) als bei den nicht-NOMI-Patienten (331 pg/ml [IQR: 242 - 489], p<0,001). Signifikante Korrelationen konnten auch zwischen prä- und postoperativem CRP und dem NOMIScore gezeigt werden $(p<0,001 / p=0,001)$. Weitere Auswertungen zeigten, dass postoperative Presepsinwerte besser zwischen milder (AUC 0.80) und schwerer NOMI (AUC 0.91) unterscheiden konnten als alle anderen getesteten Biomarker.

Schlussfolgerungen Erhöhte postoperative Presepsinwerte sind ein unabhängiger Prediktor für das Auftreten einer NOMI. Die etablierten Entzündungswerte und Presepsin korrelieren signifikant mit dem Auftreten und dem Schweregrad der NOMI.

\section{WISS 211.5 Presepsin und Entzündungswerte korrelieren mit dem Auftreten und dem Schweregrad der Nicht-Okklusiven-Mesenterialen Ischämie (NOMI) nach kardiovaskulären Operationen Bewerber für den Young Investigator Award}

Autoren Ströder $]^{1}$

Institut 1 Universitätsklinikum des Saarlandes und Medizinische Fakultät der Universität des Saarlandes, Klinik für Diagnostische und Interventionelle Radiologie, Homburg

DOI 10.1055/s-0037-1601391

Zielsetzung Prospektive Evaluierung des Zusammenhangs zwischen Presepsin und anderen Entzündungswerten mit dem Auftreten und dem Schweregrad der NOMI.

Material und Methodik Zwischen 2/2011 und 3/2012 wurden 1272 Patienten, die eine kardiovaskuläre Operation erhielten in diese prospektive Studie eingeschlossen. Patienten, die klinisch unter dem Verdacht einer NOMI standen, erhielten eine Katheterangiografie der A. mesenterica superior. Die angefertigten Aufnahmen wurden auf Konsensusbasis von zwei erfahrenen Radiologen bewertet, wobei ein standardisiertes Punktesystem verwendet wurde (Homburger-NOMI-Score, bestehend aus drei Kategorien: Gefäßmorphologie, Kontrastmittelreflux in die Aorta und Zeit bis zur Kontrastierung der Pfortader). Hierdurch ist es zudem auch möglich die NOMI in eine milde und schwere Form einzuteilen. Die erhobenen Daten der Angiografie wurden sowohl mit den prä- und postoperativ erhobenen Entzündungswerten korreliert: C-reaktives Protein (CRP), Leukozytenzahl, Procalcitonin (PCT) und Presepsin als auch mit prognostischen Daten (Tod, akutes Nierenversagen) mittels logistischer Regression und ROC-Analyse korreliert.

Ergebnisse Milde NOMI trat bei 4,5\% und schwere NOMI bei 3,2\% der Patienten auf. Mediane postoperative Presepsinwerte waren signifikant höher in diesen Gruppen (milde NOMI: 793 pg/ml [interquartile range [IQR] 411 1048], schwere NOMI: 1485 pg/ml [IQR: 709-2142]) als bei den nicht-NOMI-Patienten (331 pg/ml [IQR: 242 - 489], p <0,001). Signifikante Korrelationen konnten auch zwischen prä- und postoperativem CRP und dem NOMIScore gezeigt werden $(p<0,001 / p=0,001)$. Weitere Auswertungen zeigten, dass postoperative Presepsinwerte besser zwischen milder (AUC 0.80) und 
schwerer NOMI (AUC 0.91) unterscheiden konnten als alle anderen getesteten Biomarker.

Schlussfolgerungen Erhöhte postoperative Presepsinwerte sind ein unabhängiger Prediktor für das Auftreten einer NOMI. Die etablierten Entzündungswerte und Presepsin korrelieren signifikant mit dem Auftreten und dem Schweregrad der NOMI.

Onkologische Bildgebung/Onkologie

\section{WISS 104.2 Variabilität des standardisierten Therapy Response Assessment nach RECIST 1.1: Target lesion selection als Hauptursache?}

\author{
Autoren Alparslan $\mathrm{Y}^{1}$, Bruno $\mathrm{S}^{1}$, Jonas $\mathrm{S}^{1}$, Engelke $\mathrm{H}^{1}$, Annika $\mathrm{K}^{1}$, \\ Brümmendorf $\mathrm{T}^{2}$, Kuhl $\mathrm{C}^{1}$, Keil $\mathrm{S}^{1}$ \\ Institut 1 Uniklinik Aachen, Radiologie, Aachen; 2 Uniklinik Aachen, \\ Hämatologie, Onkologie, Stammzelltransplantation, Aachen \\ DOI 10.1055/s-0037-1600411
}

Zielsetzung Bewertungskriterien wie RECIST 1.1 und dedizierte onkologische Softwareprogramme (DOS) werden zur standardisierten Auswertung des Therapieansprechens verwendet. Hierbei sollten verschiedene Radiologen bei der Evaluierung desselben onkologischen Patienten zu gleichen Ergebnissen gelangen. Wir haben die Variabilität des Therapieansprechens nach RECIST 1.1 zwischen mehreren Radiologen bestimmt.

Material und Methodik 932 CTs wurden zwecks (Re-) Staging bei 316 Patienten mit metastasiertem Primärtumor durchgeführt. Diese Daten wurden prospektiv von drei Radiologen (Erfahrung $\geq 5$ a) unabhängig voneinander mithilfe eines DOS (MintLesion) ausgewertet. Entsprechende Zielläsionen (TL) wurden frei ausgewählt. Bestimmt wurden, wie häufig die Auswahl unterschiedlicher TL zu einer diskordanten Kategorisierung nach RECIST 1.1 führt. Um herauszufinden, wie häufig eine differente Kategorisierung zu einem Wechseldes Therapieschemas führt, wurde zudem die Variabilität nach Unterteilung in progressive (PD) vs. Nicht-progressive Disease (CR/PR/SD) bestimmt.

Ergebnisse Die Radiologen stimmten bei 40,8\% der Patienten (129/316) nach RECIST1.1 nicht überein. Bei 26,6\% (87/316) waren die Ergebnisse nach Unterteilung in PD vs. Nicht-PD diskordant. Dieselben TL wurden bei 40,5\% (128/316), verschiedene bei 59,5\% (188/316) ausgewählt. Bei 92,3\% der Patienten mit diskordanter Auswertung (119/129) haben die Auswerter unterschiedliche TL ausgewählt. Bei Wahl derselben TL führte dies in 97,7\% (125/187) der Fälle zu konkordanter Klassifizierung unterteilt nach PD vs. Non-PD, wohingegen die Wahl unterschiedlicher TL bei 44,7\% (84/188) der Patienten in einer diskordanten Kategorisierung mündete.

Schlussfolgerungen Trotz Verwendung standardisierter RECIST-1.1-Bewertungskriterien und DOS klassifizierten verschiedene Radiologen das Therapieansprechen nach PD vs. Nicht-PD bei beachtlichen $27.5 \%$ der Patienten unterschiedlich. Hierbei ist die individuelle Auswahl der TL Hauptursache für diese Diskordanz und kann nicht durch Verwendung von DOS ausgeglichen werden.

\section{WISS 104.6 3D imaging biomarkers for the prediction of survival in patients with non-small cell lung cancer brain metastases treated with stereotactic body radiation therapy}

\footnotetext{
Authors Della Seta $\mathrm{M}^{1}$, Kaul $\mathrm{D}^{2}$, Chapiro $\mathrm{J}^{3}$, Hamm B ${ }^{1}$, Collettini $\mathrm{F}^{1}$ Institute 1 Charité Universitätsmedizin Berlin, Radiologie, Berlin; 2 Charité Universitätsmedizin Berlin, Strahlentherapie, Berlin; 3 Yale School of Medicine, Radiologie, New Haven
}

DOI 10.1055/s-0037-1600412

Zielsetzung The aim of the present study was to investigate the value of 3D quantitative tissue enhancement as an early imaging biomarker for patient survival in patients with non-small cell lung cancer (NSCLC) brain metastases treated with stereotactic body radiation therapy (SBRT).

Material und Methodik This retrospective analysis included 27 patients with NSCLC brain metastasis who were treated with SBRT. Baseline contrast-enhanced MRI (ceMRI), acquired within a mean of 30 days before therapy, was used for image analysis using the qEASL tool (IntelliSpace Portal V.8, Philips Healthcare). A segmentation-based 3D quantification was performed in each patient to measure the relative tumor enhancement in each lesion. A cutoff value of $65 \%$ lesion enhancement was used to stratify the patient cohort in two groups ( $<65 \%$ and $>65 \%$ volumetric lesion enhancement). Survival was evaluated using Kaplan-Meier analysis and compared using the Mantel-Cox Log-rang test as well as proportional hazard ratios (HR).

Ergebnisse Median OS of the entire population was 6.0 months. The stratification of the cohort according to the $65 \%$ cutoff for the relative enhancing tumor volume achieved statistical significance $\mathrm{p}=0.0452(\mathrm{HR}, 2.2[95 \% \mathrm{Cl}$, $1.1-6.8]$ ). Patients with $>65 \%$ enhancing lesion volume survived significantly longer than patients with relatively less-enhancing tumor lesions (8.15 months vs. 2,09 months [HR 0.25; $95 \% \mathrm{Cl}, 0.11-0.58$ ], respectively). Stratification according to cutoffs for the total tumor volume did not achieve a statistically significant separation of survival curves.

Schlussfolgerungen As opposed to the total tumor volume which currently is one of the most commonly used staging marker, volumetric assessment of metastatic NSCLC lesion enhancement on baseline ceMRI is strongly associated with patient survival after SBRT. Specifically, patients with hyper-enhancing lesions demonstrated improved survival as compared to those with hypoenhancing lesions.

\section{WISS 402.5 Vergleich der MRT und PET/MRT für das prätherapeutische Tumorstaging von Patientinnen mit primärem Zervixkarzinom}

Autoren Grüneisen $]^{1}$, Wetter $A^{1}$, Al-bayati $M^{1}$, Sawicki $L^{2}$, Forsting $M^{1}$,

Ruhlmann $\mathrm{V}^{3}$, Umutlu $\mathrm{L}^{1}$

Institut 1 Uniklinik Essen, Radiologie, Essen; 2 Uniklinik Düsseldorf,

Radiologie, Düsseldorf; 3 Uniklinik Essen, Nuklearmedizin, Essen

DOI 10.1055/s-0037-1600413

Zielsetzung Ziel dieser Studie war der Vergleich und die Evaluation des diagnostischen Potentials der MRT und PET/MRT für das lokale Tumorstaging sowie für die Detektion von Lymphknoten und Fernmetastasen von Patientinnen mit primärem Zervixkarzinom.

Material und Methodik 47 Patientinnen mit histologisch gesichertem Zervixkarziom unterzogen sich einer Ganzkörper 18F-FDG PET/MRT Untersuchung (3T Biograf mMR, Siemens Healthcare) vor pelviner/paraaortaler Lymphadenektomie und vor Beginn der definitiven Therapie. Die Datenanalyse erfolgte durch einen Radiologen und Nuklearmediziner im Konsens. Es wurden zunächst die MRT-Daten allein und anschließend die PET/MRT-Datensätze analysiert. Die Datenanalyse beinhaltete die Beurteilung der lokalen Tumorausbreitung (T-Stadium) sowie die Detektion von Lymphknoten- und Fernmetastasen (N- und M-Stadium).

Ergebnisse Das T-Stadium wurde mittels der MRT in 41\% (87\%) Patientinnen und in der PET/MRT in 40\% (85\%) der 47 Patientinnen korrekt identifiziert. Für die Detektion von Lymphknotenmetastasen zeigte die PET/MRT höhere Werte für die Sensitivität, Spezifität, positiver predektiver Wert, negativer prediktiver Wert und diagnostische Genauigkeit (86\%, 92\%, 91\%, 89\% and 89\%) verglichen mit der MRT (73\%, 84\%, 80\%, 78\% and 79\%). Zusätzlich wurden durch die PET/MRT höhere Werte für den Nachweis von Fermetastasen im Vergleich zur MRT ermittelt ( $85 \%, 94 \%, 85 \%, 94 \%$ and $92 \%$ vs. $69 \%, 88 \%, 69 \%, 88 \%$ and $83 \%)$.

Schlussfolgerungen Die Ergebnisse demonstrieren das hohe diagnostische Potential des PET/MRT für das Ganzkörperstaging von Patientinnen mit primärem Zervixkarzinom. Insbesondere bei der Detektion von Lymphknoten- und Fernmetastasen bietet die PET/MRT eine höhere Genauigkeit im Vergleich zur 
MRT allein. Daher könnte die Kombination aus MRT und 18F-FDG PET nützliche Informationen für die Therapieplanung und Prognoseabschätzung liefern.

\section{WISS 303.1 Die Hauptkomponentenanalyse von ADC-Histogrammen als prädiktiver Faktor im HCC}

Autoren Kaissis $\mathrm{G}^{1}$, Ettlinger $\mathrm{F}^{2}$, Ahmaddy $\mathrm{F}^{1}$, Chakrabarti $\mathrm{P}^{2}$, Christ $\mathrm{P}^{2}$, Menze $B^{2}$, Weichert $W^{3}$, Rummeny $E^{1}$, Braren $R^{1}$

Institut 1 Klinikum rechts der Isar, Institut für diagnostische und interventionelle Radiologie, München; 2 Technische Universität München,

Lehrstuhl für Informatikanwendungen in der Medizin \&amp; Augmented

Reality, München; 3 Klinikum rechts der Isar, Institut für Pathologie, München DOI 10.1055/s-0037-1600414

Zielsetzung Der Einfluss der intra- und interindividuellen Tumorheterogenität als prognostischer Faktor wird zunehmend auch für das HCC diskutiert. Einzelne Studien haben die Möglichkeit der Tumorsubgruppenklassifizierung anhand der Bildgebung aufgezeigt. Ziel dieser Studie war die Anwendung einer Hauptkomponentenanalyse auf Diffusionsgewichteten-MRT (DW-MRT) Daten zur Bestimmung der Wertigkeit in Bezug auf das Gesamtüberleben.

Material und Methodik 78 HCCs wurden in kontrastverstärkten T1w (Spätphase) und DW-MRT Datensätzen identifiziert. Tumor-, Leber-, Nieren- und Aortenkonturen wurden in beiden Datensätzen manuell segmentiert und automatisch koregistriert. Histogramme der ADC Maps wurden mittels Hauptkomponentenanalyse bezüglich Intensität, Mittelwert, Standardabweichung, Skewness und Kurtosis bewertet.

Ergebnisse T1w und ADC Datensätze konnten in allen Fällen erfolgreich koregistriert werden. Die Hauptkomponentenanalyse zeigte die höchste Varianz für die Parameter "Skewness" und "Kurtosis". Anhand der Hauptkomponentenanalysen konnten Gesamtüberlebensgruppen differenziert werden.

Schlussfolgerungen Die automatische Bildkoregistrierung ist möglich und kann zur Extraktion präziser ADC-Histogramminformationen aus der Tumorregion verwendet werden. Mittels Hauptkomponentenanalyse der ADC Histogrammdaten können Gesamtüberlebensgruppen unterschieden werden. Diese Arbeit belegt, dass primär nicht-intuitive Merkmale der ADC-Map mit dem Gesamtüberleben im HCC korrelieren.

\section{WISS 104.5 Evaluation der prognostischen Signifikanz der arteriellen und venösen Gefäßummauerung und -infiltration im pankreatischen duktalen Adenokarzinom mittels präoperativer Computertomografie}

Autoren Kaissis $\mathrm{G}^{1}$, Danner $]^{1}$, Schirren $\mathrm{R}^{2}$, Friess $\mathrm{H}^{2}$, Ceyhan $\mathrm{G}^{2}$, Rummeny $E^{1}$, Braren $R^{1}$

Institut 1 Klinikum rechts der Isar, Institut für diagnostische und interventionelle Radiologie, München; 2 Klinikum rechts der Isar, Chirurgische Klinik und Poliklinik, München

DOI 10.1055/s-0037-1600415

Zielsetzung Die Prognose des Pankreaskarzinoms wird entscheidend durch das Tumorstadium bei Erstdiagnose bestimmt. Der Tumorbezug zu den arteriellen und venösen Blutleitern ist diesbezüglich entscheidend für die Klassifizierung in resektable, borderline und irresektable Tumore. Die Definition der borderline und irresektablen Tumore ist mit der Weiterentwicklung der operativen Techniken und Einführung neo-adjuvanter Therapiekonzepte einem schnellen Wandel unterworfen. Ziel dieser Studie war die Bewertung der Gefäßummauerung und -infiltration als Prognosefaktoren für das Gesamtüberleben und das postoperative Überleben nach Pankreaslinks- oder Whipple-Resektion.

Material und Methodik 314 präoperative CTs wurden ausgewertet. Die Gefäßummauerung wurde anhand einer Vierquadrantenskala quantifiziert. Die Gefäßinfiltration wurde anhand einer Konturirregularität der betroffenen Gefäße als "positiv" oder "negativ" bezeichnet. Ausgewertet wurden Truncus coe- liacus, A. mesenterica superior, A. hepatica, A. lienalis, V. mesenterica superior, V. portae und V. lienalis. Desweiteren wurden TNM- und UICC-Stadium, sowie histopathologisches Grading bestimmt. Das Gesamtsurvival und das postoperative Survival wurden kalkuliert.

Ergebnisse Über 90\% der Patienten waren $\geq$ UICC-Stadium II. Die häufigste Tumorgröße war T3 und das häufigste Grading G3. 40\% der Patienten waren R0-reseziert. Nur die venöse Infiltration zeigte ein signifikant schlechteres Überleben $(p=0.02)$.

Schlussfolgerungen Die CT basierte Diagnose einer venösen Gefäßinfiltration korreliert mit einem signifikant schlechteren Überleben, wohingegen ein arterieller Gefäßkontakt oder eine -infiltration sich nicht negativ auswirken.

\section{WISS 303.6 Prognostic value of perfusion CT in HCC treatment with sorafenib}

Authors Kaufmann $\mathrm{S}^{1}$, Thaiss $\mathrm{W}^{1}$, Kloth $\mathrm{C}^{1}$, Nikolaou $\mathrm{K}^{1}$, Bitzer $\mathrm{M}^{2}$, Horger $\mathrm{M}^{1}$

Institute 1 Universität Tübingen, Diagnostische und Interventionelle Radiologie, Tübingen; 2 Universität Tübingen, Gastroenterologie und Hepatologie, Tübingen

DOI 10.1055/s-0037-1600416

Zielsetzung To investigate the impact of sorafenib-treatment on intralesional perfusion using volume perfusion computed tomografy (VPCT) in hepatocellular carcinoma (HCC), correlate the observed changes to mRECIST at midterm for identification of their prognostic value and document potential correlations with the course of serum alpha-fetoprotein (AFP).

Material und Methodik VPCT was performed before (baseline) and after 2 months (follow-up) of sorafenib therapy in 28 consecutive HCC patients. AFP levels were registered at baseline and follow-up. Changes in tumor perfusion measurements [(blood flow (BF), blood volume (BV), mean transit time (MTT), k-trans, arterial liver perfusion (ALP) and hepatic perfusion index (HPI)) were registered in the target lesion. mRECIST measurements were performed at baseline, follow-up and every 2 months during sorafenib treatment. The receiver operating characteristic (ROC) curve analysis was performed to identify cut-off values for changes in HPI and BF as predictors of disease stabilization. Ergebnisse According to mRECIST after 2 month of treatment all patients showed stable disease, whereas after 4 month 13 patients (46\%) showed stable disease (SD) and 15 patients (54\%) showed progressive disease (PD). According to VPCT a significant decrease was found in BF, BV, k-trans, ALP and $\mathrm{HPI}$ in patients with SD as well as a significant increase in MTT $(p<0.05)$. In patients with PD a significant increase in HPI, BF and BV was observed $(p<0.05)$. There was no correlation between AFP and mRECIST or perfusion measurements, respectively. Reduction of $5.9 \%$ in $\mathrm{HPI}$ and $21.1 \%$ in BF after 2 month of treatment heralded disease stabilization with a sensitivity and specificity of $84.6 \%$ and $92.3 \%(\mathrm{HPI})$ and $100 \%$ and $92.3 \%(\mathrm{BF})$, respectively. Schlussfolgerungen Decreased intralesional perfusion after 2 months of therapy predicts disease stabilisation at mid-term (after 4 month) better than mRECIST, since non decreased intralesional perfusion indicates progressive disease at mid-term. AFP dynamics proved unreliable.

\section{WISS 402.6 Ultra-Fast 18F-FDG PET/MRT im Vergleich zu 18F-FDG PET/CT und CT beim Ganzkörper-Staging von Frauen mit Rezidiv eines Beckentumors}

Autoren Kirchner J ${ }^{1}$, Sawicki $L^{1}$, Suntharalingam $S^{2}$, Grüneisen $]^{2}$, Ruhlmann $\mathrm{V}^{3}$, Deuschl $\mathrm{C}^{2}$, Herrmann $\mathrm{K}^{3}$, Antoch $\mathrm{G}^{1}$, Umutlu $\mathrm{L}^{2}$

Institut 1 Heinrich-Heine-Universität Düsseldorf, Medizinische Fakultät, Institut für Diagnostische und Interventionelle Radiologie, Düsseldorf; 2 Universität Duisburg-Essen, Medizinische Fakultät, Institut dür Diagnostische und Interventionelle Radiologie und Neuroradiologie, Essen; 
3 Universität Duisburg-Essen, Medizinische Fakultät, Klinik für Nuklearmedizin, Essen

DOI 10.1055/s-0037-1600417

Zielsetzung Ziel der vorliegenden Arbeit war zu untersuchen, ob sich ein ultra-fast 18F-Fluordesoxyglucose Positronenemissionstomografie/Magnetresonanztomografie (18F-FDG PET/MRT) Protokoll, bestehend aus einer T2-w und einer kontrastmittelverstärkten T1-w Sequenz sowie metabolischen Informationen der PET, zur Diagnostik bei Frauen mit Verdacht auf Rezidiv eines Beckentumors eignet. Darüber hinaus erfolgte ein Vergleich mit der 18F-FDG PET/Computertomografie (18F-FDG PET/CT) und der CT.

Material und Methodik 43 Patientinnen mit Verdacht auf ein Tumorrezidiv eines Beckentumors wurden in diese Studie eingeschlossen. Bei allen Patientinnen wurde zunächst eine 18F-FDG PET/CT und im Anschluss daran eine 18F-FDG PET/MRT durchgeführt. Durch zwei unabhängige Radiologen wurden die ultra-fast 18F-FDG PET/MRT, die 18F-FDG PET/CT sowie die CT Datensätze des 18F-FDG PET/CT einzeln bezüglich Läsionsanzahl, -lokalisation und -charakterisierung ausgewertet. Die statistische Auswertung erfolgte sowohl patienten- als auch läsionsbasiert.

Ergebnisse Bei 38 der 43 Patientinnen lag ein Tumorrezidiv vor. Mittels CT konnten 25 der 38, mittels 18F-FDG PET/CT 37 der 38 Patientinnen und mittels 18F-FDG PET/MRT 36 der 38 Patientinnen mit einem Tumorrezidiv korrekt identifiziert werden. Bei der läsionsbasierten Auswertung konnte mittels 18F-FDG PET/MRT mehr Läsionen detektiert werden. Dies resultierte in einer Sensitivität, Spezifität, positivem Vorhersagewert, negativen Vorhersagewert und einer diagnostischen Präzision von 50\%, 58\%, 76\%, 31\%, und 53\% für das CT, $97 \%, 83 \%, 93 \%, 94 \%$, und $92 \%$ für das $18 \mathrm{~F}-\mathrm{FDG}$ PET/CT and $98 \%$, $83 \%, 94 \%, 94 \%$, and $94 \%$ für das 18F-FDG PET/MRT. Die durchschnittlichen Scanzeiten betrugen jeweils $18.5 \pm 1$ Minuten im 18F-FDG PET/MRT, $18.2 \pm 1$ Minuten im 18F-FDG PET/CT und 3.5 Minuten im CT.

Schlussfolgerungen Die ultra-fast 18F-FDG PET/MRT ist beim Ganzkörper-Staging von Frauen mit Verdacht auf Rezidiv eines Beckentumors der 18F-FDG $\mathrm{PET} / \mathrm{CT}$ bezüglich diagnostischer Performance und Untersuchungsdauer gleichwertig und der CT überlegen.

\section{WISS 104.7 Nutzen von Kontrastmittelgabe und DWI in der 18F-FDG PET/MRT für die Bestimmung des Tumorstadiums bei Lymphom-Patienten}

\author{
Autoren Kirchner J', Sawicki L ${ }^{1}$, Deuschl $C^{2}$, Eisen J², Ruhlmann $\mathrm{V}^{3}$, \\ Erfanian $\mathrm{Y}^{2}$, Heusch $\mathrm{P}^{1}$, Antoch $\mathrm{G}^{1}$, Umutlu $\mathrm{L}^{2}$ \\ Institut 1 Heinrich-Heine-Universität Düsseldorf, Medizinische Fakultät, \\ Institut für Diagnostische und Interventionelle Radiologie, Düsseldorf; \\ 2 Universität Duisburg-Essen, Medizinische Fakultät, Institut dür \\ Diagnostische und Interventionelle Radiologie und Neuroradiologie, Essen; \\ 3 Universität Duisburg-Essen, Medizinische Fakultät, Klinik für Nuklearmedizin, \\ Essen \\ DOI 10.1055/s-0037-1600418
}

Zielsetzung Das Ziel der vorliegenden Arbeit war die Evaluation des Nutzens einer Kontrastmittelgabe und einer Diffusionsbildgebung (DWI) in der 18FFDG PET/MRT für die Bestimmung des Ann Arbor Stadiums bei LymphomPatienten.

Material und Methodik Insgesamt wurden 91 18F-FDG PET/MRT Untersuchungen bei Lymphom-Patienten ausgewertet, die im Anschluss an die klinisch indizierte 18F-FDG PET/CT Untersuchung durchgeführt wurde. 2 Radiologen identifizierten in unterschiedlichen 18F-FDG PET/MRT Datensätzen die Lymphom-Manifestationen. Die Datensätze bestanden aus T2-HASTE und 18F-FDG PET (PET/MRT1); T2-HASTE, kontrastmittelverstärkter T1-Vibe und 18F-FDG PET sowie T2-HASTE (PET/MRT2), kontrastmittelverstärkter T1-Vibe, DWI und 18F-FDG PET (PET/MRT3). Das Ann Arbor Stadium wurde nach der Lugano Klassifikation bestimmt. Es erfolgte eine patienten- und läsionsbasierte statistische Auswertung. Die Ergebnisse der 18F-FDG PET/CT sowie alle verfügbaren histopathologischen Untersuchungen und Nachsorge-Untersuchungen dienten als Referenzstandard.

Ergebnisse Bei 48 der 91 Patienten lag eine Lymphomerkrankung vor. Mittels PET/MRT2 und PET/MRT3 wurden alle erkrankten 48 Patienten identifiziert, während mittels PET/MRT1 46 der 48 erkrankten Patienten identifiziert wurden. Insgesamt wurden 175 Lymphom-Manifestationen detektiert. Die Sensitivität, der positive Vorhersagewert, und die diagnostischen Präzision betrugen $100 \%$, $96 \%$ und $96 \%$ für das PET/MRT3 sowie $99 \%$, $96 \%$ und $95 \%$ für das PET/MRT2 und 95\%, 93\% und 90\% für das PET/MRT1. Das korrekte Ann Arbor Stadium konnte mittels PET/MRT3 in 96\%, mittels PET/MRT2 in 95\% und mittels PET/MRT1 in $88 \%$ der Fälle korrekt bestimmt werden.

Schlussfolgerungen Durch die Gabe von Kontrastmittel erfolgt eine deutliche Steigerung bei der Detektion von Lymphom-Manifestationen und damit einhergehend eine verbesserte Stadieneinteilung gegenüber einer nativen $18 \mathrm{~F}$ FDG PET/MRT. Die zusätzliche Akquise einer Diffusionsbildgebung führt lediglich zu einer geringen weiteren Steigerung.

\section{WISS 315.5 Einfluss der PET/CT auf das klinische Management bei Patienten mit high-risk und rezidiviertem Prostatakarzinom}

Autoren Pfannenberg $C^{1}$, Olthof $S^{1}$, Seith $F^{1}$, la Fougere $C^{2}$, Reischl $C^{3}$, Nikolaou $\mathrm{K}^{1}$, Zips $\mathrm{D}^{4}$, Müller $\mathrm{A}^{4}$

Institut 1 Eberhard-Karls-Universität Tuebingen, Abt. für Diagnostische und Interventionelle Radiologie, Tuebingen; 2 Eberhard-Karls-Universität

Tuebingen, Abt. für Nuklearmedizin, Tuebingen; 3 Eberhard-Karls-Universität Tuebingen, Abt. für Radiopharmazie, Tuebingen; 4 Eberhard-Karls-Universität Tuebingen, Klinik für Radioonkologie, Tuebingen

DOI 10.1055/s-0037-1600419

Zielsetzung Untersuchung des Einflusses der Cholin/PSMA-PET/CT auf das klinische Management bei Patienten mit high-risk oder rezidiviertem Prostatakarzinom (PC) im Vergleich zur konventionellen CT/MRT-basierten Therapieplanung.

Material und Methodik Bei 50 Patienten mit high-risk oder rezidiviertem PC wurde im Rahmen einer prospektiven Registerstudie eine 11C-Cholin- oder 68Ga-PSMA-PET/CT zur Planung der Radiotherapie (RT) durchgeführt. In die vorliegende Auswertung wurden ausschließlich Patienten mit konventionellem Staging (CT/MRT/BS) vor PET/CT eingeschlossen $(n=36)$, um das Management vor und nach PET/CT bezüglich Behandlungsziel (kurativ/palliativ) und Zielvolumen (ZV)-Definition vergleichen zu können.

Ergebnisse Die PET/CT führte insgesamt zu Managementänderungen bei $82 \%$ der high-risk PC ( $n=14 / 17), 66 \%$ der Rezidive nach Op $(n=8 / 12)$ und $85 \%$ der Rezidive nach Salvage RT $(n=6 / 7)$. Bei 2 Patienten, klassifiziert als M1 nach konventionellem Staging, führte die PET/CT zum Downstaging (M0) bzw. zur Erkennung einer Oligometastasierung, welches in beiden Fällen eine kurative Therapie ermöglichte. Andererseits wurde bei 12/36 Patienten durch PET/ CT nach Detektion von nodalen ( $n=3)$ oder Fernmetastasen $(n=9)$ ein Wechsel zu palliativer Therapie initiiert. Bei 2 von 7 Patienten mit Rezidiv nach Salvage RT ermöglichte die PET/CT die genaue Rezidivlokalisation und damit noch einen kurativen Therapieansatz. Insgesamt konnte die PET/CT bei 30\% $(8 / 27)$ der Patienten, die ursprünglich als kurabel klassifiziert wurden, ein Overtreatment durch frühzeitige Erkennung einer inkurablen Situation verhindern. Hauptlimitation der Studie ist die fehlende histologische Verifizierung. Schlussfolgerungen Die Cholin/PSMA-PET/CT führt zu häufigen Änderungen des geplanten Managements beim high-risk und rezidivierten PC und sollte deshalb in spezifischen klinischen Situationen bei dieser Patientengruppe Anwendung finden. 
WISS 303.5 Intravoxel Incoherent Motion (IVIM) Analyse von MR-Diffusionsbildgebung zur Bestimmung des Therapieansprechens von hepatozellulären Karzinomen nach Radioembolisation mit Harz- und Glasmikrosphären

Autoren Pieper $C^{1}$, Sprinkart $A^{1}$, Meyer $C^{1}$, Schild $H^{1}$, Kukuk $G^{1}$, Mürtz $P^{1}$ Institut 1 Universitätsklinikum Bonn, Radiologische Klinik, Bonn DOI 10.1055/s-0037-1600420

Zielsetzung Vergleich der Intravoxel Incoherent Motion (IVIM) Modell basierten Ansprechcharakteristika von hepatozellulären Karzinomen (HCC) nach Radioembolisation (RE) mit Harz- bzw. Glasmikrosphären.

Material und Methodik 37 HCC-Patienten (mittleres Alter 68 Jahre) erhielten 44 primäre RE (26 Harz, 18 Glas). Jeweils vor und vier Wochen nach RE wurde ein 1.5T Leber-MRT inklusive Diffusionsbildgebung ( $\mathrm{b} 0=0, \quad \mathrm{~b} 1=50$, b2 $=800 \mathrm{~s} / \mathrm{mm} 2)$ durchgeführt. Der Apparent Diffusion Coefficient ADC $(0,800)$, der geschätzte Diffusionskoeffizient D‘ und die Perfusionsfraktion f' wurden berechnet. Pro RE wurde ein HCC-Knoten analysiert. Die Daten wurden eingeteilt in „Responder“ (partial response/stable disease) und „Non-Responder“ (progressive disease) entsprechend der modifizierten RECIST-Kriterien im Verlauf.

Ergebnisse 27 HCCs sprachen auf die RE an (16 Harz, 11 Glas), 17 dagegen nicht (10 Harz, 7 Glas). „Responder“ auf Harz-RE hatten signifikant höhere präinterventionelle f'-Werte als „Non-Responder“ $(p=0,016)$. Demgegenüber zeigten „Responder“ auf Glas-RE signifikant niedrigere präinterventionelle ADC $(0,800)$ - und D'-Werte als „Non-Responder“ ( $p=0,0005$ bzw. $p=0,001)$. Nach Therapie stiegen bei „Respondern“ $A D C(0,800)$ - und D'-Werte an und f' fiel ab (Harz: $p=0,001, p<0,0001, p<0,0001$; Glas: $p=0,006, p=0,001, p=0,016$ ). Bei „Non-Respondern“ dagegen stieg nach Harz-RE $f^{\prime}$ signifikant an $(p=0,006)$, während nach Glas-RE ADC $(0,800)$ und D' abfielen $(p=0,023$ und $p=0,032)$. „Responder“ und „Non-Responder" ließen sich nach Harz-RE am besten Anhand von f‘-Änderungen (AUC 1,0), nach Glas-RE Anhand von D'-Änderungen (AUC 1,0) differenzieren.

Schlussfolgerungen Eine Abschätzung des Therapieerfolges einer RE von HCCs ist mittels IVIM-Analyse möglich. Ansprechende Tumoren weisen insgesamt einen Anstieg der Diffusionsparameter (Gewebenekrotisierung) und einen Abfall der Perfusionsparameter auf. Ein Ansprechen wird nach Harz-RE vor allem durch Änderungen der Perfusion („dominanter“ Embolisationseffekt) und nach Glas-RE vor allem durch Änderungen der Diffusion angezeigt.

\section{WISS 104.4 Evaluation der 68Ga-DOTATOC PET/MRT im Vergleich zur 68Ga-DOTATOC PET/CT beim Ganzkörperstaging von Patienten mit neuroendokrinen Tumoren}

Autoren Sawicki $L^{1}$, Deuschl $C^{2}$ Beiderwellen $K^{2}$ Ruhlmann $V^{3}$ Poeppel $T^{3}$ Heusch $\mathrm{P}^{1}$, Führer-Sakel $\mathrm{D}^{4}$, Bockisch $\mathrm{A}^{3}$, Herrmann $\mathrm{K}^{3}$, Antoch $\mathrm{G}^{1}$, Forsting $\mathrm{M}^{2}$, Umutlu $\mathrm{L}^{2}$

Institut 1 Universitätsklinikum Düsseldorf, Institut für Diagnostische und Interventionelle Radiologie, Düsseldorf; 2 Universitätsklinikum Essen, Institut für Diagnostische und Interventionelle Radiologie und Neuroradiologie, Essen; 3 Universitätsklinikum Essen, Klinik für Nuklearmedizin, Essen; 4 Universitätsklinikum Essen, Klinik für Endokrinologie, Essen DOI 10.1055/s-0037-1600421

Zielsetzung Evaluation des diagnostischen Nutzens der 68Ga-DOTATOC PET/ MRT im Vergleich zur 68Ga-DOTATOC PET/CT im Ganzkörperstaging von Patienten mit neuroendokrinen Tumoren (NET).

Material und Methodik Bei 30 Patienten mit histologisch gesichertem NET wurde unmittelbar anschließend an eine PET/CT die PET/MRT unter Ausnutzung der residualen 68Ga-DOTATOC Aktivität durchgeführt. PET/CT und PET/ MRT wurden separat bezüglich Anzahl, Lokalisation, Kontrast (4-Punkte-Skala) und Dignität (NET/benigne nicht-NET-Läsionen) von Läsionen ausgewertet.
Der Referenzstandard für Dignität basierte auf histologischen Untersuchungen und Verlaufsbildgebung. Die Anteile an NET- und nicht-NET-Läsionen, die mit PET/CT bzw. PET/MRT korrekt als solche bewertet wurden, wurde mittels McNemar's chi2 Test verglichen. Unterschiede zwischen PET/MRT und PET/ CT bezüglich des Kontrastes von NET-Läsionen und SUVmax bei korrespondierenden PET-positiven Läsionen wurden durch einen Wilcoxon-Test quantifiziert. Zudem wurde der SUVmax mittels Pearson Koeffizient intermodal korreliert.

Ergebnisse Insgesamt wurden 197 Läsionen im Referenzstandard beschrieben (142 NET, 55 nicht-NET). Bezüglich der Dignität ließ sich mit der PET/MRT ein höherer Anteil an korrekt bewerteten NET Läsionen identifizieren als mit der PET/CT (90.8\% vs. 86.7\%, p=0.031). Die PET/CT zeigte einen höheren Anteil an korrekt bewerteten nicht-NET-Läsionen $(94.5 \%$ vs. $83.6 \%, p=0.031)$. Der SUVmax korrelierte deutlich $(r=0.86 ; p<0.001)$ und zeigte keinen signifikanten Unterschied zwischen beiden Modalitäten $(p=0.35)$. In der PET/MRT wurden NET-Läsionen mit einem höheren Kontrast dargestellt als in der PET/CT $(p<0.01)$.

Schlussfolgerungen Mittels 68Ga-DOTATOC PET/MRT konnten mehr NET Läsionen korrekt identifiziert werden. Daher kann die 68Ga-DOTATOC PET/MRT als wertvolle Alternative zur 68Ga-DOTATOC PET/CT im Ganzkörperstaging von Patienten mit NET angesehen werden.

\section{WISS 303.2 Einfluss der Embolisate auf den Anstieg der proangiogenen VEGF-Level im Serum nach TACE}

Autoren Schicho $A^{1}$, Hellerbrand $C^{2}$, Wohlgemuth $W^{1}$, Stroszczynski $C^{1}$, Wiggermann $\mathrm{P}^{1}$, Pereira $\mathrm{P}^{3}$

Institut 1 Universitätsklinikum Regensburg, Radiologie, Regensburg; 2 Friedrich-Alexander-Universität Erlangen, Biochemie (Emil-Fischer-

Zentrum); 3 SLK Kliniken Heilbronn, Radiologie

DOI 10.1055/s-0037-1600422

Zielsetzung Bei der transarteriellen Chemoembolisation (TACE) zur Behandlung intrahepatischer Tumore werden u. a. Lipiodol, drug eluting beads (DEB) und degradable starch microspheres (DSM) als Embolisate eingesetzt. Sie unterscheiden sich wesentlich in Dynamik und Verlauf der verursachten Gefäßokklusion. Ischämie verursacht eine unerwünschte Freisetzung verschiedener proangiogener Faktoren, wie z. B. VEGF (vascular endothelial growth factor), verknüpft mit einer Begünstigung des Tumorprogress. Daher wurde der Einfluss des Embolisats auf die VEGF-Level im Serum nach TACE untersucht. Material und Methodik Patienten mit hepatozellulärem Karzinom (HCC) wurden für die TACE in eine von drei Gruppen (Lipiodol, DEB, DSM) randomisiert. Die VEGF-Level im Serum wurden vor TACE, 24 Stunden nach TACE und 1 Monat nach TACE bestimmt. Eine statistische Signifikanz wurde bei $p<0,05$ angenommen. Das Votum der Ethikkommission lag zu Untersuchungsbeginn vor.

Ergebnisse Insgesamt wurden 30 TACE an 22 Patienten beobachtet. Nach 24 Stunden waren die VEGF-Level bei Lipiodol auf $164 \%$, bei DEB auf $114 \%$ und bei DSM auf $121 \%$ gestiegen. Nach 4 Wochen betrugen die Level $170 \%$ für Lipiodol, 123\% für DEB und 124\% für DSM. Lediglich der Anstieg bei Lipiodol war sowohl nach 24 Stunden als auch nach 4 Wochen statistisch signifikant $(p<0,05)$ erhöht gegenüber dem Ausgangsniveau.

Schlussfolgerungen Der Anstieg des proangiogenen VEGF im Serum bei DEB und DSM als Embolisat bei TACE liegt deutlich unter dem des weltweit meistgenutzten Lipiodol. Um die pro-onkogene Wirkung der verursachten Ischämie zu reduzieren bietet bereits die Auswahl des richtigen Embolisates einen wirkungsvollen Ansatzpunkt. Ob dies und die mögliche zusätzliche Verwendung von VEGF-Antikörpern sich tatsächlich in einem Überlebensvorteil für die Patienten niederschlägt, bleibt in weiteren Studien zu prüfen. 


\section{WISS 104.8 Mehrwert der strukturierten Befundung bei CT-Untersuchungen im Rahmen des Primärstagings bei Patienten mit diffus großzelligem B-Zell-Lymphom}

Autoren Schöppe $F^{1}$, Sommer $W^{1}$, Dreyling $M^{2}$, Verbeek $M^{3}$, Westphalen $C^{2}$, Bogner $C^{3}$, Nörenberg $D^{1}$, Reiser $M^{1}$, Rummeny $E^{4}$, Fingerle $A^{4}$

Institut 1 Klinikum der Universität München, Institut für Klinische Radiologie, München; 2 Klinikum der Universität München, Medizinische Klinik und

Poliklinik III, München; 3 Klinikum rechts der Isar der TU München, Klinik und Poliklinik für Innere Medizin III, Hämatologie und Onkologie, München;

4 Klinikum rechts der Isar der TU München, Institut für diagnostische und

interventionelle Radiologie, München

DOI 10.1055/s-0037-1600423

Zielsetzung Ziel dieser Studie war die Evaluation, ob strukturierte Befunde (SB) einen Mehrwert gegenüber konventionellen Freitext-Befunden (FTB) haben, in der Befundung von primären Staging-Untersuchungen mittels Computertomografie (CT) bei Patienten mit diffus großzelligem B-Zell-Lymphom (DLBCL).

Material und Methodik In dieser multizentrischen Studie wurden 16 CT-Untersuchungen randomisiert ausgewählt und dazu jeweils ein FTB und ein SB akquiriert. Die SB wurden anhand einer Online-Befundvorlage erstellt, bei der durch Auswahl der entsprechenden Elemente automatisch der entsprechende Befundtext generiert wird. Die anonymisierten Befunde wurden zur Bewertung mittels eines Online-Fragebogens ( $N=128$ ausgefüllte Fragebögen) in randomisierter Reihenfolge an vier erfahrene Hämatoonkologen geschickt. Der Fragebogen enthielt hierbei Fragen zur Vollständigkeit, Struktur und Beitrag zum weiteren Vorgehen (10-Punkte Likert-Skala $(0=$ Stimme überhaupt nicht zu, 10 = Stimme vollständig zu) sowie zur allgemeinen Befundqualität 5Punkte-Skala, 0 = unzureichend, 5 = sehr gut).

Ergebnisse SB enthielten signifikant häufiger als FTB explizite Informationen über den Lymphombefall einzelner ( $95 \%$ vs. $66 \%$; $P<0.001)$ sowie suffiziente Informationen, die eine Einteilung nach der Ann-Arbor-Klassifikation ermöglichen $(89 \%$ vs. $64 \% ; \mathrm{P}=0.001)$. Die Extraktion der relevanten Informationen war nach Auffassung der Zuweiser signifikant schneller möglich bei SB als bei FTB (median rating $=9$ vs. $6 ; P=0.001$ ). SB wurden signifikant besser bewertet als FTB für die Beantwortung der klinischen Fragestellung ( 9 vs. 7 ; $P<0.001$ ) sowie für den Beitrag zur weiteren klinischen Entscheidungsfindung (9 vs. 6; $P=0.001$ ). Die Gesamtqualität von SB gegenüber FTB wurde als signifikant höher bewertet). $(\mathrm{P}<0.001)$.

Schlussfolgerungen SB von CT-Untersuchungen beim Primärstaging von Patienten mit DLBCL haben einen möglichen Mehrwert gegenüber FTB, da sie inhaltlich vollständiger sind, die Extraktion der Informationen erleichtern und besser zum weiteren Patientenmanagement beitragen können.

\section{WISS 104.3 4D MR-PET der Lunge: Effekte der Atmungsbewegungskorrektur der PET durch eine self- gated MR-VIBE auf die Rundherddetektion im belüfteten Phantom}

Autoren Sedlaczek $\mathrm{O}^{1}$, Fenchel $\mathrm{M}^{2}$, Hetzheim $\mathrm{H}^{1}$, Kiefer $\mathrm{B}^{2}$, Biederer $\rfloor^{3}$, Kauczor $\mathrm{H}^{3}$, Schlemmer $\mathrm{H}^{4}$

Institut 1 DKFZ, Heidelberg; 2 Siemens Healthcare GmbH, Diagnostic Imaging, Erlangen; 3 Uni-Heidelberg, DI-Radiologie, Heidelberg; 4 DKFZ, E010, Heidelberg

DOI 10.1055/s-0037-1600424

Zielsetzung Für die Thoraxorgane führt die Akquisition des PETs in freier Atmung zu einer unscharfen Ortsrekonstruktion und damit zu einer Unterschätzung der rekonstruierten Aktivität und einer Überschätzung des Läsionsvolumens. Ziel der Studie war die Quantifizierung des Einflusses der Atmungsbewegungsartefakte mittels einer MR-PET-basierten Bewegungskorrektur.
Material und Methodik Vier Schweine-Lungen-Explantate wurden in einem Thorax-Phantom belüftet und mit 8 - 10 artifiziellen Rundherden $(0,1-1 \mathrm{ml}$; 12,5 bis $50 \mathrm{kBq} / \mathrm{ml}$ Mischung Agarose/F-18 oder FDG) markiert. Die Hintergrund-Aktivität der Brustwand und der Leber wurde durch Dotierung (25$50 \mathrm{MBq})$ der Wand des Phantoms simuliert. Die MR-PET Daten wurden während künstlicher Atmunsexkusionen ("dynamisch", 8/min) aufgenommen und mit einem Standard-Algorithmus (dynamische PET), Atem-Gating/Listen-Modus (LM-PET) oder mit 4D-Mapping (selfgated MR-VIBE) aus den MRI-Bewegungsdaten (PETmoco) rekonstruiert. Die Nachweisbarkeit der Rundherde gegenüber dem Hintergrund wurde durch ROI-Messungen quantifiziert und ihre scheinbare Größe entlang der z-Achse des Phantoms gemessen.

Ergebnisse Das durchschnittliche Rauschen gegenüber dem Lungensignal betrug zwischen 22,7 kBq/ml (dynamisches PET), 36,7 kBq/ml für das LM-PET und $19,8 \mathrm{kBq} / \mathrm{ml}$ für PETmoco. Für PETmoco betrug das $\mathrm{S} / \mathrm{N}$-Verhältnis 6,5 ( $\min 1,3$, max 12,7, SD 3,6), für die dynamische PET, 9,4 ( $\min 3,9$, $\max 19,4$, SD 5,2) und für die LM-PET 11,7 ( $\min 2,3$, $\max 27,4$, SD 7,9). Die durchschnittliche z-Achsengröße reduzierte sich um 37,0\% (SD 5,5) für die LMPET und $31,2 \%(S D 4,3)$ für die PETmoco $(p<0,05)$ im Vergleich zur dynamischen PET.

Schlussfolgerungen Die Ex-vivo-Studie zeigt den Umfang der Fehlerkorrektur und die hohe resultierende Bildqualität der 4D MR-PET. Die bewegungskorrigierte PET mittels selfgated MR-VIBE kombiniert die Vorteile der dynamischen PET (alle Signale gehen in die Messung ein) mit guten Signal-Rausch-Verhältnis und der LM-PET (Bewegungskorrektur für räumliche Fehlregistrierung) in einer einzigen Akquisition.

\section{WISS 303.3 Therapieansprechen nach TACE im HCC: Vergleich der lod-Konzentration mit Perfusions-CT und RECIST/mRECIST}

Autoren Thaiss $W^{1}$, Haberland $U^{2}$, Kaufmann $S^{1}$, Kloth $C^{1}$, Preibsch $H^{1}$, Ketelsen $D^{1}$, Nikolaou $K^{1}$, Horger $\mathrm{M}^{1}$, Sauter $\mathrm{A}^{3}$

Institut 1 Radiologische Universitätsklinik Tübingen, Diagnostische und Interventionelle Radiologie, Tübingen; 2 Siemens Healthcare $\mathrm{GmbH}$,

Diagnostic Imaging, Computed Tomografy, Research \&amp; Development, Forchheim; 3 Universitätsspital Basel, Klinik für Radiologie und

Nuklearmedizin, Abteilung Nuklearmedizin, Basel

DOI 10.1055/s-0037-1600425

Zielsetzung Ziel der Studie war die Diskriminierung von Respondern und NonRespondern auf eine transarterielle Chemoembolisation (TACE), wobei die lodKonzentration im hepatocellulären Karzinom (HCC) aus 80 kVp Single Source CT Datensätzen mit Daten der quantitativen Perfusions-CT und der RECIST/ mRECIST-Auswertung vor und nach TACE verglichen wurden.

Material und Methodik 25 Patienten mit 33 HCC Läsionen wurden prospektiv rekrutiert und retrospektiv ausgewertet. Die Perfusions-CT Untersuchungen erfolgten unmittelbar vor- und nach TACE. lod-Karten wurden 7 Sekunden nach dem maximalen Aortenenhancement aus den $80 \mathrm{kVp}$ Datensätzen der Perfusions-CTs generiert und die lod-Konzentration in der Läsion berechnet. Perfusions-CT und die Angiografie während der TACE wurden zur KonsensusDefinition von Respondern und Non-Respondern genutzt.

Ergebnisse Die lod-Konzentration in Responder-Läsionen betrug 118.1 $\pm 47.0 \mathrm{mg} / 100 \mathrm{~mL}$ vor TACE und $17.0 \pm 25.2 \mathrm{mg} / 100 \mathrm{~mL}$ nach TACE. Der Blutfluss aus der Perfusions-Messung (BF) betrug $90.1 \pm 28.7 \mathrm{~mL} / 100 \mathrm{~mL} /$ min (vor TACE) und $32.1 \pm 19.0 \mathrm{~mL} / 100 \mathrm{~mL} / \mathrm{min}$ (nach TACE). Die Korrelation zwischen Blutfluss und berechneter lod-Konzentration betrug $r=0.8$, $\mathrm{p}<0.0001$. In Non-Respondern lag die lod-Konzentration bei 100.9 $\pm 43.6 \mathrm{mg} / 100 \mathrm{~mL}$ vor TACE und $118.8 \pm 50.6 \mathrm{mg} / 100 \mathrm{~mL}$ nach TACE. Der BF lag bei $76.8 \pm 35.6 \mathrm{~mL} / 100 \mathrm{~mL} / \mathrm{min}$ (vor TACE) und $73.5 \pm 36.1 \mathrm{~mL} / 100$ $\mathrm{mL} / \mathrm{min}$ (nach TACE). Die absoluten Unterschiede (vor/nach TACE) in der lodKonzentration der Läsion erlauben die Detektion eines Therapieansprechens mit einer Sensitivität/Spezifität von 95.8\%/88.9\%. Für mRECIST lag die Sensitivität/Spezifität bei $87.5 \% / 88.9 \%$. 
Schlussfolgerungen Die lod-Konzentration in der Läsion 7 Sekunden nach maximalem Aortenenhancement erwies sich in dieser Studie als hilfreicher Perfusionsmarker zur Evaluation des Therapieansprechens nach TACE und kann für die weitere Dosisreduktion im Perfusions-CT und zur weiteren Optimierung von Dual-Energy CT Untersuchungen in diesem Patientenkollektiv nützlich sein.

\section{WISS 303.4 Signalveränderungen von Lebermetastasen unter Bevacizumab-Therapie}

\author{
Autoren Thüring J ${ }^{1}$, Barabasch $A^{1}$, Kuhl $C^{1}$, Krämer N ${ }^{1}$ \\ Institut 1 Uniklinik RWTH Aachen, Klinik für Diagnostische und \\ Interventionelle Radiologie, Aachen \\ DOI 10.1055/s-0037-1600426
}

Zielsetzung Bevacizumab (Bevac) ist ein VEGF-Antagonist, der bei einer Vielzahl von Tumoren in der metastasierten Situation eingesetzt wird. Unsere klinische Beobachtung war, dass Bevac in der MRT zu charakteristischen Signalveränderungen führt. Ziel dieser Studie war es, das T2-Signalverhalten von Lebermetastasen unter Bevac-Therapie zu untersuchen.

Material und Methodik Longitudinale Studie an 56 Patienten (Alter $61 \pm 12$ Jahre) mit Lebermetastasen colorektaler $(n=29)$ oder Mamma-Ca $(n=27)$. Alle Patienten erhielten eine leitliniengerechte Systemtherapie, die bei 28 Patienten Bevac enthielt, bei den übrigen 28 nicht. Alle Patienten erhielten vor Therapiebeginn sowie 3, 6, und 9 Monate unter Systemtherapie ( \pm Bevac) eine Leber-MRT an 1.5T mit T2-gewichteter TSE-Sequenz. Die SI der Lebermetastasen wurde auf die SI der Milz referenziert; die resultierenden relativen Signalintensitäten (SSR) wurden intraindividuell sowie gruppenweise verglichen. Eine Reduktion der SSR um 25\% wurde als signifikant gewertet.

Ergebnisse Unter Bevac zeigte sich im Verlauf ein Abfall der SSR von im Mittel 1,01 auf 0,78 nach 3 Monaten, auf 0,54 nach 6 sowie auf 0,41 nach 9 Monaten $(p<0,001)$. Ein solcher Abfall wurde in der Kontrollgruppe nicht beobachtet (SSR 0,88/0,92/0,88/0,78; $p>0,05)$. Nach 9 Monaten bestand bei $93 \%$ (26/28) der Patienten unter Bevac, vs. 25\% (7/28) der Patienten ohne Bevac, eine signifikante Reduktion der SSR der Metastasen um im Mittel -57\% (range: $-12 \%$ bis $-84 \%$ ), gegenüber $8 \%$ (range: $-71 \%$ bis $+61 \%$ ) ohne Bevac $(p<0.001)$.

Schlussfolgerungen Lebermetastasen zeigen unter Therapie mit Bevac einen deutlichen Abfall der SI in T2-w Sequenzen. Ursächlich dürfte die sich unter anti-angiogenetischer Therapie normalisierende Gefäßpermeabilität sein, die zu einer Reduktion des interstitiellen Wassergehaltes führt. Eine Änderung des T2-Signals von Metastasen kann daher offenbar als „Imaging Biomarker“ für die Wirkung von Angiogenese-Hemmern herangezogen werden.

\section{WISS 303.7 Empirische Bestimmung volumetrischer Grenzwerte gemäß RECIST 1.1 zur Beurteilung des Therapieansprechens bei Lebermetastasen}

Autoren Winter $\mathrm{K}^{1}$, Winter $\mathrm{K}^{2}$, Hofmann $\mathrm{F}^{1}$, Thierfelder $\mathrm{K}^{1}$, Heinemann $\mathrm{V}^{3}$, Hesse $N^{1}$, Baumann $A^{1}$, Reiser $M^{1}$, Sommer $W^{1}$, D'Anastasi $M^{1}$

Institut 1 Klinikum der Ludwig-Maximilians-Universität München, Institut für Klinische Radiologie, München; 2 Ludwig-Maximilians-Universität München, Institut für Klinische Radiologie, München; 3 Klinikum der Ludwig-

Maximilians-Universität München, Medizinische Klinik und Poliklinik III, München

DOI 10.1055/s-0037-1600427

Zielsetzung Ziel der Studie war die empirische Bestimmung von Grenzwerten volumetrischer Messungen analog zu den etablierten Grenzwerten eindimensionaler Messungen gemäß RECIST 1.1 zur Beurteilung des Therapieansprechens von Lebermetastasen.

Material und Methodik Eingeschlossen wurden Patienten aus einem Kollektiv einer multizentrischen klinischen Phase-III-Studie mit metastasiertem kolorektalem Karzinom und Eingangs- sowie Nachuntersuchungen in der CT. Nach Bestimmung des größten axialen Durchmessers einer Leberläsion gemäß RE-
CIST 1.1 erfolgte eine semiautomatische Berechnung des jeweiligen Volumens. Die Summe der Durchmesser und der Volumina von $\leq 2$ Metastasen wurden jeweils mit allen vorhergehenden Untersuchungen verglichen. Die volumetrischen Grenzwerte entsprechend RECIST 1.1 (größter axialer Diameter $-30 \%$ bzw. $+20 \%$ ) wurden mittels Regressionsanalyse bestimmt. Die volumetrischen Messungen wurden gegenüber den unidimensionalen Messungen hinsichtlich ihrer Übereinstimmung der Klassifikation des Therapieverlaufs untersucht.

Ergebnisse Für die Messungen wurden 348 Patienten mit insgesamt 629 Läsionen und 1773 CT-Untersuchungen eingeschlossen. Die Regressionsanalyse ergab Grenzwerte für das Volumen von $-65,3 \%$ bzw. +64,6\% entsprechend der Diameter-basierten Grenzwerte von -30\% bzw. +20\% nach RECIST 1.1. Der Vergleich von volumetrischen mit eindimensionalen Messungen nach RECIST 1.1 ergab eine Übereinstimmung für einen signifikanten Progress $(\geq+20 \% / \geq+65 \%)$ in $98,0 \%$ und für einen nicht-signifikanten Progress $(<+20 \%$ | $<+65 \%$ ) in $87,2 \%$ der Fälle. Eine Übereinstimmung für einen signifikanten Regress ( $\leq-30 \% / \leq-65 \%$ ) bestand in $87,8 \%$ und für einen nicht-signifikanten Regress (>-30\%/>-65\%) in 94,4\% der Fälle.

Schlussfolgerungen In einem großen Kollektiv von Patienten mit Lebermetastasen ergeben sich analog zu RECIST 1.1 (Diameter $+20 \%$ bzw. -30\%) empirisch ermittelte volumetrische Grenzwerte von $+65 \%$ bzw. $-65 \%$.

\section{WISS 104.1 Dosisreduziertes CT zum Staging von Melanompatienten - Evaluation von Strahlendosis und diagnostischer Genauigkeit im Vergleich zum Standarddosis-CT}

Autoren Zinsser $\mathrm{D}^{1}$, Othman $\mathrm{A}^{1}$, Eigentler $\mathrm{T}^{2}$, Garbe $\mathrm{C}^{2}$, Nikolaou $\mathrm{K}^{1}$, Klumpp $B^{1}$

Institut 1 Universitätsklinikum Tübingen, Diagnostische und Interventionelle Radiologie, Tübingen; 2 Universitätsklinikum Tübingen, UniversitätsHautklinik, Tübingen

DOI 10.1055/s-0037-1600428

Zielsetzung Vergleich einer dosisreduzierten CT-Untersuchung mit intermediärer Röhrenspannung und einem Standarddosis-Dual-Energy-CT zum Staging von Melanompatienten bezüglich Strahlendosis, Bildqualität und diagnostischer Genauigkeit.

Material und Methodik Retrospektiv wurden 118 Melanompatienten (53 Frauen, Durchschnittsalter 60,2 \pm 14,5 Jahre) eingeschlossen, die ein Ganzkörper-Dual-Energy-CT mit 100/150 kV (Röhre A/B) erhalten hatten. Hieraus wurden ein Datensatz mit $100 \mathrm{kV}$ (dosisreduziertes CT, Röhre A) und ein gemischter Datensatz (Standdarddosis-CT, 80\% Röhre A/20\% Röhre B) rekonstruiert. Bildqualität und diagnostische Genauigkeit wurden mittels einer viergliedrigen Likert-Skala ( 1 = ungenügend, 4 = exzellent) bewertet. Zudem evaluierten wir das Ausmaß des Tumorbefalls, legten Targetläsionen gemäß RECIST 1.1 fest und berechneten das Signal-zu-Rausch-Verhältnis der Skelettmuskulatur von Hals, Thorax und Abdomen.

Ergebnisse Durch die feste Kombination beider Röhren geht die Akquisition mit $100 \mathrm{kV}$ anstelle der gemischten Dual-Energy-Akquisition mit einer Dosisreduktion von $27 \%$ einher. Die diagnostische Genauigkeit wurde für beide Datensätze ähnlich bewertet $(3,29 \pm 0,66$ vs. 3,30 $\pm 0,66, p=0,317)$. Bei der Standarddosis-Untersuchung beobachteten wir eine bessere subjektive und objektive Bildqualität des Thorax $(3,78 \pm 0,44$ vs. $3,65 \pm 0,5, p \leq 0,001$, SNR $6,69 \pm 1,02$ vs. $6,5 \pm 1,34, p=0,032$ ) und eine bessere subjektive Bildqualität des Abdomens $(3,81 \pm 0,45$ vs. $3,41 \pm 0,57, p \leq 0,001)$. Es zeigte sich eine nahezu perfekte Übereinstimmung in Anzahl und Größe der Metastasen (102 Targetläsionen, durchschnittlicher Durchmesser 33,7 $\pm 35,1 \mathrm{~mm} / 33,3$ $\pm 34,8 \mathrm{~mm}, p=0,022$, Korrelation $r \geq 0,999$, $p \leq 0,001$ ).

Schlussfolgerungen Verglichen mit dem Standarddosis-Dual-Energy-CT bietet eine dosisreduzierte CT-Untersuchung mit intermediärer Röhrenspannung für das Staging von Melanompatienten eine vergleichbare diagnostische Ge- 
nauigkeit bezüglich Anzahl und Ausmaß der Metastasen bei gleichzeitig um $27 \%$ reduzierter Strahlendosis.

Strahlenschutz

WISS 209.3 Die aktualisierten diagnostischen Referenzwerte (DRW) des Bundesamtes für Strahlenschutz: Einbettung in ein automatisches CTDosismanagementsystem und Auswirkungen auf die klinische Praxis

Autoren Appel $E^{1}$, Kröpil $P^{1}$, Aissa $]^{1}$, Heinzler $N^{1}$, Thomas $C^{1}$, Antoch $G^{1}$, Boos $]^{1}$

Institut 1 Universitätsklinikum Düsseldorf, Klinik für diagnostische und interventionelle Radiologie, Düsseldorf

DOI 10.1055/s-0037-1600429

Zielsetzung Das Bundesamt für Strahlenschutz (BfS) aktualisierte 2016 die diagnostischen Referenzwerte (DRW, jeweils CTDlvol und DLP) für die diagnostische Computertomografie (CT). Ziel dieser Studie war die Einbindung dieser neuen DRW in ein bestehendes automatisches Dosismonitoring-System und die Evaluation der klinischen Konsequenzen.

Material und Methodik Die aktualisierten DRW wurden in eine implementierte Dosismonitoring Software (Doselntelligence ${ }^{\circledR}$, Pulmokard, Herdecke) eingebunden und sämtliche CT-Protokolle den neuen DRW zugeordnet. Anschließend erfolgte eine initiale Analyse der eigenen CT-Dosiswerte in Relation zu den DRW. CT-Protokolle, welche eine systematische Überschreitung der DRW zeigten, wurden als klinische Konsequenz umgestellt (LWS: $120 \mathrm{kV}$, 150 mAs vorher/ 80 mAs nachher; Becken 100 kV, 196/147; Mittelgesicht $120 \mathrm{kV}, 125 / 100)$. Anschließend erfolgte eine erneute Auswertung im Hinblick auf die aktualisierten DRW.

Ergebnisse 31954 CT-Untersuchungen mit 349 CT-Protokollen zwischen 01/ 2016 und 11/2016 wurden analysiert. Bei der initialen Analyse betrug der durchschnittliche CTDIvol 61\% und DLP 63\% der neuen DRW. Systematische Überschreitungen fanden sich in der CT Becken (CTDlvol 112\%/DLP 105\%), der CT LWS (157\%/172\%) und der CT Gesichtsschädel (105\%/149\%). Nach der Protokollumstellung lagen die durchschnittlichen Dosiswerte bei $80 \% / 85 \%$ (Becken) und 62\%/62\% (LWS) der neuen DRW. Eine Referenzwertüberschreitung im Hinblick auf das DLP für die CT des Gesichtsschädels persistierte $(88 \% / 121 \%)$. Eine Überschreitung der empfohlenen Scanlange (15,6 $\pm 3,4 \mathrm{~cm}$; BfS: $10 \mathrm{~cm}$ ) wurde aus klinischen Gründen beibehalten.

Schlussfolgerungen Die Implementierung der 2016 aktualisierten DRW in die klinische Routine und konsekutive Anpassung von CT-Protokollen kann durch Einbindung in ein automatisches CT-Dosismmanamgementsystem zuverlässig durchgeführt werden. Klinische Anforderungen erfordern in unserem Institut eine systematische Überschreitung der DRW des Mittelgesichtes.

\section{WISS 209.4 Genauigkeit der Berechnung von größenspezifischen Dosisabschätzungen (SSDE) aus dem wasseräquivalenten Durchmesser der Mittelschicht des CT Volumens}

\footnotetext{
Autoren Boos $\mathrm{J}^{1}$, Boos $\mathrm{J}^{1}$, Kröpil $\mathrm{P}^{1}$, Bethge $\mathrm{O}^{1}$, Aissa $\mathrm{J}^{1}$, Heinzler $\mathrm{N}^{1}$, Antoch $\mathrm{C}^{1}$, Thomas $\mathrm{C}^{1}$

Institut 1 Heinrich-Heine-Universität Düsseldorf, Medizinische Fakultät, Institut für Diagnostische und Interventionelle Radiologie, Düsseldorf DOI 10.1055/s-0037-1600430

Zielsetzung In dieser Studie sollte ermittelt werden, ob der wasseräquivalente Durchmesser (Dw) der Mittelschicht des CT Volumens für die Berechnung von größenspezifischen Dosisabschätzungen (SSDE) geeignet ist.

Material und Methodik 1812 CT Serien (1583 Erwachsene: 1195 Abdominal, 388 Thorax, 61,5 \pm 15,7]ahre, Body Mass Index [BMI] 26,0 $\pm 5,6 \mathrm{~kg} / \mathrm{m}^{2} ; 229$ Kinder: 26 Abdominal, 203 Thorax, 9,3 $\pm 5,0$ Jahre, BMl 17,6 $\pm 4,3 \mathrm{~kg} / \mathrm{m}^{2}$ ) wur-
}

den in unsere retrospektive Studie eingeschlossen. Dw von jeder Schicht jedes CT Volumens wurde automatisch mithilfe einer selbstentwickelten Matlab Anwendung ermittelt (The Mathworks, Natick, MA). SSDE wurden berechnet: 1. Basierend auf Dw von der Mittelschicht und dem vollmetrischen Computertomografie-Dosis-Index (CTDlvol) der Untersuchung; und 2. Basierend auf Dw und CTDIvol von jeder einzelnen Schicht des Scanvolumens, dies diente als Referenzstandard. Der Einfluss von Patientengewicht, Größe und BMI auf die Genauigkeit der SSDE wurden ermittelt.

Ergebnisse Die mittlere absolute Differenz zwischen beiden Methoden betrug $3,9 \pm 3,4 \%$ (range $0-30,5 \%$ ) für Erwachsene in der Abdominal-CT, 5,0 $\pm 3,2 \%$ $(0-17,2 \%)$ für Erwachsene in der Thorax-CT, $3,1 \pm 2,6 \%(0-9,2 \%)$ für die pädiatrische Abdominal-CT und 2,0 $\pm 1,7 \%(0-15,5 \%)$ für die pädiatrische Thorax-CT. Die Genauigkeit bei Verwendung der Mittelschicht korrelierte mit dem BMl und Patientengewicht (BMI: $r=0,15$ in der pädiatrischen Thorax-CT bis $r=0,43$ in der Abdominal-CT von Erwachsenen; Gewicht: $r=0,26$ in der pädiatrischen Thorax-CT bis $r=0,49$ in der pädiatrischen Abdominal-CT). Es bestand eine Tendenz zur Dosisüberschätzung bei schlanken und Dosisunterschätzung bei übergewichtigen Patienten in der Abdominal $C T(r=0,26)$.

Schlussfolgerungen Die SSDE Berechnung mittels Dw der Volumenmittelschicht führt zu einem mittleren Fehler von $2-5 \%$ verglichen mit der Berechnung aus allen Schichten des Scanvolumens. Die Genauigkeit ist abhängig von Patientengewicht und BMI mit einer Dosisunterschätzung bei übergewichtigen und -Dosisüberschätzung bei schlanken Patienten in der Abdominal CT.

\section{WISS 209.7 70-kVp CT-Untersuchung des Halses zur Lymphomabklärung und Verlaufskontrolle: Beurteilung der Bildqualität und Strahlendosis}

Autoren Bucher $A^{1}$, Albrecht $M^{1}$, Vogl $T^{1}$, Martin $S^{1}$, Beeres $M^{1}$, Bodelle $B^{1}$, Scholtz J ${ }^{1}$

Institut 1 Uniklinik Frankfurt, Institut für Diagnostische und Interventionelle Radiologie, Frankfurt

DOI 10.1055/s-0037-1600431

Zielsetzung Bisherige Studien belegen einen verbesserten Kontrast der Halsweichteile in $70 \mathrm{kV}$ und $90 \mathrm{kV}$ Untersuchungen bei deutlich eingeschränkter Bildqualität im unteren Halsbereich. In dieser Studie vergleichen wir die Bildqualität und Strahlendosis von 2 Hals-CT Niedrigdosisprotokollen im Rahmen der Lymphomabklärung- und Verlaufskontrolle.

Material und Methodik Kontrastmittelgestützte Hals-CT Untersuchungen von 40 Patienten (13 weiblich, Alter: 19-57 Jahre) wurden in zwei gleich großen Gruppen ausgewertet. CTs wurden auf einem Dual-Source CT der dritten Generation mit $70 \mathrm{kV}$ (Referenz-Röhrenstrom $457 \mathrm{mAs}$ ) oder $90 \mathrm{kV}$ (Referenz-Röhrenstrom $197 \mathrm{mAs}$ ) aquiriert und in mittlerer Admire Stufe rekonstruiert. Bildqualität und Artefaktlast der oberen, mittleren und unteren Halsabschnitte wurden von zwei Radiologen bewertet. Bildrauschen und Dichte-Werte wurden vaskulär, muskulär und im Fettgewebe bestimmt und das Kontrast-zu-Rausch Verhältnis (KRV) berechnet. DLP und CTDI und die effektive Dosis wurden erhoben $(k=0.0059)$. Mann-Whitney $U$ Test und Cohen Kappa-Statistik wurden verwendet.

Ergebnisse Die Weichteilstrukturen waren in allen 3 Halsabschnitten in beiden Gruppen sehr gut beurteilbar (Oben: $p=1.000$; Mitte: $p=0.208$; Unten: $p=0.277)$ mit signifikant besseren Bewertungen bei 70-kV $(p=0.021)$. Bei 70 kV war die Halswirbelsäule in allen Fällen vollständig beurteilbar. Die Untersucher-Übereinstimmung war exzellent $(k=0.81, p<0.001)$. KRV war in $70 \mathrm{kV}$ in allen drei Halsabschnitten verbessert $(70 \mathrm{kV}: 42.0 \pm 13.1$; $90 \mathrm{kV}$ : $22.7 \pm 11.1 ; P=0,010)$. CTDI war bei $70 \mathrm{kV}$ um ca. $30 \%$ reduziert $(4,3 \pm 0.6)$ im Vergleich zu $90 \mathrm{kV}(6,1 \pm 0.6, \mathrm{P}<0,001)$ die effektive Strahlendosis um ca. $50 \%$ (70 kV: $0.6 \pm 0.1 \mathrm{mSv}$; $90 \mathrm{kV}: 1.2 \pm 0.1 \mathrm{mSv} ; \mathrm{p}<0.001)$.

Schlussfolgerungen Die aktuelle CT-Scannergeneration erlaubt eine Reduzierung der Röntgenröhrenspannung bis zu $70-k V p$ in kontrastmittelunterstützten CT-Halsuntersuchungen mit gesteigertem Weichteilkontrast ohne Limita- 
tion der Beurteilung der unteren Halsabschnitte und erlaubt eine deutliche Strahlenreduktion im klinischen Alltag.

\section{WISS 209.6 Bestimmung des Dosisreduktionspotentials iterativer Rekonstruktionen intrakranieller CTA mit einer neuartigen auf paarweisen Bildvergleichen basierenden Methode}

Autoren Ellmann $\mathrm{S}^{1}$, Kammerer $\mathrm{F}^{1}$, Allmendinger $\mathrm{T}^{2}$, Hammon $\mathrm{M}^{1}$, Brand $\mathrm{M}^{1}$, May $M^{1}$, Lell $M^{3}$, Uder $M^{1}$, Kramer $M^{1}$

Institut 1 Universitätsklinikum Erlangen, Radiologisches Institut, Erlangen;

2 Siemens Healthineers, CT Development, Forchheim; 3 Klinikum Nürnberg, Institut für Radiologie und Nuklearmedizin, Nürnberg

DOI 10.1055/s-0037-1600432

Zielsetzung Iterative Rekonstruktionen (IR) bieten Potential zur CT-Dosisreduktion durch Verringerung des Bildrauschens. Hierbei gilt es, die optimale Balance zwischen Dosisreduktion und Bildqualität zu ermitteln. Qualitative Bildanalysen erfolgen oft mittels Likert-Skalen, deren ordinaler Charakter jedoch zahlreiche statistische Auswertungen und damit exakte Berechnungen verbietet. Diese Studie diente der Entwicklung einer neuartigen Methode zur vergleichenden Bildbeurteilung mit Möglichkeiten zur exakten statistischen Auswertung, verdeutlicht an CTA des Circulus arteriosus Willisii (CAW).

Material und Methodik In 3000 verblindeten paarweisen Vergleichen wurden dosisreduzierte IR CTA des CAW von 5 Auswertern mit Volldosis gefilterten Rückprojektionen (FBP) des gleichen axialen Schnittes verglichen, die Auswerter sollten jeweils das Bild mit der besseren Gefäßdefinition bestimmen. Die Bilder unterschieden sich in der Größe des zu bewertenden Gefäßes (groß) mittel/klein), des Dosisreduktionslevels und der IR-Stärke (SAFIRE 3 vs. 5). Durch Ermittlung der Auswerterpräferenz von Volldosis-FBP- zu IR-Bildern für jedes Dosislevel wurden Regressionskurven berechnet, aus denen das Dosislevel bestimmt werden konnte, bei dem die Auswerter dosisreduzierte IR- und Volldosis-FBP-Bilder bezüglich der Gefäßdefinition als äquivalent werteten.

Ergebnisse Für den CAW zeigten sich signifikant unterschiedliche Abhängigkeiten des Dosisreduktionspotentials sowohl von der Gefäßgröße als auch von der Stärke der IR (alle $p<0.0001$ ). Große mit SAFIRE 5 rekonstruierte Gefäße boten mit $84 \%$ das höchste Potential, kleine mit SAFIRE 3 rekonstruierte Gefäße bis zu $40 \%$.

Schlussfolgerungen Diese Studie präsentiert eine neuartige Methode zur vergleichenden Bildbeurteilung, die im Gegensatz zu Likert Skalen elaborierte statistische Analysen erlaubt. Exemplarisch an CTA des CAW verdeutlicht erfolgte die Bestimmung des CT-Dosisreduktionspotentials von IR, durch die sich je nach Gefäßsituation und Fragestellung deutliche Reduktionen der Strahlendosis erreichen ließen.

\section{WISS 209.8 Etablierung eines Niedrigdosis- Abdomen-CT Protokolls anhand eines Schweinemodells und Alderson Phantoms mit einem Dual-Source-CT-Scanner der dritten Generation}

Autoren Jagoda $\mathrm{P}^{1}$, Ziegenhain $\mathrm{F}^{1}$, Bell $\mathrm{K}^{2}$, Dzierma $\mathrm{Y}^{2}$, Bücker $\mathrm{A}^{1}$, Minko $\mathrm{P}^{1}$ Institut 1 Universitätsklinik des Saarlandes, Klinik für Diagnostische und Interventionelle Radiologie, Homburg; 2 Universitätsklinik des Saarlandes, Klinik für Strahlentherapie und Radioonkologie, Homburg

DOI 10.1055/s-0037-1600433

Zielsetzung Evaluierung der effektiven Dosis und Bildqualität eines Niedrigdosis-Abdomen-CT Protokolls in einem Schweinemodell und Alderson Phantom mittels eines Dual-Source-CT-Scanners der dritten Generation (Siemens Force).

Material und Methodik Es wurden fünf unterschiedliche Niedrigdosisprotokolle im Schweinemodell untersucht (CareKV, Sn-Filter, Flash-mode PITCH 3,2 und 1,9, Dual-source). Dabei erfolgte eine graduelle Optimierung der Protokolle mit dem Ziel der geringsten möglichen Strahlendosis in Verbindung mit einer weiterhin bestehenden diagnostischen Aussagekraft. Anhand der „European Quality Criteria“ wurden die Bilddatensätze von zwei Radiologen als diagnostisch eingestuft. Anschließend erfolgte mithilfe eines 3-Punkte-Score eine subjektive Beurteilung des Bildrauschens und der Auflösung. Objektiv wurde das SNR und CNR ermittelt. An zehn definierten anatomischen Lokalisationen wurden TLDs intraabdominell platziert und die resultierende Energiedosis in den fünf Protokollen gemessen. Zur Evaluierung der effektiven Dosis der fünf Protokolle wurden die TLD-Messungen in einem Alderson-Phantom wiederholt.

Ergebnisse Alle fünf Protokolle erzielten eine diagnostisch verwertbare Bildqualität. Die vom CT-Scanner kalkulierte und die mittels TLD Messungen ermittelte Dosis zeigte, dass das Dual-Source Protokoll die signifikant niedrigste Dosis unter den fünf Protokollen aufwies (DLP: 33,5 mGy* $\mathrm{cm}$ und mediane Dosis: 478,89 $\pm 229,93 \mu S v$; $p<0,001)$. Die Literaturangaben für eine Röntgenaufnahme des Abdomens umfassen einen Bereich von 0,2-1,1 mSv. Die am Alderson-Phantom durchgeführten Messungen zeigten für alle 5 Protokolle eine effektive Dosis < 0,34 mSv.

Schlussfolgerungen Ein Niedrigdosis-Abdomen-CT-Protokoll kann bei Verwendung eines Dual-Source-CT-Scanners der letzten Generation diagnostisch verwertbare Bilddatensätze mit einer Strahlendosis entsprechend einer Abdomenübersichtsaufnahme anfertigen.

\section{WISS 209.5 Anatomisch basierte Dosiskalkulation und Abschätzung des Malignomrisikos in der Ganzkörper-Computertomografie}

Autoren Kopp $M^{1}$, Löwe $T^{2}$, Wüst $W^{3}$, Schmidt $B^{4}$, Nitsch $W^{5}$, Uder $M^{3}$, May $\mathrm{M}^{3}$

Institut 1 Universitätsklinikum Erlangen, Radiologisches Institut, Erlangen;

2 Institut für Medizinische Mikrobiologie und Hygiene TU Dresden;

3 Radiologisches Institut Universitätsklinikum Erlangen; 4 Siemens

Healthineers $\mathrm{GmbH}$, Forchheim; 5 Fraunhofer Institut für integrierte Schaltungen, Erlangen

DOI 10.1055/s-0037-1600434

Zielsetzung Für die Abschätzung des Lebenszeitrisikos (LAR) der Malignominzidenz durch die Strahlenexposition in der Computertomografie (CT) wird die effektive Dosis (ED) benötigt. Deren Berechnung mittels Dosislängenprodukt (DLP) und populationsbezogenen Konversionsfaktoren $(k)$ gilt als ungenau. Ziel dieser Studie ist es, das LAR durch eine anatomisch basierte Abschätzung der Patientendosis zu personalisieren.

Material und Methodik Mittels Monte-Carlo-Simulationen (MC) wurde die Strahlendosis in 22 Ganzkörper CT-Untersuchungen berechnet und durch manuelle Segmentierungen zu Organdosen zusammengefasst. Die EDMC wurde als Summe aller in der ICRP 103 gewichteten Organdosen bestimmt. Referenzwerte wurden durch Multiplikation des DLP mit k=0,015 mSv/mGycm berechnet (EDDLP). Das LAR wurde mittels ED und Daten aus dem B IR VII Report bestimmt. Aus dem Patientenkollektiv (5 Frauen, 17 Männer) wurde retrospektiv je ein Fall-Kontroll-Matching entsprechend Alter, Body-Mass-Index (BMI) und Geschlecht durchgeführt.

Ergebnisse Die mittlere EDMC der Ganzkörper CT war signifikant niedriger $(11,6 \pm 1,5 \mathrm{mSv})$ als die EDDLP $(13,2 \pm 4,5 \mathrm{mSv}, \mathrm{p}=0,048)$. Das Fall-KontrollPaar mit ungleichem Alter (36 und 67 Jahre) hatte ein ähnliches $\operatorname{EDDLP}(11,7$ und 11,0 mSv) und EDMC (10,9 und 10,0 mSv). Das LAR des jüngeren Patienten war um $85 \%$ höher $(72 * 10-5$ und $39 * 10-5)$. Bei ungleichem BMI $(29,9$ und $40,0 \mathrm{~kg} / \mathrm{m} 2)$ war der Unterschied in der EDDLP (15,0 und 26,3 mSv) deutlich größer als in der EDMC (11,9 und 13,8 mSv) und im LAR (29*105 und $\left.31^{*} 10-5\right)$. Bei unterschiedlichem Geschlecht war das EDDLP vergleichbar (9,5 und 9,5 mSv), jedoch zeigte sich eine um 20\% höhere EDMC (10,3 und $12,3 \mathrm{mSv}$ ) und ein um $40 \%$ höheres LAR (57*10 - 5 und $79 * 10-5$ ) bei der Frau.

Schlussfolgerungen Individuelle anatomisch basierte Dosiskalkulationen liefern insbesondere bei Frauen und bei Abweichungen vom durchschnittlichen 
BMI signifikante Unterschiede im Vergleich zur Referenzmethode. Das daraus berechnete LAR unterscheidet sich stark in Abhängigkeit von Geschlecht und Patientenalter.

\section{WISS 209.9 Effektivität der Kombination verschiedener Strahlenschutzmittel in der Interventionellen Radiologie}

Autoren König $A^{1}$, Etzel $R^{2}$, Keil $B^{2}$, Fiebich $M^{2}$, Mahnken $A^{1}$ Institut 1 Philipps-Universität Marburg, Diagnostische und Interventionelle Radiologie, Marburg; 2 Technische Hochschule Mittelhessen, Institut für Medizinische Physik und Strahlenschutz, Gießen

DOI 10.1055/s-0037-1600435

Zielsetzung In der interventionellen Radiologie ist das medizinische Personal einer nicht unerheblichen Strahlendosis ausgesetzt, welche vor allem aus Streustrahlung besteht. Um die Strahlenexposition zu vermindern, sind verschiedene Strahlenschutzmittel verfügbar, welche in dieser Studie auf ihre Effektivität hin untersucht wurden.

Material und Methodik Die Messungen wurden an einer klinischen Angiografie-Anlage (Artis Zee, Siemens Healthcare, Erlangen) unter verschiedenen Röntgenröhrenangulationen durchgeführt. Als Streukörper diente ein Thoraxphantom (Alderson RANDO Phantom, Alderson Research Laboratories Inc., Stanford, CT, USA). Die Strahlendosis wurde mittels einer lonisationskammer (U-Mo, Berthold, Bad Wildbad) an fünf repräsentativen Höhen an der Position eines Untersuchers (Augenlinse, Schilddrüse, Thorax/Lunge, Gonaden, Unterschenkel) gemessen. Der „Untersucher“ wurde am Patiententisch für einen Zugang zur A. femoralis positioniert. Folgende Strahlenschutzmittel wurden miteinander verglichen: Bleiglasscheibe (BGW: 0,5 mm), am Patiententisch montierter Bleilappen (BGW: 0,5 mm), Bleiweste und -rock (BGW vorne: 0,35 mm; BGW hinten: 0,25 mm) mit Schilddrüsenschutz (BGW: 0,5 mm) und frei hängendes Ganzkörperschutzsystem (Zero-Gravity, Biotronik, Berlin) (BGW Schürze: $1 \mathrm{~mm}$; BGW Acrylglas-Kopfschutz: 0,5 mm)

Ergebnisse Die Ergebnisse zeigen bei optimaler Verwendung der Strahlenschutzmittel im Mittel eine Reduktion der Strahlendosis um 91\%. Auf Höhe der Augenlinse konnte eine Reduktion der Strahlendosis um 97\%, in Schilddrüsenhöhe um 93\%, auf Thoraxhöhe um 98\%, auf Gonadenhöhe um 99\% und auf Unterschenkelhöhe um $68 \%$ gemessen werden. Auf Höhe der Augenlinse kann allein durch die Verwendung der Bleiglasscheibe und des Bleilappens eine Reduktion der Strahlendosis um $88 \%$ erzielt werden.

Schlussfolgerungen In dieser Studie konnte gezeigt werden, dass die Kombination aus verschieden Strahlenschutzmittel eine Strahlendosisreduktion von bis zu 99\% erzielen kann.

\section{WISS 209.2 Auswirkungen der neuen Diagnostischen Referenzwerte auf das Mammografie-Screening in Nordrhein-Westfalen}

\author{
Autoren Sommer $\mathrm{A}^{1}$, Kräling $\mathrm{T}^{2}$, Heindel $\mathrm{W}^{2}$, Lenzen $\mathrm{H}^{2}$ \\ Institut 1 Universitätsklinikum Münster, Referenzzentrum Mammografie \\ Münster, Münster; 2 Universitätsklinikum Münster, Institut für Klinische \\ Radiologie, Münster \\ DOI 10.1055/s-0037-1600436
}

Zielsetzung Im Juli 2016 hat das Bundesamt für Strahlenschutz neue diagnostische Referenzwerte (DRW) veröffentlicht. Der DRW für die Mammografie wurde von 2,5 auf 2,0 mSv (AGD) abgesenkt. In der vorliegenden Studie wurden die Auswirkungen des neuen DRW auf das Mammografie-Screening (MSP) für die unterschiedlichen Geräteklassen sowie das einzelne Gerät analysiert.

Material und Methodik Dafür wurden die Strahlenexpositionen im MSP in NRW anhand der vom Gerät angezeigten AGDs der CC- und MLO-Projektionen für den Zeitraum 01.01.-31.08.2106 ausgewertet $(\mathrm{N}=1.802 .623)$. Aufgrund des hohen Anteils an Exposition von DR-Systemen in dieser Analyse (>99\%) wurden hinsichtlich eines Vergleichs der Auswirkung auf CR-Systeme eine erweiterte Analyse ausschließlich mit diesen Geräten aus den Jahren 2013 2015 heranzogen. Insgesamt konnten so 327.285 Expositionen an CR-Systemen evaluiert werden.

Ergebnisse Die mittlere AGD aller Expositionen in der Analyse „2016“ betrug 1,44 mSv (ohne CR). Aufgeteilt nach Geräteklassen: 1,52 mSv (DR-FFDM) und 0,57 mSv (DR-Scan). In der nur für CR-Systeme durchgeführten erweiterten Analyse, betrug die mittlere AGD dieser Geräteklasse 1,66 mSv. Werden die Expositionen Hersteller abhängig aufgetragen, so ergibt sich für das ScanSystem (Philips) der niedrigste Wert (0,57 mSv) gefolgt vom FFDM-Systemen der Firma Siemens (1,18 mSv). Die DR-Systeme der Firma Hologic zeigten mit 1,79 mSv die höchsten Werte. Analysiert man die Ergebnisse bis auf die Geräte-Ebene, so liegen 13 Systeme oberhalb des neuen DRW von 2,0 mSv. Schlussfolgerungen Die Strahlenexposition im Screening in NRW liegt unabhängig von der Geräteklasse unterhalb des neuen DRW. Dies gilt überraschenderweise auch für die CR-Systeme. Weitere Analysen haben allerdings gezeigt, dass die angezeigte AGD bei CR-Systemen im Mittel ca. 20\% zu niedrig ausfällt. Es ist also davon auszugehen, dass es bei CR-Systemen vermehrt zu Überschreitungen des DRW kommen kann. An 13 Einzel-Systemen müssen Maßnahmen zur Reduktion der Strahlenexposition getroffen werden.

\section{WISS 209.1 Unsicherheitsbudget des CDMAM- Prüfverfahrens}

Autoren Sommer $\mathrm{A}^{1}$, Blendl $\mathrm{C}^{2}$, Lenzen $\mathrm{H}^{3}$

Institut 1 Universitätsklinikum Münster, Referenzzentrum Mammografie Münster, Münster; 2 Technische Hochschule Köln, Institut für Medien- und Phototechnik, Köln; 3 Universitätsklinikum Münster, Institut für Klinische Radiologie, Münster

DOI 10.1055/s-0037-1600437

Zielsetzung In der Mammografie werden hohe Anforderungen an die Bildqualität gestellt. Nach dem European Protocol for the Quality Control (EPQC) werden Kontrast-Detail-Prüfkörper (CDMAM) aufgenommen und visuell oder automatisiert ausgewertet. Das EPQC enthält keine Angaben zur Messunsicherheit und belastbare Angaben des Prüfkörper-Herstellers fehlen. Ziel dieser Studie war es die Gesamtunsicherheit (U) für das automatisierte und visuelle Verfahren abzuschätzen.

Material und Methodik Für die Bestimmung von $U$ wurden Teilunsicherheiten Ui bestimmt: bautechnische Mängel (Goldplättchen: Dicke und Fläche), Artefakte, Absorptionseigenschaften (Strahlungsqualität) und für das vis. Verfahren die der Auswerter. Die bautechnischen Ui wurden Anhand des BfS-Berichtes zur Bestimmung von Fertigungstoleranzen von Prüfkörpern ermittelt. Die dort festgestellten metrischen Schwankungen der Goldplättchen (GP) wurden durch Auswertung von CDMAM-Dosisreihen in relative Dosis-Abweichungen umgewandelt.

Ergebnisse Für die einzelnen Ui (in $\Delta$ logK) ergibt sich (2 $\sum$-Bereich): 0,03 (Absorption); 0,12 (GP-Dicke); 0,12 (GP-Durchmesser) und 0,10 (vis. Verfahren). Für das auto. Verfahren ergibt sich ein $U$ von 0,172 und für das vis. Verfahren von 0,20. Zusätzlich gilt, dass die Nenngrößen für Dicke und Durchmesser systematisch unterschritten werden, d.h. die realen Prüfanforderungen sind strenger als im EPQC festgelegt.

Schlussfolgerungen Die bautechnischen Mängel dominieren das $U$ des CDMAM Verfahrens. Die Prüfung kann am gleichen System mit unterschiedlichen Prüfkörpern zu abweichenden Ergebnissen und bei Grenzwertnahen zu einem falsch-positiven Resultat führen. Eine Trennung zwischen den Limits acchievable und acceptable ist nur mit dem automatisierten Verfahren möglich. Aufgrund unserer Analyse ist die Frage zu stellen, ob Prüfungen mit einem derart hohen $U$ für Abnahme- und Konstanzprüfungen geeignet sind. Sie sollten durch rein physikalische Prüfverfahren ersetzt werden, bei denen bautechnische Unsicherheiten des Prüfkörpers nur geringe Beiträge zu U liefern. 
Thoraxradiologie

\section{WISS 208.3 Metallartefaktreduktion in der postoperativen Thorax CT: Evaluation von drei dezidierten iterativen Algorithmen}

Autoren Aissa $]^{1}$, Boos $J^{1}$, Sawicki $L^{1}$, Heinzler $N^{1}$, Kröpil $P^{1}$, Antoch $G^{1}$, Thomas $\mathrm{C}^{1}$

Institut 1 Universitätsklinikum Düsseldorf, Institut für Diagnostische und Interventionelle Radiologie, Düsseldorf

DOI 10.1055/s-0037-1600438

Zielsetzung Das Ziel dieser Studie war es, den Einfluss dreier iterativer Algorithmen zur Metallartefaktreduktion auf die Bildqualität in der Computertomografie (CT) von Patienten mit thorakalen Metallimplantaten zu evaluieren. Material und Methodik Siebenundzwanzig Patienten $(68,1 \pm 11,1$ Jahre, 22 männlich/5 weiblich) mit thorakalen Metalimplantaten und einer postoperativen Kontroll-CT wurden retrospektiv eingeschlossen. Die Bilder wurden mit drei Metallartefaktreduktionsalgorithmen (MAR-Algo1: Herz-Algorithmus, MAR-Algo2: Schrittmacher-Algorithmus, MAR-Algo3: Coil-Algorithmus) sowie mittels gefilterter Rückprojektion (FBP) rekonstruiert, wobei FBP als Referenzstandard diente. Artefakte wurden subjektiv als mild oder schwerwiegend klassifiziert. Die Bildqualität wurde subjektiv auf einer 5-Punkt-Skala bewertet (1: schwere Artefakte, nicht diagnostisch; 5: keine Artefakte, exzellente Bildqualität). Zur objektiven Beurteilung der Bildqualität wurde die Artefaktstärke (in Hounsfield Units, HU) durch ROI-Messungen ermittelt.

Ergebnisse Alle drei iterativen Algorithmen zur Metallartefaktreduktion verminderten signifikant die Artefaktstärke verglichen mit FBP objektiv (MARAlgo1: 58,9 $\pm 36,0 \mathrm{HU}, \quad$ MAR-Algo2: $52,7 \pm 43,9 \mathrm{HU}, \quad$ MAR-Algo3: 51,9 $\pm 44,1 \mathrm{HU}$; FBP 91,6 \pm 78,3HU, $\mathrm{p}<0,01$ ) und subjektiv (MAR-Algo1: 3,8 $\pm 0,1$, MAR-Algo2 4,3 $\pm 0,8$ und MAR-Algo3: 4,2 \pm 0.7 , FBP $3.4 \pm 1,3 p<0,01)$. MARAlgo2 und MAR-Algo3 reduzierten milde Artefakte effizienter (MAR-Algo1: $3,8 \pm 0,5$, MAR-Algo2 and 3: 4,5 \pm 0.5 , FBP $3.6 \pm 0.5 p<0,01$ ) verglichen mit MAR-Algo1 und FBP $(p<0,01)$. Für schwere Artefakten waren die drei Algorithmen gleichwertig.

Schlussfolgerungen Alle drei hier untersuchten iterativen Algorithmen zur Metallartefaktreduktion führten zu einer verbesserten Bildqualität und verminderten Artefakten verglichen mit FBP in der postoperativen Thorax CT bei Patienten mit thorakalen Metallimplantaten. MAR-Algo2 und MAR-Algo3 waren dabei optimal zur Reduktion milder Artefakte.

\section{WISS 314.2 Computertomografische Varianten der Lungenfibrose bei Patienten mit mikroskopischer Polyangiitis}

Autoren Both $\mathrm{M}^{1}$, Daum $\mathrm{C}^{1}$, Holle $\mathrm{J}^{2}$, Fritzer $\mathrm{E}^{3}$, Jansen $\mathrm{O}^{1}$, Henes $\mathrm{F}^{4}$ Institut 1 Universitätsklinikum Schleswig-Holstein, Campus Kiel, Klinik für Radiologie und Neuroradiologie, Kiel; 2 Rheumazentrum Schleswig-Holstein Mitte, Neumünster; 3 Universitätsklinikum Schleswig-Holstein, Campus Kiel, Insitut für Medizinische Informatik und Statisik, Kiel; 4 Universitätsklinikum Hamburg Eppendorf, Klinik für Diagnostische und Interventionelle Radiologie, Hamburg

DOI 10.1055/s-0037-1600439

Zielsetzung Ziel dieser Studie war es, bei Patienten mit mikroskopischer Polyangiitis unterschiedliche computertomografische Muster fibrosierender Lungengerüstveränderungen zu identifizieren und diese mit der Lungenfunktionsdiagnostik zu vergleichen.

Material und Methodik Bei $n=58$ Patienten des Klinikums Bad Bramstedt, Klinik für Rheumatologie und Immunologie, mit der Diagnose einer mikroskopischen Polyangiitis (MPA), wurde mindestens eine thorakale CT durchgeführt. Die Auswertung durch zwei erfahrene Radiologen im Konsensus basierte auf einer semi-quantitativen Charakterisierung unterschiedlicher FibroseMuster auf definierten repräsentativen anatomischen Lokalisationen der Lun- ge. Typ und Ausdehnung der Fibrose wurden mit der Lungenfunktionsdiagnostik und anderen klinischen Parametern verglichen.

Ergebnisse Bei 16/58 Patienten waren in der CT fibrosierende Lungengerüstveränderungen nachzuweisen. Das häufigste Muster war eine gewöhnliche interstitielle Pneumonie (UIP) bei $n=8$ Patienten, in $n=2$ Fällen mit einem Emphysem assoziert im Sinne einer "combined pulmonary fibrosis and emphysma" (CPFE). "Airway enlargement with fibrosis" (AEF)-Muster wurde bei $n=6$ Paaienten nachgewiesen. Das Muster einer nicht-spezifischen interstitiellen Pneumonie (NSIP-Muster) lag in $n=2$ Fällen vor. Im Vergleich mit MPA-Patienten ohne Lungenfibrose war der Transferkoeffizient TLCO/VA bei Patienten mit UIP-Muster signifikant $(\mathrm{p}<0,01)$ erniedrigt.

Schlussfolgerungen Die pulmonale Fibrose bei Patienten mit mikroskopischer Plyangiitis beinhaltet Varianten wie CPFE und AEF. Ein UIP-Muster zeigte sich assoziiert mit einer reduzierten Lungenfunktion.

\section{WISS 314.6 Quantifizierung sauerstoffinduzierter hämodynamischer Veränderungen im Truncus pulmonalis von gesunden Probanden mittels zweidimensionaler Phasenkontrast-MRA}

Autoren Czerner $\mathrm{C}^{1}$, Winther $\mathrm{H}^{1}$, Zapf $\mathrm{A}^{2}$, Wacker $\mathrm{F}^{1}$, Vogel-Claussen $]^{1}$ Institut 1 Medizinische Hochschule Hannover, Institut für Diagnostische und Interventionelle Radiologie, Hannover; 2 Universitätsmedizin Göttingen, Institut für Medizinische Statistik, Göttingen

DOI 10.1055/s-0037-1600440

Zielsetzung Ziel dieser prospektiven Studie war die Untersuchung des Effekts von inhaliertem reinen Sauerstoff auf die pulmonale Hämodynamik mittels zweidimensionaler Phasenkontrast-MRA (2D PC-MRA).

Material und Methodik Bei 21 gesunden Probanden wurden Flussmessungen im Truncus pulmonalis mittels 2D PC-MRA (1,5 T) in freier Atmung und unter Atemanhalt durchgeführt. Es wurden Akzelerationszeit und -volumen, Maximal- und Minimalfläche, Flächenänderung, mittlere und maximale Durchschnittsgeschwindigkeit, Vorwärtsvolumen, Herzfrequenz sowie Blutdruck gemessen. Während der initialen Messung (Basis) atmeten die Probanden Raumluft. Nach Applikation einer geschlossen oronasalen Maske folgten drei weitere Messungen: unter Raumluft (Kontrolle), direkt nach Start der Oxygenierung mit 15l/min 100\% Sauerstoff (Oxygenierung) und nach 5 Minuten unter fortgesetzter Sauerstoffgabe (Sättigung). Die Daten wurden mit einem gemischten linearen Modell analysiert. Tukey-Kontraste mit adjustierten p-Werten wurden für die paarweisen Vergleiche verwendet.

Ergebnisse Die Inhalation von Sauerstoff beeinflusste im Vergleich zur Kontrolle mehrere hämodynamische Parameter. So sanken die mittlere Durchschnittsgeschwindigkeit (Atemanhalt: $p<0,01$ bei Oxygenierung und Sättigung, freie Atmung: $p=0,03$ bei Sättigung) und die maximale Durchschnittsgeschwindigkeit (Atemanhalt und freie Atmung: $p<0,01$ bei Sättigung). In freier Atmung kam es unter Sauerstoffgabe zu einer Zunahme von Akzelerationsvolumen ( $p=0,02$ bei Sättigung), maximaler Fläche ( $p=0,02$ bei Oxygenierung, $p=0,03$ bei Sättigung) und Flächenänderung ( $p=0,02$ bei Sättigung), während die minimale Fläche sowie das Vorwärtsvolumen keine messbaren Änderungen zeigten.

Schlussfolgerungen Die Inhalation von reinem Sauerstoff führt zu veränderter pulmonaler Hämodynamik, die mit 2D PC-MRA messbar ist. Die gezeigte Methode ermöglicht potenziell eine nichtinvasive Beurteilung der Vasoreaktivität z.B. im Rahmen der pulmonalen Hypertonie, die zukünftig für klinische Entscheidungen von Relevanz sein könnte. 


\section{WISS 314.3 Diagnose der Primären Ciliären Dyskinesie in der CT - Entwicklung eines einfachen Scores}

Autoren Dettmer $\mathrm{S}^{1}$, Vogel-Claussen $\mathrm{J}^{1}$, Ringshausen $\mathrm{F}^{2}$, Faschkami $\mathrm{A}^{1}$, Shin $\mathrm{H}^{1}$, Welte $\mathrm{T}^{2}$, Wacker $\mathrm{F}^{1}$, Rademacher $\mathrm{J}^{2}$

Institut 1 Medizinische Hochschule Hannover, Institut für Diagnostische und Interventionelle Radiologie, Hannover; 2 Medizinische Hochschule Hannover, Klinik für Pneumologie, Hannover

DOI 10.1055/s-0037-1600441

Zielsetzung Bei der Primären Ciliären Dyskinesie (PCD) kommt es genetisch bedingt zu einem abnormalen Zilienschlag, einer reduzierten mukoziliären Clearance und zur Entwicklung von Bronchiektasen. Die Diagnostik ist mit Elektronen- und Videomikroskopie sehr aufwendig. Ziel dieser Studie war es 1. charakteristische CT-Befunde bei Patienten mit PCD im Vergleich zu Bronchiektasen anderer Genese zu beschreiben und 2. auf dieser Basis einen einfachen Score für PCD zu entwickeln.

Material und Methodik 111 CT-Untersuchungen von Patienten mit Bronchiektasen wurden für bronchiale Pathologien (nach Reif), assoziierte Befunde, Situs inversus und Z.n. Lappenresektionen gescored. Bronchiektasen-Patienten mit $(n=28)$ und ohne $(n=83)$ PCD wurden mittels Man-Withney-Test für ordinale und Chi-Quadrat-Test für binäre Größen verglichen. Basierend auf diesen Ergebnissen wurde ein Score für PCD entwickelt aus Situs inversus, mittel- und unterlappenbetonten Bronchiektasen, Z.n. Mittel- oder Unterlappenresektion sowie Mittel- oder Unterlapenatelektase. Für diesen Score wurde eine ROC-Analyse zur Unterscheidung von Bronchiektase-Patienten mit und ohne PCD vorgenommen.

Ergebnisse Bronchiektasen bei Patienten mit PCD kamen häufiger in den Mittel- und Unterlappen $(p=0.046)$ vor. Ausmaß und Schwere der Bronchiektasen waren signifikant geringer in den Oberlappen $(p<0.001)$ und signifikant stärker in Mittellappen und Lingula (bis $p=0.041$ ) ausgeprägt. Emphysem $(p=0.031)$ und intralobuläre Linien $(p<0.001)$ waren seltener bei PCD. Atelektasen des Mittel- oder Unterlappens $(p=0.002)$ sowie ein Z.n. Resektion des Mittel- oder Unterlappens $(p=0.015)$ waren häufiger bei PCD. Die ROCAnalyse für den PCD-Score war mit einer Fläche unter der Kurve von 0.815 signifikant $(p<0.001)$ zur Unterscheidung von Bronchiektasen-Patienten mit und ohne PCD.

Schlussfolgerungen $\mathrm{CT}$ bei Bronchiektasen-Patienten mit und ohne PCD unterscheiden sich deutlich. Der entwickelte Score für PCD ist aufgrund seiner Einfachheit als diagnostisches Tool für PCD in der alltäglichen Praxis geeignet.

\section{WISS 314.4 Time to exhale: Evaluierung des diagnostischen Mehrwerts von Thorax CT Untersuchungen in Expiration bei Patienten mit COPD - Ergebnisse der CType Studie}

Autoren Gawlitza J ${ }^{1}$, Michels $\mathrm{J}^{2}$, Borggrefe $\mathrm{M}^{2}$, Schönberg $\mathrm{S}^{1}$, Akin $\mathrm{I}^{2}$, Saur $\mathrm{J}^{2}$, Trinkmann $\mathrm{F}^{2}$, Henzler $\mathrm{T}^{1}$

Institut 1 Universitätsmedizin Mannheim, Institut für klinische Radiologie,

Mannheim; 2 Universitätsmedizin Mannheim, 1. medizinische Klinik,

Mannheim

DOI 10.1055/s-0037-1600442

Zielsetzung Die leitliniengerechte Diagnostik der chronisch obstruktiven Lungenerkrankung (COPD) basiert neben klinischen Kriterien primär auf dem spirometrischen Nachweis einer Atemwegsobstruktion. Hierdurch wird jedoch die komplexe Pathophysiologie der COPD nur unzureichend abgebildet. Während die quantitative Computertomografie (qCT) in Inspiration bereits evaluiert wurde ist der zusätzliche diagnostische Nutzen von qCT Untersuchungen in Exspiration nur unzureichend untersucht. Ziel dieser prospektiven Studie war die systematische Evaluierung von qCT Untersuchungen des Thorax in maximaler Inspriation, maximaler Expiration sowie deren delta Werte im Vergleich zu Lungenfunktionsparametern.
Material und Methodik 46 Patienten mit bekannter COPD erhielten eine Bodyplethysmografie sowie zwei native dosisoptimierte Thorax CT Untersuchungen in maximaler In- bzw. Exspiration an einem Dual-Source-Scanner der 3. Generation (Somatom Force). Im Anschluss wurden Lungenvolumen (Vol), mittlere Lungendichte (MLD), Emphysemanteil (LAV) sowie ein Dichtedistributionsparameter (FWHM) berechnet. Diese qCT-Parameter aus In-, Exspiration und delta-Werten wurden mit sieben Lungenfunktionsparametern korreliert.

Ergebnisse In der Inspiration ließen sich schwache bis moderate Korrelationen des Vol, der MLD und des LAV mit der Lungenfunktion nachweisen. Diese konnten durch Hinzunahme der Exspirationsaufnahmen signifikant gesteigert werden $(p<0,05)$. Die FWHM korrelierte ausschließlich in den qCT-Parametern der Exspiration sowie den delta-Werten signifikant. Die übrigen delta-Werte korrelierten noch stärker mit den Lungenfunktionsparametern.

Schlussfolgerungen Exspirationsaufnahmen bieten gegenüber den etablierten Inspirationsaufnahmen zusätzliche diagnostische Informationen bei Patienten mit COPD. Nebst stärkerer Korrelationen, erlaubt der Scan in Exspiration die Berechnung der delta-Werte. Diese delta-Parameter zeigten noch stärkere Zusammenhänge mit den Lungenfunktionswerten und sollten auf ihren potentiellen Zusatznutzen weiter evaluiert werden.

\section{WISS 208.8 Strukturierte HRCT-Befundung von lungentransplantierten Patienten}

Autoren Hesse $\mathrm{N}^{1}$, Dinkel $\mathrm{J}^{1}$, Kneidinger $\mathrm{N}^{2}$, Plum $\mathrm{J}^{1}$, Sommer $\mathrm{W}^{1}$, Reiser $\mathrm{M}^{1}$, Ceelen $\mathrm{F}^{3}$

Institut 1 Klinikum der Universität München, Institut für klinische Radiologie, München; 2 LMU, Medizinische Klinik IV; 3 LMU, Medizinische Klinik IV, München

DOI $10.1055 / \mathrm{s}-0037-1600443$

Zielsetzung Evaluation der strukturierten HRCT- Befundung von lungentransplantierten Patienten in der klinischen Routine.

Material und Methodik Es wurden konventionelle und strukturierte Befunde für 49 lungentransplantierte Patienten erstellt, die im Rahmen der klinischen Routine ein HRCT bekommen haben. Das Template wurde auf die Berichterstattung von typischen Veränderungen, die im Rahmen eines akuten oder chronisches Transplantatversagens auftreten, ausgerichtet und versucht eine Differenzierung zwischen den CLAD-Subtypen (RAS/BOS). Die konventionellen Befunde waren Standard Freitextbefunde. Zwei Pulmologen bewerteten auf einer Skale von 1 (ungenügend) bis 5 (sehr gut) formelle und inhaltliche Aspekte der Befunde. Die Qualität des Befundes wurde abschließend mit einer Schulnote von 1 bis 6 bewertet. Basierend auf klinischen und radiologischen Information mussten die Kliniker hypothetisch die Therapie ändern und ggf. weitere Diagnostik anordnen. Mittels Wilcoxon- und Mc-Nemar-Test wurde getestet, ob ein signifikanter Unterschied vorlag.

Ergebnisse Strukturierte vs. konventionelle Befundung erzielt in allen Kategorien beim Vergleich der Mittelwerte bessere Bewertungen $(p<0.001)$. Übersichtlichkeit 4,7 vs. 2,9; Verständlichkeit 4,8 vs. 3,5; Beantwortung der klinischer Fragestellung 4,4 vs. 3,5, Differenzierung zwischen CLAD-Subtypen 3,9 vs. 2,9. Die strukturierten Befunde wurden im Mittel "gut" und die konventionellen Befunde „befriedigend“ bewertet (1,5 vs. 3,4). Es ergab sich kein signifikanten Unterschied zwischen den Gruppen für das weiter diagnostische Prozedere und die Therapie der Patienten.

Schlussfolgerungen Pulmologen bewerten die strukturierte HRCT-Befundung von lungentransplantierten Patienten als besser verständlich und inhaltlich stärker als die konventionelle Befundung. 


\section{WISS 208.6 Spectral-Detector-CT bei Staging Untersuchungen des Thorax: Erste Erfahrungen zur Kontrastmittelreduktion mittels monoenergetischer Rekonstruktionen}

\author{
Autoren Hickethier $\mathrm{T}^{1}$, Kroeger $\mathrm{J}^{1}$, Baeßler $\mathrm{B}^{1}$, Doerner $\mathrm{J}^{1}$, Maintz $\mathrm{D}^{1}$, \\ Chang $D^{1}$ \\ Institut 1 Uniklinik Köln, Institut für Diagnostische und Interventionelle \\ Radiologie, Köln \\ DOI 10.1055/s-0037-1600444
}

Zielsetzung Bei CT-Untersuchungen ist die Kontrastmittelgabe ein potentielles Risiko für den Patienten. Eine Reduktion der Kontrastmittelmenge bedingt allerdings bei Thoraxuntersuchungen in der parenchymalen Kontrastmittelphase eine Verschlechterung des mediastinalen Weichteilkontrasts, insbesondere zwischen Gefäßen und hilären Lymphknoten. Diese Studie untersucht das Potential monoenergetischer Rekonstruktionen (MonoE) eines neuen SpectralDetector-CT (SDCT) zur Bildqualitätsverbesserung bei deutlich reduzierter Kontrastmittelmenge.

Material und Methodik 13 Patienten erhielten im Rahmen eines Stagings eine CT-Thoraxuntersuchung an einem SDCT (IQon, Philips, $120 \mathrm{kV}$, automatische Dosismodulation) mit von 60 auf $35 \mathrm{ml}$ reduzierter Kontrastmittelmenge (350 mg lod/ml). Die Bilddaten wurden mit herkömmlicher statistischiterierten Rekonstruktionen (IR) und MonoE bei $40 \mathrm{keV}$ berechnet. Retrospektiv wurde die Bildqualität objektiv mittels Kontrast-zu-Rausch Verhältnis (CNR) eines hilären Lymphknoten (LN) sowie dessen Dichteunterschied zur angrenzenden Pulmonalarterie und subjektiv anhand einer 5 Punkte Likert-Skala (von 1 = nicht auswertbar bis 5 = exzellent) von 2 Radiologen hinsichtlich allgemeiner diagnostischer Akzeptanz sowie Kontrastierung und Abgrenzbarkeit von Lymphknoten bewertet.

Ergebnisse Die subjektive Bildqualität wurde sowohl insgesamt (QOA) als auch hinsichtlich der Beurteilbarkeit von hilären Lymphknoten (QLN) bei IR nur mäßig bewertet und konnte durch MonoE signifikant verbessert werden (QOA 4,2 vs. 3,1; $\mathrm{p}<, 01 / \mathrm{QLN}: 4,0$ vs. 2,8; $\mathrm{p}<, 01 /$ Inter-rater agreement: Kappa 0,78-0,86). Das CNR sowie der Dichteunterschied zwischen Lymphknoten und Pulmonalarterie (DLN) waren bei MonoE signifikant höher als bei IR (CNR 14,85 vs. 5,96; $\mathrm{p}<, 01$ /DLN 124,85 vs. 43,85 HU; $\mathrm{p}<, 01$ ).

Schlussfolgerungen Bei CT Staging Untersuchungen des Thorax mit deutlich reduzierter Kontrastmittelmenge lassen sich durch die Nutzung von SDCTbasierten MonoE sowohl der subjektive Bildeindruck als auch objektive Bildqualitätskriterien signifikant verbessern.

\section{WISS 314.7 Vergleich der nativen und kontrastmittelverstärkten MRT der pulmonalarteriellen Strombahn zur Detektion pulmonaler AV-Malformationen (PAVM) bei Patienten mit M. Osler}

\author{
Autoren Jagoda $P^{1}$, Stroeder $]^{1}$, Massmann $A^{1}$, Bücker $A^{1}$, Schneider $G^{1}$ \\ Institut 1 Universitätsklinik des Saarlandes, Klinik für Diagnostische und \\ Interventionelle Radiologie, Homburg \\ DOI 10.1055/s-0037-1600445
}

Zielsetzung Das Ziel dieser Studie war der Vergleich der kontrastmittelverstärkten MR-Angiografie mit einer nativen MRT-Sequenz (SPACE) zur Detektion von PAVM bei Patienten mit M. Osler.

Material und Methodik Bei 122 Patienten mit M. Osler wurden im Zeitraum von 2011 bis 2015 insgesamt 188 MRT-Untersuchungen durchgeführt. Bei jeder Untersuchung wurden eine native SPACE/3D TSE-Sequenz (TR 4,733 ms/TE $101 \mathrm{~ms} /$ FLIP $150^{\circ}$ ) und eine kontrastmittelverstärkte 3D GRE Sequenz (TR 2,87 ms/TE 1,07 ms/FLIP $25^{\circ}, 0,1 \mathrm{mmol} / \mathrm{kg}$ Körpergewicht MultiHance ${ }^{\circledR}$ (Bracco)) akquiriert. Beide Sequenzen wurden von zwei erfahrenen Radiologen begutachtet und die Anzahl der detektierten AV-Malformationen im gegenseitigen Einvernehmen erfasst. Die kontrastmittelverstärkten Se- quenzen wurden immer zuerst, die SPACE 6 Wochen später, untersucht. Die Katheterangiografien (falls durchgeführt) und die kontrastverstärkten MRT (falls keine Angiografie durchgeführt wurde) dienten als Goldstandard. Ein t-test wurde zur statistischen Evaluation genutzt.

Ergebnisse Vier Untersuchungen wurden aufgrund von in beiden Sequenzen aufgetretenen Atemartefakten ausgeschlossen. Bei 35 Patienten wurden insgesamt 60 therapiebedürftige AV-Malformationen entdeckt. Mittels der kontrastverstärkten Sequenz wurden zwar insgesamt mehr AV-Malformationen ermittelt (166 vs. 96, p <0.001), es wurde jedoch keine therapiebedürftigen AV-Malformation mit der SPACE übersehen.

Schlussfolgerungen Die Detektion klinisch relevanter PAVM kann, beispielsweise bei Patienten mit Kontraindikationen für i.v. Kontrastmittel (z. B. Schwangerschaft), auch sicher mittels nativer Aufnahmen unter Nutzung der SPACE Sequenz erfolgen.

\section{WISS 314.5 Validierung der ventilations-gewichteten Fourier Dekompositions MRT bei COPD Patienten zur Messung der regionalen Lungenfunktion}

\section{Autoren Kaireit $\mathrm{T}^{1}$, Voskrebenzev $\mathrm{A}^{1}$, Gutberlet $\mathrm{M}^{1}$, Freise $\mathrm{J}^{2}$, Welte $\mathrm{T}^{3}$,} Wacker $F^{1}$, Vogel-Claussen J ${ }^{1}$

Institut 1 Medizinische Hochschule Hannover, Institut für Diagnostische und Interventionelle Radiologie, Hannover; 2 Medizinische Hochschule Hannover, Klinik für Pneumologie, Hannover; 3 Medizinische Hochschule Hannover, Klinik für Pneumologie, Hannover

DOI 10.1055/s-0037-1600446

Zielsetzung Die klinische Beurteilung des Krankheitsverlaufes der COPD Patienten erfolgt im Wesentlichen durch den Lungenfunktionstest. Dieser ist jedoch nur ein globaler Test für die Lungenfunktion. In den letzten Jahren ist daher die Evaluierung der regionalen Lungenfunktion für ein verbessertes Monitoring von COPD Patienten von zunehmendem Interesse. Regionale Ventilationsdarstellung mittels hyperpolarisierten Gasen und Fluor-Sauerstoffgemischen im MRT ist beschrieben worden, jedoch technisch aufwendig und kostspielig. Kostengünstiger und technisch einfacher ist die regionale Ventilationsanalyse mittels Fourier Dekomposition (FD-MRT). Ziel dieser Studie war es, zu evaluieren, ob die regionale fraktionelle Ventilation der FD-MRT mit der dynamischen Fluorgas-MRT und dem Lungenfunktionstest korreliert.

Material und Methodik Acht Patienten mit COPD wurden im 1,5 T MRT untersucht. 4 repräsentative koronare Schichten der Lunge wurden mit einer 2D GRE FLASH Sequenz in freier Atmung akquiriert. Nach Anwendung eines Registrierungsalgorithmus wurde die fraktionelle Ventilation (FV) errechnet. Zusätzlich wurde die regionale Ventilation mittels Fluor-Washout nach der Inhalation von 30I eines Gasgemisches mit 79\% Fluorgas (C3F8) und 21\% Sauerstoff untersucht und korrespondierende Schichten ausgewählt. Auch wurde ein Lungenfunktionstest durchgeführt. Die ermittelten Messwerte wurden mittels Spearman-Korrelation verglichen.

Ergebnisse Sowohl die mittels FD-MRT ermittelte regionale fraktionelle Ventilation als auch die mittels Fluor ermittelte fraktionelle Ventilation korrelierten mit der FEV1: FV-FD vs. FEV1 $r=0,76 p=0,03$; FV-Fluor vs. FEV1 $r=0,81 p=0,02$ ). Die mittels FD-MRT ermittelte regionale fraktionelle Ventilation als auch die mittels Fluor ermittelte fraktionelle Ventilation korrelierten ebenfalls signifikant: FV-FD vs. FV-Fluor $r=0,93 p=0,0009$.

Schlussfolgerungen Die ventilationsgewichtete FD-MRT ermöglicht eine kostengünstige und strahlungsfreie regionale Quantifizierung der fraktionellen Ventilation bei Patienten mit COPD. 


\section{WISS 314.8 Funktionelle native Lungen-MRT (SENCEFUL) zur Ermittlung pulmonaler Perfusionsdefizite bei Cystischer Fibrose}

Autoren Kunz $A^{1}$, Weng $A^{1}$, Wirth $C^{1}$, Kestler $C^{1}$, Hebestreit $H^{2}$, Segerer $F^{2}$, Köstler $\mathrm{H}^{1}$, Bley $\mathrm{T}^{1}$, Veldhoen $\mathrm{S}^{1}$

Institut 1 Universitätsklinikum Würzburg, Institut für Diagnostische und Interventionelle Radiologie, Würzburg; 2 Universitätsklinikum Würzburg,

Kinderklinik und Poliklinik, Würzburg

DOI 10.1055/s-0037-1600447

Zielsetzung Identifikation pulmonaler Perfusionsdefizite bei cystischer Fibrose (CF) mittels funktioneller Lungen MRT in SENCEFUL (SElf-gated Non-ContrastEnhanced FUnctional Lung Imaging) Technik.

Material und Methodik Die native SENCEFUL-MRT erfolgte bei 1,5T bei 19 Patienten mit gesicherter CF und 19 gesunden Probanden in freier Atmung. Rekonstruierte farbkodierte pulmonale Organkarten in koronarer Schichtführung zeigten semiquantitative Werte für Lungenperfusion und Perfusionsphase (d.h. zeitlich aufgelöste Blutflüsse). 4 geblindete Auswerter sichteten 1644 Karten von jew. $10 \mathrm{~mm}$ Schichtdicke. Die Lungen wurden auf jeder Karte Quadranten-basiert nach einer 4-Punkt-Likert-Skala bewertet ( $1=$ gesund, $2=$ Defekt von $<25 \%$ der Quadrantenfläche, $3=$ Defekt von $>50 \%, 4=$ Defekt von $>50 \%)$. Perfusions- und Phasekarten wurden unabhängig voneinander sowie abschließend in Zusammenschau bewertet. Die Werteverteilung der Perfusionsphase wurde in einem Histogramm aufgetragen und einem GaußFit unterzogen. „Peak-to-offset“-Verhältnisse wurden definiert als Maximum der gefitteten Kurve geteilt durch den Mittelwert der äußeren 10\% der Phasenwerte. Diese wurden den subjektiven Auswertungen der Reader gegenüber gestellt.

Ergebnisse In den Perfusionskarten wurden 56,5\% der Quadranten des CFKollektivs pathologische Scores zugewiesen vs. 25,3\% der Kontrollgruppe ( $p<0,05)$. In der Perfusionsphase wurden 56,1\% vs. 20,9\% mit pathologischen Ratings versehen $(p<0,05)$. Die Konsensus-Auswertung von Perfusions- und Phasenkarte ergab 53,6\% vs. 15,1\% ( $<<0,05)$. Der Intraklassen-Korrelationstest zeigte eine exzellente Übereinstimmung der Rater (Perfusion: 0,851; Phase: 0,805; Konsensus: 0,822). Die objektive Auswertung (Peak-to-offset) ergab einen Median von 63,8 in der Kontrollgruppe vs. 15,3 im CF-Kollektiv $(p<0,05)$.

Schlussfolgerungen SENCEFUL-MRT identifiziert Perfusionsdefizite der Lunge mit guter räumlicher Auflösung und zeigt signifikante Unterschiede zwischen Kontrollgruppe und CF-Kollektiv auf, welche primär dem Euler-Liljestrand-Mechanismus zuzuschreiben sind.

\section{WISS 208.4 90-kV CT-Angiografie in Kombination mit ADMIRE: Auswirkung auf Strahlendosis, Bildqualität und diagnostische Zuverlässigkeit zur Beurteilung von Lungenarterienembolien}

Autoren Lenga $\mathrm{L}^{1}$, Beeres $\mathrm{M}^{1}$, Leithner $\mathrm{D}^{1}$, Martin $\mathrm{S}^{1}$, Albrecht $\mathrm{M}^{1}$, Wichmann $]^{1}$, Vogl $T^{1}$, Scholtz $\mathrm{J}^{1}$

Institut 1 Universitätsklinikum Frankfurt, Institut für Diagnostische und Interventionelle Radiologie, Frankfurt

DOI 10.1055/s-0037-1600448

Zielsetzung Ziel ist es 90-kV Niedrigröhrenspannung Bildserien in Kombination mit fortgeschrittenen iterativen Rekonstruktionsalgorithmen (ADMIRE) und linear blended dual-energy Bildserien zur Beurteilung von Lungenarterienembolien (LAE) zu vergleichen hinsichtlich Strahlendosis, Bildqualität und diagnostischer Zuverlässigkeit

Material und Methodik 40 Patienten mit V.a. Lungenarterienembolie haben eine CT-Angiografie erhalten (21 männlich, 19 weiblich, Durchschnittsalter: 56 Jahre). Ein intraindividueller Vergleich von 90-kV und linearly blended M_0,6 (60\% 90-kV und 40\% 150-kV Spektrum) Bildserien wurde durchgeführt. Signaldämpfung und Bildrauschen des Truncus pulmonalis wurden gemessen. Signal-zu-Rausch und Kontrast-zu-Rausch Verhältnis wurden berechnet. Drei unabhängige Radiologen haben das Vorliegen einer zentralen oder segmentalen LAE und die Visualisierung der Pulmonalarterien beurteilt. Die Übereinstimmung zwischen den Betrachtern wurde mittels intraclass correlation coefficient (ICC) kalkuliert, die Strahlendosis wurde als Dosis-Längen-Produkt (DLP) erfasst.

Ergebnisse Schwächungsprofil und Bildrauschen des Truncus pulmonalis waren in den 90-kV Bildserien signifikant höher verglichen zu den linear blended Bildserien, so dass ein signifikant erhöhtes Kontrast-zu-Rausch Verhältnis in den $90-k V$ Serien entstand $(15.4 \pm 6.3$ vs. $11.3 \pm 4.6, \mathrm{P}<0.001)$. Zentrale und segmentale LAE wurden in beiden Serien exzellent erfasst ohne signifikanten Unterschied. Die Übereinstimmung unter den Betrachtern war exzellent (mean ICC 0.83). Insgesamt wurde die Eignung zur Beurteilung einer LAE für beide Bildserien exzellent bewertet mit leichten, signifikant geringeren Bewertungen für 90-kV Bildserien. Das kalkulierte DLP war um 37,2\% geringer in der 90 kV-Serie $(77.5 \pm 44$ vs. $123.4 \pm 51.1 \mathrm{mGy} \bullet \mathrm{cm}, \mathrm{P}<0.001)$.

Schlussfolgerungen $90-\mathrm{kV}$ CT-Angiografie in Kombination mit ADMIRE führt zu einem erhöhten Kontrast-zu-Rausch Verhältnis mit exzellenter diagnostischer Zuverlässigkeit zur Detektion einer LAE. Die Strahlendosis wird dabei signifikant erniedrigt.

\section{WISS 208.9 Lungenkrebsscreening mit MRT im Vergleich zur Niedrigdosis-CT: Ergebnisse nach dem ersten Jahr}

Autoren Meier-Schroers $\mathrm{M}^{1}$, Homsi $\mathrm{R}^{1}$, Gieseke $\mathrm{J}^{2}$, Dabir $\mathrm{D}^{1}$, Kütting $\mathrm{D}^{1}$, Feißt $A^{1}$, Schild $\mathrm{H}^{1}$, Thomas $\mathrm{D}^{1}$

Institut 1 Uniklinik Bonn, Radiologie, Bonn; 2 Philips, Philips Healthcare, Hamburg

DOI 10.1055/s-0037-1600449

Zielsetzung Festzustellen, ob sich die native MRT als Methode für ein Lungenkrebsscreening eignet.

Material und Methodik Es wurden 224 Teilnehmer eines Lungenkrebsscreenings mittels Niedrigdosis-CT und 1,5 Tesla-MRT untersucht, jeweils ohne intravenös appliziertes Kontrastmittel. Für die MRT wurden T2-gewichtete MultiVane XD-, steady state free precession-, T1-gewichtete Doppelechound diffusionsgewichtete Sequenzen angefertigt mit einer max. Untersuchungszeit von 20 Minuten. Die MRT wurde ohne Kenntnis des CT-Befundes hinsichtlich fokaler Läsionen ausgewertet. Es wurden Läsionen mit einer Mindestgröße von $6 \mathrm{~mm}$ bewertet, weil diese zur Beurteilung ihrer Dignität laut LungRADS-Kriterien weiter abgeklärt werden müssen. Bei Teilnehmern mit Läsionen $\geq 6 \mathrm{~mm}$ wurde eine vorzeitige Kontrolle nach 3 oder 6 Monaten, eine $P E T / C T$, eine Biopsie oder ein chirurgischer Eingriff durchgeführt. Bei den anderen Teilnehmern wurde eine Verlaufskontrolle nach 12 Monaten eingeplant.

Ergebnisse In der CT/MRT konnten folgende Läsionen nachgewiesen werden: 17/16 Läsionen mit Größen von 6-7 mm, 12/12 Läsionen mit Größen von 8-14 mm und 4/4 Läsionen $\geq 15 \mathrm{~mm}$. Die Sensitivität/Spezifität der MRT ergab $94 \% / 100 \%$ für $6-7 \mathrm{~mm}$ und $100 \% / 100 \%$ für $\geq 8 \mathrm{~mm}$. Dementsprechend gab es in 224 MRT-Untersuchungen einen falsch-negativen Befund für Läsionen $\geq 6 \mathrm{~mm}(0,5 \%)$, jedoch keine falsch-positiven Ergebnisse. Sieben Läsionen mit Größen zwischen $7 \mathrm{~mm}$ und $35 \mathrm{~mm}$ waren histologisch gesicherte Bronchialkarzinome, fünf davon wurden kurativ reseziert. Die einzige, in der MRT nicht nachweisbare Läsion ( $6 \mathrm{~mm}$ ) lag inmitten einer Belüftungsstörung. Es gab eine Größendiskrepanz bei zwei $7 \mathrm{~mm}$ großen Läsionen, welche in der MRT mit 8 mm bzw. 9 mm gemessen wurden (dies hätte Kontrolluntersuchungen nach 3 anstatt nach 6 Monaten zur Folge gehabt, wenn nur das MRT zur Auswertung herangezogen worden wäre).

Schlussfolgerungen Die native MRT hat eine sehr hohe Sensitivität/Spezifität für Läsionen $\geq 6 \mathrm{~mm}$ und eignet sich daher für die Durchführung eines Lungenkrebsscreenings. 
WISS 314.1 Automatisierte 3D-Volumetrie von CTAngiografien zur nicht-invasiven Bestimmung des pulmonalarteriellen Drucks bei Patienten mit Verdacht auf pulmonale Hypertonie

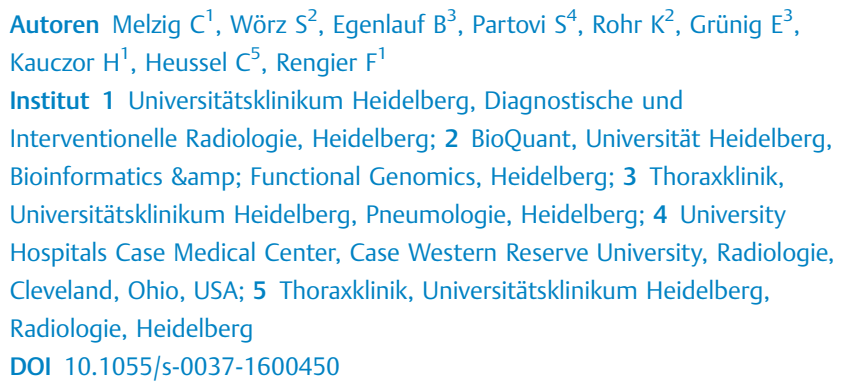

Zielsetzung Bei Verdacht auf pulmonale Hypertonie $(\mathrm{PH})$ wird neben dem Rechtsherzkatheter (RHK) häufig eine Lungen-CT-Angiografie (CTA) durchgeführt um andere Ursache für bestehende Symptome abzuklären. Ziel des Projekts ist die nicht-invasive Bestimmung des mittleren pulmonalarteriellen Drucks (mPAP) basierend auf automatisierter 3D-Volumetrie der zentralen Pulmonalarterien in der CTA um das Vorliegen einer PH vorherzusagen.

Material und Methodik Es wurden die Daten von 73 aufeinanderfolgenden Patienten (Durchschnitt 66 Jahre, 50 weiblich) die in unserer Klinik bei Verdacht auf PH mittels RHK, CTA sowie Echokardiografie untersucht wurden retrospektiv ausgewertet. Patienten mit chronisch-thromboembolischer $\mathrm{PH}$ wurden ausgeschlossen. Die Patienten wurden in eine Ableitungs- und eine Validierungskohorte randomisiert. Eigens entwickelte Software wurde für die automatisierte CTA-Segmentierung verwendet. Das Volumen von Pulmonalishauptstamm sowie rechter und linker Pulmonalarterie wurde berechnet und auf die jeweilige Gefäßlänge sowie Körperoberfläche bezogen (MPA-Volumen, RLPA-Volumen). Ein lineares Regressionsmodell zur Bestimmung des mPAP wurde unter Einbeziehung des echokardiografischen pulmonalarteriellen systolischen Drucks (PASP) in der Ableitungskohorte etabliert und die diagnostische Genauigkeit im Vergleich mit dem RHK in der Validierungskohorte überprüft.

Ergebnisse Der mittlere mPAP betrug $29,2 \mathrm{~mm} \mathrm{Hg}$ in der Ableitungskohorte ( $n=36$, Durchschnitt 66 Jahre) und $28,9 \mathrm{~mm} \mathrm{Hg}$ in der Validierungskohorte ( $n=37$, Durchschnitt 66 Jahre). Die Regressions-Analyse erbrachte folgende Formel: berechneter mPAP $=-2,353+(11,04 \times$ MPA-Volumen $)+(13,82 \times$ RLPA-Volumen $)+(0,489 \times$ PASP $)(r=0,92)$. Angewandt auf die Validierungskohorte betrugen Sensitivität und Spezifität für das Vorliegen einer $\mathrm{PH}$ (mPAP > $=25 \mathrm{~mm} \mathrm{Hg}$ ) 86\% und 100\%.

Schlussfolgerungen Die nicht-invasive Bestimmung des mPAP mittels CTAbasierter automatisierter 3D-Volumetrie der zentralen Pulmonalarterien kann das Vorliegen einer PH mit hoher Sensitivität und Spezifität vorhersagen.

\section{WISS 208.5 Bildqualität der Dual Energy Lungenperfusion in pulmonalarterieller und portalvenöser Kontrastmittelphase}

\author{
Autoren Prael I ${ }^{1}$, Wüst $\mathrm{W}^{2}$, Uder $\mathrm{M}^{2}$, Geissler $\mathrm{F}^{1}$, Köhnen J ${ }^{1}$, May $\mathrm{M}^{1}$ \\ Institut 1 Universitätsklinik Erlangen, Radiologie, Erlangen; \\ 2 Universitätsklinik Erlangen, Radiologie, Erlangen \\ DOI 10.1055/s-0037-1600451
}

Zielsetzung Die Dual Energy Computertomografie (DECT) ist für die Abklärung der Lungenembolie ein etabliertes Verfahren. Ziel dieser retrospektiven Studie war die Lungenperfusionsanalyse in portalvenösen Kontrastmittelphasen zu evaluieren.

Material und Methodik Aus 99 pulmonalarteriellen DECT (Referenzgruppe R) und 99 portalvenösen DECT des Thorax (Studiengruppe S) wurden neben den Mischbildern zusätzlich Lungenperfusionskarten mit identischer Fensterung (W80/C40) rekonstruiert. Die Kontrastmittelanreicherung des Lungenparenchyms wurde mittels automatischer Segmentierung erfasst. Die Lungen- perfusionskarten wurden bezüglich der diagnostischen Bildqualität auf einer 5-stufigen Likert Skala (1: unzweifelhaft; 5: unbrauchbar) bewertet, die Bildartefakte auf einer dichotomen Skala in 5 Gruppen unterteilt: Rotationsartefakte, Streifenartefakte, Rezessusartefakte, Dual-Source Artefakte, subpleurale Artefakte.

Ergebnisse Die mittlere Kontrastmittelanreicherung war mit $20 \pm 11 \mathrm{HU}$ in R und $23 \pm 13 \mathrm{HU}$ in S trotz unterschiedlicher Zeitpunkte vergleichbar. Die Unterschiede in der diagnostischen Bildqualität waren zwischen $S$ und R nicht signifikant $(p=0,394)$. Rotationsartefakte und Streifenartefakte traten in beiden Gruppen am häufigsten und in jedem Patienten in mindestens einer Lokalisation auf. In der Subgruppenanalyse der Streifenartefakte waren in R Aufhärtungen entlang kontrastierter Gefäße (54\%) und in S entlang der Wirbelsäule (80\%) am jeweils häufigsten. Rezessusartefakte waren in S signifikant erhöht (63 vs. $24 \%$ ), Dual Source Artefakte dagegen in beiden Gruppen gleich häufig (5\%). Subpleurale Artefakte wurden nur in S beobachtet (8\%).

Schlussfolgerungen Die Dual Energy Lungenperfusionsanalyse liefert in der portalvenösen CT des Thorax bei vergleichbarem lodgehalt im Lungenparenchym klinisch verwertbare und mit Aufnahmen in pulmonalarterieller Kontrastmittelphase vergleichbare Bilder. Die Kenntnis der unterschiedlichen Dynamik und Verteilung von Bildartefakten kann bei der Befundung zur Vermeidung falsch positiver Befunde hilfreich sein.

\section{WISS 208.7 Einfluss von Bildmatrix und Schichtdicke auf die subjektive Bildqualität in der HR-CT der Lunge: Untersuchung an einem Ultra-High-Resolution Ganzkörper-Prototyp-CT}

Autoren Rogalla $\mathrm{P}^{1}$, Prokop $\mathrm{M}^{2}$, Blum $\mathrm{A}^{3}$, Chen $\mathrm{M}^{4}$, Kandel $\mathrm{S}^{5}$, Farrell $\mathrm{C}^{6}$, Hoppel $B^{6}$

Institut 1 University of Toronto, Medical Imaging, Toronto; 2 Radboud University Nijmegen, Radiologie, Nijmegen; 3 Centre University Hospital Nancy, Radiologie, Nancy; 4 National Institutes of Health Clinical Center, Kardiologie, Washington D.C.; 5 UHN, Radiologie, Toronto; 6 Toshiba, Forschung, Toronto DOI 10.1055/s-0037-1600452

Zielsetzung Ziel der Untersuchung war herauszufinden, ob eine Schichtdicke von 0.25 mm und 1024-Bildmatrix (1k) die Bildqualität in der HR-CT der Lunge verbessert.

Material und Methodik 59 Patienten erhielten ein Thorax-CT an einem 128Zeilen Ganzkörper-Prototyp-CT (TSX-304R, Toshiba) mit: 0.25 mm Schichtdicke, 0.5 s Rotation, Dosismodulation (SD: $25 \mathrm{HU}$ ), $120 \mathrm{kV}$, AIDR-3D und Filter FC81. 6 Serien wurden rekonstruiert (Schichtdicke in mm/Bildmatrix): $0.25 /$ $1 \mathrm{k}, 0.25 / 512,0.5 / 1 \mathrm{k}, 0.5 / 512,2 / 1 \mathrm{k}$ und 2/512. Zwei Radiologen mit 21 und 4 Jahren Erfahrung beurteilten ohne Kenntnis der Parameter die Serien an einem 4K-Monitor (6:1-Aufteilung) mittels Forced-Ranking und freier Skala (1 = exzellent, 10 = schlecht) in den Kategorien: (K1) Darstellung von Bronchien, (K2) Gefäßen, (K3) Septen und Fissuren, (K4) Bildschärfe, (K5) RauschTextur, (K6) Artefakte und (K7) persönliche Präferrenz. Das Bildrauschen (SD in ROIs) wurde in der Aorta und in Luft auf allen 6 Serien gemessen, das DLP notiert. Die Statistik erfolgte mittels ANOVA für Haupt- und Interaktionseffekt und mittels Kendall-Koeffizient für Konkordanz (KKC).

Ergebnisse Haupt- und Interaktionseffekt der Bildmatrix und Schichtdicke waren in allen Kategorien signifikant bei $p<0.05$, wobei die Bildmatrix generell einen stärken Effekt hatte als die Schichtdicke. Die 1k-Matrix wurde der 512-Matrix in K1-K3 vorgezogen, beide Befunder bevorzugten $0.25 \mathrm{~mm}$ gegenüber 0.5 und $2 \mathrm{~mm}$ (Forced Ranking). $2 \mathrm{~mm}$ mit $1 \mathrm{k}$-Matrix wurde für Bildrauschen- und Artefakte-unterdrückung bevorzugt, 0.25 mm mit 512-Matrix erhielt die schlechteste Bewertung. Auf der freien Skala erhielt $0.5 \mathrm{~mm}$ mit $1 \mathrm{k}$ (n.s.) die beste und $2 \mathrm{~mm}$ mit 512-Matrix die schlechteste Bewertung $(p<0.001)$. Der KKC lag bei $0,89-0,93$ in $\mathrm{K} 1-\mathrm{K} 3$ und $0,66-0.83$ in K4-K7. Das mittlere DLP war 377,9 mGy* $\mathrm{cm}$. SD variierte zwischen $58.1 \mathrm{HU}(0.25$ / $1 \mathrm{k})$ und $25.8 \mathrm{HU}(2 / 512)$. 
Schlussfolgerungen In der HR-CT der Lunge verbessert die 1024-Bildmatrix die Bildqualität im Vergleich zur 512-Matrix; $0.25 \mathrm{~mm}$ Schichtdicke wird überwiegend einer $0.5 \mathrm{~mm}$ Schichtdicke vorgezogen

\section{WISS 314.9 Die selbstnavigierte und kontrastmittelfreie funktionelle Lungen-MRT zur quantitativen Ventilationsanalyse bei Patienten mit cystischer Fibrose}

Autoren Veldhoen $\mathrm{S}^{1}$, Weng $\mathrm{A}^{1}$, Knapp $\mathrm{J}^{1}$, Kunz $\mathrm{A}^{1}$, Stab $\mathrm{D}^{2}$, With $\mathrm{C}^{1}$, Segerer $\mathrm{F}^{3}$, Hebestreit $\mathrm{H}^{3}$, Malzahn $\mathrm{U}^{4}$, Köstler $\mathrm{H}^{1}$, Bley $\mathrm{T}^{1}$

Institut 1 Universitätsklinikum Würzburg, Institut für Diagnostische und Interventionelle Radiologie, Würzburg; 2 University of Queensland, Centre of Advanced Imaging, Brisbane; 3 Universitätsklinikum Würzburg, Kinderklinik und Poliklinik, Würzburg; 4 Universitätsklinikum Würzburg, Zentrale für Klinische Studien, Würzburg

DOI 10.1055/s-0037-1600453

Zielsetzung Die klinische Evaluation der SENCEFUL-MRT (SElf-gated Non-Contrast-Enhanced FUnctional Lung imaging) zur quantitativen Ventilationsanalyse bei Patienten mit cystischer Fibrose (CF).

Material und Methodik Zwanzig Patienten mit cystischer Fibrose und 20 gepaarte Kontrollprobanden durchliefen eine native funktionelle Lungen-MRT in freier Atmung an einem 1,5T Scanner. Die Ventilationsmessungen wurden mit dem SENCEFUL-Ansatz, einer Weiterentwicklung der Fourier-Dekompositionsmethode durchgeführt, mit der durch Sortierung selbstnavigierter Daten einer 2D-FLASH Sequenz morphologische Bildserien vollständiger Atem- und Herzzyklen rekonstruiert werden können. Die Lungen wurden manuell segmentiert und die quantitative Ventilation (QV) in Milliliter Gasaustausch pro Milliliter Lungenvolumen $(\mathrm{ml} / \mathrm{ml})$ automatisiert berechnet. Die QV-Werte der CF-Patienten wurden mit Parametern der Lungenfunktionsprüfung korreliert und zwischen Patienten und Probanden verglichen. Drei Radiologen bewerteten die farbkodierten Organkarten hinsichtlich vorhandener Ventilationsdefizite. Ergebnisse Die QV der Lungen war bei den CF-Patienten geringer (0.09 vs. $0.11 \mathrm{ml} / \mathrm{ml}, \mathrm{p}<0.01)$. In Übereinstimmung mit der CF-Prädilektion der Oberlappen waren die QV-Quotienten zwischen oberen und unteren Lungenhälften bei den Patienten geringer (rechts, 0.84 vs. 1.16, $p<0.001$; links, 0.88 vs. 1.11, $\mathrm{p}=0.02$ ). Die mittlere Atemfrequenz war bei den CF-Patienten erhöht (20 vs. 16 Atemzyklen/Min, $\mathrm{p}<0.01$ ). Die QV-Werte der CF-Patienten korrelierten mit der Vitalkapazität $(r=0.7, p=0.01)$, dem Residualvolumen (Überblähungsmarker, $r=-0.8, p<0.01)$ und der forcierten Einsekundenkapazität (Obstruktionsmarker, $r=0.7, p=0.02$ ). Die Ventilationskarten zeigten disseminierte kleine Defizite als typisches Muster in 40\% der CF-Patienten.

Schlussfolgerungen Mit der SENCEFUL-MRT gelingt die ortsaufgelöste quantitative Ventilationsanalyse der Lungen ohne Kontrastmittelapplikation und in freier Atmung. Die Technik könnte sich somit für ein strahlenfreies Krankheitsmonitoring eignen.

\section{WISS 208.1 Thorax-Röntgenuntersuchung von Flüchtlingen: eine Notwendigkeit?}

Autoren Weinrich $\mathrm{J}^{1}$, Roland $\mathrm{D}^{2}$, Sauer $\mathrm{M}^{1}$, Frank Oliver $\mathrm{H}^{1}$, MeywaldWalter $\mathrm{K}^{3}$, Adam $\mathrm{G}^{1}$, Bannas $\mathrm{P}^{1}$

Institut 1 Zentrum für Radiologie und Endoskopie des Universitätsklinikums Hamburg-Eppendorf, Klinik und Poliklinik für Diagnostische und

Interventionelle Radiologie und Nuklearmedizin, Hamburg; 2 LungenClinic Großhansdorf, Pneumologie, Hamburg; 3 Gesundheitsamt Hamburg-Mitte, Tuberkulosebekämpfung, Hamburg

DOI 10.1055/s-0037-1600454

Zielsetzung Das Infektionsschutzgesetz sieht für alle nach Deutschland kommenden Asylsuchenden, die das 15. Lebensjahr vollendet haben, mit Ausnahme von Schwangeren, eine verpflichtende Thorax-Röntgenuntersuchung zum Ausschluss einer Lungentuberkulose (LT) vor. Ziel dieser Studie war es, die
Anzahl der detektierten Fälle von aktiven LT während der Flüchtlingskrise 2015 in Hamburg zu ermitteln.

Material und Methodik Es wurden alle Thorax-Röntgenuntersuchungen von Flüchtlingen, die 2015 in Hamburg am Screening teilnahmen, ausgewertet. Hieraus wurde die Prävalenz, die number needed to screen (wie viele Röntgenaufnahmen nötig sind um eine aktive TB zu detektieren) und die Genauigkeit des Röntgenbildes eine aktive LT zu erkennen, bestimmt. Als Referenzmethode galt, das für Krankenhäuser und Labore verpflichtende, TuberkuloseMelderegister des Gesundheitsamtes. Hiermit konnte die Anzahl der in Hamburg als Tuberkulose gemeldeten Fälle ermittelt und mit dem Screening abgeglichen werden.

Ergebnisse Insgesamt wurden 17487 Flüchtlinge im Rahmen des Screenings geröntgt. Die Prävalenz der aktiven Tuberkulose betrug hierbei 0,103\%. Es waren 1749 Thorax-Röntgenuntersuchungen notwendig um eine aktive LT zu detektieren. Die Thorax-Röntgenuntersuchung zeigte hierbei eine Sensivität von 55,6\% (95\%-Konfidenzintervall (KI): 30,8-78,5) und eine Spezifität von 98,3\% (Kl: 98,1 - 98,5) in der Erkennung einer aktiven Tuberkulose.

Schlussfolgerungen Trotz einer hohen Spezifität zeigte sich bei der Reihen Thorax-Röntgenuntersuchung während der Flüchtlingskrise 2015 eine niedrige Prävalenz der aktiven LT. Aufgrund der hohen number needed to screen, erscheint es sinnvoll das aktuell auf Röntgenuntersuchungen basierende Screening kritisch zu betrachten und weitere Screening-Maßnahmen zu diskutieren. Es benötigt weiterer Daten um die vorgestellten Ergebnisse zu verstärken.

WISS 208.2 Thoraxradiografie: Güte von Befundung, Beurteilung und Selbsteinschätzung in Abhängigkeit von Fallschwierigkeit, realem Vorgehen während der Bildbefundung (eye-tracking) und des Weiterbildungsstands

Autoren Wirth $\mathrm{S}^{1}$, Krupp $\mathrm{J}^{1}$, Bolzer $\mathrm{M}^{2}$, Reiser $\mathrm{M}^{1}$, Fischer $\mathrm{M}^{2}$

Institut 1 Ludwigs-Maximilians-Universität München, Institut für Klinische Radiologie, München; 2 Ludwigs-Maximilians-Universität München, Institut für medizinische Didaktiv und Ausbildungsforschung, München

DOI 10.1055/s-0037-1600455

Zielsetzung Als wesentlicher Bestandteil radiologischer Routinetätigkeit sind bei der Radiografie des Thorax Korrektheit von erhobenen Befunden, klinischer Konsequenz und Selbsteinschätzung der Befunder wichtige Qualitätsmerkmale. Ziel der Studie ist es mögliche Einflussparameter zu zu bewerten. Material und Methodik 20 Radiolog/Innen („Novice“ mit >3 Monaten und „Expert“ mit > 4 Jahren Erfahrung) befundeten 12 Radiografien des Thorax mit unterschiedlichem Schwierigkeitsgrad im Abstand von mindestens einem Monat zweimal: einmal mit und einmal ohne Verwendung eines anatomisch orientierten Befundschemas ( $n=480$ ). Das Betrachtungsverhalten wurde mittels Eye-tracking objektiviert und als Kontrolle zwei weitere, zufällige Fälle ein drittes Mal ohne die Apparatur bewertet. Der Vergleich erfolgte am GoldStandard, für den Befunde in 12 Pathologiegruppen eingeteilt und jeweils ordinal (0: normal bis 5: maximal) anhand einer CT im Konsensus von 3 Fachärzten bewertet wurden. Die Qualität der Beurteilung wurde in vergleichbarer Weise festgelegt ( 0 : perfekt, 1 : falsch, aber klinische Konsequenz korrekt bis 5 : falsch, Patientenschaden wahrscheinlich). Hinsichtlich der Selbsteinschätzung wurden die Auswerter befragt, ob diese gar nicht, bei Gelegenheit oder sofort zusätzliche Expertise hinzuziehen würden und ob sie eine CT empfehlen würden.

Ergebnisse Einerseits überschätzten sich mehr als 80\% der Auswerter und andererseits wurde in weit mehr als der Hälfte eine CT empfohlen. Keines der Qualitätsmerkmale korrelierte mit Fallschwere, Weiterbildungsstand, Grad der Bildabdeckung, Befundschema oder Befundungsdauer. Der einzig signifikante Effekt ( $p<0.05$, Wilcoxon) wurde für das theoretische Setting einer zweimaligen Befundung durch Novizen und Experten festgestellt. Die EyeTracking Apparatur hatte keinen nachweisbaren Einfluss auf die Ergebnisse. 
Schlussfolgerungen Die Befundung von Radiografien ist oft schwierig, unvollständig und fehleranfällig. Wann immer machbar, scheint es empfehlenswert ein Vier-Augen-Prinzip zur Befundung einzuhalten.

Uroradiologie/Urogenitaldiagnostik

\section{WISS 402.7 Morphologische Veränderungen des Beckenbodens Erstgebärender nach vaginaler Entbindung}

Autoren Alt $\mathrm{C}^{1}$, Hampel $\mathrm{F}^{2}$, Radtke $\mathrm{J}^{3}$, Hallscheidt $\mathrm{P}^{4}$ Schlehe $\mathrm{B}^{2}$, Sohn $\mathrm{C}^{2}$, Brocker $\mathrm{K}^{2}$

Institut 1 Heinrich-Heine-Universität Düsseldorf, Medizinische Fakultät, Institut für Diagnostische und Interventionelle Radiologie, Düsseldorf;

2 Universitätsklinikum Heidelberg, Frauenklinik, Heidelberg;

3 Universitätsklinikum Heidelberg, Urologische Klinik, Heidelberg; 4 Alice-

Hospital Darmstadt, Radiologie, Darmstadt

DOI 10.1055/s-0037-1600456

Zielsetzung Detektion früher morphologischer Veränderungen des Beckenbodens bei Erstgebärenden nach vaginaler Entbindung mittels 3T MRT.

Material und Methodik Prospektive Machbarkeitsstudie mit Durchführung einer nativen 3T MRT des Beckens innerhalb 1 Woche nach zeitgerechter vaginaler Entbindung und vorliegender Einverständniserklärung unter Verwendung einer hochauflösenden T2 turbo spin echo (tse) Sequenz in 3 Ebenen, einer T2 tse mit Fettsättigung und einer nativen T1 tse jeweils in transversaler Ebene. Dokumentation aller sichtbaren morphologischen Veränderungen (Ödeme, Hämatome, muskuläre oder ligamentäre Ein- bzw. Abrisse) und Erhebung von Messparametern für die Unversehrtheit des Beckenbodens im Vergleich zu einer Kontrollgruppe von Frauen, die noch kein Kind geboren haben. Die Differenzanalyse erfolgte mittels ungepaartem t-Test mit Definition der statistischen Signifikanz bei $p<0,05$. Es erfolgte eine Befragung der Studienpatientinnen 3 Jahre nach der MRT hinsichtlich nach der Entbindung aufgetretener Beckenbodensymptome.

Ergebnisse 25 Erstgebärende zeigten multiple oberflächliche als auch tief im Becken gelegene morphologische Veränderungen. Signifikante Änderungen der Messparameter zeigten der anteroposteriore und der laterolaterale Hiatus, die hiatale Zirkumferenz und Fläche, die maximale Muskeldicke und der Abstand zwischen Levator und Urethra. In der 3-Jahres-Befragung gaben von 20 Frauen nur 6 Frauen zumeist temporär aufgetretene Blasenprobleme an, keine der Frauen beklagte Stuhlbeschwerden oder eine Beckenorgansenkung. 10 Frauen gaben keinerlei Beschwerden an, obwohl die MRT und die klinische Untersuchung postpartal morphologische Veränderungen gezeigt hatten.

Schlussfolgerungen Die 3T MRT kann zur Diagnostik postpartaler Veränderungen und der Beurteilung der Unversehrtheit des Beckenbodens zuverlässig eingesetzt werden. Eine direkte Korrelation detektierter morphologischer Veränderungen zu symptomatischen Beckenbodenbeschwerden kann zum jetzigen Zeitpunkt noch nicht bestätigt werden.

\section{WISS 315.2 Verteilung falsch positiver Befunde im PI- RADSv2 Lexikon für Prostata-MRT: Unterschiedliche Ergebnisse für periphere Zone und Transitionalzone}

Autoren Benndorf $\mathrm{M}^{1}$, Hahn $\mathrm{F}^{1}$, Lenz $\mathrm{P}^{1}$, Soschynski $\mathrm{M}^{1}$, Krönig $\mathrm{M}^{2}$, Jilg $\mathrm{C}^{2}$, Langer $\mathrm{M}^{1}$

Institut 1 Universitätsklinikum Freiburg, Klinik für Radiologie, Freiburg;

2 Universitätsklinikum Freiburg, Klinik für Urologie, Freiburg

DOI 10.1055/s-0037-1600457

Zielsetzung Es wird untersucht ob sich die Verteilungen falsch positiver Befunde zwischen peripherer Zone (PZ) und Transitionalzone (TZ) der Prostata im aktuellen PI-RADS (Prostate Imaging Reporting and Data System, version 2: PI-RADSv2) Lexikon unterscheiden.
Material und Methodik Wir analysieren die Ergebnisse von 78 konsekutiv in unserer Institution mittels Prostata-MRT untersuchter und anschließend mittels transperinealer MRT-Ultraschall Fusionsbiopsie (MonaLisa, Biobot) diagnostizierter Patienten. Nicht betrachtet werden Patienten mit gesichertem Prostatakarzinom und mit posttherapeutischen Veränderungen der Prostata. Alle Patienten haben ein erhöhtes Karzinomrisiko (PSA $>4 \mathrm{ng} / \mathrm{ml}$ ). Es werden 176 Läsionen in multiparametrischen MRT Untersuchungen (1,5 Tesla) markiert und histopathologisch verifiziert. Alle Untersuchungen werden nach dem PI-RADSv2 Lexikon beurteilt, detektierte Läsionen erhalten eine Bewertungskategorie (2 bis 5). Anhand der Ergebnisse der Fusionsbiopsie werden die positiven prädiktiven Werte (PPW) für die PI-RADSv2 Kategorien berechnet. Der PPW wird als Maß für falsch positive Befunde angesehen: Je niedriger der PPW, desto mehr falsch positive Befunde werden generiert. Vergleiche werden mittels Chi-Quadrat Test geführt.

Ergebnisse Es werden 86 Läsionen in der PZ und 90 Läsionen in der TZ detektiert. In der PZ werden 33 Malignome (38,4\%), in der TZ 21 Malignome diagnostiziert (23,3\%), P<0.05. In der PZ beträgt der PPW für PI-RADS 2, 3, 4 und 5 11,1\% (1/9), 27,6\% (8/29), 46,9\% (15/32) und 56,3\% (9/16). In der TZ beträgt der PPW für PI-RADS 2, 3, 4 und 5 10\% (1/10), 10,2\% (5/49), 34,8\% $(8 / 23)$ und $87,5 \%(7 / 8)$. Kategorie 3 zeigt in der TZ mehr falsch positive Befunde als in der $\mathrm{PZ}, \mathrm{P}<0,05$.

Schlussfolgerungen Falls in Zukunft eine Handlungsempfehlung mit den PIRADS Kategorien verknüpft werden soll (ähnlich dem BI-RADS Lexikon für die Mamma-Diagnostik), scheint der Unterschied des PPW zwischen PZ und TZ relevant. Möglich wäre eine Modifikation des Lexikons um den PPW für PZ und TZ anzugleichen.

\section{WISS 315.8 Detektionsrate der [68Ga]PSMA I\&T PET/ CT bei Patienten mit biochemischen Prostata- karzinomrezidiv und Vergleich mit publizierten Detektionsraten von [68Ga]PSMA HBED-CC}

Autoren Berliner $\mathrm{C}^{1}$, Tienken $\mathrm{M}^{2}$, Kobayashi $\mathrm{Y}^{1}$, Kirchner $\mathrm{U}^{1}$, Klutmann $\mathrm{S}^{1}$, Budäus $\mathrm{L}^{3}$, Wester $\mathrm{H}^{4}$, Mester $\mathrm{J}^{1}$, Adam $\mathrm{G}^{1}$, Bannas $\mathrm{P}^{1}$

Institut 1 Universitätsklinikum Hamburg-Eppendorf, Klinik und Poliklinik für Diagnostische und Interventionelle Radiologie und Nuklearmedizin, Hamburg; 2 Universitätsklinikum Hamburg-Eppendorf, Klinik und Poliklinik für Diagnostische und Interventionelle Radiologie und Nukleardeizin, Hamburg; 3 Universitätsklinikum Hamburg-Eppendorf, Martini-Klinik, Hamburg;

4 Technische Universität München, Radiochemie und Pharmazeutische Radiochemie, Garching DOI 10.1055/s-0037-1600458

Zielsetzung Bestimmung der Detektionsrate der [68Ga]PSMA I\&T PET/CT bei biochemischen Prostatakarzinomrezidiv und Vergleich mit publizierten Detektionsraten der [68Ga]PSMA HBED-CC PET/CT.

Material und Methodik Retrospektive Analyse von 83 [68Ga]PSMA I\&T PET/ CT bei Patienten mit gesichertem biochemischen Prostatakarzinomrezidiv nach radikaler Prostatektomie. Die PET/CT Untersuchungen wurden hinsichtlich eines Lokalrezidivs, Lymphknotenmetastasen und Fernmetastasen ausgewertet. Die relativen Ergebnisse positiver PET/CT Untersuchungen wurden für sechs Untergruppen mit jeweils ansteigendem prostataspezifischem Antigen (PSA) Blutwert berechnet ( $<0,5 \mathrm{ng} / \mathrm{ml}, 0,5$ bis $<1,0 \mathrm{ng} / \mathrm{ml}, 1,0$ bis $<2,0 \mathrm{ng} / \mathrm{ml}$, 2,0 bis $<5,0 \mathrm{ng} / \mathrm{ml}, 5,0$ bis $<10,0, \geq 10,0 \mathrm{ng} / \mathrm{ml}$ ). Zusätzlich wurde die Detektionsrate von [68Ga]PSMA I\&T mittels exaktem Fisher Test statistisch mit den publizierten Detektionsraten von [68Ga]PSMA HBED-CC verglichen.

Ergebnisse Der mediane PSA-Wert war 0,81 (Spanne: 0,01-128) ng/ml. Bei 58/83 Patienten (70\%) wurde mindestens eine [68Ga]PSMA I\&T positive Läsion detektiert. 18 Patienten (22\%) zeigten ein Lokalrezidiv, 29 Patienten (35\%) Lymphknotenmetastasen und 15 Patienten (18\%) Fernmetastasen. Die Tumordetektionsrate korrelierte positiv mit einem ansteigendem PSA-Wert. Hierbei zeigten sich Detektionsraten von $52 \%(<0,5 \mathrm{ng} / \mathrm{ml}), 55 \%(0,5$ bis $<1,0 \mathrm{ng} / \mathrm{ml})$, $70 \%$ ( 1,0 bis $<2,0 \mathrm{ng} / \mathrm{ml}), 93 \%$ (2,0 bis $<5,0 \mathrm{ng} / \mathrm{ml}), 100 \%$ (5,0 bis $<10,0 \mathrm{ng} /$ 
$\mathrm{ml}$ ) und 100\% ( $\geq 10,0 \mathrm{ng} / \mathrm{ml})$. Zwischen den Detektionsraten von [68Ga]PSMA I\&T und den publizierten Detektionsraten von [68Ga]PSMA HBED-CC zeigte sich kein signifikanter Unterschied ( $\mathrm{p}$ immer $>0.05$ ).

Schlussfolgerungen Die [68Ga]PSMA I\&T PET/CT hat eine hohe Detektionsrate des biochemischen Rezidivs bei Prostatakarzinom, welche vergleichbar mit der Detektionsrate von [68Ga]PSMA HBED-CC ist.

\section{WISS 315.7 Vergleich von Radiomics, quantitativen ADC Messungen und PI-RADS zur Detektion des signifikanten Prostatakarzinoms}

Autoren Bonekamp D , Bonekamp D¹, Hadaschik B², Wiesenfarth $\mathrm{M}^{3}$, Wieczorek $\mathrm{K}^{4}$, Götz $\mathrm{M}^{5}$, Schlemmer $\mathrm{H}^{1}$, Maier-Hein $\mathrm{K}^{5}$

Institut 1 Deutsches Krebsforschungszentrum, Radiologie, Heidelberg; 2 Universitätsklinikum Heidelberg, Urologie, Heidelberg; 3 Deutsches Krebsforschungszentrum, Biostatistik, Heidelberg; 4 Universitätsklinikum Heidelberg, Pathologie, Heidelberg; 5 Deutsches Krebsforschungszentrum, Medizinische Informatik, Heidelberg

DOI 10.1055/s-0037-1600459

Zielsetzung Durch Prostata-MRT kann die Überdiagnose insignifikanter Prostatakarzinome (PC) potentiell verringert werden, bei vermehrter Detektion signifikanter PC gegenüber der 12-fach Biopsie. Eine kleine aber wichtige Zahl von PC wird aber weiterhin von MRT oder gezielten MRT/TRUS Fusionsbiopsien übersehen. Hier vergleichen wir den zusätzlichen Nutzen von Radiomics und quantitativen ADC Messungen über klinische PI-RADS Befundung.

Material und Methodik 16954 radiomische Parameter wurden aus den Bilddaten 197 konsekutiv bei 3 Tesla untersuchten Patienten mit klinischem Verdacht auf PC bestimmt. Klinische PI-RADS Befundungen sowie eine unter Kenntnis der durch MRT/TRUS Fusions- und Sättigungsbiopsien gesicherten Histologie erstellte Segmentation und retrospektive Likert-Beurteilung lagen zum Vergleich vor. Nach Vorfilterung wurden aus den verbleibenden 938 Parametern ein 13 und ein 29 Parameter Lasso und Elastic Net (Enet) Vorhersagemodell konstruiert und durch +.632 bootstrapping validiert. Die Modelle wurden mit dem Monoparameter mittlerer ADC Wert (meanADC) verglichen.

Ergebnisse Ein klinischer PI-RADS score von 3 oder mehr hatte eine true positive rate (TPR) von $95 \%$, allerdings bei einer false positive rate (FPR) von $74 \%$ und einer accuracy von $52 \%$. Dagegen zeigten die Lasso/Enet Modelle eine accuracy von $83 \% / 84 \%$ und ADCmean von $85 \%$, verglichen mit $85 \%$ für Likert >=3. Für die ROC AUC lagen die Modelle mit $89 \% / 90 \%$ über Likert $(87 \%)$ aber gering unter meanADC $(92 \%)$.

Schlussfolgerungen ADC und Radiomics führten zu einer geringen Verbesserung der Prädiktion signifikanter PC gegenüber retrospektivem Likert Scoring. Die Performance war deutlich besser als für prospektive PI-RADS Beurteilung. Mean ADC kann die qualitative Auswertung des MRT verbessern. Retrospektives Likert Scoring kann als Obergrenze der PI-RADS Performance interpretiert werden. Ein zusätzlicher Nutzen komplexer Radiomics-Signaturen konnte hier nicht gezeigt werden, ist aber bei Weiterentwicklung der Parameterselektion und Modellkonstruktion nicht ausgeschlossen.

\section{WISS 402.3 Einfluss der Zeitauflösung in der dynamischen kontrastmittelunterstützten renalen MR- Perfusionsbildgebung}

Autoren Budjan $]^{1}$, Riffel $\mathrm{P}^{1}$, Grimm R${ }^{2}$, Block $\mathrm{K}^{3}$, Schönberg $\mathrm{S}^{1}$, Attenberger $\mathrm{U}^{1}$, Hausmann $\mathrm{D}^{1}$

Institut 1 Universitätsmedizin Mannheim, Institut für Klinische Radiologie und Nuklearmedizin, Mannheim; 2 Siemens Healthcare GmbH, Erlangen; 3 Center for Advanced Imaging Innovation and Research (CAI2R), Department of Radiology, New York University School of Medicine, New York, USA DOI 10.1055/s-0037-1600460

Zielsetzung Die dynamische kontrastmittelunterstützte (DCE) PerfusionsMRT ist ein etabliertes Verfahren zur Bestimmung der renalen Perfusion. Die
Literatur ist in Hinblick auf die für eine verlässliche quantitative Auswertbarkeit notwenige minimale Zeitauflösung uneinheitlich. In dieser Studie wurden Perfusionsanalysen in-vivo anhand von Datensätzen mit unterschiedlichen Zeitauflösungen intra-individuell verglichen.

Material und Methodik Bei 22 Patienten (7 weiblich; 58,7 \pm 21,2 Jahre) wurde eine kontrastmittelverstärkte, T1-gewichtete, radiale golden-angle stack-ofstars Sequenz (GRASP) über einen Zeitraum von 3 min in freier Atmung bei 3T akquiriert. Aus dieser Akquisition wurden 4 Datensätze mit unterschiedlicher Zeitauflösung (9,7 s, 5,9 s, 3,6s und 1,3 s) rekonstruiert. Der renale Plasmafluss (rPF) wurde mittels eines voxel-by-voxel Dekonvolutionsalgorithmus für das manuell segmentierte Nierenvolumen berechnet. Die rPF-Parameter wurden mittels gepaartem t-Test und Blant-Altmann-Analysen verglichen.

Ergebnisse In der Auswertung des Datensatzes mit der höchsten Zeitauflösung $(1,3 \mathrm{~s})$ ergab sich ein mittlerer rPF von $216 \pm 93 \mathrm{ml} / 100 \mathrm{ml} / \mathrm{min}$. Dies liegt im Bereich der bisherigen Literaturangaben zum mittels DCE ermittelten rPF. Ähnliche Werte ergab auch die Auswertung der Datensätze mit geringerer Zeitauflösung $(219 \pm 97 \mathrm{ml} / 100 \mathrm{ml} / \mathrm{min}$ bei $3,6 \mathrm{~s} ; 217 \pm 96 \mathrm{ml} / 100 \mathrm{ml} / \mathrm{min}$ bei $5,9 \mathrm{~s}$ und $209 \pm 80 \mathrm{ml} / 100 \mathrm{ml} / \mathrm{min}$ bei $9,7 \mathrm{~s}$ ). Statistisch signifikante Unterschiede zwischen den Gruppen fanden sich nicht ( $p>0.09$ für alle Paare).

Schlussfolgerungen Eine zeitliche Auflösung von $1,3 \mathrm{~s}$ bis $9,7 \mathrm{~s}$ führt in der renalen DCE Perfusions-MRT zu vergleichbaren rPF-Werten. Eine Erhöhung der Zeitauflösung scheint die rPF-Parameter nicht relevant zu beeinflussen. Die Wahl der Zeitauflösung erscheint damit von untergeordneter Bedeutung für quantitative rPF-Analysen.

\section{WISS 315.3 PI-RADS v2 versus PI-RADS v1: Reliabilität und Zeitaufwand - Wert einer strukturierten Befundung im klinischen Alltag und in der Weiterbildung}

Autoren Garmer $\mathrm{M}^{1}$, Karpienski $\mathrm{J}^{2}$, Busch $\mathrm{M}^{1}$, Mateiescu $\mathrm{S}^{1}$, Grönemeyer $\mathrm{D}^{1}$ Institut 1 Institut für Mikrotherapie, Radiologie, Bochum; 2 Universität Witten/Herdecke

DOI 10.1055/s-0037-1600461

Zielsetzung Eine strukturierte Befundung anhand eines Klassifizierungsschemas (hier PI-RADS für die multiparametrische MRT der Prostata) liefert möglicherweise Vorteile in einer strukturierten Weiterbildung unerfahrener Kollegen. Gleichzeitig ist der Zeitaufwand im Alltag gesteigert. Wir vergleichen die Effizienz des modifizierten PI-RADS Version 2 (v2) im Hinblick auf diagnostische Verwertbarkeit und Zeitaufwand im Vergleich zur Version 1 (v1). Material und Methodik Retrospektiv erfolgte eine Analyse der multiparametrischen MRT von 688 Prostatataläsionen bei 180 Patienten durch einen erfahrenen Radiologen sowie eine eingewiesene studentische Kraft nach PI-RADS v2 und v1. Die Ergebnisse wurden mit Ergebnissen der MRT-gesteuerten Biopsie korreliert. Sensitivität, Spezifität und korrekt klassifizierte Stanzen wurden für verschiedene Schwellenwerte in einer ROC-Analyse bestimmt. Für die Bestimmung der Übereinstimmung zwischen Radiologe und Student wurden entsprechend der klinischen Konsequenz (unauffällig, kontrollbedürftig, biopsiebedürftig) 3 Kategorien zusammengefasst. Der Zeitaufwand der studentischen Kraft wurde gemessen.

Ergebnisse Die ROC Analyse zeigte eine Fläche unter der Kurve von 0,845 für PI-RADSv1 und 0,853 für PI-RADSv2. Der Schwellenwert $\geq$ PI-RADS 3 zeigte eine Sensitivität, Spezifität und richtige Klassifizierung in $95 \%$, 30\% und $53 \%$ für PI-RADSv1 sowie $88 \%, 62 \%$ und $72 \%$ für PI-RADSv2; der Schwellenwert $\geq$ PI-RADS 4 zeigte eine Sensitivität, Spezifität und richtige Klassifizierung in $80 \%, 77 \%$ und $78 \%$ für PI-RADSv1 sowie $83 \%, 84 \%$ und $84 \%$ für PI-RADSv2. Die Übereinstimmung zwischen Radiologe und Student zeigte einen Cohens kappa Koeffizienten von 0,386 für PI-RADSv1 und 0,412 für PI-RADSv2. Der mediane Zeitaufwand pro Läsion betrug 4:34 min für PI-RADSv1 und 2:00 min für PI-RADSv2 ( $<<0.001)$. 
Schlussfolgerungen PI-RADSv2 zeigt gegenüber PI-RADSv1 ein höheres diagnostisches Potenzial und eine bessere Übereinstimmung zwischen erfahrenem Radiologen und eingewiesener studentischer Kraft bei einem geringeren Zeitaufwand.

\section{WISS 402.1 Einfluss iterativer, Modell-basierter Rekonstruktionsalgorithmen (IMR) auf die Bildqualität von ultra-low-dose Steinsuche $\mathrm{CT}$ in adipösen Patienten}

Autoren Große Hokamp N ${ }^{1}$, Slebocki $\mathrm{K}^{1}$, Mammadov $\mathrm{K}^{1}$, Salem $\mathrm{I}^{2}$, Herden $\mathrm{J}^{2}$, Maintz $D^{1}$, Chang $D^{1}$

Institut 1 Uniklinik Köln, Institut für Diagnostische und Interventionelle

Radiologie, Köln; 2 Uniklinik Köln, Klinik für Urologie, Uro-Onkologie, spezielle urologische und roboter-assistierte Chirurgie, Köln

DOI 10.1055/s-0037-1600462

Zielsetzung Ultra-Low-Dose CT Protokolle (ULD-CT) sind in der Abklärung einer Ureterolithiasis etabliert. Limitationen ergeben sich vor allem bei adipösen Patienten aufgrund des konsekutiv erhöhten Bildrauschens. Diese Studie untersucht den Einfluss von IMR auf die Bildqualität bei ULD-CT zur Steinsuche in adipösen Patienten.

Material und Methodik Retrospektiv wurden im Rahmen der klinischen Routine akquirierte ULD-CT (100 kVp, $50 \mathrm{mAs})$ in adipösen Patienten (BMI $\geq 25 \mathrm{~kg} / \mathrm{m}^{2}$ ) mit der Frage nach Ureterolithiasis identifiziert und die Bilder unter Verwendung von gefilterter Rückprojektion (FBP), iterativer Rekonstruktion (iDose) und IMR rekonstruiert. Die Auswertung erfolgte objektiv durch die ROI-basierte Berechnung von Kontrast-zu-Rausch-Verhältnis (CNR) und subjektiv durch die unabhängige Bewertung durch zwei Radiologen auf einer 4-Punkte-Skala in Bezug auf diagnostische Sicherheit, Abgrenzbarkeit der Ureteren, Schärfe und Rauschen.

Ergebnisse 20 Patienten (BMI: $31,6 \pm 7,4 \mathrm{~kg} / \mathrm{m}^{2}$ ) mit Steinnachweis in $85.7 \%$ der Fälle wurden in die Studie eingeschlossen. Das durchschnittliche DosisLängen-Produkt betrug 106,8 $\pm 34,3 \mathrm{mGy} / \mathrm{cm}$. Die Verwendung von IMR führte zu einer Verbesserung der Bildqualität um $370 \%$ im Vergleich zu FBP und $122 \%$ im Vergleich zu iDose $(p>0,001)$. Auch in der subjektiven Analyse hat IMR bessere Ergebnisse als iDose bzw. FBP erzielt, insbesondere auch hinsichtlich der diagnostischen Sicherheit (3,2/4 gegenüber $2,2 / 4$ und 1,6/4, $\mathrm{p}<0,05)$.

Schlussfolgerungen ULD-CT Untersuchungen von adipösen Patienten unter Verwendung von IMR ermöglichen eine signifikant verbesserte Bildqualität gegenüber iDose und FBP. Der Einsatz von IMR sollte in diesen Untersuchungen bevorzugt werden.

\section{WISS 315.1 Subdifferenzierung von intermediären Läsionen (PI-RADS 3) in der multiparametrischen Prostata-MRT}

Autoren Hansen $\mathrm{N}^{1}$, Koo $\mathrm{B}^{2}$, Warren $\mathrm{A}^{3}$, Kastner $\mathrm{C}^{4}$, Barrett $\mathrm{T}^{2}$

Institut 1 Uniklinik Köln, Diagnostische und Interventionelle Radiologie, Köln; 2 Addenbrooke's University Hospital, Department of Radiology, Cambridge, UK; 3 Addenbrooke's University Hospital, Department of Pathology, Cambridge, UK; 4 Addenbrooke's University Hospital, Department of Urology, Cambridge, UK

DOI 10.1055/s-0037-1600463

Zielsetzung Wir untersuchten ob intermediäre Läsionen (PI-RADS 3) in der multiparametrischen Prostata-MRT mittels Analyse der T2- und Diffusionsgewichteten Bildeigenschaften weiter subdifferenziert werden können um die daraus folgende Entscheidung für oder gegen eine Biopsie zu erleichtern.

Material und Methodik 143 Männer hatten eine mpMRT mit intermediärem Befund nach PIRADS. Alle erhielten eine transperineale MR/US Fusions-Biopsie mit im Median 26 Stanzen von 2013 - 2015. Die Biopsie-Daten wurden innerhalb einer prospektiven MR basierten transperinealen Biopsie-Datenbank erfasst. 2 Radiologen mit 2 und 7 Jahren Erfahrung in der Prostata-MRT-Befun- dung führten eine geblindete retrospektive Zweit-Befundung jeder PI-RADS 3 Läsion durch, bewerteten diese anhand verschiedener T2- und Diffusionsgewichteten Bildeigenschaften und gaben eine Empfehlung ab zu „benötigt Biopsie“ oder „benötigt keine Biopsie“. Der positive prädiktive Wert (PPW) für das Vorliegen eines signifikanten Karzinoms mit GS $7-10$ ( \pm 95\% Konfidenzintervall) wurde berechnet für die jeweilige Biopsieempfehlung und mittels Exact Fisher Test verglichen.

Ergebnisse Insgesamt waren 61 (43\%) der Biopsien maligne, mit GS $7-10$ in $30(21 \%)$ Fällen. Bildeigenschaften wie Form, Berandung, Homogenität und Diffusionseinschränkung beeinflussten signifikant den PPV, abhängig von der Lokalisation der Läsion in Transitions- oder Peripherer Zone. Bei der Biopsieempfehlung stimmten die Zweit-Befunder in 62\% (89/143) der Fälle überein. Mit „benötigt keine Biopsie“ ( $n=66$ ) lag der PPW für ein Karzinom mit GS 6 10 bei $0.21( \pm 0.10)$, mit „benötigt Biopsie“ $(n=77)$ bei $0.61( \pm 0.11)$; $p=0.0001$. Der PPW für ein Karzinom mit GS 7-10 betrug mit „benötigt keine Biopsie“ $0.08( \pm 0.07)$ und mit "benötigt Biopsie“ $0.32( \pm 0.10)$; $\mathrm{p}=0.0003$.

Schlussfolgerungen Die Identifizierung bestimmter Bildmerkmale sowie eine subjektive Biopsieempfehlung durch einen erfahrenen Radiologen kann dabei helfen den PPW für PI-RADS 3 Läsionen zu steigern und zu entscheiden welche Patienten eine Biopsie benötigen.

\section{WISS 402.2 Kann die Nierenkortexvolumetrie anhand von CT Datensätzen die MAG3-Szintigrafie zur Beurteilung der seitengetrennten Nierenfunktion ersetzen?}

Autoren Houbois $\mathrm{C}^{1}$, Püsken $\mathrm{M}^{2}$, Merkt $\mathrm{M}^{2}$, Hellmich $\mathrm{M}^{3}$, Mainz $\mathrm{D}^{2}$, Haneder $S^{2}$

Institut 1 Uniklinik Köln, Institut für Diagnostische und Interventionelle Radiologie, Köln; 2 Institut für Diagnostische und Interventionelle Radiologie, Köln; 3 Institut für Medizinische Statistik, Informatik und Epidemiologie, Köln DOI 10.1055/s-0037-1600464

Zielsetzung Der aktuelle Goldstandard für die Berechnung der seitengetrennten Nierenfunktion (SRF) ist die MAG3-Szintigrafie. Ein alternatives Verfahren stellt die Volumetrie des Nierenkortex (renal cortex volumetry $=\mathrm{RCV}$ ) anhand von Kontrastmittel (KM)-verstärkten CT-Datensätzen dar. In einzelnen Studien konnten für beide Verfahren gleichwertige Ergebnisse erzielt werden, allerdings in selektionierten Patientenkollektiven aus Nierengesunden. Ziel dieser Arbeit ist die weitere Evaluation der RCV gegenüber dem Goldstandard in einem großen, unselektionierten Patientenkollektiv.

Material und Methodik Retrospektiv wurden 279 (132 Männer, 147 Frauen; Alter +/- 54,2 Jahre (SD 12,9)) Patientendatensätze, bei denen sowohl eine MAG3-Szintigrafie als auch eine KM-verstärkte $\mathrm{CT}$ des Abdomens in der früharteriellen Phase in einem Abstand von \pm 2 Wochen vorlag, ermittelt. Sämtliche Datensätze wurden von 2 Radiologen (R1/R2) unabhängig voneinander, je einmal mit dem semiautomatischen Volumetrie-Tool der Software Intellispace Portal (Philips Healthcare, Best, Niederlande) ausgewertet. Die statistische Analyse der Übereinstimmung nach Bland-Altman wurde mit dem Programm SPSS Statistics 24 (IBM Corp., Armonk, NY, USA) durchgeführt.

Ergebnisse In 249 Fällen mit normaler Nierenfunktion zeigte sich bzgl. der Differenz der SRF (rechts minus links, „delta“) für R 1 (R 2) ein bias von $-4 \%$ (-5\%) mit den limits of agreement $-25 \%$ bis $17 \%$ (-23\% bis $16 \%)$. Die Korrelation (nach Pearson) zwischen delta MAG3-Szintigrafie und RCV betrug 0.34 (0.46). Bzgl. der klinisch-relevanten Differenz von 20\% zeigte die RCV keinen (einen) falsch-positiven Fall und keinen (keinen) falsch-negativen. Bei den 30 Fällen mit eingeschränkter Nierenfunktion erwies sich die Übereinstimmung als unzureichend mit 1 (2) falsch-positiver Fall, 11 (12) falsch-negative. Schlussfolgerungen Die RCV anhand von CT Datensätzen ist eine vielversprechende, zuverlässige Untersuchungsmethode zur Bestimmung der SRF, welche allerdings bei Patienten mit einer Hydronephrose nicht aussagekräftig ist. 
WISS 402.4 Einfluss der rauschoptimierten virtuellen monoenergetischen Dual-EnergyComputertomografie auf die Bildqualität bei Patienten mit Nierenzellkarzinomen

\author{
Autoren Martin $\mathrm{S}^{1}$, Albrecht $\mathrm{M}^{1}$, Scholz $J^{1}$, Leithner $\mathrm{D}^{1}$, Lenga $\mathrm{L}^{1}$, Vogl $\mathrm{T}^{1}$, \\ Wichmann J' \\ Institut 1 Zentrum der Radiologie, Institut für Diagnostische und \\ Interventionelle Radiologie, Frankfurt \\ DOI 10.1055/s-0037-1600465
}

Zielsetzung Ziel der Studie ist es, den Einfluss des rauschoptimierten virtuellen monoenergetischen Rekonstruktionsalgorithmus ( $\mathrm{VMI+}$ ) auf die quantitative und qualitative Bildqualität bei Patienten mit Nierenzellkarzinomen (RCC) in der Dual-Energy Computertomografie (DECT) zu untersuchen.

Material und Methodik Es wurden die kortikomedulläre und nephrogener Phase von DECT-Datensätze von 52 Patienten (33 Männer, 61,5 \pm 13,6 Jahre) mit RCC mit standardmäßigen linear gewichteten Rekonstruktionen (M_0.6) sowie VMI+ und traditionelle virtuelle monoenergetische (VMI) Algorithmen in 10-keV Intervallen von 40 bis $100 \mathrm{keV}$ rekonstruiert. Region-of-intrest (ROI) Messungen wurden in RCC-Läsionen und im Nierenkortex durchgeführt, um das objektiven Kontrast-zu-Rauschen (CNR) Verhähltnis, sowie das Tumor-zuKortex-Verhältnis für kortikomedulläre und nephrogene Phasenbilder zu berechnen. Die subjektive Bildqualität wurde von drei Radiologen mit unterschiedlicher Erfahrung beurteilt.

Ergebnisse Die CNR-Werte waren am höchsten in den 40-keV VMI+ Bildern, sowohl in den kortikomedullären $(8,95 \pm 4,99)$, als auch in den nephrogenen Phasenbilder $(7,10 \pm 4,68)$, und waren signifikant höher im Vergleich zu allen anderen Rekonstruktionen $(P<0,001)$. Das Tumor-zu-Kortex-Verhältnis war am höchsten für VMI+ Rekonstruktionen bei $40 \mathrm{keV}(2,19 \pm 3,59)$ in der nephrogenen Phase und deutlich besser als alle anderen Serien $(P<0,001)$. Die qualitativen Bildparameter wiesen die höchsten Werte für VMI+ Bildrekonstruktionen bei $50 \mathrm{keV}$ in der kortikimedullären Phase, sowie bei $60-\mathrm{keV}$ in der nephrogenen Phasen auf, mit signifikanten Unterschieden gegenüber herkömmlichen VMI Rekonstruktionen und linear gewichteten M_0.6-Serien $(\mathrm{P} \leq 0,031)$.

Schlussfolgerungen Niedrig-keV VMI+ Rekonstruktionen haben eine deutlich höhere quantitative und qualitative Bildqualität von kortikomedullären und nephrogenen DECT Phasenbilder bei Patienten mit RCC.

\section{WISS 308.1 Detektion von Prostatakarzinomen in der multiparametrischen MRT: Einfluss der sexuellen Aktivität}

\author{
Autoren Marx $C^{1}$, Meier-Schroers $\mathrm{M}^{2}$, Schmeel $\mathrm{C}^{2}$, Block $\mathrm{W}^{2}$, Träber $\mathrm{F}^{2}$, \\ Sprinkart $\mathrm{M}^{2}$, Schild $\mathrm{H}^{2}$, Kukuk $\mathrm{G}^{2}$ \\ Institut 1 Universität Bonn, Radiologie, Bonn; 2 Radiologische \\ Universitätsklinik Bonn \\ DOI 10.1055/s-0037-1600466
}

Zielsetzung Vor einer multiparametrischen MRT-Untersuchung der Prostata wird meist eine sexuelle Abstinenz von 3-4 Tagen empfohlen. Wissenschaftlich belastbare Daten für diese Empfehlung liegen bislang nicht vor. Ziel der Studie war es, zu untersuchen, ob sexuelle Aktivität innerhalb von 8 Stunden vor der MRT einen Einfluss auf die MRT-Bildqualität und die Detektionsrate von Prostatakarzinomen hat.

Material und Methodik In einer prospektiven Studie wurden 18 Patienten (Altersdurchschnitt 64,4 Jahre \pm 8 Jahre) mit Verdacht auf ein Prostatakarzinom nach mpMRT entsprechend der ESUR-Leitlinien (sexuelle Abstinenz von mindestens 4 Tagen) eingeschlossen. Vor MR-gesteuerter Biopsie erhielten alle Patienten eine zweite mpMRT innerhalb von 8 Stunden nach sexueller Aktivität. Beide Untersuchungsprotokolle waren abgesehen von einer Kontrastmittelgabe in der ersten MRT identisch. Zwei erfahrene Radiologen klassifizierten alle Läsionen nach PI-RADS. Zudem wurde für alle Läsionen eine quantitative ROI-basierter Analyse des Kontrastes durchgeführt.
Ergebnisse In 18 Patienten wurden insgesamt 18 Läsionen detektiert. 14 Patienten $(77,8 \%)$ wiesen in ihrer Biopsie ein Prostatakarzinom auf. In der qualitativen Auswertung wurden alle Karzinome sowohl vor als auch nach sexueller Aktivität mit unverändertem PI-RADS Score bewertet. In der quantitativen Auswertung wurden signifikant höhere ADC-Werte in 15 Läsionen nach sexueller Aktivität (15/18 Läsionen, durchschnittlich $142 \mathrm{~mm} 2 / \mathrm{s}$, $(16 \%)(p<0.05))$ gemessen. In der T2-gewichteten Sequenz konnten keine statistisch signifikanten Signalveränderungen zwischen beiden Untersuchungen nachgewiesen werden (durchschnittlicher Kontrast der Läsionen zum umgebenden Prostatagewebe 1. MRT 0,66 vs. 2. MRT 0,69).

Schlussfolgerungen Sexuelle Aktivität vor einer multiparametrischen MRT der Prostata hat bei Patienten mit Verdacht auf ein Prostatakarzinom keinen Einfluss auf die PI-RADS-Klassifikation von Karzinomen. Es konnte jedoch eine geringfügige Erhöhung des ADC-Wertes nach sexueller Aktivität nachgewiesen werden.

\section{WISS 308.4 MultiVane XD für T2-gewichtete MRT- Aufnahmen der Prostata bei 3 Tesla: Auswirkungen auf die Abgrenzbarkeit von Läsionen, auf die PI-RADS- Klassifikation und auf Artefakte und Bildqualität}

Autoren Meier-Schroers $\mathrm{M}^{1}$, Marx $\mathrm{C}^{1}$, Schmeel $\mathrm{F}^{1}$, Rauch $\mathrm{M}^{1}$, Wolter $\mathrm{K}^{1}$, Block $W^{1}$, Sprinkart $A^{1}$, Träber $F^{1}$, Willinek $W^{2}$, Schild $H^{1}$, Kukuk $G^{1}$ Institut 1 Uniklinik Bonn, Radiologie, Bonn; 2 Brüderkrankenhaus Trier, Radiologie, Neuroradiologie, Sonografie und Nuklearmedizin, Trier DOI 10.1055/s-0037-1600467

Zielsetzung Zu klären, ob die MultiVane XD (MVXD)-Technik die Standard Turbo Spin Echo (TSE)-Technik für T2-gewichtete MRT-Aufnahmen der Prostata ersetzen kann.

Material und Methodik Es wurden MRT-Aufnahmen bei 3 Tesla von 50 Patienten mit 55 karzinomsuspekten Läsionen prospektiv ausgewertet. Bei allen Patienten wurden T2-gewichtete MVXD- und TSE-Sequenzen, diffusionsgewichtete Sequenzen, eine Kontrastmitteldynamik und eine MR-Spektroskopie angefertigt. Die Echozeiten der T2 MVXD und T2 TSE waren gleich. Die PIRADS-Scores (Version 2) wurden getrennt für TSE und MVXD erhoben. Die MVXD-Sequenz wurde in Bezug auf Artefakte, Bildqualität, PI-RADS-Scores sowie Größe, T2-Signalintensität und T2-Kontrast der Läsionen mit der TSESequenz verglichen. Die Befunde wurden mit den Ergebnissen der histopathologischen Untersuchung nach MRT-gesteuerter Prostatabiopsie korreliert.

Ergebnisse 41 der 55 Läsionen waren histologisch gesicherte Prostatakarzinome (75\%). Mittels T2 TSE waren 37 von 41 Karzinomen nachweisbar (90\%), mittels T2 MVXD 40 von 41 (98\%). In der T2 TSE konnten 4 Karzinome (10\%) aufgrund von ausgeprägten Artefakten nicht nachgewiesen werden. Das eine in der T2 MVXD nicht nachweisbare Karzinom war auch in der TSE nicht abgrenzbar. Die T2 MVXD zeigte signifikant weniger Artefakte und eine signifikant bessere Bildqualität (jeweils $p<0,001$ ). Zwischen der MVXD- und der TSE-Sequenz gab es keine Unterschiede beim PI-RADS-Einzelscore für T2-gewichtete Sequenzen $(p=0,106)$ und keine Unterschiede beim PI-RADS-Gesamtscore ( $p=0.107)$. Die Größe der Läsionen unterschied sich ebenfalls nicht $(p=0,105)$. Karzinomsuspekte Läsionen zeigten eine niedrigere T2-Signalintensität und einen höheren T2-Kontrast in der TSE-Sequenz (jeweils $p<0,001$ ). Schlussfolgerungen Die MVXD ist für die Detektion des Prostatakarzinoms aufgrund der Robustheit gegenüber Bewegungsartefakten, der besseren Bildqualität und der höheren Detektionsrate karzinomsuspekter Läsionen der TSE überlegen. Daher kann die MVXD die TSE in der T2-Bildgebung der Prostata ersetzen. 
WISS 315.9 MRT als bildgebendes Verfahren zur Identifizierung von Sentinel-Lymphknoten (SLN) bei Prostatakarzinomen (PCA) nach Applikation superparamagnetischer Eisenoxid-Nanopartikel (SPION)

Autoren Paulo $\mathrm{T}^{1}$, Winter $\mathrm{A}^{2}$, Kowald $\mathrm{T}^{1}$, Goos $\mathrm{P}^{2}$, Engels $\mathrm{S}^{2}$, Gerullis $\mathrm{H}^{2}$, Wawroschek $\mathrm{F}^{2}$, Charan $\mathrm{A}^{1}$

Institut 1 Klinikum Oldenburg, Institut für Interventionelle und Diagnostische Radiologie, Oldenburg; 2 Klinikum Oldenburg, Universitätsklinik für Urologie, Oldenburg

DOI 10.1055/s-0037-1600468

Zielsetzung Derzeit erfolgt die präoperative SLN Diagnostik bei Prostata-Ca überwiegend mittels Lymphszintigrafie nach Applikation eines RadionuklidTracers. Ziel der Studie ist es, die Eignung der MRT als neues Schnittbildverfahren zur Identifizierung von SLN nach zuvor erfolgter interstitieller, intraprostatischer Injektion eines SPION-Tracers darzulegen.

Material und Methodik 50 Patienten mit Mittel- und Hochrisikoprostatakarzinom wurden präoperativ am Tag vor (nativ) und nach intraprostatischer Injektion mit SPIO Nanopartikeln bei 1,5 Tesla untersucht. Erstellt wurden u. a. STIR,T1-, T2- und T2* -gewichtete Sequenzen des Beckens in transversaler und coronarer Ausrichtung. Die Identifizierung der Sentinel Lymphknoten in der MRT erfolgt über den Signalverlust der drainierenden LK im Lymphabflussgebiet in den $12^{*}$ w Sequenzen. Die Auswertung erfolgte durch 2 Fachärzte für Radiologie, Anzahl und anatomische Lage wurden dokumentiert. Am Tag nach der Injektion erfolgte die radikale Prostatektomie und pelvine Lymphadenektomie, intraoperativ wurden die SLN mit einem Handmagnetometer aufgesucht und entfernt.

Ergebnisse In allen MRT konnten SLN visualisiert werden. Insgesamt wurden 890 SLN (median 17.5; IQR 12 - 22.5) detektiert und ihrer anatomischen Lage zugeordnet. Hierbei entfielen 16,63\% auf die Region Arteria iliaca communis, 19,21\% Arteria iliaca externa, 13,82\% A. iliaca interna, 15,84\% Fossa obturatoria, $12,02 \%$ perirektal, $12,13 \%$ praesakral, 3,71\% periprostatisch, 2,25\% paravesikal und 4,38\% auf sonstige Regionen.

Schlussfolgerungen Die Identifizierung von SLN in der MRT nach intraprostatischer Injektion von SPION ist eine effektive und zuverlässige Methode und kann eine Alternative zur Lymphszintigrafie darstellen. Darüber hinaus liefert sie zusätzliche Informationen über die individuelle Beckenanatomie, was zu einer präziseren Operationsplanung beitragen kann.

\section{WISS 308.7 Kombination klinischer und MR- tomografischer Parameter zur Vorhersage signifikanter Prostatakarzinome und extrakapsulärer Tumorausdehnung}

Autoren Radtke $\rfloor^{1}$, Bonekamp $D^{1}$, Kesch $C^{2}$, Freitag $M^{1}$, Alt $C^{3}$, Celik $K^{2}$, Distler $\mathrm{F}^{2}$, Roth $\mathrm{W}^{4}$, Wieczorek $\mathrm{K}^{4}$, Duensing $\mathrm{S}^{2}$, Roethke $\mathrm{M}^{1}$, Teber $\mathrm{D}^{2}$, Schlemmer $\mathrm{H}^{1}$, Hohenfellner $\mathrm{M}^{2}$, Hadaschik $\mathrm{B}^{2}$

Institut 1 Deutsches Krebsforschungszentrum, Abteilung Radiologie, Heidelberg; 2 Universitätsklinikum Heidelberg, Urologische Universitätsklinik, Heidelberg; 3 Universitätsklinikum Düsseldorf, Institut für Diagnostische und Interventionelle Radiologie, Düsseldorf; 4 Ruprechts-Karl-Universität Heidelberg, Pathologisches Institut, Heidelberg

DOI 10.1055/s-0037-1600469

Zielsetzung Die multiparametrische MRT (mpMRT) gewinnt zur Diagnostik von Prostatakarzinomen ( $\mathrm{PCa}$ )zunehmend an Bedeutung und verbessert die Diagnose von signifikanten Prostatakarzinomen (SPCa) (Gleason-score $\geq 3+4$ ) und der extraprostatischen Tumorausdehnung (EPE). Zudem kann die Entscheidungsfindung zur Biopsie basierend auf European Randomised study of Screening for PC (ERSPC) Risikokalkulatoren (RC) helfen, Limitationen des PSAScreenings zu attenuieren. Wir kombinierten die präbioptische mPMRT einerseits mit ERSPC RC-Parametern und entwickelten ein Risikomodell (RM) um das individuelle Patientenrisiko vor Biopsie und andererseits mit klinischen Parametern um die seitenspezifische EPE vor radikaler Prostatektomie (RP) vorherzusagen.

Material und Methodik Für beide Fragestellungen wurden mittels multivariater Regressionsanalysen klinische und MR-tomografische Parameter anhand von Trainingsdatensätzen (755 Patienten für die Vorhersage des SPCa und 132 Patienten für die EPE-Prädiktion) zu Nomogrammen kombiniert und diese Nomogramme dann mit klinischen RCs mittels ROC Kurvenanalysen sowohl auf den Trainingsdatensätzen, als auch auf prospektiven Validierungskohorten (404 bzw. 132 Patienten) verglichen.

Ergebnisse PSA, Prostatavolumen, Digital-rektale Untersuchung und PI-RADS waren signifikante Prädiktoren eines SPCA und wurden in das RM inkludiert. Die ROC Area under the curve (AUC) des RM war sowohl in der Trainings- als auch in der Validierungskohorte signifikant größer als die ERSPC RCs und PIRADS allein. Zur seitenspezifischen EPE-Prädiktion war das kombinierte Modell (bestehend aus primäre Gleason Pattern der Biopsie, die ESUR MRT Kriterien der seitenspezifischen Läsion, die PSA-Dichte, DRU, das Volumen der Tumorläsion in Milliliter und die Kapselkontaktlänge der Läsion in Millimeter in der MRT) zwei etablierten klinischen Nomogrammen und der MRT allein ebenfalls in beiden Patientenkohorten signifikant überlegen.

Schlussfolgerungen Das RM, das ERSPC RC Parameter und PI-RADS kombiniert, war in vorliegender Analyse signifikant besser als RC und mpMRT allein und zeigte einen messbaren Benefit in der Entscheidungsfindung zur Prostatabiopsie bei Männern mit Verdacht auf ein Prostatakarzinom. Zur seitenspezifischen EPE Vorhersage zeigte sich durch das entwickelte RM ebenfalls ein signifikanter Vorteil vor RP.

\section{WISS 308.6 Die präoperative Vorhersagegenauigkeit der multiparametrischen Prostata-MRT in der Detektion der Gefäßnervenbündel-Infiltration mithilfe von PI-RADS Version 2}

Autoren Sauer $\mathrm{M}^{1}$, Weinrich $\mathrm{J}^{1}$, Salomon $\mathrm{G}^{2}$, Tennstedt $\mathrm{P}^{2}$, Adam $\mathrm{G}^{1}$, Beyersdorff $D^{1}$

Institut 1 Universitätsklinikum Hamburg Eppendorf, Diagnostische und interventionelle Radiologie und Nuklearmedizin, Hamburg;

2 Universitätsklinikum Hamburg Eppendorf, Martini Klinik, Hamburg DOI 10.1055/s-0037-1600470

Zielsetzung Die Analyse der Vorhersagegenauigkeit der multiparametrischen Prostata-MRT (mpMRT) mithilfe des revidierten Befundungsstandards PI-RADS V2 in Bezug auf die Infiltration der Gefäßnervenbündel vor radikaler Prostatektomie.

Material und Methodik Die lokale Ethikkomission hat der Durchführung der retrospektiven Studie zugestimmt. 198 Patienten erhielten vor radikaler Prostatektomie eine standardisierte mpMRT, einschließlich High Resolution T2wTSE in 3 Ebenen, T1w-TSE, DWI mit ADC-Karte, PDW-TSE und Kontrastmitteldynamik bei 3 Tesla. Für jeden Befund wurde das TNM Stadium festgesetzt und die Infiltration der Gefäßnervenbündel wurde für beide Seiten präoperativ bestimmt $(n=396)$. Weiterhin wurde ein maximaler PI-RADS V2 Wert für jedes posterolaterale Drüsenareal, angrenzend an die Gefäßnervenstränge, festgelegt. Diese präoperativen mpMRT-Ergebnisse wurden mit den postoperativen Pathologiebefunden als Referenzstandard korreliert.

Ergebnisse Sensitivität, Spezifität, positiver prädikativer Wert (PPW) und negativer prädikativer Wert (NPW) in der Bestimmung des generellen TNM-Stadiums betrugen $64,4 \%, 89,2 \%, 82,4 \%$ bzw. 76,2\%. Die Infiltration der Gefäßnervenstränge pro Patientenseite wurde erfasst mit 75,2\% und 94,0\% Sensitivität bzw. Spezifität (80,2\% PPW und 92,1\% NPW). 13 Gefäßnervenstränge, deren angrenzende Drüsenareale keinen suspekten PI RADS V2 Score aufwiesen, zeigten dennoch postoperativ eine Tumorinfiltration. Hiermit mussten $14 \%$ falsch negative Vorhersagen anerkannt werden.

Schlussfolgerungen Die präoperative Feststellung der Infiltration der Gefäßnervenbündel mithilfe der mpMRT zeigt eine hohe Spezifität und eine mode- 
rate Sensitivität und sollte bei der Entscheidung über eine Nerven-erhaltende Operationstechnik gewürdigt werden. Dennoch können unauffällige Drüsenareale in der Detektion mithilfe von PI-RADS V2 kapselüberschreitende Tumore in sich bergen.

\section{WISS 308.5 Wert der multiparametrischen Prostata MRT bei Patienten mit Gleason 3+3 Stanzbiopsie unter Active Surveillance oder vor radikaler Prostatektomie}

Autoren Sauer $\mathrm{M}^{1}$, Strölin $\mathrm{P}^{2}$, Salomon $\mathrm{G}^{2}$, Budäus $\mathrm{L}^{3}$, Adam $\mathrm{G}^{1}$,

Beyersdorff $D^{1}$

Institut 1 Universitätsklinikum Hamburg Eppendorf, Diagnostische und interventionelle Radiologie und Nuklearmedizin, Hamburg; 2 Martini Klinik, Universitätsklinikum Hamburg Eppendorf, Hamburg; 3 Universitätsklinikum Hamburg Eppendorf, Martini Klinik, Hamburg

DOI 10.1055/s-0037-1600471

Zielsetzung Der Vergleich von Tumorabgrenzbarkeit und -morphologie in der multiparametrischen MRT (mpMRT) von zwei Patientengruppen mit Gleason Score (GS) 3+3 Befund in der Stanzbiopsie: (1) unter Active Surveillance und (2) vor radikaler Prostatektomie (RP) mit anschließender Korrelation von Bildgebung und Pathologie.

Material und Methodik Die lokale Ethikkomission hat der Durchführung der retrospektiven Studie zugestimmt. 77 Patienten erfüllten die Kriterien zur Teilnahme an der PRIAS (Prostate Cancer Research International Active Surveillance) Studie (max. GS $3+3$, max. 2/ 10 positive Biopsien, PSA $\leq 10 \mathrm{ng} / \mathrm{ml}$ ) und erhielten im Zeitraum von 07/2014 bis 03/2016 eine mPMRT. 40 von diesen 77 entschieden sich für eine Active Surveillance (AS), während sich 37 einer radikalen Prostatektomie (RP) unterzogen. Alle Untersuchungen wurden in der klinischen Routine gemäß TNM-Klassifikation und PI-RADS V2 befundet und retrospektiv miteinander verglichen. Die mpMRT Befunde der Gruppe vor RP wurden mit den postoperativen Pathologieergebnissen korreliert.

Ergebnisse Von 40 AS Patienten entfiel keiner gemäß PI-RADS V2 auf die Kategorie 5. 31/40 wurden als PI-RADS 4 eingestuft, 8/40 als PI-RADS 3 und $1 / 40$ als PI-RADS 2. Von 37 RP Patienten entfielen 6 gemäß PI-RADS V2 auf die Kategorie 5. 29/37 wurden als PI-RADS 4 eingestuft und 2/37 als PIRADS 3. Der Mittelwert der RP Patienten war signifikant höher $(p<.0005)$ im Vergleich zu den AS Patienten (4,10 vs. 3,75). 22/37 (59,5\%) RP Patienten wurden postoperativ pathologisch upgegradet (18/22 auf $3+4 ; 2 / 22$ auf $4+3$; $1 / 22$ auf $4+5 ; 1 / 22$ auf $5+4)$.

Schlussfolgerungen PI-RADS Kategorie 4 ist das dominante Muster im mpMRT bei stanzbioptischem GS von 3+3. Patienten, die sich einer RP unterzogen, zeigten jedoch signifikant höhere PI-RADS Scores gegenüber Patienten unter AS. Im Umkehrschluss scheint somit ein signifikanter Einfluss der Bildgebung auf die Wahl des Therapieverfahrens gegeben. Mehr als die Hälfte dieser Patienten erfuhren ein Upgrading auf $\mathrm{GS} \geq 7$ in der postoperativen Histologie.

\section{WISS 315.4 PI-RADS v1 versus v2 zur Vorhersage eines Prostatakarzinoms: Analyse an Patienten mit Großflächen-Histologie als Goldstandard}

Autoren Schaudinn $A^{1}$, Mucha $S^{1}$, Linder $N^{1}$, Garnov $N^{1}$, Moche $\mathrm{M}^{1}$, Stolzenburg $\mathrm{J}^{2}$, Horn $\mathrm{L}^{3}$, Kahn $\mathrm{T}^{1}$, Busse $\mathrm{H}^{1}$, Gawlitza J

Institut 1 Universitätsklinikum Leipzig, Klinik für Diagnostische und Interventionelle Radiologie, Leipzig; 2 Universitätsklinikum Leipzig, Klinik für Urologie, Leipzig; 3 Universitätsklinikum Leipzig, Institut für Pathologie, Leipzig DOI 10.1055/s-0037-1600472

Zielsetzung Das strukturierte Befundungsschema für die multiparametrische MRT (mpMRT) der Prostata, PI-RADS (Prostate Imaging Reporting and Data System), liegt in seiner zweiten Version (v2) vor. Ziel unserer Studie war der Vergleich von PI-RADS v2 mit v1 hinsichtlich der Detektion eines Prostatakarzinoms (PCa).
Material und Methodik MRT-Daten von 28 Patienten ( $\varnothing 65$ Jahre) mit erhöhtem PSA-Wert ( $\varnothing 12,1 \mathrm{ng} / \mathrm{ml}$ ) und positiver transrektal Ultraschall-gestützter Biopsie wurden retrospektiv von einem geblindeten Auswerter (6 Jahre Erfahrung) mit PI-RADS v1 und v2 hinsichtlich aller PCa-suspekten Läsionen bewertet. Unmittelbar vor Prostatektomie unterzogen sich alle Patienten einem mpMRT der Prostata bei 3 T mit Endorektalspule (T2-Wichtung, DiffusionWichtung, Dynamische Kontrastmittel-Sequenz). Als Goldstandard diente die Histologie aus Großflächenschnitten des Prostatektomiepräparats. Verglichen wurden v1 und v2 hinsichtlich ihrer patienten- und läsionsbezogenen Sensitivität, dem positiv prädiktiven Wert (PPV) sowie der für die Bewertung dominanten Sequenz (höchster Einzelscore bei v1, zonenbezogen bei v2).

Ergebnisse Insgesamt wurden 41 Läsionen, 29 in der peripheren Zone und 12 in der Transitionalzone, beschrieben ( $\varnothing$ Gleasonscore: 6,5 ). Mit PI-RADS $\geq 4$ wurden in v1 34 und in v2 26 Läsionen bewertet. Die läsionsbezogene Sensitivität lag damit für v1 bei $82,1 \%$ und für v2 bei $64,1 \%$, der PPV für das Vorliegen eines PCa bei 94,1\% (v1) bzw. 96,2\% (v2). Im patientenbezogenen Ansatz ergaben sich Sensitivitäten von $85,7 \%$ für v1 und $64,3 \%$ von v2. Die dominante Sequenz aus v1 entsprach in $44 \%$ der Fälle der führenden (zonenbezogenen) Sequenz in v2.

Schlussfolgerungen Bei strikter Auslegung des zonenbestimmten Modells zur Bewertung der Wahrscheinlichkeit des Vorliegens eines Prostatakarzinoms zeigte PI-RADS v2 eine geringere Sensitivität als v1.

\section{WISS 315.6 Benötigen Patienten mit einem PI-RADS- Gesamtscore 3 eine sofortige (Re-)Biopsie?}

Autoren Schimmöller $L^{1}$, Ullrich $T^{1}$, Dietzel $F^{1}$, Schmaltz $A^{1}$, Quentin $M^{1}$,

Arsov $C^{2}$, Blondin $D^{3}$, Rabenalt $R^{2}$, Albers $P^{2}$, Antoch $G^{1}$

Institut 1 Heinrich-Heine-Universität Düsseldorf, Medizinische Fakultät,

Institut für Diagnostische und Interventionelle Radiologie, Düsseldorf;

2 Heinrich-Heine-Universität Düsseldorf, Medizinische Fakultät, Urologische

Klinik, Düsseldorf; 3 Städtische Kliniken Mönchengladbach GmbH, Elisabeth-

Krankenhaus Rheydt, Klinik für Radiologie, Gefäßradiologie und

Nuklearmedizin, Mönchengladbach

DOI 10.1055/s-0037-1600473

Zielsetzung Diese Studie evaluiert die diagnostische Genauigkeit der multiparametischen Prostata-MRT in Patienten mit einem PI-RADS-Gesamtscore von 3 hinsichtlich der Prostatakarzinomdetektion.

Material und Methodik Insgesamt 120 konsekutive Patienten mit einem PIRADS-Gesamtscore von 3 nach multiparametrischer 3-Tesla-MRT (T2WI, DWI, DCE-MRI) und anschließender gezielter MRT-gestützter Prostatabiopsie (Gruppe A: direkte MRI-(in-bore)-Biopsie; Gruppe B: MR/US-Fusions- plus systematischer transrektaler Ultraschallbiopsie) wurden retrospektiv in diese Studie eingeschlossen. Die Daten wurden hinsichtlich der Prostatakarzinomdetektion und der Detektion höhergradiger Karzinome (Gleason-Score $\geq 4+3=7 \mathrm{~b}$ ) analysiert. Darüber hinaus wurden diverse Parameter (Prostatavolumen, PSAWert, Anzahl negativer Vorbiopsien etc.) mit Biopsie-positiven Patienten korreliert.

Ergebnisse In der Gruppe A war die Biopsie in 35 von 39 und in der Gruppe B in 73 von 81 Patienten negativ (90\%). In 10 Patienten $(8,3 \%)$ wurde ein Niedrig-Risiko-Karzinom (Gleason-Score $3+3=6$ oder $3+4=7 a$ ) detektiert. In der Gruppe A konnte kein höhergradiges Prostatakarzinom nachgewiesen wurden, in der Gruppe B zeigten 2 Patienten ein Prostatakarzinom mit einem Gleason-Score von $4+3=7 b(1,7 \%)$. Die Prostatakarzinome wurden häufiger durch die zusätzlichen systematischen Stanzen detektiert. Das Prostatavolumen war niedriger in Patienten mit positiver Biopsie, die PSA-Werte und die Anzahl negativer, vorheriger TRUS-Biopsien unterschied sich in diesen Patienten jedoch nicht signifikant.

Schlussfolgerungen In Patienten mit einem PI-RADS-Gesamtscore von 3 liegen sehr selten höhergradige Prostatakarzinome vor, Niedrig-Risiko-Tumoren können jedoch vorkommen. Eine MRT-Verlaufs- bzw. Kontrolluntersuchung (z.B. nach 12 Monaten) erscheint primär angebracht, sodass diesen Patienten 
eine sofortige (Re-)Biopsie erspart bleiben kann. Voraussetzung ist eine qualitative Durchführung und Auswertung der multiparametrischen Prostata-MRT (analog PI-RADS v2).

\section{WISS 308.2 Prospektiver Vergleich der Prostata-MRT bei 1,5 versus 3,0 Tesla}

Autoren Ullrich $T^{1}$, Schimmöller $L^{1}$, Oelers $C^{1}$, Dietzel $F^{1}$, Arsov $C^{2}$, Rabenalt $\mathrm{R}^{2}$, Albers $\mathrm{P}^{2}$, Blondin $\mathrm{D}^{1}$, Antoch $\mathrm{C}^{1}$, Quentin $\mathrm{M}^{1}$

Institut 1 Heinrich-Heine-Universität Düsseldorf, Medizinische Fakultät, Institut für Diagnostische und Interventionelle Radiologie, Düsseldorf;

2 Heinrich-Heine-Universität Düsseldorf, Medizinische Fakultät, Urologische Klinik, Düsseldorf

DOI 10.1055/s-0037-1600474

Zielsetzung Diese prospektive Studie vergleicht die objektive Bildqualität (IQ), die subjektive IQ und die diagnostische Performance der Prostata-MRT bei 1,5 und 3 Tesla ohne Endorektalspule im selben Patienten.

Material und Methodik 63 konsekutive Patienten (64 9 Jahre; Prostatavolumen $63 \pm 34 \mathrm{ml}$ ) mit erhöhten PSA-Werten (Median 7,7 ng/ml; IQR 5,2$13 \mathrm{ng} / \mathrm{ml}$ ) erhielten jeweils eine MRT-Untersuchung der Prostata bei 3T (T2, DWI, DCE) und 1,5T (T2, DWI). Diese Nichtunterlegenheitsstudie wurde auf 80\% gepowert einen Unterschied des Signal-zu-Rausch-Verhältnisses (SNR) und des Kontrast-zu-Rausch-Verhältnisses (CNR) von $\leq 10 \%$ zwischen $1,5 \mathrm{~T}$ und 3T zu belegen. Sekundäre Endpunkte waren die subjektive IQ (5-Punkte-Skala) und die PI-RADS-v2-Bewertung durch zwei verblindete (Uro-)Radiologen.

Ergebnisse Das SNR und CNR der T2-gewichteten Sequenzen war vergleichbar zwischen 1,5T und 3T $(p=0,7-1)$, jedoch in der Diffusionsbildgebung (DWI) signifikant niedriger bei 1,5T $(p<0,01)$. Die subjektive IQ war sowohl für T2 als auch für DWI signifikant besser bei $3 T(p<0,01)$. Die PI-RADSEinzelscores und Gesamtscores unterschieden sich nicht signifikant zwischen den beiden Magnetfeldstärken $(p=0,5-1)$. Die Interrater-Reliabilität war exzellent, sowohl bei subjektiver IQ, als auch bei dem PI-RADS-Scoring ( $k=0,9$ 1).

Schlussfolgerungen Die MRT der Prostata bei 1,5T kann vergleichbare Bildqualität in den T2-gewichteten Sequenzen liefern, ist jedoch in der Diffusionsbildgebung (DWI) und auch in der subjektiven Bildqualität gegenüber 3T unterlegen. Nichtsdestotrotz ist unter optimierten Aufnahmeparametern bei 1,5T eine vergleichbare diagnostische Performance (mittels PI-RADS) bei beiden Magnetfeldstärken ohne Endorektalspule möglich.

\section{WISS 308.3 Simultane Mehrschichtakquisition zur Beschleunigung der MR-Diffusionsbildgebung an der Prostata - eine Machbarkeitsstudie zur Beurteilung der klinischen Anwendung}

Autoren Weiß I $^{1}$, Martirosian $\mathrm{P}^{2}$,Taron $\mathrm{J}^{2}$ Othman $\mathrm{A}^{2}$, Künstner $\mathrm{T}^{3}$, Bedke $\mathrm{J}^{4}$, Bamberg $\mathrm{F}^{2}$, Nikolaou $\mathrm{K}^{2}$, Notohamiprodjo $\mathrm{M}^{2}$

Institut 1 Uni Klinik Tübingen, Radiologie, Tübingen; 2 Radiologische Klinik, Diagnostische und Interventionelle Radiologie, Tübingen; 3 Universität Stuttgart, Signalprozessierung und Systemtheorie, Stuttgart; 4 Urologische Klinik, Urologische Klinik, Tübingen

DOI 10.1055/s-0037-1600475

Zielsetzung Beurteilung der simultanen Mehrschichtakquisition (SMS) zur Beschleunigung der MR-Diffusionsbildgebung an der Prostata in der klinischen Anwendung.

Material und Methodik Alle Untersuchungen wurden an einem 3T System durchgeführt. Für Phantommessungen wurde ein Diffusionsphantom mit verschiedenen Zuckerkonzentrationen $(0-40 \%)$ verwendet. In vivo Messungen wurden an 10 gesunden Probanden und 16 Patienten mit V.a. Prostatakarzinom durchgeführt. Bei allen Messungen wurde eine Prototypsequenz zur simultanen Mehrschichtdiffusionsbildgebung (DW-EPISMS; Akquisitionszeit 3:14 min) mit einer konventionellen single-shot Diffusionssequenz (DW-EPISS;
Akquisitionszeit 6:12 min) als Referenzstandard verglichen. Die Bildqualität wurde sowohl qualitative (Bildqualität, anatomische Differenzierbarkeit, Abgrenzbarkeit von Läsionen, Rauschen, Verzerrung, 2 unabhängige Reader, 5Punkteskala, 5 =exzellent) und quantitative (Coefficient-of-Variation (CV); ADC-Werte) beurteilt.

Ergebnisse Die DW-EPISMS ermöglicht eine deutliche Verkürzung der Akquisitionszeit um ca. 50\%. Die Bildqualität, anatomische Differenzierbarkeit, Abgrenzbarkeit von Läsionen und Distorsion von b1000 gewichteten Bilder und ADC-Karten zeigten keine signifikanten Unterschiede zwischen den Sequenzen $(p \geq 0.2)$. Lediglich in der Probandenstudie wurde das Rauschen der DW-EPISS höher gewertet als in der DW-EPISMS $(p=0.05)$. Keine signifikanten Unterschiede zeigten die Messungen der ADC-Werte und der Signalintensitäten in den b1000 gewichteten Bildern ( $p \geq 0.3$ ). Nur die CV-Berechnung in den b1000 gewichteten Bildern der DW-EPISMS zeigte signifikant höher Werte als bei der DW-EPISS $(p<0.001)$.

Schlussfolgerungen Die simultane Mehrschichtakquisition ist zur Beschleunigung der Diffusionsbildgebung an der Prostata im klinischen Alltag anwendbar und ermöglicht bei deutlicher Verkürzung der Akquisitionszeit eine vergleichbare qualitative und quantitative Bildqualität wie die Standardsequenz.

\section{Poster (Wissenschaft)}

\section{Bildverarbeitung/IT/Software/Gerätetechnik/ \\ Qualitätsmanagement}

\section{Poster 7.2 Stand-alone Auswerte-Tool für CT-Perfusion der Leber}

Autoren Busse $\mathrm{H}^{1}$, Garnov $\mathrm{N}^{1}$, Brandmaier $\mathrm{P}^{1}$, Seider $\mathrm{D}^{1}$, Alhonnoro $\mathrm{T}^{2}$, Pollari $\mathrm{M}^{2}$, Kahn $\mathrm{T}^{1}$, Moche $\mathrm{M}^{1}$

Institut 1 Universitätsklinikum Leipzig, Klinik und Poliklinik für Diagnostische und Interventionelle Radiologie, Leipzig; 2 Aalto University, Department of Neuroscience and Biomedical Engineering, Helsinki, FI

DOI 10.1055/s-0037-1600476

Zielsetzung CT-Perfusionsparameter können für die Evaluation der Leberdurchblutung (z.B. nach LTX oder Resektion) oder zur Planung von Interventionen hilfreich sein. Bei der Analyse stellen die duale Blutversorgung sowie die Atembeweglichkeit der Leber besondere Anforderungen. Momentan gibt es einige kommerzielle Pakete der jeweilige CT-Gerätehersteller. Hier soll eine Stand-alone Analyse-Software beschrieben werden, die herstellerunabhängig arbeitet.

Material und Methodik Ein Matlab-basiertes Tool erlaubt das Einlesen der Perfusionsdaten verschiedener Hersteller im DICOM-Format. Die Bewegungskorrektur erfolgt über eine nichtrigide 3D-Registrierung. Die Daten werden wahlweise als zeitliche Einzelbilder oder als Maximum-Intensitäts-Projektion dargestellt mit der Möglichkeit, durch die Schichten und Zeitpunkte zu scrollen. Der Nutzer muss interaktiv jeweils eine ROI in der Arterie, Pfortader und Milz definieren. Die Perfusionsanalyse findet mittels Maximum-Slope-Methode vollautomatisiert oder mit Nutzer-Interaktion statt. Für drei beliebige ROIs im Lebergewebe werden dann die arterielle Leberperfusion (ALP), Pfortaderperfusion (PVP), die Summe der beiden (TLP = ALP+PVP) und der hepatische Perfusionsindex (HPI) berechnet. Das Tool wurde an 12 Perfusionsdatensätzen mit einer kommerziellen Software (CT Body Perfusion, Siemens) verglichen.

Ergebnisse Die aktuelle Version kann Datensätze von drei Geräteherstellern verarbeiten (Philips, Siemens, Toshiba). Die Bewegungskorrektur eines Datensatzes mit 32 Schichten und 30 Zeitpunkten dauerte an einem PC mit einer 6Kern CPU etwa 8 Min. Die mittleren relativen Abweichungen von PVP und TLP betrugen $10,4 \%$ bzw -11,6\% (vs. kommerzielle Software).

Schlussfolgerungen Das vorgestellte Stand-alone CT-Perfusionstool ermöglicht eine bewegungskorrigierte Auswertung der CT-Perfusionsdaten verschiedener Hersteller. 
Poster 9.2 Implementierung eines Computersimulationsmodells zur Generierung von Lungenemphysemen auf der Basis von Thorax-CTDatensätzen Lungengesunder

\author{
Autoren Kartal $\mathrm{R}^{1}$, Limburg $\mathrm{R}^{1}$, Krombach $\mathrm{G}^{1}$, Obert $\mathrm{M}^{1}$ \\ Institut 1 Universitätsklinikum Gießen, Klinik für Diagnostische und \\ Interventionelle Radiologie, Gießen \\ DOI 10.1055/s-0037-1600477
}

Zielsetzung Zur Beurteilung der Leistungsfähigkeit von quantitativen Bildanalyseparametern wie z.B. dem Emphysemindex werden bislang subjektiv beurteilte Bilder von Radiologen als Goldstandard herangezogen. In dieser Studie werden mit objektivierbaren numerischen Methoden CT-Bilder modifiziert, in die durch Computerberechnungen verschiedene Emphysemschweregrade hineinsimuliert wurden.

Material und Methodik Emphyseme wurden kreisförmig simuliert und der Schweregrad anhand der Kreisanzahl variiert. Die Software durchläuft folgende Schritte für alle CT-Schichten eines Patienten $(n=8)$ : Jede CT-Schicht wird eingelesen, die Lungenhälften werden segmentiert und zur Modifikation zwischengespeichert. Danach werden Pseudozufallszahlen für Parameter wie die Größe der Kreisradien oder die prozentuale Änderung für die HE-Minderung innerhalb der Kreise erzeugt. Anhand der Parameter wird das Emphysembild berechnet. Der Modifikationsvorgang wird solange wiederholt, bis die gewünschte Kreisanzahl erreicht ist. Entstehende Artefakte in Gefäß- und Bindegewebsarealen werden weitgehend entfernt. Die Kreisanzahl und der Emphysemindex (EI) werden in Dateien gespeichert. Originale und veränderte CTBilder werden im Lungenfenster gesichert.

Ergebnisse Die Software durchläuft alle CT-Schnittbilder mehrerer Patienten und sichert alle Daten verlustfrei. Ein erfahrener Radiologe bestätigte stichprobenartig den Emphysemcharakter. Die Trachea wurde als Teil der Lungenhälften erkannt, sodass entsprechende Daten bei der Auswertung keine Verwendung fanden. Für die verbleibenden 850 CT-Schichten wurden die Kreisanzahl und die El-Differenz der veränderten und originalen Lungenhälften korreliert, der Spearman Korrelationskoeffizient $r$ beträgt 0.926, $p=0.01$.

Schlussfolgerungen Mit der erstellten Software können Lungenemphyseme automatisiert und streng reproduzierbar simuliert werden. Somit ist die Grundlage für die objektive Evaluierung verschiedener Methoden zur Quantifizierung von Emphysemen geschaffen worden.

\section{Poster 6.3 Automatisierte Auswertung von multiparametrischen MRTs für die Prostatakrebs Detektion: Eine ausreichende Alternative zur Biopsie? Ergebnisse von 104 histologisch gesicherten Läsionen}

Autoren Papageorgiou $I^{1}$, Thon $A^{2}$, Teichgräber $U^{3}$, Kovacevic $D^{4}$,

Wintzler $S^{4}$, Malich $A^{4}$

Institut 1 Südharz Klinikum Nordahusen, Insitut für Radiologie, Nordhausen; 2 Südharz Klinikum Nordhausen gGmbH, Dr.-Robert-Koch str. 39, D-99734 Nordhausen, Institut für Radiologie, Nordhausen; 3 Jena University Hospital, Friedrich-Schiller University, Jena, Institut für Diagnostische und

Interventionelle Radiologie, Abteilung Experimentelle Radiologie, Jena;

4 Südharz Klinikum Nordahusen, Insitut für Radiologie, Nordahusen

DOI 10.1055/s-0037-1600478

Zielsetzung Die quantitative Untersuchung von multiparametrischen MRTs mit Computer- assistierten Diagnosesystemen (CADs) stellt eine Herausforderung in der Diagnostik von Prostatakrebs (PCa) dar. Unser Ziel war die Bewertung der Genauigkeit von Watson Elementary ${ }^{\mathrm{TM}}$ CAD-Software auf Basis der Datenbank von MRT-gestützt-bioptierten Prostataläsionen aus unserer Klinik. Material und Methodik Wir überprüften retrospektiv 104 Läsionen (47 PCa, 57 benigne) von insgesamt 79 Patienten, im Alter von 64,61 \pm 6,64 Jahren, welche gemäß $3 \mathrm{~T}$ T2w, ADC und dynamisch- kontrastmittelgestützten MRTs untersucht wurden. Watson Elementary ${ }^{\mathrm{TM}}$ kalkuliert einen „malignacy atten- tion index“ (MAI), welche mit den zugehörigen histologisch gesicherten Bereichen verglichen wurde.

Ergebnisse Ergebnisse: Die PCa Läsionen zeigten eine signifikante ADC Signalsenkung von ca. 31,57\% (von 950/340 auf 650/295 Median/IQR), $\mathrm{P}<0.001$, ranksum. Das CAD- System fand 36/104 Läsionen, dies entspricht einer Sensitivität von 46,80\% für PCa mit einer falsch negativen Rate von $0,53 /$ pt. Die Spezifität liegt bei $75,43 \%$ mit einem positiv prädiktiven Wert von $61,11 \%$, einem negativ prädiktiven Wert von $63,23 \%$ und falsch detektierter Rate von 38,89\%. Watson Elementary ${ }^{\mathrm{TM}}$ erkannte maligne und benigne Läsionen mit gleicher Wahrscheinlichkeit (P 0.06, ï $\ddot{z}^{1} / 2 \mid K 2$ test) und der MAI lässt keine signifikante Korrelation mit dem Gleason Score (P 0.60, Pearson correlation) erkennen. Die ROC gibt einen schwachen Vorhersagewert für den MAI mit AUC 0,63 \pm 0.06 (MAI median/SEM), P 0.02. Der MAI cut-off Wert wurde mit 0.535 geschätzt, dies entsprach einem Sensitivität/Spezifiät Verhältis von $61,70 \%$ (Cl 46,38-75,49\%) und 68,42\% (Cl 54,76-80.09\%).

Schlussfolgerungen Schlussfolgerung: MAl eignet sich nicht als Gleason Vorhersage in unserer Untersuchung. Divergenzen mit vorausgegangenen Publikationen wurden erörtert. Keine Interessenkonflikte zu erklären.

Gastro- und Abdominaldiagnostik

\section{Poster 7.1 T1 mapping in der Gd-EOB-DTPA- gestützten MRT zur Detektion und Graduierung von Leberfibrose}

Autoren Haimerl $\mathrm{M}^{1}$, Utpatel $\mathrm{K}^{2}$, Verloh $\mathrm{N}^{1}$, Fellner $\mathrm{C}^{1}$, Stroszczynski $\mathrm{C}^{1}$, Wiggermann $\mathrm{P}^{1}$

Institut 1 Universitätsklinikum Regensburg, Institut für Röntgendiagnostik, Regensburg; 2 Universität Regensburg, Institut für Pathologie, Regensburg DOI 10.1055/s-0037-1600479

Zielsetzung Ziel dieser Studie ist die Differenzierung histopathologisch aufgearbeiteter Leberfibrosegrade mithilfe Gd-EOB-DTPA- gestützter T1 Relaxometrie.

Material und Methodik 65 Patienten mit histologisch untersuchten Leberbiopsien bzw. Leberteilresektaten unterzogen sich einer Gd-EOB-DTPA-gestützten 3T MRT einschließlich MR-Relaxometrie. Der Grad der Leberfibrose wurde nach dem METAVIR score 5 Gruppen zugeordnet: Patienten ohne Leberfibrose ( $F 0 ; n=10)$; geringgradiger Leberfibrose $(F 1 ; n=14)$; fortgeschrittener Leberfibrose (F2; $n=15$ ); schwerwiegender Leberfibrose ( $F 3 ; n=12$ ) und Leberzirrhose ( $F 4 ; n=14)$. Um T1 maps zu generieren wurde eine 3D VIBESequenz vor und 20 Minuten nach iv Gd-EOB-DTPA-Gabe akquiriert und die Reduktionsrate der T1 Relaxationszeiten (rrT1) zwischen nativen und Gd-EOBDTPA-gestützten Sequenzen berechnet. Mittels ROC-Analyse wurden die optimalen cut-off Werte für die einzelnen Fibrosegrade bestimmt.

Ergebnisse Mit steigendem Grad der Leberfibrose (F0- F4) zeigte sich ein konstanter, signifikanter Abfall der rrT1 (\%): F0, 69.4 2.6 ; F1, $59.1 \pm 3.7$; F2, $55.3 \pm 4.6$; F3, 42.2 \pm 0.10 ; und F4, $28.8 \pm 8.2$. In einer einfachen Regressionsanalyse zeigte sich somit ein signifikanter Zusammenhang zwischen rrT1 und dem Grad der Leberfibrose $(r=-0.906, p<0.001)$. In der ROC- Analyse zeigte die T1 Relaxometrie hinsichtlich der Graduierung einzelner Leberfibrosegrade gute Ergebnisse mit folgenden AUC - Werten: 1.0 für Fibrosegrad $\geq F 1,0.92$ für Fibrosegrad $\geq F 2,0.98$ für Fibrosegrad $\geq F 3$ und 0.96 für Fibrosegrad F4. Schlussfolgerungen Die Gd-EOB-DTPA-gestützte T1 Relaxometrie stellt eine nützliche Methode dar unterschiedliche Leberfibrosegrade zu differenzieren. Insbesondere zeigen die Ergebnisse der ROC- Analyse die hohe Trennschärfe zur Detektion initial fibrotischer Veränderungen des Leberparenchyms auf. 
Herzdiagnostik/Gefäßdiagnostik

\section{Poster 10.2 Inzidentelle thrombotische Ereignisse in der CT bei Intensivstation-Patienten}

Autoren Meyer $\mathrm{H}^{1}$, Schramm $\mathrm{D}^{2}$, Bach $\mathrm{A}^{2}$, Surov $\mathrm{A}^{2}$

Institut 1 Universitätsklinikum Leipzig, Klinik und Poliklinik für Diagnostische und Interventionelle Radiologie, Leipzig; 2 Klinik und Poliklinik für Radiologie, Halle (Saale)

DOI 10.1055/s-0037-1600480

Zielsetzung Patienten auf der Intensivstation stellen eine Risikogruppe für die Entstehung von Thrombosen und Thromboembolien dar. Das Ziel dieser Studie war es die Frequenz und die Lokalisation von klinisch stummen thrombotischen Ereignissen (TE) von Patienten einer Intensivstation an Hand der Computertomografie (CT) zu analysieren.

Material und Methodik Von 2006 bis 2013 wurden 370 Patienten (135 Frauen, 235 Männer) der Intensivstation unseres Universitätsklinikum mittels Kontrastmittel verstärkter CT untersucht. Diese CTs wurden zur Suche von septischen Foki durchgeführt. Die CT-Untersuchungen beinhalteten die cervikale, thorakale, abdominale und pelvikale Region. Alle CT-Aufnahmen wurden von 2 Radiologen im Konsens erneut ausgewertet. Es wurden lediglich inzidentelle TE in die Analyse aufgenommen, bekannte Thrombosen wurden ausgeschlossen. Die Patienten wurden in eine chirurgische Patientengruppe und eine nicht-chirurgische Patientengruppe eingeteilt.

Ergebnisse Bei 31,9\% der Patienten wurden verschiedene Arten von TE diagnostiziert. Diese waren venöse Thrombosen (89,8\%), pulmonale Embolien (7,6\%) und kardiale Thrombosen (2,6\%). Am häufigsten war die Vena jugularis, gefolgt von der Vena brachiocephalica und der Vena iliaca betroffen. Es ergab sich keine statistisch signifikante Differenz in der Frequenz der TE zwischen der chirurgischen Patientengruppe und der nicht-chirurgischen Patientengruppe, jedoch wurden in der chirurgischen Gruppe statistisch signifikant häufiger Thrombosen in den Extremitäten gefunden. Insgesamt wurden 61,9\% aller TE zum Zeitpunkt der damaligen CT-Untersuchung nicht korrekt diagnostiziert.

Schlussfolgerungen 31,9\% der Intensivstation-Patienten zeigten in der CT inzidentelle TE. Die häufigsten TE waren venöse Thromben. Deswegen sollte der Radiologe bei einer CT-Untersuchung eines Patienten einer Intensivstation sorgfältig auf die verschiedenen Arten von TE achten.

\section{Poster 10.1 Erhöhte Wandschubspannung und Wandschubspannungsgradient im 4D-Fluss MRT bei Patienten nach klappenerhaltendem Aortenwurzelersatz}

Autoren Schultz $\mathrm{V}^{1}$, Oechtering $\mathrm{T}^{1}$, Sieren $\mathrm{M}^{2}$, Scharfschwerdt $\mathrm{M}^{3}$, Hennemuth $A^{4}$, Hüllebrand $M^{4}$, Sievers $H^{5}$, Barkhausen $J^{1}$, Frydrychowicz $A^{1}$ Institut 1 Universitätklinikum Schleswig-Holstein, Klinik für Radiologie und Nuklearmedizin, Lübeck; 2 Universitätsklinikum Schleswig-Holstein, Radiologie, Lübeck; 3 Universitätsklinikum Schleswig-Holstein, Klinik für Herzund thorakale Gefäßchirurgie, Lübeck; 4 Fraunhofer, Mevis, Bremen; 5 Universitätklinikum Schleswig-Holstein, Klinik für Herz- und thorakale Gefäßchirurgie, Lübeck

DOI 10.1055/s-0037-1600481

Zielsetzung Nach klappenerhaltendem Ascendensersatz mit anatomisch geformter Sinusprothese wurde eine nahe-physiologische Hämodynamik im Aortenbulbus bestätigt. Distal der Prothese fanden sich jedoch vermehrt sekundäre Flussmuster. Ziel war daher die Analyse der von der Hämodynamik abgeleiteten Wandschubspannung (WSS).

Material und Methodik 12 Patienten (1w, Alter $55 \pm 15$ Jahre) mit Sinusprothese (Uni-Graft ${ }^{\circledR}$ SINUS, Braun) und 12 altersgematchte Probanden (10w, Alter $55 \pm 6$ Jahre) wurden mit 4D-Fluss-MRT an einem 3T-MRT (Philips Achieva) untersucht. Die Untersuchungsparameter beinhalteten retrospektive EKG-
Triggerung und Atemgating, eine von 2,4 mm auf 2,0 mm interpolierte Ortsauflösung und 20 rekonstruierte Herzphasen. Zur WSS Analyse wurden auf 8 Ebenen der Aorta (AO) mit je 8 Segmenten in GTFlow (GyroTools, CH) zeitliches WSS-Maximum, das Minimum und Maximum des WSS je Segment und der WSS-Gradient (maxWSSseg - minWSSseg) berechnet. Mittels t-Test wurde auf statistische Signifikanz $(p<0,05)$ geprüft.

Ergebnisse Das WSS-Maximum war in Prothesennähe mit Gesunden vergleichbar oder geringer (prox. AO acendens: $0,57 \pm 0,18$ vs. $0,66 \pm 0,12 \mathrm{~N} /$ $\mathrm{m} 2$ ) und zeigte distal der Prothese eine Tendenz zu höherem WSS (dist. AO ascendens: $0,74 \pm 0,19$ vs. $0,68 \pm 0,15 \mathrm{~N} / \mathrm{m} 2$ ). Gesteigerter segmentaler WSS bei Patienten ab der prox. $\mathrm{AO}$ ascendens unterstützte diese Tendenz; in der distalen $A O$ ascendens $(1,21 \pm 0,29$ vs. $0,92 \pm 0,16 \mathrm{~N} / \mathrm{m} 2)$ und Aortenbogen $(1,22 \pm 0,45$ vs. $0,90 \pm 0,16 \mathrm{~N} / \mathrm{m} 2)$ erreichten die Differenzen statistische Signifikanz $(p<0,05)$. Der WSS-Gradient bei Patienten zeigte allseits höhere Werte mit einem Maximum in der $A O$ ascendens $(1,03 \pm 0,43$ vs. $0,72 \pm 0,26 \mathrm{~N} / \mathrm{m} 2)$. Schlussfolgerungen Nach Sinusprothesenimplantation sind die WSS-Veränderungen nahe der Prothese geringer als distal der Prothese, was gut mit den sekundären Flussmustern vereinbar scheint. Der WSS-Gradient hingegen zeigte durchgehend ausgeprägte Unterschiede zwischen Patienten und Probanden, was ihn zu einem interessanten Parameter für zukünftige Studien macht.

Interventionelle Radiologie

\section{Poster 2.3 Experimentelle Untersuchungen zur Effizienz verschiedener aspirationsgestützter Thrombektomiesysteme in der Behandlung thrombembolischer Verschlüsse der femoropoplitealen Strombahn im Flussmodell}

Autoren Aludin $\mathrm{S}^{1}$, Schäfer $\mathrm{P}^{1}$, Groß $J^{2}$, Rusch $\mathrm{R}^{2}$, Jansen $\mathrm{O}^{1}$, Trentmann $]^{1}$ Institut 1 UKSH, Campus Kiel, Klinik für Radiologie und Neuroradiologie, Kiel; 2 UKSH, Campus Kiel, Klinik für Herz- und Gefäßchirurgie, Kiel DOI 10.1055/s-0037-1600482

Zielsetzung Untersuchung der Effizienz verschiedener aspirationsgestüzter Thrombektomiesysteme in der Behandlung akuter Verschlüsse der femoropoplitealen Strombahn an verschiedenen Thrombusentitäten im Flussmodell. Material und Methodik In einem Flussmodell der arteriellen Becken-Beinstrombahn wurde ein standardisierter thrombembolischer femoropoplitealer Verschluss mit drei unterschiedlichen, aus humanem Blut hergestellten, Thrombusentitäten simuliert. Vier verschiedene Aspirationssysteme wurden in jeweils fünf Durchgängen pro Thrombusentität bezüglich des Thrombektomieerfolges und des Auftretens distaler Makroembolien ( $\geq 3 \mathrm{~mm}$ ) gegeneinander getestet. Zur Verwendung kamen: Einfache Aspiration, Stentretriever + Aspiration, lasergestützte Thrombusdegradation + Aspiration, mechanische Fragmentierung + Aspiration.

Ergebnisse Unser Modell erlaubt die standardisierte Herstellung makroskopisch und mechanisch differenter Thromben, mit denen in vitro ein arterieller femoropoplitealer Gefäßverschluss simuliert werden kann. Der First-Pass-Rekanalisationserfolg für die verschiedenen Thromben (Grau/Rot/Weiß) betrug für Aspiration $0 \% / 80 \% / 80 \%$, für Stentretriever + Aspiration 60\%/100\%/100\%, für Laserdegradation + Aspiration 20\%/20\%/20\%, für mechanische Fragmentierung + Aspiration 100\%/100\%/100\%. Die Embolierate für Aspiration betrug $40 \% / 0 \% / 0 \%$, für Stentretriever + Aspiration $80 \% / 40 \% / 20 \%$, für Laserdegradation + Aspiration $40 \% / 80 \% / 0 \%$, für mechanische Fragmentierung + Aspiration $60 \% / 60 \% / 40 \%$.

Schlussfolgerungen Die Effizienz der getesteten Aspirationssysteme unterscheidet sich in Abhängigkeit von der Thrombusentität. Im Allgemeinen kann eine Steigerung des First-Pass-Rekanalisationserfolges durch zusätzliche Anwendung von Stentretrievern oder mechanischer Fragmentierung erzielt werden; dies jedoch jeweils mit Steigerung der Embolierate. Hinsichtlich des Rekanalisationserfolges und der Embolierate ist die zusätzliche Laserdegradation in unserem Modell insgesamt am ineffizientesten. 


\section{Poster 2.1 Interventional treatment of peripheral vascular malformations - Long-term results with special emphasis on Quality of Life aspects}

Authors Greling $\mathrm{B}^{1}$, Meila $\mathrm{D}^{2}$, Grieb $\mathrm{D}^{3}$, Melber $\mathrm{K}^{3}$, Schmitz $\mathrm{T}^{3}$, SchlunzHendann $\mathrm{M}^{3}$, Brassel $\mathrm{F}^{3}$, Loose $\mathrm{DA}^{4}$

Institute 1 Sana Kliniken Duisburg, Radiologie und Neuroradiologie, Duisburg; 2 Sana Kliniken Duisburg, Medizinische Hochschule Hannover, Klinik für Radiologie und Neuroradiologie, Institut ful’^r Diagnostische und Interventionelle Neuroradiologie, Duisburg; 3 Sana Kliniken Duisburg, Klinik für Radiologie und Neuroradiologie, Duisburg; 4 Bereich Gefäßchirurgie und Angiologie, Die Facharztklinik Hamburg und Klinik Fleetinsel Hamburg, Hamburg

DOI $10.1055 / \mathrm{s}-0037-1600483$

Zielsetzung We present our long-term results of patients with peripheral venous malformations (PVM) and peripheral arteriovenous malformations (PAVM) after interventional treatment.

Material und Methodik We retrospectively analyzed the medical files of 100 patients with PVM (41) and PAVM (59) that were treated between 2002 and 2014 at our department. The interventional procedure of choice for PAVM was primarily a transarterial superselective embolization using liquid embolic agents, and percutaneous sclerotherapy with Polidocanol for PVM. Patients answered a symptoms based Quality of Life (QoL) questionnaire before and after treatment.

Ergebnisse 30 patients with PVM and 44 patients with PAVM answered the questionnaire. In 407 therapeutic sessions (PVM: 112, PAVM: 295) 16 minor complications directly related to the treatment were encountered. $70 \%$ in PVM-group reported an improvement in motion pain, 54\% in PAVM-group. $70 \%$ in PVM-group reported an improvement in rest pain, 50\% in PAVMgroup. $50 \%$ in PVM-group reported an improvement in functional impairment, $52 \%$ in PAVM-group. $40 \%$ in PVM-group reported an improvement in skin deformity, 27\% in PAVM-group. 56\% in PVM-group reported an improvement in swelling, 50\% in PAVM-group. 34\% in PVM-group reported an improvement in sensibility disorders, $13 \%$ in PAVM-group. 53\% in PVM-group reported an improvement in impairment in daily life, 50\% in PAVM-group. More than $95 \%$ of patients with PAVM and PVM were very satisfied with their treatment in general.

Schlussfolgerungen Interventional treatment is a well-tolerated therapy for PAVM an PVM with a low complication rate. Positive subjective results and improvement in different aspects of QoL can be achieved.

\section{Poster 3.3 Evaluation of radiation exposure to the examiner in the angiografy in routine clincal practice using a $\mathrm{x}$-ray protictive glove}

Authors Kamusella $\mathrm{P}^{1}$, Scheer $\mathrm{F}^{2}$, Lüdtke $\mathrm{C}^{3}$, Wiggermann $\mathrm{P}^{4}$, Wissgott $\mathrm{C}^{2}$, Andresen $\mathrm{R}^{2}$

Institute 1 Röntgenpraxis Heide, Heide; 2 Westküstenklinikum Heide, Institut für Diagnostisch und Interventionelle Radiologie/Neuroradiologie Akademisches Lehrkrankenhaus der Universitäten Hamburg, Kiel und Lübeck, Heide; 3 Klinikum Nordfriesland, Radiologisches Zentrum, Husum;

4 Universitätsklinum Regensburg, Radiologie, Regensburg

DOI 10.1055/s-0037-1600484

Zielsetzung Purpose of the study was to evaluate the radiation exposure to the examiner in the angiografy in routine clinical practice without and withxrayprotective gloves using a direct dosimeter.

Material und Methodik Phantom memeasurements were conducted in the direct $x$-ray beam and in the area of scattered Radiation without and with protection. Patient measuremnts were determined in interventional angiografies of the lower limb in antegrade puncture technique via the femoral artery. in $24 / 50$ interventions, an elastic natural rubber latex glove with lead-free metal shielding against Radiation was used. All measurements were per- formed with a direct Dosimeter. At the end of the investigations, the glove in relation to sensomotor characteristicswas evaluated.

Ergebnisse Phantom measurements: under application oft he protective glove a significant increase oft he dose area product 1084.2 to 1603.8 $\mathrm{mGy}^{*} \mathrm{~cm}^{2}(\mathrm{p}<0.001)$ and of the examiners hand dose 143 to $221.8 \mu \mathrm{Sv}$ $(p<0.001)$ can be found in the direct $x$-ray beam. A significant reduction oft he examiners hand dose is verifiable for scattered radiation 1.76 to $0.75 \mu \mathrm{Sv}$ $(p<0.001)$. Patient measurements: under application oft he protective glove a significant increase oft he dose area product 6183.2 to $10462.9 \mathrm{mGy}^{*} \mathrm{~cm}^{2}$ $(p<0.05)$ and a decrease of the average dose rate 0.76 to $0.43 \mathrm{mSv} / \mathrm{h}$ fort he entire procedurewas determined. The tactile sensitivity was assed as not restricted (18/24).

Schlussfolgerungen The new generation of protective gloves are characterized by a shielding effect against X-ray diffused radiation, without restricting the sense of touch. However, an absolute recommendation for the use of protective gloves cannot be made, since this/these cause(s) an increase in the dose both for the patient and the examiner, especially in the area of the direct radiation exposure (in the path to be punctured)

\section{Poster 2.2 Korrelation zwischen der Absenkung des hepatischen portal-venösen Druckgradienten nach TIPSS Implantation und der Änderung der intrahepatischen venösen Arborisation}

Autoren Tealip $S^{1}$, Diamantis $I^{2}$, Aschenbach $R^{2}$, Teichgräber $U^{2}$

Institut 1 Univeritätsklinikum Jena, Institut für Diagnostische und

Inerventionelle Radiologie IDIR, Jena; 2 Universitätsklinikum Jena, Institut für Diagnostische und Interventionelle Radiologie, Jena

DOI 10.1055/s-0037-1600485

Zielsetzung Untersuchung der Korrelation zwischen Absenkung des hepatischen portal-venösen Druckgradients (HVPG) und der intrahepatischen portalvenösen Arborisation nach TIPSS-Implantation.

Material und Methodik Von Insgesamt 116 TIPSS-Intervention in der Zeit zW. 01/2011 und 04/2015, sind 43 Patienten (27 今., 16 \%; Durchschnittsalter 62 \pm 9 ,8 Jahre) eingeschlossen worden. HPVG wurde vor und nach TIPSS Implantation bestimmt. Direkt Portografie via Pigtail- Katheter positioniert im Konfluenz des V. portae wurde durchgeführt. Basiert auf den KM-Füllungsgrad der intrahepatischen Verzweigung der Pfortaderäste ist eine Klassifikation für die intrahepatische Arborisation entwickelt worden; 0: keine Visualisierung der portalvenösen Äste; 1: Visualisierung der Hauptäste, 2: Teil-Visualisierung des Gefäßbaum, 3: komplette Darstellung des Gefäßbaumes inklusiv der peripheren Äste. Abhängigkeit zwischen beiden Parametern wurde statistisch nach Pearson-Koeffizient geprüft.

Ergebnisse Durchschnittliche HVPG vor TIPSS Implantation lag bei 22,5 $\pm 6,1 \mathrm{~mm} \mathrm{Hg}$, nach TIPSS $11,9 \pm 6,4 \mathrm{~mm} \mathrm{Hg}$. Nach unserer Klassifikation ist die Arborisation im Durchschnitt von 2.6 vor TIPSS auf 0,72 nach TIPSS gesunken. Pearsons Korrelationskoeffizient vor TIPSS $(r=-0,45)$, nach TIPSS $(r=-0,106)$, und zwischen HVPG Differenz und Absenkung des Verzweigungsgrad $(r=0,038)$. Damit besteht keine Korrelation zwischen den 2 Parametern. Jedoch könnte ein Zusammenhang zwischen den Grad der Arborisation und das Auftreten von hepatischer Enzephalopathie beobachtet werden (bei 8 Patienten davon 7 mit 0 Arborisation). Das Risiko eines TIPSS-Verschluss steigt bei einer Arborisation von 2 (bei 5 Patienten war eine Dilatation erforderlich davon 4 mit Arborisation $\geq 2$ ).

Schlussfolgerungen Eine Reduktion des HVPG hat keinen direkten Einfluss auf die postinterventionelle interhepatische Arborisation. Der Grad der Arborisation stellt einen guten prognostischen Faktor für das Risiko einer hepatischen Enzephalopathie oder eines TIPSS-Verschlusses dar und ist dabei dem HVPG überlegen. 
Kinderradiologie

\section{Poster 9.3 Röntgenaufnahmen des pädiatrischen Thorax im Kontext der aktuellen Leitlinien der Europäischen Kommission - Sind die Vorgaben in der täglichen Routine realisierbar?}

\author{
Autoren Krüger $\mathrm{P}^{1}$, Schmidt $\mathrm{F}^{1}$, Otto $S^{1}$, Lange $\mathrm{A}^{2}$, Langner $S^{1}$ \\ Institut 1 Universitätsmedizin Greifswald, Institut für Diagnostische \\ Radiologie und Neuroradiologie, Greifswald; 2 Universitätsmedizin Greifswald, \\ Klinik und Poliklinik für Kinder- und Jugendmedizin, Greifswald \\ DOI 10.1055/s-0037-1600486
}

Zielsetzung In der pädiatrischen Bevölkerung ist die akkurate Einblendung zur Reduktion einer unnötigen Strahlenexposition und verbesserter Bildqualität von immenser Bedeutung. Für die pa/ap Röntgenaufnahmen des Thorax gibt die neue Leitlinie 16261 der Europäischen Kommission eine minimale Feldgröße mit einer altersabhängigen Toleranz vor. Die Qualitätskriterien dieser Leitlinie wurden in einer Universitären Kinderradiologie qualitativ und quantitativ evaluiert.

Material und Methodik In einem Zeitraum von 12 Monaten wurden 1238 Röntgenaufnahmen des Thorax (58\% männlich, $42 \%$ weiblich, Alter $0-17$ Jahre) mithilfe eines semi-automatischen Algorithmus ausgewertet. Qualitätskriterien, minimale Feldgröße und Überbelichtung wurden aufgrundlage der EC-Leitlinien beurteilt.

Ergebnisse Insgesamt erfüllten nur 4,4\% aller Aufnahmen die EC-Leitlinien vollständig. Die am häufigsten korrekten Aufnahmen (14,1\%) wurden von Kleinkindern angefertigt. Allgemein wurden $46,3 \%$ als unter- und $43,9 \%$ als überbelichtet gewertet. Es wurden zwischen mittlerer Überbelichtung und Gewebeüberbelichtung unterschieden. Es erfolgte eine semi-automatische Überprüfung der Qualitätskriterien tiefe Inspiration und Rotation, die übrigen Kriterien wurden visuell von zwei unabhängigen Radiologen überprüft. In Abhängigkeit des Alters verbesserten sich beide Kriterien (Inspiration Neugeborene: $21,2 \%$, Adoleszente: $81,6 \%$ ). Darüberhinaus wurden alle Aufnahmen unter Berücksichtigung der angefertigten Abteilung ausgewertet. Eine signifikant vermehrte Überbelichtung konnte bei Aufnahmen aus der neonatologischen Intensivstation $(69,9 \%)$ im Vergleich zur päd. Intensivstation $(56,7 \%)$ festgestellt werden.

Schlussfolgerungen Die Leitlinien der Europäischen Kommission sind unverzichtbar, um Kinder vor unnötiger Strahlenexposition zu schützen. In der täglichen Routine erscheint die Realisierbarkeit insbesondere auf den Intensivstationen eingeschränkt möglich. Eine semi-automatische Qualitätskontrolle ist eine schnelle und zuverlässige Methode die Patientensicherheit zu verbessern.

\section{Kopf/Hals-Diagnostik}

\section{Poster 8.1 Quantifizierung parodontaler Knochensubstanzdefekte mittels Dental-MRT}

Autoren Heil $A^{1}$, Rütters $\mathrm{M}^{2}$, Kim $\mathrm{T}^{2}$, Bendszus $\mathrm{M}^{1}$, Heiland $\mathrm{S}^{1}$, Hilgenfeld $\mathrm{T}^{1}$ Institut 1 Universitätsklinikum Heidelberg, Neuroradiologie, Heidelberg; 2 Universitätsklinikum Heidelberg, Poliklinik für Zahnerhaltungskunde, Sektion Parodontologie, Heidelberg

DOI 10.1055/s-0037-1600487

Zielsetzung Durch anwendungsoptimierte Technologien gewinnt die Magnetresonanztomografie (MRT) zunehmend an Bedeutung für Fragestellungen der Parodontologie. Die Standardbildgebung parodontaler Knochensubstanzdefekte erfolgt mittels projektionsradiografischer Zahnfilm (ZF)-Aufnahmen, welche u.a. die Quantifizierung der sog. Restknochenhöhe (RKH) erlauben. Ziel dieser prospektiven In-vivo-Machbarkeitsstudie war es, am Beispiel der RKH zu evaluieren, ob die MRT gegenüber dem ZF eine gleichwertige Quantifizierung parodontaler Knochensubstanzdefekte ermöglicht.
Material und Methodik Diese prospektive Studie wurde von der lokalen Ethikkommission genehmigt. Unter Anwendung der Bezugspunkte Zahnapex, Defektboden und Pulpenhorn wurde ein Quotient (q) etabliert, welcher die RKHBestimmung sowohl im ZF als auch in der MRT erlaubt. In fünf Patienten mit fortgeschrittener Parodontitis wurde mithilfe dieser Methode die RKH von insgesamt 41 parodontalen Defekten im ZF und in der MRT (3 Tesla: T1w TSE fatsat + Gd) bestimmt. Die Messungen erfolgten durch zwei unabhängige Untersucher (Inv.1, Inv.2) an zwei Zeitpunkten (Intervall > vier Wochen). Die statistische Analyse erfolgte mittels Pearson-Korrelationskoeffizient ( $r$ ), BlandAltman-Analyse sowie Wilcoxon-Rangsummentest.

Ergebnisse Insgesamt zeigte sich für beide Modalitäten eine sehr gute Reliabilität der Messungen und es ergab sich ein hohes Maß an Übereinstimmung der RKH-Quotienten (q) im Vergleich ZF vs. MRT. Die Intraobserver-Reliabilität lag bei $r=0,928 / 0,993$ (Inv.1/Inv.2) für ZF und bei $r=0,995 / 0,989$ (Inv.1/ Inv.2) für MRT. Die Interobserver-Reliabilität betrug $r=0,968$ für ZF und $r=0,978$ für MRT. Im Vergleich ZF vs. MRT ergaben sich Korrelationen von $r=0,951 / 0,971$ (Inv.1/Inv.2) sowie Bias-Werte in der Bland-Altman-Analyse von -0,006/-0,002 (Inv.1/Inv.2). Der Wilcoxon-Rangsummentest zeigte keine signifikanten Unterschiede zwischen den beiden Modalitäten ( $p>0.05)$.

Schlussfolgerungen Die Dental-MRT erscheint zur Bestimmung des parodontalen Knochenabbaus geeignet.

\section{Molekulare Bildgebung}

\section{Poster 4.3 A novel apelin-receptor targeted contrast agent for molecular ultrasound imaging of tumor angiogenesis: in vitro evaluation}

Authors Stiglbauer $A^{1}$, Hegedüs $B^{2}$, Döme $B^{3}$, Laszlo $V^{4}$, Helbich $T^{1}$ Institute 1 Medizinische Universität Wien, Universitätsklinik für Radiologie und Nuklearmedizin, Wien; 2 Medizinische Universität Wien, Universitätsklinik für Chirurgie, Wien; 3 Medizinische Universität Wien, Universtitätsklinik für Chirurgie, Wien; 4 Medizinische Universität Wien, Universitätsklinik für Radiologie und Nuklearmedizin, Universitätsklinik für Chirurgie, Wien DOI 10.1055/s-0037-1600488

Zielsetzung To evaluate the binding specificity of a novel apelin-receptortargeted contrast agent for molecular ultrasound imaging of tumor angiogenesis in vitro. Bracco Posterpreis

Material und Methodik Apelin-receptor (APJ) expression of human umbilical vein endothelial cells (HUVECs) was confirmed with immunofluorescent staining of HUVECs with a biotinylated anti-APJ antibody and a streptavidin-conjugated fluorescent dye. Target-ready microbubbles (Vevo MicroMarker, Visualsonics, Toronto, Canada) were either conjugated with the biotinylated anti-APJ antibody or a biotinylated isotype-matched control antibody. Concentration and size of both types of targeted microbubbles were assessed. HUVECs were grown on microscopic glass slides and incubated with either APJtargeted microbubbles or control microbubbles for $5 \mathrm{~min}$, then cells were washed with phosphate buffered saline and the binding of each type of microbubble per cell was visually assessed under an inverted brightfield microscope by counting microbubbles manually. An unpaired t-test was used for statistical analysis of binding specificity for each type of microbubble.

Ergebnisse Mean number of microbubbles bound to HUVEC was 5.57 per cell for APJ-targeted and 1.78 for control microbubbles, respectively. APJ-targeted microbubbles showed a significantly higher binding specificity to HUVECs than control microbubbles $(p=0.021)$. Binding of microbubbles corresponded to APJ expression proven by immunohistochemistry.

Schlussfolgerungen APJ expression of HUVECS targeted by a novel contrast agent for molecular ultrasound imaging may serve as a potential imaging biomarker in tumor angiogenesis. 
Muskuloskelettale Radiologie

\section{Poster 1.3 Vorhersage des Ausheilens von Pseudarthrosen einer Extremitätenfraktur mithilfe klinischer Scores, des kontrastverstärkten Ultraschalls (CEUS) und der dynamischen kontrastverstärkten MRT (DCE-MRT)}

Autoren Weber $\mathrm{M}^{1}$, Bruckner $\mathrm{T}^{2}$, Schmidmaier $\mathrm{G}^{3}$, Kauczor $\mathrm{H}^{4}$, Fischer $\mathrm{C}^{3}$ Institut 1 Universitätsklinikum Heidelberg, Diagnostische und Interventionelle Radiologie, Heidelberg; 2 Universität Heidelberg, Institut für Medizinische Biometrie und Informatik, Heidelberg; 3 Universitätsklinikum Heidelberg, Orthopädie und Unfallchirurgie, Heidelberg;

4 Universitätsklinikum Heidelberg, Diagnostische und Interventionelle Radiologie, Heidelberg

DOI 10.1055/s-0037-1600489

Zielsetzung Prospektive Beurteilung, ob die dynamische kontrastverstärkte MRT (DCE-MRT) und der kontrastverstärkte Ultraschall (CEUS) die Konsolidierung einer Pseudarthrose (PA) nach einer Revisionsoperation vorhersagen können.

Material und Methodik Die Vaskularisierung innerhalb des PA-Spalts wurde präoperativ bei 205 Patienten (im Mittel: 51,5 Jahre, 76 Frauen) mit einer im CT nachgewiesenen PA einer Extremitätenfraktur vor einer Revisionsoperation und $6,12,26$, sowie 52 und mehr Wochen postoperativ quantifiziert. Die mittels 3-Tesla DCE-MRT unter i.v. Gabe von Gadotersäure und einer ROIAnalyse ermittelten Signalintensitätszeitkurven wurden anhand der umgebenden gesunden Muskulatur normiert und die PA in gut oder schlecht vaskularisiert eingeteilt. Eine entzündliche Genese der PA wurde mittels mikrobiologischer Kulturen des resezierten PA-Gewebes überprüft. Zusätzlich wurden 43 Patienten (46,8 Jahre, 14 Frauen) mittels CEUS nach i.v. Bolusgabe von 2,4 ml Schwefelhexafluorid-Mikrobläschen untersucht. Die Fähigkeit, eine knöcherne Konsolidierung in der CT vorherzusagen, wurde für Parameter des CEUS und der DCE-MRT sowie für den Body-Mass-Index (BMI) und das NonUnion Scoring System (NUSS) mittels ROC-Analyse bewertet.

Ergebnisse 103 der 169 im Verlauf verfügbaren Patienten (61\%) zeigten schließlich eine Heilung der PA und diese PA hatten eine bessere Vaskularisierung als jene PA ohne Konsolidierung zur $6(p=0,023), 12(p=0,025)$ und 26 $(p=0,009)$ Wochen Verlaufskontrolle (Sensitivität/Spezifität der DCE-MRT nach 26 Wochen: 75/87\%, Falschklassifikationsrate: 19\%). 50\% aller PA konsolidierten innerhalb von 14 Monaten. Ein niedriger BMI $(p=0,041)$ und NUSS $(p<0,0001)$ waren mit einer Konsolidierung im Verlauf assoziiert. Der CEUS detektierte präoperativ erhöhte Perfusionskinetiken in Infekt-PA ( $n=16 / 43$, Rise Time $(p=0,024)$, Time-to-Peak $(p=0,011)$, Sensitivität/Spezifität: 93 / $65 \%)$.

Schlussfolgerungen Die DCE-MRT prädiziert 26 Wochen postoperativ die Konsolidierung einer PA und präoperativ sind CEUS, NUSS und BMI prognostisch wichtig.

\section{Neuroradiologie}

\section{Poster 8.4 Systemische Luftembolien nach Endoskopie - eine Übersicht}

Autoren Voigt $\mathrm{P}^{1}$, Schob $\mathrm{S}^{1}$, Gottschling $\mathrm{S}^{2}$, Kahn $\mathrm{T}^{3}$, Surov $\mathrm{A}^{3}$ Institut 1 Universitätsklinikum Leipzig, Abteilung Neuroradiologie, Leipzig; 2 Herzzentrum Leipzig, Abteilung für Radiologie, Leipzig;

3 Universitätsklinikum Leipzig, Klinik für Diagnostische und Interventionelle Radiologie, Leipzig

DOI 10.1055/s-0037-1600490

Zielsetzung Systemische Luftembolien sind eine seltene Komplikation nach Endoskopien. Dabei sind vor allem zerebrale Luftembolien aufgrund ihrer prognostischen Bedeutung besonders ernstzunehmende Ereignisse. Ziel der Ar- beit war es, einen Überblick über die veröffentlichten Fälle systemischer Luftembolien nach Endoskopie zu geben.

Material und Methodik Methode: In einer systematischen Recherche wurde PubMed nach beschriebenen Fällen systemischer Luftembolien durchsucht. Es wurden aus diesen Fällen Lokalisation der Embolie(n), Vorhandensein eines persistierenden Foramen ovale, Therapie und Outcome eruiert. Zusammen mit einem Fall aus dem eigenen Patientenkollektiv umfasst die vorliegende Auswertung 40 Patienten.

Ergebnisse Die überwiegende Mehrzahl der in die Auswertung eingeschlossenen Patienten erhielt eine ERCP (60\%) oder Gastroskopie (33\%), der Rest sonstige endoskopische Prozeduren. Zerebrale Luftembolien wurden weitaus am häufigsten beobachtet (73\%). In annähernd der Hälfte der Patienten konnte ein persistierendes Foramen ovale bestätigt werden (46\%). Therapeutisch kam meist die hyperbare Oxygenierung zur Anwendung. Das Outcome war insgesamt schlecht $-45 \%$ der beschriebenen Patienten verstarben infolge der systemischen Luftembolien.

Schlussfolgerungen Systemische Luftembolien sind - insbesondere aufgrund der hohen Rate zerebraler Embolien - eine ernstzunehmende Komplikation endoskopischer Prozeduren und gehen mit einer hohen Morbidität und Letalität einher. Besonders Patienten mit bekanntem persistierendem Foramen ovale sollten vor Endoskopien über die Möglichkeit paradoxer Luftembolien und das damit einhergehende Schlaganfallrisiko aufgeklärt werden. Bei Risikopatienten ist zudem ein zurückhaltender Einsatz sedierender Medikation während der Endoskopie zu erwägen, um neurologische Frühsymptome nicht zu maskieren.

\section{Poster 8.3 Intralabyrinthäre Schwannome, eine unterschätzte Entität}

Autoren Zirkler $\mathrm{E}^{1}$, Plontke $\mathrm{S}^{2}$, Rahne $\mathrm{T}^{2}$, Kösling $\mathrm{S}^{1}$

Institut 1 Universitätsklinikum Halle (Saale), Radiologie, Halle (Saale);

2 Universitätsklinikum Halle (Saale), HNO, Halle (Saale)

DOI 10.1055/s-0037-1600491

Zielsetzung Intralabyrinthäre Schwannome (ILS) sind selten. Diese benignen Tumoren entwickeln sich von Schwann-Zellen nervaler Strukturen im Innenohr und fallen im MRT durch eine intralabyrinthäre Kontrastmittel-Anreicherung auf. Bei einer Schichtdicke von $\geq 3 \mathrm{~mm}$ können ILS in der MRT übersehen werden.

Material und Methodik Wir berichten über 21 Patienten mit intralabyrinthären Schwannomen, die im Zeitraum von 2008 bis 2016 in unserer Einrichtung vorstellig wurden. Es handelte sich um 10 Frauen und 11 Männer, ihr medianes Alter betrug 53 Jahre. Die Indikation zur operativen Therapie wurde bei 7 Patienten gestellt, die Diagnose konnte in diesen Fällen histologisch bestätigt werden. In den übrigen Fällen wurde die Verdachtsdiagnose durch Kontrolluntersuchungen erhärtet.

Ergebnisse Klinisch klagten die Patienten vom akuten Hörsturz mit Tinnitus, akuten Schwindel über schleichende Schwerhörigkeit bis hin zur vollständigen Ertaubung. Die rechte Seite war doppelt so häufig betroffen wie die linke. Die Tumoren lagen bei 11 Patienten intrakochleär: basale Windung $(W)(n=3)$, basale und mittlere $W(n=2)$, apikale $W(n=1)$, apikale und mittlere $W(n=3)$, alle $W(n=2)$. Bei 4 Patienten lag eine intravestibuläre Manifestation, bei 5 eine vestibulokochleäre und bei 1 Patienten eine transotische vor.

Schlussfolgerungen Trotz existierender Studien sind ILS wenig bekannt. Bei entsprechender Klinik sollte daher im Rahmen der gezielten Diagnostik der Region des Kleinhirnbrückenwinkels auch an ILS gedacht werden. Da ILS wie Vestibularisschwannome sehr langsam wachsen, sind bei fehlender Interventionsindikation MRT-Kontrollen im Abstand von 6 Monaten, danach 1 Jahr und 2 Jahr vorzuschlagen. 
Thoraxradiologie

\section{Poster 9.1 Charakterisierung von Milchglastrübung bei Patienten mit chronisch thromboembolischer pulmonaler Hypertonie im Vergleich zur pulmonalen Hypertonie anderer WHO-Klassifikation mittels Dual- Energy CT}

\author{
Autoren Leifheit J $]^{1}$, Moritz-Tugral $\mathrm{R}^{1}$, Krombach $\mathrm{G}^{1}$ \\ Institut 1 Universitätsklinikum Justus-Liebig-Universität Gießen, \\ Diagnostische und Interventionelle Radiologie, Gießen \\ DOI 10.1055/s-0037-1600492
}

Zielsetzung Bestimmung typischer Veränderungen des Lungenparenchyms bei pulmonaler Hypertonie $(\mathrm{pH})$ durch CT-Datensätzen des Dual-Energy-CT (DECT), sowie „Perfused Blood Volume“ (PBV)- und "Virtual Non-Contrast" (VNC)-Rekonstruktionen.

Material und Methodik Es wurden 29 Patienten, davon 20 mit CTEPH $(8 \mathrm{~m}$, $12 \mathrm{w}, 64 \mathrm{~J}$.) und $19 \mathrm{mit} \mathrm{pH}$ anderer Genese ( $10 \mathrm{~m}, 9 \mathrm{w}, 69 \mathrm{~J}$.), mittels DECT bei 80 und $140 \mathrm{kV}$ untersucht. Es wurden die Ausdehnung (Skala 0 -4) und Ausprägung von Milchglastrübung (Skala 0-3),sowie die Perfusion in den PBVDatensätzen (Skala 1-3) subjektiv bewertet. Der jeweilige Punktewert für Ausdehnung und Ausprägung wurde pro Patient zu einem Gesamtscore addiert. Die objektive Auswertung wurde durch HE-Messungen der hyper- und hypodensen Bereiche an jeweils identischen Positionen der drei Rekonstruktionen vorgenommen.

Ergebnisse Bei CTEPH lag der mittlere Score der Ausdehnung von Milchglastrübung bei 15,1 auf der rechten und 14 auf der linken Seite und bei 5,8 auf der rechten und 5,6 auf der linken Seite in der PH-Gruppe. Der mittlere Score der Ausprägung bei CTEPH lag bei 10,8 auf der rechten und 10 auf der linken Seite, während die Werte der PH-Gruppe mit 3,7 auf der rechten und 4 auf der linken Seite deutlich niedriger waren. Auch die farbcodierten Perfusionsbilder zeigten deutlich mehr Perfusionsunterschiede in der CTEPH-Gruppe. Beim Vergleich der verschiedenen Rekonstruktionen (PBV, VNC) konnte man Perfusionsdefekte, sowie hyperperfundierte Areale exakt zuordnen. Der Vergleich zwischen den beiden Gruppen in den hyperdensen und hypodensen Arealen zeigte signifikante Werte mit $p<0,0001$ in allen hypodensen Arealen der drei Rekonstruktionen.

Schlussfolgerungen Durch den Vergleich von KM-CT- und PBV-Datensätzen konnten Milchglastrübungen aufgrund einer Hyperperfusion von solchen anderer Genese abgegrenzt und damit als spezifisches Zeichen für CTEPH erkannt werden. Bei der objektiven Messung ist es nötig hypodense und hyperdense Areale gezielt zu erkennen und die Bereiche getrennt zu untersuchen, um Unterschiede feststellen zu können.

Ultraschall

\section{Poster 1.4 Kontrastmittel-verstärkter Ultraschall (CEUS) zur Beurteilung der Nierenmikrovaskularisation vor und nach Embolisation mit resorbierbaren experimentellen Stärkemikrosphären}

\footnotetext{
Autoren Do $\mathrm{T}^{1}$, Fard $\mathrm{N}^{2}$, Vollherbst $\mathrm{D}^{3}$, Schlett $\mathrm{C}^{1}$, Gockner $\mathrm{T}^{4}$, Flechsig $\mathrm{P}^{5}$, Heim $J^{1}$, Pereira $\mathrm{P}^{6}$, Macher-Göppinger $\mathrm{S}^{7}$, Kauczor $\mathrm{H}^{1}$, Sommer $\mathrm{C}^{1}$ Institut 1 Universitätsklinikum Heidelberg, Diagnostische und interventionelle Radiologie, Heidelberg; 2 DKFZ, Radiologie, Heidelberg; 3 Universitätsklinikum Heidelberg, Neuroradiologie, Heidelberg; 4 Universitätsklinikum Mainz, Diagnostische Radiologie, Mainz; 5 Universitätsklinikum Heidelberg, Nuklearmedizin, Heidelberg; 6 SLK Kliniken Heilbronn, Klinik für Radiologie, minimalinvasive Therapien und
}

Nuklearmedizin, Heilbronn; 7 Universitätsklinikum Mainz, Institut für Allgemeine Pathologie, Mainz

DOI 10.1055/s-0037-1600493

Zielsetzung Bracco Posterpreis. Dynamische Evaluation und Quantifizierung der Nierenmikrovaskularisation vor und nach Embolisation mit resorbierbaren experimentellen Stärkemikrosphären mit Kontrastmittel-verstärktem Ultraschall (CEUS).

Material und Methodik CEUS erfolgte bei 3 Hausschweinen jeweils vor, 5 Minuten und 7 Tage nach transarterieller selektiver Nieren-Embolisation. Bei 2 Tieren wurden resorbierbare experimentelle Stärkemikrosphären (Typ L1 und $L 2$, jeweils $0.3 \mathrm{ml}$ ) und bei einem Tier nicht-resorbierbare etablierte Mikrosphären (Typ ES 500 - 700, $0.3 \mathrm{ml}$ ) verwendet. Die quantitative Analyse der Nierenmikrovaskularisation erfolgte mittels spezifischer Perfusionsparameter (u.a. Peak-Enhancement (PE), Time-to-peak (TTP)) für drei unterschiedliche Lokalisationen: Nierenarterie (NArt), Nierenparenchym mit maximaler (NMax) und minimaler (NMin) Kontrastierung. Zudem wurde der Blutfluss (RI) in der Nierenarterie mit gepulstem Doppler bestimmt.

Ergebnisse Das PE nahm direkt nach der Embolisation ab und zeigte eine partielle Erholung nach 7 Tagen für NArt (24439 AU/29279 AU), NMax (9628 AU/11583 AU) sowie NMin (3980 AU/7037 AU). TTP verkürzte sich stetig für NMin (25 s, $18 \mathrm{~s}$ und $14 \mathrm{~s})$ wohingegen sich TTP von NArt nach 7 Tagen normalisierte (10 s, $16 \mathrm{~s}$ und $11 \mathrm{~s}$ ). Das Wash-out zeigte nach initialem Abfall eine Reperfusion in NMax und NMin (465594 AU/651493 AU bzw. $65747 \mathrm{AU} / 131203 \mathrm{AU}$ ) sowohl bei den resorbierbaren als auch den nichtresorbierbaren Partikeln. PE und TTP von NArt sowie RI zeigten eine gute Korrelation.

Schlussfolgerungen CEUS ermöglicht die differenzierte quantitative Beurteilung der Nierenmikrovaskularisation vor und nach Embolisation mit resorbierbaren experimentellen Stärkemikrosphären. Verglichen mit nicht-resorbierbaren etablierten Mikrosphären ergeben sich unterschiedliche Mikrovaskularisationsmuster, die am ehesten auf unterschiedliche Größenverteilungen und Resorptionscharakteristika zurückzuführen sind.

\section{Poster 1.2 Kontrastmittelsonografie (CEUS) als neues bildgebendes Verfahren in der Diagnostik von Muskelverletzungen: Vergleichende Analyse von konventioneller B-Bild-Sonografie, CEUS und MRT}

Autoren Heiß $R^{1}$, Wildner $D^{2}$, Swoboda $B^{3}$, Gelse $K^{4}$, Grim $C^{5}$, Strobel $D^{2}$, Hotfiel $\mathrm{T}^{3}$

Institut 1 Universitätsklinikum Erlangen, Diagnostische Radiologie, Erlangen; 2 Medizinische Klinik 1, Universitätsklinikum Erlangen, Erlangen;

3 Orthopädische Universitätsklinik, Friedrich-Alexander-Universität ErlangenNürnberg, Orthopädische Rheumatologie, Erlangen; 4 Unfallchirurgische Abteilung, Universitätsklinikum Erlangen, Erlangen; 5 , Unfallchirurgie und Orthopädie, Klinikum Osnabrück, Osnabrück

DOI 10.1055/s-0037-1600494

Zielsetzung "Bracco-Posterpreis" Die Graduierung des Schweregrades von Muskelverletzungen mithilfe bildgebender Verfahren ist die Grundvoraussetzung für die Einleitung einer stadien- und zielgerichteten Therapie. In der vorliegenden Studie wurde die diagnostische Wertigkeit der Kontrastmittelsonografie (CEUS) zur Beurteilung von Muskelverletzungen verschiedener Schweregrade im Vergleich zu etablierten bildgebenden Verfahren evaluiert.

Material und Methodik Insgesamt wurden 14 Patienten nach ereigneter indirekter Muskelverletzung an der unteren Extremität untersucht. Innerhalb von 24-48 Stunden nach Verletzungsereignis erfolgten eine konventionelle B-Bild-Sonografie sowie eine CEUS. Im unmittelbaren Anschluss an die Sonografie wurde eine MRT als „Goldstandard“ durchgeführt, um eine Graduierung der Verletzung vorzunehmen und das räumliche Ausmaß der Läsion als Referenzgröße zu bestimmen. Die Stadieneinteilung erfolgte nach der von Ekstrand et al. modifizierten, vierstufigen Peetrons-Klassifikation. 
Ergebnisse Alle 14 Verletzungen konnten in der MRT sowie in der CEUS, jedoch nur 9 Verletzungen in der konventionellen Sonografie, detektiert werden. Sieben Muskelverletzungen wurden in der MRT als Grad I, vier als Grad II und drei als Grad III Verletzung eingeteilt. Die in der konventionellen Sonografie nicht detektierbaren Grad I Verletzungen stellten sich im CEUS alle als gut abgrenzbare, umschriebene Minderperfusionen dar. Diese korrelierten in ihrer räumlichen Ausdehnung mit dem korrespondierendem Ödemsignal in der MRT. Zudem zeigten sich in Abhängigkeit des Schweregrades signifikante Unterschiede in der erfassten Läsionsgröße zwischen der konventionellen Sonografie und der CEUS.

Schlussfolgerungen Die CEUS scheint ein zusätzliches sensitives Verfahren in der bildgebenden Diagnostik von akuten Muskelverletzungen zu sein. In unserer Studie zeigte sie sich in der Detektion von niedriggradigen Muskelverletzungen gegenüber der konventionellen Sonografie überlegen, was bisher ausschließlich der MRT vorbehalten war.

\section{Poster 1.7 Role of CEUS in multiparametric prediction in prostate cancer}

Authors Marticorena Garcia $\mathrm{S}^{1}$, Maxeiner $\mathrm{A}^{2}$, Baur $\mathrm{A}^{3}$, Fischer $\mathrm{T}^{3}$ Institute 1 Charité, Institut für Radiologie, Berlin; 2 Charité Universitätsmedizin Berlin, Klinik für Urologie, Berlin; 3 Charité Universitätsmedizin Berlin, Institut für Radiologie, Berlin DOI 10.1055/s-0037-1600495

Zielsetzung To compare MRI/US fusion guided and conventional biopsies and to investigate whether a mulitparametric ultrasound (mpUS) score can further characterize mPMRI suspicious lesions according to the prostate MRI reporting and data system (PI-RADS) score.

Material und Methodik From January 2012 to January 2014, 169 patients with at least one negative conventional prostate biopsy (PBX) before mulitparametric magnetic resonance imaging (mpMRI) and consistently elevated PSA levels were prospectively included. Real-time MRI/US fusion was used to biopsy the mpMRI targeted lesions. MpUS, including B-mode, Power Doppler, elastografy and CEUS was performed to further characterize those lesions. Afterwards, a conventional 10-core PBX was performed. The results of targeted and conventional PBX were compared and single US modalities were analyzed within the mpUS score. The mpUS score was further investigated for PI-RADS score prediction.

Ergebnisse Among 169 patients 71 prostate cancer (PCa) (42\%) were detected. From these 71 cases clinically significant PCa ( $\geq$ Gleason $4+3=7$ ) were detected exclusively by MRI/US fusion guided biopsy in 22 from 25 cases (88\%). The highest sensitivity was observed in US elastografy (85\%) and CEUS (80\%) and their combination (92\%) applied on PI-RADS classified lesions. The mpUS score predicts PCa and PI-RADS score 5 lesions with an overall accuracy of $86 \%$ and $80 \%$, respectively.

Schlussfolgerungen MpUS may present a novel tool to predict PCa targeted prostate biopsy. Real-time MRI/US fusion guided PBX detects more clinically significant PCa compared with conventional TRUS. The initiated mpUS score could confirm this with a comparable accuracy within PI-RADS predefined lesions.

\section{Poster 1.5 Quantitative Perfusionsanalyse mittels Kontrastmittelsonografie (CEUS) zur Differenzierung eines aggressiven Prostatakarzinoms}

\author{
Autoren Marticorena Garcia $S^{1}$, Schwabe $J^{2}$, Jung $E^{2}$, Fischer $T^{2}$ \\ Institut 1 Charité, Institut für Radiologie, Berlin; 2 Charité - \\ Universitätsmedizin Berlin, Institut für Radiologie, Berlin \\ DOI 10.1055/s-0037-1600496
}

Zielsetzung ,Bracco Posterpreis‘ Ziel der Studie ist es den Verlauf der mittels Kontrastmittelsonografie (CEUS) gewonnenen Kontrastmittelkurve mithilfe einer quantitativen Perfusionsanalyse zu objektivieren und Parameter zu erhe- ben, die für eine erhöhte Aggressivität (ab Gleason Score 4+3) und damit Behandlungsrelevanz von Prostatakarzinomen sprechen.

Material und Methodik 170 Patienten mit Verdacht auf ein Prostatakarzinom unterzogen sich von 2012 bis 2015 einer MRT (Magnetresonanztomografie)US (Ultraschall) fusionierten Stanzbiopsie der Prostata. Nach intravenöser Applikation des Ultraschallkontrastmittels SonoVue wurde der Kontrastmittelverlauf als DICOM-Clip auf einem high-end Ultraschallgerät (Aplio500, Toshiba) aufgezeichnet. Anschließend erhielten die Patienten eine randomisierte Biopsie bestehend aus einer systematischen 10-fach Biopsie und max. 2 gezielten Fusionsstanzen zur Ermittlung des Gleason Scores. Die quantitative Perfusionsanalyse erfolgte als verblindete Auswertung mit der Software VueBox (Bracco). Für die Auswertung markiert wurden 4 ROls. ROI 1 liegt im Bereich der MRT-Fusion, während ROI 2 - 4 Referenzgewebe abgrenzt.

Ergebnisse Von 170 Patienten (Durchschnittsalter 66 Jahre) wurden 158 in die Auswertung einbezogen. Bei 26 dieser Patienten ergab die histologische Untersuchung einen aggressiven Karzinombefund (Gleason 4+3 oder höher), bei 49 einen risikoarmen Karzinombefund und bei 84 einen benignen Befund. Die Subgruppenanalyse ergab in Bezug auf das Peak Enhancement (PE), Washin und die Wash-out Rate einen signifikanten Unterschied zwischen aggressiv malignen und risikoarm malignen und benigner Läsionen $(P<0.05)$.

Schlussfolgerungen Die Parameter PE, Wash-in und Wash-out erlauben die Differenzierung aggressiver Prostatakarzinome von risikoarmen Malignomen bzw. benignen Befunden, wodurch diese gezielt identifiziert und therapiert werden können.

\section{Poster 1.1 Präoperative Differenzierung von Schilddrüsenadenomen und Schilddrüsenkarzinomen mittels kontrastmittelgestütztem Ultraschall (CEUS)}

Autoren Schleder $S^{1}$, Stroszczynski $C^{1}$, Schreyer $A^{1}$, Jung $E^{1}$

Institut 1 Universitätsklinikum Regensburg, Institut für Röntgendiagnostik, Regensburg

DOI 10.1055/s-0037-1600497

Zielsetzung Ziel der Studie war die Evaluation der kontrastmittelverstärkten Ultraschalldiagnostik (CEUS) für die präoperative Differenzierung von Schilddrüsenadenomen und Schilddrüsenkarzinomen. "Bracco Posterpreis"

Material und Methodik Zu diesem Zweck wurden 101 Patienten (55 weiblich, mittleres Alter 54 Jahre) evaluiert, welche aufgrund eines Schilddrüsenadenoms oder -karzinoms operiert werden mussten. Alle Patienten erhielten präoperativ von einem erfahrenen Untersucher eine CEUS-Untersuchung mittels multifrequenter Linearsonde (6-9 oder 6-15 MHz). Das akquirierte Bildmaterial wurde digital gespeichert und von zwei erfahrenen Radiologen in Konsensus retrospektiv ausgewertet. Der definitive histopathologische Befund wurde bei allen Patienten als Goldstandard herangezogen.

Ergebnisse Insgesamt wurden 75 Schilddrüsenadenome und 26 Schilddrüsenkarzinome evaluiert. Der Unterschied in der Mikrozirkulation von Schilddrüsenadenomen und -karzinomen war statistisch hochsignifikant $(p<0,01)$. Diagnostische Kriterien für das Schilddrüsenadenom waren entweder das fehlende Auswaschphänomen oder ein perisitiernender hypervaskularisierter Randsaum bis in die späte Phase. Diagnostisches Kriterium für das Schilddrüsenkarzinom war eine inhomogene Hypervaskularisation und ein irreguläres komplettes Auswaschphänomen. Die Berechnung der Sensitivität, Spezifität, des positiv (PPV) und negativ prädiktiven Wertes (NPV) ergab 81\%, 92\%, 97\% bzw. $63 \%$ für die Unterscheidung von gutartigen und bösartigen Knoten mittels CEUS.

Schlussfolgerungen Die dynamische Beurteilung der Mikrozirkulation mithilfe CEUS ermöglicht dementsprechend eine zuverlässige präoperative Unterscheidung zwischen Schilddrüsenadenomen und Schilddrüsenkarzinomen. 


\section{Poster 1.6 Perkutane Behandlung bei malignen Leberläsionen: Evaluation des Therapieerfolgs mittels CEUS und Perfusionssoftware}

\author{
Autoren Wiesinger $\mathrm{I}^{1}$, Zausig $\mathrm{N}^{2}$, Lück $\mathrm{C}^{1}$, Salzberger $\mathrm{B}^{1}$, Beyer $\mathrm{L}^{1}$, \\ Wiggermann $P^{1}$, Stroszczynski $C^{1}$, jung $E^{1}$ \\ Institut 1 Universitätsklinikum Regensburg, Regensburg; \\ 2 Universitätsklinikum Regensburg, Regensburg \\ DOI 10.1055/s-0037-1600498
}

Zielsetzung Ziel war es den Therapieerfolg nach der perkutanen Therapie bei malignen Leberläsionen mittels einer neuen Perfusionssoftware und CEUS zu evaluieren. Bracco-Posterpreis.

Material und Methodik Retrospektive Analyse von 88 Patienten (74 m, 14 w; 30 - 84 Jahre) mit 165 malignen Leberläsionen. Die Läsionen waren im Vorfeld als Metastasen in 57 Fällen und HCCs in 108 Fällen klassifiziert worden. Der Therapieerfolg der interventionellen Behandlung (IRE $n=47$; RFA $n=38$; MWA $\mathrm{n}=44$; TACE $\mathrm{n}=36$ ) wurde mittels CEUS und Perfusionssoftware (VueBox ${ }^{\circledR}$ ) evaluiert. CEUS wurde nach i.v. Injektion von 1 - $2.4 \mathrm{ml}$ Schwefel-HexafluoridMikrobläschen (SonoVue ${ }^{\circledR}$ ) mit einer $1-5 \mathrm{MHz}$ Konvexsonde durchgeführt. Regions of Interest (ROI) wurden im Zentrum und am Rand der behandelten Läsion platziert und ebenso im umgebenden Gewebe. Mittels VueBox ${ }^{\circledR}$ wurden Peak, Time to Peak (TTP), mean Transit Time (mTT) und Rise-Time (RT) berechnet. Verglichen wurden die Ergebnisse mit dem follow-up MRT bzw. CT, die bis zu 6 Monate nach der Behandlung erstellt wurden.

Ergebnisse Es zeigten sich signifikante Unterschiede in Bezug auf Peak, mTT und RT $(p<0.001)$ in allen Fällen für den direkten Vergleich zwischen Zentrum und Rand der Ablationsstelle. Alle Parameter waren signifikant unterschiedlich zwischen Läsionen, die vollständig therapiert und abladiert wurden und solchen, die im Verlauf ein Rezidiv entwickelten.

Schlussfolgerungen Eine Kombination aus CEUS und einer Perfusionsanalyse ermöglicht eine kritische Beurteilung des Erfolges nach interventionellen Behandlungen bei malignen Leberläsionen.

\section{Poster (Fortbildung)}

\section{Ganzkörperdiagnostik/Kohortenstudien}

\section{Poster 4.1 Vergleich tumor- versus therapieassoziierter neurologischer Komplikationen der spinalen Achse bei Non-Hodgkin Lymphomen unter besonderer Berücksichtigung des Plasmozytoms}

\author{
Autoren Fahnert $\mathrm{J}^{1}$, Borte $\mathrm{G}^{1}$, Quäschling $\mathrm{U}^{2}$, Kahn $\mathrm{T}^{1}$, Pönisch $\mathrm{W}^{3}$ \\ Institut 1 Universitätsklinikum Leipzig AÖR, Klinik und Poliklinik für \\ Diagnostische und Interventionelle Radiologie, Leipzig; 2 Universitätsklinikum \\ Leipzig AÖR, Abteilung für Neuroradiologie, Leipzig; 3 Universitätsklinikum \\ Leipzig AÖR, Abteilung Hämatologie, Internistische Onkologie, \\ Hämastaseologische Ambulanz, Leipzig \\ DOI 10.1055/s-0037-1600499
}

Kurzfassung Non-Hodgkin-Lymphome (Inzidenz 8/100000) und insbesondere die Subtypisierung des Multiplen Myeloms (syn. Plasmozytom) neigen häufig zu einer diffusen ossären und extraossären Infiltration. Vor allem in fortgeschrittenen Krankheitsstadien kann dies bei entsprechender lokaler spinaler oder cerebraler Manifestation zu schwerwiegenden neurologischen Komplikationen führen. Die Schnittbilddiagnostik erscheint vor dem Hintergrund einer oftmals extraossären Tumorausbreitung das Mittel der Wahl zur Evaluation des Lokalbefundes und der Prädiktabilität von instabilen bzw. frakturgefährdeten Befunden. Prädilektionsstellen in der Verursachung neurologischer Symptomkomplexe sind in erster Linie die spinale Achse, das Os sacrum unter Einbeziehung der Neuroforamina sowie periphere Läsionen im Verlauf von GefäßNerven-Strängen (z.B. auf Höhe des Kniegelenks). Wichtige differentialdia- gnostische Überlegungen sollten jedoch in allen Fällen autoimmunologisch (z.B. chronische inflammatorisch demyelinisierende Polyneuropathie) oder therapeutisch (z. B. nach Chemotherapie) bedingte Polyneuropathien mit generalisierter Nervenbeteiligung einschließen. Die gewählten Fallbeispiele demonstrieren anhand von MRT und CT - Untersuchungen kritische Befundkonstellationen und -verläufe, die bei progredienter Grunderkrankung bzw. unter Therapie zu gravierenden neurologischen Komplikationen führten.

Lernziele

- Typische MR-morphologische Veränderungen intra- und extraossären Tumorbefalls bei NHL

- Prädiktabilität der Wirbelkörperstabilität bei Tumorinfiltration

- „häufige“ neurologische Komplikationen bei intra- und extraspinaler Tumormanifestation

\section{Poster 4.2 Management von Zufallsergebnissen in der NAKO Gesundheitsstudie}

Autoren Sprengel $S^{1}$, Schlett $C^{1}$, Stackelberg $\mathrm{O}^{1}$, Kauczor $\mathrm{H}^{1}$, Bertheau $\mathrm{R}^{1}$, Hirsch $\mathrm{J}^{2}$, Bamberg $\mathrm{F}^{3}$, Günther $\mathrm{M}^{2}$, Weckbach $\mathrm{S}^{1}$

Institut 1 Universitätsklinik Heidelberg, Diagnostische und Interventionelle Radiologie, Heidelberg; 2 Fraunhofer-MEVIS, Bremen; 3 Universitätsklinik Tübingen, Diagnostische und Interventionelle Radiologie, Tübingen DOI 10.1055/s-0037-1600500

Kurzfassung Die NAKO Gesundheitsstudie (NAKO) ist eine multizentrische Kohortenstudie, die die Erforschung der Ursachen für die Entstehung von Volkskrankheiten zum Ziel hat. In die Studie sollen dabei 200.000 Probanden im Alter von 20 bis 69 Jahren eingeschlossen werden. Neben diversen körperlichen Untersuchungen und dem Sammeln von Bioproben erhält eine Untergruppe von 30.000 Probanden eine Ganzkörper-Magnetresonanztomografie (GK-MRT) in einem der fünf dezidierten Studienzentren in Deutschland. Um bei diesem großen Patientenkollektiv eine hohe Reproduzierbarkeit und gleichbleibend hohe Bildqualität zu gewährleisten, erfolgt eine zentrale Qualitätssicherung durch einen sogenannten „MR imaging core”, welcher aus vier Untereinheiten besteht: einem Zentrum für Datenverarbeitung (Bremen), für Studienkoordination und Training (München), einem Zentrum für Qualitätskontrolle (Greifswald) und einem Zentrum für das Management der Zufallsergebnisse (Incidental Findings, IF) (Heidelberg). Je nach Studiendesign zeigen ca. $10 \%$ der Probanden relevante IF in GK-MRT-Untersuchungen. Hier stellt sich das grundsätzliche Problem zu entscheiden, welche dieser Ergebnisse den Teilnehmern mitgeteilt werden sollen. Um bei der großen Anzahl der beteiligten Radiologen einen einheitlichen Umgang mit IFs zu gewährleisten, wurde ein komplexes System zur Erfassung und Mitteilung von Zufallsergebnissen entwickelt: Die Studienradiologen wählen, nachdem sie ein mehrstufiges Training durchlaufen haben, in einem eigens entwickelten, web-basierten Betrachtungsprogramm aus einer vorgegebenen Liste von relevanten Zufallsergebnissen das betreffende IF aus und markieren es, so dass dann je nach Dringlichkeit eine telefonische oder postalische Ergebnisübermittlung an die Probanden erfolgt.

Lernziele Unser Poster wird eine ausführlichere Übersicht über das Management von Zufallsbefunden in der NAKO geben und anhand von Bildbeispielen verschiedene Kategorieren von Zufallsbefunden illustrieren. 
Interventionelle Radiologie

\section{Poster 3.2 Selektive Embolisation aortaler Seitenäste vor EVAR als Strategie zur Verhinderung relevanter Typ 2 Endoleaks}

Autoren Petersen $\mathrm{T}^{1}$, Fuchs $\mathrm{J}^{2}$, Bremicker $\mathrm{K}^{2}$, Maiwald $\mathrm{B}^{2}$, Kahn $\mathrm{T}^{2}$, Moche $\mathrm{M}^{2}$ Institut 1 Universitätsklinikum Leipzig, Klinik und Poliklinik für Diagnostische und Interventionelle Radiologie, Leipzig; 2 Uniklinik Leipzig, Klinik und Poliklinik für Diagnostische und Interventionelle Radiologie, Leipzig DOI 10.1055/s-0037-1600501

Kurzfassung Ein Risiko der endovaskulären Aortenrekonstruktion (EVAR) ist die persistierende Perfusion des Aneurysmasacks (Endoleak). Typ 2 Endoleaks (T2EL) treten dabei in $6-42 \%$ der Fälle als Folge einer Flussumkehr in aortalen Seitenästen auf. Von den frühen T2EL zeigen $80 \%$ eine spontane Regression. Bestehen T2EL jedoch länger als 6 Monaten ( $5-33 \%$ ), bilden sich nur noch 6\% spontan zurück. Dabei sind nur die persistierende Endoleaks mit einem erhöhten Risiko für ein weiteres Aneurysmawachstum verbunden. Nach bereits durchgeführter EVAR kann die Embolisation der verantwortlichen Seitenäste jedoch zu äußerst komplexen und langwierigen Prozeduren führen. Mehrere Studien haben gezeigt, dass eine systematische Embolisation der aortalen Seitenäste die T2EL-Rate deutlich reduziert. Allerdings verursacht nicht jeder Seitenast ein Endoleak. Mehrere Arbeitsgruppen haben Prädiktoren, bezogen auf die aortalen Seitenäste, für die Entstehung relevanter T2EL bestimmt: Die Anzahl der perfundierten Lumbalarterien (0-3: $13 \%$; $\geq 6$ : $50 \%)$, der Durchmesser der Lumbalarterien (>2-2,4 mm) und die Offenheit und der Durchmesser der A. mesenterica inferior $(>3,8 \mathrm{~mm})$ sind relevante Parameter.

Lernziele Die systematische prä- oder periprozedurale Embolisation von aortalen Seitenästen bei geplanter EVAR reduziert das Auftreten von klinisch relevanten Typ 2 Endoleaks. Dabei lassen sich die relevanten Seitenäste anhand weniger Parameter identifizieren und dann gezielt embolisieren.

\section{Poster 3.1 Der radiale Zugang bei interventionsradiologischen Prozeduren}

Autoren Schotten $\mathrm{S}^{1}$, Düber $\mathrm{C}^{1}$, Pitton $\mathrm{M}^{2}$

Institut 1 Universitätsmedizin Mainz, Klinik für Diagnostische und

Interventionelle Radiologie, Mainz; 2 Universitätsmedizin Mainz,

Diagnostische und Interventionelle Radiologie, Mainz

DOI 10.1055/s-0037-1600502

Kurzfassung Der radiale Zugang hat sich in mehreren randomisierten kardiologischen Studien gegenüber dem femoralen Zugang als überlegen erwiesen, was die zugangsbedingten Komplikationsraten bei Koronarinterventionen betrifft. Daher wird das radiale Vorgehen von der europäischen Fachgesellschaft für Kardiologie mittlerweile als Standard empfohlen. Darüber hinaus bietet der radiale Zugang einen deutlich höheren Patientenkomfort, da die Immobilisation weitgehend entfällt. Im Bereich der Interventionsradiologie findet der radiale Zugang noch relativ wenig Beachtung, obwohl er auch hier die prinzipiellen Vorteile gegenüber dem femoralen Zugang bietet. Mit aktuell verfügbaren längeren Kathetersystemen sind Eingriffe im Bereich der Viszeralarterien (z. B. TACE, SIRT, Stentimplantation) und Beckenarterien (z. B. UFE, PAE) problemlos durchführbar. Während der Zugang bei Koronarinterventionen überwiegend von rechts erfolgt, bietet sich für interventionsradiologische Prozeduren die linke Seite an, da hier der Abstand zu den Viszeralarterien kürzer ist und das Risiko für neurologische Komplikationen als geringer angesehen wird. Um ischämische Komplikationen zu vermeiden, sollte vor der Radialispunktion die Kollateralisierung über die A. ulnaris überprüft werden (z. B. mittels Barbeau Test). Die Verwendung hydrophil beschichteter Schleusen, die i.a. Verabreichung eines "Cocktails" spasmolytischer Substanzen (Nitroglycerin, Verapamil), sowie die nichtokklusive Kompression der Punktionsstelle minimieren das Risiko eines, meist asymptomatisch verlaufenden, Verschluss der A. radialis. Der radiale Zugang lässt sich relativ einfach erlernen, erfordert keine grö- ßeren Änderungen im räumlichen Setup und ist geeignet das interventionsradiologische Repertoire gewinnbringend zu erweitern.

Lernziele

- Potential des radialen Zugangs für interventionsradiologische Prozeduren

- Tipps und Tricks für die erfolgreiche Durchführung, das Raumsetup und Strahlenschutzaspekte

Kopf/Hals-Diagnostik

\section{Poster 8.2 Dynamische MRT der Halswirbelsäule: Technik, Anwendungen und Vorteile}

Autoren Mallouhi A ${ }^{1}$, Marik W

Institut 1 Medizinische Universität Wien, Universitätsklinik für Radiologie und Nuklearmedizin, Abteilung für Neuro- und Muskuloskeletale Radiologie, Wien DOI 10.1055/s-0037-1600503

Kurzfassung Die Bildgebung mittels MRT erfolgt in Neutralstellung der Halswirbelsäule (HWS) und gilt als Goldstandart. Dennoch korrelieren nicht alle von den Patienten angegeben Beschwerden mit den erhobenen Befunden in der MRT. Die dynamische MRT der Halswirbelsäule in Anteflexion, Retroflexion, lateral Flexion und/oder Rotation erlaubt die Detektion sowie auch die genaue Quantifikation von Stenosen des Spinalkanals und der Neuroforamina. Dies erlaubt eine bessere Beurteilung von neuralen und ligamentären Veränderungen. Diese Technik erscheint besonders hilfreich in der Beurteilung von morphologischen Veränderungen der degenerativen Wirbelsäule (knöcherne Strukturen und Bandscheiben), Instabilität, posttraumatische Verletzungen, postoperative mechanische Veränderungen und gewisse angeborene Deformitäten. Die dynamische MRT der HWS erscheint insbesondere hilfreich bei Patienten bei denen im Routine-MRT keinen ausreichenden Grund für die angegeben Beschwerden gefunden werden konnte. Hier kann die dynamische MRT der HWS Pathologien aufdecken helfen, die in Neutralstellung nicht erkennbar sind. Sie kann daher zur Vermeidung von Spätschäden wie Myelopathie oder weitere Operation führen.

Lernziele Es sollen verschieden pathologische Veränderungen des zervikalen Halsmarks im dynamischen MRT gezeigt werden. Dies dient dazu um die Vorteile gegenüber dem routinemäßig durchgeführten statischen MRT zu zeigen sowie die Relevanz in der weiteren Therapieplanung.

Muskuloskelettale Radiologie

\section{Poster 5.1 Plantarvenenthrombose, eine seltene Differentialdiagnose des Fußschmerzes - MR Bildgebung}

Autoren Kamusella $\mathrm{P}^{1}$, Andrea $\mathrm{B}^{2}$

Institut 1 Röntgenpraxis Heide, Heide; 2 Röntgen Praxis Heide, Heide DOI 10.1055/s-0037-1600504

Kurzfassung Die Plantarvenenthrombose ist eine seltene Ursache des Fußschmerzes. Als Ursache finden sich Faktoren, die typischerweise im Rahmen der Tiefenbeinvenenthrombose zu finden sind, wie zum Beispiel eine vorausgegangene Operation, aber auch ein Trauma oder eine Immobilisation können evaluiert werden. Charakteristische Zeichen dieser Entität finden sich nicht, sodass in der Regel nach anderen Ursachen für die unspezifischen Beschwerden/Schmerzen gesucht wird. Nicht selten werden die Patienten nach klinischer Untersuchung einem bildgebenden Verfahren zugeführt. Hierbei spielt die MR-Bildgebung eine besondere Rolle mit ihren herausragenden Eigenschaften der Weichteildarstellung. Durch eine der Fragestellung angepassten Bildakquisition ist es möglich eine Vielzahl an Ursachen zu diagnostizieren bzw. auszuschließen. Da der plantare Venenplexus eher ein Randerscheinung bei der Befundung darstellt, ist es unerlässlich diese gezielt zu beurteilen, um charakteristische Zeichen einer Venenthrombose im MRT zu erkennen. So lässt sich der intraluminale Thrombus als Signalveränderung abgrenzen. Zu- 
dem finden sich venöse Ektasien und ein Umgebungsödem. Seltener lassen sich Kollateralvenen erkennen. Insbesondere nach einer intravenösen Kontrastmittelapplikation finden sich ein perivaskuläres Enhancemen und ein venöser Füllungsdefekt.

Lernziele In der MR-Bildgebung der schmerzhaften Plantarregion ist es wichtig sich an die Plantarvenenthrombose zu erinnern und die charakteristischen MR Informationen zu kennen.

\section{Poster 5.2 Intermodale Bildgebung der plantaren Fibromatose (M. Ledderhose)}

Autoren Reichardt B ${ }^{1}$, Nicolas $V^{2}$

Institut 1 BG Universitätskrankenhaus Bergmannshein Bochum, Institut für diagnostische und Interventionelle Radiologie und Nuklearmedizin, Bochum;

2 BG Universitätsklinikum Bergmanssheil, Institut für Diagnostische

Radiologie, Interv. Radiologie und Nuklearmedizin

DOI 10.1055/s-0037-1600505

Kurzfassung Morbus Ledderhose ist eine gutartige Erkrankung des Bindegewebes der Füße (Plantar-Aponeurose) aus der Gruppe der Fibromatosen und verwandt mit dem häufiger auftretenden Morbus Dupuytren. Ein schubweiser, über mehrere Jahre dauernder Krankheitsverlauf ist typisch. Beim Morbus Ledderhose bilden sich in der unter der Sohlenhaut liegenden Plantaraponeurose Knoten und gelegentlich (selten) auch Stränge. Die Gehfähigkeit beeinträchtigen. Eine Abklärung der Plantarfibromatose kann durch die kontrastmittelgestützte Kernspintomografie oder durch die Sonografie erfolgen. Beide Modalitäten bieten die gleiche Aussagekraft vor chirurgischer Intervention haben, wobei die Ultraschalldiagnostik unkomplizierter und zeitsparender ist. Methodik Ultraschall: 10 Mhz linearen Oberflächenschallkopf mit Vorlaufplatte von $5 \mathrm{~mm}$ Stärke um eine ebene Schallauflage zu gewährleisten. Die Firbromatose stellt sich als hypoechogene bis intermediäre Raumforderung mit fusiformer bis multinodulärer Struktur in der Plantarfaszie ohne Kontakt zur calcanaeren Insertion dar. Im Farbdoppler und Powerdoppler sind nur schwache randständige und teils zentrale signale erkennbar. MRT: T1: iso- bis hypointenses Signal im Vergleich zur umliegenden Muskulatur Fettgesättigte PDw: hyperinten T1: intermediäres bis starkes KM Emhancement.

Ergebnisse Das MRT scheint die Methode der Wahl, da multilokale Manifestationen einfacher zu detektieren sind. Ebenso ist die räumliche Zuordnung einzelner Lokalisationen im MRT aufgrund der übersichtlicheren Anatomie für die chirurgische Versorgung relevant. Eine MR-Bildgebung mit Kontrastmittel erscheint jedoch unerlässlich, da ansonsten Weichteiltumore wie die aggressive Fibromatose übersehen werden könnte. Die Ultraschalldiagnostik bietet eine gute Bildgebungsmöglichkeit. Ergebnisse über KM-gestützte Sonografien und Doppleruntersuchungen der Microvaskularisation stehen aus, scheinen aber vielversprechend.

Lernziele Erkennen und Diagnose der plantaren Fibromatose.

Notfalldiagnostik / Intensivmedizin

\section{Poster 1.1 Darstellung des Stichkanals mittels Kontrastmittel bei penetrierenden Verletzungen im CT bei fraglicher Eröffnung von Körperhöhlen}

\author{
Autoren Reichardt $B^{1}$, Nicolas $V^{1}$ \\ Institut 1 BG Universitätsklinikum Bergmannsheil Bochum, Diagnostische \\ und Interventionelle Radiologie und Nuklearmedizin, Bochum \\ DOI 10.1055/s-0037-1600506
}

Kurzfassung Stichverletzungen am Bauch und Thorax ziehen bei oberflächlichen Weichteilverletzungen eine explorative Eröffnung des Abdomens bzw. des Thorax nach sich.

Bei grenzwertigen Befunden, unbekannter Eindringtiefe des penetrierenden Objektes ist eine Darstellung des Stichkanals mit Kontrastmittel - angelehnt an eine Fisteldarstellung - möglich und können somit eine explorative Operation vermeidbar machen.

Patienten mit Penetrationsverletzungen am Hals, Thorax und Abdomen wurden nach initialen Trauma CT-Scan bei fraglicher Durchstoßung der inneren Bauchwand und der Pleura erneut auf Läsionshöhe nach Injektion von Kontrastmittel mittels Knopfkanüle im CT eruiert.

Eingeschlossen wurden 9 Patienten mit Stichverletzungen, 1 Patient mit Penetrationsverletzung durch ein Amierungseisen sowie 1 Patient mit einem großen Holzspan.

Insgesamt wurden 21 Stichwunden versorgt, wobei $13(N=13)$ eine fragliche Eröffnung der Körperhöle vorwiesen.

Kontrastmittel breitete sich entlang der Bauchwand dorsal epifaszial der Muskulatur in 8 Fällen aus ohne KM-Übertritt in den Pleuraspalt, ins Mediastinum oder nach intraperitoneal. Bei einer Stichverletzung war aufgrund der Lage (laterodorsale Flanke) und ihrer Größe ein adäquates Auffüllen mit KM nicht möglich.

2 Stichkanäle zeigten einen minimalen Kontrastmittelübertritt in den Bauchraum.

Lernziele Bei fraglicher Eröffnung der Körperhöhlen bei penetrierenden Verletzungen ist eine direkte KM-Gabe in den Stichkanal je nach Lage und Größe möglich und kann für eine chirurgische Exploration oder das folgende Behandlungsregime ausschlaggebend sein.

Problematisch sind jedoch sehr große oder ungünstig (dorsal) gelegene Stichverletzungen, da eine adäquate KM-Auffüllung nicht möglich ist.

Bei kleinen Stichkanälen und zu geringem Druck beim Auffüllen mittels KM darf ein „Kulissenphänomen“ nicht unterschätzt werden, wobei sich nahe gelegene Weichteilstrukturen wieder aneinander legen und zur Demaskierung des Stichkanals führen können.

Uroradiologie/Urogenitaldiagnostik

\section{Poster 6.1 Befallsmuster bei malignen lymphoproliferativen Erkrankungen der Nieren}

Autoren Beeskow $A^{1}$, Leifels $L^{2}$, Fahnert $J^{2}$, Bach $A^{3}$, Beimler $M^{2}$, Surov $A^{2}$ Institut 1 Universitätsklinikum Leipzig, Abteilung für Kinderradiologie,

Leipzig; 2 Universitätsklinikum Leipzig, Klinik und Poliklinik für Diagnostische und Interventionelle Radiologie, Leipzig; 3 MLU Halle, Klinik für Diagnostische Radiologie, Halle

DOI 10.1055/s-0037-1600507

Kurzfassung Die renale Manifestation maligner lymphoproliferativer Erkrankungen ist die zweithäufigste extranodale Manifestation. In Autopsiestudien wird sie mit einer Inzidenz von 34-62\% beschrieben, in der Bildgebung jedoch mit nur 3-8\%. Sie zeigt bildmorphologisch vier sehr unterschiedliche Befallsmuster, die von anderen Differentialdiagnosen abgegrenzt werden müssen. Allen gemeinsam ist, dass sie sich in der nativen Bildgebung iso- oder leicht hyperdens darstellen und als hypovaskularisierte Tumoren kein signifikantes KM- Enhancement zeigen. Die häufigste Form ist die Manifestation als multiple oder solitäre Rundherde. Ebenfalls häufig auftretend ist die perirenale Infiltration. Das Lymphom ummauert die Niere und breitet sich entlang des Urogenitaltraktes aus, ohne diesen zu infiltrieren. Ein selteneres Befallsmuster ist die, häufig bilaterale Nephromegalie. Hier wird das renale Interstitium von lymphoproliferativen Zellen infiltriert, was sich in einer Nephromegalie mit verminderter Mark-Rindendifferenzierung äußert. Das letzte Befallsmuster stellt die perihiläre Infiltration dar, hier infiltriert das Lymphom das Nierenbeckenkelchsystem sowie die ableitenden Harnwege und ummauert die renalen Gefäße.

Lernziele Die renale Manifestation maligner lymphoproliferativer Erkrankungen geht automatisch mit einer Ann-Arbor Klassifikation Stadium IV einher. Dies ist für den Patienten und seine Prognose von entscheidender Bedeutung, daher ist es für den Radiologen wichtig, die unterschiedlichen Befallsmuster zu kennen. Die vier verschiedenen Befallsmuster, die solitäre oder multiple Herd- 
Manifestation, die perirenale Infiltration, die Nephromegalie und die perihiläre Infiltration müssen erkannt und von anderen Differentialdiagnosen abgegrenzt werden.

\section{Poster 6.2 Die diagnostische Wertigkeit der Hysterosalpingografie in der Sterilitätsdiagnostik}

\author{
Autoren Schramm D ${ }^{1}$, Bach $A^{2}$ \\ Institut 1 Universitätsklinikum Halle, Diag. Radiologie, Halle; \\ 2 Universitätsklinik und Poliklinik für Radiologie Halle, Diagnostische \\ Radiologie, Halle
}

DOI 10.1055/s-0037-1600508

Kurzfassung Die Hysterosalpingografie (HSG) ist eine diagnostische Methode zur Darstellung der inneren weiblichen Geschlechtsorgane, welche im Rahmen der Sterilitätsdiagnostik angewendet wird. Durch eine kontrastmittelgestützte röntgenologische Darstellung der Gebärmutterhöhle ermöglicht sie neben der Darstellung von Uterusfehlbildungen und der Durchgängigkeitsprüfung der Tuben im Rahmen obstruierender Erkrankungen (z.B. entzündliche Verklebung, Tubenendometriose) auch die Erfolgskontrolle nach Tubensterilisation. Von 2006 bis 2012 wurden insgesamt 174 Patientinnen im Alter zwischen 20 und 42 Jahren hysterosalphingografisch in unserer Universitätsklinik untersucht. Zu den am häufigsten diagnostizierten Uterusanomalien zählte neben dem Uterus arcuatus auch der Uterus bicornis unicollis. Uterus duplex, Uterus unicornis und Uterus subseptus konnten als Uterusfehlbildung nur sehr selten nachgewiesen werden. In Zusammenschau mit den wichtigsten Arbeiten der vergangenen Jahre soll diese Studie dem Leser einen Überblick über die oft unterschätzte diagnostische Wertigkeit HSG in der Sterilitätsdiganostik geben sowie typische radiologische Befunde aufzeigen.

Lernziele Die Hysterosalpingografie hat eine hohe diagnostische Wertigkeit in der Sterilitätsdiagnostik. Der Radiologe sollte neben ihrer Bedeutung auch die Indikationen und Kontraindikationen sowie die Nebenwirkungen und Komplikationen der HSG kennen und zwischen den wichtigsten Uterusanomalien differenzieren können.

\section{Nachtrag Poster-Ausstellung (Wissenschaft)}

Bildverarbeitung/IT/Software/Gerätetechnik/

Qualitätsmanagement

\section{Beurteilung von Rippenfrakturen mittels automatischer Rippensegmentierung und multiplanarer Reformation im Rahmen der Traumadiagnostik}

Autoren Beyer $L^{1}$, Steer $S^{1}$, Platz Batista da Silva $N^{1}$, Stroszczynski $C^{1}$, MüllerWille $\mathrm{R}^{1}$

Institut 1 Universitätsklinikum Regensburg, Institut für Röntgendiagnostik, Regensburg

DOI 10.1055/s-0037-1602598

Zielsetzung Evaluation einer softwaregestützten Rippensegmentierung mit multiplanaren Reformation (MPR) bezüglich der diagnostischen Genauigkeit bei der Erkennung von Rippenfrakturen im Vergleich zu herkömmlichen axialen Rekonstruktionen.

Material und Methodik In die Studie wurden retrospektiv 75 polytraumatisierte Patienten eingeschlossen (53 Männer, 22 Frauen, Durchschnittsalter 45 \pm 20 Jahre). MPR und herkömmliche axiale Schichten mit $5 \mathrm{~mm}$ Schichtdicke wurden in Hinblick auf das Vorliegen von Rippenfrakturen beurteilt. Der Goldstandard wurde im Konsensusreading von zwei erfahrenen Radiologen definiert.
Ergebnisse Insgesamt waren 107 der 1800 ausgewerteten Rippen frakturiert. Bei separater Betrachtung jeder einzelnen Rippe wurde in den axialen Schichten gegenüber den MPR eine signifikant höhere Sensitivität erreicht (76\% vs. 93\%; $p=0.001$ ). Bei patientenzentrierter Sichtweise (min. 1 Fraktur pro Patient) wurde ebenfalls eine höhere Sensitivität in den axialen Schichten erreicht, jedoch ohne dass der Unterschied signifikant wäre ( $84 \%$ vs. $92 \%$; p =0.63). Schlussfolgerungen Die herkömmlichen axialen Rekonstruktionen scheinen den multiplanaren rippenzentrierten Reformationen bei der Erkennung von Rippenfrakturen überlegen zu sein.

\section{Physikalische Quantifizierung der Bildqualität in der Computertomographie - eine zwei Jahres Analyse}

Autoren Höhne $\mathrm{M}^{1}$, Schulz-Menger $\mathrm{J}^{2}$, Herold $\mathrm{T}^{1}$

Institut 1 HELIOS Klinikum Berlin Buch, Radiologie, Berlin; 2 HELIOS Klinikum Berlin Buch, Kardiologie, Berlin

DOI 10.1055/s-0037-1602599

Zielsetzung Die quantitative Beurteilung der Bildqualität in der CT wird durch regelmäßige Ermittlung der physikalischen Parameter Rauschen, Kontrast und Auflösung gewährleistet.Ziel der Studie war es, die Abweichungen der Parameter Rauschen, MTF sowie die Ortsauflösung über einen Zeitraum von zwei Jahren zu beurteilen.

Material und Methodik Am CT Sensation16 (Siemens) wurden Messungen mit typ. CT Protokollen und herstelltyp. Phantomen in axialer Ausrichtung ausgeführt. Die Datenakquisition für das Rauschen erfolgte aus Scanparametern des Schädel- und Körperprotokolls an einem Wasserphantom. Es wurde die Standardabweichung von HU in einer Region (ROI) ausgewertet. Zur Ermittlung der Ortsauflösung wurden Daten an einem dünnen Wolframdraht in einem Acrylphantom generiert. Drei unterschiedliche Scan-Protokolle mit Rekonstruktionsalgorithmen für den Kopf, Körper und in hochauflösender Darstellung wurden verwendet. Aus den Bildern ergab sich die Punktbildfunktion, daraus wurde über die Fourier Transformation die MTF berechnet. Die Ortsauflösung wurde bei einem 50\%, 10\%, 2\% MTF Abfall ermittelt. Alle Messwerte wurden mit den Referenzwerten verglichen und ausgewertet.

Ergebnisse Für das Rauschen mit Scanparametern des Kopfes wurde bei einer durchschnittlichen Spannung $119.55 \pm 0.09 \mathrm{kV}$ eine Standardabw. von 7.10 SD von HU,beim Körperprotokoll $139.51 \pm 0.10 \mathrm{kV}$ eine Standardabw. von 14.24 SD von HU erreicht. Die Abweichungen zu den Referenzwerten waren kleiner als $1 \%$. Bei der Ermittlung der MTF wurden Rekonstruktionen mit H30 s, B30 s und U95 u Faltungskernen ausgeführt. Die ermittelten Ortsauflösungen ergaben Abweichungen zu den Referenzwerten von maximal 3\%.

Schlussfolgerungen Der Vgl. über 24 Monate zeigt, dass die ermittelten Parameter zu den Referenzwerten über einen langen Zeitraum im Toleranzbereich liegen und somit konstant sein können.Davon ausgehend, lassen diese Ergebnisse eine hohe Stabilität und Zuverlässigkeit erwarten. Dieser Ansatz sollte bei QS-Standards an unterschiedlichen CT Nutzungskonzepten (hybrid, diagnost) diskutiert werden.

\section{Diagnostisches Potenzial der Clear-Read CT-Software in der präoperativen CT-Detektion und Differenzierung pulmonaler Rundherde}

Autoren Papageorgiou I', Wagner $A^{2}$, Teichgräber $U^{3}$, Winzler $S^{4}$, Predel $D^{2}$, Malich $\mathrm{A}^{2}$

Institut 1 Südharz Klinikum Nordahusen, Insitut für Radiologie, Nordhausen; 2 Südharz Klinikum Nordahusen, Insitut für Radiologie, Nordahusen; 3 Institut für Diagnostische und Interventionelle Radiologie, Friedrich Schiller Universität Jena, Abteilung für Experimentelle Radiologie, Jena; 4 Südharz Klinikum Nordhausen, Institut für Radiologie, Nordhausen

DOI 10.1055/s-0037-1602600

Zielsetzung Die Differenzierung von pulmonalen Rundherden anhand von CTBildern bleibt eine Herausforderung und erfordert oft eine Biopsie. Ziel: Be- 
wertung der Genauigkeit der Clear read ${ }^{\mathrm{TM}}$ CAD-Software (Riverain) auf Basis von CT-detektierten, histologisch gesicherten pulmonalen Rundherden aus unserem Klinikum.

Material und Methodik Wir überprüften 50 Rundherde (30 maligne, 20 benigne) von 50 Patienten. Die CAD- Analysen erfolgten auf Basis der Weichteilund Lungenfensterung (WF/LF) in 3 mm Schichtdicke mit Clear Read ${ }^{\mathrm{TM}}$ CT (V1) und Clear Read ${ }^{\mathrm{TM}} \mathrm{CT}$ vessel suppression (V2).

Ergebnisse V1 fand im LF 33/50 bzw. im WF 34/50 Rundherden und V2 fand im LF 32/50 Rundherden bzw. im WF 31/50 Rundherden, was einer Sensitivität von $66 \%$ bzw. $68 \%$ (LF/WF) in V1 und $64 \%$ bzw. $62 \%$ in V2 entspricht, mit einer falsch negativen Rate von 0.34 bzw. 0.32 /pt (LF/WF) in V1 und 0.36 bzw. $0.38 / p t$ in V2. Clear read ${ }^{\mathrm{TM}}$ detektierte sowohl die benignen als auch die malignen Rundherde, ggf. mit einer Präferenz für die benignen Rundherde $\left(P<0,01 ; X^{2}\right.$ - Test). Die malignen Läsionen zeigten allerdings eine enge Dichtenverteilung. Mittels ROC Analyse konnte die Detektionsschwele für malingnen Herde bei $-148.50 \mathrm{HU}$ und $50 \mathrm{ml}$ errechnet werden (V1 WF). Hierunter wurden maligne Rundherde mit einer Sensitivität von $91.18 \%$ bzw. $82.35 \%$ und einer Spezifität von $83.33 \%$ detektiert. Die V2 und die LF Rekonstruktion ergaben ähnliche Ergebnisse. Die Dignität der falsch positiven (FP) Markierungen hat sich mit V2 trotz der integrierten Gefäßsuppression nicht modifiziert; die Mehrheit der FP (ca. 50\%) entsprach Verdichtungen und in nur 5\% Gefäßanschnitten, unabhängig vom Rekonstruktionskernel und CAD-Version. Schlussfolgerungen Mit einer Sensitivität von ca. 65-68\% ist das Clear read'M für eine „standalone“ Rundherdendetektion nicht geeignet. Die schnelle Errechnung des Volumens und der Dichte der Rundherde, welche mit einer signifikanten Korrelation für Malignität einhergehen, könnte für die Selektion der biopsiebedürfigen Läsionen bei Multifokalität nützlich sein.

\section{Entwicklung und Evaluation eines semiautomatischen Segmentierungsprogramms und einer modifizierten Ellipsoidformel zur volumetrischen Analyse von Nieren in nativen T2-gewichteten MR-Datensätzen}

\author{
Autoren Seuß $H^{1}$, Janka $R^{1}$, Prümmer $M^{2}$, Cavallaro $A^{1}$, Theis $R^{1}$, \\ Sandmair $\mathrm{M}^{1}$, Amann $\mathrm{K}^{3}$, Bäuerle $\mathrm{T}^{1}$, Uder $\mathrm{M}^{1}$ \\ Institut 1 Universitätsklinikum Erlangen, Radiologisches Institut, Erlangen; \\ 2 Chimaera GmbH, Erlangen; 3 Pathologisches Institut, Nephropathologische \\ Abteilung, Erlangen \\ DOI 10.1055/s-0037-1602601
}

Zielsetzung Zur Volumetrie von Nieren in nativen T2-gewichteten MR-Datensätzen, sollte ein semiautomatisches Segmentationsprogramm und eine modifizierte Ellipsoidformel (mEF) entwickelt und evaluiert werden.

Material und Methodik Ein semiautomatisches, auf Schwellenwerten basierendes, Segmentationsprogramm wurde entwickelt und von drei Untersuchern evaluiert. Dazu segmentierten diese die Nierenvolumina von 24 gesunden Probanden (48 Nieren) in axialen, nativen, T2-gewichteten MR-Datensätzen je zweimal. Des Weiteren maßen die Untersucher die Niere in drei Dimensionen (Länge, Breite, Tiefe) aus und verwendeten verschiedene Formeln zur Berechnung des Gesamtvolumens. Eine manuelle Segmentierung diente als Referenzstandard. Die Volumina der unterschiedlichen Methoden wurden verglichen und die Zeitdifferenz zur Datenerhebung berechnet.

Ergebnisse Es lag kein signifikanter Unterschied zwischen den semiautomatischen und den manuell ermittelten Volumina vor $(p=0.31)$. Die mittlere Volumendifferenz lag bei $0,3 \mathrm{ml}$ (95\% Konfidenzintervall (KI): 10,1 bis 10,7 ml). Die semiautomatische Segmentierung war im Durchschnitt $188 \mathrm{~s}$ schneller als die manuelle Segmentierung ( 220 s gegenüber 408 s; $p<0,05$ ). Die Volumina differierten nicht signifikant zwischen den Untersuchern. Die Berechnung des Volumens über eine $\mathrm{mEF}$ (Ellipsoides Volumen $\times$ 0.85) unterschied sich nicht signifikant vom Referenzvolumen (mittlere Volumendifferenz: 0,3 ml). Der mittlere Fehler lag jedoch dreimal so hoch (95\% Kl: 31,1 bis 30,9 ml). Die mEF war, mit durchschnittlich $41 \mathrm{~s}$, die schnellste Methode zur Ermittlung des Nierenvolumens.
Schlussfolgerungen Das entwickelte semiautomatische Segmentationsprogramm lieferte eine akkurate und reproduzierbare Volumetrie von Nieren in T2-gewichteten MR-Datensätzen und war signifikant schneller als eine manuelle Segmentierung. Eine modifizierte Ellipsoidformel kann zur schnell Schätzung des Nierenvolumens verwendet werden.

\section{Experimentelle Radiologie}

\section{Abhängigkeit der in der Lunge gemessenen T1- Relaxationszeit von der Echozeit und Sauerstoffkonzentration}

\author{
Autoren Jobst $\mathrm{B}^{1}$, Wielpütz $\mathrm{M}^{1}$, Kauczor $\mathrm{H}^{1}$, Jakob $\mathrm{P}^{2}$, Triphan $\mathrm{S}^{1}$ \\ Institut 1 Universitätsklinikum Heidelberg, Diagnostische und \\ Interventionelle Radiologie, Heidelberg; 2 Universität Würzburg, \\ Experimentelle Physik 5, Würzburg \\ DOI 10.1055/s-0037-1602602
}

Zielsetzung Die T1-Relaxationszeit (T1) stellt einen potenziellen Biomarker für den Gesundheitszustand der Lunge dar, da erkranktes und gesundes Lungengewebe eine unterschiedlich lange T1 aufweisen. Darüber hinaus zeigt sich bei Ventilation mit reinem Sauerstoff (O2) eine Verkürzung der T1, was Rückschlüsse auf die Lungenventilation erlaubt. Ferner ist bekannt, dass die gemessene T1 von der verwendeten Echozeit (TE) abhängt. Ziel dieser Studie war, den Einfluss der O2-Konzentration auf die TE-Abhängigkeit der T1 zu untersuchen.

Material und Methodik Mittels einer 2D Ultra-Short Echo-Time (UTE) Sequenz (Triphan et al., JMRI 2015) wurden T1-Karten bei 5 verschiedenen TE entlang einer Inversion Recovery Kurve erzeugt. Dies wurde an 7 gesunden Probanden zunächst während Inhalation von Raumluft und anschließend bei 100\% O2 durchgeführt. Die T1 wurde jeweils über den gesamten Querschnitt einer koronaren Einzelschicht gemittelt.

Ergebnisse Die jeweils bei Raumluft gemessene mediane Relaxationsrate 1/ T1 (TE1 - 5) $=(950 \pm 22 ; 801 \pm 30 ; 746 \pm 22 ; 748 \pm 26 ; 721 \pm 39) \mu$ s- 1 zeigte unter Inhalation von 100\% O2 einen Anstieg auf 1/T1 (TE1 - 5)=(1042 $\pm 30 ; 879$ \pm 36 ; $826 \pm 23 ; 813 \pm 30 ; 762 \pm 54) \mu \mathrm{s}-1$ mit signifikanten Änderungen bei TE1 $-4(p<0,001)$ und bei TE5 $(p<0,05)$. Die $1 / T 1$ bei Raumluft und bei $100 \%$ O2 nahmen zusammen mit der TE ab, ebenso die Differenz zwischen diesen Werten, $\Delta 1 / \mathrm{T} 1 \quad(\mathrm{TE} 1-5)=(95 \pm 14 ; 95 \pm 17 ; 78 \pm 19 ; 65 \pm 16 ; 45$ $\pm 22) \mu s-1 . \Delta 1 / T 1$ war signifikant verschieden von TE1 nach TE4 und TE5 $(p<0,0005-0,005)$, jedoch nicht von TE1 - $3(p<0,1-0,8)$.

Schlussfolgerungen Da das beobachtete T1 von der TE abhängig ist und der Effekt auf die unterschiedlichen Beiträge von extra- und intravaskulären Protonen zurückzuführen ist, sollte sowohl bei T1-Messungen unter Raumluft ebenso wie unter Ventilation mit zusätzlichem $\mathrm{O} 2$ eine Angabe der verwendeten TE erfolgen. Da der O2-Effekt auf die gemessene T1 bei der kürzesten TE am stärksten war, erscheinen UTE-Sequenzen für die Beobachtung von O2-Effekten am besten geeignet.

\section{Messung der pulmonalen T1-Relaxationszeit in Abhängigkeit von der Echozeit bei Patienten mit zystischer Fibrose}

Autoren Jobst $\mathrm{B}^{1}$, Wielpütz $\mathrm{M}^{1}$, Stahl $\mathrm{M}^{2}$, Eichinger $\mathrm{M}^{3}$, Kauczor $\mathrm{H}^{1}$, Mall $\mathrm{M}^{2}$, Triphan $\mathrm{S}^{1}$

Institut 1 Universitätsklinikum Heidelberg, Diagnostische und Interventionelle Radiologie, Heidelberg; 2 Universitätsklinikum Heidelberg, Translational Pneumology (TLRC), Heidelberg; 3 Thoraxklinik am Universitätsklinikum Heidelberg, Diagnostische und Interventionelle Radiologie mit Nuklearmedizin, Heidelberg DOI 10.1055/s-0037-1602603

Zielsetzung Bislang wurde gezeigt, dass die bei gesunden Probanden und COPD-Patienten gemessene pulmonale T1-Relaxationszeit (T1) von der ver- 
wendeten Echozeit (TE) abhängt. Diese Abhängigkeit wurde darauf zurückgeführt, dass das MR-Signal innerhalb einzelner Voxel durch unterschiedliche Beiträge von Protonen im Blut und Protonen des umgebenden Lungengewebes bestimmt wird. Ziel der Studie war die genauere Untersuchung dieses T1-Verhaltens im Zusammenhang mit pathologisch veränderter Lungenperfusion und Gewebezusammensetzung bei Patienten mit zystischer Fibrose (CF).

Material und Methodik Mithilfe einer 2D-Ultra-Short-Echo-Time (UTE) Sequenz (Triphan et al., JMRI 2015) wurde jeweils eine coronare Schicht zentral in der Lunge bei TE1 $-5=(70 ; 500 ; 1200 ; 1650 ; 2300) \mu s$ im Verlauf einer Inversion Recovery Kurve bei 30 pädiatrischen CF-Patienten im Alter von 1-18 Jahren aufgenommen. Für jedes TE wurden T1-Karten berechnet. Der Median der T1-Zeit wurde für die komplette abgebildete Lungenfläche berechnet und mit vorher veröffentlichten Werten für gesunde Probanden verglichen (unabhängiger t-Test).

Ergebnisse Der Median über alle Patienten war T1(TE1 - 5)=(1089 $\pm 79 ; 1155$ $\pm 83 ; 1223 \pm 90 ; 1243 \pm 89 ; 1306 \pm 94) \mathrm{ms}$, verglichen mit bisher berichteten T1(TE1 - 5) $=(1050 ; 1238 ; 1318 ; 1345 ; 1366) \mathrm{ms}$ für gesunde Probanden. Dies entspricht einem Anstieg von TE1 zu TE5 um ca. 20\% ( $p<0.001)$. Die intraindividuelle Variation von T1 ist wie erwartet groß, aber eine deutliche Zunahme mit TE ist bei allen Patienten in ähnlichem Ausmaß erkennbar. Der Vergleich mit dem T1 der gesunden Probanden ergibt ein $p<0,02$ für die Differenz bei TE2 - 4, nicht jedoch für TE1 und TE5.

Schlussfolgerungen Pädiatrische CF-Patienten unterscheiden sich hinsichtlich der T1-Zeit bei einer TE von 500 - $1650 \mu$ s deutlich von gesunden Probanden. Interessanterweise zeigen CF-Patienten einen langsameren Anstieg von T1 bei ansteigender TE. Die Ursachen für o.g. Effekte und die mögliche klinische Anwendbarkeit bedürfen der weiteren Untersuchung.

\section{In-vitro-Validierung der Fettquantifizierung im MR}

Autoren Linder $\mathrm{N}^{1}$, Rakete $\mathrm{T}^{1}$, Garnov $\mathrm{N}^{1}$, Schaudinn $\mathrm{A}^{1}$, Solty $\mathrm{K}^{1}$, Michel $\mathrm{S}^{1}$, Dipper $\mathrm{N}^{1}$, Stange $\mathrm{R}^{1}$, Kahn $\mathrm{T}^{1}$, Busse $\mathrm{H}^{1}$

Institut 1 Universitätsklinikum Leipzig AöR, Klinik und Poliklinik für

Diagnostische und Interventionelle Radiologie, Leipzig

DOI 10.1055/s-0037-1602604

Zielsetzung Die MR-Technologie ist auf dem Gebiet der Quantifizierung von Fett etabliert. Neben der MR-Bildgebung, die vorrangig modifizierte DixonSequenzen nutzt, kommt die MR-Spektroskopie, z. B. in Form der Stimulated echo acquisition mode (STEAM)- oder Point resolved spectroscopy (PRESS)Sequenzen, zum Einsatz. Wie genau die genannten Sequenzen in der Quantifizierung von definierten in-vitro-Lösugen von Fett sind, ist Gegenstand dieses Projektes.

Material und Methodik Zwei Reihen unterschiedlicher Fettemulsionen (E1 und E2) von verschiedenen Herstellern lieferten in 10\% Schritten Fettkonzentrationen von $0 \%$ bis $100 \%$. Die Messungen im 1,5 T MR (Archieva, Philipps, Best, NL) erfolgten mittels folgenden Sequenzen: 2-Punkt-Dixon (Schichtdicke $10 \mathrm{~mm}$, FOV $530 \times 530 \mathrm{~mm}$, TE1 3,45 ms, delta TE 1,44 ms), 11-Punkt-Dixon $(10 \mathrm{~mm}, 384 \times 288 \mathrm{~mm}, 0,93 \mathrm{~ms}, 0,77 \mathrm{~ms})$, STEAM (VOI $20 \times 20 \times 20 \mathrm{~mm}, \mathrm{TE}$ $10 \mathrm{~ms}$, TR $4000 \mathrm{~ms}$ ) und PRESS $30 \times 30 \times 30 \mathrm{~mm}, 29 \mathrm{~ms}, 3500 \mathrm{~ms})$. Die Ergebnisse wurden mittels Bestimmtheitsmaß $\left(R^{2}\right)$ einer linearen Regression durch den Ursprung und Bland Altman Blot verglichen.

Ergebnisse Insgesamt zeigten die Ergebnisse der MR Messungen eine moderate bis sehr gute Übereinstimmung mit den bekannten Fettkonzentrationen, mit der genauesten Messung in der 11-Punkt Dixon Sequenz. Die $R^{2}$ Werte für 2-Punkt Dixon, 11-Punkt Dixon, STEAM und PRESS waren 0,75, 0,98, 0,34 und 0,98 für E1 bzw. 0,81, 0,99, 0,34 und 0,93 für E2.

Schlussfolgerungen Sowohl 11-Punkt Dixon als auch PRESS sind genaue MRSequenzen zur Quantifizierung von Fett über einen breiten Bereich unterschiedlicher Konzentration.

\section{Visualisierung des $\mathrm{Y}$-H2AX-Epitops durch eine innovative Methode zur DNA-Doppelstrangbruch (DSB)-Analyse in der Radiologie}

Autoren Ruprecht N ${ }^{1}$, Hungerbühler $\mathrm{M}^{1}$, Heverhagen J ${ }^{1}$, Böhm I Institut 1 Inselspital, Universitätsspital Bern, Universitätsinstitut für Diagnostische, Interventionelle und Pädiatrische Radiologie (DIPR), Bern DOI 10.1055/s-0037-1602605

Zielsetzung Strahleninduzierte und sonstige genotoxische Einflüsse können in eukaryotischen Zellen anhand von DNA Doppelstrangbrüchen (DSB) mit der $\mathrm{Y}-\mathrm{H} 2 \mathrm{AX}$ Immunfluoreszenz-Methode indirekt in Zellkernen nachgewiesen werden. Im Kontext der radiologischen Diagnostik kann das Verfahren zur Abschätzung der induzierten Strahlenschäden dienen. Entscheidender Nachteil dieser Methode ist die Tatsache, dass die Dreidimensionalität des Zellkerns nicht berücksichtigt wird. Im Rahmen des vorgestellten Projektes haben wir daher die etablierte mit der konfokalmikroskopischen Methode verglichen.

Material und Methodik MRC-5 Lungenfibroblasten wurden mit einer 44CsQuelle bestrahlt. Die Zellen wurden in vitro folgenden Strahlendosen ausgesetzt: 200mGy, 500mGy und 1000mGy. Anschließend wurden die MRC-5 fixiert, mit einem anti-y-H2AX Antikörper (Maus) inkubiert und mit anti-Maus Alexa 488 gefärbt. Visualisierung und Ermittlung vorhandener Foci erfolgte mittels konventioneller Fluoreszenzmikroskopie (Zeiss Axiovert) und Konfokalmikroskopie (Zeiss LSM 880 mit Airyscan).

Ergebnisse Bei konventioneller Fluoreszenzmikroskopie wird die Gesamtheit der Fluoreszenzsignale als Integral visualisiert, ohne dass eine einwandfreie Abgrenzung der einzelnen Foci erfolgt. Durch Einsatz der konfokalen Mikroskopie konnten die vorhandenen $\mathrm{\gamma}$-H2AX-Foci in ihrer tatsächlichen Größe und räumlichen Anordnung innerhalb des Zellkerns einzeln dargestellt werden. Folglich fanden wir zwischen beiden Methoden einen statistisch signifikanten Unterschied identifizierter Foci $(p<0.001)$.

Schlussfolgerungen Konfokales Imaging ermöglicht als semi-quantitative Methode die korrekte Enumerierung vorhandener $\mathrm{y}-\mathrm{H} 2 \mathrm{AX}$-Foci, auch und vor allem bei hoher Focidichte pro Kern. Im Vergleich dazu ermöglicht die konventionelle Fluoreszenzmikroskopie im Wesentlichen eine Grobabschätzung. Damit ist die konfokale Mikroskopie der etablierten Methode deutlich überlegen.

\section{Oxygen sensitive MRI: Comparison of TOLD and pO2 measurements in experimental rat tumors during inspiratory hypoxic challenge}

Authors Svatko $Z^{1}$, Gündel $D^{2}$, Thews $O^{3}$, Knörgen $M^{1}$

Institute 1 Universitätsklinikum, Klinik für Radiologie, Halle (Saale);

2 Universitätsklinikum, Abteilung für Nuklearmedizin, Halle (Saale); 3 MartinLuther-Universität, Institut für Physiologie, Halle (Saale)

DOI 10.1055/s-0037-1602606

Zielsetzung Establishment of TOLD (“Tissue Oxygenation Level Dependent”) measurements in rats with implanted syngeneic mammary carcinoma. TOLD: T1-VIBE, dependent on molecular O2-concentration in tissue. The relative change of tumor oxygenation obtained by TOLD MRI during variation of the breathing gas mixture was compared to simultaneous quantitative measurements by Oxylite, an invasive fluoro-optic oximetry method.

Material und Methodik Tumor models: Walker-256 mammary carcinoma in Wister rats and prostate carcinoma AT1 in Copenhagen rats, subcutaneously implanted on the hind foot. MRI: 3T Magnetom Skyra (Siemens). Animal coil: Quadrature volume coil, $\varnothing 70 \mathrm{~mm}$ (Stark Contrast). Pulse sequences: T1-VIBE (TOLD). Tissue oxygenation monitoring: Oxylite 2000 (Oxford Optronics Ltd., Oxford, UK), fluoro-optical E-Series sensor placed in the center of tumor. The O2-concentration of the inspiratory gas was changed sequentially: From $21 \%$ (normoxia) to $8 \%$ (hypoxia) to $100 \%$ (hyperoxia) to $21 \%$. Tumor segmentation and calculation of intensity mean values were performed with MATLAB. 
Ergebnisse Both TOLD signal and Oxylite data showed dependence on oxygen concentration, but they are not strictly correlated. Partly this can be attributed to the heterogeneity of tumor tissue and the local character of data collection by the Oxylite probe. Furthermore, the Oxylite measurements show prominent signal increase when breathing pure oxygen compared to normoxic gas. The dynamics of MR measurements were faster than those of the Oxylite system. Comparing both tumor models, the AT1 model showed a diminished response on change of inspiratory $\mathrm{O} 2$ content, and oxygenation in the AT1 model was lower than with the W256 model.

Schlussfolgerungen Oxygenation levels of two tumor models under normoxia, hypoxia and hyperoxia were assessed non-invasively by TOLD MRI. Comparison with fluorometric oximetry measurements proved TOLD MRI to be a robust and simple method appropriate to estimate oxygenation of tumor tissue.

\section{Ganzkörperdiagnostik/Kohortenstudien}

\section{Radiale VIBE-Sequenz in der pädiatrischen Bildgebung: Evaluation der diagnostischen Wertigkeit}

\section{Autoren Stanescu $\mathrm{N}^{1}$, Cario $\mathrm{H}^{2}$, Beer $\mathrm{M}^{3}$, Schmidt $\mathrm{S}^{3}$}

Institut 1 Universitätsklinikum Ulm, Diagnostische und Interventionelle Radiologie, Ulm; 2 Klinik für Kinder- und Jugendmedizin, Ulm; 3 Klinik für Diagnostische und Interventionelle Radiologie, Ulm DOI 10.1055/s-0037-1602607

Zielsetzung Die radiale VIBE (rVIBE, Radial Volume Interpolated Breathhold Examination) stellt eine neue Technik der MR-K-Raum-Auslesung dar, um artefaktarmes und dennoch hochauflösendes Bildmaterial zu erhalten. Anhand von Untersuchungen verschiedener Körperregionen bei Kindern (3T MRT) soll die diagnostische Wertigkeit der rVIBE im Gegensatz zu herkömmlich akquirierten VIBE- Sequenzen beurteilt werden.

Material und Methodik Insgesamt wurden 29 Kinder zwischen 1 und 17 Jahren in die retrospektive Studie eingeschlossen. Zusätzlich zur herkömmlichen VIBE wurde auch eine rVIBE erstellt. Das Untersuchungsvolumen erstreckte sich auf verschiedene Körperregionen wie Schädel $(n=2)$, Hals $(n=1)$, Thorax $(n=13)$, Ober- und Mittelbauch $(n=11)$, Unterbauch und Becken $(n=1)$ sowie Wirbelsäule $(n=1)$. Die Bilddatensätze wurden von fünf erfahrenen Radiologen unabhängig voneinander befundet und die diagnostische Wertigkeit anhand einer 5-stufigen likelihood-Skala quantifiziert. Hierbei wurden folgende Beurteilungskriterien berücksichtigt: allgemeiner Bildeindruck, Auftreten von Artefakten, Abgrenzbarkeit der Organkonturen, Abgrenzbarkeit des Gefäßsystems und Abgrenzbarkeit einer evt. Pathologie.

Ergebnisse In allen Körperregionen konnten Vorteile der radialen gegenüber der herkömmlichen VIBE-Sequenz festgestellt werden, was sich insbesondere im Bereich des Thorax auch als statistisch signifikant erwies. Hier ergaben sich mittlere Bewertungs-Scores für den allgemeinen Bildeindruck mit 2,1 für die rVIBE versus 2,5 für die herkömmliche Sequenz $(p=0,032)$, dem Auftreten von Artefakten mit einem mittleren Bewertungsscore von 2,3 versus 2,8 $(p=0,001)$ und der Abgrenzbarkeit von Organkonturen mit 2,2 versus 2,4 $(p=0,034)$.

Schlussfolgerungen Die radiale VIBE-Sequenz kann in der pädiatrischen Bildgebung durch geringere Artefakte und verbesserte Abgrenzbarkeit von Organkonturen zu einer signifikanten Steigerung der Bildqualität führen, insbesondere im Bereich des artefaktanfälligen Thorax.
Gastro- und Abdominaldiagnostik

\section{D Multi-Echo Dixon in der Evaluierung einer pathologischen Eiseneinlagerung und Verfettung der Leber: erste klinische Resultate}

Autoren Henninger $\mathrm{B}^{1}$, Zoller $\mathrm{H}^{2}$, Kannengiesser $\mathrm{S}^{3}$, Zhong $\mathrm{X}^{4}$, Jaschke $\mathrm{W}^{1}$, Kremser $C^{1}$

Institut 1 Medizinische Universität Innsbruck, Department Radiologie, Innsbruck; 2 Medizinische Universität Innsbruck, Department Innere Medizin, Innsbruck; 3 Siemens Healthcare, MR Applications Development, Erlangen; 4 Zhong, 4MR R\&amp;D Collaborations, Atlanta

DOI 10.1055/s-0037-1602608

Zielsetzung Prospektive Evaluierung einer neuen 3D-Multi-Echo-Dixon (3D ME DIXON) Sequenz in der Quantifizierung von Lebereisen und -fett in der klinischen Routine.

Material und Methodik 120 Patienten wurden mittels 1.5T MagnetresonanzTomographie der Leber zwischen Dezember 2013 und Juni 2015 mit den folgenden drei Sequenzen untersucht: 3D ME DIXON mit Inline-Berechnung von R2* und der „Proton Density Fat Fraction“ (PDFF), Single-Voxel-Spektroskopie (SVS), 2D Multi Gradienten-Echo Sequenz (2D ME GRE). SVS und 2D ME GRE wurden als Referenz für die PDFF und R2* verwendet. R2* $(1 / \mathrm{s})$ - und PDFF (\%)-Werte der 3D ME Dixon wurden mit jenen der Referenzsequenzen verglichen. Eine lineare Regressionsanalyse, Bland-Altman-Plots und Übereinstimmungsparameter wurden berechnet.

Ergebnisse 103 Patienten konnten in die Studie eingeschlossen werden (87 Männer und 16 Frauen, mittleres Alter 51 Jahre). 17/120 wurden aufgrund von „Fat/Water-Swaps“ oder wegen R2*-Werte über dem für die 3D ME Dixon festgelegten Limit von $4001 / \mathrm{s}$ ausgeschlossen. Eine starke Korrelation $(r=0.992, p<0.001)$ zwischen den R2*-Werten der 3D ME Dixon und der Referenz 2D ME GRE wurde gefunden. Die Bland-Altman-Analyse ergab systematisch niedrigere Werte für die 3D ME Dixon (16,499\%). Durch Verwendung eines angepassten Schwellenwertes von 58 1/s wurde für die 3D ME Dixon eine positive/negative prozentuale Übereinstimmung (PPA/NPA) von 84,4\%/ $93,1 \%$ in der Detektion einer pathologischen Eisenüberladung der Leber berechnet. Bezogen auf die Leberverfettung wurde ebenfalls eine starke Korrelation zwischen der 3D ME Dixon und der Referenz SVS festgestellt werden $(r=0,957, p<0,001)$, der PPA/NPA lag bei 88,3\%/91,4\%.

Schlussfolgerungen Die 3D ME Dixon-Sequenz ist ein wertvolles Werkzeug für die Evaluierung von Lebereisen und -fett unter klinischen Bedingungen. „Fat/Water-Swaps“ bleiben ein Nachteil sodass Verbesserungen bei der Sequenzimplementation erforderlich sind - bei einem eventuellen Auftreten sollten herkömmliche Sequenzen als zusätzliche Option vorhanden sein.

Herzdiagnostik/Gefäßdiagnostik

\section{Advanced Myocardial Tissue Characterization by a multi-component CMR Protocol in Patients with Rheumatoid Arthritis}

Authors Mayr $A^{1}$, Greulich $S^{2}$, Kitterer $D^{3}$, Latus $J^{3}$, Henes $J^{4}$, Vecchio $F^{2}$, Kaesemann $\mathrm{P}^{2}$, Patrascu $\mathrm{A}^{2}$, Greiser $\mathrm{A}^{5}$, Groeninger $\mathrm{S}^{5}$, Romeo $\mathrm{F}^{6}$, Braun $\mathrm{N}^{3}$, Alscher $D^{3}$, Sechtem $U^{7}$, Mahrholdt $H^{7}$

Institute 1 Medizinische Universität Innsbruck, Univ.-Klinik für Radiologie, Innsbruck; 2 Robert-Bosch-Medical Center, Division of Cardiology, Stuttgart; 3 Robert-Bosch-Medical Center, Division of Nephrology, Stuttgart; 4 University Hospital Tuebingen, Centre for Interdisciplinary Clinical Immunology, Tuebingen; 5 Siemens Healthcare GmbH, Erlangen; 6 Universita 
degli Studi di Roma „Tor Vergata“, Division of Cardiology, Rome, Italy: 7 Robert-Bosch-Medical Center, Division of Cardiology, Stuttgart DOI 10.1055/s-0037-1602609

Zielsetzung Rheumatoid arthritis (RA) patients are at increased risk of suffering from adverse cardiovascular events. These potential life-threatening consequences often face a subclinical presentation, normal left ventricular ejection fraction (LV-EF) and normal ECG. Hence, advanced myocardial tissue characterization in early, potential modifiable stages is highly desirable. Late gadolinium enhancement (LGE)-cardiovascular magnetic resonance (CMR) alone with its ability to detect focal myocardial lesions may not be sufficient since RA patients are also known to show diffuse inflammation/fibrosis. Therefore, CMR mapping techniques might be appropriate tools to complement LGE for the assessment of myocardial involvement. Aim of this study was to perform advanced myocardial tissue characterization in RA patients by a multi-component CMR protocol, including LGE and T1/T2 mapping sequences.

Material und Methodik 22 RA patients were prospectively enrolled and underwent CMR, 20 volunteers served as controls.

Ergebnisse Mean LV-EF was 66\%; prevalence of LGE was $18 \%$. RA patients had increased native T1 ( 985 vs. $959 \mathrm{~ms}, \mathrm{p}=0.03$ ), expanded extracellular volume (ECV) ( 27 vs. $25 \%, p=0.02$ ), and higher T2 values ( 52 vs. $49 \mathrm{~ms}, \mathrm{p}<0.001$ ) compared to controls irrespective of the presence of LGE. T2 mapping showed the highest prevalence of values beyond the $95 \%$ percentile of controls.

Schlussfolgerungen RA patients demonstrated higher T1, ECV, and T2 values compared to controls, with most significant differences for T2. Since these results seem to be independent of the presence of LGE, advanced myocardial tissue characterization including CMR mapping techniques in addition to LGECMR might be useful in the evaluation of myocardial involvement in RA patients.

\section{Diagnostische Genauigkeit und Nutzen der koronaren CT-Angiographie unter Berücksichtigung von unklaren Befunden: Meta-Analyse mit Intention-to-diagnose}

Autoren Menke J'

Institut 1 Universitätsmedizin Göttingen, Institut für Diagnostische und

Interventionelle Radiologie, Göttingen

DOI 10.1055/s-0037-1602610

Zielsetzung Meta-analyse von diagnostischer Genauigkeit, test yield und Nutzen der koronaren CT-Angiograpie (CCTA) mit einem intention-to-diagnose Ansatz unter Berücksichtigung unklarer Befunde.

Material und Methodik Vier Datenbanken wurden von 2005 bis 3/2013 durchsucht. Geeignete prospektive Studien hatten bei KHK-Patienten 16- bis 320-Zeilen-CT oder dual-source CT verwendet und berichteten $3 \times 2$ Patienten-level Daten von CCTA (positives, negatives oder unklares Ergebnis) versus Katetherangiographie (positiv oder negativ) für die Diagnose von > $50 \%$-igen Koronarstenosen. Die Meta-Analyse erfolgte mit multivariaten Bayesian random-effects Modellen.

Ergebnisse Es wurden 30 Studien mit insgesamt 3422 Patienten eingeschlossen. Im Vergleich zu 16- bis 40-Zeilen-CTs haben test yield und diagnostische Genauigkeit bei $>=64$-Zeilen-CTs signifikant zugenommen $(P<0.05)$. Mit $>=64$-Zeilen-CTs hatten zirka $2.5 \%$ der erkrankten Patienten und $7.5 \%$ der nicht-erkrankten Patienten unklare CCTA-Ergebnisse. Die positive likelihoodratio von $8.9(95 \%-\mathrm{Cl}, 6.1-13.5)$ zeigte eine moderate Eignung der CCTA für die Identifikation erkrankter Patienten, die in der Katheterangiographie $>=50 \%$-ige Koronarstenosen hatten. Die negative likelihood-ratio von 0.022 (0.01-0.04) zeigte eine exzellente Eignung der CCTA für Identifikation von Patienten, die in der Katheterangiographie keine $>=50 \%$-igen Koronarstenosen hatten. CCTAs mit unklarem Ergebnis hatten eine indifferente likelihoodratio von $0.42(0.22-0.71)$. In der Kosten-Nutzen-Analyse war CCTA bei intermediären Prätest-Wahrscheinlichkeiten von 16 - 70\% sinnvoll.

Schlussfolgerungen Die koronare CT-Angiographie ist bei intermediärer Prätest-Wahrscheinlichkeit für KHK sinnvoll. Positive CCTAs benötigen eine Veri- fikation, um eine KHK zu bestätigen, unklare CCTAs erfordern alternative Diagnostik, und negative CCTAs schließen eine KHK mit hoher Wahrscheinlichkeit aus. Es erscheint sinnvoll, CCTA-Ergebnisse mit einem BIRADS-ähnlichen Score zu beschreiben $(1-2=$ negativ, $3=$ unklar und $4-5=$ positiv für relevante Koronarstenosen).

\section{Assessing the internal consistency of cardiac output measurements in phase contrast MRI by comparison to other systemic flows}

Authors Menke J $]^{1}$

Institute 1 Universitätsmedizin Göttingen, Institut für Diagnostische und Interventionelle Radiologie, Göttingen

DOI 10.1055/s-0037-1602611

Zielsetzung In cardiac magnetic resonance imaging (MRI) cardiac output measurements in the ascending aorta with phase contrast (PC) velocity encoding can be compromised e.g. by susceptibility artifacts. This study investigated, whether such cardiac output measurements can be internally validated by comparison to flow measurements in other systemic vessels.

Material und Methodik 50 consecutive patients with clinical indication for pulmonary/aortic functional valve assessment were included. The systemic cardiac output was measured by 1.5 Tesla MRI with PC imaging in the proximal ascending aorta. In the same image sets the flows in the descending aorta, superior vena cava and right azygos vein were measured and combined for comparison (comparator flow). This comparison was done by Bland-Altman and regression analysis. Flows were calculated in $\mathrm{L} / \mathrm{min}$, and as index in relation to the body surface area in $\mathrm{L} / \mathrm{min} / \mathrm{m} 2$.

Ergebnisse The patients' cardiac output index (measured in the ascending aorta) ranged from 1.9 to $6.6 \mathrm{~L} / \mathrm{min} / \mathrm{m} 2$ (mean, $3.4 \mathrm{~L} / \mathrm{min} / \mathrm{m} 2$ ). The comparator flow correlated well to the cardiac output $(r=0.98)$. The $95 \%$-limits of agreement of the comparator flow index versus the ascending aortic flow index ranged from -0.38 to $0.17 \mathrm{~mL} / \mathrm{min} / \mathrm{m} 2$, corresponding to flow index ratios of 0.89 to 1.05 (mean, 0.97).

Schlussfolgerungen PC-MRI measurements of the cardiac output in the ascending aorta can be well consistency-checked by comparison to the combined flow of the descending aorta, superior vena cava and right azygos vein.

\section{Nachsorge infrarenaler Bauchaortenaneurysmen nach endovaskulärer Aneurysmareparatur (EVAR): Wie gut ist die subjektive Einschätzung der Größenzunahme im Verlauf im Vergleich zur Aneurysmavolumetrie?}

Autoren Schnitzbauer $\mathrm{M}^{1}$, Stroszczynski $\mathrm{P}^{2}$, Pfister $\mathrm{P}^{3}$, Wohlgemuth $\mathrm{P}^{4}$, Müller-Wille $\mathrm{P}^{4}$

Institut 1 HELIOS Klinikum Berlin/Buch, Institut für Röntgendiagnostik, Berlin; 2 Institut für Röntgendiagnostik, Regenburg; 3 , Abteilung für Gefäßchirurgie, Regensburg; 4 Institut für Röntgendiagnostik, Regensburg DOI 10.1055/s-0037-1602612

Zielsetzung Vergleich von Aneurysmavolumetrie und subjektiver Größeneinschätzung zur Beurteilung der Größenzunahme des Aneurysmasacks bei infrarenalen Aneurysmen in der Nachsorge nach EVAR.

Material und Methodik Bei 98 Patienten (9 Frauen, 89 Männer, Durchschnittsalter 70,6 $\pm 7,6$ ) mit infrarenalem Aortenaneurysma wurde retrospektiv die Größenzunahme des Aneurysmasacks durch die Bestimmung des totalen Aneurysmavolumens und durch subjektive Größeneinschätzung analysiert (größer, gleich, kleiner). Cut-off-Wert für die Größenveränderung in der Volumetrie waren $5 \%$.

Ergebnisse Anhand der Volumetriedaten nahm das Aneurysmavolumen bei 24,5\% (24/98) der Patienten zu, stagnierte bei 29,6\% (29/98) der Patienten und nahm bei 45,9\% (45/98) Patienten ab. Die subjektive Einschätzung zeigte eine Zunahme bei 14,3\% (14/98), eine Stagnation bei 54\% (53/98), und eine Abnahme bei 31,6\% (31/98). Die Sensitivität/Spezifität für die korrekte Detek- 
tion einer Größenzunahme mittels subjektiver Einschätzung lag bei 37,5\% (9/ 24)/93\% (69/74). Die Interobserver und Intraobservervariabilität (ICC = Intraclass correlation coefficient) für die totalen Volumenmessungen betrugen 0,987 und 1. Die Volumetrie dauerte im Schnitt $8,4+-1,1$ min.

Schlussfolgerungen Die subjektive Größeneinschätzung ist der reproduzierbaren Aneurysmavolumetrie deutlich unterlegen.

Interventionelle Radiologie

\section{Verwendung von funktionalisierten Kalziumphosphatpartikeln als potentielles Embolisat für die Behandlung von Lebertumoren}

\author{
Autoren Grözinger $\mathrm{G}^{1}$, Schmehl J $]^{1}$, Nikolaou $\mathrm{K}^{1}$, Elser $\mathrm{S}^{1}$ \\ Institut 1 Universitätsklinikum Tübingen, Department Radiologie, Abteilung \\ für Diagnostische und Interventionelle Radiologie, Tübingen \\ DOI 10.1055/s-0037-1602613
}

Zielsetzung In diesem Versuchsansatz wurden Kalziumphosphatpartikel in verschiedenen Größenbereichen als alternatives Embolisat zur Chemoembolisation (TACE) getestet. Sowohl aggregierte CaP-Nanopartikel wie auch makroskopische CaP-Partikel $>(80-200 \mu \mathrm{m})$ wurden in vitro auf ihre Zytotoxizität getestet. Im VX2-Kaninchentumormodell wurde die Wirksamkeit/Effektivität der mit Chemotherpeutika gekoppelten CaP-Partikel bei der Embolisation gezeigt.

Material und Methodik Die Zytotoxizität von dispergierte CaP-Nanopartikel $(100-200 \mathrm{~nm})$ wurden in vitro auf verschiedene HCC-Ziellinien mittels Zellvitalitätsassay untersucht. Auch die Kopplung verschiedener Chemotherapeutika an CaP-Partikel wurde in vitro getestet. Die aggregierten CaP-Nanopartikel wurden auf ihre Stabilität nach einer Mikrokatheterpassage untersucht. Im VX2- Kaninchentumormodell wurden CaP-Partikel im Größenbereich von $80-200 \mu \mathrm{m}$ als Embolisat eingesetzt. Prä- und Post- Embolisation wurde dabei die Leberperfusion mittels Volumenperfusions CT (VPCT) gemessen.

Ergebnisse In nanopartikulärer, dispergierter Form zeigen die CaP-Partikel konzentrationsabhängig einen zytotoxischen Effekt auf verschiedene etablierte humane HCC-Zell-Linien. Stabile Kopplungsprodukte entstehen bei der Verwendung von Epirubicin bzw. Doxorubicin. Die Instabilität der aus CaP-Nanopartikeln zusammengesetzten Aggregate unterbindet aktuell deren Einsatz als Embolisat. Beim Einsatz der makroskopischen CaP- Partikel konnte gezeigt werden, dass diese Partikel als neuartiges Embolisat in Betracht kommen und in den embolisierten Lebersegmenten zu einer Stase mit entsprechendem Perfusionsausfall führen.

Schlussfolgerungen In vitro konnte ein zytotoxischer Effekt von CaP beobachtet werden. Grundsätzlich eignet sich von den beiden getesteten CaPFormulierungen bisher nur die makroskopische Form für eine Embolisation. Um auch den Einsatz von nanopartikulären CaPhosphat-Partikeln als Embolisat zu gewährleisten, müssen weiterführende chemische Ansätze zur Erhöhung der Stabilität der CaP-Aggregate verfolgt werden.

\section{Anatomische Darstellung des sympathischen Granzstranges, seiner nervalen Seitenäste/Varianten, der Signalintensität und der benachbarten Strukturen vor Sympathikolyse beim CRPS}

Autoren Reichardt $B^{1}$

Institut 1 BG Universitätskrankenhaus Bergmannshein Bochum, Institut für diagnostische und Interventionelle Radiologie und Nuklearmedizin, Bochum DOI 10.1055/s-0037-1602614

Zielsetzung Anatomische Darstellung des sympathischen Granzstranges, seiner nervalen Seitenäste/Varianten, der Signalintensität und der benachbarten Strukturen vor Sympathikolyse beim CRPS.
Material und Methodik 10 Patienten wurden im 3T MRT Philips Achiva mit 36 Kanal TXL Spule in Rückennage untersucht. Alle Patienten bekamen vor und nach diagnostischer Sympathicusblockade mittels 0,5\% Bubivacain, sowie nach erfolgreicher Sympathikolyse mit 99\%igem Ethanol eine MRT Neurographie des sympathischen Grenzstranges von Lwk 1- SWK 2. MRT Protokoll: T1 tse coronar, T2 tse coronar, STIR axial, STIR cor, T2 fs axial, DWIBS tra, T1 und T2 sag, PDW fs cor.

Ergebnisse Die sympathischen Ganglien der betroffen Seite zeige vor Blockade eine geringfügige ödematöse Verschwellung und Signalsteigerung in den protonensensitiven Sequenzen. sie sind jedoch klar abgrenzbar. Deine Diffusionsstörung konnte nicht sicher nachgewiesen werden. Nach Blockade und Sympathikolyse zeigten sich unterschiedliche Verteilungsmuster der applizierten Substanzen. Die Umgebungsreizung reichte beim $1 \mathrm{ml}$ Ethanol an die Faszie des Psoas und an die $\mathrm{VCl}$ respektive die Aortahinterwand heran und verteilte sich bis an den Urether. eine nervale Reizung des lumbalen Grenzstranges trat nicht auf; ebenso keine Uretherschädigung. Bei allen Patienten trat eine Schmerzminderung im Mittel um 50\% (3-4 Schmerzpunkte auf einer 0 - 10 Skala) und eine Tempertursteigerung um $3{ }^{\circ} \mathrm{C}$ im Mittel der betroffen Extremität auf.

Schlussfolgerungen MR Neurographie des sympathischen Grenzstranges ist möglich. Applizierte geringe Mengen von Lokalansästheticum und dem des Ethanol zeigen ein entschieden unterschiedliches Verteilungsmuster bei größerer flächiger Ausbreitung des Alkohols in die Nachbarstrukturen bei schon $1 \mathrm{ml}$. Eine adäquate und regelrechte Platzierung des Alkohols in die anatomischen Strukturen vermeidet Nebenwirkungen wie Nervenschäden, Impotenz und Urtherstrikturen.

\section{Der Truncus hepatogastricus - ein Ausschlusskriterium für eine SIRT des linken Leberlappens?}

Autoren Zimmermann $\mathrm{M}^{1}$, Liebl $\mathrm{M}^{1}$, Schulze-Hagen $\mathrm{M}^{1}$, Isfort $\mathrm{P}^{1}$, Pedersoli $F^{1}$, Kuhl $C^{1}$, Bruners $P^{1}$

Institut 1 Uniklinik RWTH Aachen, Klinik für Diagnostische und Interventionelle Radiologie, Aachen

DOI 10.1055/s-0037-1602615

Zielsetzung Für die arterielle Anatomie der Oberbauchorgane sind diverse Normvarianten bekannt. Die Transposition der A. hepatica sinistra bzw. der Segment II und III-Arterie auf die A. gastrica sinistra (sog. Truncus hepatogastricus) ist eine regelmäßig auftretende Variante. Im Rahmen der Durchführung einer SIRT besteht hier das theoretische Risiko einer Fehlembolisation in die Magenwand mit den daraus resultierenden Komplikationen. Das Ziel war daher retrospektiv die Sicherheit einer SIRT des linken Leberlappens bei Patienten mit einem Truncus hepatogastricus zu untersuchen.

Material und Methodik Sechs Patienten mit multifokalem HCC bzw. multifokalen hepatischen Metastasen wurden zwischen 2011 und 2015 mittels SIRT (SIR-Spheres ${ }^{\circledR}$, Sirtex) des linken Leberlappens über einen Truncus hepatogastricus behandelt. Fünf der Patienten erhielten eine lobäre SIRT, während beim sechsten Patienten in drei Sitzungen jeweils selektiv die Segmente II, III und IV behandelt wurden. Die Position der Katheterspitze wurde bei der Embolisation jeweils min. $3 \mathrm{~cm}$ distal des letzten extrahepatischen Gefäßabgangs positioniert. Es erfolgte eine retrospektive Auswertung der Patientendaten hinsichtlich peri- und postinterventioneller Komplikationen.

Ergebnisse Alle acht Interventionen verliefen komplikationslos und die Patienten waren während des gesamten postinterventionellen Verlaufs beschwerdefrei. Die applizierte Aktivität wurde gemäß Herstellervorgaben bestimmt (keine zusätzliche Dosisreduktion) und betrug durchschnittlich $672 \pm 226 \mathrm{~Bq}$. Das postinterventionell durchgeführte Bremsstrahl-PET zeigte jeweils eine regelrechte tumorbezogene intrahepatische Nuklidanreicherung ohne Nachweis extrahepatischer Speicherungen. In der postinterventionellen Bildgebung (MRT oder PET/CT) 4 Wochen nach SIRT zeigte sich jeweils ein gutes Therapieansprechen der behandelten Areale. 
Schlussfolgerungen Die SIRT des linken Leberlappens über einen Truncus hepaticogastricus erscheint sicher - in dieser Fallserie ergaben sich keine Hinweise auf ein erhöhtes Komplikationsrisiko.

Mammadiagnostik

\section{Vergleich MonteCarlo berechneter Dosisverteilungen für Mammographiesysteme mit TLD-Messungen}

Autoren Babalik $\mathrm{A}^{1}$, Buchgeister $\mathrm{M}^{1}$

Institut 1 Beuth-Hochschule für Technik Berlin, Fachbereich II, MathematikPhysik-Chemie, Berlin

DOI 10.1055/s-0037-1602616

Zielsetzung Mittels MonteCarlo-Simulationen (MC) sollen mit Thermolumineszenzdosimetern (TLD) in drei speziellen Mammaphantomen aus gewebeähnlichem Bolusmaterial an einem Philips MicroDose L30 und Siemens Mammomat Novation DR [1] gemessene Dosisverteilungen verifiziert werden. Material und Methodik Für die MC-Simulationen wurde PENELOPE 2014 [2] verwendet. Das für die TLD-Messungen verwendete weiche Bolusmaterial der drei Phantome $\left(10 \times 10 \times 2 \mathrm{~cm}^{3}, 15 \times 15 \times 4 \mathrm{~cm}^{3}\right.$ und $\left.20 \times 20 \times 6 \mathrm{~cm}^{3}\right)$ wurde anhand von gemessenen Schwächungsdaten aus den Elementen des ICRUWeichgewebes für die MC-Simulation in seiner Zusammensetzung optimiert. Die Röntgenspektren der Strahlqualitäten (kV, bzw. Anodenmaterial/Filter) der TLD-Messungen wurden mit dem Online-Tool Simulation of X-ray Spectra von Siemens erstellt [3]. Die MC-Simulationen und TLD-Daten wurden im Hinblick auf die sich aus den Dosisverteilungen ergebende mittlere Parenchymdosis (AGD) verglichen.

Ergebnisse Die Ergebnisse der AGD der MC-Simulationen sind in guter Übereinstimmung mit den TLD-Daten. Die Abweichungen der TLD-Messungen mit dem Siemens System (W/Rh) betragen 9,4\% für das kleine, 2,2\% für das mittlere und 1,3\% für das große Phantom. Für die Messungen mit dem Philips System (W/Al) sind die Abweichungen 15,9\% für das kleine, 0,0\% für das mittlere und $12,1 \%$ für das große Phantom. Weiter konnten Abweichungen von bis zu 69,4\% zwischen der MC berechneten bzw. der TLD-gemessenen AGD und der Angabe im DICOM-Header des Philips-Systems registriert werden.

Schlussfolgerungen In dieser Arbeit konnte gezeigt werden, dass die real gemessenen Dosisverteilungen mit MC-Simulationen auf Plausibilität überprüft werden konnten. Besondere Aufmerksamkeit ist bei der MC-Simulation vor allem auf die Berücksichtigung des Heel-Effekts und die exakte Nachbildung der Messgeometrie im MC-Code zu legen. [1] M. Buchgeister, R. Leben, M. Roll, R. Juran; RöFo, 185 (2013) S274. [2] F. Savat, PENELOPE 2014, www. oecd-nea.org/tools/abstract/detail/nea-1525 [3] www.oem-products.siemens. com/x-ray-spectra-simulation

\section{Multicenterstudie: Radiologische Befunde bei primären Brustsarkomen}

Autoren Wienbeck $S^{1}$, Meyer $\mathrm{H}^{2}$, Dr. med. Herzog $A^{3}$, Dr. med. Nemat $S^{4}$, Dr. med. Teifke $A^{5}$, Prof. Dr. med. Heindel $W^{6}$, Prof. Dr. med. Schäfer $F^{7}$, PD Dr. med. Kinner $S^{8}$, Prof. Dr. med. Müller-Schimpfle $M^{9}$, PD Dr. med. Surov $A^{10}$ Institut 1 Universitätsmedizin Göttingen, Institut für Diagnostische und Interventionelle Radiologie, Göttingen; 2 Universitätsklinikum Halle, Klinik und Poliklinik für Radiologie, Halle; 3 Universitätsklinikum Jena, Institut für Diagnostische und Interventionelle Radiologie, Jena; 4 Universitätsklinikum des Saarlandes, Klinik für Diagnostische und Interventionelle Radiologie, Homburg Saar; 5 Universitätsmedizin Mainz, Klinik und Poliklinik für Diagnostische und Interventionelle Radiologie, Mainz; 6 Universitätsklinikum Münster, Institut für Klinische Radiologie, Münster; 7 Universität Kiel, Klinik für Radiologie und Neuroradiologie, Kiel; 8 Universitätsklinikum Essen, Institut für Diagnostische und Interventionelle Radiologie und Neuroradiologie, Essen; 9 Klinikum Frankfurt Höchst, Institut für Radiologie, Neuroradiologie und
Nuklearmedizin, Frankfurt am Main/Höchst; 10 Universität Leipzig, Klinik und Poliklinik für Diagnostische und Interventionelle Radiologie, Leipzig

DOI 10.1055/s-0037-1602617

Zielsetzung Es wurden die unterschiedlichen radiologischen Befunde bei Patienten mit primärem Brustsarkomen (PBS) evaluiert.

Material und Methodik In einer retrospektiven klinischen Studie erfolgte eine Datenbankrecherche an 10 deutschen radiologischen Instituten im Zeitraum von 2000 bis 2011. Alle histopathologisch gesicherten Befunde von PBS wurden in die Studie eingeschlossen. Es lagen bei 31 Patienten (33 Läsionen) Mammographien und bei 24 Patienten (24 Läsionen) Sonographien der Brust vor. Bei 10 Patienten (14 Läsionen) war eine Magnetresonanztomographie (MRT) der Brust durchgeführt worden. Sämtliche erhobenen Brustbefunde wurden anhand des BI-RADS ${ }^{\circledR}$ Atlas (5th edition) klassifiziert und mittels deskriptiver Statistik ausgewertet.

Ergebnisse Es konnten 42 Frauen (mittleres Alter 62,0 Jahre, 30 - 86 Jahre) in die Studie eingeschlossen werden. In allen Fällen lag ein schmerzloser Tastbefund vor. Mammographisch fand sich überwiegend ein hyperdenser, irregulärer (53,3\% [16/30]) oder ovalärer (30,0\% [9/30]) Herdbefund mit unscharfer (73,3\% [22/30]) oder mikrolobulierter (10\% [3/30]) Randbegrenzung. Assoziierte Verkalkungen waren sehr selten (3,3\% [1/30]). Sonographisch konnten häufig irreguläre (79,2\% [19/24]) und echoarme (62,5\% [15/24]) Herdbefunde mit unscharfer Randbegrenzung (79,2\% [19/24]) und dorsaler Schallauslöschung (79,2\% [19/24]) gesehen werden. Im MRT zeigten sich ebenfalls irregulär begrenzte Herdbefunde (81,8\% [9/11]), die in der Kinetik einen schnellen initialen Signalanstieg und ein verzögertes wash-out aufwiesen.

Schlussfolgerungen PBS weisen keine pathognomonischen Merkmale in der Bildgebung auf und können prinipiell ein invasives Karzinom nachahmen. Das MRT der Brust ist im Rahmen der weiteren Bildgebungsdiagnostik hilfreich. Bei einem irregulär oder ovalär konfiguriertem Herdbefund mit unscharfer oder mikrolobulierter Randbegrenzung, sollte differentialdiagnostisch an das Vorliegen eines PBS gedacht werden.den.

\section{Molekulare Bildgebung}

\section{Zusammenhänge zwischen Perfusionsparametern und histopathologischen Befunden bei Plattenepithelkarzinomen der Kopf-Hals-Region}

Autoren Surov $\mathrm{A}^{1}$, Meyer $\mathrm{H}^{2}$, Gawlitza $\mathrm{M}^{3}$, Höhn $\mathrm{A}^{4}$, Kahn $\mathrm{T}^{3}$, Stumpp $\mathrm{P}^{3}$ Institut 1 Universitätsklinikum Leipzig, Institut für Diagnostische und Interventionelle Radiologie, Leipzig; 2 Martin-Luther-Universität HalleWittenberg, Radiologie, Halle; 3 Universität Leipzig, Radiologie, ; 4 Universität Leipzig, Pathologie,

DOI 10.1055/s-0037-1602618

Zielsetzung Dynamic contrast-enhanced magnetic resonance imaging (DCE MRI) kann die Perfusion und die Vaskularisation von Geweben charakterisieren. Es wurde bereits gezeigt, dass die DCE MRI-Parameter zwischen malignen und benignen Läsionen unterscheiden und den Tumorgrad vorhersagen können. Das Ziel der Studie war es mögliche Korrelationen zwischen DCE MRIund histopathologischen Parametern in Kopf und Hals-Plattenepithelkarzinomen (HNSCC) zu ermitteln.

Material und Methodik 16 Patienten mit histologisch gesichertem HNSCC (11 Patienten mit primärem Tumor und 5 Patienten mit lokalem Tumorrezidiv) wurden in diese Studie eingeschlossen. Folgende Parameter der DCE-Bildgebung wurden ermittelt: Ktrans, Ve, Kep, and iAUC. Der Proliferationsindex und die Zellzahl der Tumoren wurden auf Ki67 gefärbten histologischen Schnitten ermittelt. Die Gefäßdichte (gefärbte Gefäßfläche, totale Gefäßfläche, Gefäßanzahl und mittlerer Gefäßdurchmesser) wurden an Hand von CD 31 gefärbten Schnitten untersucht. Spearman Korrelationskoeffizienten wurde für die Korrelationsanalyse verwandt.

Ergebnisse Die Mittelwerte der DCE-Parametern waren wie folgt: Ktrans $0,189 \pm 0,056 \mathrm{~min}-1$, Kep 0,390 $\pm 0,160 \mathrm{~min}-1$, Ve $0,548 \pm 0,119 \%$, and iAUC 
$22,40 \pm 12,57$. Statistisch signifikante Korrelationen wurden zwischen Kep und der gefärbten Gefäßfläche $(r=0,51, p=0,041)$ und totalen Gefäßfläche $(r=0,51, p=0,043)$; zwischen Ve und dem mittleren Gefäßdurchmesser $(r=-0,59, p=0,017)$ gefunden. Die Zellzahl zeigte eine Tendenz mit Ve $(r=-0,48, \quad p=0,058)$ und dem mittleren Gefäßdurchmesser $(r=0,45$, $p=0,08)$ zu korrelieren. In der Analyse nur der primären Tumoren konnte eine signifikante inverse Korrelation zwischen Ktrans and KI 67 gezeigt werden $(r=-0,62, p=0,041)$.

Schlussfolgerungen Unsere Analyse ermittelte signifikante Korrelationen zwischen DCE-Parametern und der Gefäßdichte in HNSCC.

\section{Muskuloskelettale Radiologie}

\section{Multi-modale Bildgebung zur Tumorlokalisierung bei Onkogener Osteomalazie}

Autoren Peldschus $\mathrm{K}^{1}$, Bahls $\mathrm{C}^{2}$, Derlin $\mathrm{T}^{3}$, Adam $\mathrm{G}^{1}$, Amling $\mathrm{M}^{4}$, Breer $\mathrm{S}^{5}$ Institut 1 Universitätsklinikum Hamburg-Eppendorf, Diagnostische und Interventionelle Radiologie, Hamburg; 2 Universitätsklinikum HamburgEppendorf, Klinik für Nuklearmedizin, Hamburg; 3 Medizinische Hochschule Hannover, Klinik für Nuklearmedizin, Hannover; 4 Universitätsklinikum Hamburg, Institut für Osteologie und Biomechanik, Hamburg;

5 Universitätsklinikum Hamburg-Eppendorf, Institut für Osteologie und Biomechanik, Hamburg

DOI 10.1055/s-0037-1602619

Zielsetzung Retrospektive Evaluation von Patienten mit onkogener Osteomalazie, bei denen zur Lokalisation der verursachenden Tumore verschiedene Bildgebungsverfahren eingesetzt wurden.

Material und Methodik Es konnten fünf Patienten mit onkogener Osteomalazie identifiziert werden, die durch Resektion des verursachenden Tumors erfolgreich behandelt wurden. Alle Patienten wurden mittels 111ln-Octreotid Szintigraphie, 68Ga DOTATATE PET/CT und MRT untersucht. Die Analyse der Bilddaten beinhaltete Detektion, Tracer-Anreicherung, Größe und Kontratmittelverhalten der Tumore. Es erfolgte eine Beurteilung durch Nuklearmediziner und Radiologen.

Ergebnisse Von den fünf Patienten (50,4 \pm 7,3 Jahre, 3 w, 2 m) gelang mit der $111 \mathrm{ln}$-Octreotid Szintigraphie nur in 2 Fällen eine Tumorlokalisation. Mit der 68Ga DOTATATE PET/CT konnte in allen fünf Fällen ein Tumor lokalisiert werden (SUVmax 14.9 \pm 10.2 ). Mit der MRT konnten die tumorsuspekten nuklearmedizischen Befunde bestätigt und die Tumore näher charakterisiert werden. Es ergab sich ein mittlerer Tumor-Diameter von $2.5 \pm 1.6 \mathrm{~cm}$. Die Größe der beiden Tumore, die mit der $111 \mathrm{ln}$-Octreotid Szintigraphie detektiert wurden $(4.5 \pm 0.6 \mathrm{~cm})$ unterschied sich signifikant von den drei Tumoren, die nur mit der 68Ga DOTATATE PET/CT detektiert wurden $(1.2 \pm 0.1 \mathrm{~cm})$. Alle fünf Tumore konnten vollständig chirurgisch reseziert werden. Die Histopathologie ergab in allen Fällen die Expression des Somatostatinrezeptor-Subtyp 2. Drei Tumore wurden als mesenchymale mixed connective tissue-Varianten klassifiziert und zwei Tumore als odontogene Fibrome, endothelium-rich Typ.

Schlussfolgerungen Bei Patienten mit onkogener Osteomalazie konnten mit der 68Ga DOTATATE PET/CT signifikant kleinere Tumore als mit der $111 \mathrm{ln}$ Octreotid Szintigraphie detektiert werden. In allen Fällen war eine MRT zur Charakterisierung der tumorsuspekten nuklearmedizinischen Befunde sowie zur OP-Planung notwendig.

\section{D-Doppel-Echo Steady-State Sequenz zur Bewertung des Gelenkknorpels und Labrums des Hüftgelenks bei 3 Tesla: Vergleichende Analyse von Magnetresonanztomographie und intraoperativen Daten}

Autoren Schleich $C^{1}$, Bittersohl $B^{2}$, Hesper $T^{2}$, Aissa $J^{1}$, Boos J $J^{1}$, Sawicki $L^{1}$, Schaarschmidt $B^{1}$, Müller-Lutz $A^{1}$, Tschischka $A^{1}$, Antoch $C^{1}$

Institut 1 Heinrich-Heine-Universität Düsseldorf, Medizinische Fakultät, Institut für Diagnostische und Interventionelle Radiologie, Düsseldorf; 2 Universitätsklinikum Düsseldorf, Klinik für Orthopädie, Düsseldorf DOI 10.1055/s-0037-1602620

Zielsetzung Zur Bestimmung der diagnostischen Genauigkeit einer hochauflösenden, dreidimensionalen (3D) double-Echo Steady-State (DESS) Sequenz mit radialen Bildgebung bei 3 Tesla $(T$ ) für den Knorpel und des Labrums des Hüftgelenks. Bracco Posterpreis

Material und Methodik Die Magnetresonanztomographie (MRT) Daten wurden bei 3T aufgenommen, daraus wurden radiale Reformationen angefertigt. Die DESS Daten wurden mit intraoperativen Daten nach Hüftgelenksarthroskopie von 45 Patienten (mittleres Alter: $42 \pm 13$,7 Jahre) verglichen. Der acetabuläre Knorpel, der zentrale Knorpel des Femurkopfes und das Labrum wurden analysiert. Sensitivität, Spezifität, Genauigkeit sowie negative und positive prädiktive Werte wurden bestimmt.

Ergebnisse Sensitivität, Spezifität und Genauigkeit der DESS Technik waren 96,7\%, 75\% und 93,7\% für die Detektion von Knorpelläsionen und 98\%, 76,2\% und 95,9\% für die Detektion von Labrumläsionen. Die positiven und negativen Vorhersagewerte für den Nachweis oder um Knorpelläsionen auszuschließen waren $96 \%$ und $78,9 \%$. Für Labrumläsionen waren die positiven und negativen prädiktiven Werte $97,5 \%$ und $80 \%$.

Schlussfolgerungen Die hochauflösende 3D-DESS Technik mit radialer Bildgebung bei 3T zeigte eine hohe Genauigkeit für die Detektion von Hüftknorpel und -labrumläsionen im Vergleich mit der Hüftgelenksarthroskopie.

\section{Neuroradiologie}

\section{SWI als Nachweismethode der akuten und reversiblen Migräne-Manifestation}

Autoren Fedders $D^{1}$, Ehrlich $S^{2}$, Sokolowski $\mathrm{P}^{2}$, Köhler $\mathrm{W}^{2}$

Institut 1 Universitätsklinikum Carl Gustav Carus, Institut und Poliklinik für Radiologische Diagnostik, Dresden; 2 Klinik für Neurologie und neurologische Intensivmedizin, Wermsdorf

DOI 10.1055/s-0037-1602621

Zielsetzung Die suszeptibilitätsgewichtete Sequenz nutzt die paramagnetischen Eigenschaften von Blutprodukten. Typischerweise wird die Sequenz zum Nachweis von Mikroblutungen genutzt. Sie ist jedoch auch für die Darstellung kleiner venöser Gefäße prädestiniert, da sie auf räumlich hochauflösenden 3D-Gradienten-Echo-Sequenzen beruht, deren Daten einer Phasen-Nachbearbeitung unterzogen werden. Oxygeniertes und desoxygeniertes Hämoglobin haben unterschiedliche Suzeptibilitäten, die zu differenten Phasen-Effekten führen, die bildmorphologisch Areale mit und ohne desoxygeniertem Blut deutlich unterscheidbar machen. Typischerweise werden diese in der Bildgebung arteriovenöser Fehlbildungen genutzt. Aufgrund der Nutzung als Nachweissequenz von Microbleeds im Rahmen der Schlaganfall- und Kopfschmerzdiagnostik sahen wir in einer relevanten Untergruppe von Patienten mit dem Verdacht auf Migräne-bedingte Kopfschmerzen prägnante Unterschiede bei der Darstellung der kleinen venösen Hirngefäße, die im Falle des akuten Migräneanfalls mit entweder deutlich seitenbezogener KopfschmerzSymptomatik und/oder sensomotorischen Symptomen ipsilateral eine deutliche Aufweitung der kleinen intrasulcalen venösen Gefäße aufzeigte. 
Material und Methodik Wir möchten drei, u.a. pädiatrische Patienten mit unterschiedlichen klinischen und bildmorphologischen reversiblen Manifestationen einer Migräne vorstellen (1.5 T, Routine-SWI-3D-GE-Sequenz).

Ergebnisse In den im anfallsfreien Intervall durchgeführten Kontroll-Untersuchungen (typischerweise 1 Tag nach Aufnahme) stellten sich die ausgeprägten Veränderungen vollständig reversibel dar mit einer dann seitengleichen unauffälligen Gefäßdarstellung.

Schlussfolgerungen Der Fokus in der akuten Migränediagnostik verschiebt sich für einen Teil der Patienten von der reinen Ausschlussdiagnostik der Ursachen eines ausgeprägten Kopfschmerzes wie Hirnblutung, Tumor oder Entzündung hin zu einem bildgebenden Nachweis eines funktionell reversiblen Substrats der Migräne, nämlich der manchesmal ausgeprägten venösen Vasodilation.

\section{Distance to thrombus in Korrelation zu cerebraler Perfusion und CTA-Kollateralscores bei Patienten mit akuten Verschlüssen der Arteria cerebri media}

\author{
Autoren Lobsien $D^{1}$, Raviolo $\mathrm{M}^{2}$, Schaudinn $\mathrm{A}^{3}$, Hobohm $\mathrm{C}^{4}$, Gawlitza $\mathrm{M}^{5}$, \\ Hoffmann $K^{1}$, Friedrich $B^{6}$ \\ Institut 1 Universitätklinikum Leipzig, Neuroradiologie, Leipzig; \\ 2 Universitätsklinikum Leipzig, Neuroradiologie, Leipzig; \\ 3 Universitätsklinikum Leipzig, Klinik und Poliklinik für Diagnostische und \\ Inverventionelle Radiologie, Leipzig; 4 Carl von Basedow Klinikum Saalekreis, \\ Neurologie, Merseburg; 5 Centre Hospitalier Universitaire Reims, Service de \\ Neuroradiologie, Reims; 6 Technischem Universität München, \\ Neuroradiologie, München \\ DOI 10.1055/s-0037-1602622
}

Zielsetzung Es konnte kürzlich gezeigt werden, dass die in der CTA gemessene Distanz bis zum Thrombus (Distance to thrombus, DT) bei Patienten mit akuten Verschlüssen der A. cerebri media signifikant mit dem Outcome nach systemischer Lyse mit rtPA korreliert. In dieser Arbeit untersuchen wir die Zusammenhänge zwischen DT und der CT Perfusion als auch dem in der CTA bestimmten Kollateralstatus vor Therapie.

Material und Methodik Retrospektiv wurden Patienten mit akutem Schlaganfall und in der CTA bewiesenem Verschluss der A. cerebri media bei denen eine vollständige CT-Perfusionsmessung vorlag eingeschlossen. Auf Parameterkarten des CBV, CBF, MTT und TTP der präinterventionellen PerfusionsCTs wurden Läsionsvolumina quantitativ (1 Reader) und Perfusions-ASPECTS (pASPECTS) (2 Reader, verblindet) bestimmt. Der Kollateralstatus wurde mittels des Miteff Scores (2 Reader, verblindet) auf den initalen CTAs bestimmt. Ergebnisse 130 Patienten wurden eingeschlossen (77w, Alter 78J (Median, Range 27 -96). DT zeigte eine signifikante Korrelation mit den Läsionsvolumina (CBV $\rho=-0,157 ; p<0,001 / C B F \quad \rho=-0,32 ; \quad p<0,001 /$ MTT $\rho=-0,428$; $p<0,001 /$ TTP $\rho=-0,393 ; p<0,001)$. Des Weiteren fanden sich signifikante Korrelationen zwischen DT und pASCPECTS (CBV $\rho=0,289 ; p=0,001 / C B F$ $\rho=0,426 ; p<0,001 /$ MTT $\rho=0,563 ; p<0,001 /$ TPP $\rho=0538 ; p<0,001$ ). Der Kollateralisationsgrad bestimmt mittels Miteff Score korrelierte signifikant mit DT $(p=0,386 ; p<0,001)$.

Schlussfolgerungen DT korreliert signifikant mit der cerebralen Perfusion und dem cerebralen Kollateralisationsgrad entsprechend dem Miteffscore und ist somit ein wichtige Parameter zur Evaluation des akuten ischämischen Schlaganfalls.
Onkologische Bildgebung/Onkologie

\section{Prognostische Bedeutung fokaler \\ Knochenmarksläsionen bei Patienten mit multiplem Myelom vor und nach allogener Stammzelltransplantation}

Autoren Mosebach $\mathrm{J}^{1}$, Shah $\mathrm{S}^{2}$, Fard $\mathrm{N}^{2}$, Goldschmidt $\mathrm{H}^{3}$, Schönland $\mathrm{S}^{2}$, Hegenbart $\mathrm{U}^{2}$, Schlemmer $\mathrm{H}^{1}$, Delorme $\mathrm{S}^{1}$, Hillengass $\mathrm{J}^{2}$

Institut 1 DKFZ, Radiologie, Heidelberg; 2 Medizinische Klinik, Universitätsklinikum Heidelberg, Innere Medizin V, Heidelberg; 3 Medizinische Klinik, Universitätsklinikum Heidelberg, Innere Medizin V, Heidelberg

DOI 10.1055/s-0037-1602623

Zielsetzung Mittels Ganzkörper-MRT vor und nach allogener Stammzelltransplantation (SZT) sollen Prädiktoren des Überlebens von Patienten mit multiplem Myelom untersucht werden.

Material und Methodik Insgesamt 79 Patienten mit multiplem Myelom (mind. Stadium 2 nach Salmon und Durie) erhielten eine GK-MRT bei 1,5T vor allogener STZ, 63 davon nach erfolgter Therapie. Die Tumorlast wurde anhand der Anzahl Myelom-typischer Läsionen bestimmt, definiert als korrespondierende fokale T1w-hypointense, sowie T2wTIRM hyperintense Signalveränderungen mit einer Größe > 5 mm. Die statistische Analyse beinhaltete uniund multivariate Cox-Regressionsmodelle. Der Log-rank-Test wurde für die Berechnung des Einflusses der fokalen Läsion auf die Überlebenszeit genutzt. Ergebnisse Patienten mit fokalem Infiltrationsmuster nach erfolgter Therapie hatten ein geringeres Progressionsfreies-Überleben (PFS; HR 2,52; $p=0,035$ ) und Gesamtüberleben (OS; HR 3,8; $p=0,032$ ) als Patienten ohne fokale Tumorläsionen. Prognostisch ungünstig war zudem eine höhere Anzahl an Läsionen zum vergleichbaren Zeitpunkt (OS: Baseline HR 1,02; $p=0,013$ und Follow-up HR 1,04; p = 0,009). Die 5-Jahresüberlebensrate betrug 31\% für Patienten mit fokaler Knochenmarksinfiltration und 74\% für Patienten ohne fokale Läsionen (Log-rank p=0,02).

Schlussfolgerungen Ein höheres Progressionsrisiko und geringeres Gesamtüberleben fand sich für Patienten mit mindestens einer suspekten Läsion, so dass diese von einer kontinuierlichen Konsolidierungstherapie profitieren könnten. Zur Detektion dieser prognostisch relevanten Läsionen, kann die GK-MRT vor und nach allogener SZT empfohlen werden.

\section{CT-basierte Tumorheterogenitätsanalye von Pankreaskarzinomen erlaubt Aussagen zur Tumorprogression}

Autoren Steinacker $\mathrm{J}^{1}$, Stanescu-Siegmund $\mathrm{N}^{2}$, Ettrich $\mathrm{T}^{3}$, Baumhauer $\mathrm{M}^{4}$, Barth $\mathrm{T}^{5}$, Kornmann $\mathrm{M}^{6}$, Beer $\mathrm{A}^{7}$, Beer $\mathrm{M}^{8}$, Schmidt $\mathrm{S}^{8}$

Institut 1 Universitätsklinik Ulm, Diagnostische und Interventionelle Radiologie, Ulm; 2 Klinik für Diagnostische und Interventionelle Radiologie, Ulm; 3 Klinik für Innere Medizin I, Ulm; 4 mint-medical, Mannheim; 5 Allgemeine Pathologie, Ulm; 6 Klinik für Allgemein- und Viszeralchirurgie, ; 7 Klinik für Nuklearmedizin, Ulm; 8 Diagnostische und Interventionelle Radiologie, Ulm DOI 10.1055/s-0037-1602624

Zielsetzung Die Bildgebung von Pankreaskarzinomen zur Therapieevaluation bringt Herausforderungen mit sich. Tumorgröße und Kontrastierungsverhalten sind weithin als messbare Parameter in Baseline und Follow-Up-Untersuchungen verbreitet, jedoch limitiert durch unscharfe Tumorränder und fehlende qualitative Parameter. Wir testeten einen neuen CT-basierten Ansatz zur bildmorphologischen Tumorheterogenitätsanalyse im Rahmen des Therapiemonitorings.

Material und Methodik 13 Patienten mit einem inoperablen Adenokarzinom des Pankreas wurden mittels kontrastgestützter CT (portalvenöse Phase) als Baseline- sowie unter Therapie als Follow-up untersucht. Alle erhielten eine 
systemische Chemotherapie (FOLFIRINOX/Gemcitabin), ein Progress wurde als neue Läsion (6 Patienten) oder lokale Tumorausdehnung (3 Patienten) definiert, die Auswertung erfolgte retrospektiv. Die Tumorheterogenitätsanalyse erfolgte mit mintLesion ${ }^{\circledR}$. Erhobene Parameter waren Tumorvolumen, Entropie, Kurtosis, Mittlere Positivität von Pixeln (MPP), Schiefe/Skewness, Uniformität der Verteilung von positive Pixeln (UPP), und Uniformität der Pixel. Statistische Analysen erfolgten mittels Spearmans Rangkorrelation und Mann-Whitney-U-Test.

Ergebnisse Im Follow-up zeigte das Tumorvolumen keine signifikanten Unterschiede zwischen den Gruppen mit und ohne Progress (lokal und/oder systemisch, $p=0,661$ ). Dagegen waren die MPP-Werte bei Patienten ohne Progress im Vergleich zu Patienten mit Progress signifikant höher $(p=0,030)$. Zudem zeigte sich eine signifikant negative Korrelation zwischen Veränderungen der Kurtosis und der Zeit bis zum lokalen $(p=0,008)$ oder systemischen ( $p=0,017)$ Tumorprogress.

Schlussfolgerungen Die Ergebnisse legen nahe, dass die Tumorheterogenitätsanalyse wertvolle Zusatzinformationen zur klinischen Routinediagnostik bieten kann; eine Implementierung im klinischen Alltag ist leicht möglich. Die Heterogenitätsanalyse könnte helfen, das Therapieansprechen besser zu beurteilen und so wichtige Therapieänderungen frühzeitiger einzuleiten.

Ultraschall

\section{Korrelation der kontrastmittelverstärkten Sonographie der Brust mit dem immunhistochemisch bestimmten Profil von Brusttumoren}

Autoren Kapetas $\mathrm{P}^{1}$, Woitek $\mathrm{R}^{1}$, Clauser $\mathrm{P}^{1}$, Pinker $\mathrm{K}^{1}$, Bernathova $\mathrm{M}^{1}$, Helbich $\mathrm{T}^{1}$, Baltzer $\mathrm{P}^{1}$

Institut 1 Medizinische Universität Wien, Universitätsklinik für Radiologie und Nuklearmedizin, Wien

DOI 10.1055/s-0037-1602625

Zielsetzung ,Bracco Posterpreis‘ Untersuchung ob die funktionelle Erfassung der Durchblutung von Brusttumoren anhand von quantitativen Parametern der kontrastmittelverstärkten Sonographie (KMUS) mit dem immunhistochemisch bestimmten Tumortyp assoziiert ist.

Material und Methodik 64 Patientinnen mit histologisch gesichertem Brustkrebs wurden im Rahmen dieser prospektiven, durch die lokale Ethikkommission bewilligten Studie mit KMUS untersucht. Die akquirierten Datensätze wurden mit einer dedizierten Software ausgewertet. Folgende quantitative Parameter wurden gemessen: Peak Enhancement (PE), Wash-in Area Under the Curve (WiAUC), Rise Time (RT), mean Transit Time (local) (mTTI), Time To Peak (TTP), Wash-in Rate (WiR), Wash-in Perfusion Index (WiPI), Wash-out AUC (WoAUC), Wash-in-Wash-out AUC (WiWoAUC), Fall Time (FT) and Wash-out Rate (WoR). Die histologische Untersuchung bestimmte den Tumorgrad, den Östrogen- (ÖR) und Progesteronrezeptorstautus (PR), den human epidermal growth factor receptor 2-Status (HER2), den Ki67-Proliferationsindex und das Tumorprotein p53. Mittels Spearmans Rangkorrelationskoeffizient wurde die Korrelation von quantitativen KMUS-Indizes mit immunhistochemischen Parametern bewertet.

Ergebnisse Eine signifikante aber schwache negative Korrelation zwischen ÖR-Positivität und PE ( $r s=-0,258, p<0,05)$, WiAUC ( $r s=-0,329, p<0,01)$, WiWoAUC ( $r s=-0,309, p<0,05)$ und WoAUC $(r s=-0.294, p<0,05)$ wurde beobachtet; ähnlich auch zwischen PR-Positivität und PE ( $r s=-0.248, p=0,05$ ), WiWoAUC ( $r s=-0,279, p<0,05)$ und WoR ( $r s=-0,294, p<0,05)$.

Schlussfolgerungen Mehrere quantitative KMUS-Parameter zeigen eine signifikante, wenn auch schwache Korrelation mit dem immunhistochemischen Profil von Brusttumoren. Die klinische Relevanz dieses Befundes sollte weiter untersucht werden.

\section{Intraoperativer Kontrastmittelultraschall (CEUS) zur Steuerung und Erfolgskontrolle von intraoperativen Radiofrequenzablationen während elektiver Lebertumoroperationen}

Autoren Platz Batista da Silva $\mathrm{N}^{1}$, Wiggermann $\mathrm{P}^{1}$, Scherer $\mathrm{M}^{2}$, Hornung $\mathrm{M}^{2}$, Jung $E^{1}$

Institut 1 Universitätsklinikum Regensburg, Institut für Röntgendiagnostik, Regensburg; 2 Universitätsklinikum Regensburg, Klinik und Poliklinik für

Chirurgie, Regensburg

DOI 10.1055/s-0037-1602626

Zielsetzung „Bracco Posterpreis“ Ziel der Studie war es, das Potential des intraoperativen Kontrastmittelultraschalls (io-CEUS) zum Monitoren von intraoperativen Radiofrequenzablationen (io-RFA) und zur unmittelbaren postinterventionellen Erfolgskontrolle zu evaluieren, welche während elektiver Lebertumoroperationen durchgeführt wurden.

Material und Methodik Retrospektive Analyse von digital gespeicherten UItraschalldaten von 14 Patienten mit 20 Leberläsionen, die zwischen 02/2011 und 03/2016 im Rahmen einer elektiven Lebertumoroperation eine zusätzliche intraoperative RFA erhielten. Io-CEUS wurde mittels Bolusinjektionen von bis zu $15 \mathrm{ml}$ Sulfurhexaflourid-Mikrobläschen und $20 \mathrm{ml} \mathrm{NaCl}$ in einen zentralen Venenkatheter durchgeführt. Die Kontrastmittelkinetik von Leberläsionen wurde prä- und postinterventionell während arterieller, portalvenöser und Spätphase analysiert. Die Sondenplatzierung zur Durchführung einer io-RFA erfolgte im Doppelbildmodus mit B-Bild und io-CEUS. Pro Läsion erfolgten bis zu 3 Zyklen über max. 14 min. bei $100-180^{\circ} \mathrm{C}$. Eine avaskuläre Nekrose unmittelbar postinterventionell wurde als vollständige Ablation gewertet. Zur Kontrolle des io-CEUS-Befundes wurden die CT- und MRT-Befunde im 3-Monats-Follow up herangezogen.

Ergebnisse 14 Patienten (im Mittel 60,7 Jahre, 11 männlich, 3 weiblich) mit 20 malignen Leberläsionen $(0,4-3 \mathrm{~cm}$, im Mittel $1,59 \mathrm{~cm})$ wurden in die Studie eingeschlossen. Eine unmittelbar postinterventionelle Kontrolle der ioRFA ergab in 17/ 20 Fällen eine avaskuläre Nekrose nach 1 Zyklus (85\% primäre Erfolgsrate) und in 3 Fällen eine Restvaskularisation, so dass weitere Zyklen bis zum Erreichen einer avaskulären Nekrose angeschlossen wurden. Im Follow up mittels CT und/oder MRT ergaben sich in den behandelten Arealen in keinem Fall Hinweise für einen Tumorrest oder -Rezidiv.

Schlussfolgerungen Intraoperativer CEUS ist eine geeignete und effektive Methode, um eine io-RFA während Lebertumoroperationen zu steuern und eine unmittelbar postinterventionelle Erfolgskontrolle durchzuführen.

Uroradiologie/Urogenitaldiagnostik

\section{Validierung der automatisierten Messung des Diameters und Volumens von Harnsteinen}

Autoren Neubauer $]^{1}$, Benndorf $\mathrm{M}^{1}$, Fritz $\mathrm{B}^{1}$, Kotter $\mathrm{E}^{1}$, Langer $\mathrm{M}^{1}$, Wilhelm $\mathrm{K}^{2}$

Institut 1 Universitätsklinik Freiburg, Radiologie, Freiburg;

2 Universitätsklinik Freiburg, Urologie, Freiburg

DOI 10.1055/s-0037-1602627

Zielsetzung Die Behandlungsmöglichkeiten von Harnsteinen sind in erster Linie abhängig von ihrem Volumen, welches aus der Messung des Diameters in CT Bildern geschätzt werden kann. Eine neue Software ermöglicht die automatisierte Bestimmung des Diameters und des Volumens von Harnsteinen aus CT Bildern. Das Ziel der Studie war eine Validierung der Software.

Material und Methodik Der Diameter und das Volumen von 96 Nieren- und Blasensteine wurden von drei Urologen gemessen. Der Mittelwert der Messungen wurde jeweils als Referenzwert angenommen. Die Steine wurden in einem anthropomorphem Phantom positioniert und in Dual-Energy-Technik mit $100 \mathrm{kV} / \mathrm{Sn} 140 \mathrm{kV}$ in einem 128 Zeilen CT untersucht. Axiale Rekonstruktionen wurden mit $0,75 \mathrm{~mm}$ Schichtdicke und -abstand angefertigt. Drei Ra- 
diologen maßen unabhängig voneinander den maximalen Diameter und das Volumen mittels der Software. Nach einer Pause von mindestens 4 Wochen maßen die Radiologen den Diameter der Steine in den multiplanar rekonstruierten CT Bildern von Hand. Aus diesen Messungen wurde mit der Formel $V=4 / 3^{*} \pi^{*} r 3$ ein zu erwartendes Volumen berechnet. Die Statistik wurde mit Intraclass-, Pearson-Korrelationskoeffizient und Pearson und Filon's Test durchgeführt.

Ergebnisse Die Interrater-Korrelation für die Referenzmessungen betrug 0,99 für Diameter und Volumen. Der mittlere Referenzdiameter betrug $13,3 \mathrm{~mm}$. Das mittlere Referenzvolumen betrug $1,18 \mathrm{ml}$. Die Korrelationen des automatisiert bestimmten Diameters und der radiologischen Messung von Hand mit den Referenzwerten betrugen 0,91 und 0,99 $(p=<0,001)$. Die Korrelationen des automatisiert bestimmten Volumens und des errechneten Volumens mit den Referenzwerten betrugen 0,99 und 0,97 ( $p=<0,001)$.

Schlussfolgerungen Die automatisierte Bestimmung des Harnsteinvolumens aus $C T$ Bildern ist möglich und genauer als volumetrische Berechnungen basierend auf den von Hand gemessenen Diametern. Allerdings ist die automatisierte Messung des Harnsteindiameters nicht so exakt wie die Messung von Hand, die daher weiterhin obligatorisch erscheint.

\section{PIRADS 3 - ist eine Verlaufskontrolle sinnvoll}

Autoren Steinkohl $F^{1}$, Gruber $L^{1}$, Junker $D^{1}$, Aigner $F^{2}$

Institut 1 Medizinische Universität Innsbruck, Department für Radiologie, Innsbruck; 2 Medizinische Universität Innsbruck, Department für Radiologie, DOI 10.1055/s-0037-1602628

Zielsetzung Ziel der Studie war es herauszufinden, wie viele PIRADS-3-Befunde sich in einer Verlaufskontrolle verändern und ob der Radiologe mit längerer Beobachtungszeit leichter eine Entscheidung bezüglich der Dignität treffen kann.

Material und Methodik In diese retrospektive Studie wurden 102 Patienten eingeschlossen, die zwischen 2012 und 2015 eine MRT-Untersuchung der Prostata und eine Verlaufskontrolle hatten. Die erste Untersuchung und die Verlaufskontrolle wurden von demselben Uroradiologen befundet. Die Verlaufskontrolle fand nach $12.3 \pm 5.4$ Monaten $(4.6-38.2)$ statt. Die MRT-Untersuchung wurde nach den gültigen ESUR-Guidelines an einem 3 Tesla Gerät durchgeführt.

Ergebnisse Das Alter betrug 60,8 $\pm 8,4$ Jahre. Die Zeit zwischen den MRTUntersuchungen betrug 12,3 $\pm 5,4$ Monate (4,6 - 38,2 Monate). Der mittlere PSA-Wert bei der ersten MRT-Untersuchung war $6,54 \mathrm{ng} / \mathrm{ml}$, bei der zweiten $7,43 \mathrm{ng} / \mathrm{ml}$. Bei $26,4 \%$ der 102 Patienten kam es zu keiner Änderung des PIRADS in der Verlaufskontrolle. Bei $73,5 \%$ veränderte sich der PIRADS-Befund. 46,1\% konnten auf PIRADS 2 heruntergestuft werden, 27,5\% wurden auf PIRADS 4 heraufgestuft. Reklassifikationen zu PIRADS 4 wurden nach durchschnittlich 10,3 $\pm 3,5$ Monaten vorgenommen, während Reklassifikationen zu PIRADS 2 nach durchschnittlich 13,2 \pm 5,2 Monaten und eine Befundkonstanz mit PIRADS 3 nach durchschnittlich 13,1 \pm ,8 Monaten vorkamen. Schlussfolgerungen Eine Verlaufskontrolle eines PIRADS-3-Befundes ist sinnvoll. Die Daten zeigen, dass es dem Radiologen möglich war, sich in der Verlaufskontrolle in 3/4 der Fälle bezüglich Benignität bzw. Malignität festzulegen. Eine Festlegung auf PIRADS 4 erfolgte durchschnittlich nach kürzerer Zeit als eine Festlegung auf PIRADS 2 oder eine Befundkonstanz. Auch eine längere Zeit zwischen den MRT-Untersuchungen gab dem Radiologen also nicht mehr Sicherheit in seiner Entscheidung. Die Daten legen nahe, dass eine Verlaufskontrolle nach über einem Jahr die Wahrscheinlichkeit für eine Entscheidung bezüglich Malignität oder Benignität nicht erhöht.
Ausbildung und Beruf

\section{Der Langzeiteffekt einer Ultraschallausbildung als Peer Teaching von Studierenden für Studierende im Rahmen eines Basistrainings zum studentischen Ultraschalltutor}

Autoren Engel $A^{1}$, Rösslhuemer $P^{1}$, Sachs $A^{1}$

Institut 1 Medizinische Universität Wien, Universitätsklinik für Radiologie und Nuklearmedizin, Wien

DOI 10.1055/s-0037-1602629

Zielsetzung In Zeiten abnehmender verfügbarer ärztlicher Ressourcen wird es immer schwieriger die Ausbildung von Medizinstudierenden durch ärztliches Personal sicherzustellen. Wir haben im Rahmen eines Basistrainings zum Ultraschalltutor ein Peer Teaching Konzept untersucht und dessen Langzeiteffekte auf die Ultraschall bezogenen Fertigkeiten und das theoretische Wissen der Studierenden erhoben.

Material und Methodik Nach einer durch Studierende geleiteten, 15 Stunden umfassenden, intensiven theoretischen und praktischen Basisausbildung im Ultraschall haben wir das theoretische Wissen und die Ultraschall bezogenen praktischen Fertigkeiten der studentischen Ultraschalltutoren erfasst. In Form eines Fragebogens zu 20 anatomischen Strukturen führten wir die theoretische Evaluation durch. Die Beurteilung der praktischen Fertigkeiten erfolgt mit einem selbstentwickelten 10 Punkte Score für 3 Untersuchungsaufgaben: (1) Rechte Niere, (2) Leber und Gallenblase, (3) Milz. Nach 4 Monaten wurde ein Follow Up nach gleichem Muster durchgeführt.

Ergebnisse Es zeigte sich, dass die Studierenden im Langzeiteffekt von der intensiven Basisausbildung profitieren. Wir beobachteten eine deutliche Steigerung des Kompetenzlevels bei theoretischem Wissen und praktischen Ultraschall Fertigkeiten im Follow Up nach 4 Monaten.

Schlussfolgerungen Im Rahmen eines Peer Teachings können die Grundlagen von Ultrschalluntersuchungen effektiv erlernt werden. Diese Grundlagen ermöglichen den Studierenden eine eigenständige Weiterbildung je nach Interesse, Zugänglichkeit zu Ultraschallgeräten und Fachliteratur. Als Grund für die deutliche Steigerung im 4 Monate Follow Up vermuten wir die freie Zugänglichkeit zu Ultraschallgeräten für unsere Probanden. Ein prägraduelles Peer Teaching im Ultraschall wird eine postgraduelle Ausbildung nie ersetzen, kann jedoch prägraduell anatomisches und physiologisches Lernen effektiver gestalten. Des Weiteren fördert es den sinnvollen Einsatz der diagnostischen Methode, sowohl als zukünftiger Untersucher oder Zuweiser.

\section{Current Radiation Dose Reduction (RDR) Practices of Diagnostic Radiology Technologists in Pakistan}

Authors Syed $A^{1}$, Masood $K^{2}$

Institute 1 Dow University Hospital, Radiology, Karachi; 2 Dow University

hospital, Radiology, Karachi

DOI 10.1055/s-0037-1602630

Zielsetzung Although digital radiography is one of the lowest dose imaging tests, unnecessary radiation exposure is of particular concern. Recent advances in radiation safety practices emphasizes on RDR to prevent unnecessary exposure. „Image gently“ campaign has gained significant momentum in this regard, creating educational and awareness opportunities for parents, patients, and medical professionals. „Image Gently Digital Radiography Safety Checklist" developed by the Alliance for Radiation Safety in Pediatric Imaging, describes the critical steps of digital radiography that, if omitted, could potentially result in harm to the patient. We thus aim to assess current RDR practices of diagnostic technologists at the radiology institutes of Pakistan; and explore determinants of poor practice among them, using „Image Gently“ guidelines.

Material und Methodik A total of 100 radiation technologists employed at several radiology departments of the city were surveyed through structured 
questionnaire using modified "Image Gently Digital Radiography Safety Checklist”. The checklist assessed RDR practices „prior to starting”, „image capture”, "image critique”, and "completion of examination“ through 20 question items. Poor RDR score was used as outcome variable (score $<15=$ Poor, and $\geq 15$ as Good score). Regression analyses were used to find determinants of poor RDR score (the outcome variable) among them. Results are reported as frequency for descriptive characteristics, and Odds ratio with $95 \%$ confidence interval $(\mathrm{Cl})$ for regression analysis.

Ergebnisse Almost half of all participants $(n=46)$ scored poorly on the RDR score. Participants who worked in public sector setups (OR $=5.35,95 \% \mathrm{Cl}$ $(2.26-12.65)$, $p$-value $<0.01)$, and were of the opinion that staff at their institute were overworked $(\mathrm{OR}=3.96,95 \% \mathrm{Cl}(0.44-35.18)$, p-value 0.04$)$; and reported that the management did not promote RDR practices ( $O R=6.61,95 \% \mathrm{Cl}(1.69-25.77)$, p-value $<0.01)$ were more likely to score poorly on the RDR score.

Schlussfolgerungen The results of this study indicate poor RDR practices among Pakistan diagnostic radiology technicians; and the findings may be used to identify areas of weakness, and set goals for improvement planning. The findings highlight the patient radiation safety issue in developing country context.

\section{Nachtrag Poster-Ausstellung (Fortbildung)}

Gastro- und Abdominaldiagnostik

\section{Milz oder Nicht-Milz - das ist die Frage: Stellenwert von MRT und Szintigraphie bei der detektion und Identifizierung dystopen Milzgewebes}

\section{Autoren Grieser $\mathrm{T}^{1}$, Blodow $\mathrm{V}^{2}$}

Institut 1 Klinikum Augsburg, Klinik f. Diagnostische und Interventionelle Radiologie u. Neurorad., Augsburg; 2 Klinikum Augsburg, Nuklearmedizin, Augsburg

DOI 10.1055/s-0037-1602631

Kurzfassung Dystopes Milzgewebe stellt eine diagnostische Herausforderung dar, wenn es sich um onkologische Patienten handelt. Bzgl. überzähligen oder fehlenden Milzgewebes unterscheidet man: 1. Splenunkulose; 2. Splenosis; 3. Polysplenie-Syndrom; 4. Asplenie-Syndrom (Ivemark-Syndrom). Es werden fünf Patienten vorgestellt, bei denen dystopes bzw. überzähliges Milzgewebe z.T. erhebliche diagnostische Probleme in der Abgrenzung zu Tumoren bzw. Metastasen darstellten, welche einerseits durch anamnestische Eruierungen, andererseits durch den Einsatz nuklearmedizinischer Methoden gelöst werden konnten. a) 52-jähriger Holländer mit Oberbauchschmerzen infolge Thrombosen der Pfortader, der V. mesenterica sup und der V. lienalis. Darstellung mehrerer, bis $1,5 \mathrm{~cm}$ großer runder Formationen im Oberbauch, die szintigraphisch als Milzregenerate identifiziert werden konnten (anamn. Z.n. Motorradunfall); b) 50-jähriger Mann mit Zufallsbefund einer ovalen RF im Pankreasschwanzbereich: Vd.a. neuroektodermalen Tumor. 63Ga-DOTATATE Szintigraphie ergab in Kombination mit weiteren Befunden die Diagnose intrapankreatischen ektopen Milzgewebes (Diskussion Spezifität der DOTATATESzintigraphie notwendig); c) 38-jähriger türkischer Patient, der mit einer großen runden RF im linken Mittelbauch auffiel; keine B-Symptomatik etc., milzszintigrapisch eindeutiger Nachweis von Milzgewebe i.S. einer großen Regeneratmilz bei Z.n. Splenektomie als Kind in der Türkei; d) 60-jähriger Mann mit bekanntem Nierenzelltumor: im CT zahlreiche runde Gewebeformationen an atypischen Lokalisationen für LK-Metastasen: Identifizierung als Milzregenerate bei Z.n. Splenektomie; e) 58-jährige Patientin mit einem bekannten malignen Tumorleiden sowie bekannter Splenektomie: vorbekannte runde Nebenmilz, aber weitere Gewebeformationen entsprachen metastatischem Tumorgewebe (FDG-PET-CT) und nicht - wie angenommen - Nebenmilzen.
Lernziele

1. beachte DD Nebenmilzen vs. Tumore oder Metastasen;

2. Ery-Szintigraphie hilfreich;

3. Anamnese beachten.

\section{MR Bildgebung der akuten primären Appendagitis epiploicae}

Autoren Kamusella $\mathrm{P}^{1}$, Heidelbach $\mathrm{M}^{2}$, Früchtnicht $\mathrm{S}^{1}$

Institut 1 Röntgenpraxis Heide, Heide; 2 Röntgenpraxis Heide, Heide SH DOI 10.1055/s-0037-1602632

Kurzfassung Die Appenagitis epiploicae ist eine seltene, selbstlimitierende, jedoch klinisch schwierig zu diagnostizierende Erkrankung. Es handelt sich um eine Ischämie einer der Appendices epiploicae des Colons. Zu unterscheiden ist eine primäre und eine sekundäre Form. Bei der primären Form findet sich keine andere Ursache für die Fettanhängseltorsion oder spontane Thrombosierung der drainierenden Vene. Klinisch präsentieren die Patienten einen plötzlichen abdominellen Schmerz, selten einhergehend mit Übelkeit und Erbrechen. In der Blutanalyse können die Entzündungszeichen leicht erhöht sein. Um invasive therapeutische Maßnahmen zu verhindern ist eine optimale Bildgebung und dezidierte Bildanalyse notwendig. In der Notfalldiagnostik kommen in der Regel die Sonographie und die Computertomographie zum Einsatz. Die Magnetresonanztomographie (MRT) sollte insbesondere beim jungen Patienten und bei nicht wegweisendem sonographischen Befund eingesetzt werden und liefert weitere Charakteristika, die zur Diagnosefindung beitragen. Für die Appendagitis epiploicae typische Befunde in der MR Bildgebung sind eine oval geformte Läsion (bis zu $5 \mathrm{~cm}$ ) unmittelbar am Colon angrenzend ohne relevante Beteiligung der Darmwand, im T2 und T1 gewichteten Bild mit hyperintensem Zentrum und einem hypointensem Saum, im T1 gewichteten Bild weniger signalreich im Vergleich zum umgebenden Fettgewebe. In den fettsuppremierten Sequenzen zeigt sich eine typische Fettsättigung der Läsion. In der Diffusionsbildgebung findet sich kein Zeichen einer Restriktion. Nach intravenöser Kontrastmittelapplikation lässt sich ein geringes Enhancement im Randbereich nachweisen.

Lernziele Ziel muss es sein, die Charakteristika einer Appendagitis epiploicae zu erkennen, um diese von schwerwiegenden Erkrankungen differenzieren zu können. Mit der MR Bildgebung steht eine Methode zur Verfügung, die bei unklarem Sonographiebefund, insbesondere für den jungen Patienten auch im Notfall eine präzise Diagnosefindung erlaubt.

\section{CT-Diagnostik der akuten Appendizitis und ihrer Komplikationen}

Autoren Nell C $C^{1}$, Kühn J $]^{1}$, Partecke $L^{2}$, Hosten N ${ }^{1}$, Mensel B Institut 1 Universitätsmedizin Greifswald, Institut für Diagnostische Radiologie und Neuroradiologie, Greifswald; 2 Universitätsmedizin Greifswald, Klinik und Poliklinik für Allgemeine Chirurgie, Viszeral-, Thorax- und

Gefäßchirurgie, Greifswald

DOI 10.1055/s-0037-1602633

Kurzfassung Die akute Appendizitis gehört zu den häufigsten Diagnosen in der chirurgischen Notaufnahme und führt in vielen Fällen zu einer notfallmäßigen Laparoskopie oder Laparotomie. Während ca. zwei Drittel der Fälle klinisch-anamnestisch sicher zu diagnostizieren sind, stellen sich in ca. einem Drittel die körperliche Untersuchung und Anamnese inkonkludent dar. Die Standardbildgebung ist dann die Abdomensonographie, diese kann allerdings nicht immer die Verdachtsdiagnose bestätigen bzw. sicher ausschließen. In diesen Fällen sowie bei vermuteten Komplikationen (z. B. perityphlitischer Abszess, Perforation) können vor einer Operation Schnittbildverfahren die Diagnose stützen bzw. Differentialdiagnosen oder Komplikationen nachweisen. Die CT-Diagnostik des Abdomens bei der akuten Appendizitis bietet eine exzellente Sensitivität und Spezifität. Das Poster zeigt neben den typischen 
bildmorphologischen Befunden einer akuten Appendizitis auch atypische Befunde und häufige Differentialdiagnosen.

Lernziele Nach Studium des Posters soll ein/e Weiterbildungsassistent/in der Radiologie in der Lage sein, die Indikation für die CT-Diagnostik bei der Frage nach akuter Appendizitis zu stellen, die richtige Untersuchungstechnik anzuwenden, typische und atypische Befunde einer Appendizitis zu erkennen und schließlich von wichtigen Differentialdiagnosen abzugrenzen. Außerdem sollen die Möglichkeiten einer alternativen Bildgebung sowie der CT-gestützte Therapie des perityphlitischen Abszesses dargestellt werden.

Herzdiagnostik/Gefäßdiagnostik

\section{Ungewöhnlicher Fall einer Malformation der Vena cava inferior mit Ektasie, Umgehungskreisläufen und partieller Thrombosierung}

\author{
Autoren Schramm D ${ }^{1}$, Dober $]^{2}$ \\ Institut 1 Universitätsklinikum Halle, Diagnostische Radiologie, Halle; \\ 2 Universitätsklinik und Poliklinik für Radiologie Halle, Diagnostische \\ Radiologie, Halle \\ DOI 10.1055/s-0037-1602634
}

Kurzfassung Wir berichten über einen ungewöhnlichen Fall eines aufgrund plötzlich einsetzender Rückenschmerzen in unserer ZNA vorstellig gewordenen jungen Patienten mit erhöhtem CRP, D-Dimeren, Leberwerten und Leukozytose. Der Patient hatte keine Vorerkrankungen. Zum Ausschluss eines Aneurysmas, einer Dissektion und eines Infektfokus wurde eine EKG-getriggerte Angio-Computertomtographie von Thorax, Abdomen und Becken durchgeführt, in der sich eindrucksvolle Bilder einer Malformation der Vena cava inferior mit Ektasie, Umgehungskreisläufen und partieller Thrombosierung zeigten. Die Differentialdiagnosen zu diesem Krankheitsbild sind vielfältig. Fehlbildungen der V. cava inferior stellen mit einer Prävalenz von etwa $1 \%$ der Allgemeinbevölkerung eine seltene Ursache für Thrombosen der unteren Extremität bzw. der V. cava inferior und Iliakalvenen dar. Gerade bei jüngeren Patienten ohne Vorerkrankungen wie im vorliegenden Fall sollte diese Möglichkeit aber nicht außer Acht gelassen werden, da sie hier in etwa 5\% der Fälle beobachtet werden kann. Trotz zum Teil ausgeprägter Kollateralen zur V. cava superior über das Azygos-System und die oberflächlichen epigastrischen Venen sowie kavoportaler Kollateralen über die rektalen Venen des Plexus haemorrhoidalis, begünstigt ein insuffizienter venöser Rückstrom die Entstehung von Thromben. Therapeutisch kommen die kathetergestütze Thrombolyse und lebenslange systemische Antikoagulation in Betracht.

Lernziele Mit diesem eindrucksvollen Fall möchten wir auf das im radiologischen Alltag ungewöhnliche aber klinisch bedeutsame Krankheitsbild einer Malformation der Vena cava inferior aufmerksam machen. Neben den vielfältigen Differentialdiagnosen (die jeder Radiologe kennen sollte) werden auch alle notwendigen radiologischen bildgebenden Verfahren zur Abklärung dieser Gefäßpathologie aufgezeigt.

\section{Verlaufskontrolle nach TAVI-Intervention mittels Multidetektor-CT: technische Durchführung, reguläre Befunde und typische Komplikationen}

\footnotetext{
Autoren Soschynski $\mathrm{M}^{1}$, Capilli $\mathrm{F}^{1}$, Ruile $\mathrm{P}^{2}$, Langer $\mathrm{M}^{3}$, Krauss $\mathrm{T}^{1}$ Institut 1 Klinik für Radiologie, Universitätsklinikum Freiburg, Sektion Kardiovaskuläre Radiologie, Freiburg; 2 Universitäts-Herzzentrum Freiburg Bad Krozingen, Klinik für Kardiologie und Angiologie II, Bad Krozingen; 3 Klinik für Radiologie, Universitätsklinikum Freiburg, Ärztlicher Direktor, Freiburg DOI 10.1055/s-0037-1602635

Kurzfassung Die Transkatheter-Aortenklappen-Implantation (TAVI) hat sich in den letzten Jahren als Alternative für Patienten mit hohem Operationsrisiko etabliert. Trotz technischer Optimierungen und verbessertem Patientenscreening besteht immer noch ein Risiko für Komplikation während und nach
}

dem Eingriff. Die EKG-getriggerte Multidetektor-CT (MDCT) ist essentiell für die prä-interventionelle Planung zur Auswahl der korrekten Prothese und des Zugangsweges. Für die post-interventionelle Kontrolle der Klappenprothese spielt die MDCT eine wichtige Rolle in der frühzeitigen Diagnostik von Komplikationen. Die Darstellung der Klappenprothese über den gesamten Herzzyklus erlaubt eine Funktionsanalyse der bewegten Klappe ähnlich der Echokardiographie, welche dem CT in der morphologischen Diagnostik der stentgetragenen Klappe zum Ausschluss einer Thrombose unterlegen ist. Das Fortbildungsposter erklärt die technisch korrekte Durchführung der EKG-getriggerten MDCT zur Kontrolle nach TAVI-Implantation. Anhand mehrerer Fallbeispiele werden post-interventionelle Normalbefunde verschiedener Klappentypen sowie klassische und seltenere Komplikationen illustriert.

Lernziele

- Technische Durchführung der Untersuchung einer TAVI-Klappenprothese mittels EKG-getriggerter MDCT.

- Step-by-step Befundung.

- Normalbefunde und Erkennen von Komplikationen.

\section{Interventionelle Radiologie}

\section{CT-Diagnostik und interventionelle Therapieoptionen von Endoleaks nach EVAR}

Autoren Bremicker $\mathrm{K}^{1}$, Maiwald $\mathrm{B}^{1}$, Fuchs $\mathrm{J}^{1}$, Moche $\mathrm{M}^{1}$, Kahn $\mathrm{T}^{1}$, Petersen $\mathrm{T}^{1}$ Institut 1 Universitätsklinikum Leipzig, Klinik und Poliklinik für Diagnostische und Interventionelle Radiologie, Leipzig

DOI 10.1055/s-0037-1602636

Kurzfassung Die Mehrheit abdomineller Aortenaneurysmen werden mittlerweile endovasculär mittels Stentgraft-Implantation (EVAR) therapiert. Bei bis zu 44\% der EVAR besteht eine persitierende Perfusion des Aneurysmasacks (Endoleak), welche zum weiteren Aneurysmawachstum mit Stentgraftdislokation oder Ruptur führen kann. Die Diagnostik erfolgt mittels kontrastmittelgestütztem Ultraschall (CEUS), mehrphasiger Computertomographie (CT) oder Magnetresonanztomographie (MRT). Neben der Detektion ist aufgrund unterschiedlicher therapeutischer Konsequenzen vor allem die genaue Klassifikation der Endoleaks entscheidend. Endoleaks Typ I und III zeichnen sich durch direkte Übertragung des arteriellen Drucks auf die Aneurysmawand aus und sind meist mit deutlicher Größenzunahme und erhöhtem Rupturrisiko assoziiert. Sie bedürfen daher dringlicher interventioneller Therapie z. B. mittels perkutaner transluminaler Angioplastie (PTA), Verlängerung des Stents oder Implantation eines Cuffs. Typ II Endoleaks sind mit bis zu 30\% am häufigsten und resultieren aus einer Flussumkehr in den Lumbalarterien oder der Arteria mesenterica inferior (AMI). Interventionelle Therapie ist wegen des harmloseren Verlaufs nur bei weiterer Größenzunahme des Aneurysmasacks notwendig, dann aber sehr komplex und aufwendig. Ursächliche Gefäße können mittels Sondierung über Kollateralen (z. B. Riolan-Anastomose) oder über eine Sondierung des Aneurysmasacks mit einem Katheter zwischen Stentgraft und Aneurysmawand gezielt embolisiert werden. Auch eine perkutane Direktpunktion des perfundierten Aneurysmasacks mit anschließender Embolisation ist möglich. Endoleaks Typ IV und V sind mit den aktuellen Stentgrafts zu Raritäten geworden und bedürfen gleichfalls nur einer Therapie bei GröBenprogess des Aneurysmasacks.

Lernziele

- Klassifikation der Endoleaks (Typ I-V) und Kenntnis der Dringlichkeit der Endoleak-Behandlung abhängig vom Typ

- Darstellung interventioneller Therapieoptionen der Endoleak-Typen mit Bildbeispielen (CT/Angiographie) 


\section{Tricks und Tipps für schwierige abdominelle Biopsien}

Autoren Kettenbach J ${ }^{1}$

Institut 1 Universitätsklinikum St. Pölten, Klinisches Institut für Medizinische Radiologie, Diagnostik, Intervention, St. Pölten

DOI 10.1055/s-0037-1602637

Kurzfassung Darstellung alternativer Ansätze zur Biopsie von Abdominalorganen wie Leber, Milz, Magen, Pankreas und Intestineum, wenn deren Läsionen aufgrund ihrer Lage oder Unzugänglichkeit üblicherweise nicht zur Biopsie geeignet sind. Dazu zählen: (a) eine adäquate Auswahl der Bildmodalität zur Nadelführung; (b) adäquate Patientenlagerung; (c) geeignete Wahl des perkutanen Zuganges; (d) Verlagerung interponierter Strukturen durch Insufflation oder Instillation; (e) Versiegelung des Stichkanals.

Lernziele

1. Kennenlernen alternativer Zugangswege sowie Punktionstechniken für methodisch schwierige Biopsien im Abdominalbereich.

2. Tricks und Tipps zur Vermeidung klinisch relevanter Komplikationen.

\section{Kinderradiologie}

\section{Neuroendocrine Cell Hyperplasia of Infancy (NEHI) - Charakteristische Zeichen in der CT-Bildgebung}

Autoren Beeskow $A^{1}$, Roth $C^{1}$, Prenzel $F^{2}$, Hirsch $F^{1}$

Institut 1 Universitätsklinikum Leipzig, Abteilung für Kinderradiologie,

Leipzig; 2 Universitätsklinikum Leipzig, Klinik und Poliklinik für Kinder- und

Jugendmedizin, Leipzig

DOI 10.1055/s-0037-1602638

Kurzfassung Die neuroendokrine Zellhyperplasie (NEHI) ist eine erstmalig 2005 beschriebene Erkrankung des frühen Kindesalters aus der Gruppe der diffusen parenchymatösen Lungenerkrankungen (DLPD). Sie geht mit einer Proliferation von neuroendokrinen Zellen im Epithel der distalen Atemwege einher, deren Ursache bislang ungeklärt ist. Sie hat eine gute Prognose und einen selbstlimitierenden Verlauf im Alter von 18-24 Monaten. Daher ist es wichtig, sie von anderen, progredient verlaufenden diffusen parenchymatösen Lungenerkrankungen, wie der pulmonalen interstitiellen Glykogenose und der lymphofollikulären Bronchiolitis, abzugrenzen. Sie geht mit einer typischen Klinik in Form von Tachypnoe, Knisterrasseln, persistierenden Einziehungen und Hypoxämie einher. In der Computertomographie lassen sich charakteristische Befunde nachweisen. Das Hauptkriterium ist eine landkartenartige Milchglasverschattung in mindestens vier Lungenlappen, vornehmlich im Mittellappen und der Lingula sowie geographische Air Trapping-Phänomene. Weitere Pathologien in der CT sind selten. In Zusammenschau von typischer Klinik und der charakteristischen Bildgebung kann die Diagnose einer NEHI gestellt werde. Daher ist es für den Radiologen wichtig, die charakteristischen Befunde in der Computertomographie zu kennen.

Lernziele Ziel ist das sichere Identifizieren der NEHI-charakteristischen Veränderungen in der Bildgebung mit Milchglasverschattung, insbesondere im Mittellappen und der Lingula sowie geographische Air Trapping-Phänomene. Die NEHI soll anderen pädiatrischen Krankheitsentitäten aus der Gruppe der interstitiellen Lungenerkrankungen bildmorphologisch gegenübergestellt und von ihnen abgegrenzt werden.

\section{Fallbericht einer Hirnstamm-Diskonnektion: Schwierigkeiten und Lösungsansätze der Diagnosestellung mittels MRT}

Autoren Leicht $\mathrm{J}^{1}$, Bock $\mathrm{C}^{1}$, Rüdiger $\mathrm{S}^{2}$, Stock $\mathrm{S}^{3}$, Stock $\mathrm{K}^{1}$ Institut 1 Städtisches Klinikum Dessau, Klinik für Diagnostische und Interventionelle Radiologie und Neuroradiologie, Dessau-Roßlau; 2 Universitätsklinikum Halle (Saale), Institut für Anatomie und Zellbiologie,
Halle; 3 Krankenhaus St. Elisabeth und St. Barbara Halle, Praxis für Radiologie, Halle

DOI 10.1055/s-0037-1602639

Kurzfassung Hirnstamm-Diskonnektion (engl. Brainstem Disconnection) ist die Bezeichnung für eine extrem seltene angeborene Fehlbildung, die mit schweren psychomotorischen Behinderungen verbunden ist. Die Ursachen der Hirnstamm-Diskonnektion sind weiterhin unklar. Lediglich 14 Fallberichte wurden bis zum Oktober 2016 gemacht. Die MRT liefert wertvolle Hinweise für die Diagnose und Differentialdiagnose der Brainstem Disconnection sowie anderer Fehlbildungen von Rhomb- und Mesenzephalon. Insbesondere hochauflösende 3D-Sequenzen (z. B. 3D-CISS) können die genaue Morphologie des pathologisch veränderten Hirnstamms aufzeigen. In unserem Fall war noch eine dünne strangartige Verbindung zwischen Hirnstamm und Medulla oblongata nachweisbar und nicht, wie in früheren Fallberichten, eine völlig fehlende Verbindung.

Lernziele Diagnose und Differentialdiagnose von Entwicklungsstörungen der hinteren Schädelgrube am Beispiel der BD Vorteile hochauflösender 3D-Sequenzen zur genauen Darstellung der pathologischen Anatomie

Muskuloskelettale Radiologie

\section{Muskuloskelettale MRT bei Kindern}

Autoren Harth $\mathrm{S}^{1}$, Roller $\mathrm{F}^{1}$, Schneider $\mathrm{C}^{1}$, Krombach $\mathrm{G}^{1}$ Institut 1 Universitätsklinikum Gießen des UKGM, Klinik für Diagnostische und Interventionelle Radiologie, Gießen

DOI 10.1055/s-0037-1602640

Kurzfassung Die muskuloskelettale MRT bei Kindern erfordert angepasste Untersuchungsprotokolle unter Berücksichtigung eines kindgerechten Ablaufes und spezieller Risiken. So ist häufig eine Minimierung der Untersuchungszeit sinnvoll, ferner müssen die auf das Körpergewicht bezogen größere Körperoberfläche und die Unreife der Thermoregulation bei Neugeborenen und Säuglingen bedacht werden. Die bei kleinen Kindern häufig unspezifische Klinik und die damit unspezifischen klinischen Angaben führen außerdem zu erschwerten Bedingungen. Für die Untersuchungsbefundung sind Kenntnisse über typische pädiatrische Erkrankungsbilder sowie die normale Skelettentwicklung und deren Erscheinungsbild in der MRT nötig. Insbesondere die Beurteilung des Knochenmarks sowie der Epiphysen und Apophysen kann Anlass für Interpretationsschwierigkeiten bieten. Es werden wichtige Fälle aus der pädiatrischen muskuloskelettalen MRT vorgestellt zu Normvarianten in der Skelettentwicklung sowie zu pädiatrischen Krankheitsbildern aus den Bereichen Traumatologie (z. B. Epiphysenverletzungen), infektiöse und inflammatorische Erkrankungen (z.B. Osteomyelitis), Weichteiltumoren und Knochentumoren (z.B. Osteosarkom, Ewing-Sarkom) mit typischen Bildbefunden sowie kurzen Erläuterungen zu Klinik und MRT-Indikationen bzw. MRT-Protokollen.

Lernziele Kenntnisse über wichtige Besonderheiten der muskuloskelettalen MRT bei Kindern von der Untersuchungsplanung bis zur Befundung.

\section{Maligne und benigne Läsionen der skelettalen Muskulatur}

Autoren Leifels $L^{1}$, Kahn $T^{1}$, Surov $A^{1}$

Institut 1 Klinik und Poliklinik für Diagnostische und Interventionelle

Radiologie, Leipzig

DOI 10.1055/s-0037-1602641

Kurzfassung Es gibt eine Vielzahl an Tumoren und tumorartigen Veränderungen mit unterschiedlichem biologischem Verhalten, die die Skelettmuskulatur befallen und sich unterschiedlich manifestieren (solide, liquide/semiliquide, fettäquivalent), diffuse Muskelauftreibungen und Muskelkalzifikationen. Darüber hinaus gibt es Läsionen mit Mischverhalten der o.g Veränderungen. Benigne und maligne Veränderungen können die gleichen radiologischen Muster 
aufweisen, warum eine sichere Differenzierung häufig schwierig ist. Benigne Tumoren der skelettalen Muskulatur sind viel seltener als maligne Tumoren. Eine systematische radiologische Beschreibung und Einordnung bzgl. Ätiologie/Dignität ist notwendig um konsekutive Probeentnahmen empfehlen bzw. verhindern zu können. Radiologisch lassen sich folgende Muster differenzieren: Intramuskuläre Raumforderungen -solide -maligne (Metatase, Sarkome) -benigne (fokale Myositis, Elastofibrom, Aneurysmata intramuskulärer Gefäße, Neurofibrom, Neurom, Hämatom) -liquide/semiliquide -maligne (Metastase, Sarkome) -benigne (Abszess, intramuskuläres Myxom, synoviale Zyste) -lipomatös -maligne (Liposarkom) -benigne (Lipom, Lipoblastom, Hibernom, Myositis) Diffuse Muskelauftreibungen -maligne (Lymphom, Sarkome, Metastase) -benigne (Myositis) Muskelkalzifikationen -maligne (Metastase (vor allem unter systemischer Therapie), Sarkome) -benigne (kalzifizierte Myonekrose, Myositis ossificans) Mischmuster der o.g. Veränderungen -maligne (Sarkome) -benigne (Hämangiome)

Lernziele Es gibt eine große Vielzahl muskuloskelettaler Tumoren unterschiedlicher Dignität. Verschiedene maligne und benigne muskuläre Läsionen können sich mit identischen radiologischen Mustern präsentieren. Mit Ausnahme einiger gutartiger Weichteil-Läsionen (z. B. Lipom), lässt sich nicht sicher zwischen gutartigen und bösartigen Tumoren unterscheiden. Somit ist eine histologische Sicherung in vielen klinischen Konstellationen notwendig, vor allem bei Nachweis malignitätssuspekter Zeichen.

\section{Radiologische Konstellationen beim Kippel-Feil-Syndrom}

Autoren Reichardt $B^{1}$, Pätzholz $S^{2}$, Prof. Dr. med, Nicolas $V^{2}$ Institut 1 BG Universitätskrankenhaus Bergmannshein Bochum, Institut für diagnostische und Interventionelle Radiologie und Nuklearmedizin, Bochum; 2 BG Universitätsklinikum Bergmanssheil, Institut für Diagnostische Radiologie, , Interv. Radiologie und Nuklearmedizin, Bochum DOI 10.1055/s-0037-1602642

Kurzfassung Das Klippel-Feil-Syndrom ist eine seltene Erkrankung, welche 1912 erstmalig beschrieben wurde. Charakteristisch ist bei diesem kongenitalen Defekt ein tiefer Haaransatz und die Formation oder Segmentation des HWS mit konsekutiver Verkürzung des Nackens mit eingeschränkter Beweglichkeit der HWS. Das Klippel-Feil-Syndrom geht mit einer hohen Anzahl an assoziierten Varianten anderer Organsysteme einher. Diese werden in dieser Übersicht an unserem Patientengut beispielhaft aufgeführt. Assoziierte Defomitäten des Skelettsystems sind die Sprengel Deformität, SKoliose, die Spina bifida, Rippen- und Nierenveränderungen, Spina bifida, Lippen-Kiefer-Gaumenspalte und Minderwuchs, Oligo- und Hypodentie, Herzfehler. Eine Übersicht mit Röntgen und CT.

Lernziele Die seltene Erkrankung des Klippel-Feil-Syndroms erkennen und assozierte Deformitäten des Skelettsystems und der Organe im Kontext erkennen können.

\section{Neuroradiologie}

\section{Intraspinale Blutungen in der Schnittbildgebung}

Autoren Lobsien $D^{1}$, Hoffmann $\mathrm{K}^{1}$, Fahnert $\mathrm{J}^{2}$

Institut 1 Universitätklinikum Leipzig, Neuroradiologie, Leipzig;

2 Universitätsklinikum Leipzig, Klinik und Poliklinik für Diagnostische und

Interventionelle Radiologie, Leipzig

DOI 10.1055/s-0037-1602643

Kurzfassung Intraspinale Blutungen sind seltene Erkrankungen, ihre klinische Symptomatik ist oft uneindeutig, die korrekte Diagnose ist von hoher Relevanz. Da die initiale diagnostische Modalität in diesen Situationen meist die MRT der Wirbelsäule darstellt, ist es wichtig intraspinale Blutungen gegenüber anderen deutlich häufigeren Diagnosen, die eine ähnliche Symptomatik hervorrufen, abzugrenzen. Gezeigt werden fallbasiert Beispiele aus der MRT und
CT Schnittbildgebung an Hand derer die verschiedenen Blutungstypen verdeutlicht werden, mit einem deutlichen Schwerpunkt in der MRT Diagnostik. Lernziele Gewinnen eines Überblicks über die intraspinalen Blutungen und ihre Diagnostik in der Schnittbildgebung

\section{Neuroradiologische Diagnostik und Therapie bei spontanem Liquorverlustsyndrom}

Autoren Neumann $A^{1}$, Helmchen $C^{2}$, Eckey $T^{1}$, Kemmling $A^{1}$, Schramm $P^{1}$ Institut 1 UKSH, Campus Lübeck, Institut für Neuroradiologie, Lübeck; 2 UKSH, Campus Lübeck, Klinik für Neurologie, Lübeck

DOI 10.1055/s-0037-1602644

Kurzfassung Ein spontanes Liquorverlustsyndrom sollte klinisch Differentialdiagnose des länger andauernden, insbesondere positionalen Kopfschmerzes sein. Betroffen sind vor allem Patienten mittleren Alters. Hauptlokalisation für den spontanen Liquorverlust stellen die Nervenscheiden des zervikothorakalen Überganges dar. Ätiologisch abzugrenzen ist der iatrogene Liquorverlust nach Punktionen oder chirurgischer Duraeröffnung. Beim spontanen Liquorverlust sind Mechanismus und Prädispositionen letztlich unklar, vorrangig werden Insuffizienzen des Bindegewebes diskutiert. Die neuroradiologische Diagnostik des spontanen Liquorverlustsyndroms erfolgt üblicherweise im MRT, evtl. auch unter Zuhilfenahme einer intrathekalen KM-Gabe. Sichtbar sind zum einen lokale Veränderungen an der Wirbelsäule mit pathologischem Liquoraustritt unmittelbar epidural bzw. perineural, aber auch in den angrenzenden Weichteilen, zum anderen intrakranielle Befunde in Form entlastungsbedingter Hygrome und mechanisch bedingter Duraverdickungen mit KM-Enhancement. Im diagnostischen Algorithmus des spontanen Liquorverlustsyndroms kommt dem Verständnis des schmerzlosen Lokalbefundes an der Wirbelsäule als Ursache und des intrakraniellen Befundes als dessen Folge und Korrelat der Kopfschmerzen besondere Bedeutung zu. Die Therapie des spontanen Liquorverlustsyndroms erfolgt in der Regel zunächst medikamentös mit Koffein, ggf. kommen ein bildgesteuerter Eigenblutpatch und die lokale Anwendung von Fibrinkleber zur Anwendung.

Lernziele Kenntnis oder Weiterentwicklung der Pfade neuroradiologischer Diagnostik und Therapie bei spontanem Liquorverlustsyndrom.

\section{Meningeale Blutungsquellen als Ursache der Superfiziellen Siderose}

Autoren Neumann $A^{1}$, Eckey $T^{1}$, Kemmling $A^{1}$, Schramm $P^{1}$ Institut 1 UKSH, Campus Lübeck, Institut für Neuroradiologie, Lübeck DOI 10.1055/s-0037-1602645

Kurzfassung Die Superfizielle Siderose ist ein klinisch und bildmorphologisch mannigfaltiges Krankheitsbild, häufig zu sehen bei cerebraler Amyloidangiopathie. Weniger Beachtung finden derzeit noch Zusammenhänge dieser Entität mit pathologischen Veränderungen der Meningen wie Zysten, Divertikeln oder Duplikaturen sowie Herniationen. Diese sind potentiell als Blutungsquelle anzusehen, wobei wiederum hiefür ein primärer Liquorverlust als Ursache diskutiert wird. Die betroffenen Patienten können Symptome wie einen durch Hirnnervenaffektion bedingten progredienten Hörverlust oder eine Ataxie aufweisen, welche primär häufig fehlgedeutet werden. Bildgebendes Verfahren der Wahl bei Superfizieller Siderose ist die MRT mit intrakraniell und ggf. auch spinal der Darstellung von flächigen subpialen Hämosiderinablagerungen in Form von Signalminderungen in T2- bzw. suszeptibilitätsgewichteten Sequenzen. An der spinalen Achse finden sich zudem mitunter sehr subtile Veränderungen der oder angrenzend an die Dura, deren Deutung als Blutungsquelle eine genaue Kenntnis der pathophysiologischen Zusammenhänge und Übung verlangt. Die frühzeitige Detektion einer Blutungsquelle kann wichtig für die Indikationstellung zur chirurgischen Sanierung sein.

Lernziele Verständnis der komplexen pathophysiologischen Zusammenhänge zwischen spinalen Blutungsquellen und Superfizieller Siderose sowie deren Abbildung im craniellen und spinalen MRT. 


\section{Steady State-Bildgebung der Syringomyelie}

Autoren Reichardt B ${ }^{1}$, Prof. Dr. med. Nicolas V²

Institut 1 BG Universitätskrankenhaus Bergmannshein Bochum, Institut für diagnostische und Interventionelle Radiologie und Nuklearmedizin, Bochum;

2 BG Universitätsklinikum Bergmanssheil, Institut für Diagnostische

Radiologie, , Interv. Radiologie und Nuklearmedizin, Bochum

DOI 10.1055/s-0037-1602646

Kurzfassung Die Darstellung der Pathophysiologie und Struktur intraspinaler Defekte wie die Syringomyelie unterliegen bei der Wahl T2 gewichteter Sequenzen in der MRT Diagnostik Problemen im Auffinden von subarachnoidalen Gewebegrenzen und Septierungen. 50 Patienten mit bekannter Syringomyelie an 2 Institutionen mit Neurotraumatologischen Versorgungszentren und Paraplegiezentren wurden prospektiv einer kompletten neurologischen Untersuchung im Rahmen von Verlaufskontrollen und bei Symptomverschlechterung analysiert. Das Untersuchungsprotokoll umfasste im Routineprogramm T2 Flair, T1 und T2 gewichtete Sequenzen in koronarer, sagittaler und transversaler Akquisationsrichtung. Zusätzlich wurden 3D- CISS Sequenzen in sagittaler Richtung angefertigt. Die Auswertung umfasste die Analyse von Liquorflussartefakten, das quantitative und qualitative Verhältnis von MyeIon und Liquorkontrast und Delinetion sowie die Bewegungsartefakte. Ergebnisse: Die 3D-CISS Sequenz zeigte ein signifikant höheres Contrast to noise Verhältnis mit höherer Detailgenauigkeit im Vergleich zu den Standartsequenzen bezüglich der Ausdehnung und der subarachnoidalen Septierungen als in der T2 bei weniger Flussartefakten. Limitationen waren die Artefaktanfälligkeit bei Bewegungen und die verlängerte Untersuchungszeit von ca: 7 min.

Lernziele CISS MR Sequenzen erlauben eine höhere Genauigkeit in der Beurteilung der Syrinxmorphologie und der diagnostischen Aussagekraft intrasyringealer subarachnoidaler Septen und ermöglichen die Identifizierung von operationswürdigen bzw. interventionsbedürftigen Liqourpolstern.

Thoraxradiologie

\section{Unilaterale Agenesie der Arteria pulmonalis als seltene Differenzialdiagnose - akut einsetzender Hämoptysen - Interventionell-endovaskuläre Therapie und alternative Behandlungsoptionen}

Autoren Rückner D ${ }^{1}$, Petermann, Dr. med. $C^{2}$, Burmeister, PD. Dr. med. $\mathrm{H}^{3}$, Gross-Fengels, Prof. Dr. med. W ${ }^{3}$

Institut 1 AK Hamburg-Harburg, Diagnostische und Interventionelle

Radiologie, Hamburg; 2 AK Hamburg-Harburg, Lungenheilkunde

Thoraxzentrum, Hamburg; 3 AK Hamburg-Harburg, Diagnostische und

Interventionelle Radiologie, Hamburg

DOI 10.1055/s-0037-1602647

Kurzfassung Die unilaterale Agenesie der Arteria pulmonalis (UAPA) ist eine seltene kongenitale Anomalie, welche durch eine Malformation des sechsten Kiemenbogens während der Embryogenese entsteht. Die UAPA ist häufig mit kardialen Fehlbildungen, wie der Fallot-Tetralogie, Septumdefekten und einem persistierenden Ductus arteriosus vergesellschaftet. Sie kann jedoch insbesondere bei der selteneren isolierten Form (IUAPA) bis ins hohe Alter unentdeckt bleiben. Die Leitsymptome können Dyspnoe, rezidivierende pulmonale Infekte und unspezifische Brustschmerzen sein. Schwere Hämoptysen (in bis zu 20\% d.F.) sind eine seltene, jedoch lebensbedohliche Komplikation der IUAPA. In unserer Fallvorstellung berichten wir über einen 73-Jährigen männlichen Patienten mit akut aufgetretenen schweren Hämoptysen bei IUAPA sowie deren erfolgreiche endovaskuläre Behandlung mittels Coil- und ergänzender Partikelembolisation. Klinische relevante alternative Behandlungkonzepte von Komplikationen der IUAPA werden diskutiert.

Lernziele

- Inzidenz, klinische Relevanz, Komplikationen und Differentialdiagnosen der isolierter unilateraler Agenesie der Arteria pulmonalis (IUAPA).
- Diagnosemöglichkeiten bei IUAPA.

- Erfolgreiche Therapie lebensbedrohlicher Hämoptysen bei IUAPA anhand des vorliegenden Fallbeispiels und alternative Therapieoptionen.

Notfalldiagnostik/Intensivmedizin

\section{Darstellung des Stichkanals mittels Kontrastmittel bei penetrierenden Verletzungen im CT bei fraglicher Eröffnung von Körperhöhlen}

Autoren Reichardt B ${ }^{1}$, Prof. Dr. med. Nicolas $\mathrm{V}^{2}$ Institut 1 BG Universitätskrankenhaus Bergmannshein Bochum, Institut für diagnostische und Interventionelle Radiologie und Nuklearmedizin, Bochum; 2 BG Universitätsklinikum Bergmannsheil Bochum, Diagnostische und Interventionelle Radiologie und Nuklearmedizin, Bochum DOI 10.1055/s-0037-1602648

Kurzfassung Stichverletzungen am Bauch und Thorax ziehen bei oberflächlichen Weichteilverletzungen eine explorative Eröffnung des Abdomens bzw. des Thorax nach sich. Bei grenzwertigen Befunden, unbekannter Eindringtiefe des penetrierenden Objektes ist eine Darstellung des Stichkanals mit Kontrastmittel - angelehnt an eine Fisteldarstellung - möglich und können somit eine explorative Operation vermeidbar machen. Patienten mit Penetrationsverletzungen am Hals, Thorax und Abdomen wurden nach initialen Trauma CT-Scan bei fraglicher Durchstoßung der inneren Bauchwand und der Pleura erneut auf Läsionshöhe nach Injektion von Kontrastmittel mittels Knopfkanüle im CT eruiert. Eingeschlossen wurden 9 Patienten mit Stichverletzungen, 1 Patient mit Penetrationsverletzung durch ein Amierungseisen sowie 1 Patient mit einem großen Holzspan. Insgesamt wurden 21 Stichwunden versorgt, wobei $13(N=13)$ eine fragliche Eröffnung der Körperhöle vorwiesen. Kontrastmittel breitete sich entlang der Bauchwand dorsal epifaszial der Muskulatur in 8 Fällen aus ohne KM-Übertritt in den Pleuraspalt, ins Mediastinum oder nach intraperitoneal. Bei einer Stichverletzung war aufgrund der Lage (laterodorsale Flanke) und ihrer Größe ein adäquates Auffüllen mit KM nicht möglich. 2 Stichkanäle zeigten einen minimalen Kontrastmittelübertritt in den Bauchraum. Lernziele Bei fraglicher Eröffnung der Körperhöhlen bei penetrierenden Verletzungen ist eine direkte KM-Gabe in den Stichkanal je nach Lage und Größe möglich und kann für eine chirurgische Exploration oder das folgende Behandlungsregime ausschlaggebend sein. Problematisch sind jedoch sehr große oder ungünstig (dorsal) gelegene Stichverletzungen, da eine adäquate KMAuffüllung nicht möglich ist. Bei kleinen Stichkanälen und zu geringem Druck beim Auffüllen mittels KM darf ein „Kulissenphänomen“ nicht unterschätzt werden, wobei sich nahe gelegene Weichteilstrukturen wieder aneinander legen und zur Demaskierung des Stichkanals führen können. 


\section{Namenverzeichnis}

A

Abdul-Khaliq H $\mathrm{S49}$

Abdullayev N S13, S70, S71, S73, S80

Achterhold K S10

Ackermann $\mathrm{H} \quad \mathrm{S} 49$

Adam G S9, S12, S14, S31, S32, S52, S61, S64 S100, S101, S105, S106, S125

Adams L S66

Afat S S72

Ahmaddy F 587

Ahmadzadehfar $\mathrm{H} \quad \mathrm{S} 44$

Aigner F S128

Aissa J S4, S13, S15, S64, S65, S92, S95, S125

Akin I 596

Al-Bayati M S73, S86

Albers P S106, S107

Albert N S62

Albrecht M S28, S29, S65, S83, S92, S98, S104

Albrecht T S37

Alejandre-Lafont E S27

Alfke $\mathrm{H} \quad \mathrm{S} 61$

Alhonnoro T S107

Alikhani B S8

Allkemper T S21

Allmendinger $\mathrm{T} \quad \mathrm{S93}$

Alparslan Y S86

Alscher D $\quad$ S121

Alt C S101, S105

Altaf N S30

Altmeyer K S24, S49

Aludin S $\mathrm{S} 109$

Amann K S119

Amling M S125

Anastasios M S68

Andrea B S116

Andresen J $\mathrm{S} 65$

Andresen R S45, S65, S110

Annika K S86

Antoch G S4, S13, S15, S55, S62, S64, S65, S71,

S87, S88, S89, S92, S95, S106, S107, S125

Anton S S4, S37

Apfaltrer G S22

Apfaltrer P S22

Appel E S15, S92

Arendt C S23

Arnemann J 559

Arsov C S106, S107

Aschenbach R S35, S36, S40, S110

Attenberger U S17, S102

Auer D $\quad$ S30

Austein F $\mathrm{S} 76$

Auweter S S10, S17, S31, S34

Avanesov M S31, S32

Ayyoub N S56

$$
\text { B }
$$

Baader S S13

Babalik A S124

Bach A S109, S117, S118

Baeßler B S26, S83, S97

Bahls C S125

Ballhausen $\mathrm{H} \quad \mathrm{S} 48$

Baltzer P S58, S127

Bamberg F S17, S31, S34, S84, S107, S115

Bannas P S55, S64, S100, S101

Barabasch A S35, S91

Baraliakos X $S 71$

Bardehle F S7

Barkhausen J S4, S23, S31, S37, S47, S109

Bar-Ness D S18, S24

Barrett T S103

Barth T S70, S126

Bassing T S2

Basten L S49

Bauer R S83
Bäuerle T S10, S119

Bauknecht C S54

Baum P $\quad 567$

Baum T $\quad 572$

Baumann A S23, S91

Baumann S S34

Bäumer P S73, S77

Baumhauer M S70, S126

Baur A $\mathrm{S} 114$

Bayer L S37

Bayerl C $\quad 517$

Becker A 563

Bedke J S107

Beer A S126

Beer M S5, S70, S121, S126

Beeres M S28, S92, S98

Beeskow A S117, S131

Béhé M S61

Behnke S S83

Behr V S11

Behrmann C S15

Beiderwellen K S18, S89

Beimler M S117

Bell K 593

Beller C S27

Bendszus M S53, S73, S77, S111

Bennani-Baiti B S58

Benndorf M S101, S127

Bennett D S83

Benrath 」 $\mathbf{5 8 0}$

Berkefeld J 576

Berkemeyer S S21

Berlin C S23, S31

Berliner C S52, S101

Bernathova M S58, S127

Bertheau R S115

Beseoglu K S81

Bethge O S4, S92

Bettinger D S36

Beyer $F$ S21

Beyer L S115, S118

Beyer S S78

Beyersdorff D S105, S106

Biederer 」 $\mathbf{S 9 0}$

Bier G S9

Bietenbeck M S63

Bippus R $\quad$ S72

Bischoff B S23

Bittersohl B S64, S125

Bitzer M S87

Blasenbrey $T$ S5

Blendl C S94

Bley T S11, S25, S41, S98, S100

Bliemsrieder E S11

Block K S102

Block W S44, S77, S104

Blodow V $\mathrm{S129}$

Blondin D S106, S107

Blum A 599

Bock C 5131

Bock J S23

Bockisch A 589

Böckler D S33

Bodelle B S28, S65, S83, S92

Bogner C $S 90$

Böhm I S53, S120

Bohnen S S31, S32

Böker S S66

Bokhof B S58

Bolzer M S100

Bomberg H S85

Bonekamp D S102, S105

Bongers $\mathrm{M}$ SS

Boos D 545

Boos J S4, S15, S64, S65, S92, S95, S125
Borggrefe 」 S13, S70, S71, S73, S79, S80

Borggrefe M S96

Börm P $\quad$ S75

Borte G $\mathbf{S 1 1 5}$

Both M S61, S95

Böttcher 」 S51

Brand M S93

Brandmaier P S107

Brandts B S25

Braren R S2, S11, S18, S57, S87

Bräsen 」 S14

Brassel F $\quad S 110$

Braun N S121

Braun C S10

Breer S S125

Breinl J S15

Breithecker A S27

Bremer C S21

Bremicker K S63, S116, S130

Brinkmann $S$ S30

Britzen-Laurent N S10

Brocker K S101

Brockmann C 572

Brockmann M S72

Bruckner T S112

Bruhn H S6, S55

Brümmendorf $\mathrm{T} \quad \mathrm{S} 86$

Bruners P S36, S39, S41, S123

Brüning R $\quad S 48$

Brunkhorst T S26

Bruno S 586

Buchbender C S62, S71

Bucher A S56, S65, S83, S92

Buchgeister M S124

Bücker A S5, S24, S46, S49, S85, S93, S97

Budäus L S101, S106

Budjan 」S17, S102

Buerke B S21

Bunck A S6, S26

Burck I S38

Bürckenmeyer $F \quad$ S35

Burmeister, PD. Dr. med. H $\$ 133$

Burmeister $\mathrm{H} \quad \mathrm{S} 38, \mathrm{~S} 43$

Busch M S102

Busse H S8, S106, S107, S120

Byrtus J S3, S35, S36

c

Cadeddu R S13

Callan J $\mathrm{S69}$

Capilli F $\mathbf{S 1 3 0}$

Cario H S121

Carr C S34

Cartes M S8

Caspers J S55, S64, S65, S74, S81

Cavallaro A S2, S119

Ceelen F 596

Celik K S105

Ceyhan G S18, S87

Chakrabarti P $\quad 587$

Chanchek D 567

Chang D S83, S97, S103

Chapiro J 586

Charan A $\mathbf{S 1 0 5}$

Chen M S99

Chen R S14

Cho C S55

Christ P 587

Cieslik E S74

Ciritsis A S35

Clauser P S58, S127

Collettini $\mathrm{F} \quad \mathrm{S} 86$

Cyran C S62

Czerner C S9, 595

Czwikla R S23 


$$
\text { D }
$$

Dabew R S24

Dabir D S27, S28, S98

Daerr H S18, S19, S24

D’Anastasi M S91

Dangelmaier J S2, S24, S81

Danner J 587

Darakananda K S69

Daum C $\mathrm{S95}$

Daum H S38, S43

De Cecco C S28, S33, S34

de Marco F S10

Déan-Ben X S62

Della Seta M S86

Delles M S27

Delorme S S126

Dendl L S16, S37, S84

Denecke T S54

Derlin T S125

Dettmer S S96

Deuschl C S87, S88, S89

Diamantis I S35, S36, S110

Dickmann C S13

Dieckhoff J S9, S14

Dietz C S55

Dietzel F S106, S107

Dietzel M S58

Dillmann R S27

Dinkel J $\mathrm{S96}$

Dipper N S120

Dirrichs T S59, S66

Distler F S105

Do P S84

Do T S113

Dober 」 $\mathrm{S} 130$

Doerner J S26, S28, S97

Dohmen C 579

Döme B S111

Domin M S54

Dorn F $\quad \mathrm{S79}$

Dörner J S3, S36

Douek P S18, S19, S24

Dr. med. Herzog A S124

Dr. med. Nemat S S124

Dr. med. Teifke A S124

Dralle H S15

Dreyling M S90

Drömann D S4, S23, S31

Düber C S40, S42, S116

Duensing S S105

Duguay T S28

Dutschke A S22

Düwel S S11

Duwendag D S61

Dzierma Y S93

E

Eckardt N S40

Eckey T S132

Egenlauf B S99

Ehrlich S S125

Eiberger B S13

Eichhorn L S77

Eichinger M S119

Eichler K S38

Eickhoff S S74

Eigentler T S91

Eisele P $\mathrm{S} 80$

Eisen」 $\mathbf{S} 88$

Eisenblätter M S63

Elger C S82

Elisabeth A S4

Ellmann S S10, S93

Elser S S123

Endrich O S53

Engel A S128

Engelke C S48

Engelke H S86
Engels S S105

Enk A S48

Enkirch J 577

Erfanian Y S32, S66, S88

Erhardt J S4

Ernst L S82

Erpenbach S S46

Ertl-Wagner B S51, S57, S74, S76

Ettlinger $\mathrm{F} \quad \mathrm{S} 87$

Ettrich T S126

Etzel R S94

Exner M S63

Ezziddin S $\mathrm{S} 43$

$\mathrm{F}$

Faber C S63

Fabian H S50

Fabritius M S74, S78, S79

Fahnert J S115, S117, S132

Fakler J S84, S85

Farag M S27

Fard N S113, S126

Farrell C S99

Farschtschi S S73

Faschkami A $\mathbf{S 9 6}$

Fassbender $\mathrm{K} \quad \mathrm{S} 83$

Fedders D $\mathbf{S 1 2 5}$

Feißt A $\mathbf{S 9 8}$

Fellner C S16, S108

Felten A S47

Fenchel M $\mathbf{S 9 0}$

Feuerriegel G S67

Fiebich M S94

Fiedler $\mathrm{E} \quad \mathrm{S} 82$

Fingerle A S2, S10, S18, S19, S81, S90

Fink C S33

Fink G $\quad 579$

Finsterbusch 」 S12

Fischbach F S67

Fischer C S112

Fischer E S14

Fischer F S10, S14

Fischer M S57, S100

Fischer S S27, S47

Fischer T S114

Fischer U S60

Flechsig P S113

Fleck S S75

Foldyna B S24

Förster A S74

Forsting M S3, S25, S86, S89

Franiel T S22

Frank Oliver H S100

Freise J $\mathrm{S} 97$

Freitag M S105

Frenzel T S12

Friebe B $\mathrm{S} 67$

Friedrich B S82, S126

Fries P S24, S46, S49

Friess H S18, S87

Fritz B S127

Fritzer E S61, S95

Fritzsch D S82

Früchtnicht S S129

Frydrychowicz A S4, S23, S31, S109

Fuchs J S116, S130

Führer-Sakel D S89

Fumey W S64

Fürst P S45

\section{G}

Gabmeyer P S6

Galle P S40, S42

Garbe C S91

Gärber I S14

Garmer M S25, S102

Garnov N S8, S106, S107, S120

Garthe T S23
Gassenmaier T S25

Gawlitza J S96, S106

Gawlitza M S82, S124, S126

Gdaniec N S61

Gebauer B S48

Gebrekidan L S11

Geier A S41

Geis C S68

Geisel D S54

Geissler F 599

Gelse K S113

Gerhard A S55

Gersing A S67, S72

Gerullis H S105

Getzin T S10

Giese D S30

Gieseke J S13, S27, S77, S98

Gleich B S10

Glüer C S61

Gockner T S113

Godel T S73, S77

Godenschweger F S67

Goerg F S36

Goessmann H S16, S37, S52

Gold R S77

Goldschmidt H S126

Goltz J S4, S37, S47

Goos P S105

Gordron Y S27

Gottschling S S112

Götz M S102

Götze O S41

Grabe H S41

Gran S S63

Gräter T S16

Greiser A S121

Greling B S110

Gremse F S14

Greulich S S121

Grieb D $\quad$ S110

Grieser T $\quad$ S129

Grim C S113

Grimm R S102

Grinspoon S S24

Groden C S74

Groeninger S S121

Groesdonk H S85

Gromann L S10

Grönemeyer D S25, S102

Groß 」 S109

Große Hokamp N S3, S13, S20, S68, S70, S71, S80, S103

Gross A 537

Grosser A S8

Gross-Fengels, Prof. Dr. med. W S133

Gross-Fengels W S38, S43

Groth M S52

Grothe F $\mathrm{S75}$

Grothe M S54

Grothe S S75

Grözinger G S123

Gruber H $\mathrm{S} 69$

Gruber L S128

Gruber O S73

Gruber T S38

Gruber-Rouh T S23, S48

Grüneisen 」 S66, S86, S87

Grüner B S16

Grünig E S99

Guberina N $\mathbf{S 2 5}$

Gueler F S14

Guenette G S20

Gündel D S120

Günther M S115

Günther R S15

Gutberlet M S10, S14, S97

Güttler F S4, S6 
$\mathrm{H}$

Haack M S57

Haas H S62

Haase R S53

Haberland U S72, S90

Hacker U S8

Hadaschik B S102, S105

Hadizadeh D S11, S46, S77

Hahn F S101

Haimerl M S16, S21, S108

Hallscheidt P S101

Hamm B S15, S66, S86

Hamm N S66

Hammerstingl R S48

Hammon M S93

Hampel F S101

Haneder S S3, S30, S80, S103

Hansen N S103

Harth M S38

Harth S S131

Hasembank S S53

Haslerud T S43

Hatopp A S46

Hattingen E S13, S77, S82

Hau P S77

Haubenreisser H S7

Haubold J S3, S68

Hauger M S3

Hausmann D S17, S80, S102

Hautzel H S62

Havel M S57

Havla L S78, S79

Heber S S17

Hebestreit H S98, S100

Hedderich D S69, S75

Heermann P S26

Hegedüs B S111

Hegenbart U S126

Heidelbach M S129

Heidinger O S60

Heidrich J $\mathbf{S 6 0}$

Heil A S111

Heiland S S77, S111

Heilmeier U S69

Heim 」 5113

Heindel W S21, S26, S58, S60, S63, S94

Heinemann V S91

Heinrich A S4, S6

Heinzel A S35

Heinzler N S4, S92, S95

Heiß R S17, S34, S113

Helbich T S58, S111, S127

Hellbach K S10

Hellerbrand C S89

Hellmich M S103

Helmchen C S132

Henes F $\$ 95$

Henes J S121

Henes O S55

Heneweer C S3, S61

Hennemuth A S109

Henning P S38

Henninger B S121

Hense $\mathrm{H}$ S60

Hensen B S8, S47

Henzler C S52

Henzler T S7, S96

Herden J S103

Herold T S118

Herrmann J S52

Herrmann K S18, S53, S66, S87, S89

Herz S S11, S41

Herzen J S10

Hesper T S125

Hesse N S91, S96

Hetterich H S17, S31, S34

Hetzheim H S90

Heusch P S13, S15, S62, S88, S89
Heussel C $\$ 99$

Heverhagen 」S53, S120

Hickethier T S3, S6, S26, S83, S97

Hildner K S10

Hilgenfeld T S111

Hillengass 」 $\mathbf{S 1 2 6}$

Hinrichs J S20, S26, S42

Hirsch F S131

Hirsch 」 $\$ 115$

Hobohm C S126

Hoeper M S42

Hoepker M S26

Hoffmann K S15, S82, S84, S126, S132

Hoffmann R S44

Hoffmann U S24

Hoffstaedter F $\mathrm{S74}$

Hofmann F S69, S91

Hofmann M S61

Hofmockel T S44

Hohenfellner M S105

Höhn A S15, S63, S124

Höhne C S74, S78

Höhne M S118

Holle J 595

Holl-Ulrich K S61

Holl-Wieden A S50

Holwein D S67

Homsi R S27, S28, S98

Hopf-Jensen S S38, S39, S75

Hoppel B S20, S99

Horger M S9, S87, S90

Horn L S106

Horn M S47

Hornung M S127

Hosten N S41, S129

Hotfiel T S113

Houbois C S103

Huber A S29

Huber C S10

Huber T S78

Hubois C S3

Hudak A S8

Hueper K S10, S14

Huhndorf M S76

Hüllebrand M S109

Humbert J S61

Hundshammer C S11

Hundt C S9

Hundt W S39, S61

Hungerbühler M S120

Hunink M S78

Hunold P S31$$
\text { I }
$$

Imhoff M S14

Imhoff $\mathrm{P}$ S67

Immenschuh S S14

Ingrisch M S76

Isfort P S14, S39, S41, S123

Ittermann T S41, S54

Ittrich H S9, S12, S14, S61

I

Jacob F S4

Jagoda P S5, S93, S97

Jakob P $\$ 119$

Jan B 568

Janka R S119

Jansen O S61, S76, S79, S95, S109

Jaschke W S121

Jenes J 563

jilg C S101

Jobst B S119

Jonas S S86

Joseph D $\mathrm{S67}$

Joseph G S69

Josip E 569

Jost G S11
Josten C S84, S85

Jung C S9, S12, S14, S61

Jung E S114, S115, S127

Jungblut L S62

Jungmann P S67

Junker D $\quad$ S128

Jurcoane A S82

K

Kabbasch C S73, S79

Kachel P S15

Kaesemann P $\quad$ S121

Kaesmacher J S75

Kahn 」 S54

Kahn T S8, S56, S84, S85, S106, S107, S112,

S115, S116, S120, S124, S130, S131

Kaireit T S9, S97

Kaissis G S11, S12, S18, S57, S87

Kalkan A $\$ 79$

Kallenbach K S27, S81

Kaltenbach B S39

Kammerer F $\mathrm{S93}$

Kammerer S S21, S76

Kampf T S11

Kamusella P S110, S116, S129

Kandel S S99

Kannengiesser S S121

Kapetas P S58, S127

Karampinos P S67

Karck M S81

Karmonik C S33

Karpienski J S102

Kartal R S108

Kastner C S103

Katenkamp K S22

Katoh M S85

Kauczor H S27, S33, S81, S90, S99, S112, S113 S115, S119

Kaufmann S S87, S90

Kaul D $S 86$

Kaul M S9, S12, S14, S61

Kaup M S65

Kazmierczak P S62

Keil B S94

Keil S S86

Keil V S11, S13, S77

Keim D $\quad$ S11

Keller P S53

Keller T S49

Kemmling A S132

Kentouche K S53

Kesch C S105

Kestler C S98

Ketelsen D $\mathbf{S 9 0}$

Kettenbach 」 S40, S131

Keulers A S59

Khil L S60

Kickingereder $\mathrm{P} \quad \mathrm{S} 53$

Kickuth R S41

Kiefer B S90

Kieslich P S53

Kildal D S5

Kim T S111

Kimm M S62

Kirchner J S62, S66, S71, S87, S88

Kirchner U S101

Kirschke J S69, S72, S81

Kirschke P S67

Kitterer D S121

Klapp Oliger M S46

Klapsch F S17

Klaus T S27

Klee D S55

Kleemann M S37

Kleinbach S S41

Kleine J $\mathrm{S75}$

Klein-Wiele O S25

Kloeckner R S8, S40, S42 
Kloth C S87, S90

Klumb C S40

Klumpp B S91

Klutmann S S52, S101

Knapp J $\mathbf{S 1 0 0}$

Knaudt 」 S58

Kneidinger $\mathrm{N}$ S96

Knopp T S9, S12, S14, S61

Knörgen M S120

Kobayashi Y S101

Koch S S14, S40, S42, S48

Koch-Nolte F S64

Koehler T S10

Koerdt S S57

Kohle C S65

Köhler M S21

Köhler W S125

Köhnen J S99

Kolb M S19, S22

König A S94

Konstandin S S80

Koo B S103

Kooi M S30

Kopp F S10, S72

Kopp M S93

Körber F S49

Körner H S83

Kornetzky N S6

Kornmann M S126

Körsmeier K S68

Kösling S S112

Kosmala A S25

Köstler H S50, S98, S100

Kotelis D S33

Kotter E S127

Kottlors J 573

Kovacevic D S7, S108

Kowald T S105

Kraemer B S73

Kraff O S68

Kräling T S94

Kramer M $\mathrm{S93}$

Krämer N S35, S91

Krämer P S2

Kratzer W S16

Kraus C S83

Krauss T S130

Kremp K S49

Kremser C S121

Krenn T S49

Kretzschmar M S69

Kribs A S49

Kriner F S10

Kroeger J S26, S97

Kröger 」 S6

Krombach G S7, S27, S108, S113, S131

Kromrey M S41, S54

Kröncke T S45

Krönig M S101

Kronlage M S77

Kropf S S67

Kröpil P S4, S13, S15, S45, S65, S92, S95

Krüger P S111

Krumbein I S53

Krumm P S63

Krupp J S100

Kuetting D S27

Kuhl C S35, S36, S39, S41, S50, S59, S66, S70,

S71, S72, S86, S91, S123

Kühn J S41, S54, S129

Kukuk G S44, S89, S104

Kümpel S S51

Kunick V S64

Künstner T S107

Kunz A S25, S98, S100

Kunz J S11, S41

Kunz W S62, S74, S78, S79

Kurz P S46
Kütting D S28, S98

L

la Fougere C S88

la Fougère $C \quad S 63$

Lampe N S44

Landgraeber S S68

Lange A $\$ 111$

Langenbach M S48

Langer M S101, S127, S130

Langer $\mathrm{V}$ S10

Langhorst J S18

Langner S S54, S75, S111

Laniado M S44

Lanzman P S45

Lanzman R S13

Laszlo V S111

Latus J S121

Laukamp K S13, S68, S70

Lazik-Palm A $\mathrm{S} 68$

Leberer L S38

Legler K S24

Lehmann T S22

Lehmkuhl L S36

Lehrenfeld C S25

Lehrke D S75

Leicht J S131

Leidenberger $\mathrm{T} \quad \mathrm{S} 27$

Leifels L S56, S117, S131

Leifheit J $\$ 113$

Leißner M S39

Leithner D S23, S28, S29, S83, S98, S104

Leitner E S16

Lell M S93

Lenga L S28, S29, S98, S104

Lennartz S S13, S70

Lenz P S101

Lenzen H S20, S94

Leone $\mathrm{F} \quad \mathrm{S} 58$

Leonhard G 569

Lethaus B S56

Li Y S18

Liebig T S54, S79

Liebl M S39, S41, S123

Liedtke K S54

Limburg R S7, S108

Lin $\mathrm{H} \quad \mathrm{S} 62$

Linder N S8, S106, S120

Link P S67

Link T S69

Lisson C S70

Liu F $\$ 69$

Liu S 517

Ljimani A S13

Lo J S24

Loberg C S56

Lobsien D S82, S126, S132

Loewe C S22

Lohöfer F S18, S57, S62

Lohse A $\mathrm{S75}$

Loizides A S69

Loose DA $\$ 110$

Lorbeer R S17, S31, S34

Lorch H S6

Lotz J $\mathrm{S60}$

Löwe T S93

Lu M S24

Lück C S115

Lüdtke C S110

Luedtke C \$45

Luerken L S18

Luetkens J S27, S28, S46

Lund G S31, S32

Lürken L S43

Lurz M S17

Lütje S S71

Lynch J S69
M

Maack H S10

Machann J S17

Macher-Göppinger S S113

Madjidyar J $\mathrm{S79}$

Mädler B S77, S82

Madyaningrana K S14

Maegerlein C S69

Maehringer-Kunz A S42

Mahnken A S32, S39, S94

Mahrholdt H S121

Mähringer-Kunz A S40

Maier-Hein K S76, S81, S102

Maintz D S3, S6, S13, S20, S26, S30, S36, S68 S70, S71, S73, S80, S83, S97, S103

Mainz D $\$ 103$

Maiwald B S84, S116, S130

Makowski M S66

Malich A S7, S108, S118

Mall M S119

Mallouhi A S116

Malouhi A S6, S42

Malzahn U S100

Mammadov K S83, S103

Manns M S20

Mao K S20

Marik W S116

Marques L S39

Marticorena Garcia S S114

Martin S S28, S29, S92, S98, S104

Martirosian P S19, S22, S107

Marx C S11, S27, S104

Maschke S S42

Maschke T S44

Maslova M S47

Maßmann A $\$ 46$

Masood K S128

Massmann A $\$ 97$

Masthoff M S63

Mateiescu S S102

Mathys C S74, S81

Matuschek E S44

Maurer K S43

Maurer M S84

Maus V S73, S79

Mautner V S73

Maxeiner A S114

May M S10, S17, S34, S93, S99

May R S81

Mayer-Steinacker R S70

Mayr A S121

Mbapte Wamba J 569

McCulloch C S69

McCulloch P S67

MD Partovi S S33

Mehler K S49

Mehrmann M S46

Mei K S72

Meier M S14

Meier R S62, S70, S84

Meier-Schroers M S27, S98, S104

Meila D $\quad$ S110

Meinel F $\mathrm{S78}$

Meiser N S50

Melber K S110

Melzig C S99

Menke J $\$ 122$

Mensel B S41, S129

Mentzel H S50, S51, S53

Menze B S87

Merkt M S103

Messerli M S81

Mester J S101

Metz F S25

Mey Lien M S7

Meyer B S26, S42

Meyer C S43, S44, S46, S89

Meyer H S15, S63, S82, S109, S124 
Meyer M S7

Meyer P S61

Meywald-Walter K S100

Michel S S120

Michels G \$26

Michels J 596

Mikat C S32

Mildenberger $\mathrm{P}$ S8

Minko P S5, S85, S93

Mirza M S73

Moche M S106, S107, S116, S130

Modabber A S56

Mohamed N S38

Mohammad Q S7

Möhlhenrich S S56

Mohr 」 S10

Molwitz I S12

Moody A S30

Moritz-Tugral R S113

Morris E S58

Mosebach 」 S126

Mpotsaris A S13, S71, S73, S79, S80

Mucha S S106

Muecke T S57

Muenzel D S81

Mühl-Benninghaus R S83

Müller A S61, S77, S88

Müller F S50

Müller M S72

Müller-Eschner M S27, S33, S76

Müller-Hülsbeck P S38, S39, S75

Müllerleile K S31, S32

Müller-Lutz A S64, S125

Müller-Schimpfle M S60

Müller-Wille P S122

Müller-Wille R S43, S52, S118

Mummert T S9

Münch 」 S31

Münzel D S2, S10, S18, S19, S24

Mürtz P S44, S89

$$
\text { N }
$$

Naas O S81

Nadine Tischer N S61

Nadjiri 」 S29

Naehle C S28

Nagel A S2

Naguib N S49

Nairz K S53

Nance Jr. J S28

Nassenstein K S32

Natho 0 S43

Nebelung S S70, S71, S72

Neff K S52

Negrao de Figueiredo G S76

Nell C S129

Nensa F S3, S18

Neubauer H S50

Neubauer 」 $\$ 127$

Neuhaus $V$ S13, S68, S70, S71, S73, S80

Neumann A $\mathrm{S} 132$

Neumann」 $\mathbf{S 6 9}$

Nevitt M S69

Nevitt P S67

Nickel D 516

Nickel M S19, S22

Nicolas V S117

Niessen C S16

Nikolaou K S9, S17, S19, S22, S31, S63, S72, S84,

S87, S88, S90, S91, S107, S123

Nikoubashman O S72

Nitsch W S93

Noel P S18, S19, S81

Noël P S2, S10, S18, S24, S57, S72

Norajitra T S81

Nörenberg D S76, S90

Notohamiprodjo M S19, S22, S84, S107

Nour-Eldin N S39, S49
Nowak J S51

Nunez 」 S33

Nuran A $\mathbf{S 6 8}$

O

Obert M S7, S108

Oberthuer A $\$ 49$

Oechtering T S4, S23, S31, S109

Oelers C S107

Olsson K S26, S42

Olthof S S63, S88

Oltmann Schröder J S61

Ong M S80

Opitz S S16

Orlikowsky T S50

Otani K S33, S34

Othman A S84

Othman A S19, S22, S72, S91, S107

Otto S S111

Pahn G S6, S20, S26

Panagiotopoulos N S47

Panek A S50

Papageorgiou I S7, S108, S118

Partecke L S129

Partovi S S99

Patrascu A S121

Patten-Hamel M S31

Pätzholz S S132

Paulo T 5105

PD Dr. med. Kinner S S124

PD Dr. med. Surov A S124

Pedersoli F S39, S123

Peldschus K S12, S125

Penzlin S S67

Pereira P S89, S113

Persigehl T S20

Petermann, Dr. med. C $\$ 133$

Peters A S17, S31, S34

Petersen T S84, S116, S130

Petritsch B S25

Pfannenberg C S63, S88

Pfeifer 」 $\$ 49$

Pfeiffer F S10, S18, S19, S24

Pfeil A S51

Pfister K S43

Pfister P $\quad \mathrm{S} 122$

Philipp D S11

Pieper C S29, S30, S43, S44, S89

Pietsch H S11

Pietsch T S77

Pineda Vidal L S79

Pinker K S58, S127

Pino-Lopez L S74

Pinto dos Santos D S8

Pitarokoili K S77

Pitton M S40, S42, S116

Plaikner M S69

Planert M S47

Platz Batista da Silva N S118, S127

Plontke S S112

Plum J $\mathrm{S} 96$

Poeppel T S89

Pollari M S107

Polzer P S58

Pomschar A 557

Pönisch W S115

Pöppel T S66

Poppert H S30

Posch H S54

Poschenrieder F S37

Post M S70

Prael I S99

Pralow T S10

Predel D S118

Pregler B S16

Preibsch H $\mathrm{S} 90$
Preiß M S38, S39, S75

Prenzel F S131

Prof. Dr. med. Heindel W S124

Prof. Dr. med. Müller-Schimpfle M S124

Prof. Dr. med, Nicolas V S132, S133

Prof. Dr. med. Schäfer F S124

Prokop M S99

Proksa R S10, S18, S19, S24

Proquitté H S50

Prümmer M S119

Purz S S56

Püsken M S103

O

Quack V S66

Quadrat A S41

Quäschling U S82, S115

Quentin M S106, S107

Quick H S71

$\mathrm{R}$

Raatschen H S8

Rabenalt R S106, S107

Radbruch A S53

Rademacher C S55

Rademacher 」 $\mathbf{S 9 6}$

Radmer S S65

Radosa C S44

Radosa J $\mathrm{S44}$

Radtke J S101, S105

Radunksi U S32

Radunski U S31

Rahne T S112

Rakete T S120

Rasper M S29

Rathmann E S75

Rathmann W S31

Rattansingh A S20

Rau R S20, S71

Rauch M S11, S104

Rausch V S55

Raviolo M S82, S126

Razansky D $\mathrm{S} 62$

Regier M S52

Reh C S77

Reichardt B S117, S123, S132, S133

Reinartz S S14

Reischl G S88

Reiser M S10, S17, S23, S30, S34, S51, S57, S62, S74, S76, S78, S79, S90, S91, S96, S100

Reith W S83

Renger B S10

Rengier F S27, S33, S81, S99

Renz D S50, S51

Renz M S2, S10, S81

Rheinwald M S57

Richter G S46

Richter M S67

Ricke J S67

Rieder C $\mathbf{S 4 8}$

Riederer I S2, S81

Riffel P S17, S102

Rindt K S10

Ringe K S20, S47, S48

Ringelstein A S3, S25

Ringl H S22

Ringshausen F S96

Roessl E S18

Roest M S20

Roethke M S105

Rogalla P S20, S99

Rohr K S99

Roland D $\quad$ S100

Roll W S21

Roller F S27, S131

Roman A S39

Romeo F $\mathrm{S} 121$

Rominger A S62 
Rossi A S51

Rössle M S36

Rösslhuemer P S128

Roth C S131

Roth J S63

Roth W S105

Rottenbach C S6

Rubbert C S74, S81

Rüber T S82

Rückert M S11

Rückner D S133

Rüdiger S S131

Rudolph B S15

Ruff C S19, S22

Ruhlmann V S71, S86, S87, S88, S89

Ruhnke H S45

Ruhparwar A S33

Ruile P $\mathrm{S130}$

Rummeny E S2, S10, S11, S18, S19, S24, S29, S57, S72, S81, S87, S90

Rummeny P S67

Ruprecht N S120

Rusch R S109

Rütters M S111

$S$

Saam T S30

Sabet A 543

Sachs A $\mathrm{S} 128$

Salamon J S9, S12, S14, S61

Salem 」 S103

Salomon G S105, S106

Salzberger B S115

Sandmair M S119

Säring D S32

Sauer A $\quad$ S50

Sauer M S100, S105, S106

Saur J 596

Sauter A S2, S81, S90

Sawicki L S15, S62, S64, S65, S71, S86, S87, S88, S89, S95, S125

Schaarschmidt B S45, S62, S125

Schabel C S9

Schad L S80

Schaefer A S47

Schäfer P S109

Schäfer S S41

Schäfers H S85

Schaible T S52

Schaper 」 S55

Scharfschwerdt M S109

Schaudinn A S8, S82, S106, S120, S126

Scheck J S39

Scheef L S77

Scheer F S45, S110

Scheller B S24

Scherer K S10

Scherer M S16, S127

Schicho A S89

Schievelkamp A 582

Schild H S11, S13, S27, S28, S30, S43, S44, S46, S77, S82, S89, S98, S104

Schilling F S11

Schimmöller L S106, S107

Schindler A S30

Schindler C S10

Schindler R S23

Schinner R S30

Schirren R S87

Schleder S S18, S84, S114

Schlehe B S101

Schleich C S64, S125

Schleicher C S6

Schlemmer H S53, S73, S90, S102, S105, S126

Schlesinger-Irsch U S28

Schlett C S17, S31, S34, S46, S113, S115

Schlichtenbrede F $\mathrm{S74}$

Schlunz-Hendann M S110
Schmaltz A S106

Schmeel C S104

Schmeel F S11, S28, S46, S104

Schmeel L S46

Schmehl J $\$ 123$

Schmidbauer M S10, S14

Schmidmaier G $\mathbf{S 1 1 2}$

Schmidt A S80

Schmidt B $\$ 93$

Schmidt C S15

Schmidt F S111

Schmidt S S5, S70, S121, S126

Schmidtmann I S40, S42

Schmiedberger 」 S16

Schmitz D S5

Schmitz T S110

Schnackenburg B S32

Schnapauff D S15

Schneider C S27, S77, S131

Schneider G 597

Schneider H S59

Schneider M S76

Schnitzbauer M S122

Schnitzler A S74

Schnorr J S15

Schob S S15, S63, S82, S112

Schober H S65

Schoenberg S S7, S52

Schoepf J S28

Schoepf U S33, S34

Scholtz J S28, S29, S65, S83, S92, S98

Scholz A S34

Scholz」 S104

Schönberg S S17, S80, S96, S102

Schönland S S126

Schöppe F S51, S57, S90

Schorn F S54

Schotten S S116

Schrading S S50, S59, S66

Schramm D S109, S118, S130

Schramm P $\mathrm{S} 132$

Schreyer A S16, S18, S37, S84, S114

Schriewer L S64

Schröder W S30

Schroeder T S13

Schröter T S10

Schüer M S85

Schuler F S74, S78, S79

Schülke C S21, S26

Schultheiss M S70

Schultz V S109

Schulze J S20

Schulze L S41

Schulze-Hagen M S39, S41, S123

Schulz-Menger 」 S31, S118

Schulz-Wendtland R S60

Schumann C S48

Schütze K S64

Schwabe J S114

Schwaiger B S2, S72

Schwaiger D S67

Schwaiger M S11

Schwarz D 577

Schwarz F $\$ 23$

Schwarz M S57

Schwehr V S77

Schwenzer N S63

Sechtem U S121

Sedlaczek O S90

Segerer F S98, S100

Seidel R S24, S46, S49

Seider D S107

Seith F S88

Seufferlein T S16

Seuß H $\$ 119$

Seyfer P S32

Shah S $\$ 126$

Shin H S9, S96
Siedek F S3, S30

Siemens P S38, S43

Sieren M S23, S31, S109

Sievers H S109

Sigl B S74

Simgen A S83

Simon B $\$ 46$

Simon L S68

Sinn M S32

Skalla E S69

Slebocki K S20, S103

Sohn C S101

Sohns 」 S26

Sokolowski P S125

Solty K S120

Sommer A $\mathrm{S94}$

Sommer C S46, S113

Sommer N S57

Sommer W S57, S74, S78, S79, S90, S91, S96

Sorantin E S22

Sorokin L S63

Soschynski M S101, S130

Speck O S67

Spielmann R $\mathrm{S} 82$

Sprengel S S115

Sprinkart A S27, S28, S44, S89, S104

Sprinkart M S77, S104

Stab D $\mathbf{S 1 0 0}$

Stackelberg O S115

Stahl M S119

Stahlberg E S37, S47

Stahnke V S60

Stanescu N S121

Stanescu-Siegmund N S126

Stange R S8, S120

Starekova 」 S32

Stáreková J S31

Stärke C S67

Stechele M S15

Steer S S118

Steffen I S54

Stehning C S32

Steiger K S11

Steinacker J S126

Steinbach S S39

Steinkohl F S128

Stiglbauer A $\quad$ S111

Stock K S131

Stock S S131

Stocker G S8

Stöcklein S S51

Stoffels M S52

Stolzenburg 」 S106

Stolzenburg N S15

Storz C S17, S31

Sträter A S29

Streitparth F S15, S51

Strobel D S113

Strobel K S59

Ströder 」 $\mathbf{5 8 5}$

Stroeder J S24, S97

Strölin P $\$ 106$

Stroszczynski C S16, S18, S21, S37, S43, S84, S89, S108, S114, S115, S118

Stroszczynski P $\quad$ S122

Stumpp P S56, S84, S85, S124

Stürzl M $\$ 10$

Suchorska B S76

Suchowierski A S67

Südmeyer M S74

Suntharalingam S S32, S87

Surov A S15, S56, S63, S82, S109, S112, S117. S124, S131

Sutton E S58

Svatko Z $\$ 120$

Swargulski P $\mathrm{S} 61$

Swoboda B S113

Syed A S128 
Szolar D S22

$$
\text { T }
$$

Tahir E S31, S32

Taron J S19, S22, S107

Tealip S S110

Teber D S105

Teichgräber U S4, S6, S7, S22, S35, S36, S40, S51, S55, S108, S110, S118

Tennstedt P $\mathrm{S} 105$

Tesche C S34

Teschner E S14

Teusch V S37

Thaiss W S87, S90

Thäns N S23

Theis R $\quad$ S119

Thews O $\mathrm{S} 120$

Theysohn J $\mathrm{S} 68$

Thierfelder K S74, S78, S79, S91

Thomas C S4, S15, S65, S92, S95

Thomas D S27, S28, S44, S98

Thomas K S46

Thomas P $\mathbf{S 4 5}$

Thon A S108

Thüring J $\$ 91$

Tibussek D S55

Tienken M S101

Titschert A $\$ 47$

Tiwari S S61

Tjiong R S32

Tolba R S14

Tombach B S21

Tonn J $\mathrm{S76}$

Topping G S11

Träber F S46, S77, S104

Trebicka J S36

Trentmann J S109

Treppels-Kottek S S50

Trinkmann F S96

Triphan S $\$ 119$

Tritt S S76

Trojan M S33

Trommer 」 S28

Truhn D S70, S71, S72

Trumm C S23

Tschischka A S64, S125

Turowski B S55, S74, S81

$$
\text { U }
$$

Uder M S10, S17, S34, S60, S93, S99, S119

Uller W S37, S52

Ullich T S106, S107

Umutlu L S18, S66, S71, S86, S87, S88, S89

Unger M S83

Utpatel K S21, S108

$$
\text { V }
$$

van der Heijden $\mathrm{H} \quad$ S10

Vasyukov M S13

Vatankhah D S38

Vecchio $F \quad S 121$

Veldhoen S S98, S100

Verbeek M S90

Verloh N S16, S21, S43, S108

\section{Vijith $\mathrm{V}$ S14}

Vliegenthart R S33

Vo Chieu V S47, S48

Vogel P S11

Vogel-Claussen J S9, S95, S96, S97

Vogl T S23, S28, S29, S33, S34, S38, S39, S48. S49, S65, S83, S92, S98, S104

Vogler N S7

Vogt F S4

Voigt P S15, S112

Voigt W S46

Vollherbst D S113

von Baer A S70

von Bestenbostel M S8

von Fintel E S60

von Stritzky A S31

von Tengg-Koblink H S33

Voskrebenzev A S97

$$
\text { W }
$$

Wachsmuth L S63

Wacker F S8, S9, S10, S14, S20, S26, S42, S47, S48, S95, S96, S97

Wagenhofer K S38, S43

Wagenpfeil S S85

Waginger M S51

Wagner A $\mathrm{S} 118$

Walter S S83

Wang L S14

Warmuth-Metz M S77

Warren A S103

Wawroschek F S105

Weber M S112

Weber W S47

Weberling L S53

Wechtenbruch J S57

Weckbach S S115

Weichert W S11, S18, S57, S87

Weigel S S58, S60

Weinmann A S40, S42

Weinrich J S31, S100, S105

Weis C S27

Weiß 」 S22, S84, S107

Weis M S52

Weiss \ $\$ 19$

Weiss K S30

Weiss N S44

Well L S55

Welte T S96, S97

Wendt S S4

Weng A S98, S100

Wengenroth L S49

Wenger N S33

Wenz H S74

Werncke T S26, S47

Wester H S101

Westphalen C S90

Wetter A S86

Wichmann J S23, S28, S29, S33, S34, S83, S98,

$$
\text { S104 }
$$

Wick W S53

Wickenhauser C S15

Wieberneit N S10

Wieczorek K S102, S105
Wiedner M S37

Wiegand S S39

Wielpütz M S119

Wienbeck S S60, S124

Wieners B S13

Wienke A S82

Wiesenfarth M S102

Wiesinger I $\mathrm{S} 115$

Wiesmann M S72

Wiesmüller M S17, S34

Wiggermann P S16, S21, S89, S108, S110, S115, S127

Wildgruber M S52, S62, S63

Wildner D S113

Wilhelm K S44, S127

Willer K S10

Willinek W S11, S44, S48, S104

Winter A S105

Winter K S23, S91

Winther H S9, S95

Wintzler S S108

Winzler S S118

Wirth C S98, S100

Wirth S $\$ 100$

Wissgott C S45, S110

Wittgenstein $\mathrm{H} \quad \mathrm{S} 15$

Wohlgemuth P S122

Wohlgemuth W S43, S52, S89

Woitek R S58, S127

Wolter K S104

Wörz S S99

Wurschi G S53

Wurzinger E S22

Wüst W S17, S34, S93, S99

Wutschke M S22

Wybranski C S3

X

Xenitidis T S63

Yaroshenko A S10

Yilmaz U S83

Yoon M S77

You S S79

Z

Zapf A $\mathbf{S 9 5}$

Zarnowski J S67

Zausig N S115

Zhang $X \quad S 63$

Zhao Q S58

Zhong $X \quad S 121$

Ziegenhain $\mathrm{F} S 93$

Zilles D $\mathrm{S73}$

Zimmer C S69, S75

Zimmermann M S39, S41, S123

Zinsser D S84, S91

Zips D S88

Zirkler E S112

Zitzelsberger T S34

Zoller H S121 